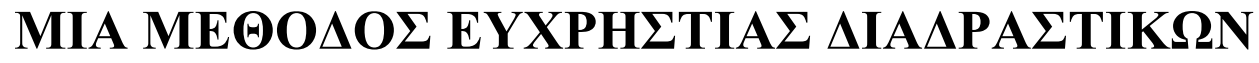

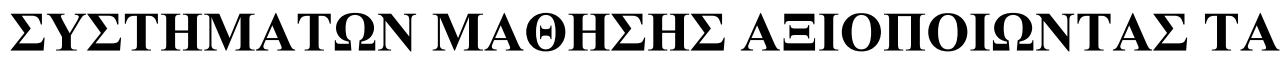 $\Sigma$ XE $\Delta$ IA $\Sigma$ TIKA XNAPIA
}

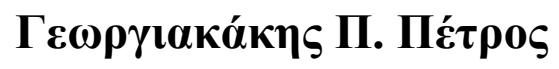

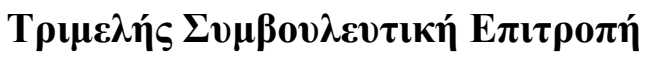

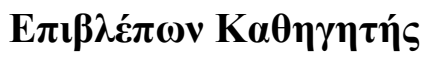

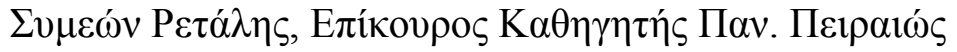

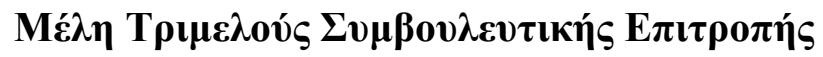

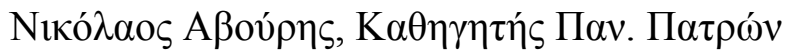

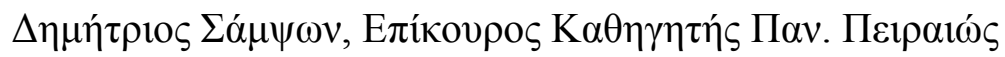


A 


\section{Пєрі́ $\eta \psi \eta$}

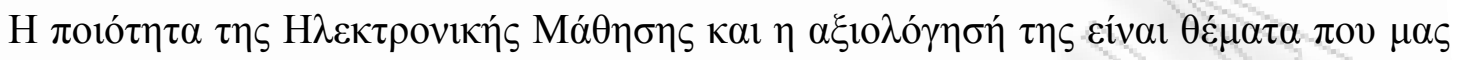

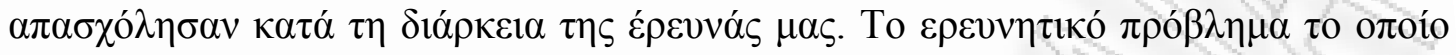

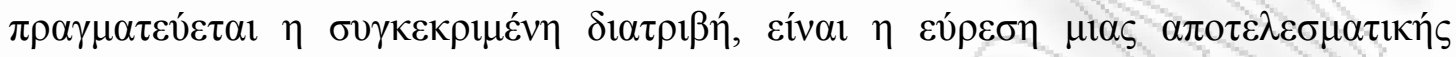

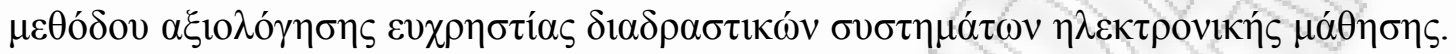

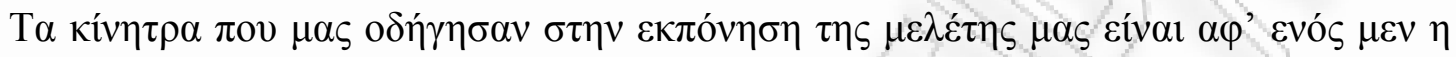

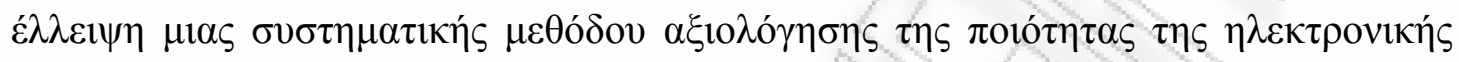

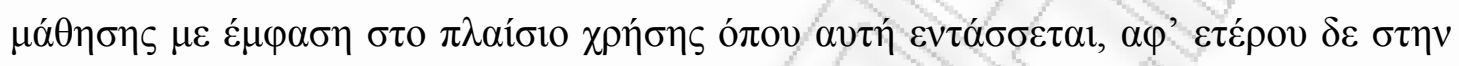

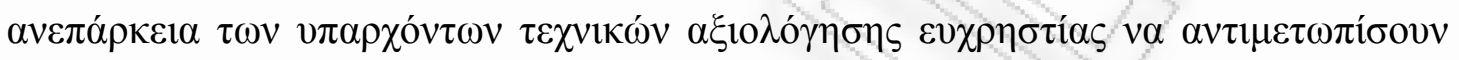

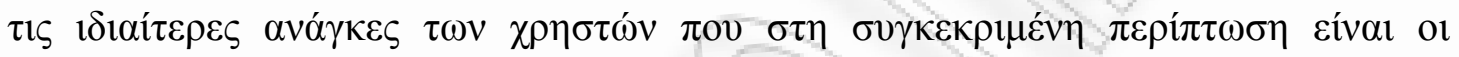
$\varepsilon \kappa \pi \alpha 1 \delta \varepsilon v o ́ \mu \varepsilon v o 1$.

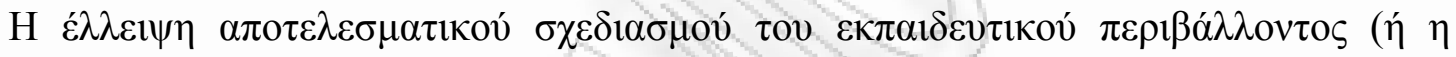

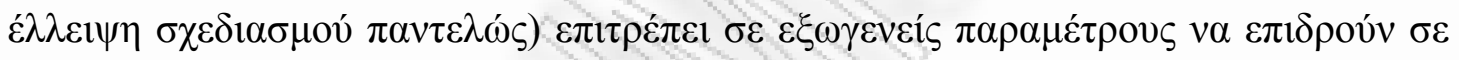

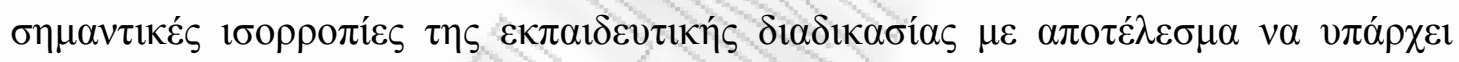

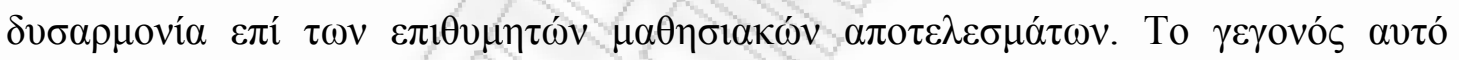

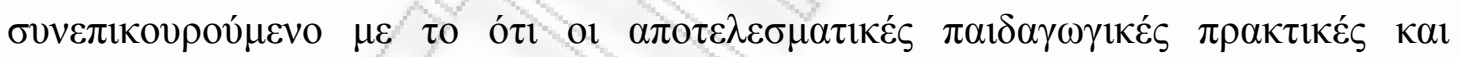

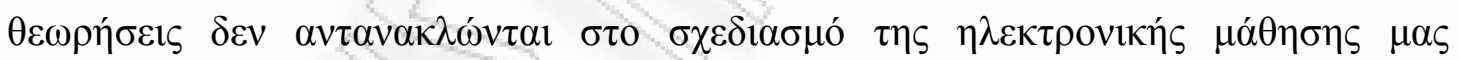

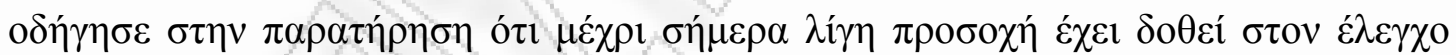

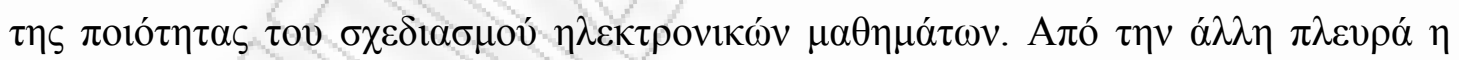

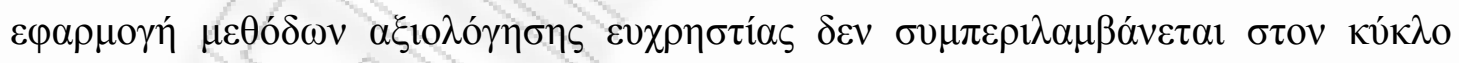

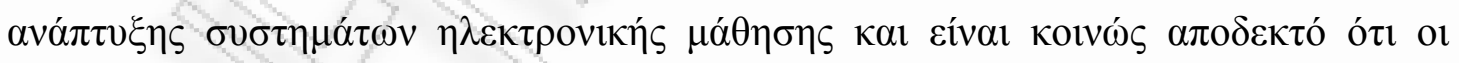

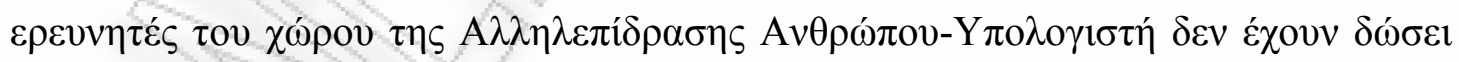

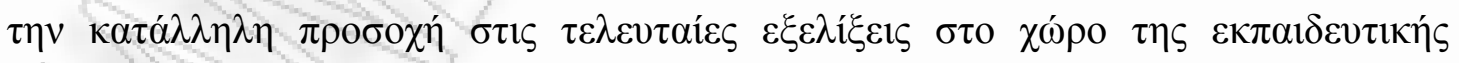

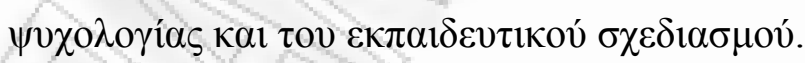

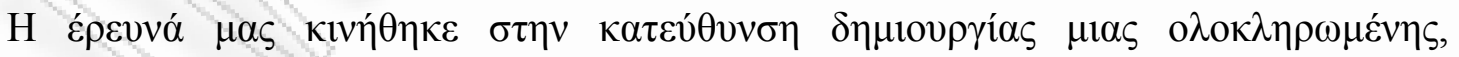

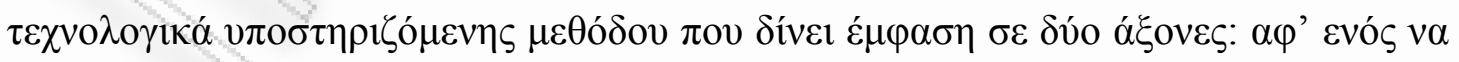

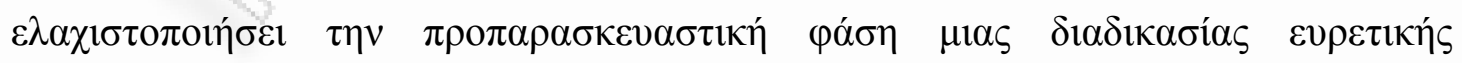

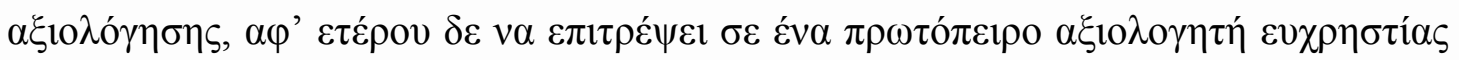

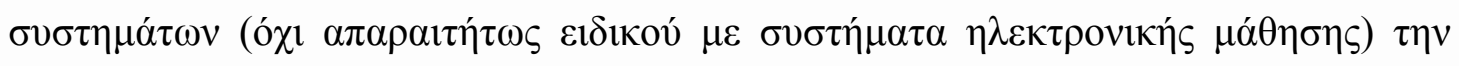

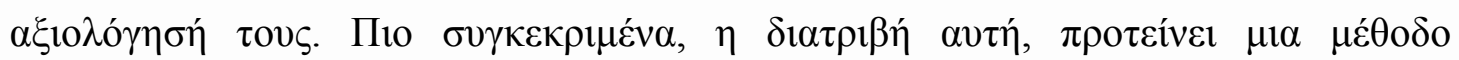




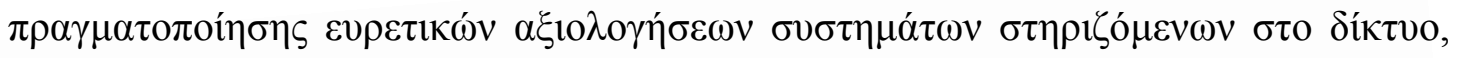

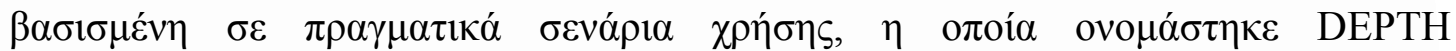

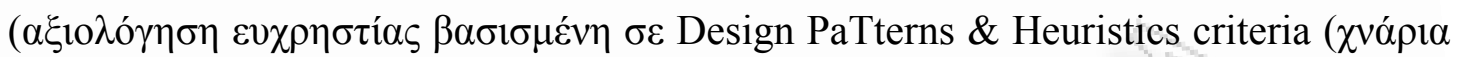

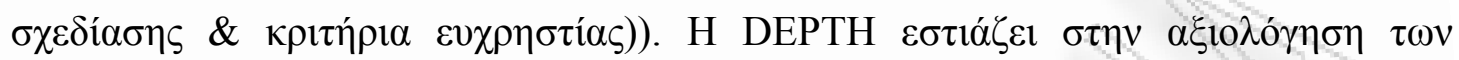

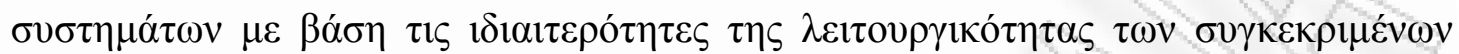

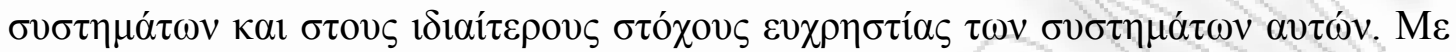

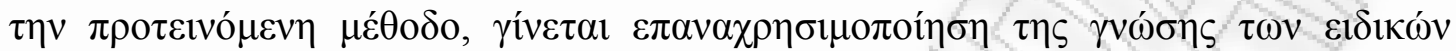

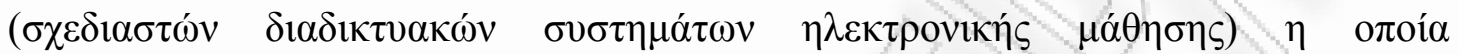

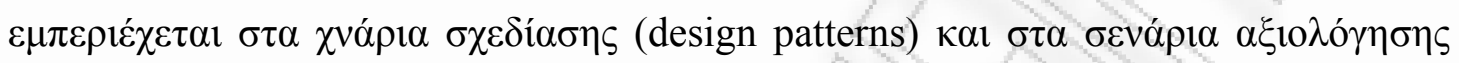

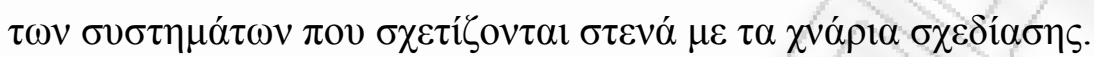

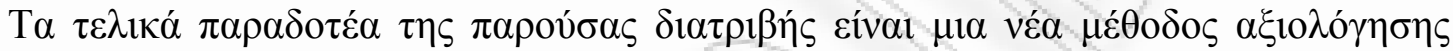

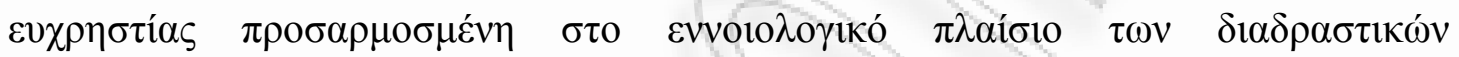

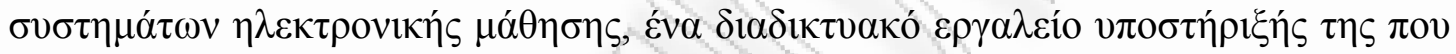

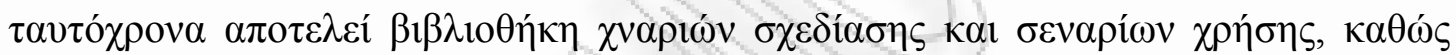

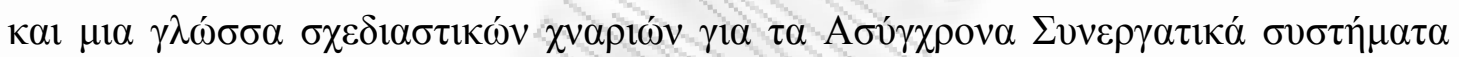

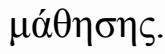




\begin{abstract}
In the area of education there are a great number of e-learning systems available today, both commercial and open source, providing various integrated services. However, the varying needs of instructors and institutions along with the plethora of the systems available today, create a need for investigating their potential and appropriateness in the context of pedagogy, open learning and instructional design. The educational community has much to learn regarding how effectively and in what way e-learning systems can enhance the instructional process. One of the most critical factors towards this direction is the identification of their affordances in conjunction to their usability in the specific e-learning context. Quality of e-learning systems has been questioned and this has been attributed mainly to the lack of attention to their design using user-centered/learner-centered approaches.

A lot of evaluation studies and tools of various genres of e-learning systems are available in the literature as well as on the Web. Most of them are comparative reviews of the systems functionality. All these studies present lists of features offered by selected e-learning systems. However, the usability of an e-learning system is not supported by the mere enumeration of the features offered, but how well these features are implemented. Usability addresses the quality of the functionality of a system in the context of use.

Among the various usability evaluation approaches, usability inspection methods are very popular because they cost less than lab-based usability evaluations which involve users. Usability inspection methods involve expert evaluators only, who inspect the system and, based on their knowledge and expertise, provide judgments about the usability of the different application elements. Heuristic evaluation is by far the most well known usability inspection method due to its simplicity. But allowing a usability expert, who does not know a lot about the e-learning domain, to evaluate an elearning system is not safe since one might not be able to spot usability problems which have to do with the learning context of use. On the other hand general purpose usability heuristics are not enough since they do not focus on the specificities of the elearning domain. This is why various sets of usability heuristics for e-learning systems have been proposed.
\end{abstract}


A key solution would be to help novice usability evaluators, who are plenty and easier to find, in performing the usability evaluation of e-learning systems more accurately. For this solution to be achieved, we need to help novice evaluator reveal as many usability problems as experts by augmenting the evaluator's existing knowledge and stimulating him/her to think about usability more broadly with the help of different perspectives.

In this thesis we describe the DEPTH (usability evaluation based on DEsign PaTterns \& Heuristics criteria) method for usability evaluation of e-learning systems. The DEPTH method eliminates the difficulties described above and provides an integrated framework where the evaluator can find and (re)use expert domain knowledge thus being aided during the evaluation. The innovative idea behind the DEPTH approach is the use of design patterns in the examination of the usability performance of a system. A design pattern describes a design problem and a solution for this problem in a particular context, together with the rationale behind this solution and the consequences (pros and cons) of using it. Our method uses design patterns in three dimensions: a) for checking the supported functionality of an e-learning system according to its specific genre, b) for the examination of the usability performance of the supported functionality according to the specific context of use, c) for the examination of the general usability performance according to heuristics criteria.

The idea of using design patterns rather than guidelines for usability evaluation lies primarily on their perspective and representation of the underlying information. Design patterns are more problem oriented, and focus on the description of both the problem and the solution along with the rationale that led to this solution, while guidelines are more general information or advice. Another fact is that we can easily generate scenarios for usability evaluation from design patterns while the same task is not as easy when we use guidelines. Scenarios provide a versatile and reproducible means of evaluating a system. A scenario is an instantiation of one or more representative work tasks and transitions linking those tasks

The final deliverables of this thesis they are a) a new method of usability evaluation adapted in the conceptual frame of e-learning systems, b) an online tool that supports this method and at the same time can be used as a library of design patterns and related scenarios of use, as well as c) a design pattern language of for the genre of Asynchronous Network-Supported Collaborative Learning systems (ANSCL). 


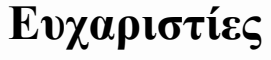

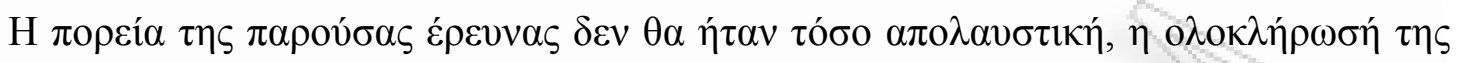

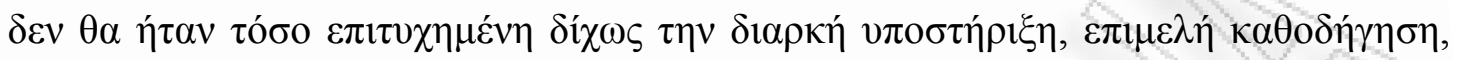

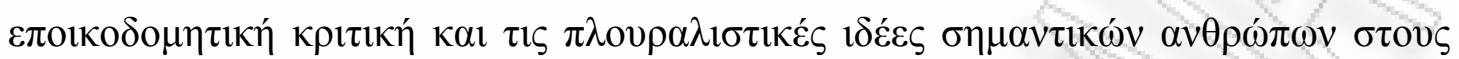

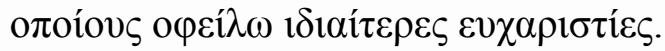

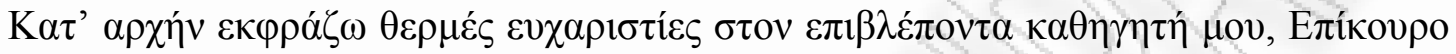

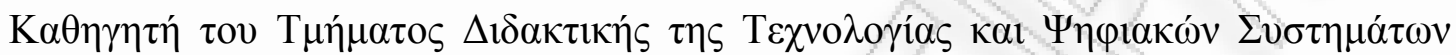

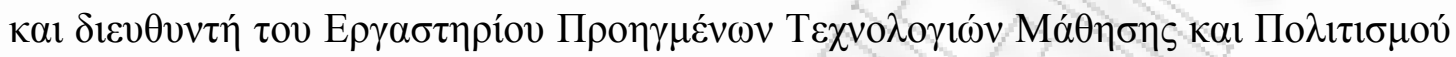

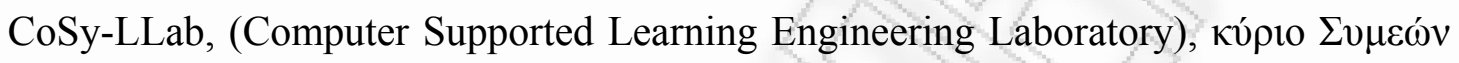

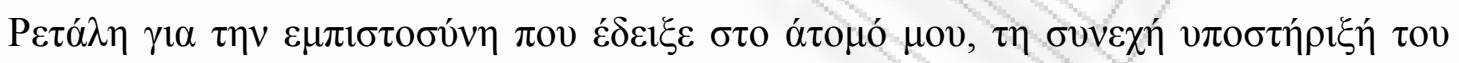

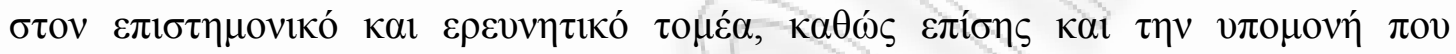

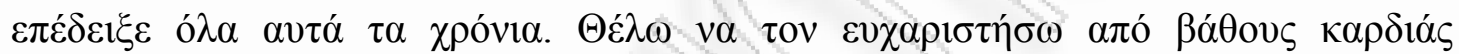

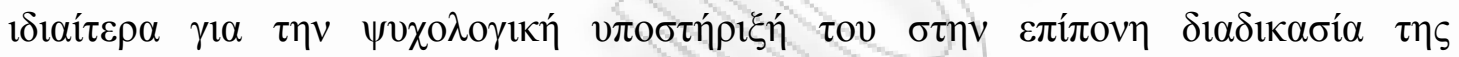

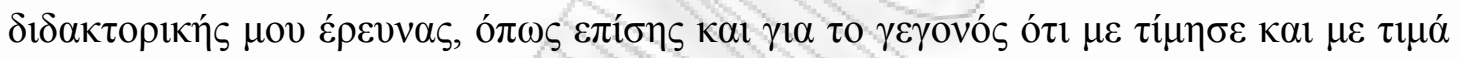

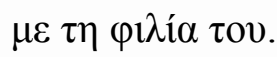

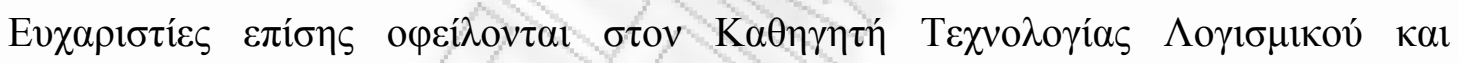

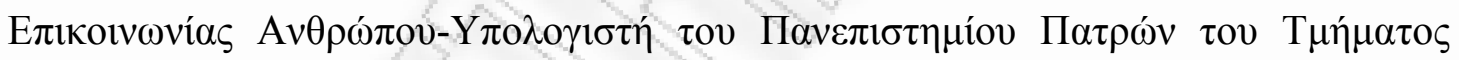

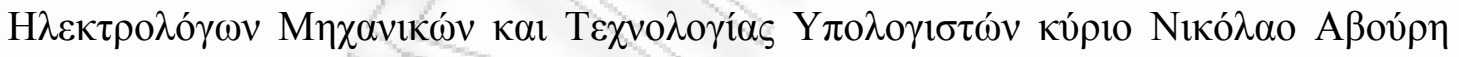

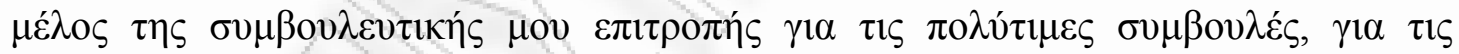

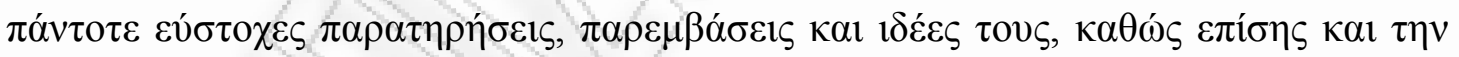

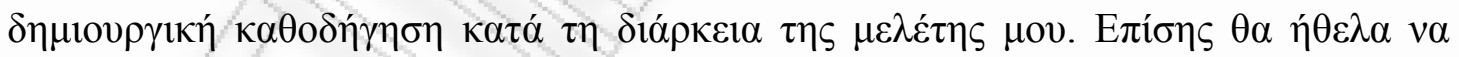

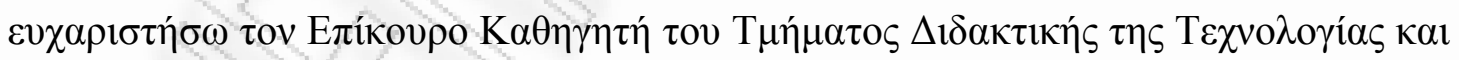

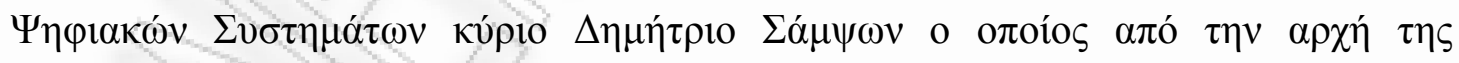

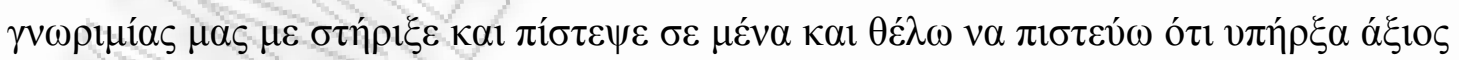

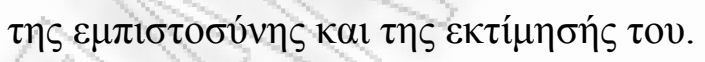

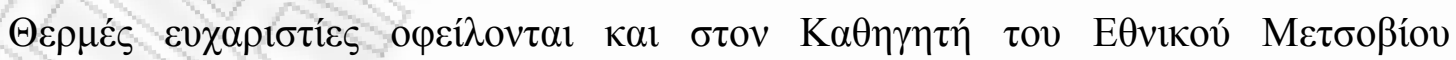

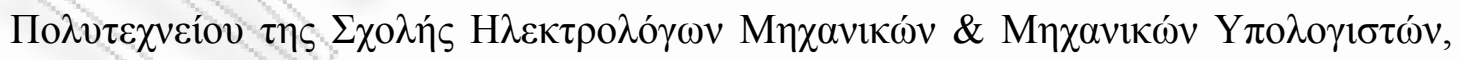

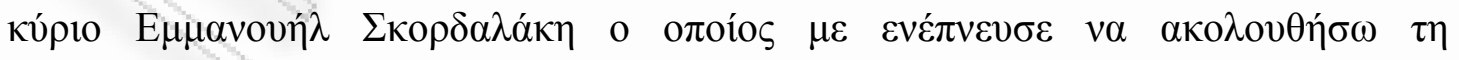

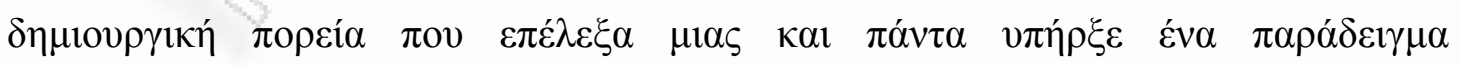

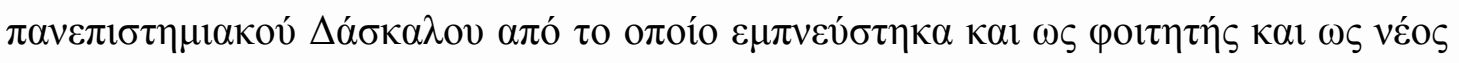

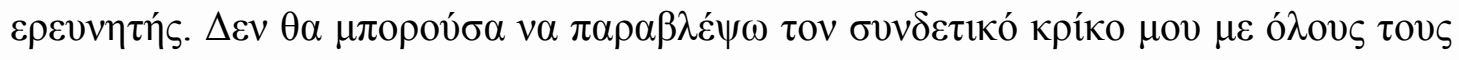

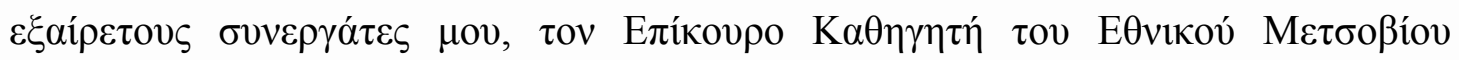




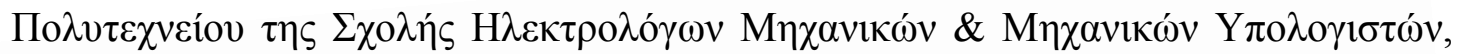

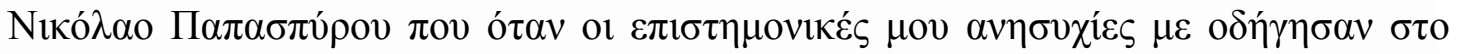

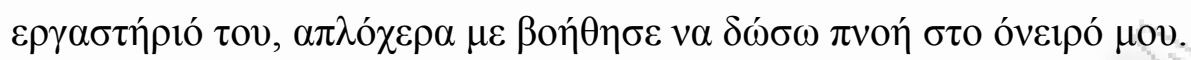

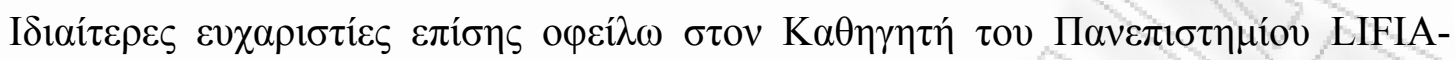

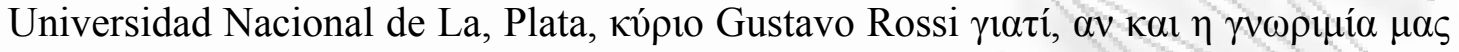

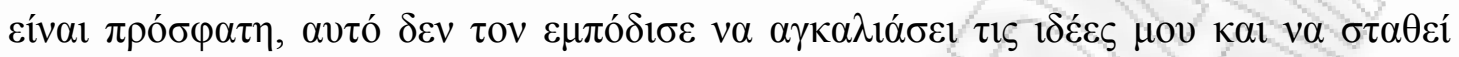

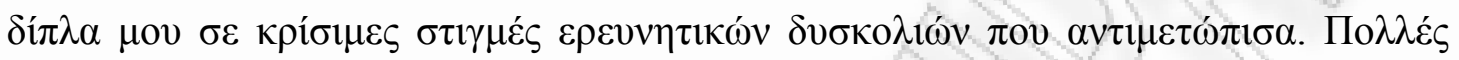

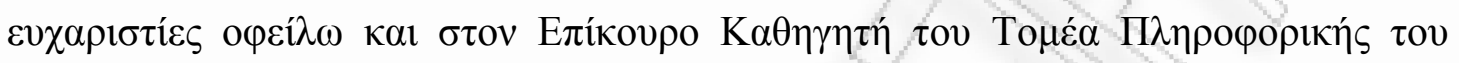

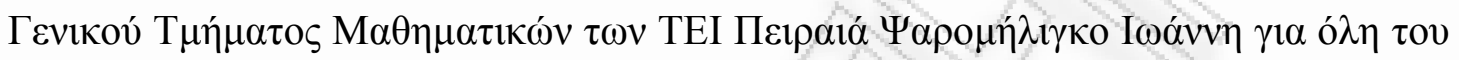

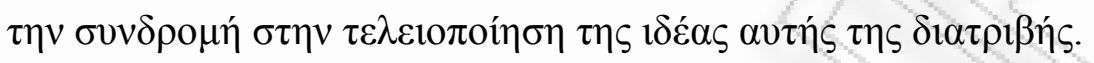

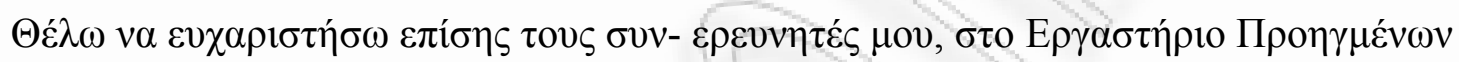

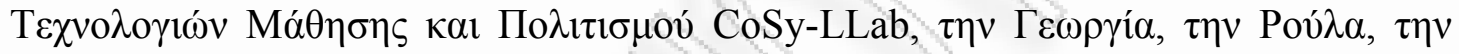

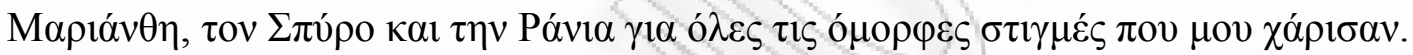

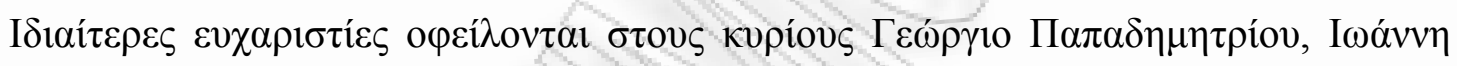

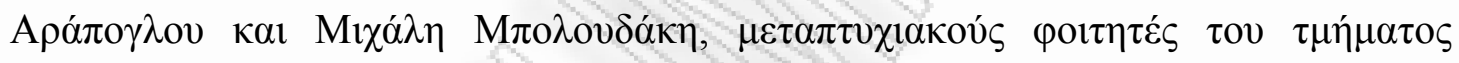

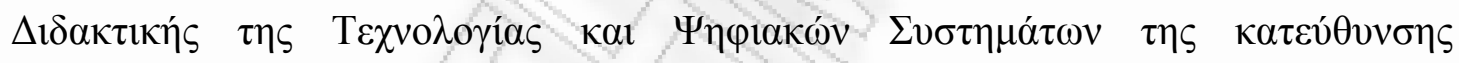

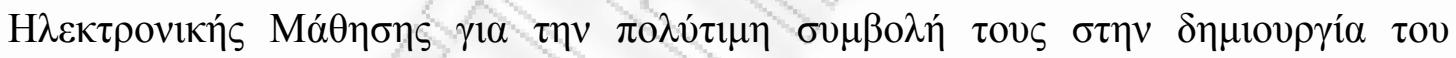

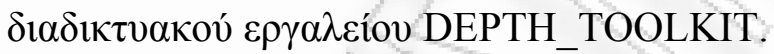

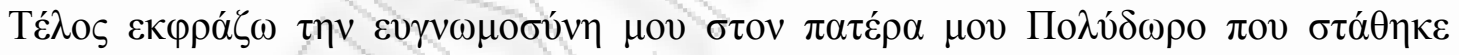

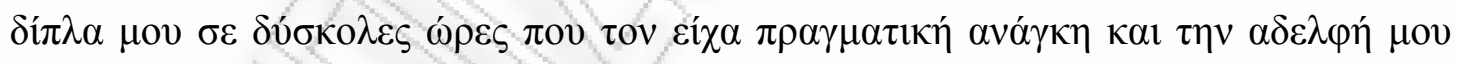

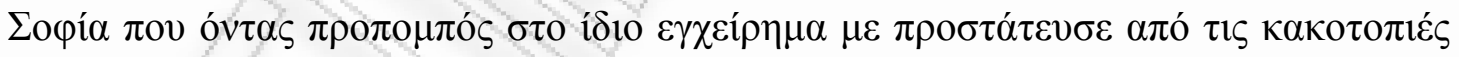

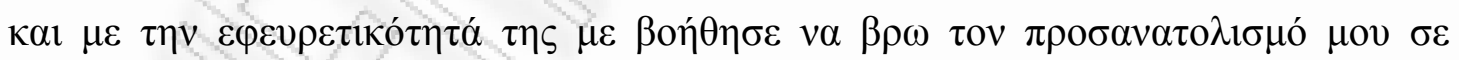

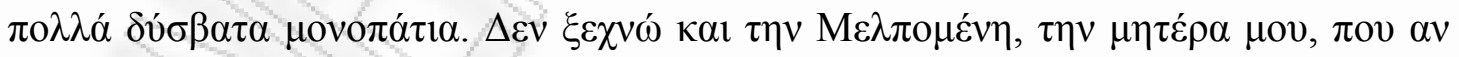

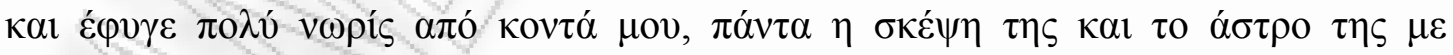

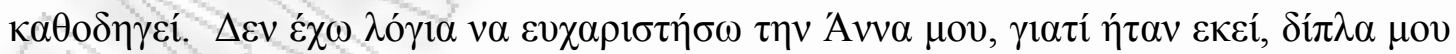

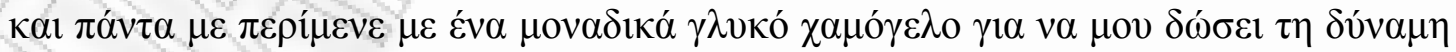

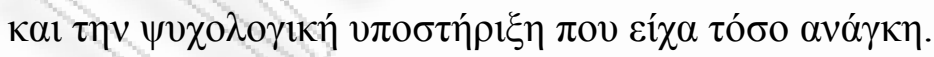




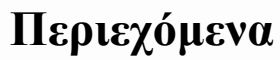

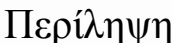

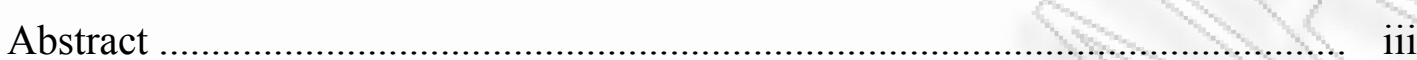

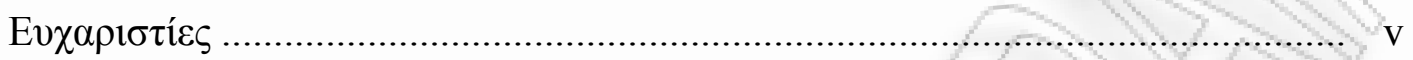

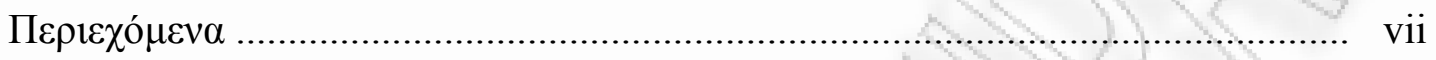

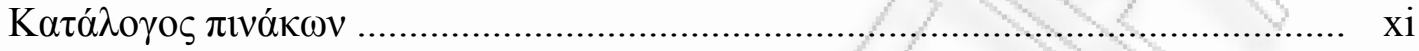

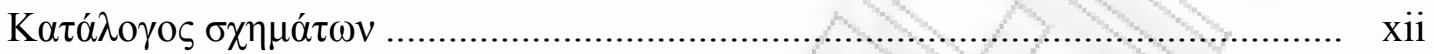

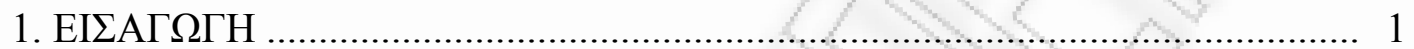

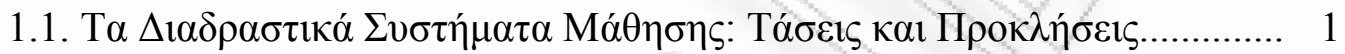

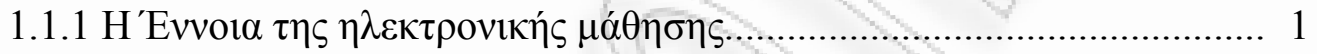

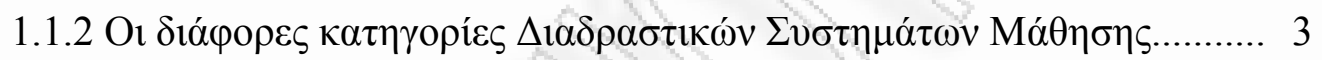

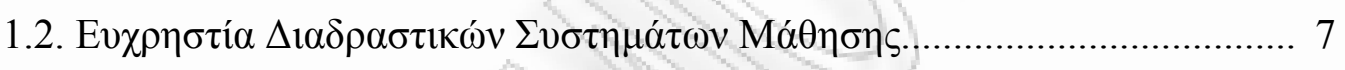

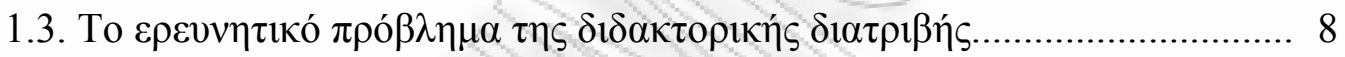

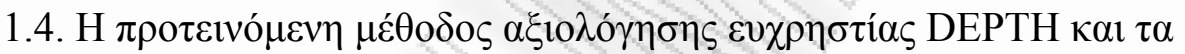

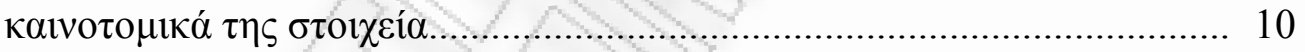

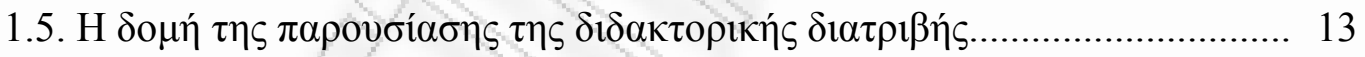

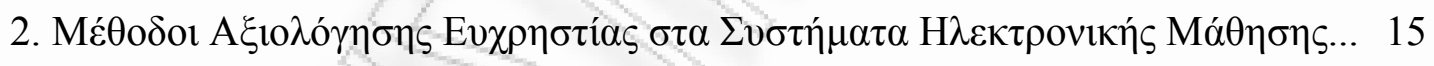

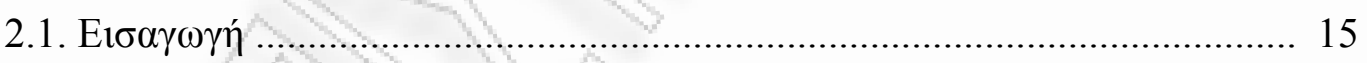

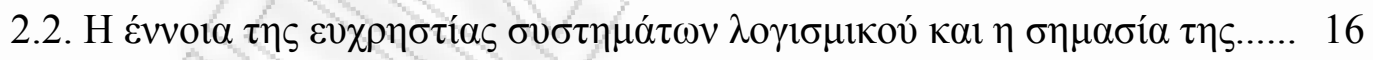

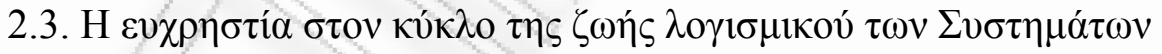

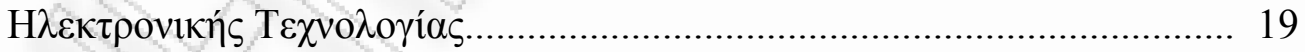

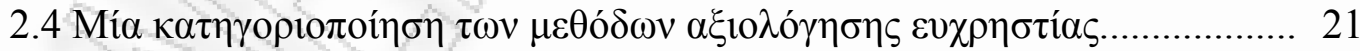

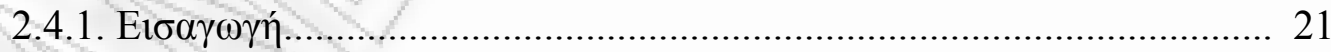

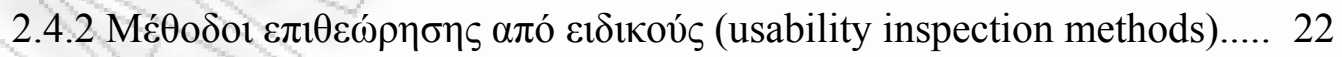

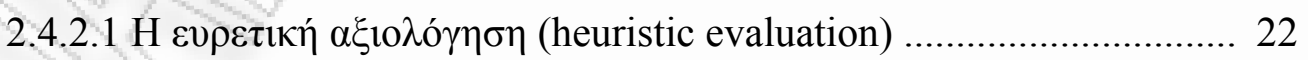

2.4.2.2 To $\gamma \nu \omega \sigma ı \alpha \kappa o ́ ~ \pi \varepsilon \rho ı \delta i \alpha ́ \beta \alpha \sigma \mu \alpha$ (cognitive walkthrough) .................... 25

2.4.2.3 По $\lambda \lambda \alpha \pi \lambda o ́ ~ \pi \varepsilon \rho 1 \delta i \alpha ́ \beta \alpha \sigma \mu \alpha$ (pluralistic walkthrough) ...................... 26

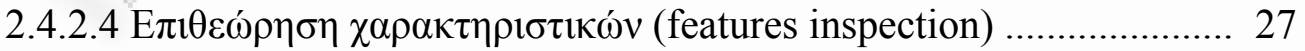

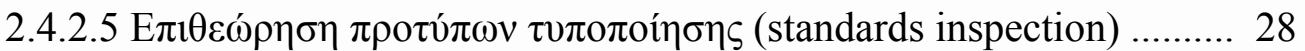

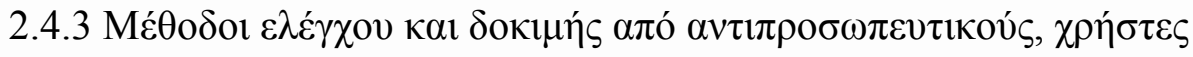

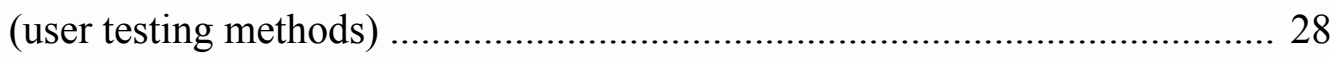




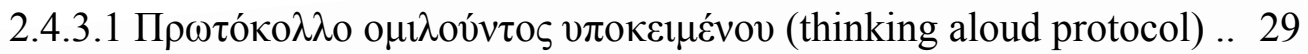

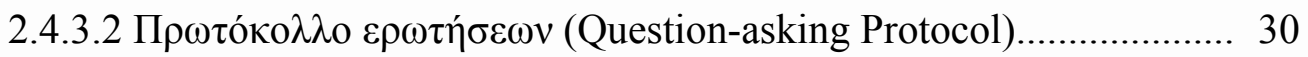

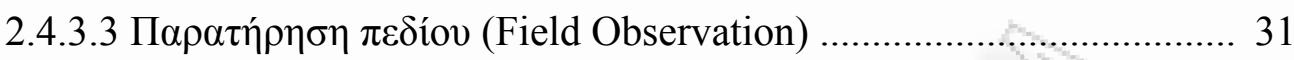

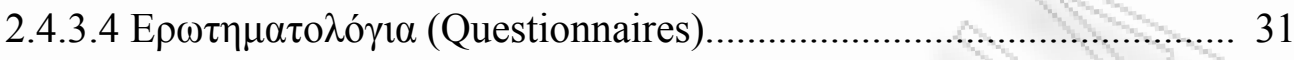

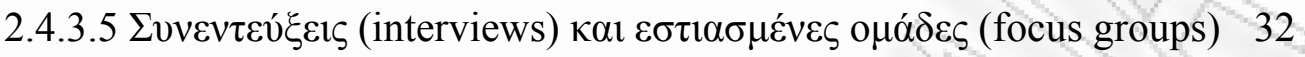

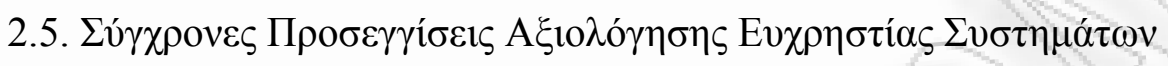

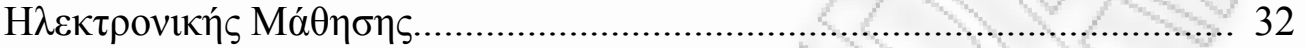

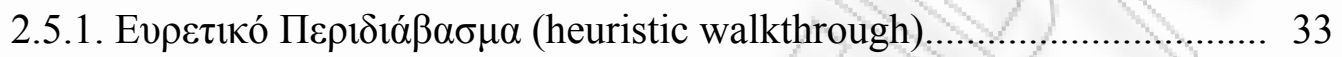

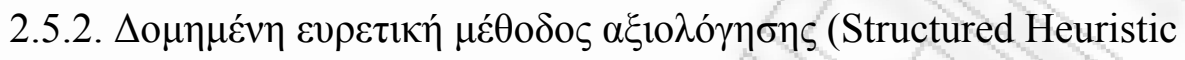

Evaluation Method)

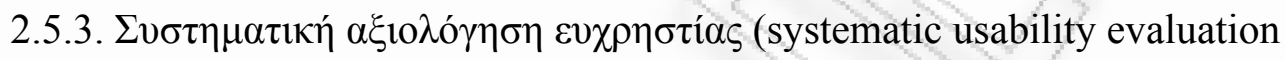

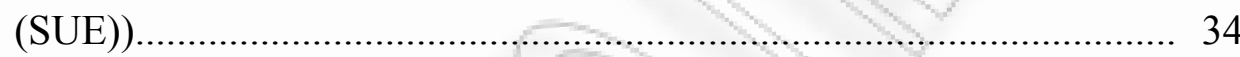

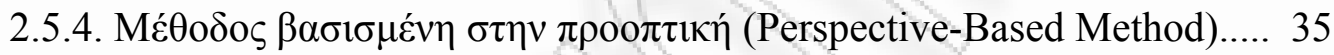

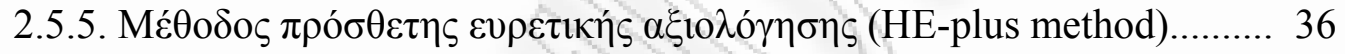

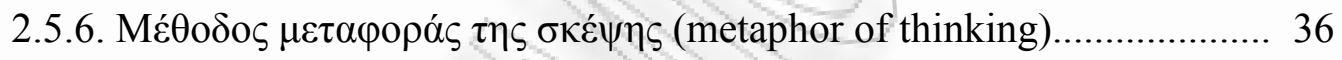

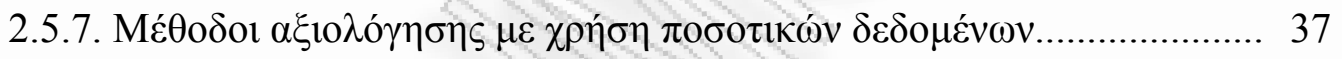

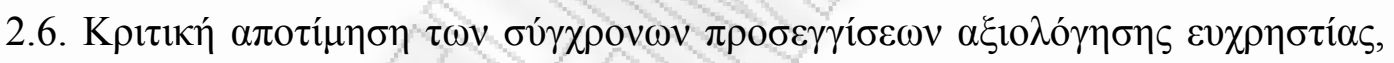

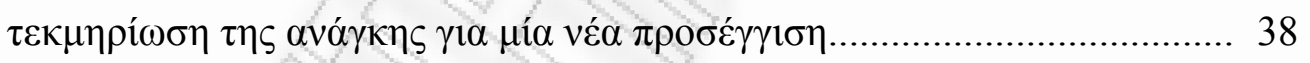

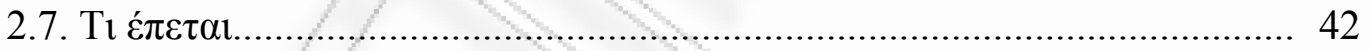

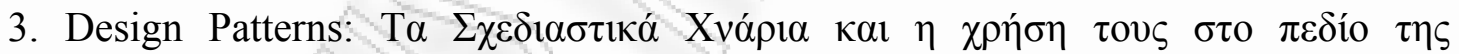

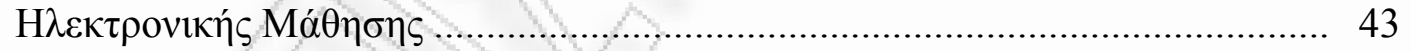

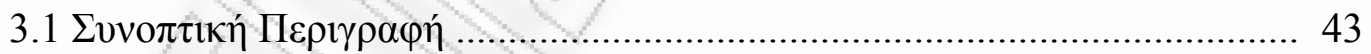

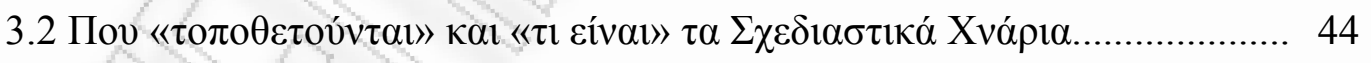

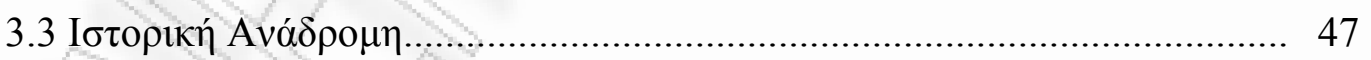

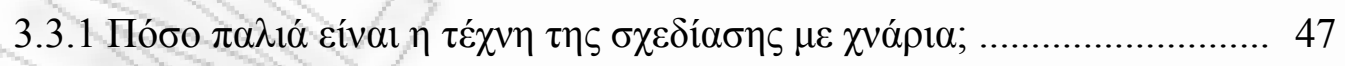

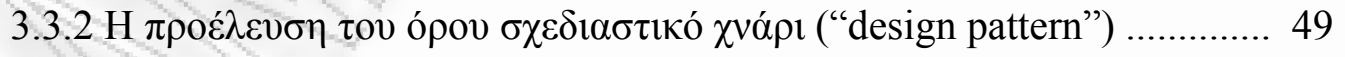

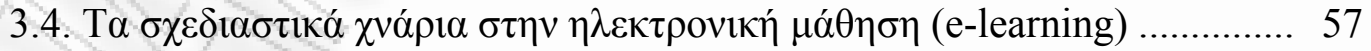

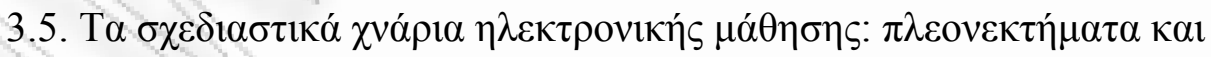

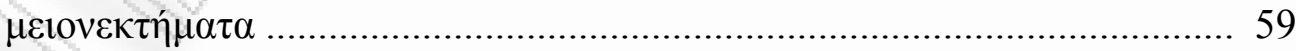

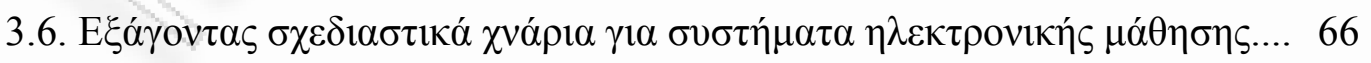

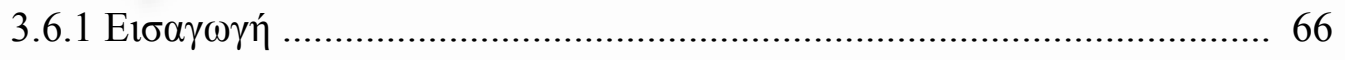

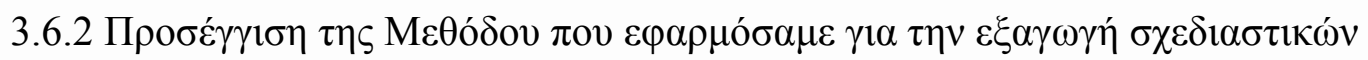
$\chi v \alpha \rho i \omega ́ v$ 68 


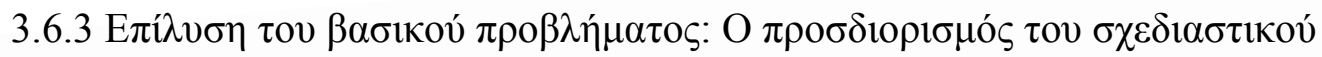

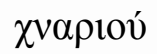

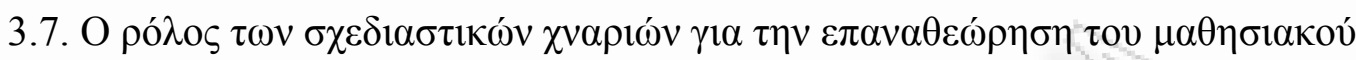
$\sigma \chi \varepsilon \delta 1 \alpha \sigma \mu o u ́$. 76

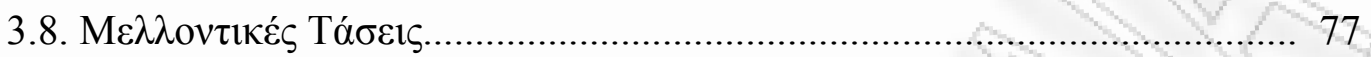

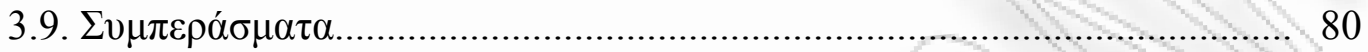

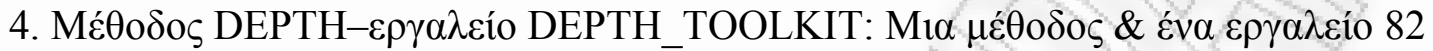

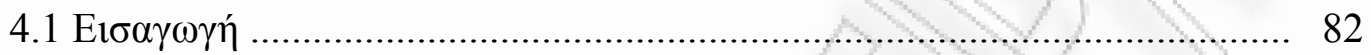

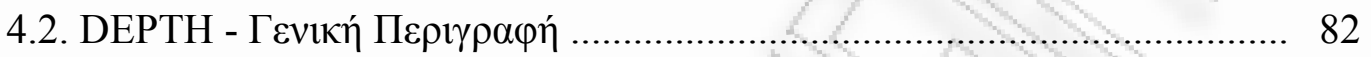

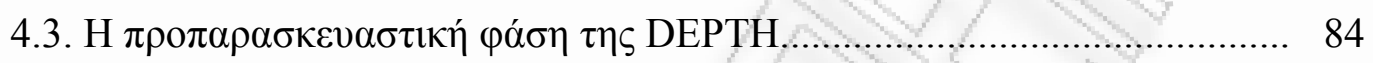

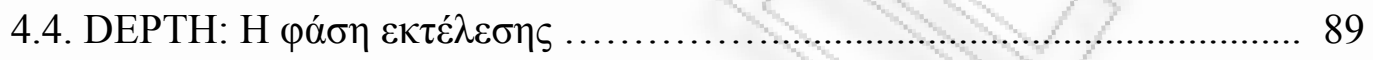

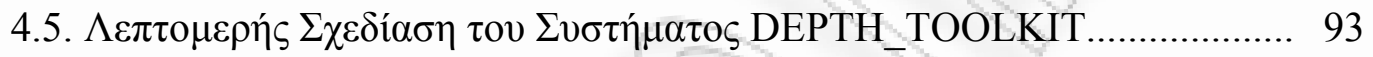

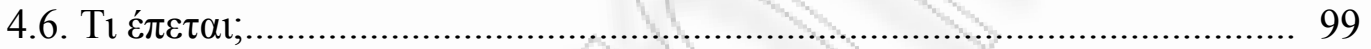

5. A $\xi$ ı૦

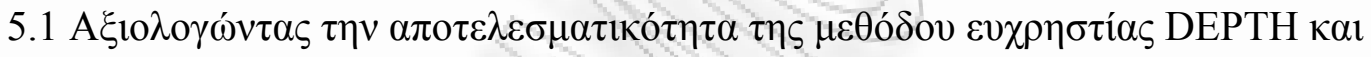

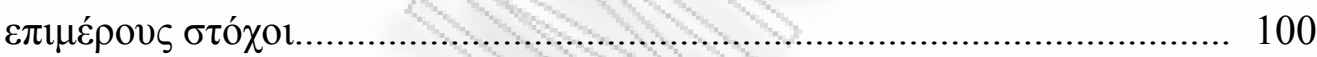

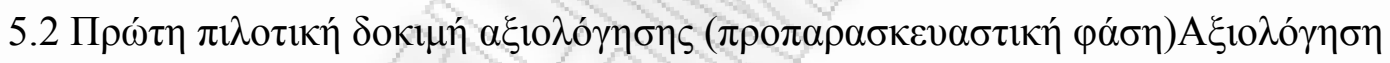

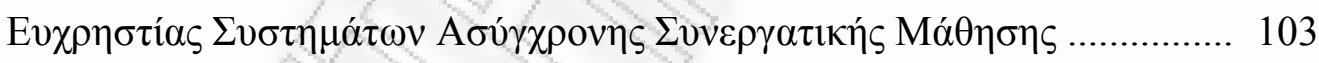

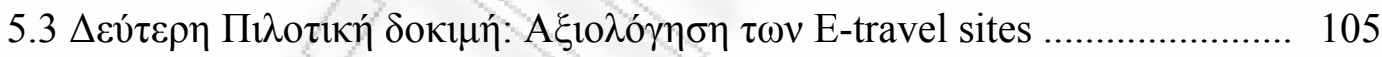

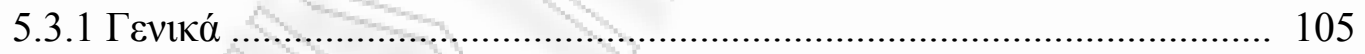

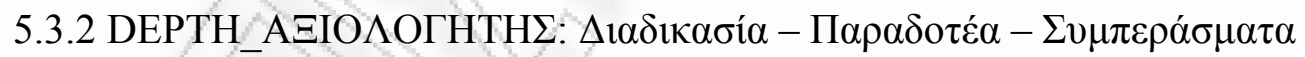

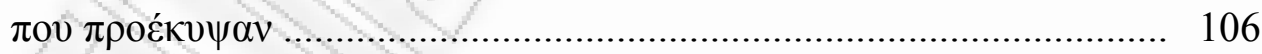

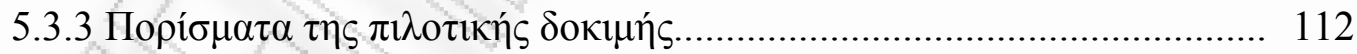

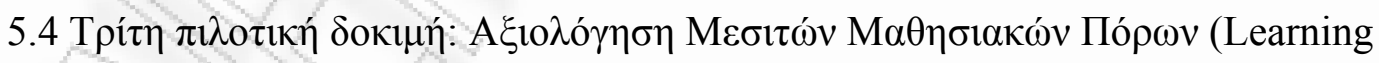

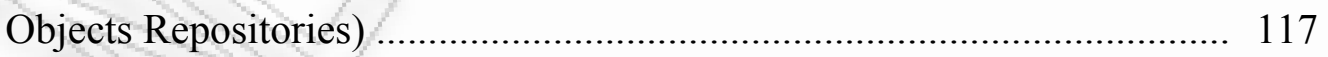

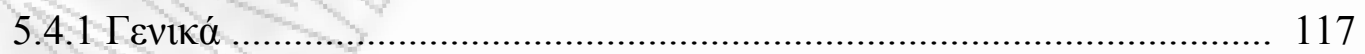

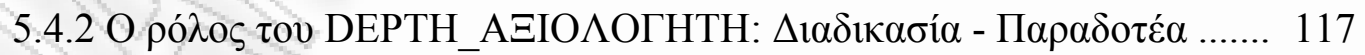

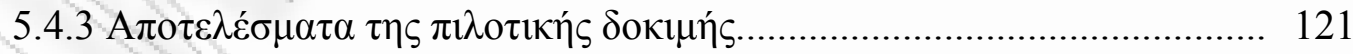

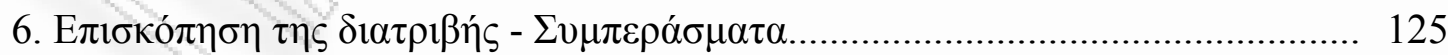

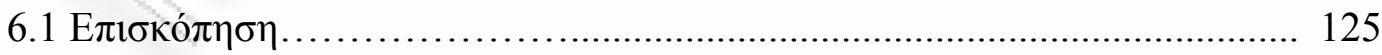

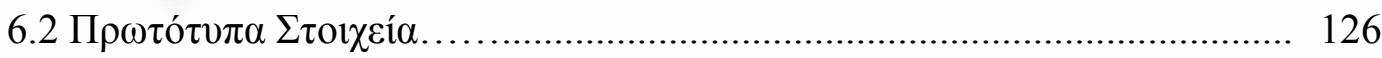

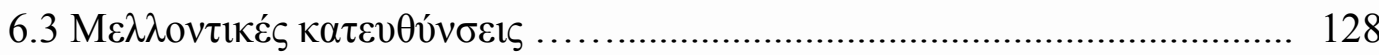

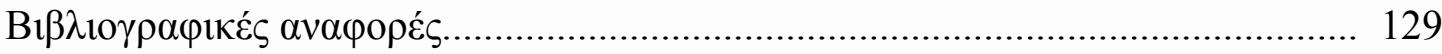




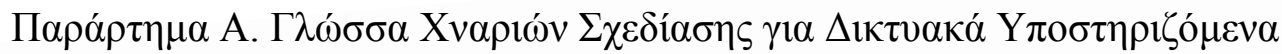

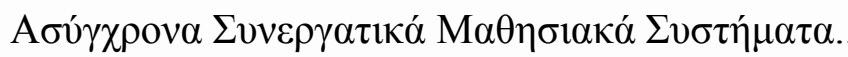

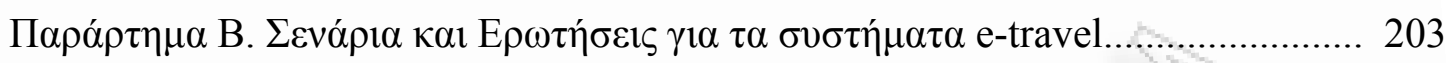

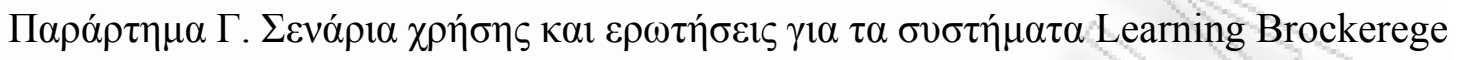
Platforms. 209 


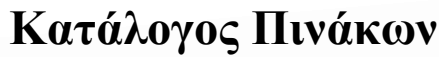

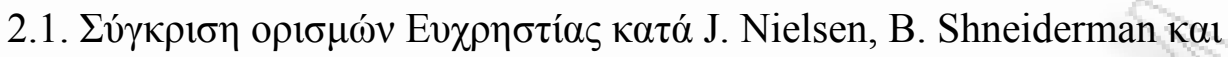
ISO9241-11

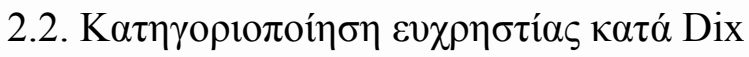
18

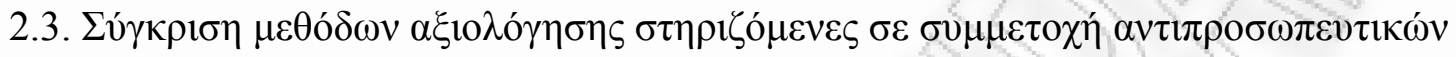

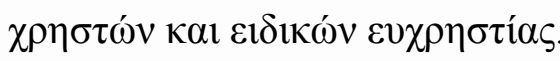
40

5.1. $\Sigma v \sigma \chi \varepsilon ́ \tau \imath \sigma \eta \Lambda \varepsilon เ \tau o v \rho \gamma \iota \omega ́ v-\Sigma \varepsilon v \alpha \rho i ́ \omega v$ 109

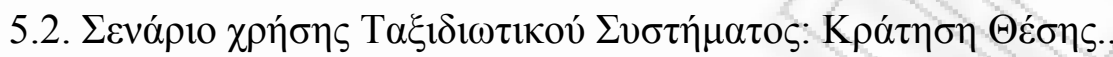
110

5.3. $\Sigma v \sigma \chi \varepsilon ́ \tau \imath \sigma \eta ~ \Lambda \varepsilon \imath \tau o v \rho \gamma \imath \omega ́ v-\Sigma \chi \varepsilon \delta i \alpha \sigma \tau \imath \kappa \omega ́ v ~ \chi v \alpha \rho i \omega ́ v$. 111

5.4. A 113

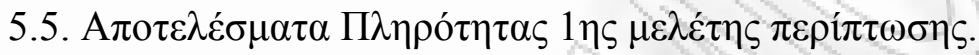
114

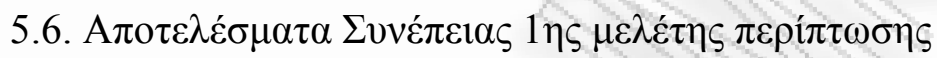

5.7. $\Sigma v \sigma \chi \varepsilon ́ \tau ı \sigma \eta \Lambda \varepsilon ı \tau o v \rho \gamma ı \omega ́ v-\Sigma \varepsilon v \alpha \rho i ́ \omega v$

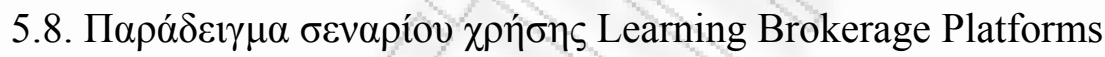

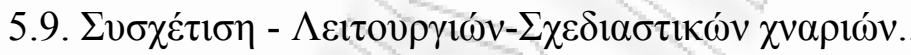
120

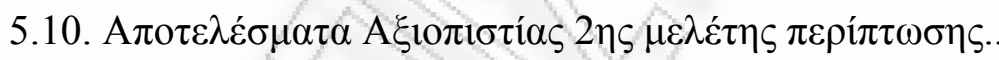

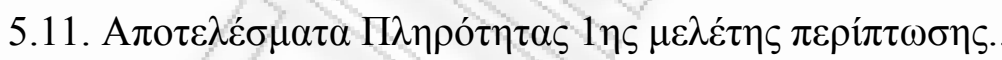
123

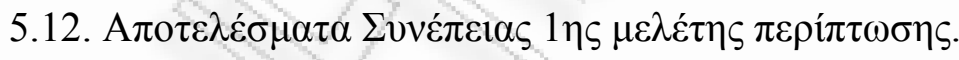




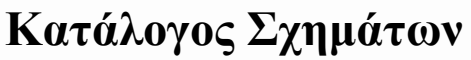

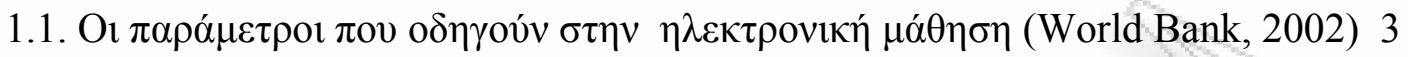

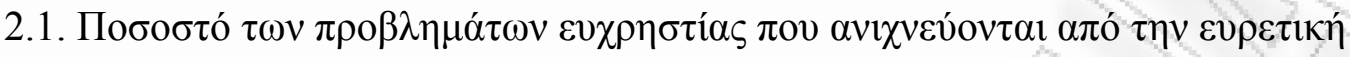

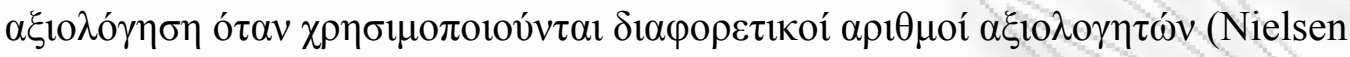
\& Landauer 1993)

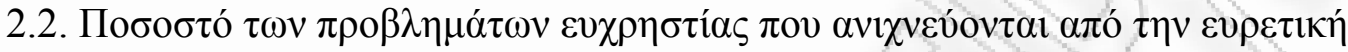

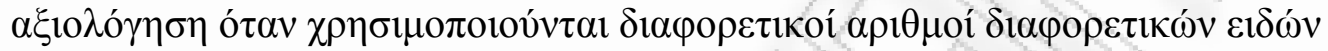

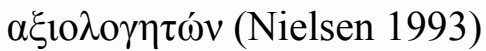

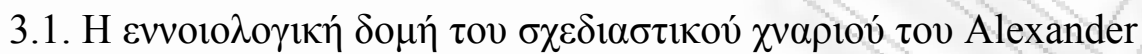

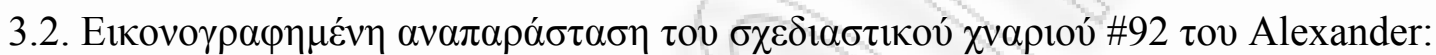
$\Sigma \tau \alpha ́ \sigma \eta \lambda \varepsilon \omega \varphi о \rho \varepsilon i ́ o v$ 54

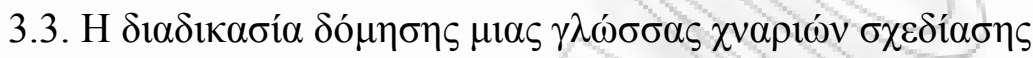

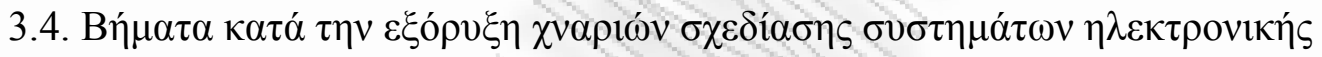
$\mu \alpha ́ \theta \eta \sigma \eta \varsigma$.

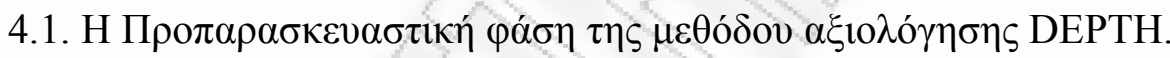
85

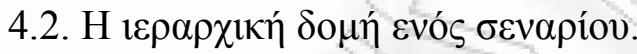

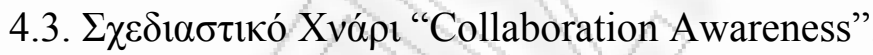
88

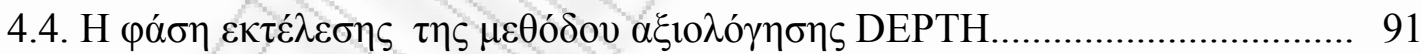

4.5. To $\delta 1 \alpha \delta \iota \kappa \tau v \alpha \kappa o ́ ~ \sigma u ́ \sigma \tau \eta \mu \alpha-\varepsilon \rho \gamma \alpha \lambda \varepsilon i ́ o$ DEPTH_TOOLKIT.................................. 93

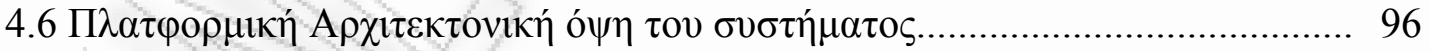

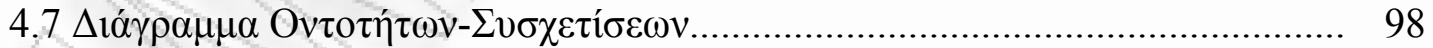

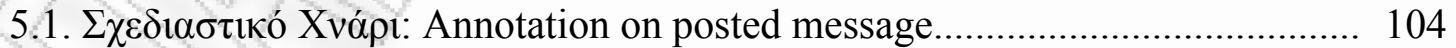

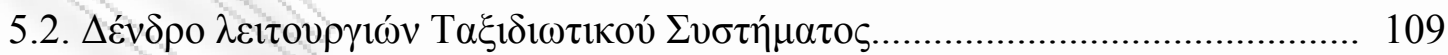

5.3. $\Delta \varepsilon ́ v \delta \rho \circ ~ \lambda \varepsilon \iota \tau o v \rho \gamma \iota \omega ́ v$ Learning Brokerage Platforms...................................... 119 


\section{КЕФААAIO 1}

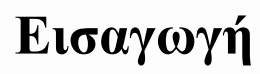

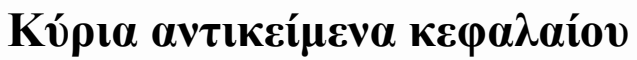

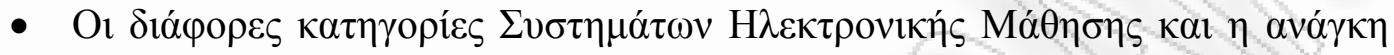

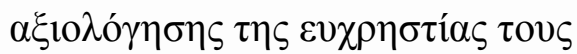

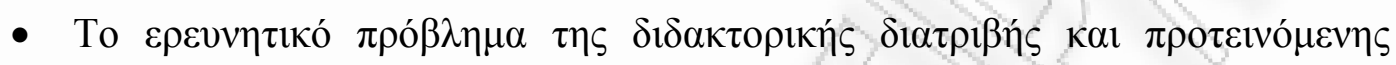

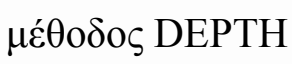

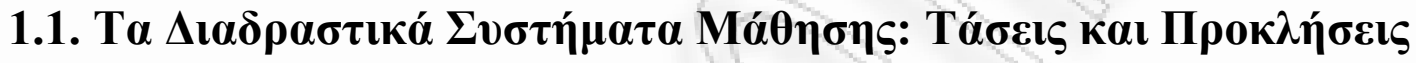

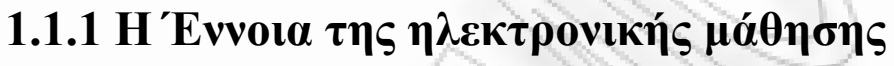

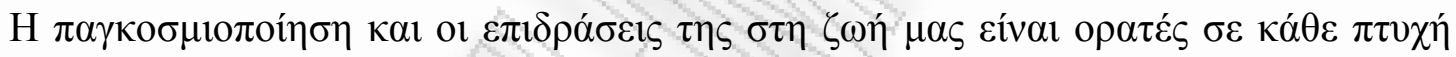

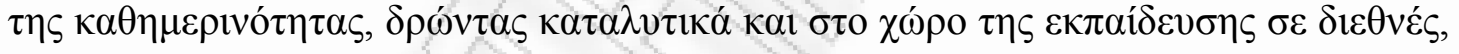

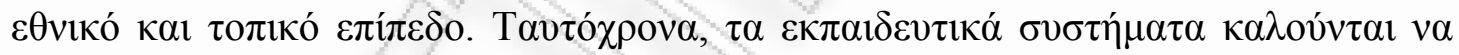

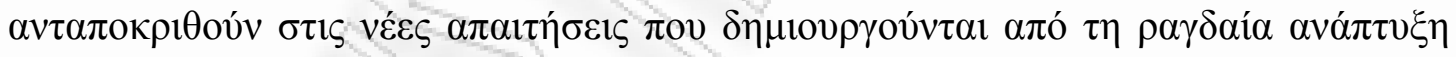

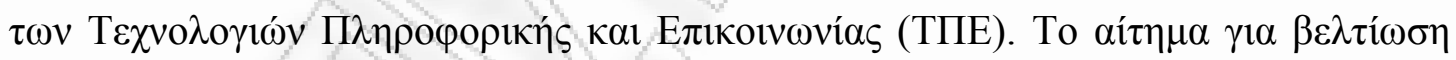

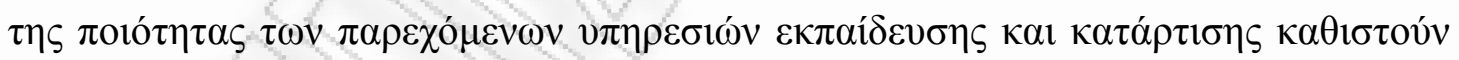

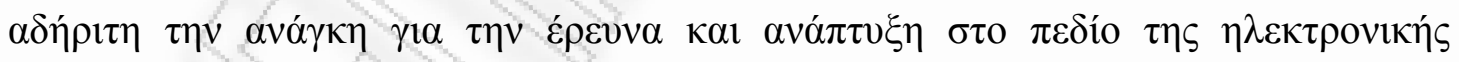

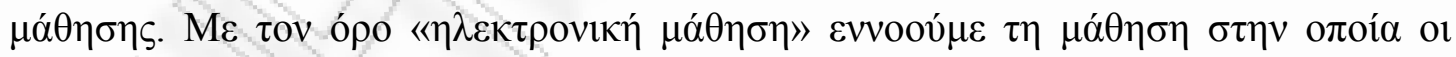

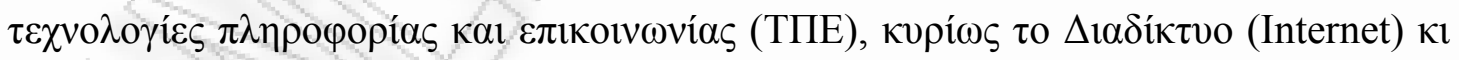

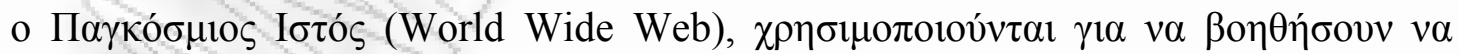

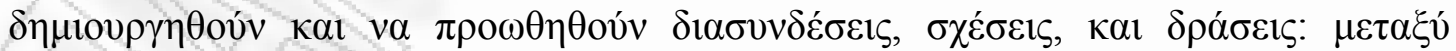

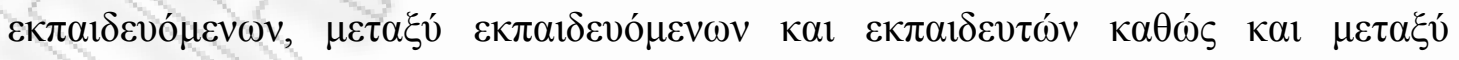

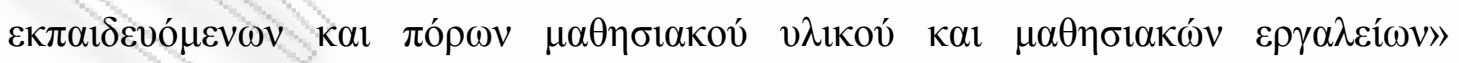
(Goodyear, et. al., 2004a).

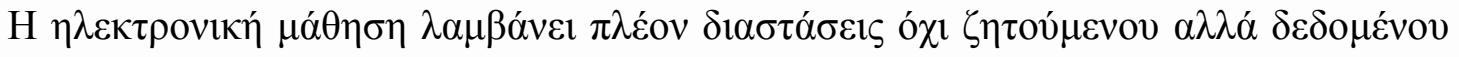

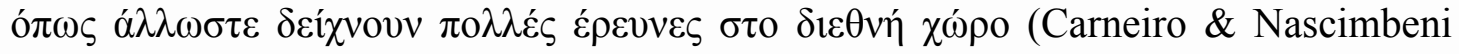

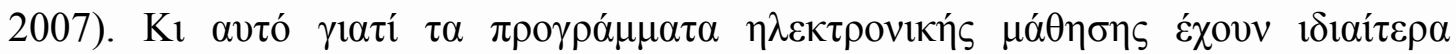




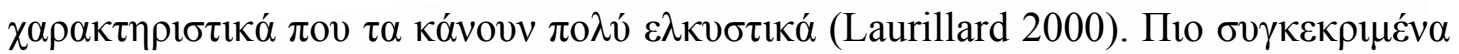

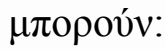

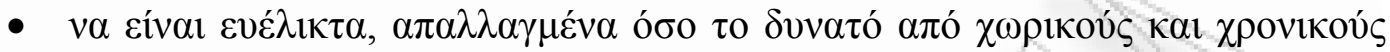

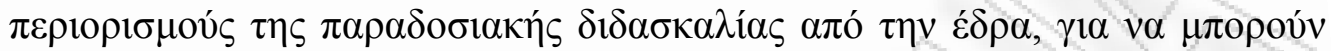

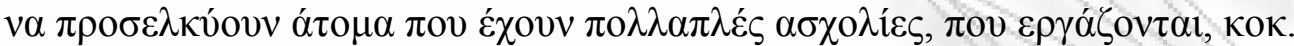

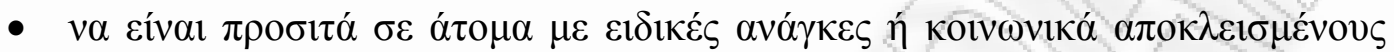

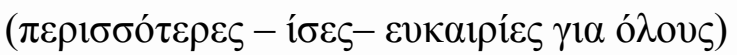

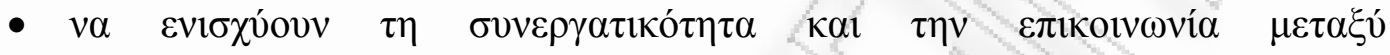

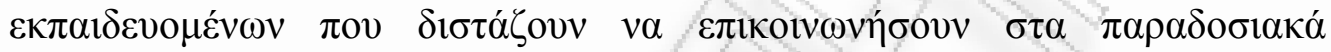

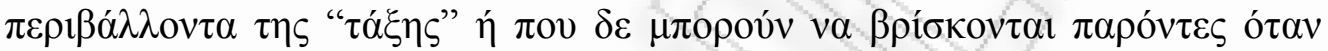

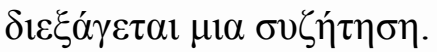

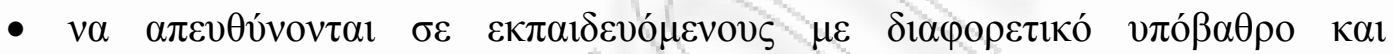

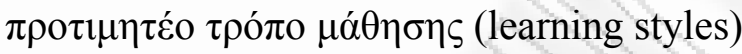

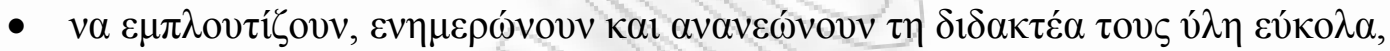

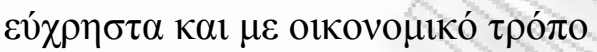

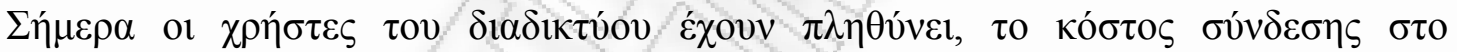

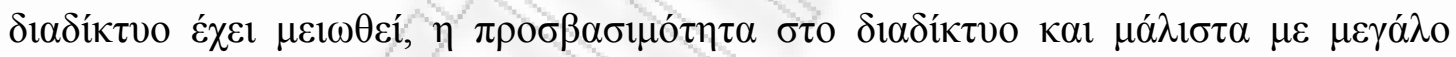

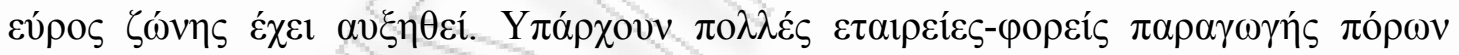

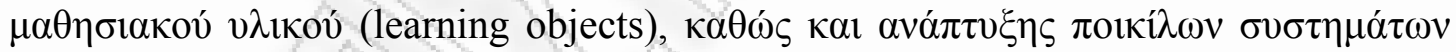

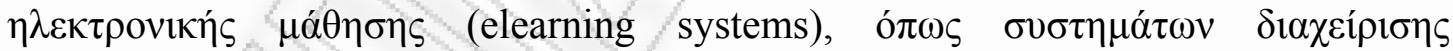

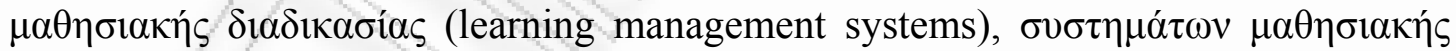

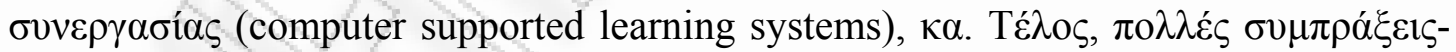

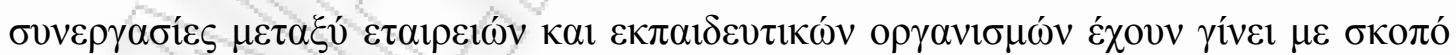

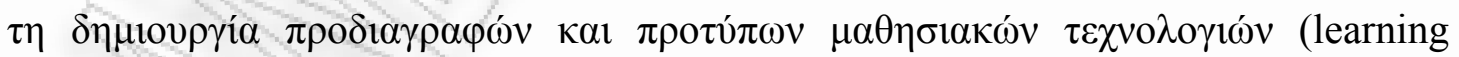

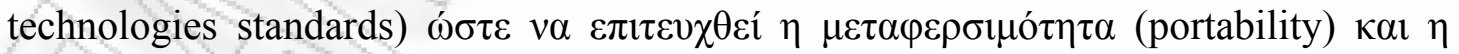

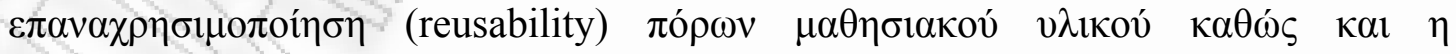

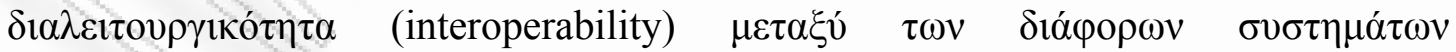

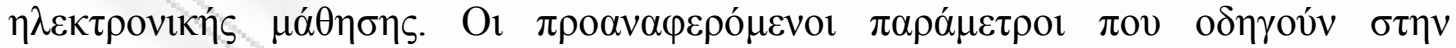

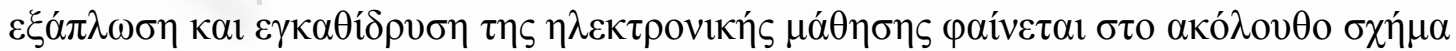
1.1 (World Bank, 2003). 


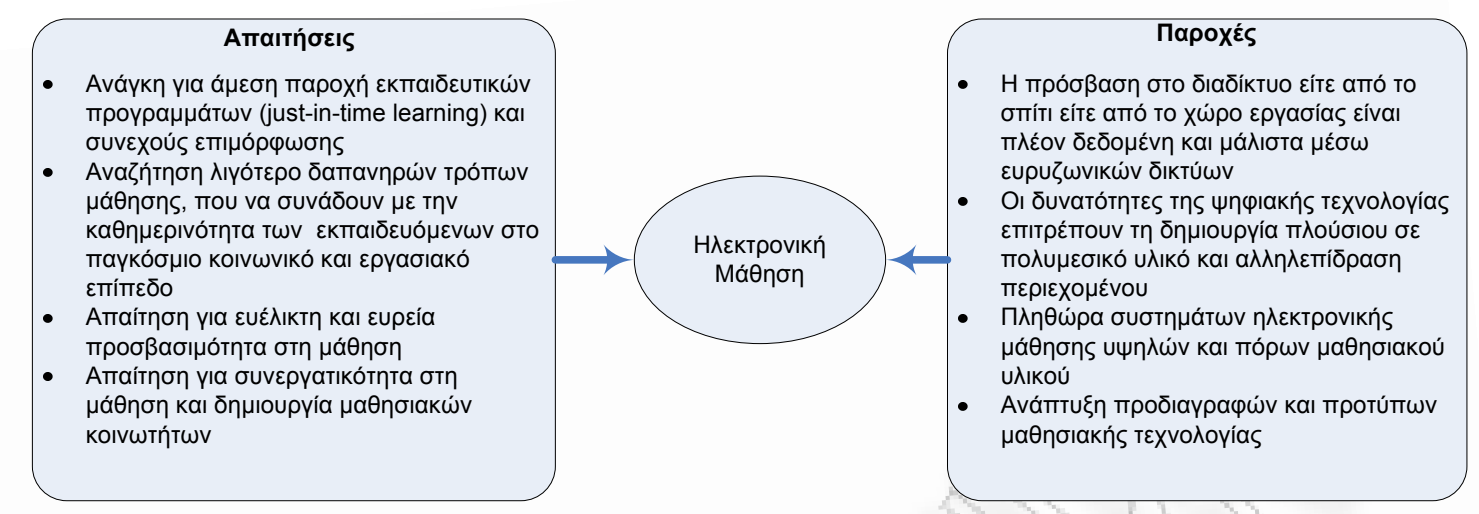

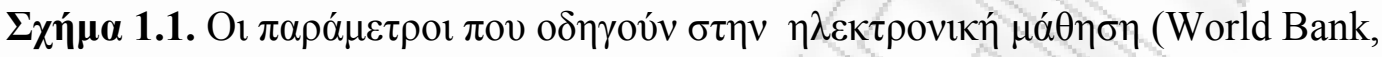
2003).

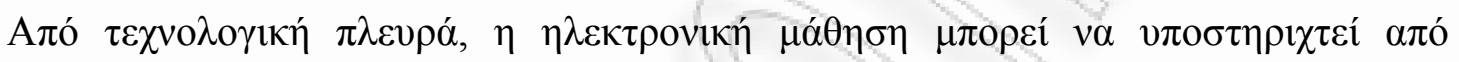

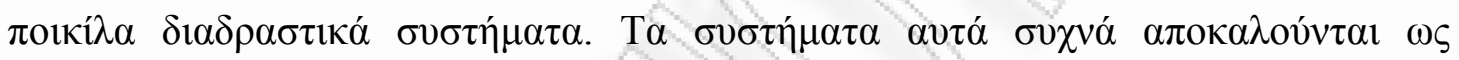

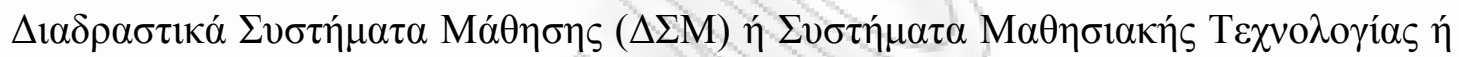

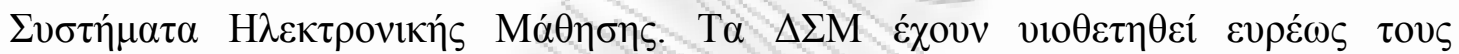

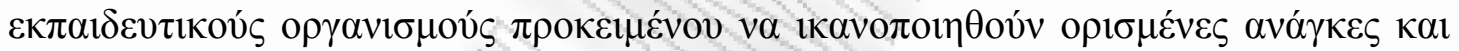

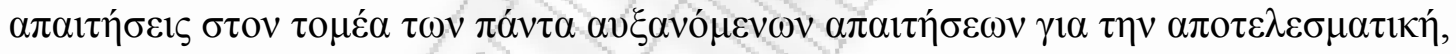

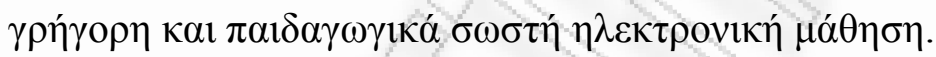

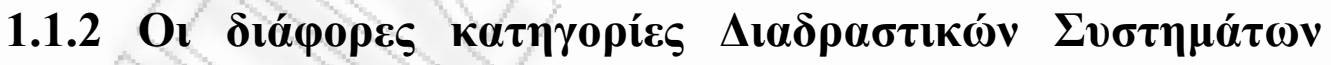

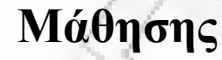

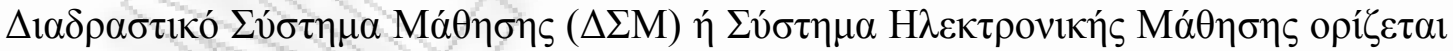

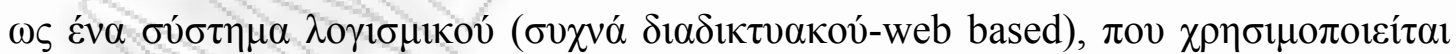

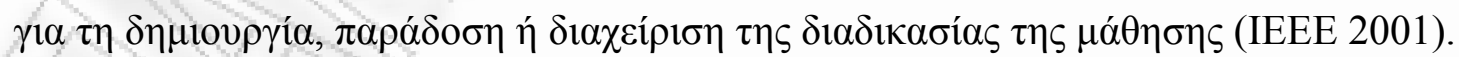

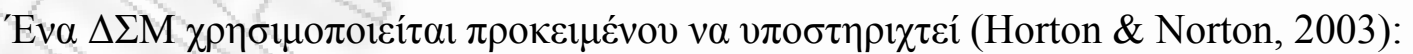

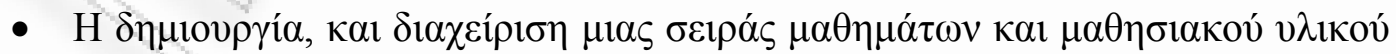
$\gamma 1 \alpha \alpha v \tau \alpha ́$.

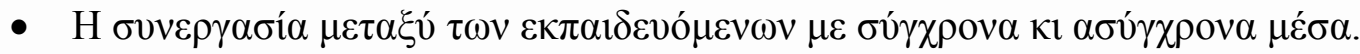




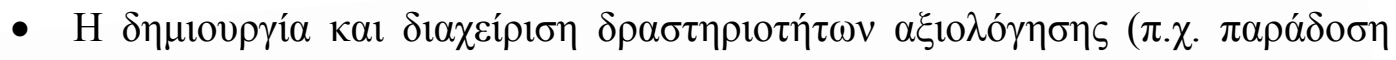

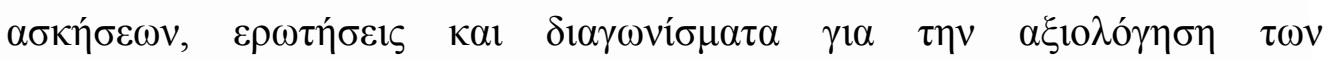
$\sigma \pi \mathrm{ov \delta} \alpha \sigma \tau \omega \dot{v}, \kappa \lambda \pi)$

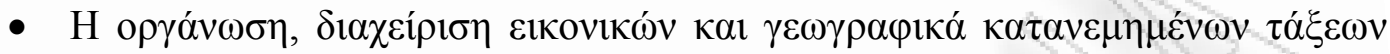

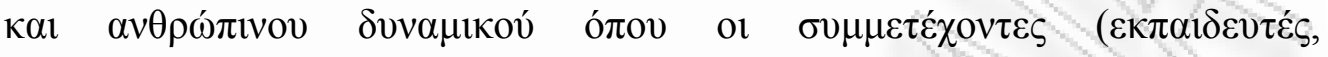

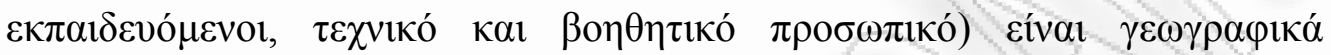

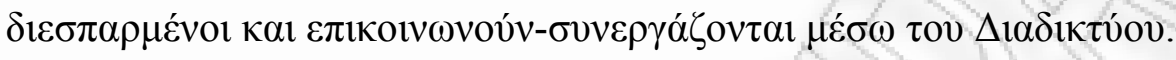

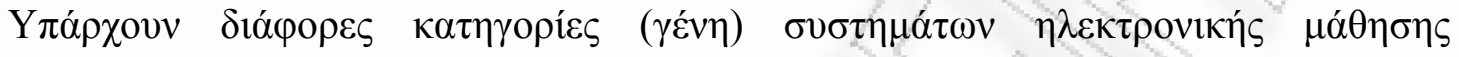
(McCormack \& Jones, 1997) ó $\pi \omega \varsigma$ :

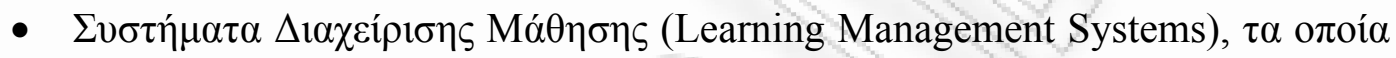

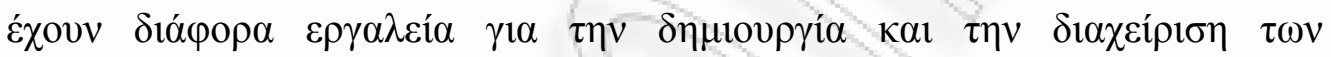

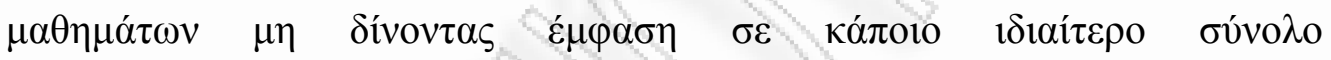

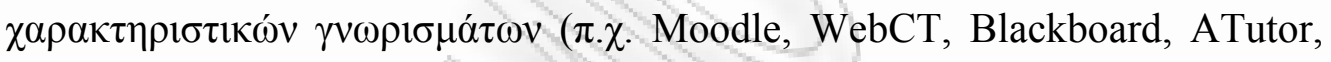
IBM LMS).

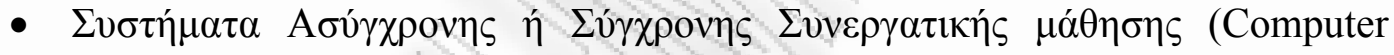

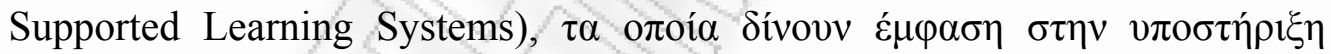

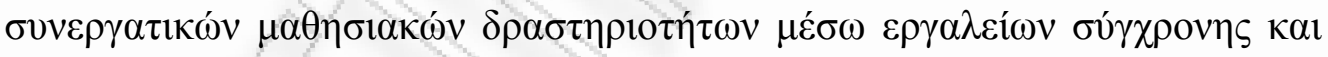

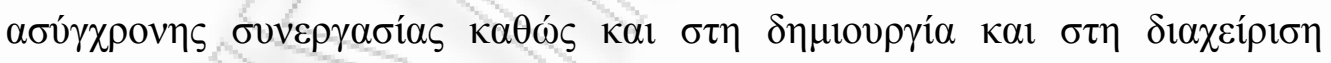

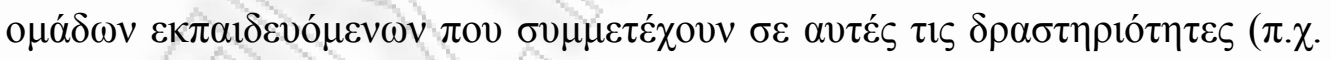
FLE3, Synergeia, Centra, Synergo).

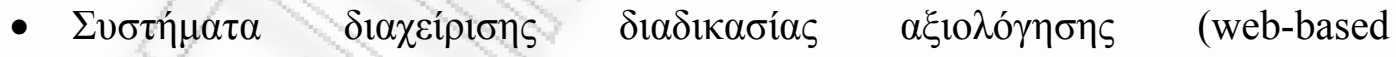

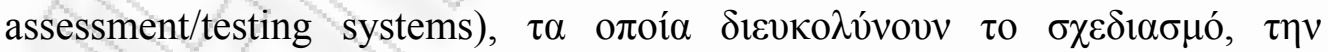

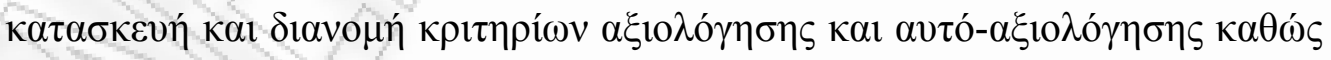

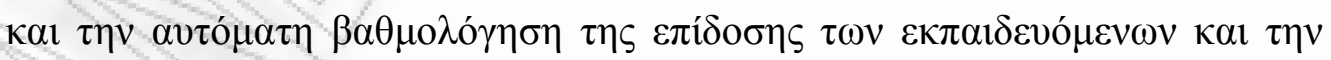

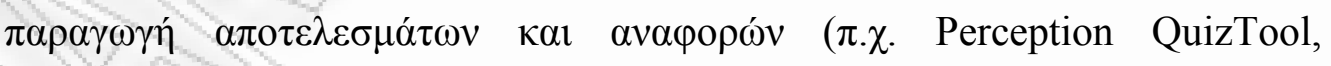
HotPotatoes, Respondus).

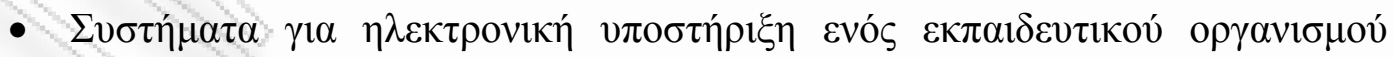

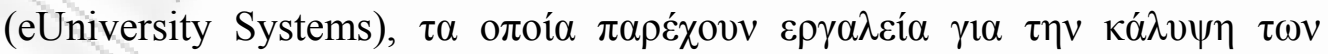

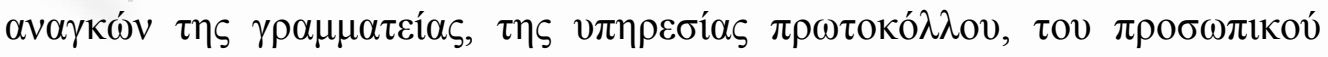

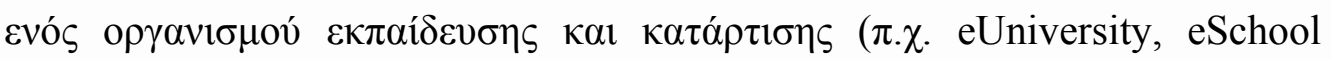
Management System). 


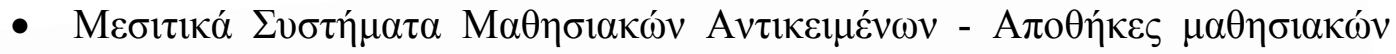
$\alpha \nu \tau \iota \kappa \varepsilon น \varepsilon ́ v \omega v$ (Learning Objects Brokerage Systems - Learning Object

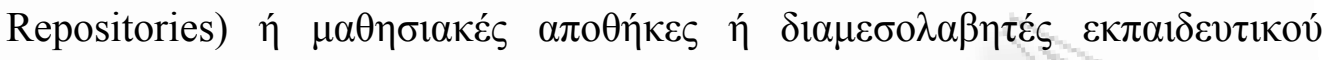

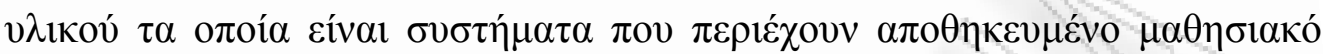

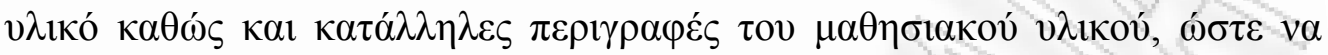

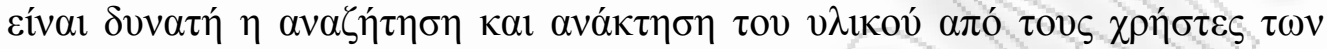

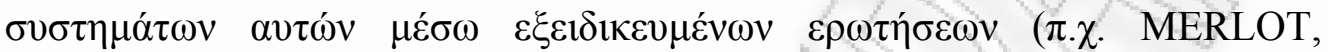
EducaNext, Ariadne).

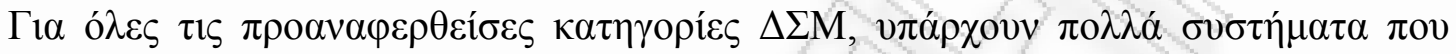

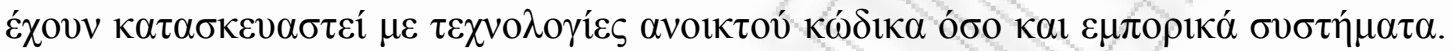

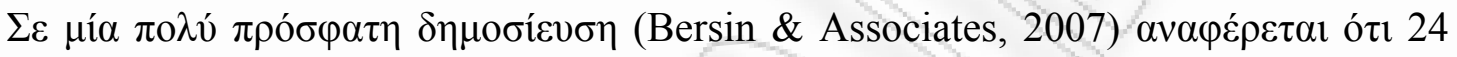

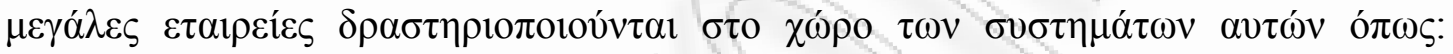
Blackboard, Cornerstone OnDemand, Generation21, GeoLearning, GeoMetrix, ElementK, KnowledgePlanet, Learn.com, Meridian KSI, NetDimensions, Oracle, Outstart, Plateau, RISC, Saba, SAP, SkillSoft, SumTotal Systems, TEDS, Thompson

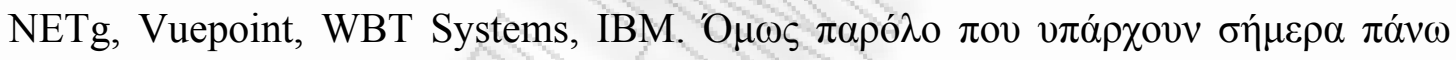

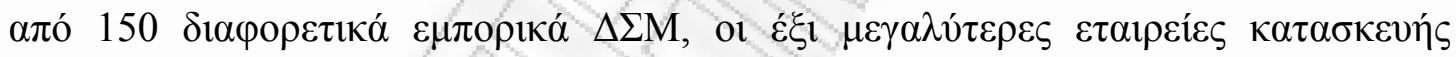

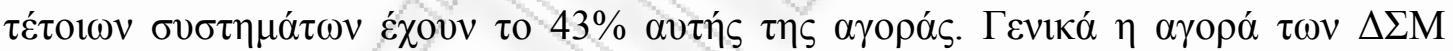

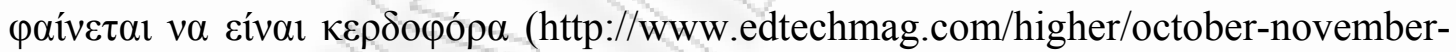
2006/tech-outlook.html).

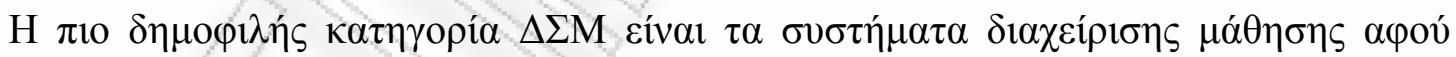

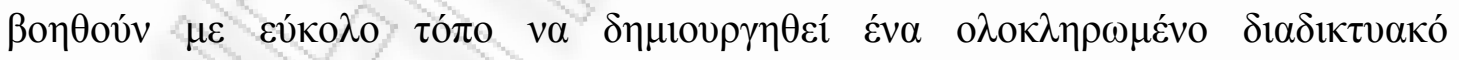

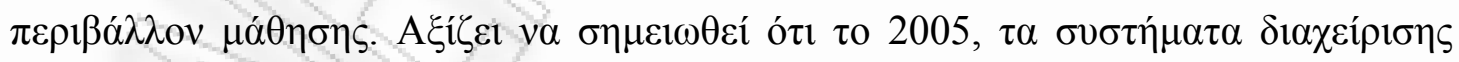

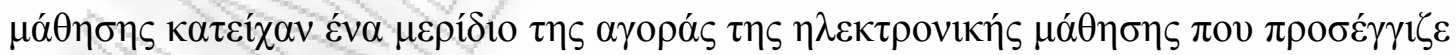

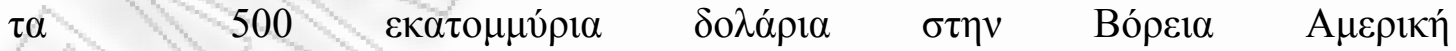

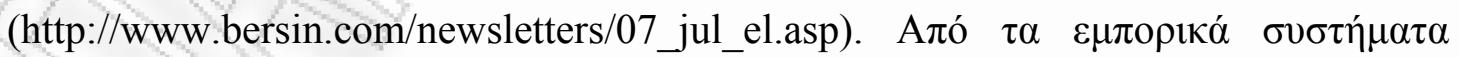

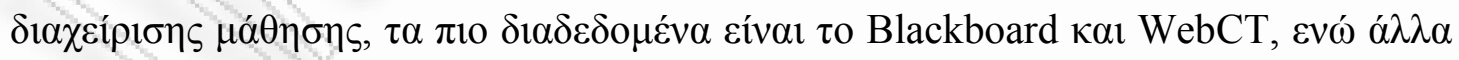

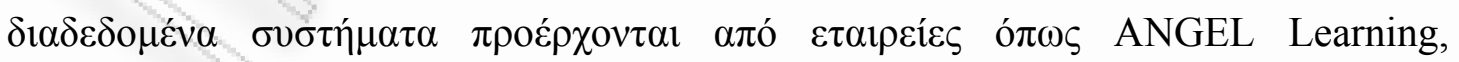
Desire2Learn, eCollege, Jenzabar and Ucompass.

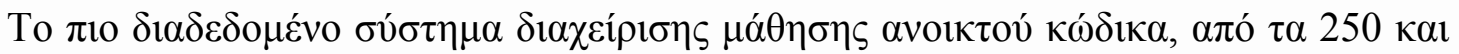

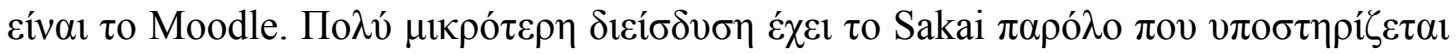




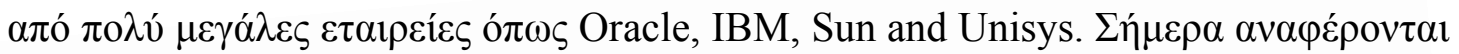

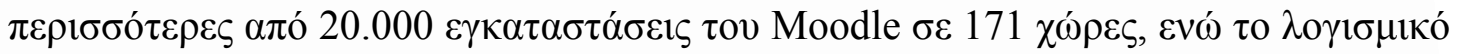

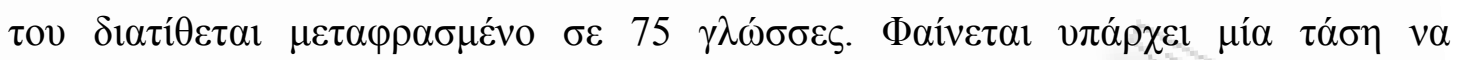

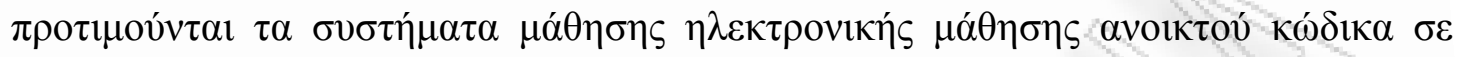

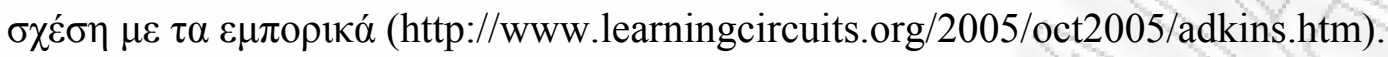

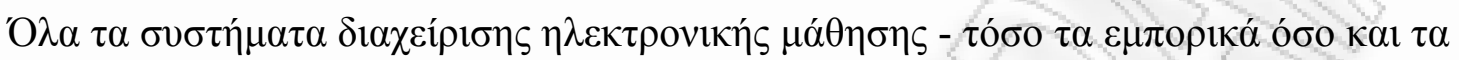

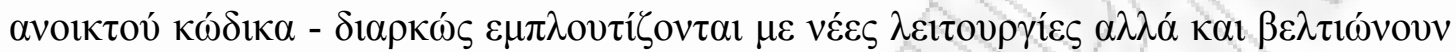

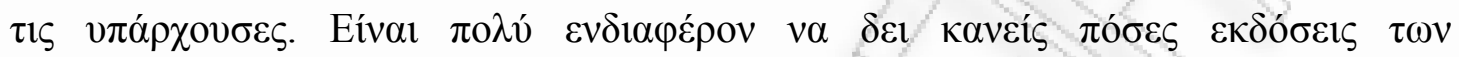

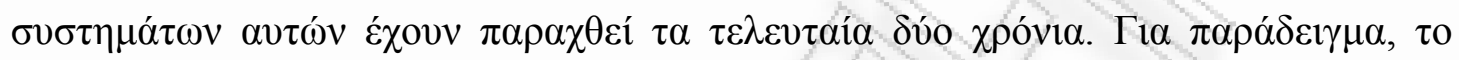

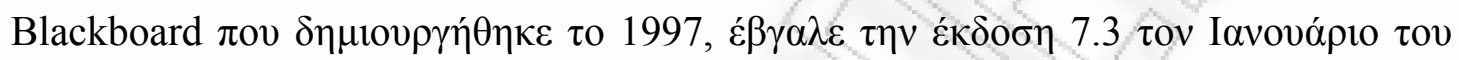

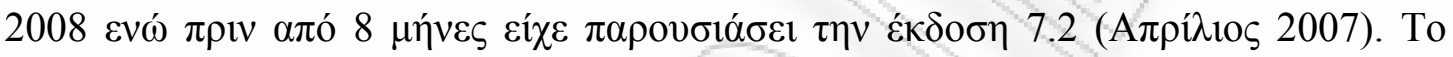

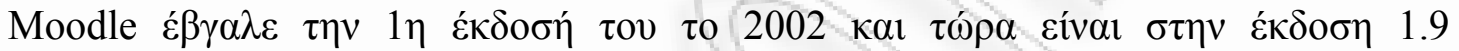

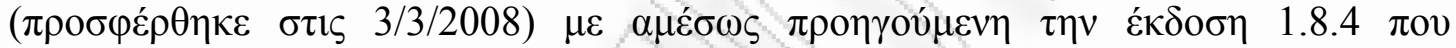

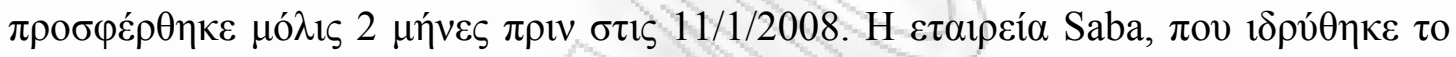

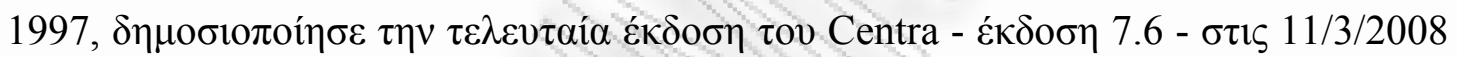

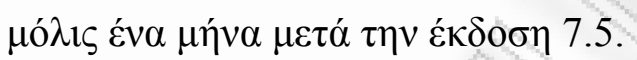

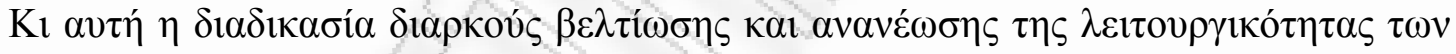

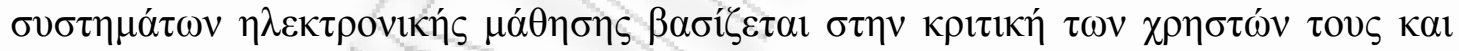

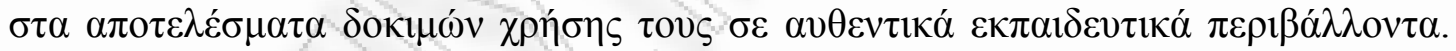

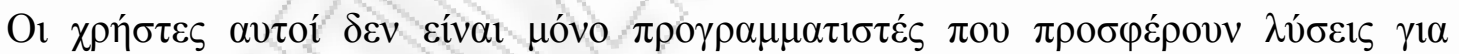

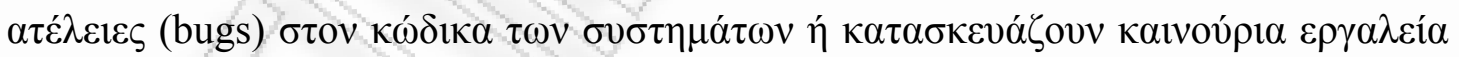

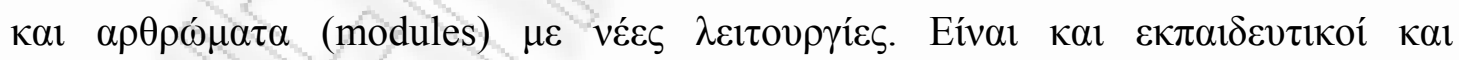

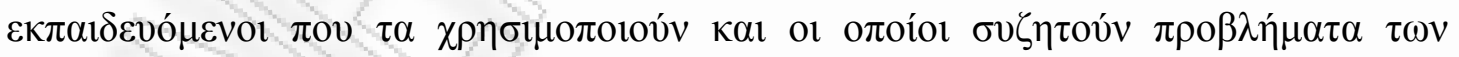

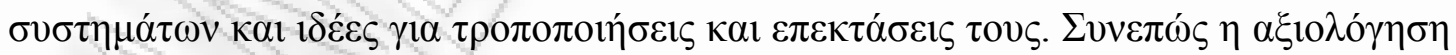

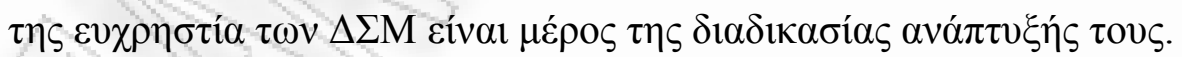

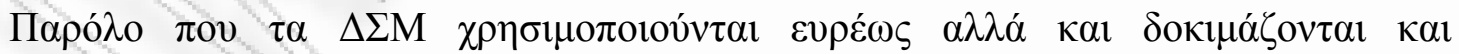

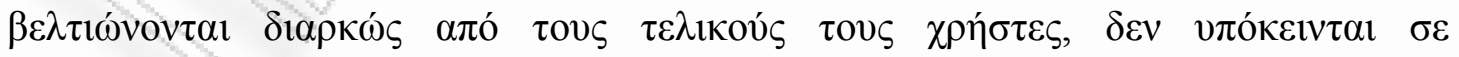

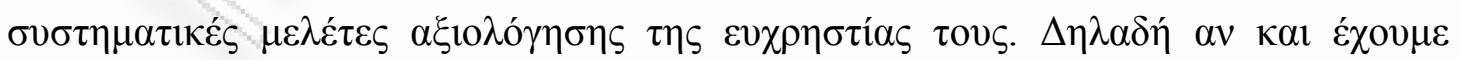

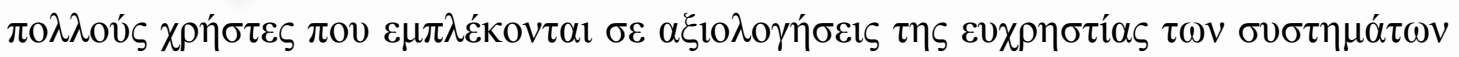

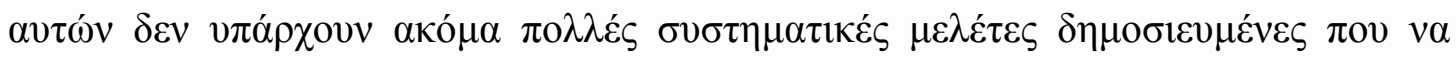

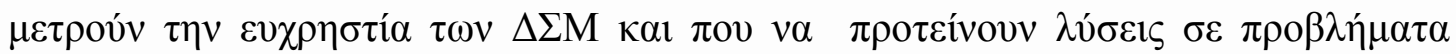

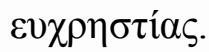




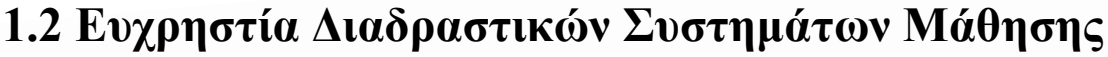

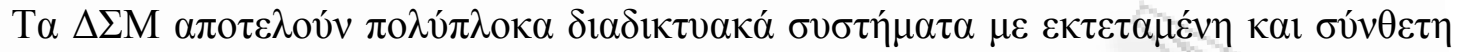

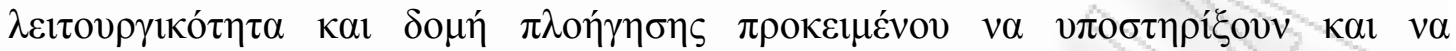

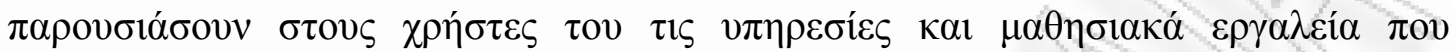

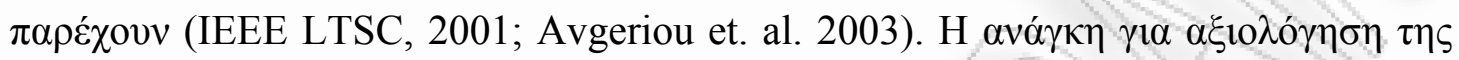

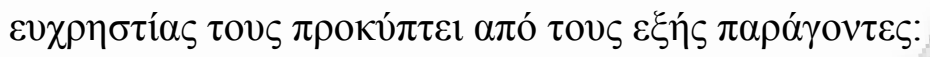

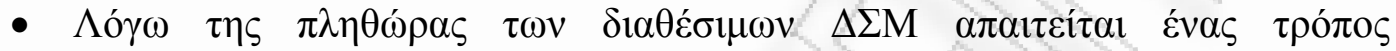

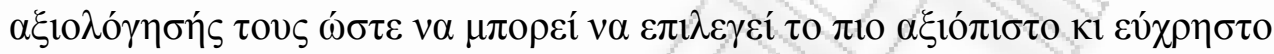

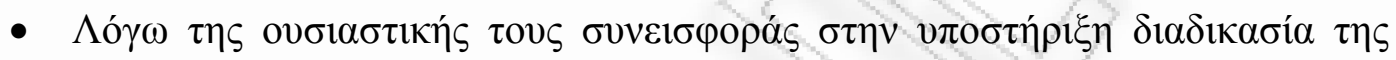

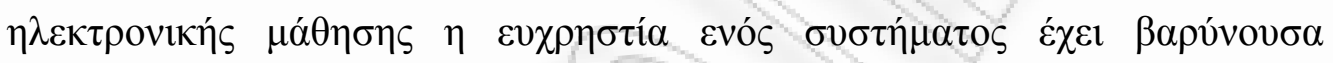

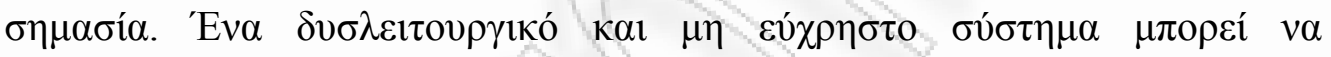

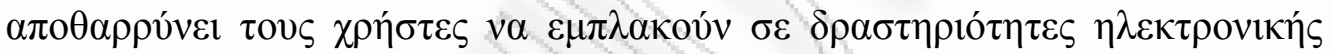

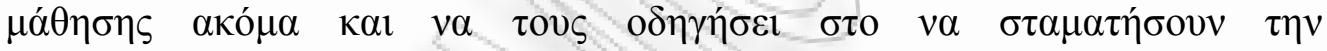

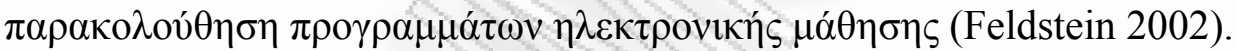

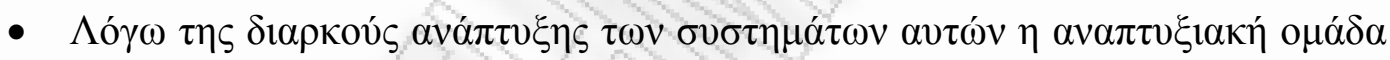

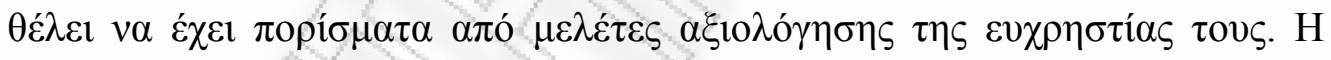

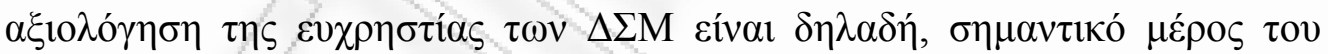

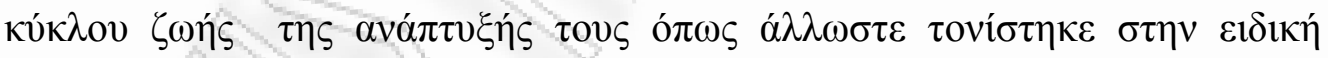

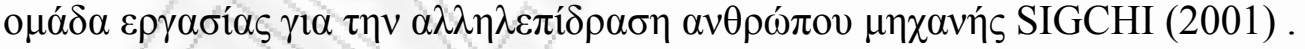

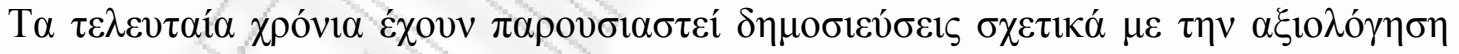

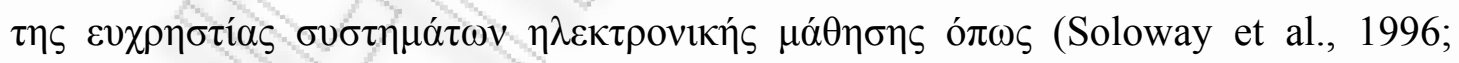
Squires, 1999; Squires \& Preece, 1999; Lohr, 2000; Notess, 2001; SIGCHI, 2001; Reeves et al., 2002; Brusilovsky et. al., 2004, Zaharias, 2004; M.J. Miller, 2005). H

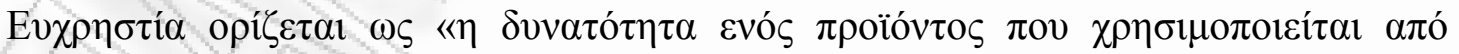

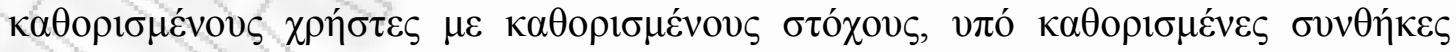

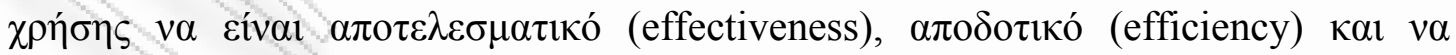

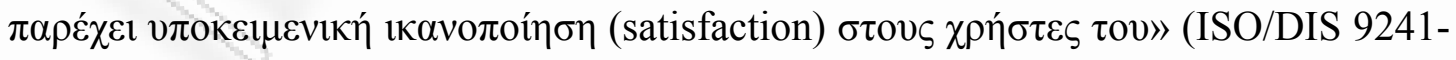
11).

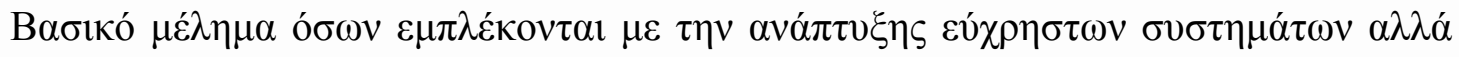

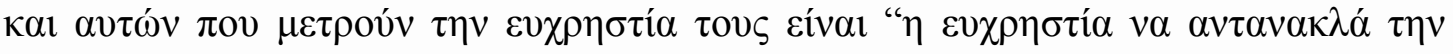

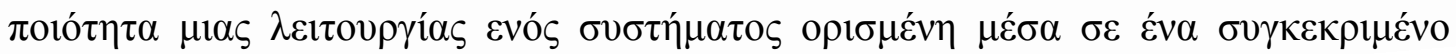




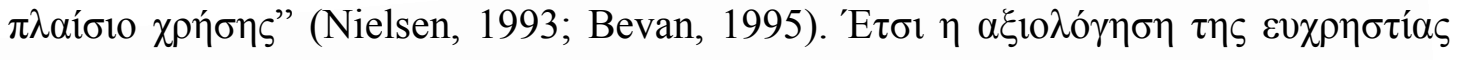

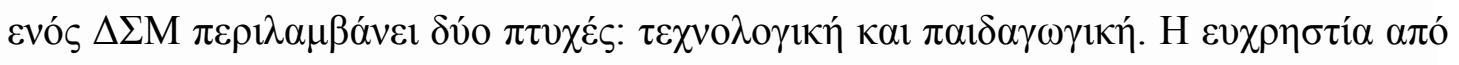

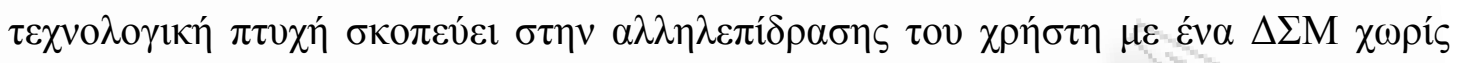

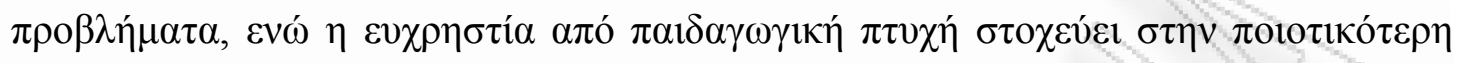

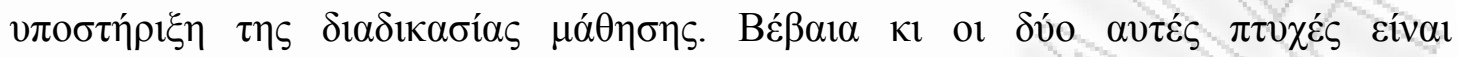
$\alpha \lambda \lambda \eta \lambda \circ \sigma v \sigma \chi \varepsilon \tau \imath \zeta o ́ \mu \varepsilon v \varepsilon \varsigma$.

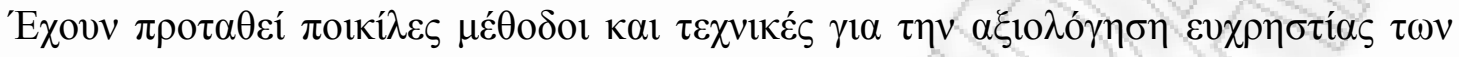

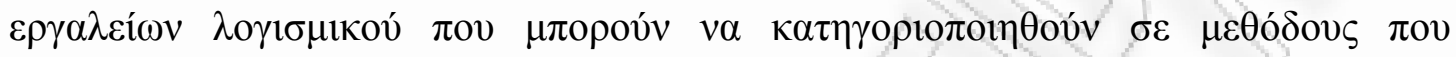

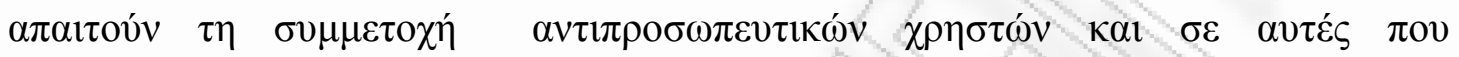

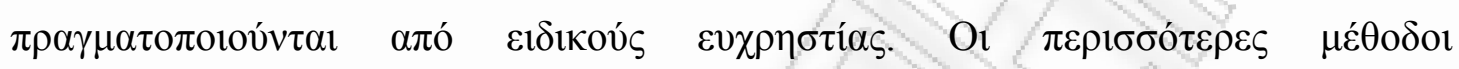

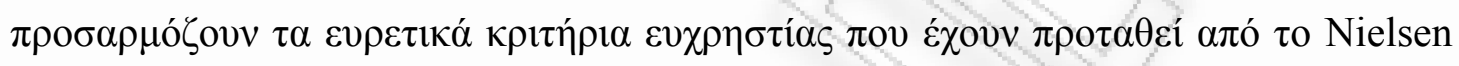

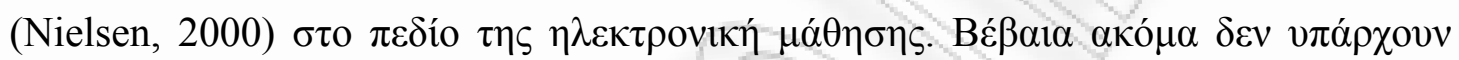

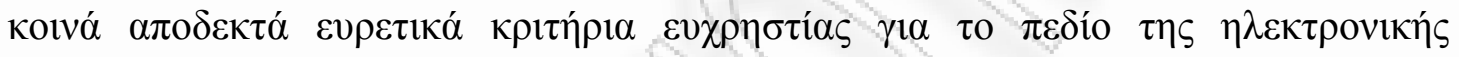

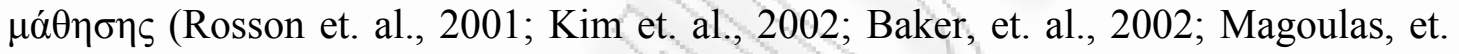

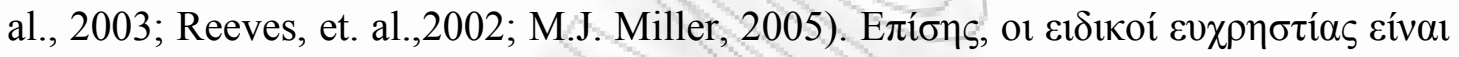

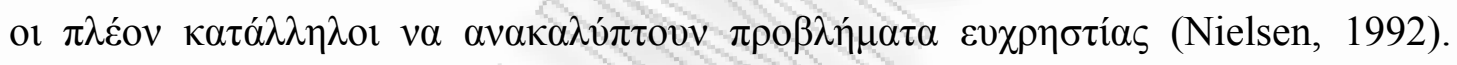

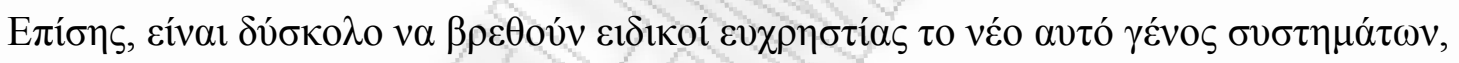

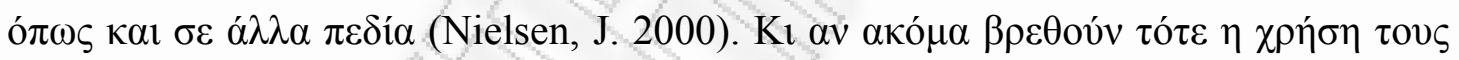

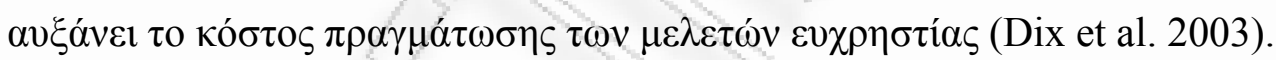

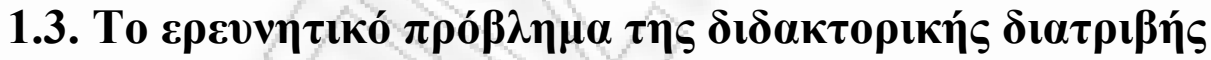

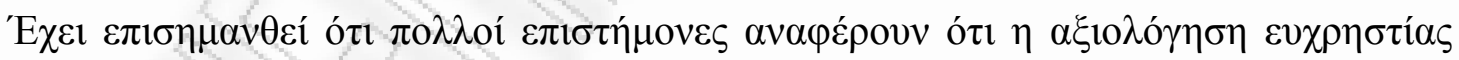

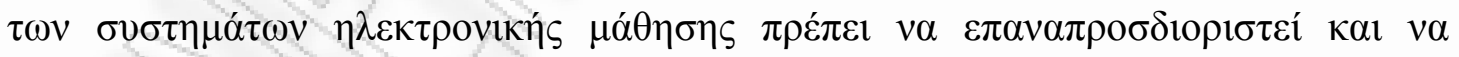

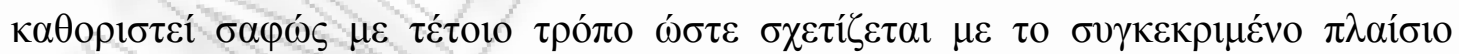

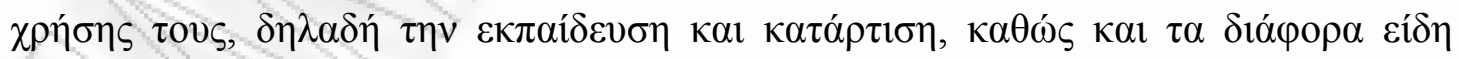

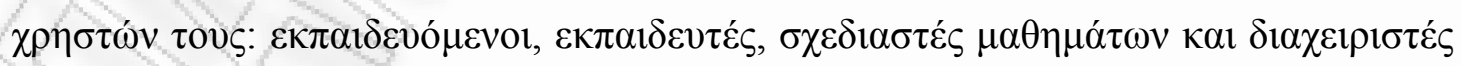
(Tselios et. al., 2008; Ardito et. al., 2006).

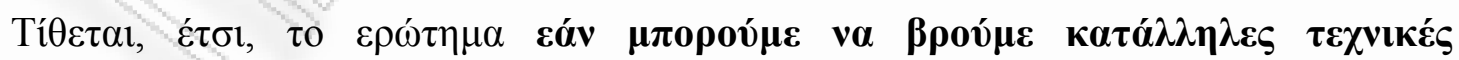

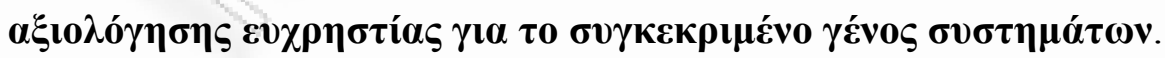

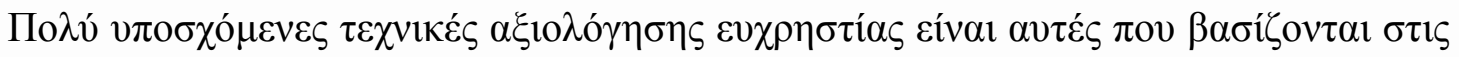

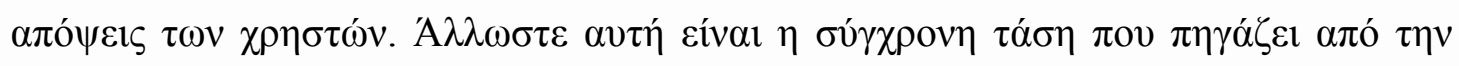




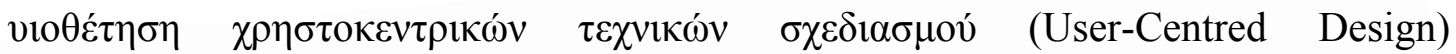

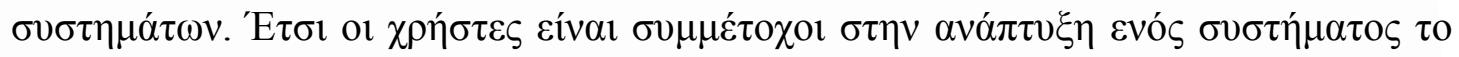

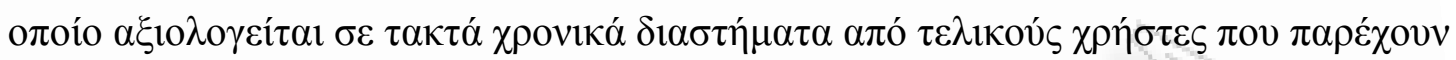

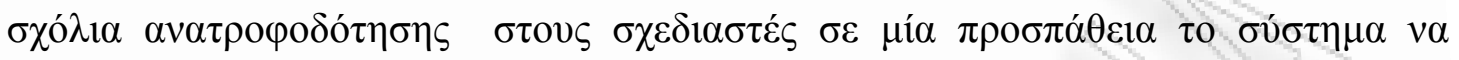

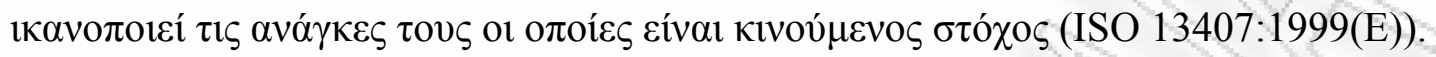

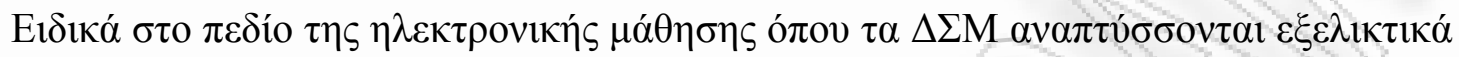

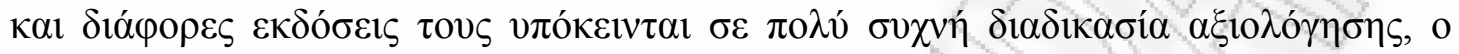

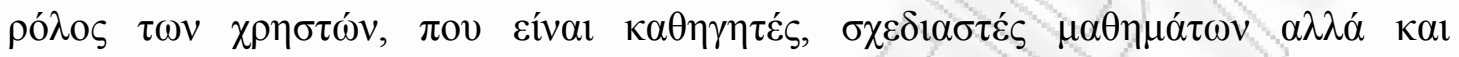

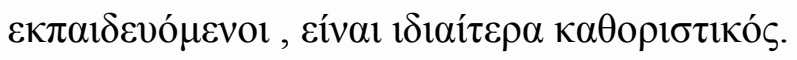

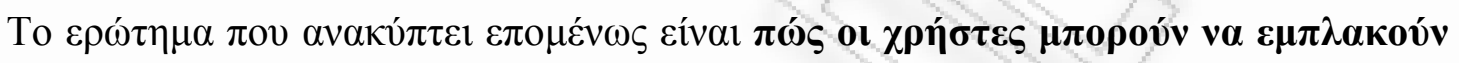

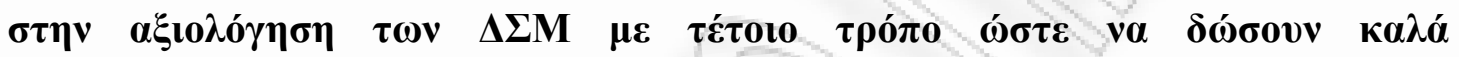

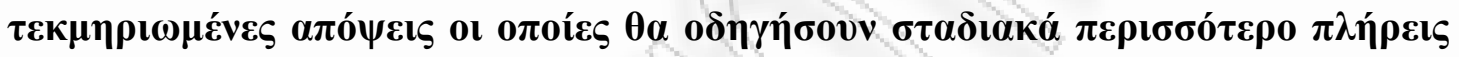

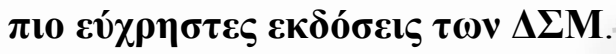

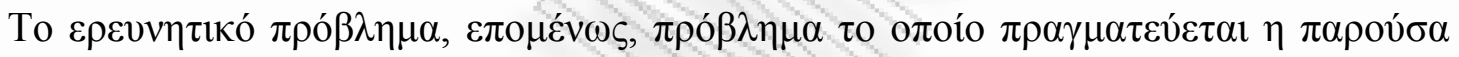
$\delta ı \alpha \alpha \tau$ о

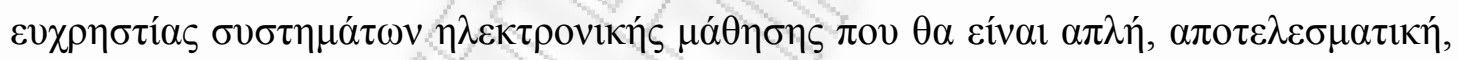

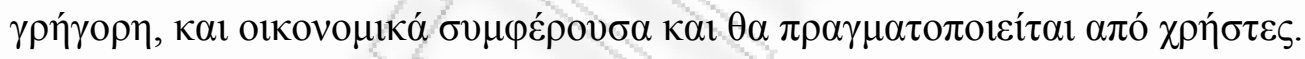

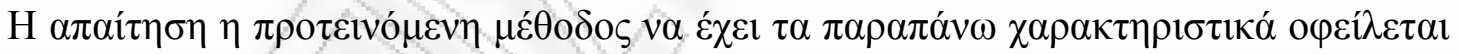

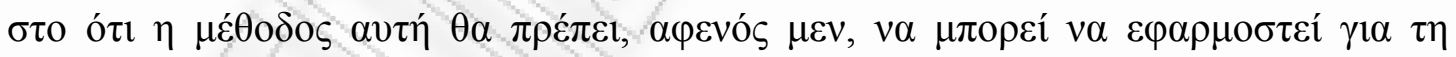

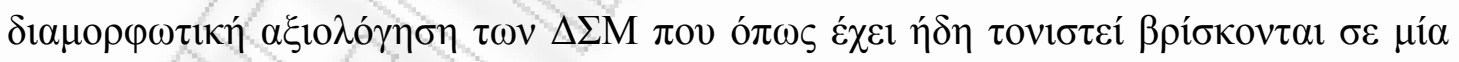

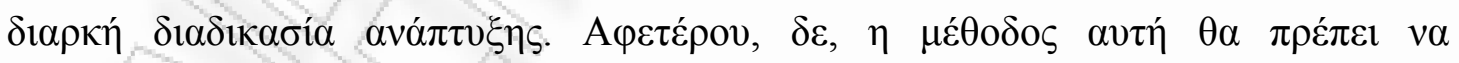
$\chi \rho \eta \sigma 1 \mu о \pi о \eta \eta \theta \varepsilon i ́ ~ \mu \varepsilon$ a

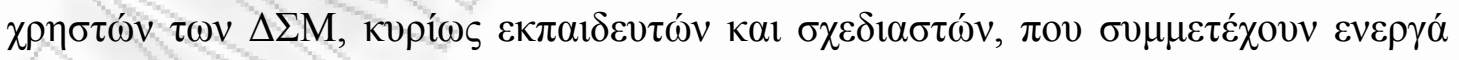

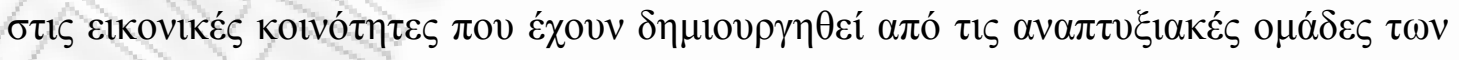
$\Delta \Sigma \mathrm{M}$.

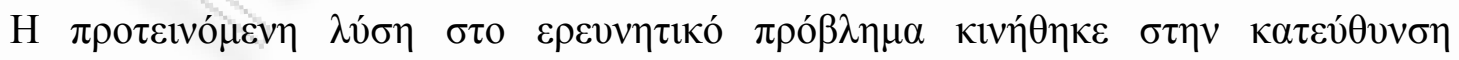

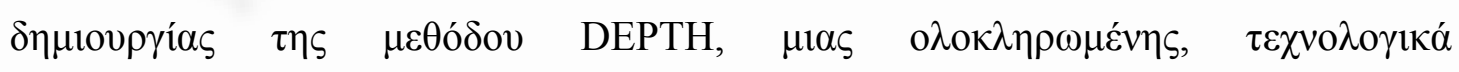

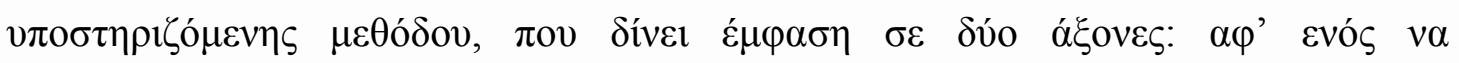

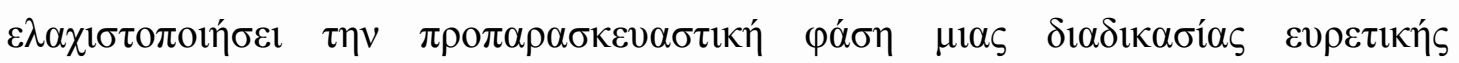

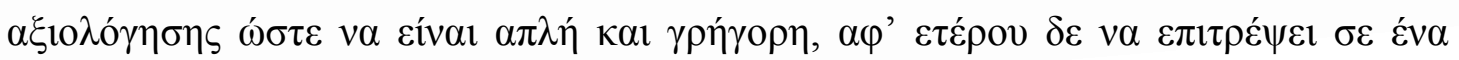




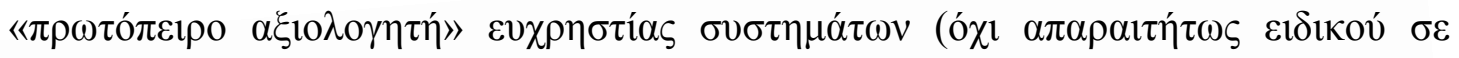

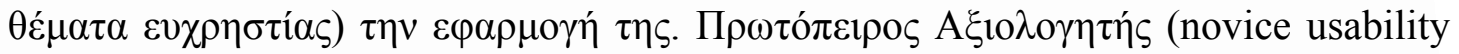

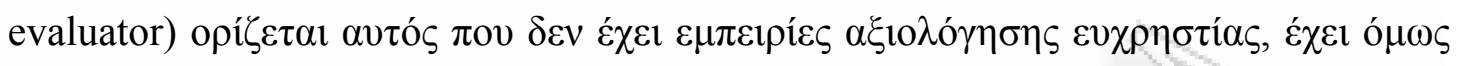

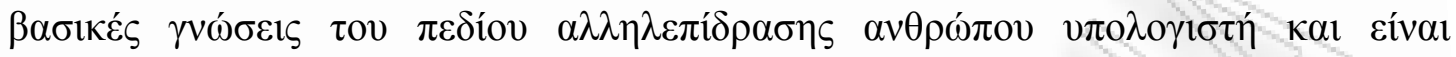

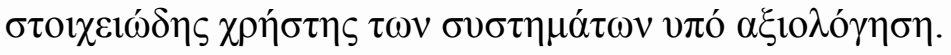

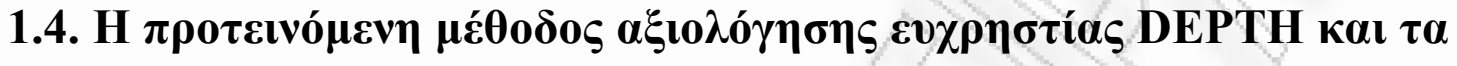

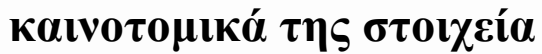

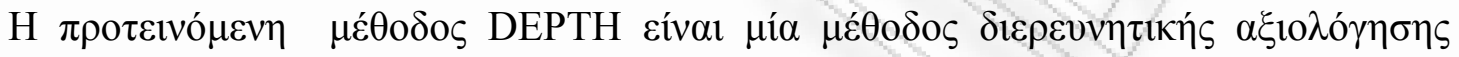

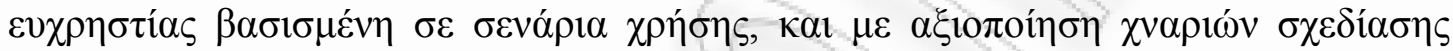

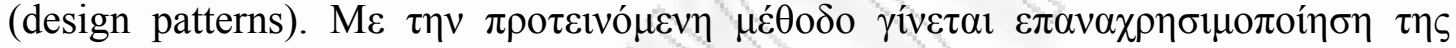

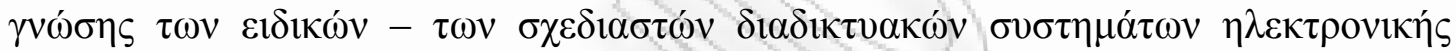

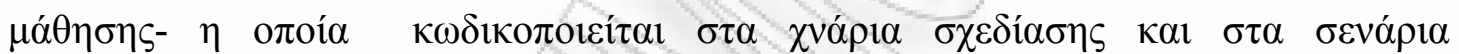

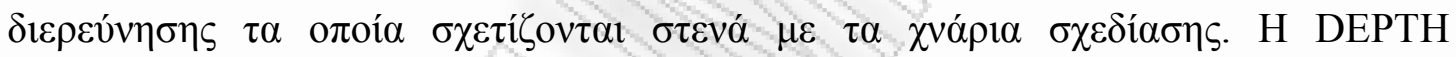

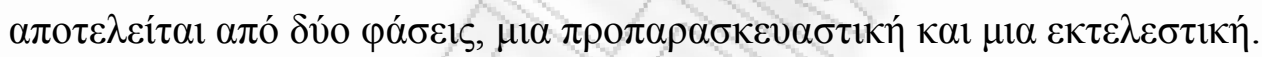

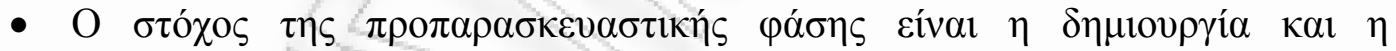

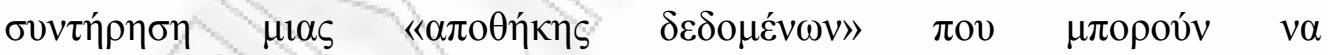

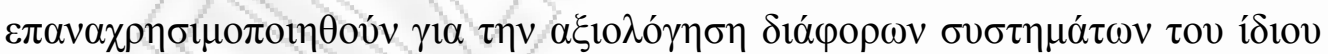

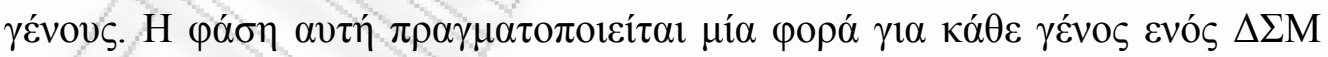

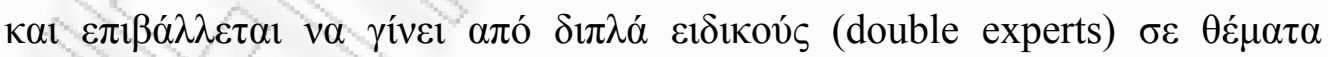

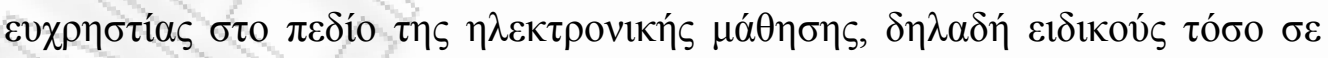

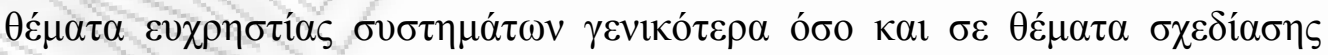

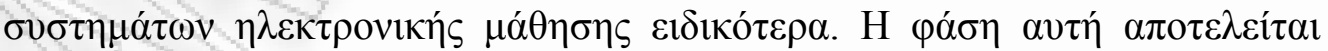

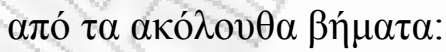

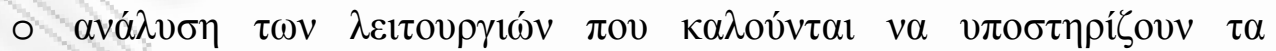

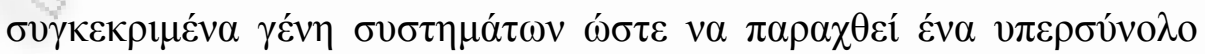
$\lambda \varepsilon ı \tau o v \rho \gamma ı \kappa o ́ \tau \eta \tau \alpha \varsigma$ (functionality superset)

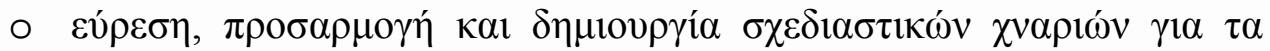

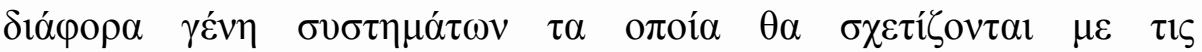
$\pi \rho \circ \alpha \nu \alpha \varphi \varepsilon \rho \theta \varepsilon i ́ \sigma \varepsilon \varsigma \lambda \varepsilon \varepsilon \imath \tau 0 \nu \rho \gamma i ́ \varepsilon \varsigma$ (related design patterns) 


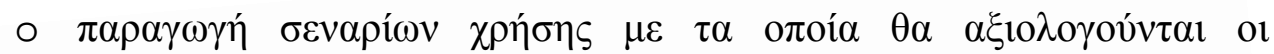

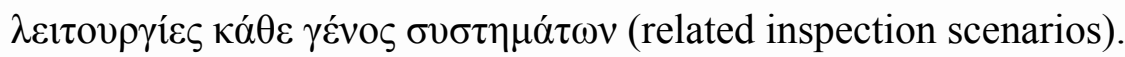

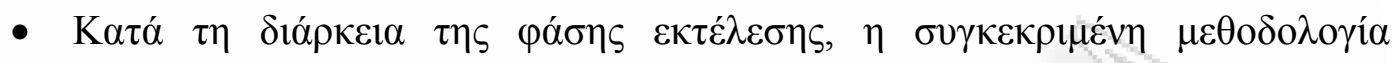

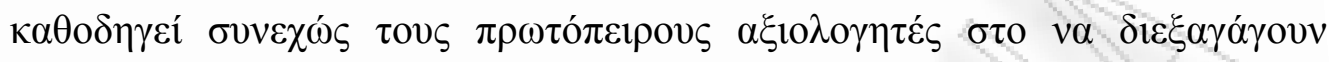

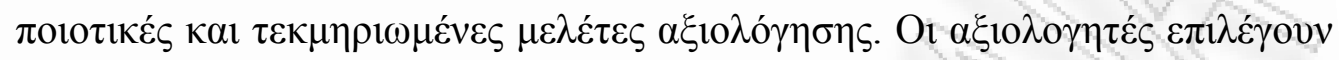

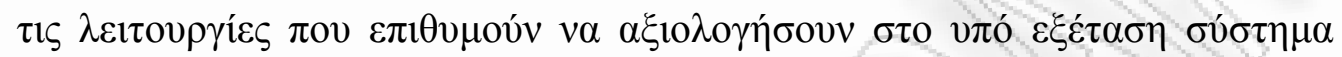

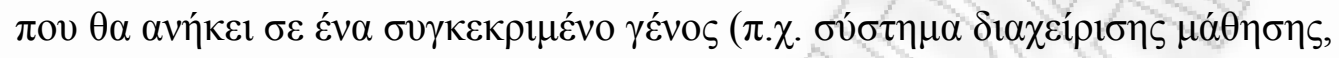

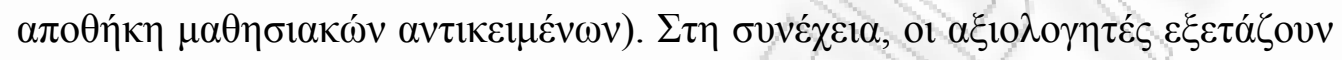

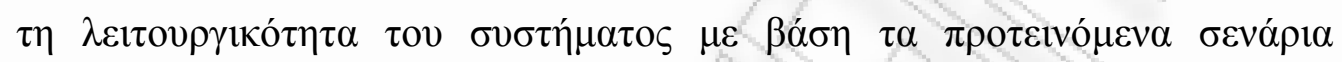

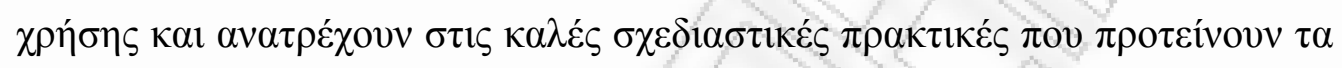

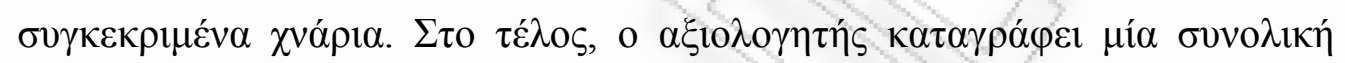

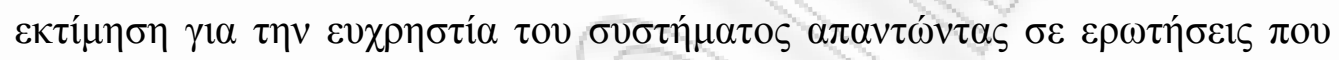

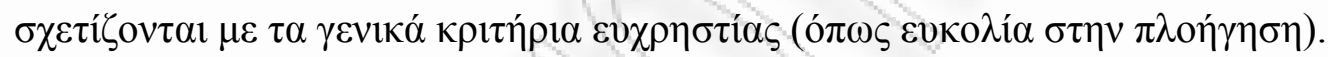

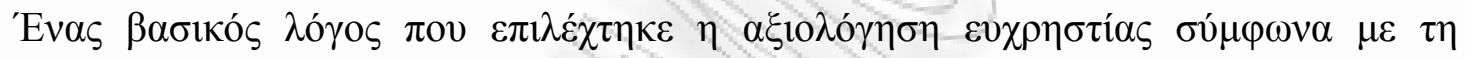

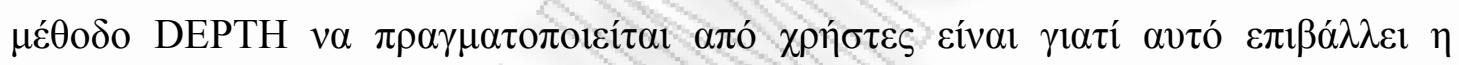

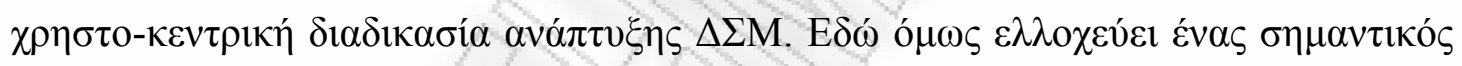

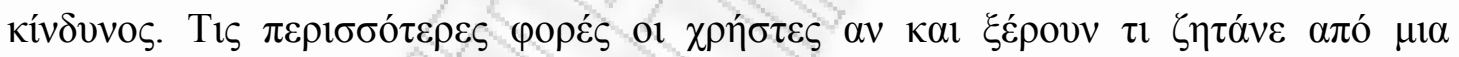

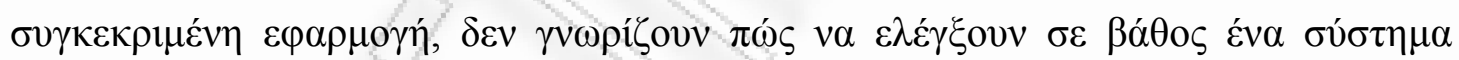

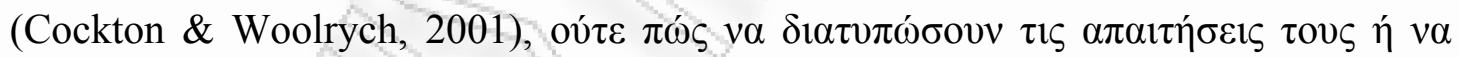

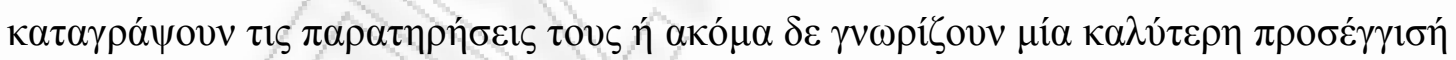

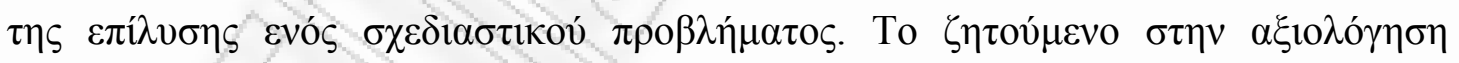

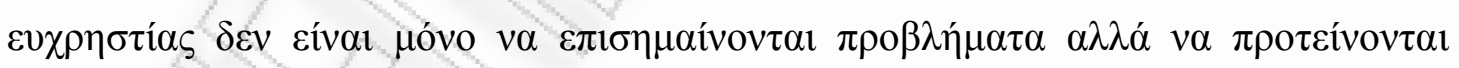
$\lambda$

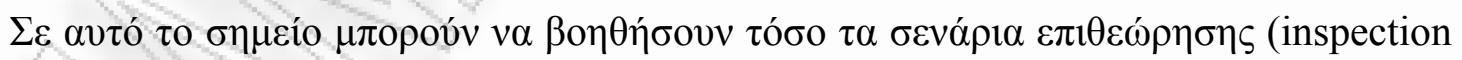

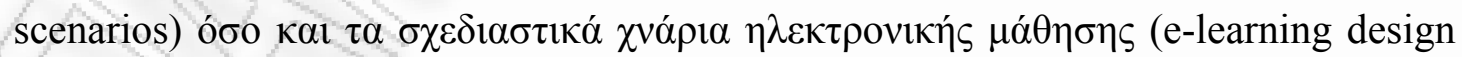

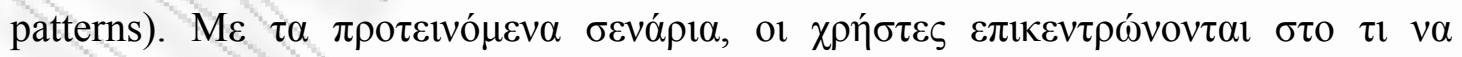

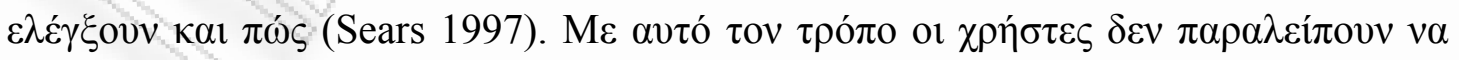

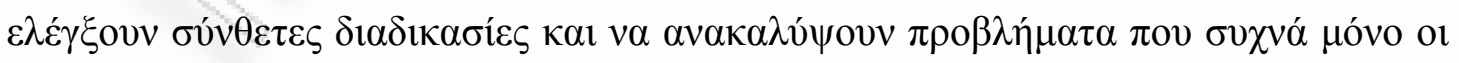

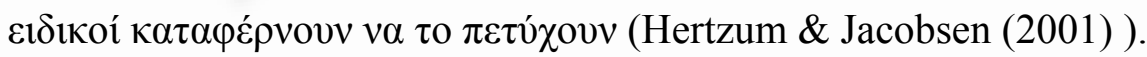

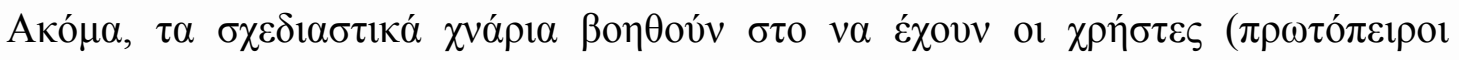

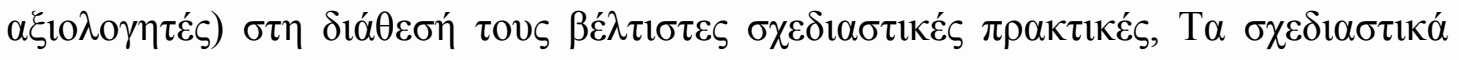

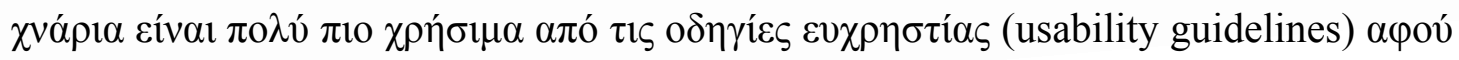




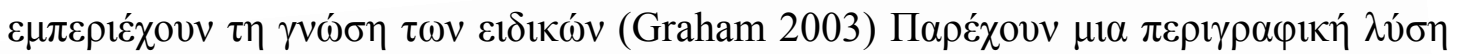

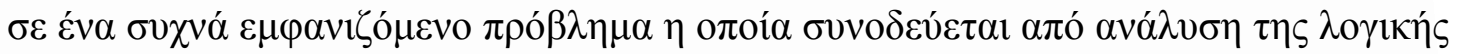

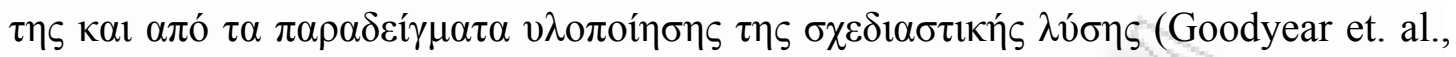
2004b).

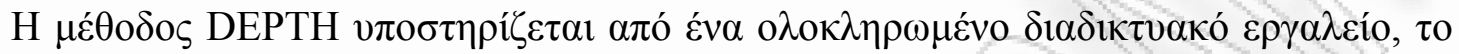

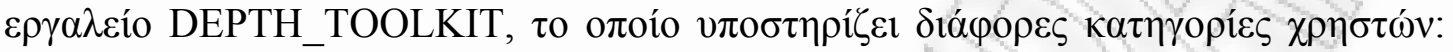

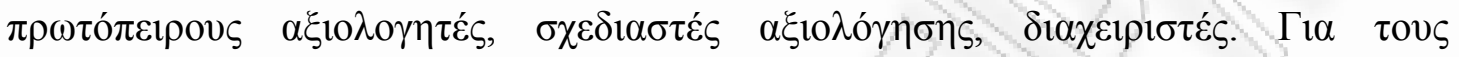

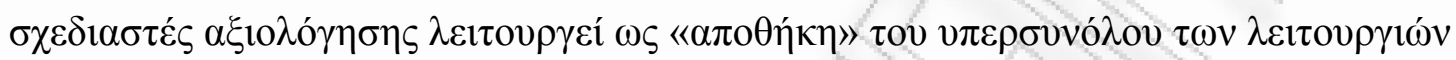

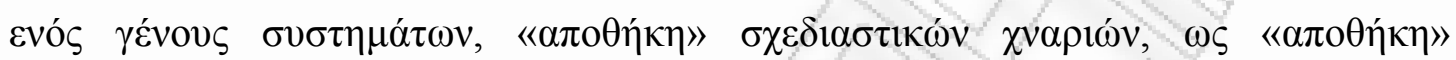

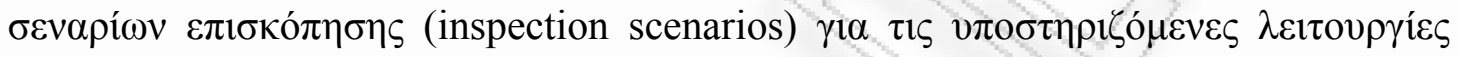

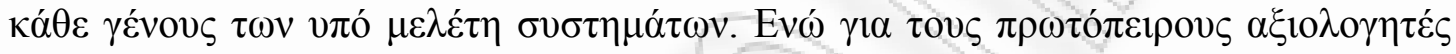

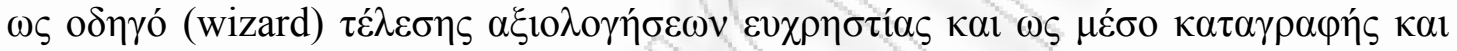

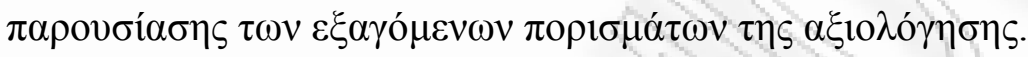

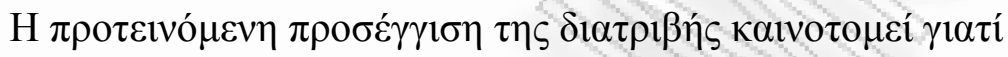

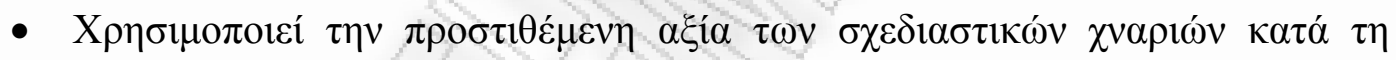

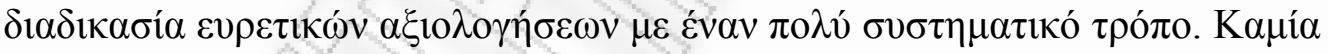

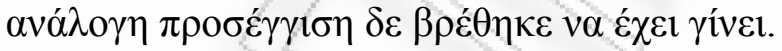

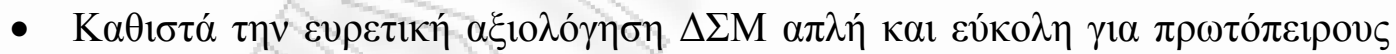

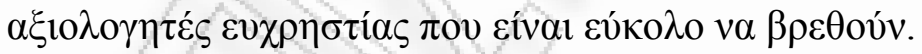

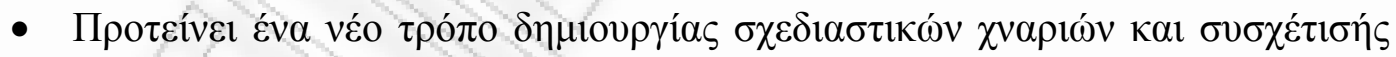

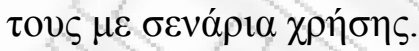

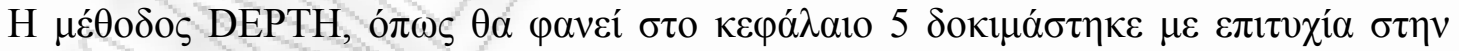

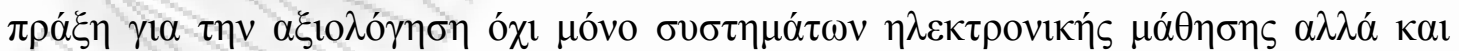

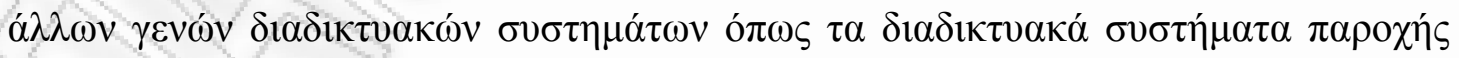

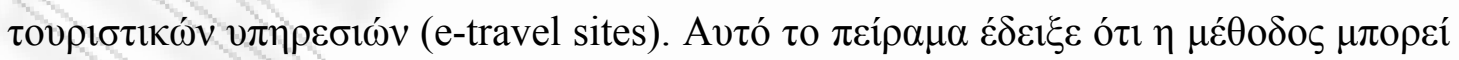

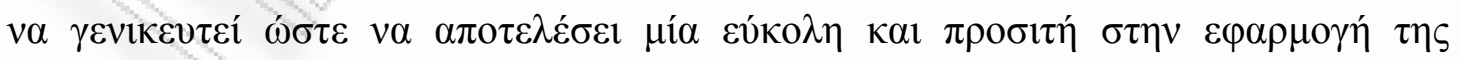

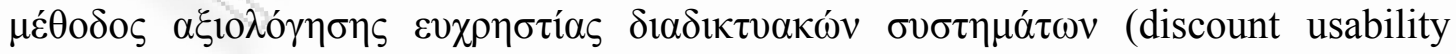

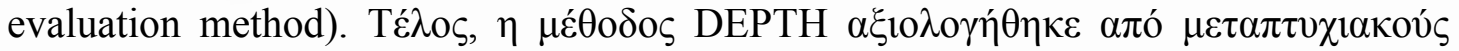

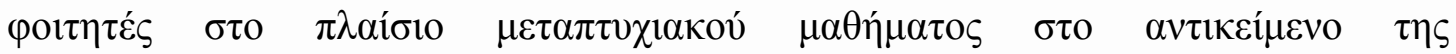




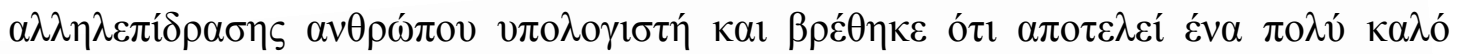

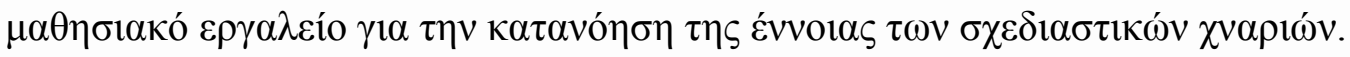

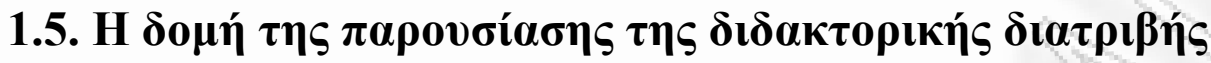

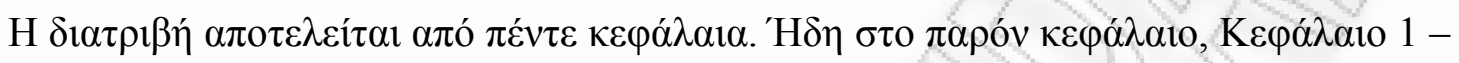

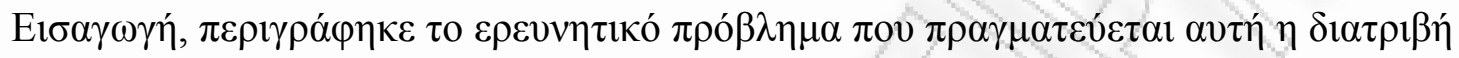

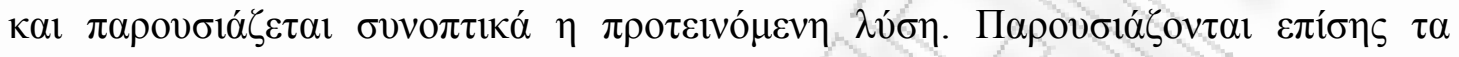

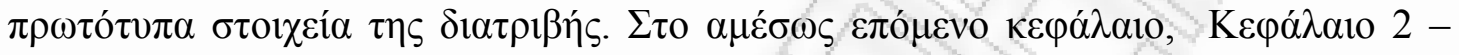

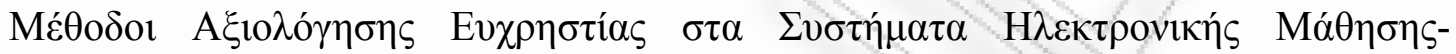

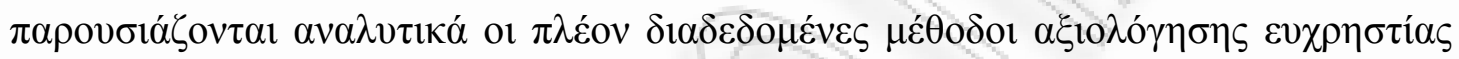

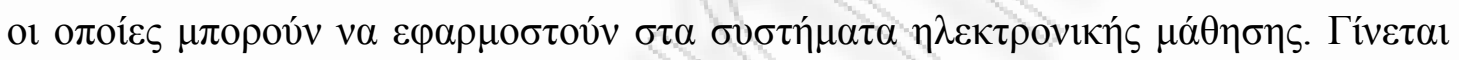

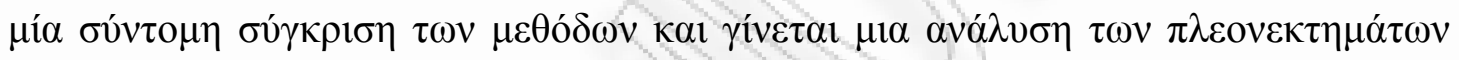

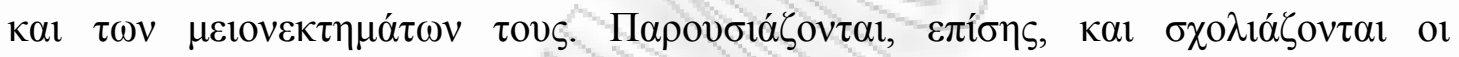

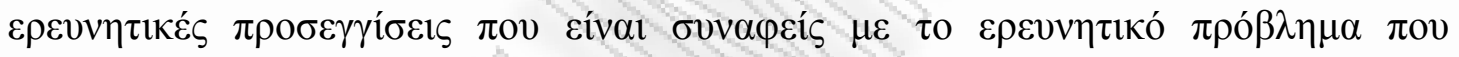

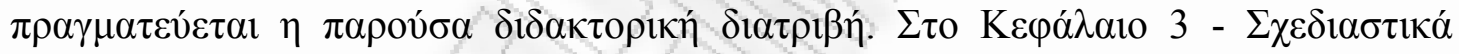

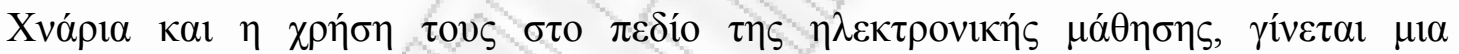

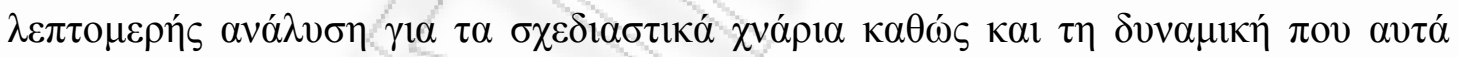

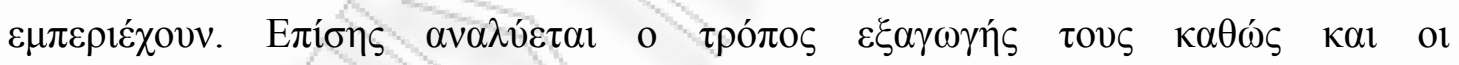

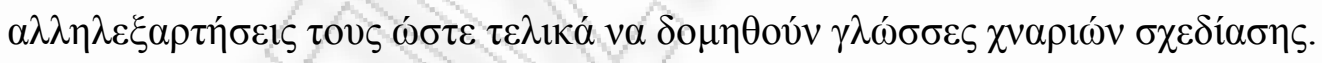

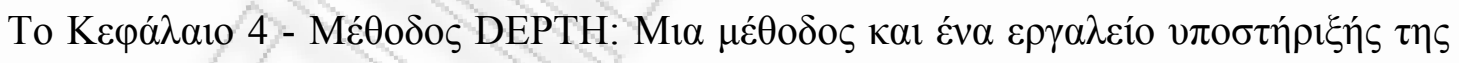

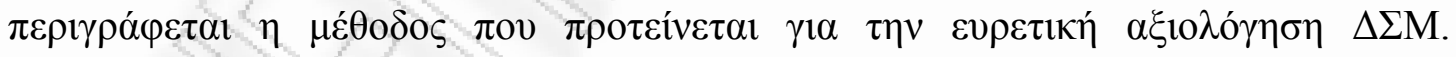

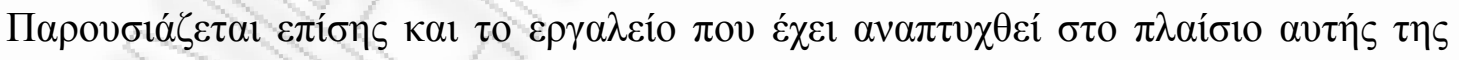

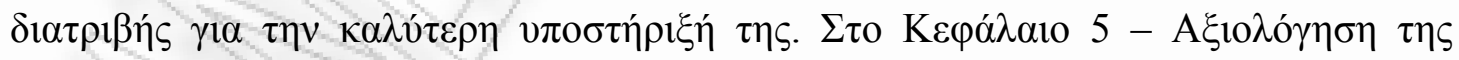

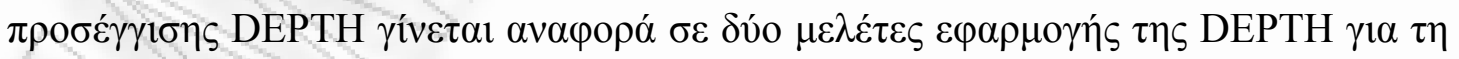

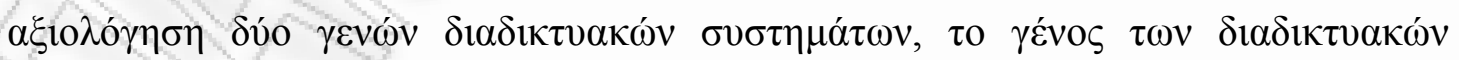

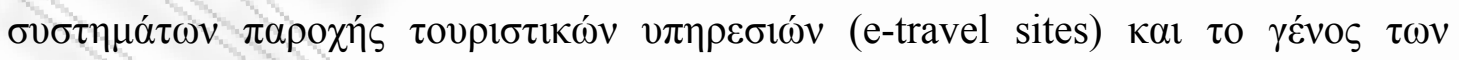

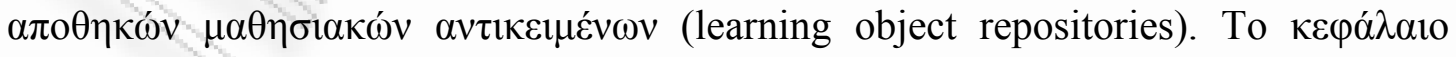

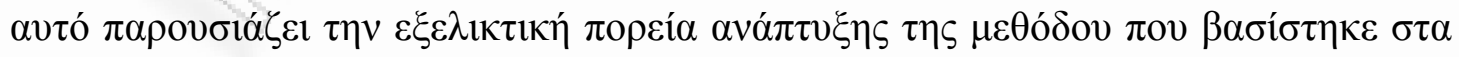

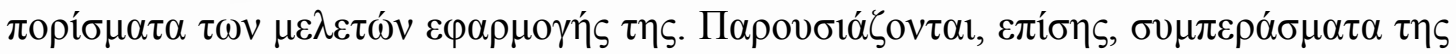

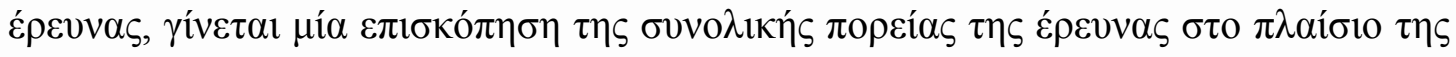

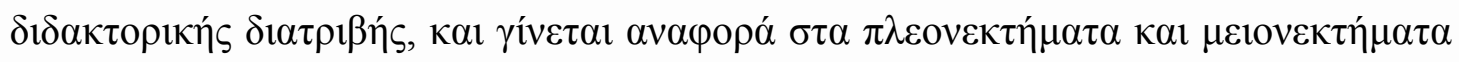




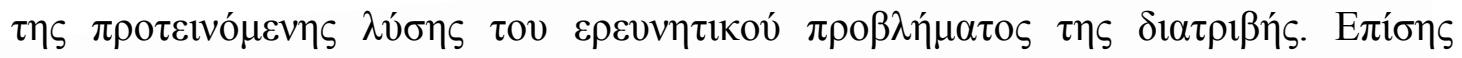

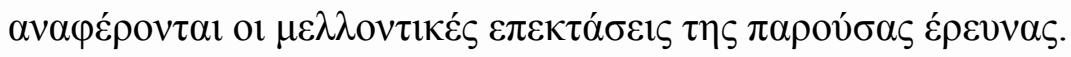

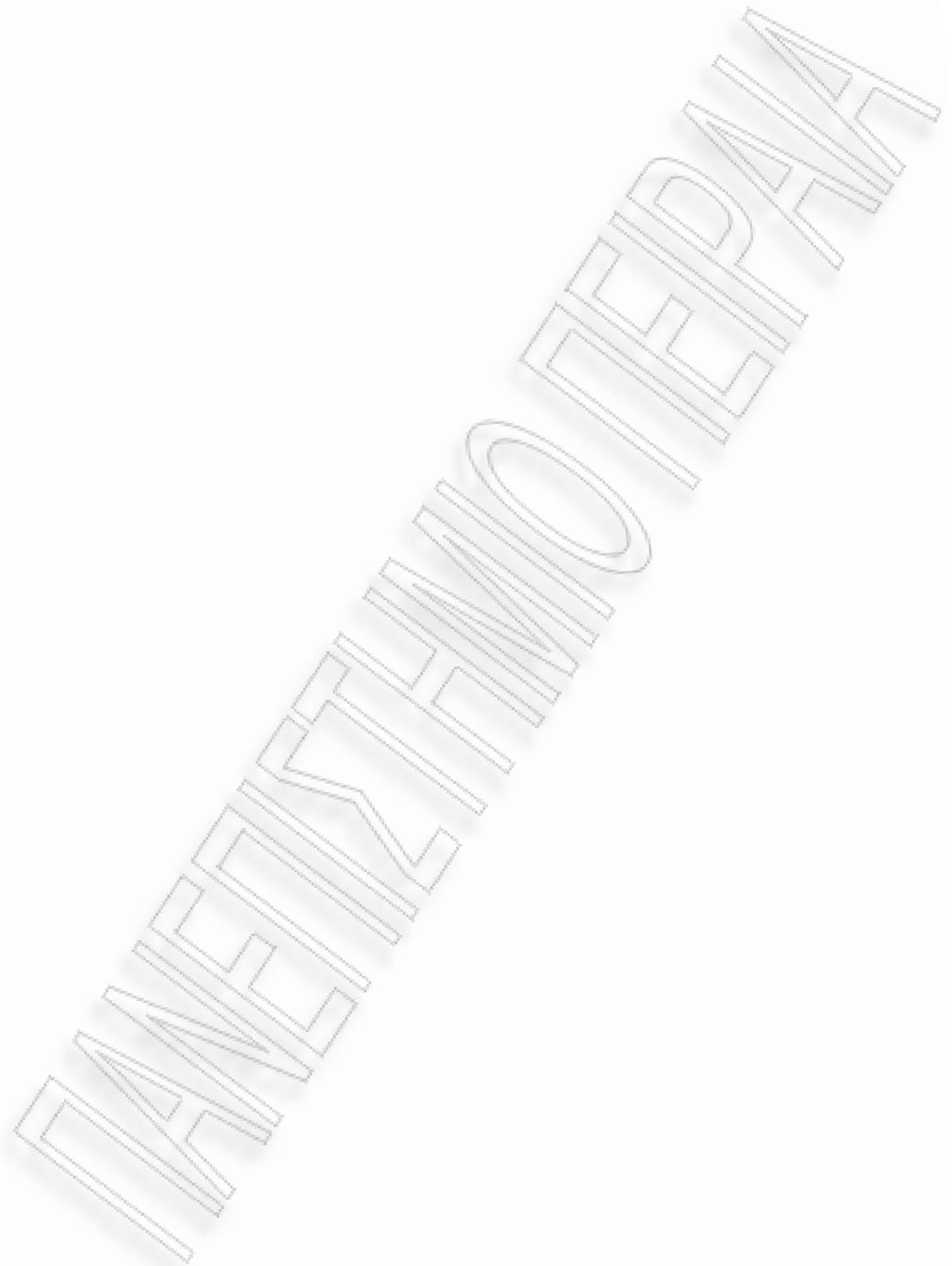




\section{КЕФАААIO 2

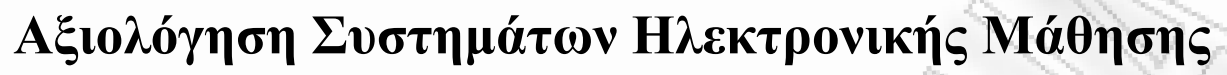

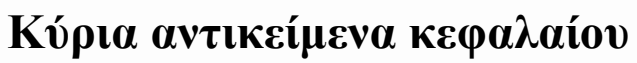

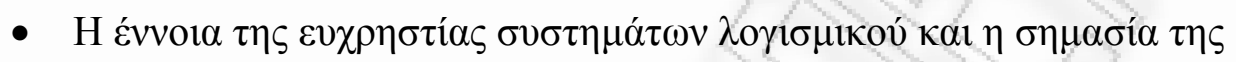

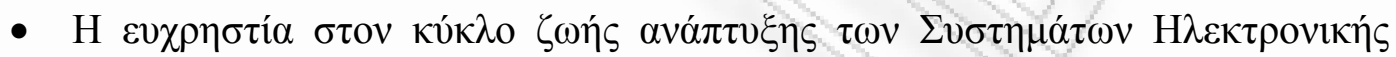

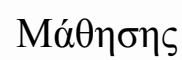

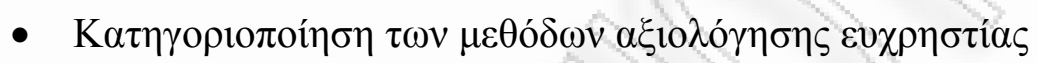

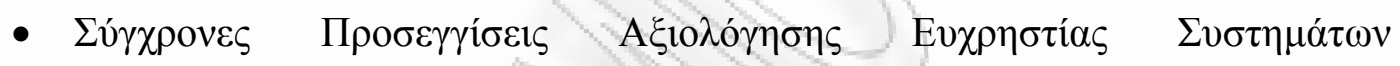

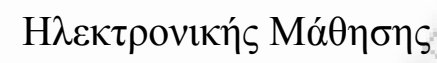

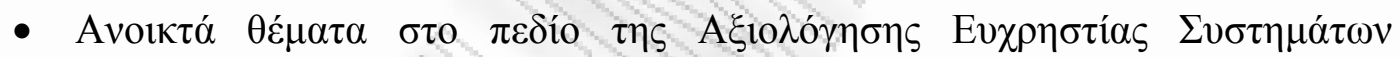

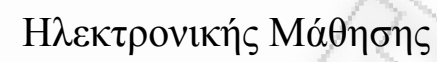

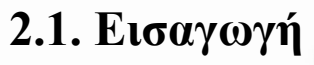

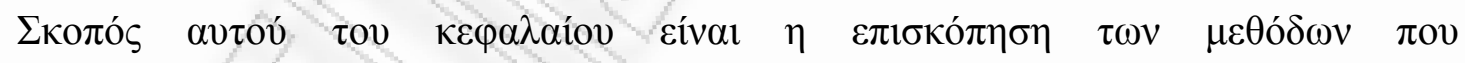

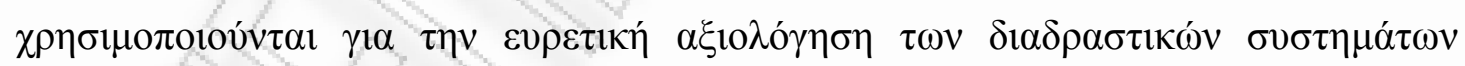

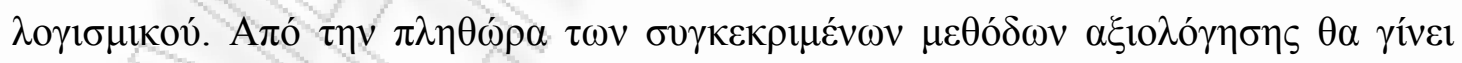

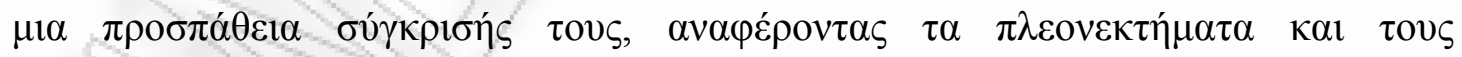

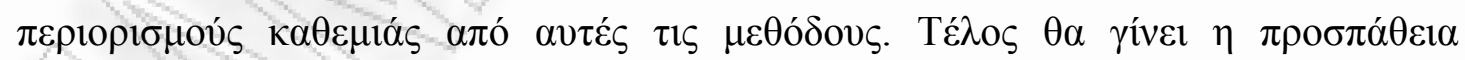

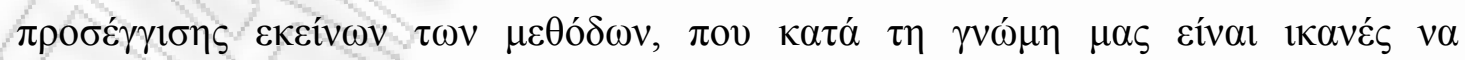

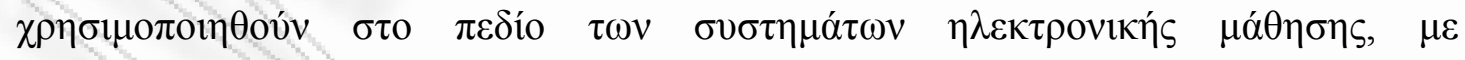

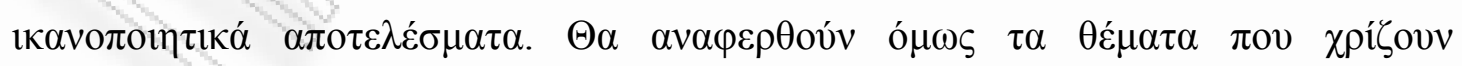

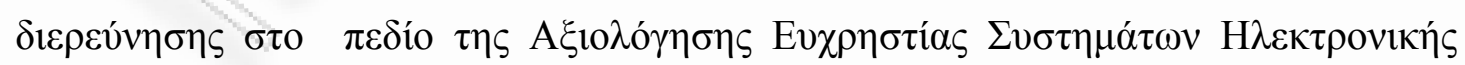

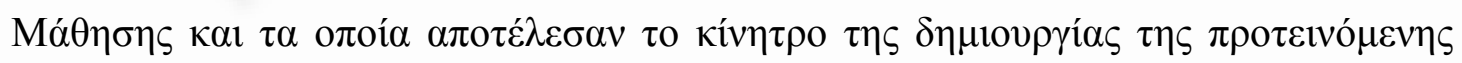

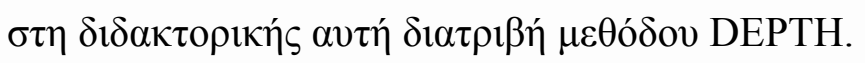




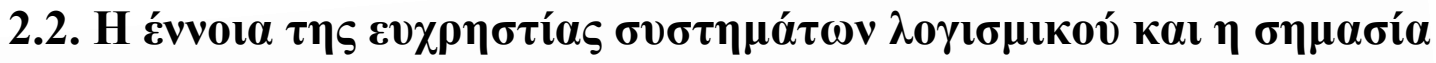 $\tau \eta \varsigma$}

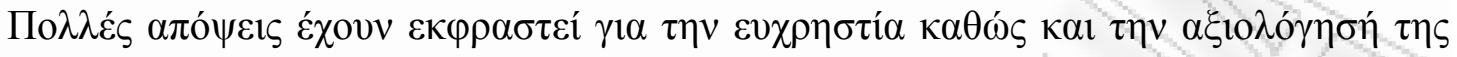

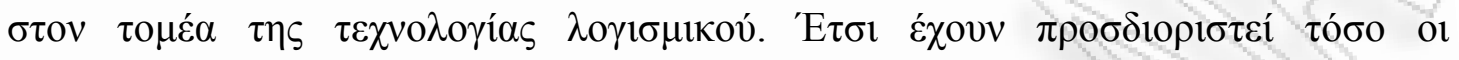

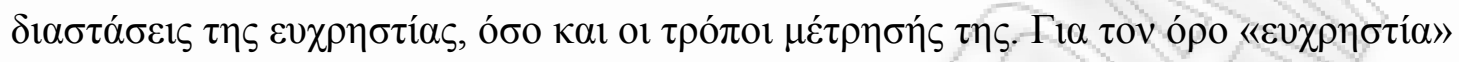

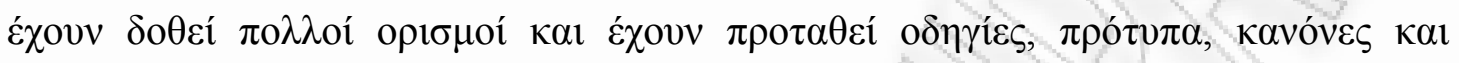

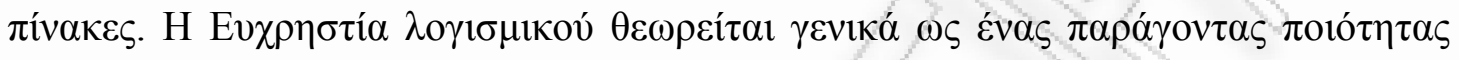

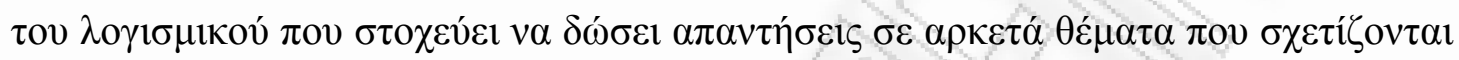

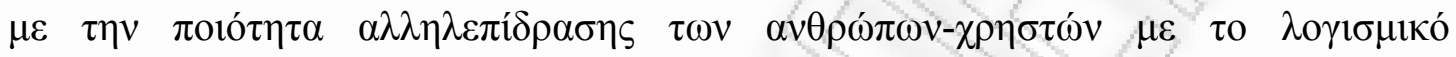
$\tau \varepsilon \chi v o \lambda$ ojías.

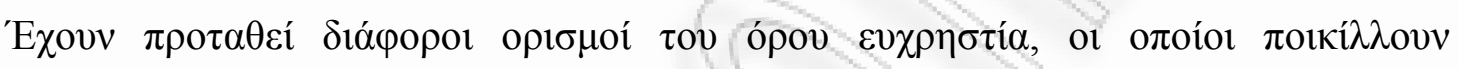

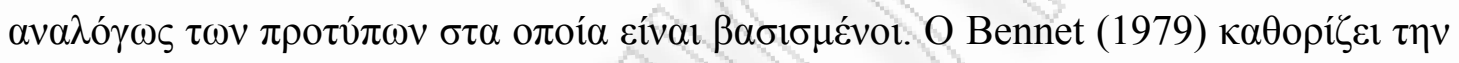

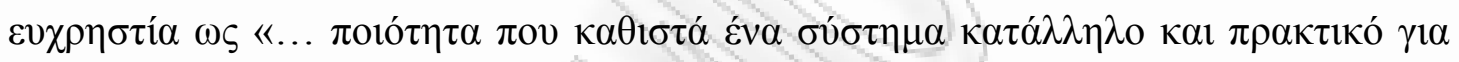

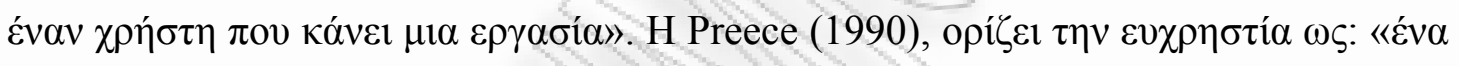

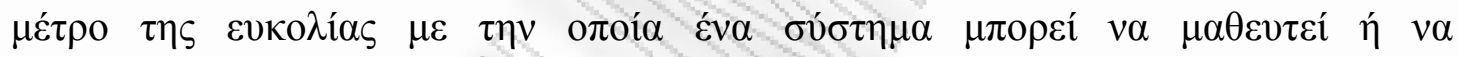

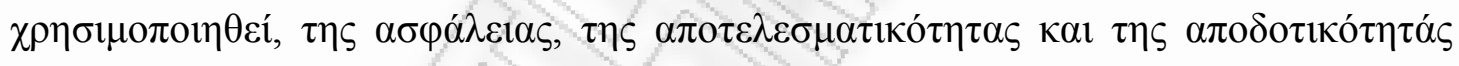

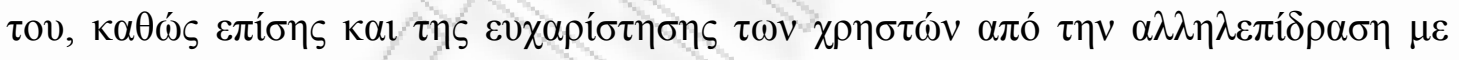

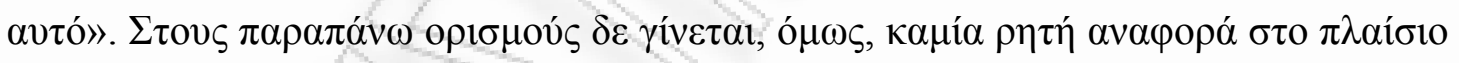

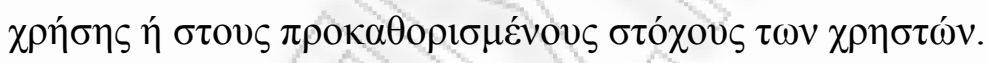

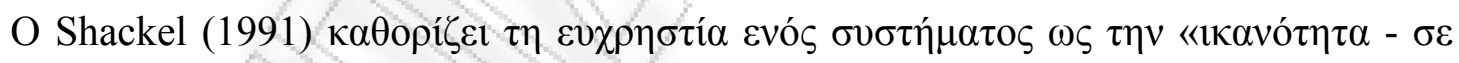

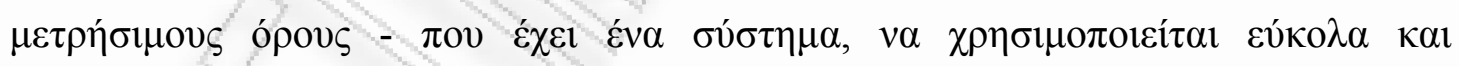

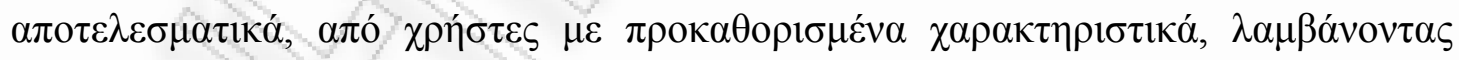

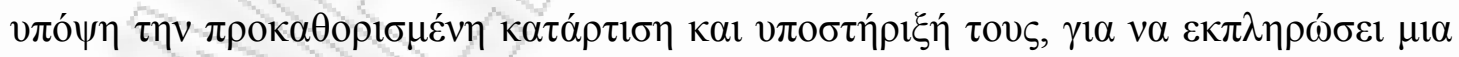

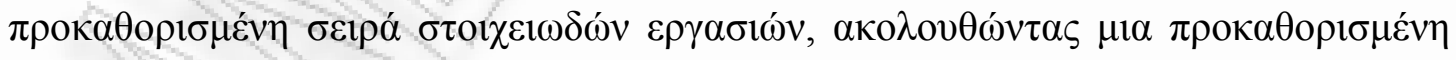

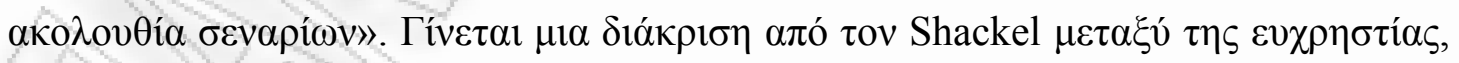

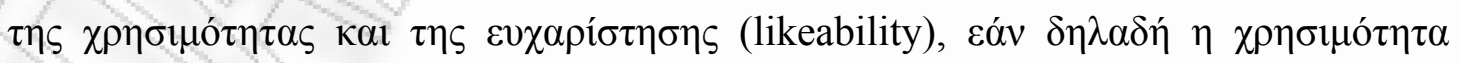

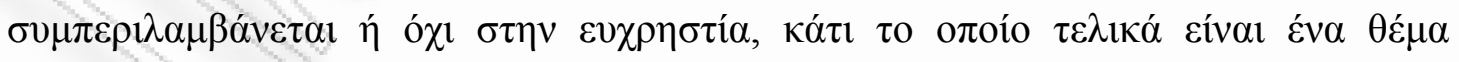

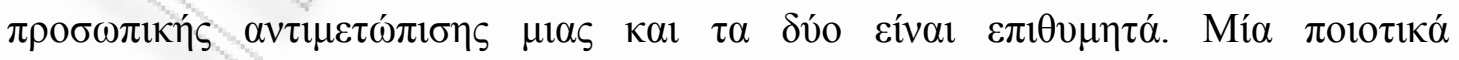

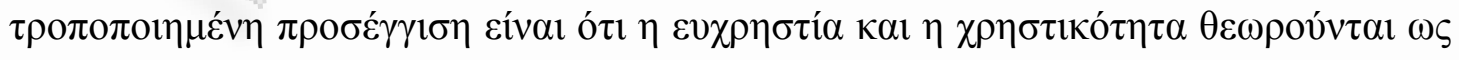

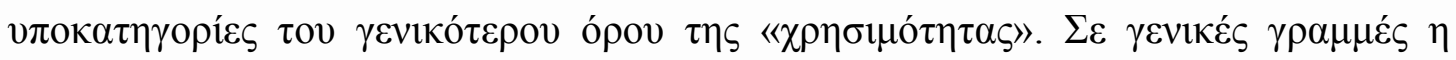

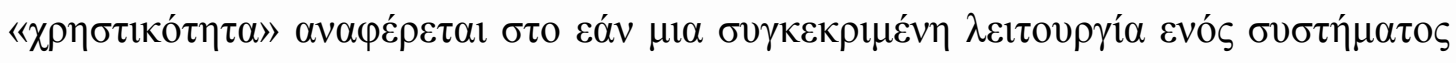

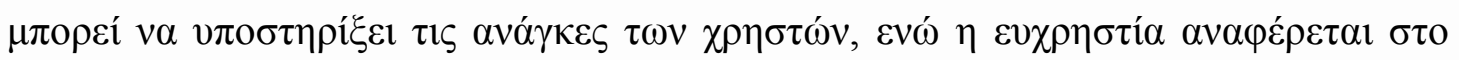




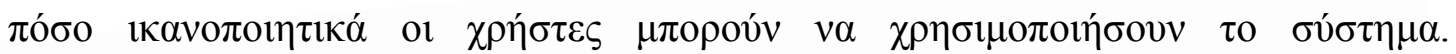

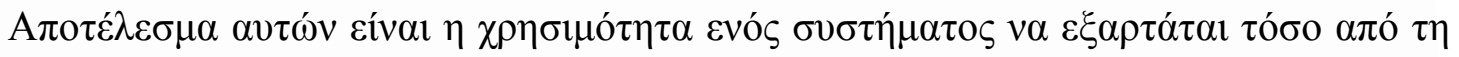

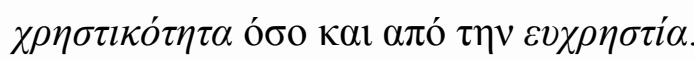

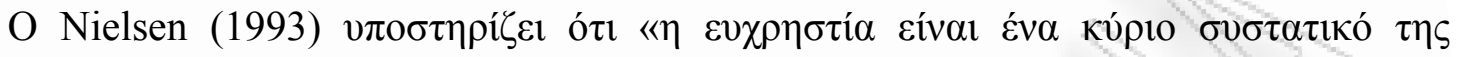

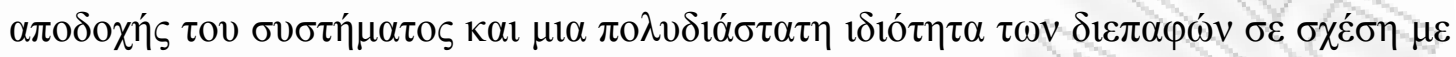

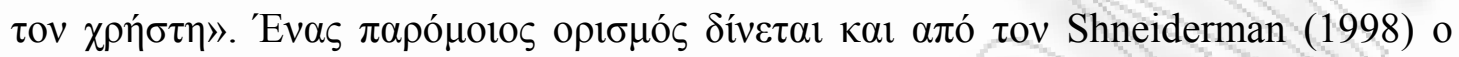

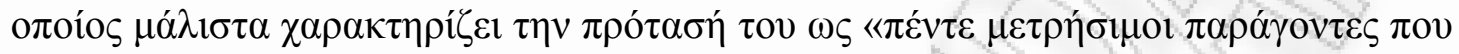

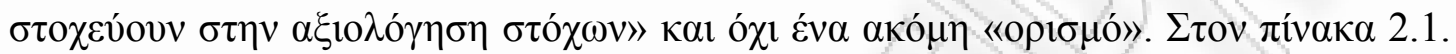

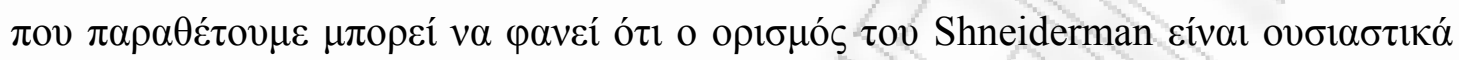

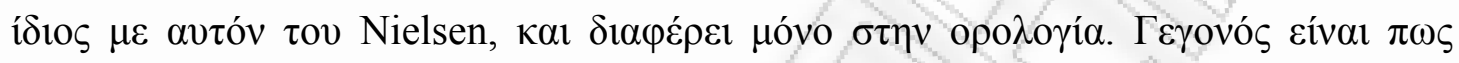

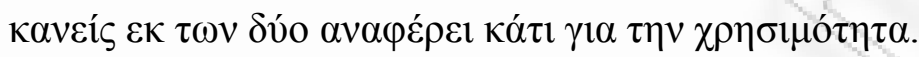

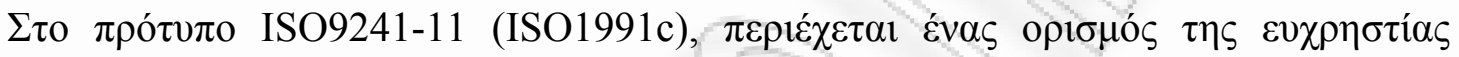

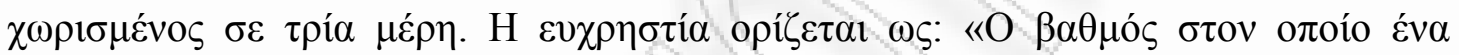

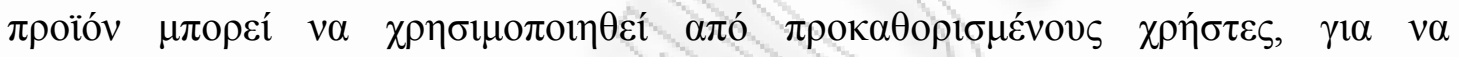

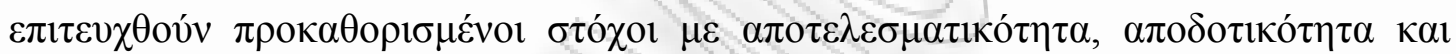

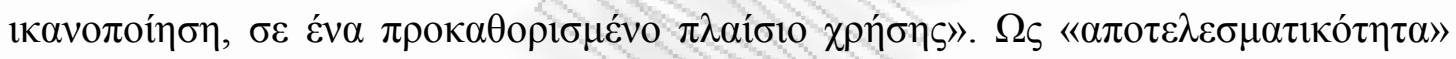

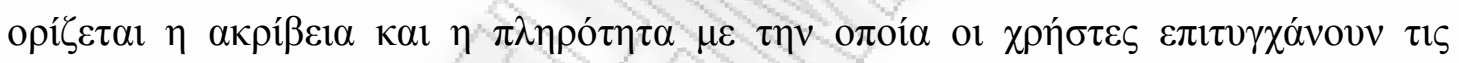

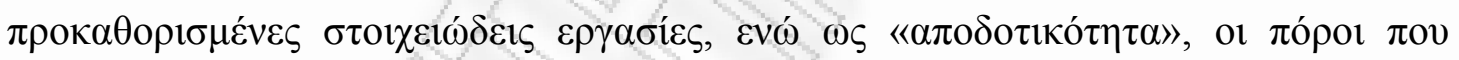

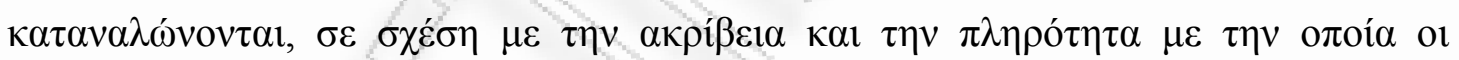

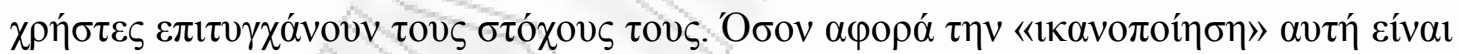

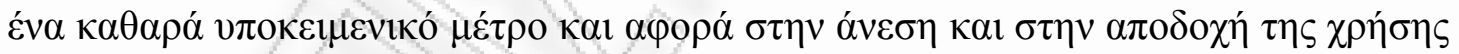

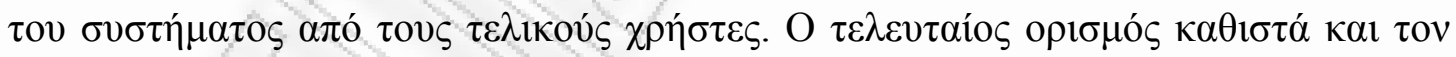

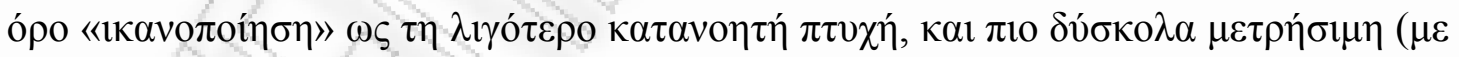

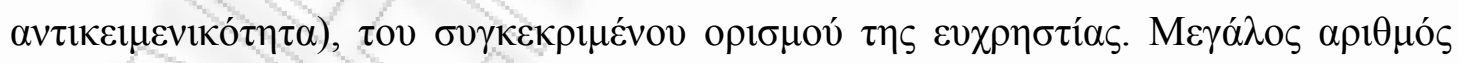

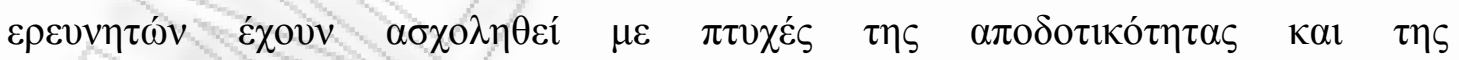

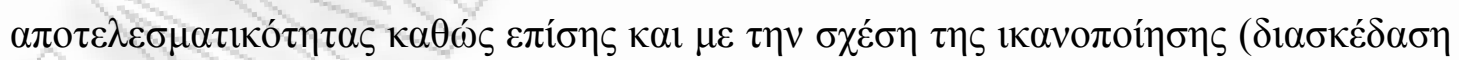

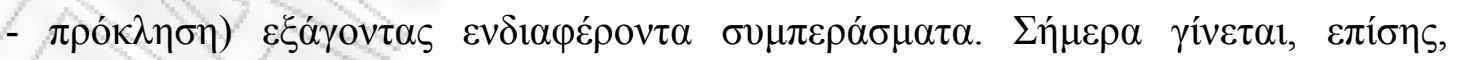

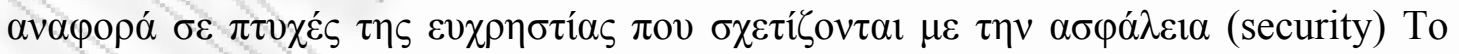

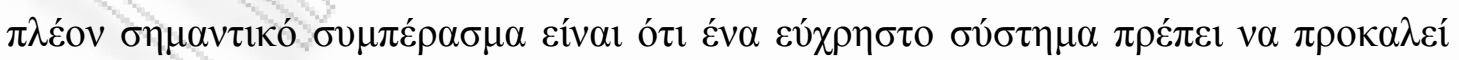

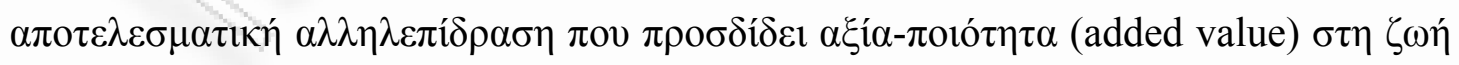

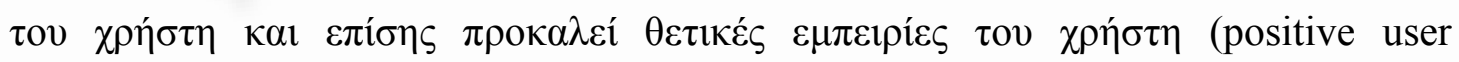
experiences). (Cockton, 2006; Mørch et. al., 2006) 


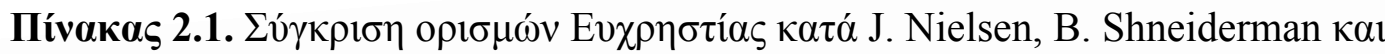
ISO9241-11.

\begin{tabular}{|c|c|c|}
\hline Nielsen & Shneiderman & ISO9241-11 \\
\hline А $\pi \mathrm{o} \delta \mathrm{o} \tau \imath \kappa o ́ \tau \eta \tau \alpha$ & 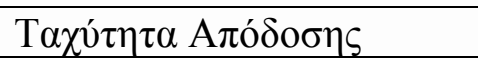 & A $\pi \mathrm{o} \delta \mathrm{o} \tau 1 \kappa o ́ \tau \eta \tau \alpha$ \\
\hline 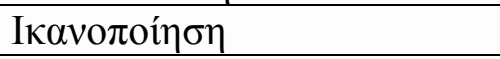 & 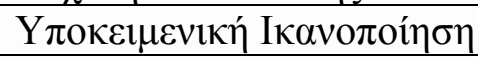 & 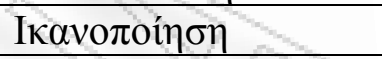 \\
\hline 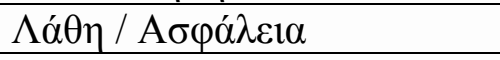 & 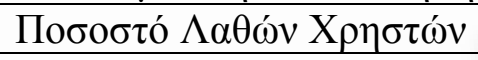 & A $\pi \mathrm{o} \tau \varepsilon \lambda \varepsilon \sigma \mu \alpha \tau 1 \kappa o ́ \tau \eta \tau \alpha$ \\
\hline 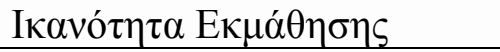 & 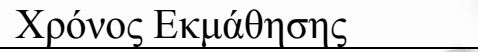 & \\
\hline 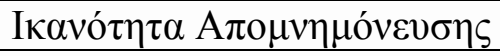 & 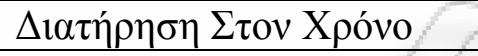 & \\
\hline
\end{tabular}

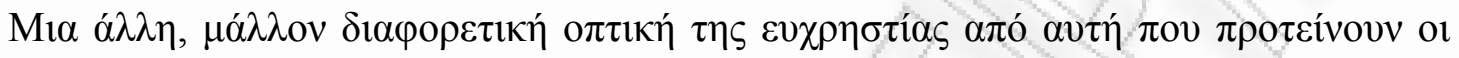

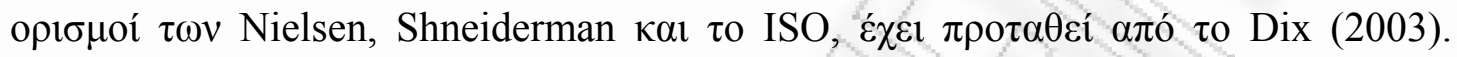

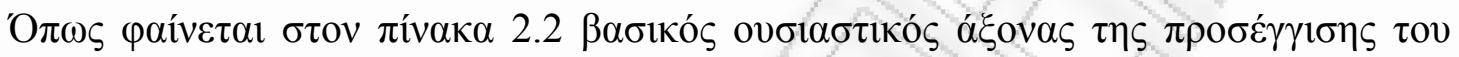

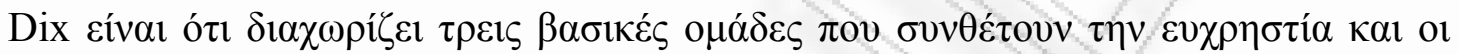

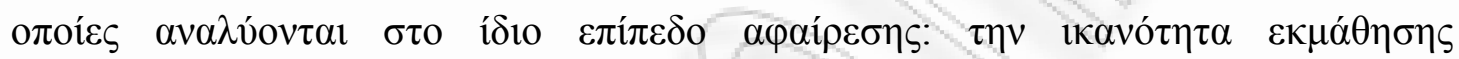

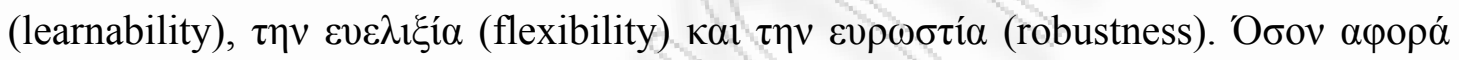

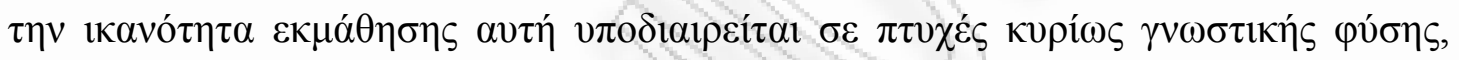

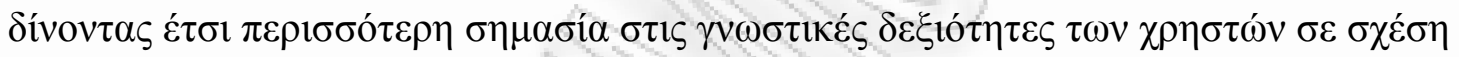

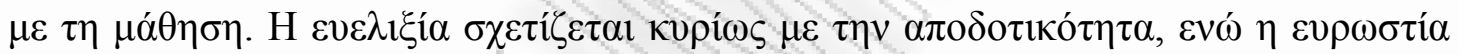

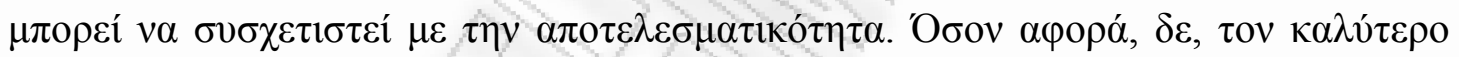

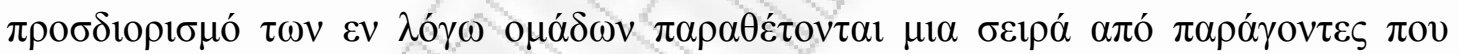

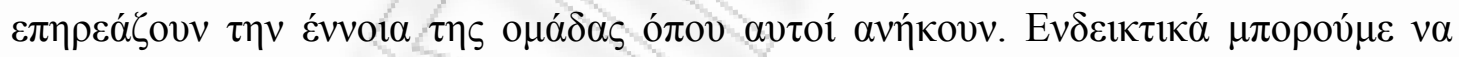

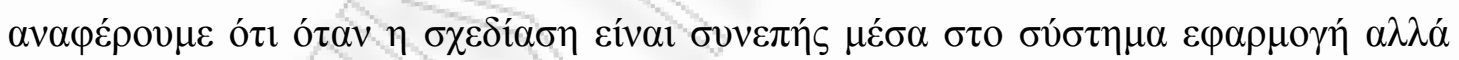

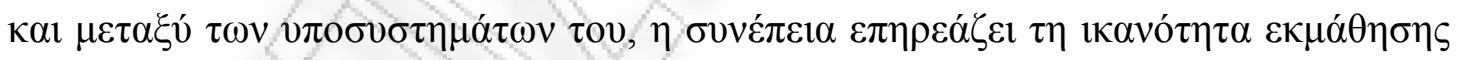

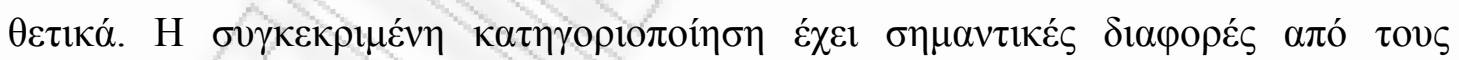

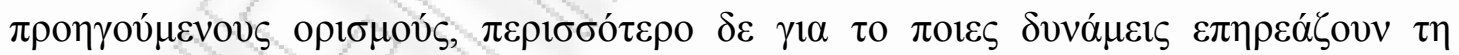

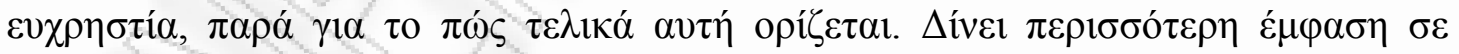

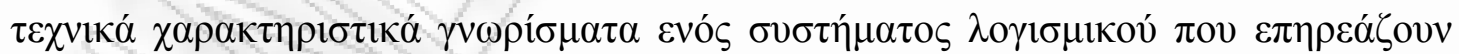

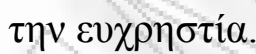

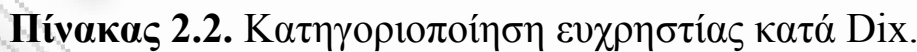

\begin{tabular}{|c|c|c|}
\hline 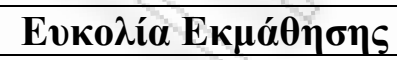 & 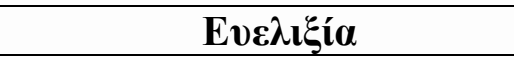 & 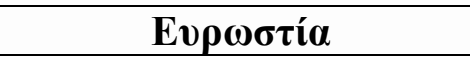 \\
\hline Про $\beta \lambda \varepsilon \psi \mu \mu{ }^{\prime} \tau \eta \tau \alpha$ & 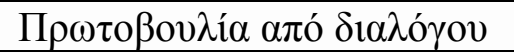 & 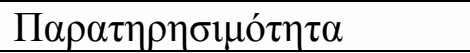 \\
\hline$\Delta v v \alpha \tau o ́ \tau \eta \tau \alpha \Sigma v ́ v \theta \varepsilon \sigma \eta \varsigma$ & 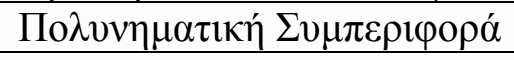 & 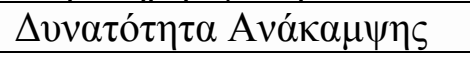 \\
\hline Оıкعió $\tau \eta \alpha$ & 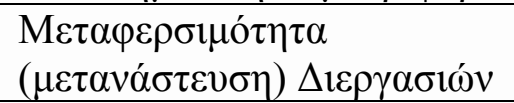 & 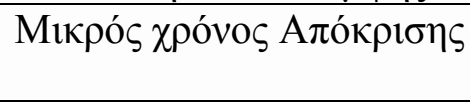 \\
\hline 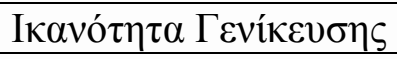 & 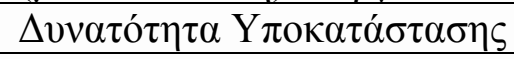 & $\Sigma v \mu \mu o ́ \rho \varphi \omega \sigma \eta \mu \varepsilon \varepsilon \rho \gamma \alpha \sigma i ́ \alpha$ \\
\hline$\Sigma v v \varepsilon ́ \pi \varepsilon i \alpha$ & 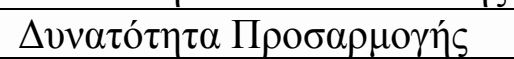 & \\
\hline
\end{tabular}




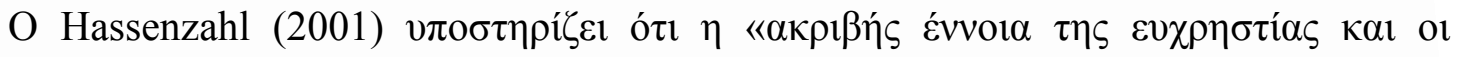

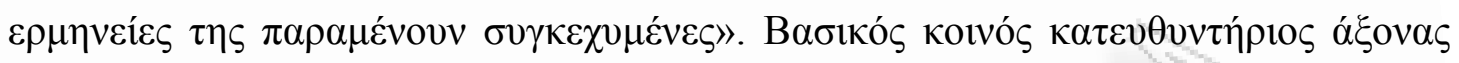

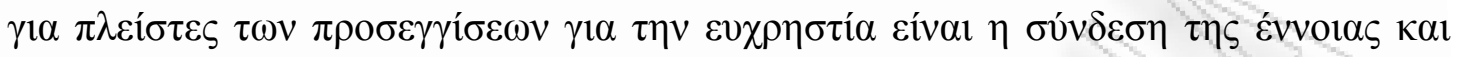

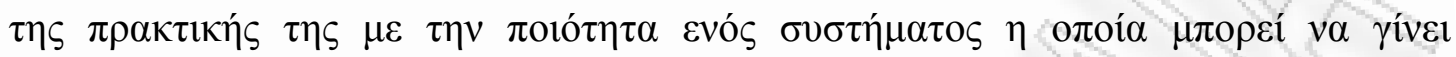

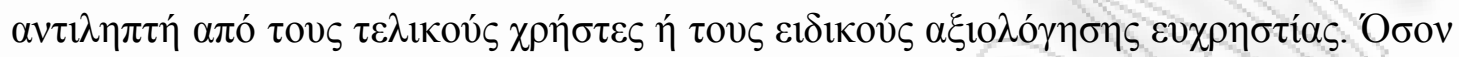

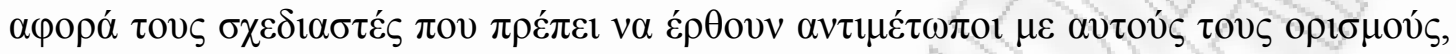

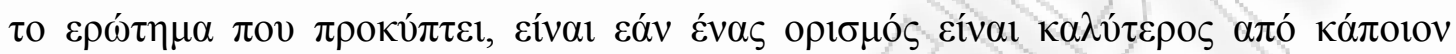

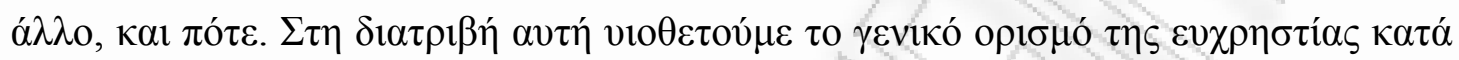

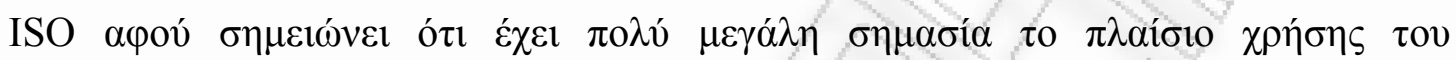

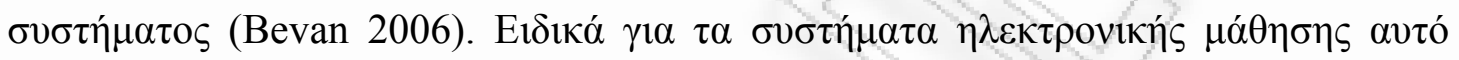

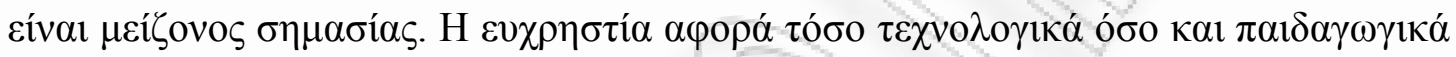
$\theta \varepsilon \dot{\varepsilon} \mu \alpha \tau \alpha$ (Tselios et. al., 2008).

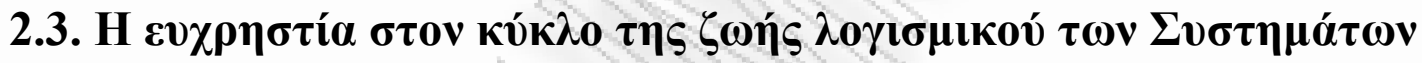

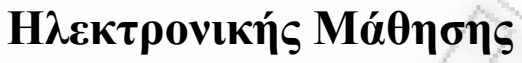

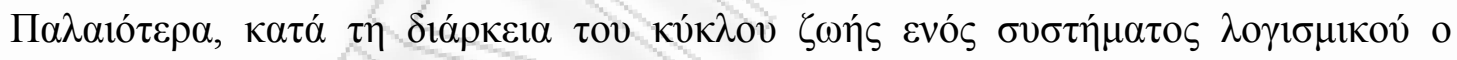

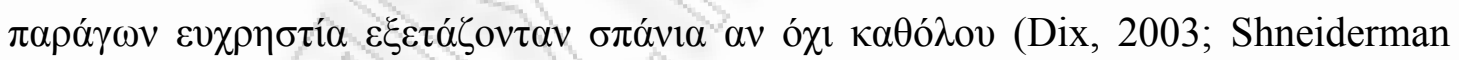

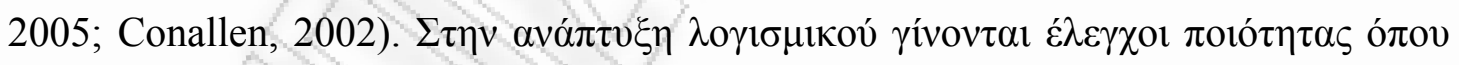

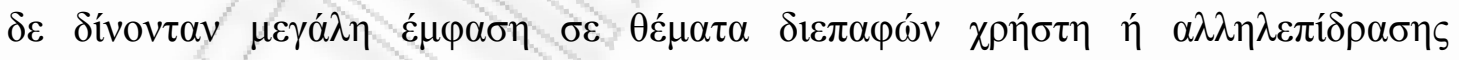

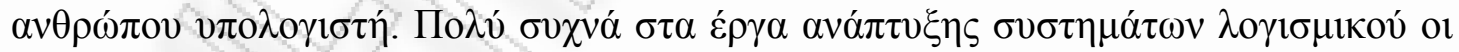

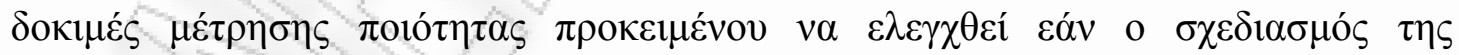

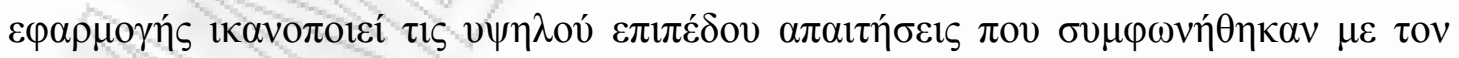

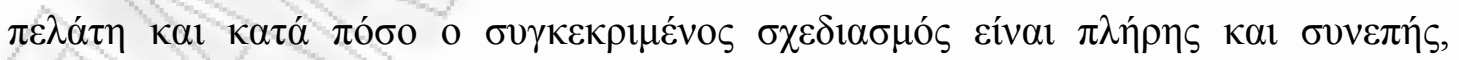

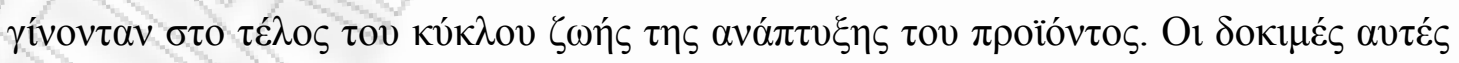

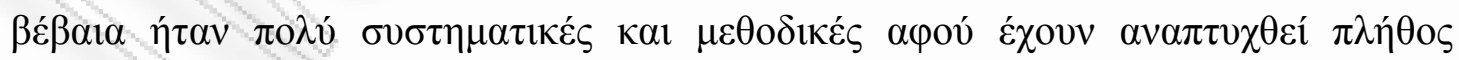

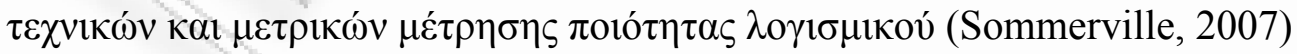

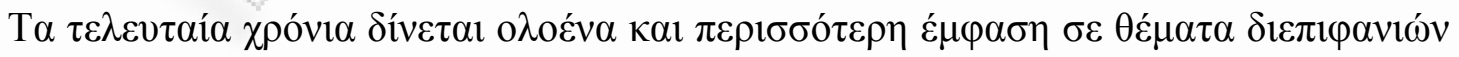

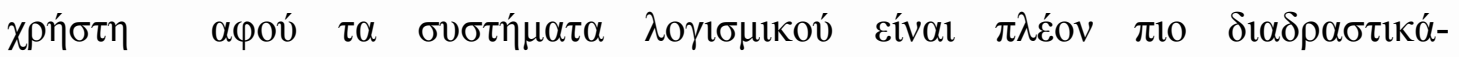

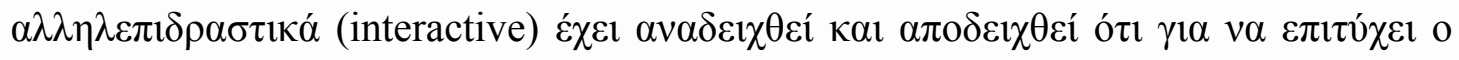

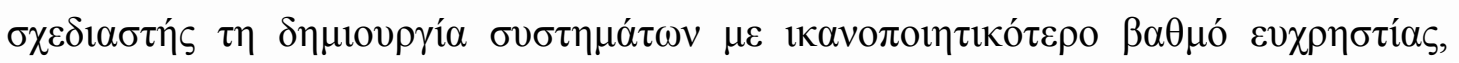




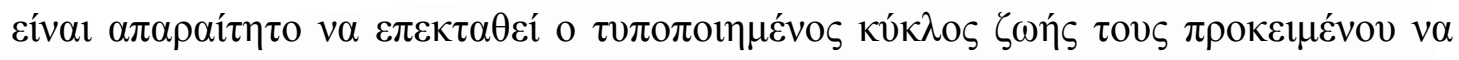

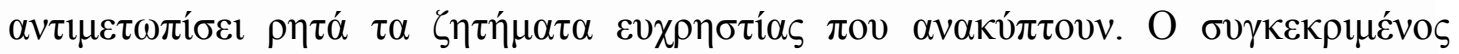

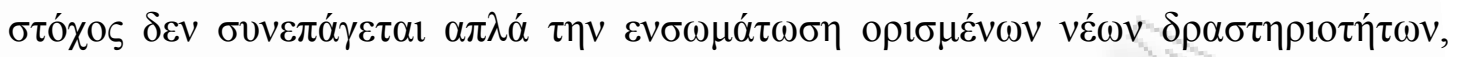

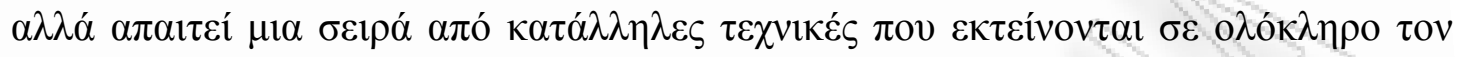

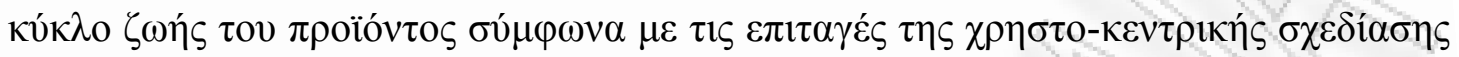
(user-centered design) (ISO 13407:1999).

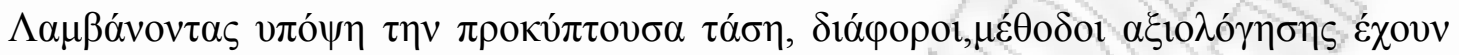

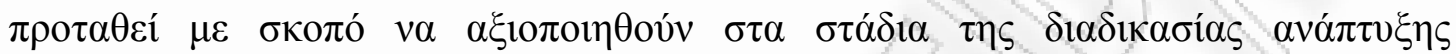

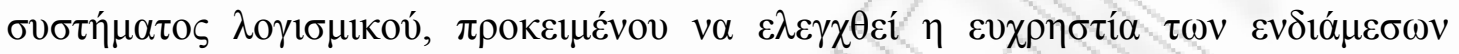

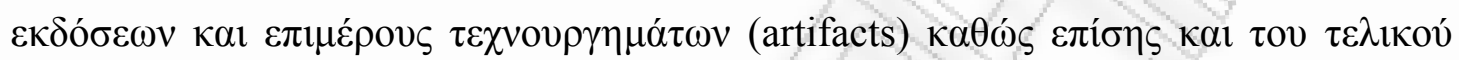

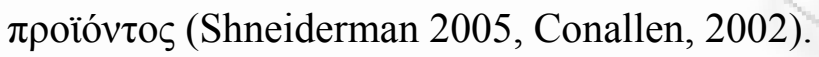

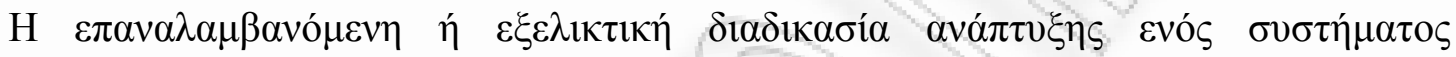

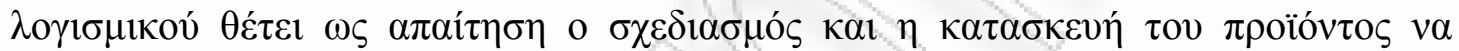

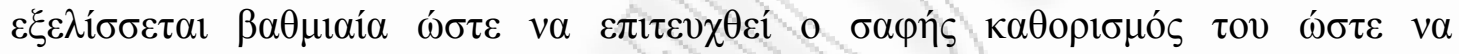

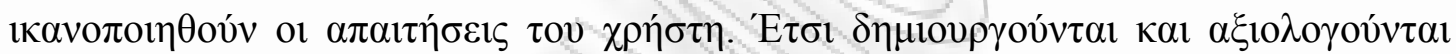

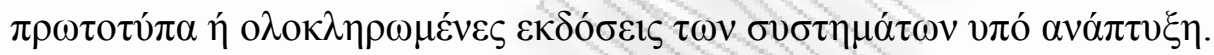

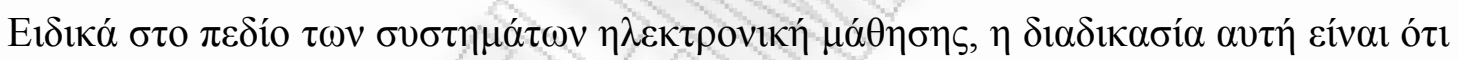

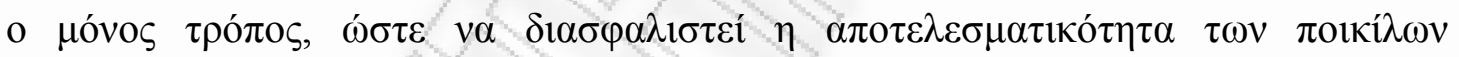

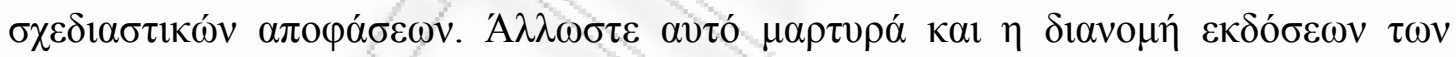

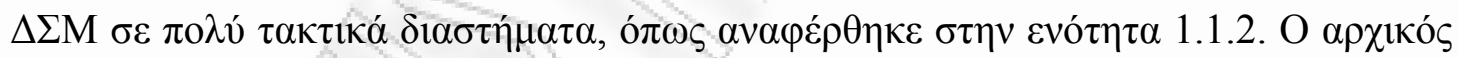

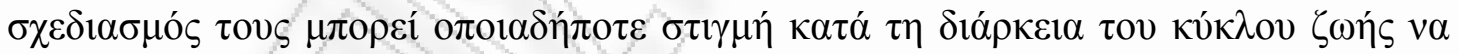

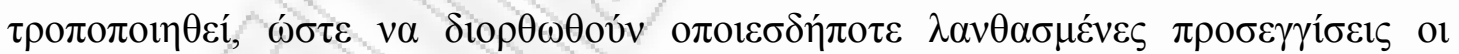

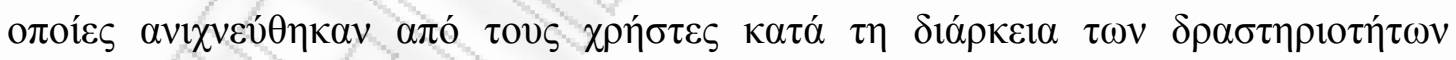

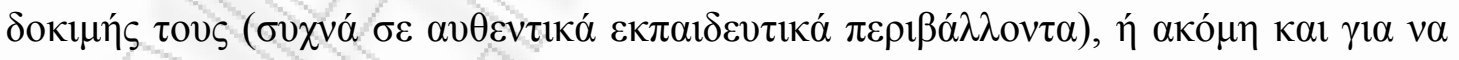

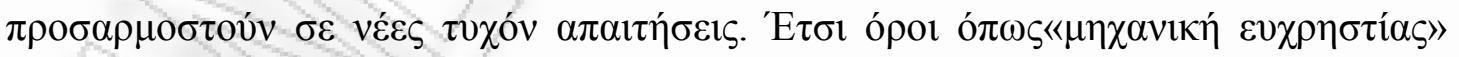

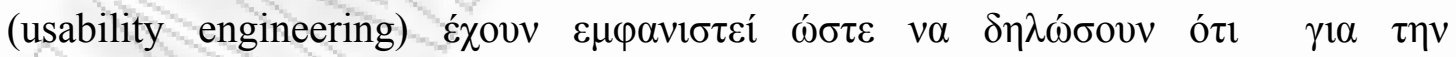

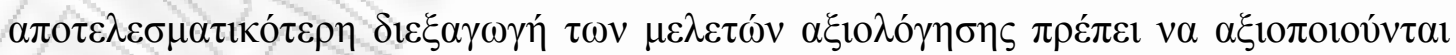

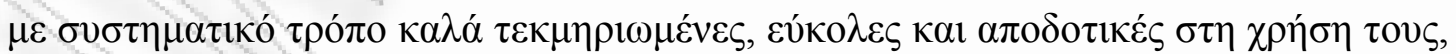

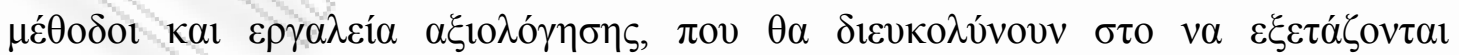

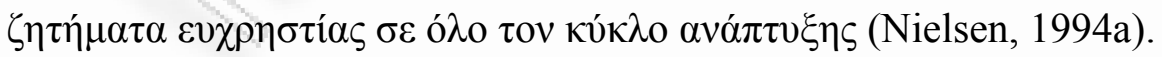

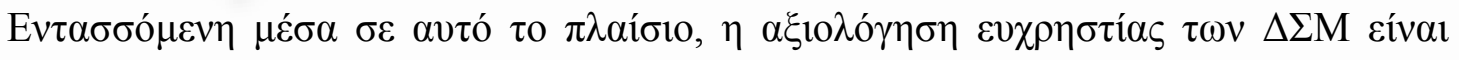

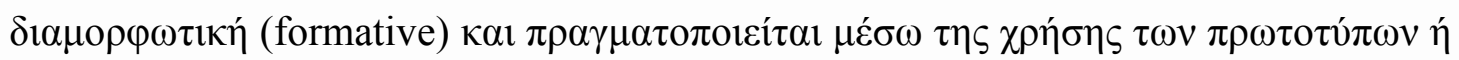

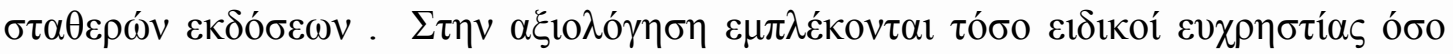

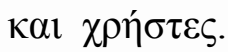




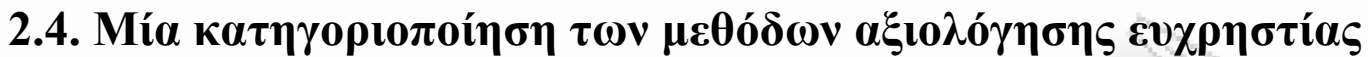

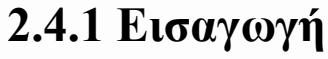

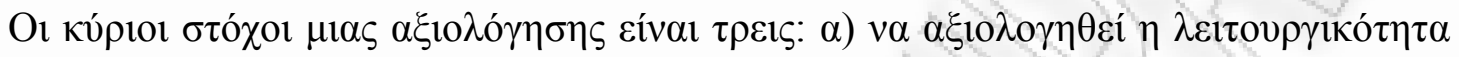

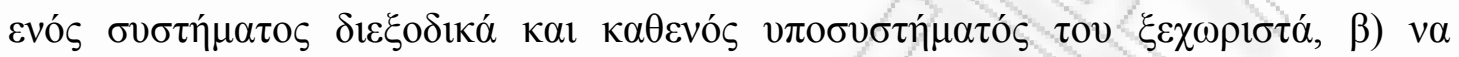

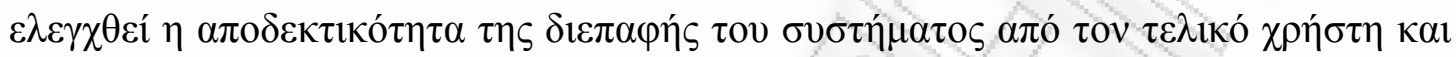

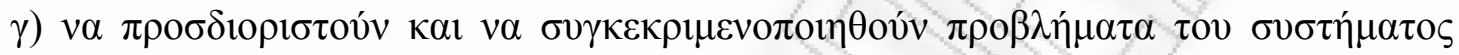

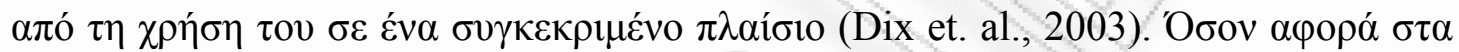

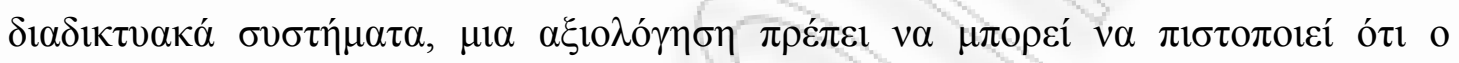

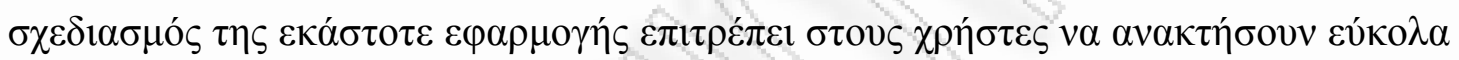

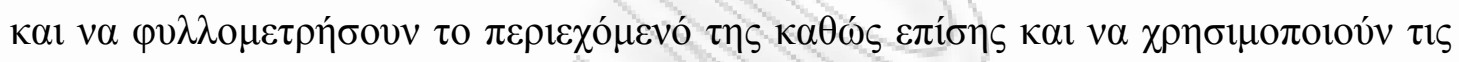

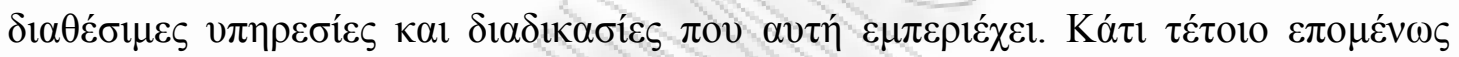

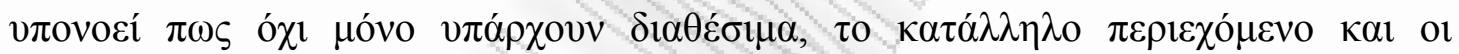

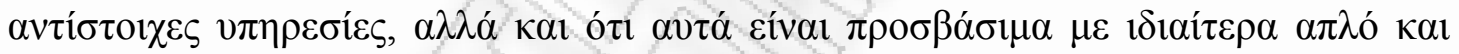

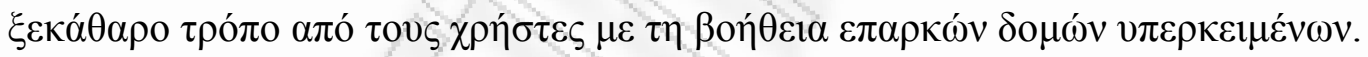

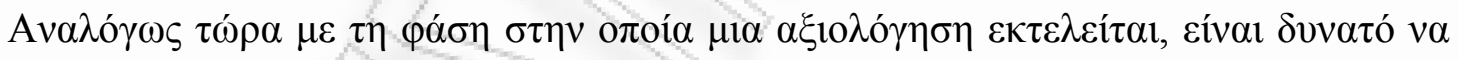

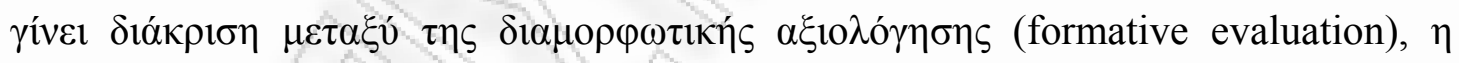

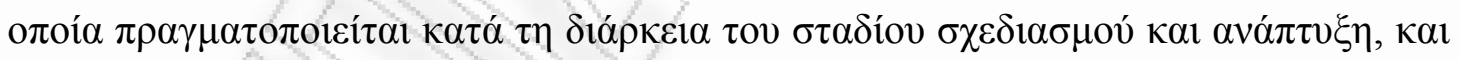

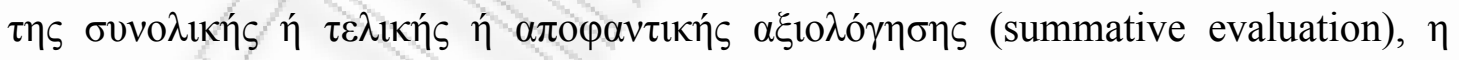

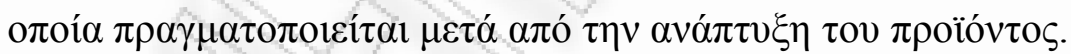

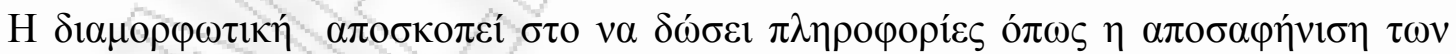

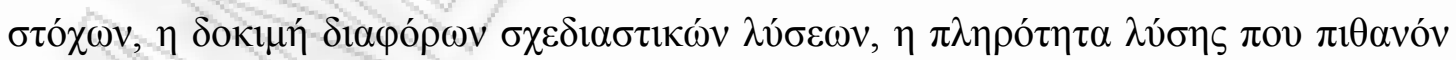

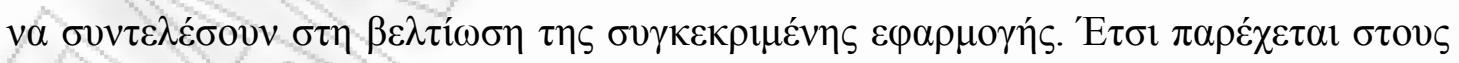

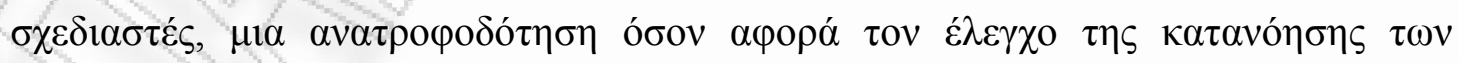

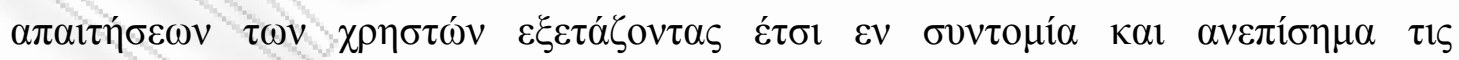

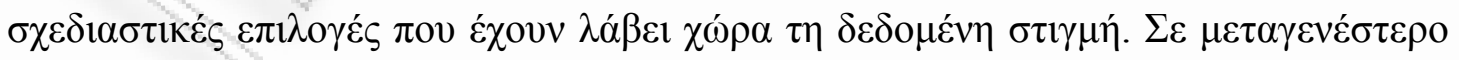

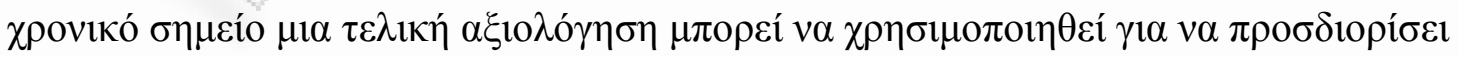

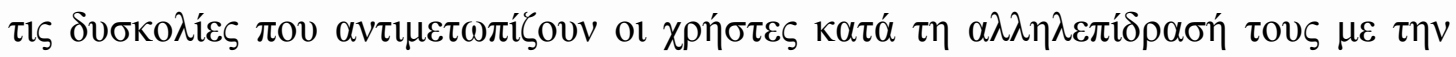

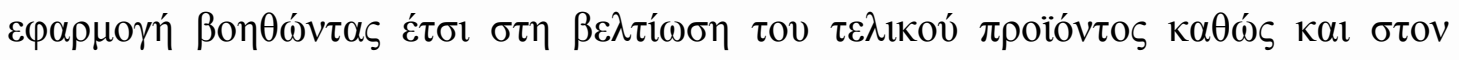

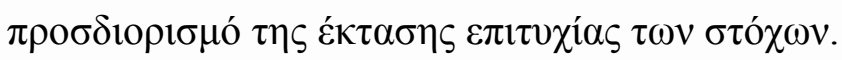




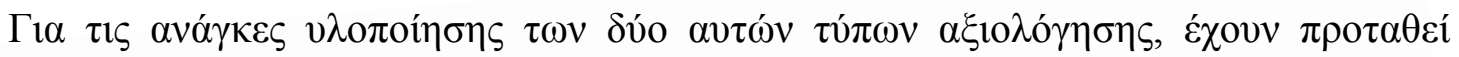

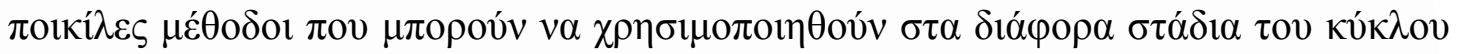

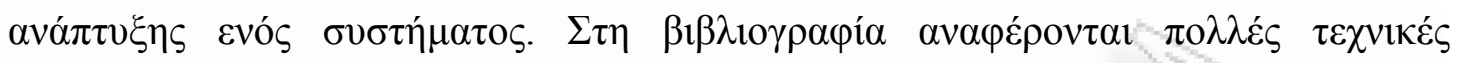

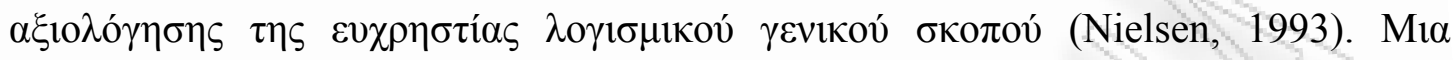

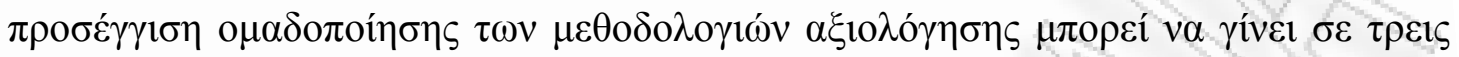

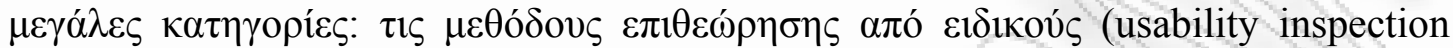

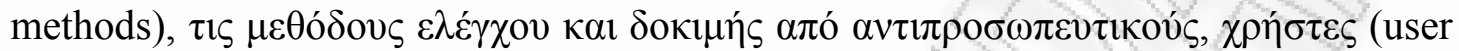

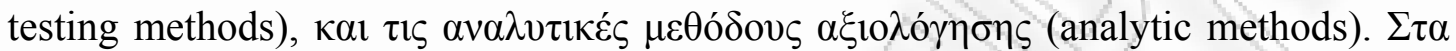

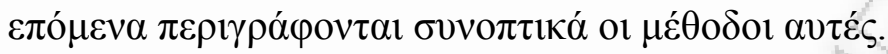

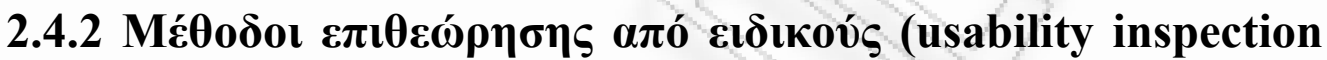 methods)}

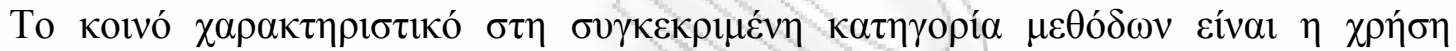

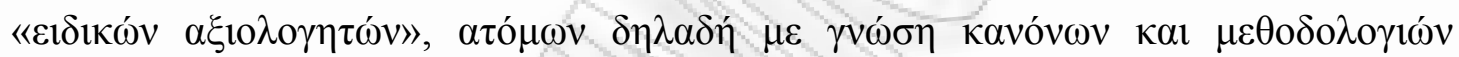

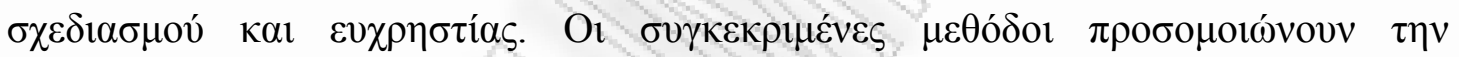

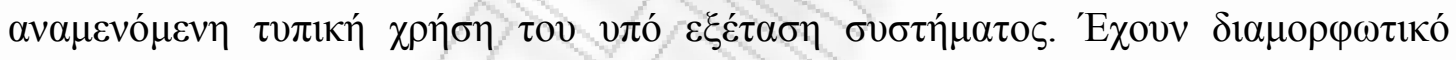

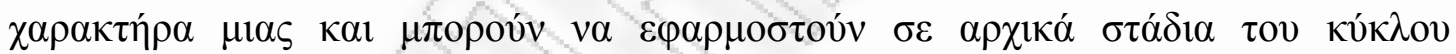

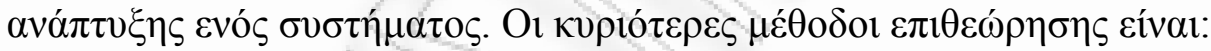

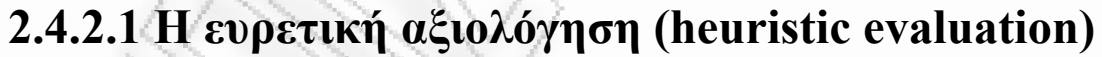

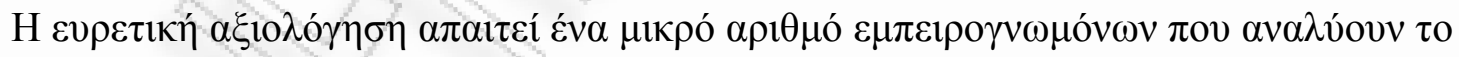

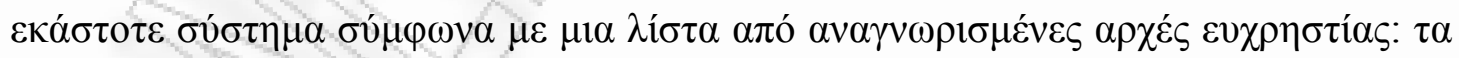

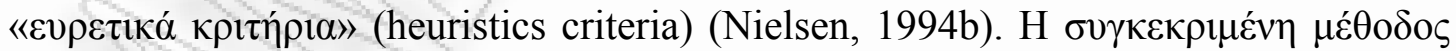

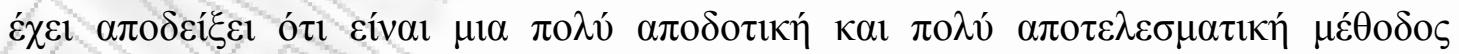

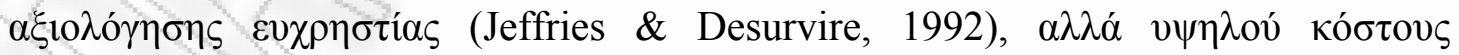
(Nielsen, 1994b).

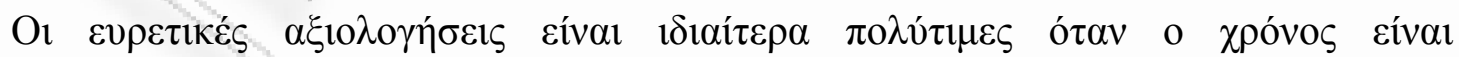

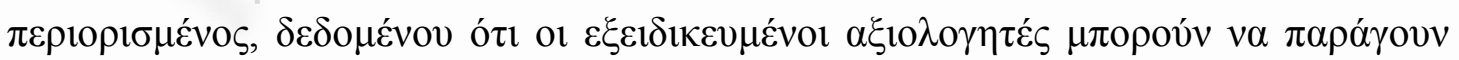

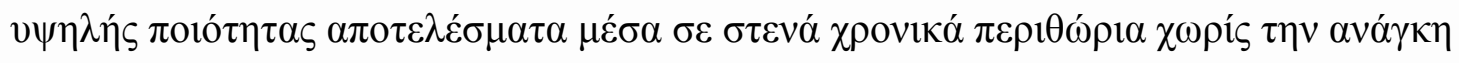

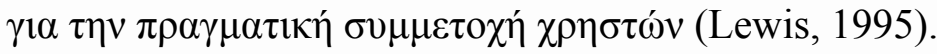




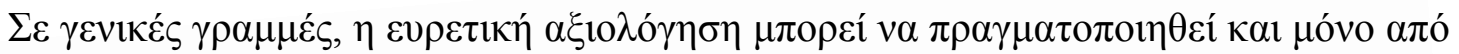

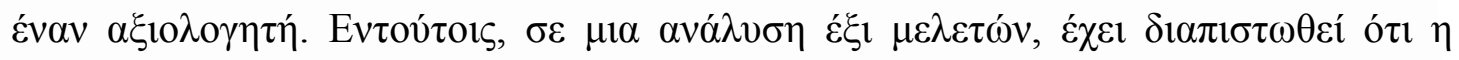

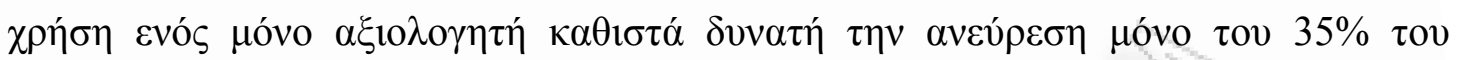

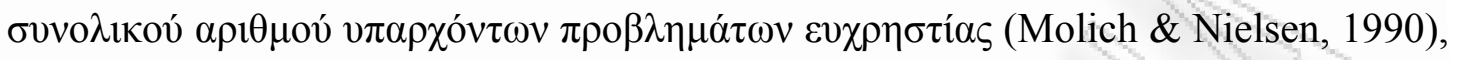

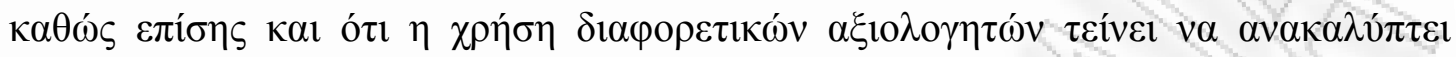

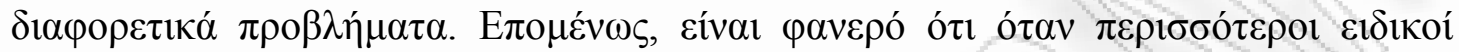

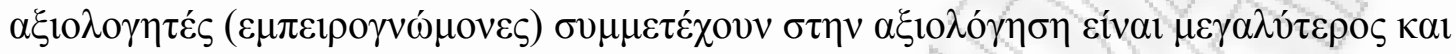

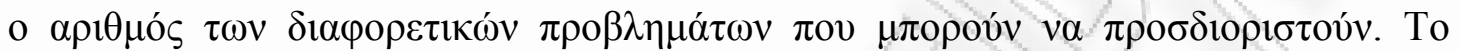

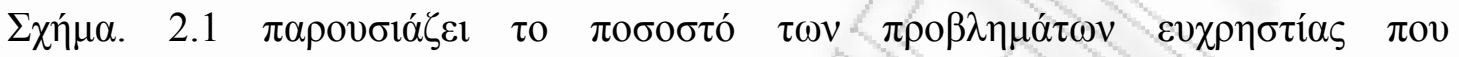

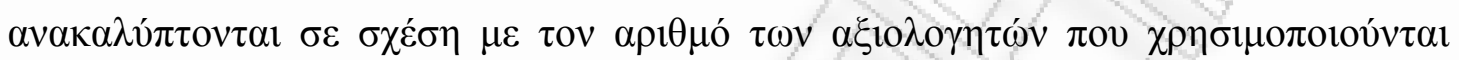

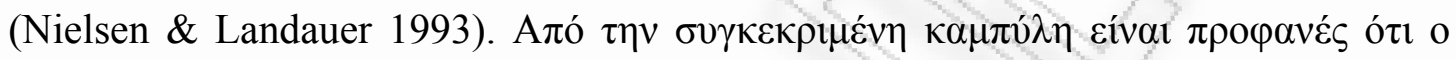

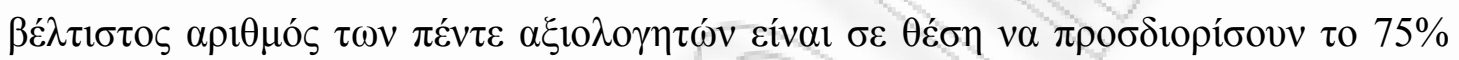

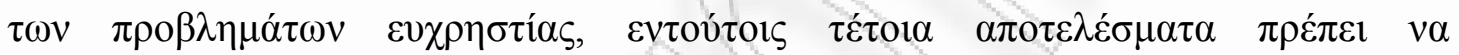

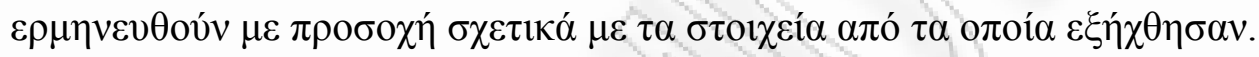

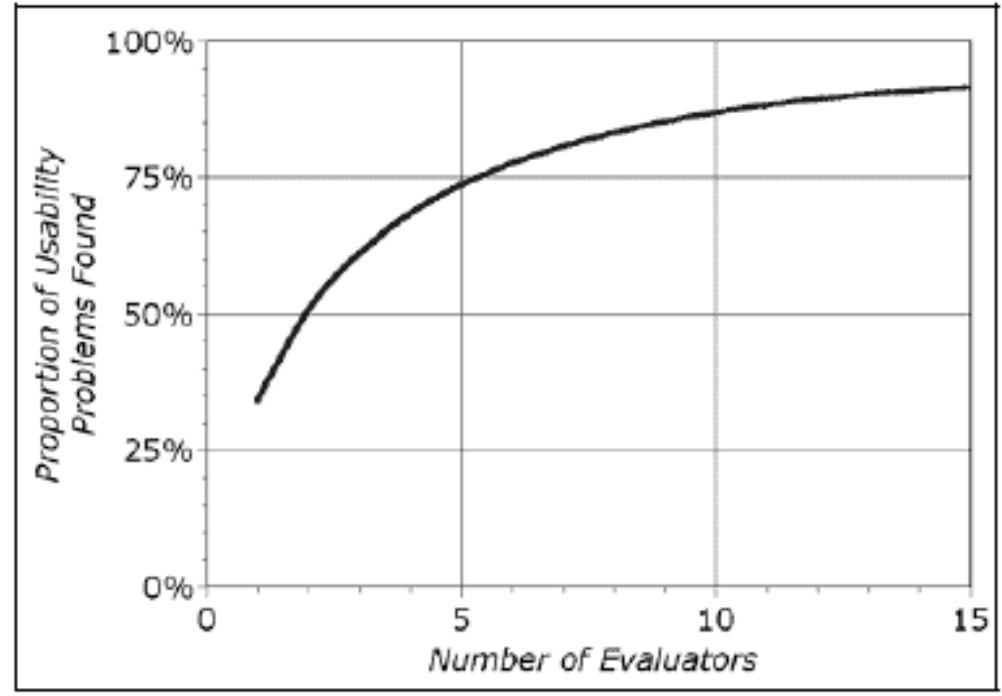

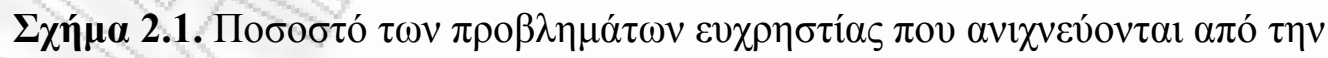

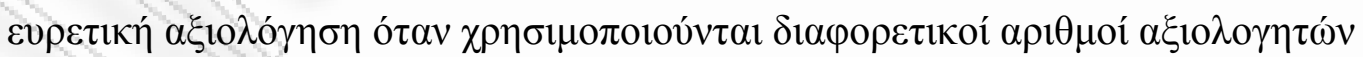
(Nielsen \& Landauer 1993)

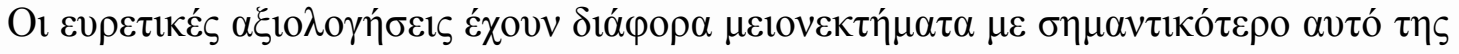

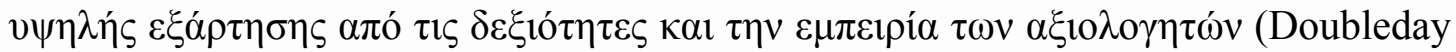


et. Al., 1997; Jeffries \& Desurvire 1992; Kantner \& Rosenbaum 1997). O Nielsen

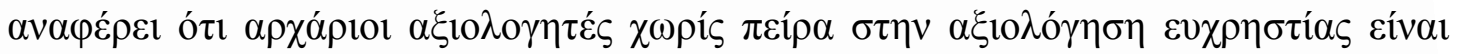

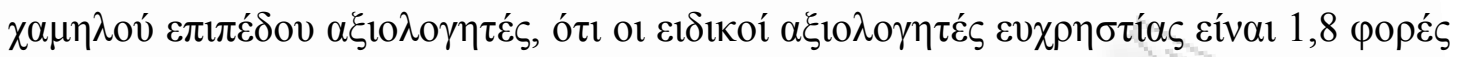

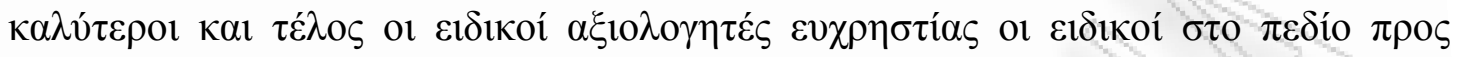

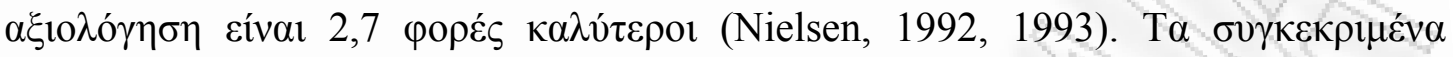

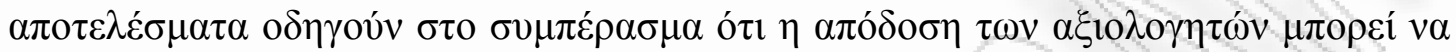

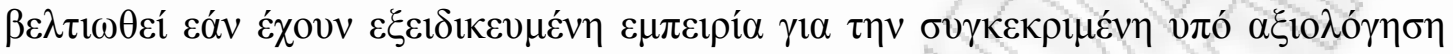

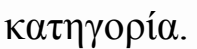

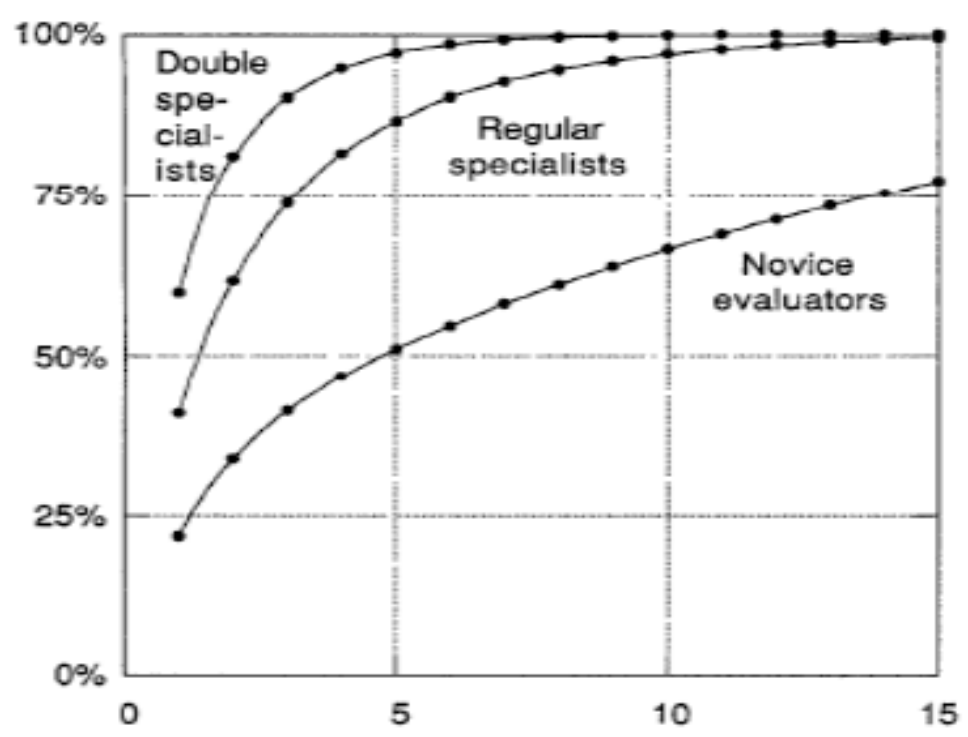

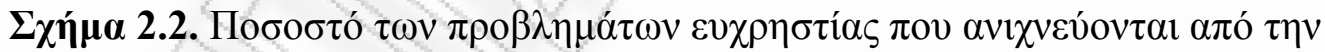

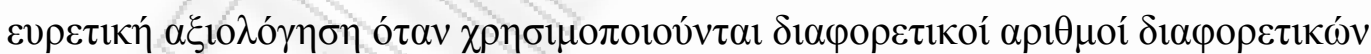

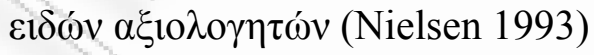

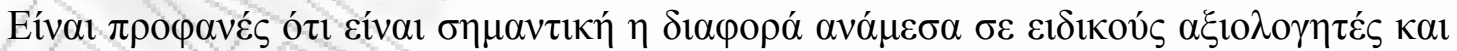

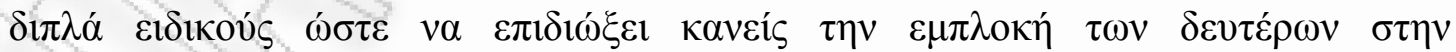

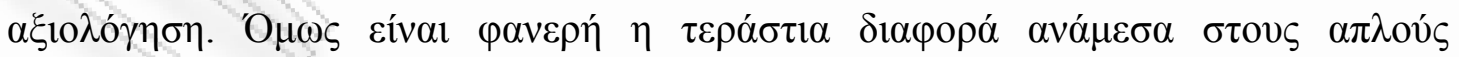

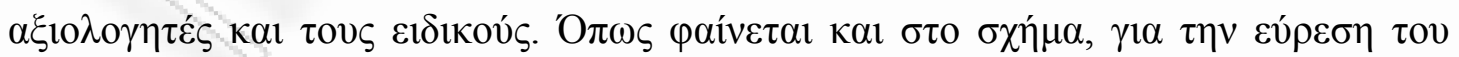

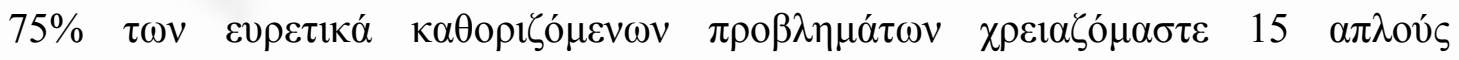

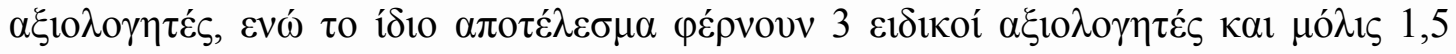

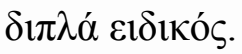




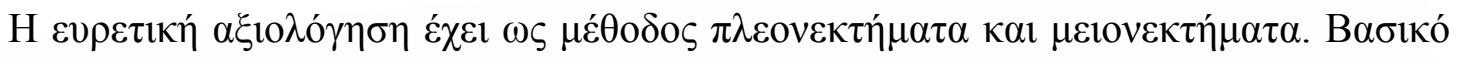

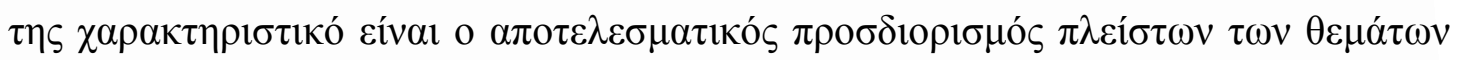

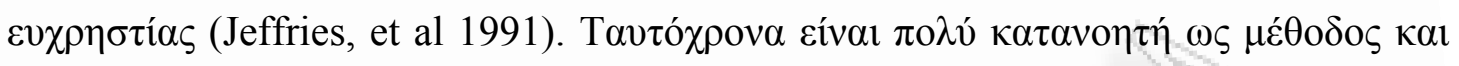

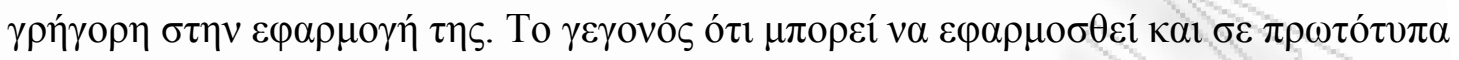

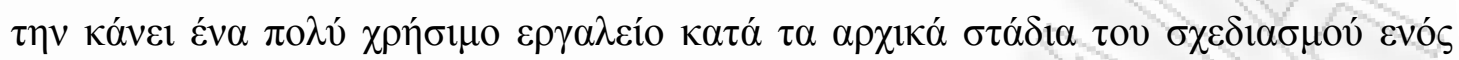

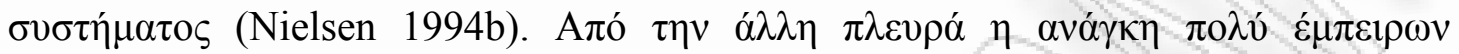

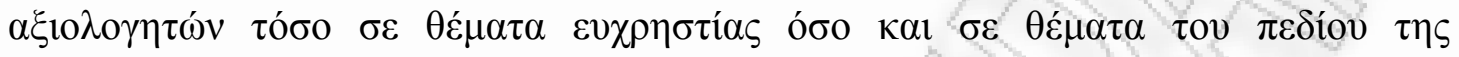

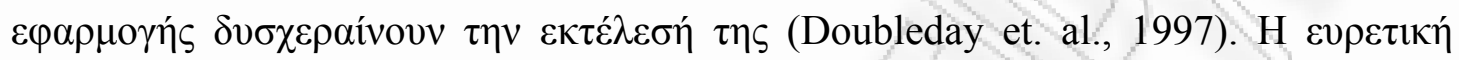

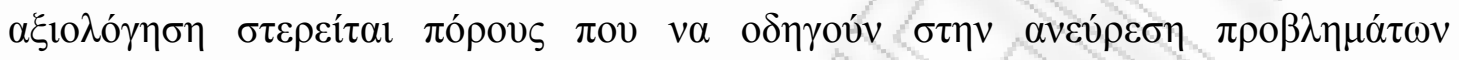

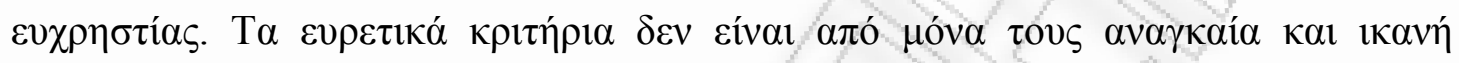

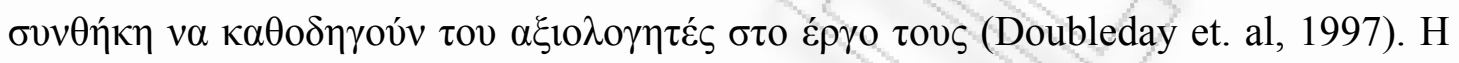

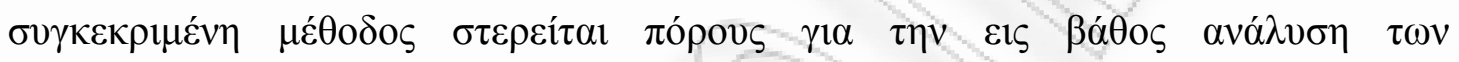

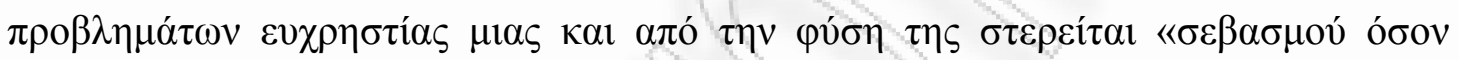

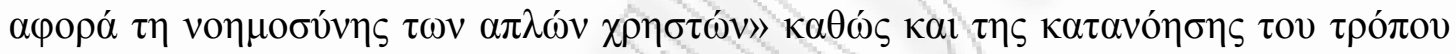

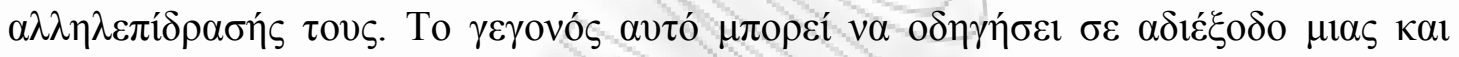

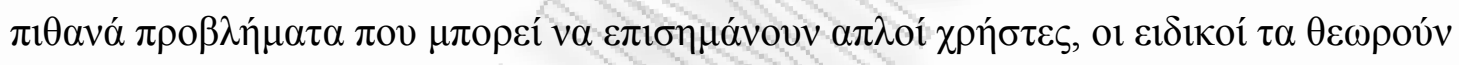

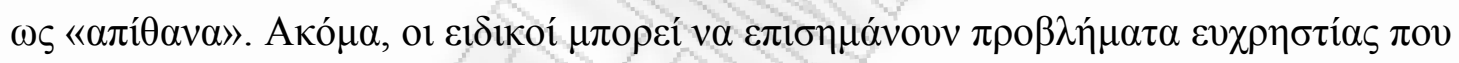

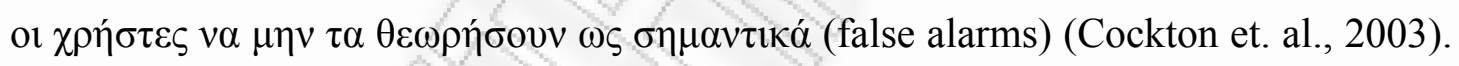

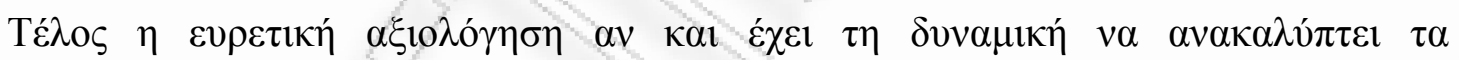

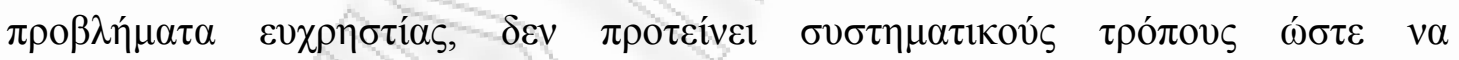

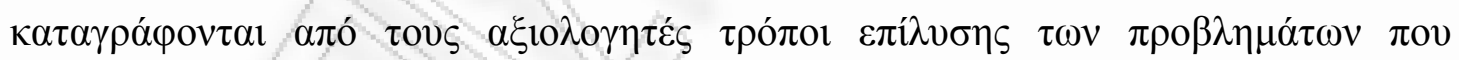

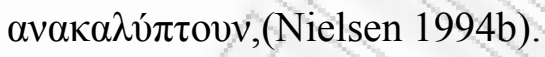

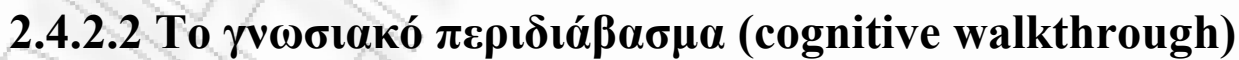

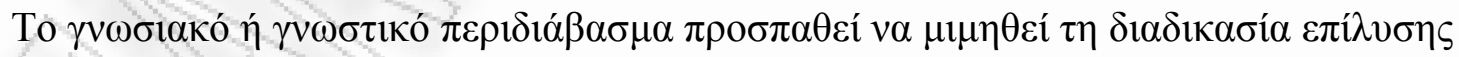

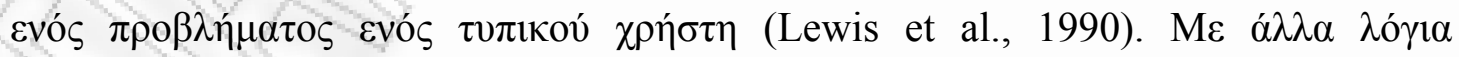

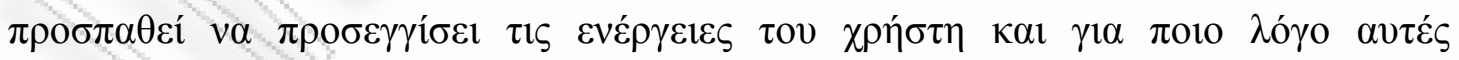

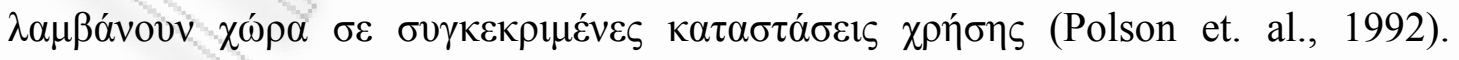

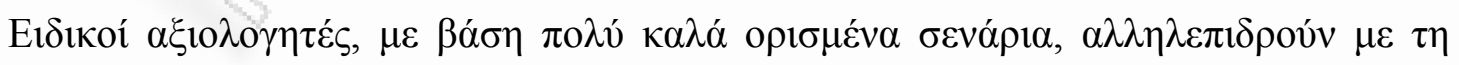

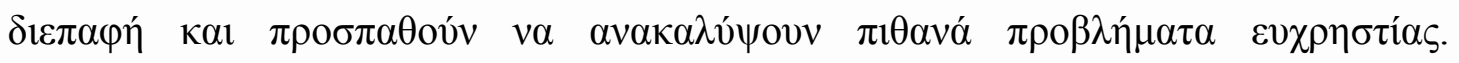

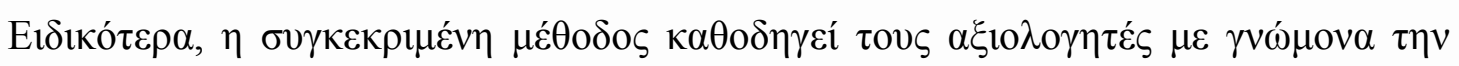

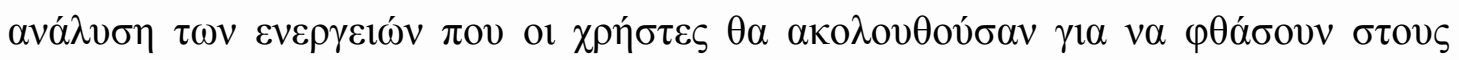




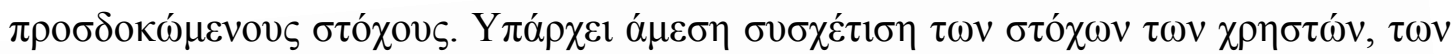

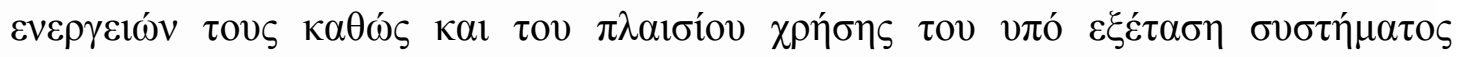

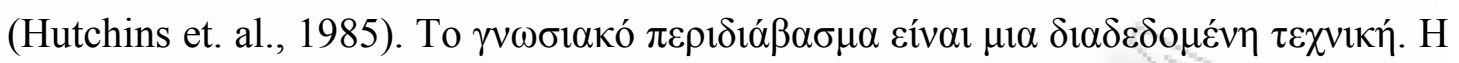

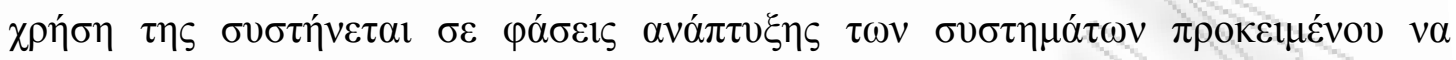

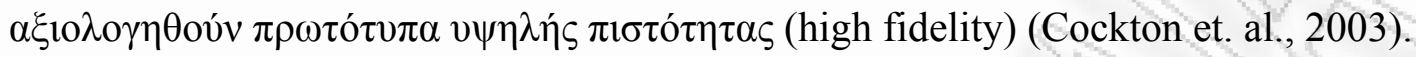

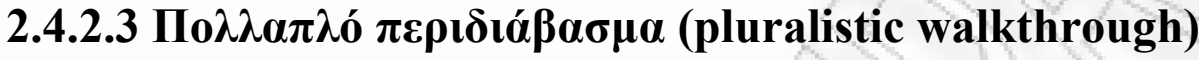

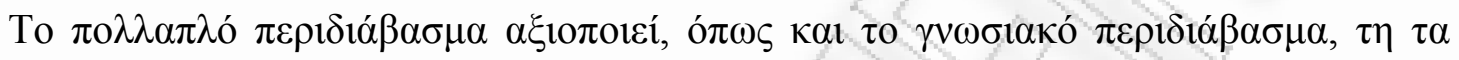

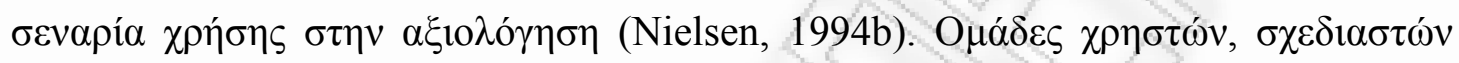

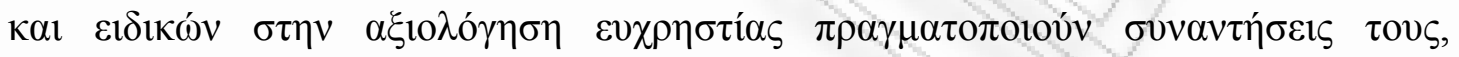

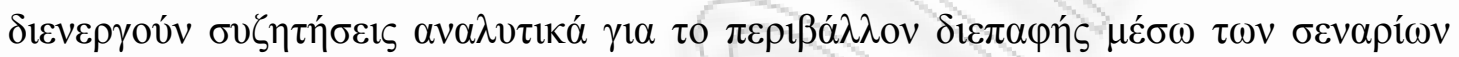

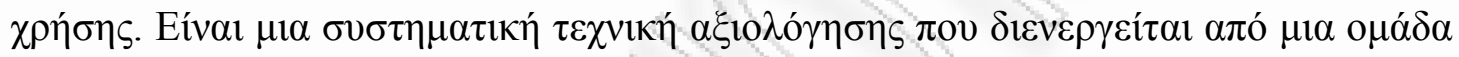

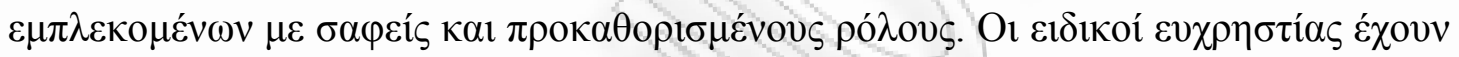

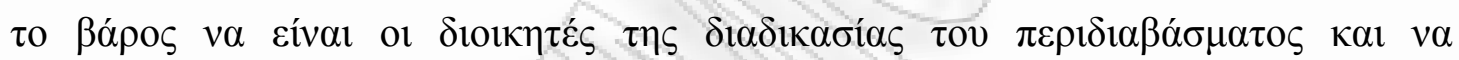

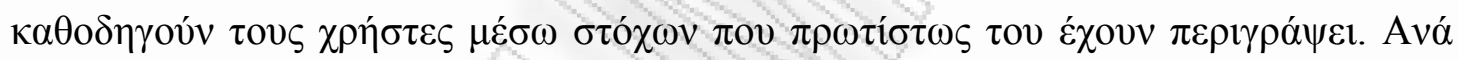

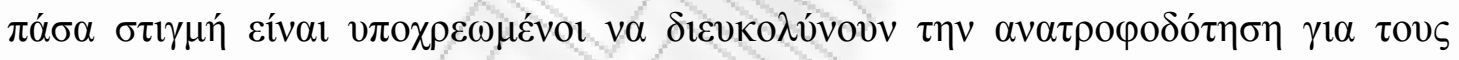

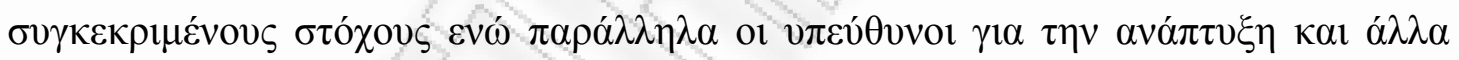

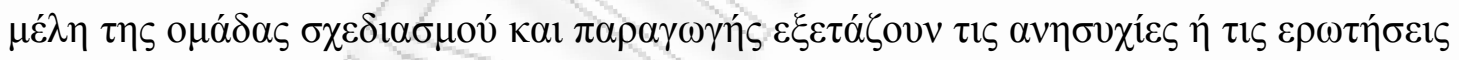

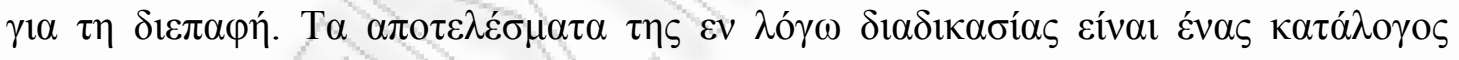

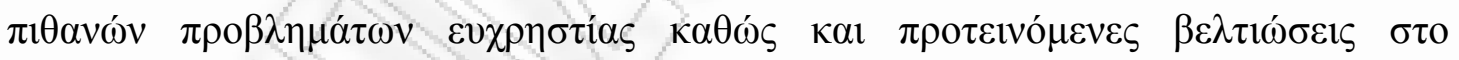

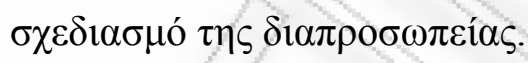

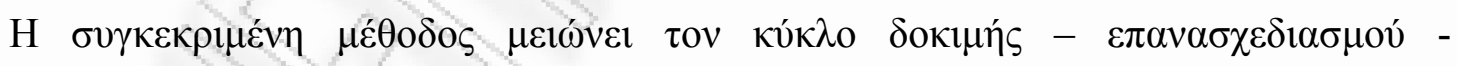

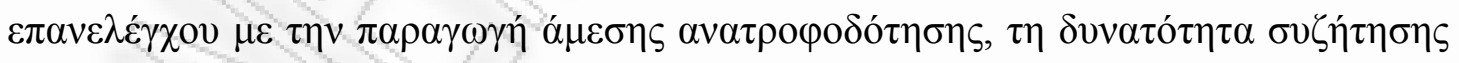

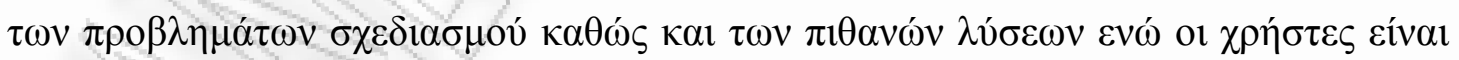

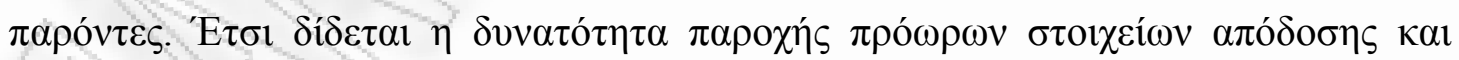

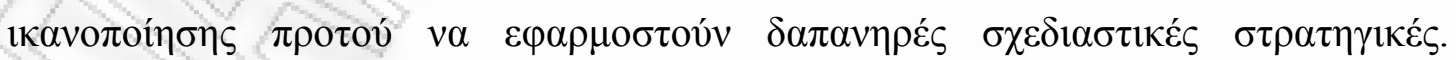

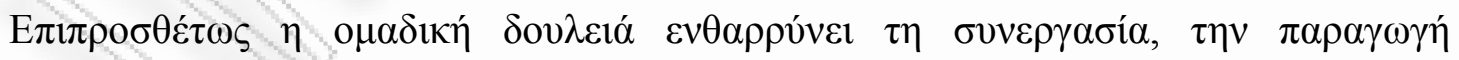

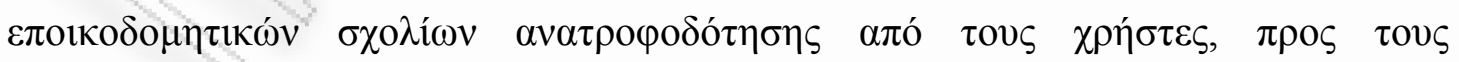

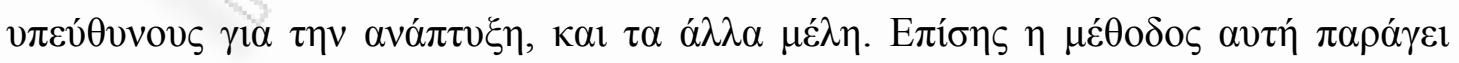

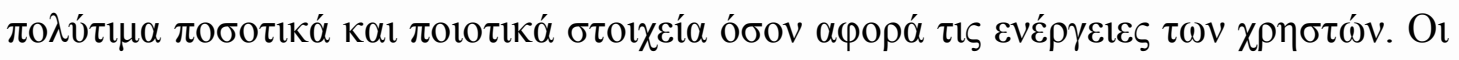

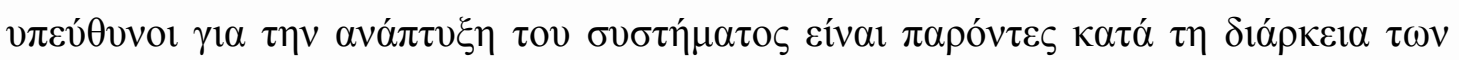




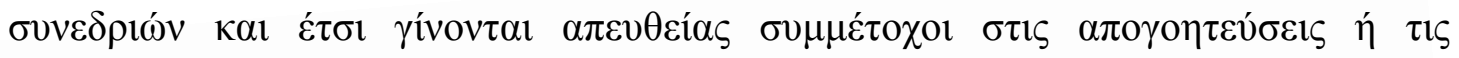

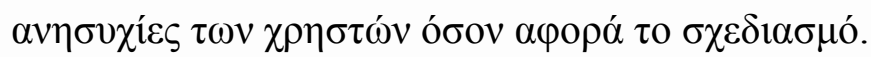

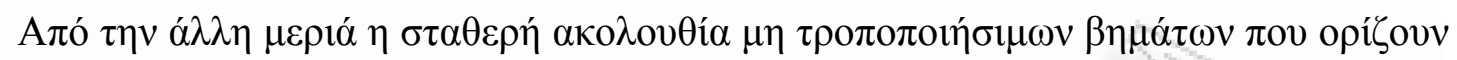

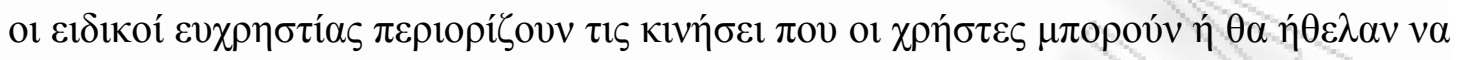

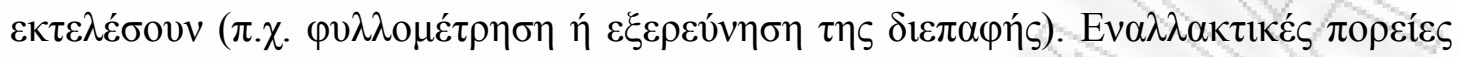

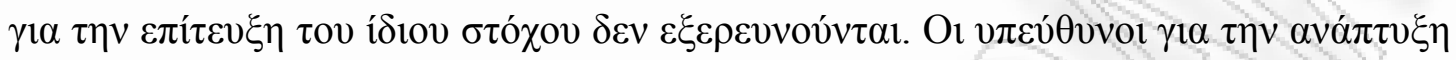

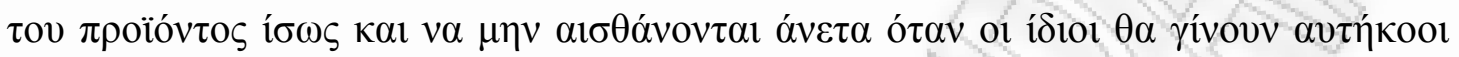

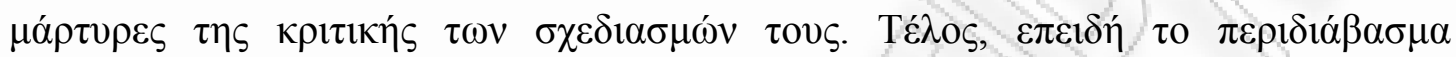

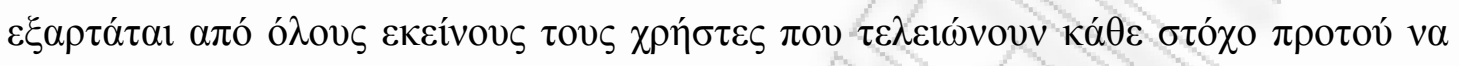

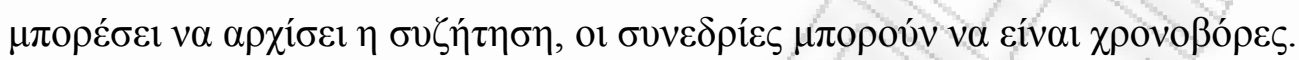

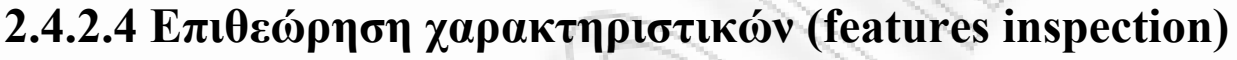

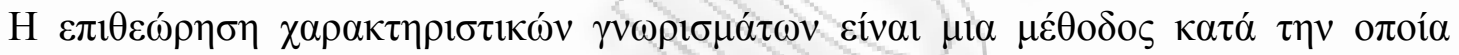

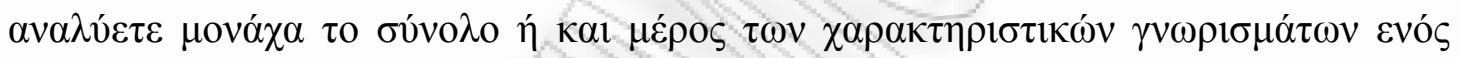

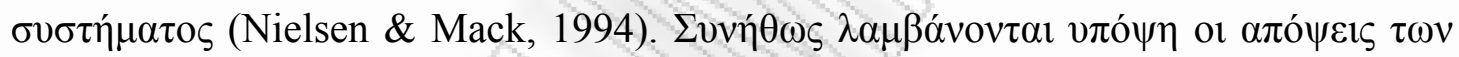

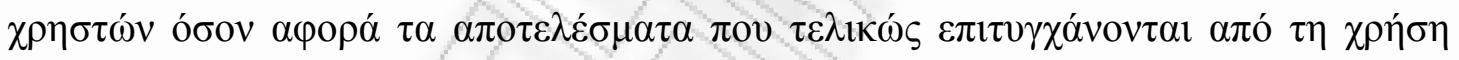

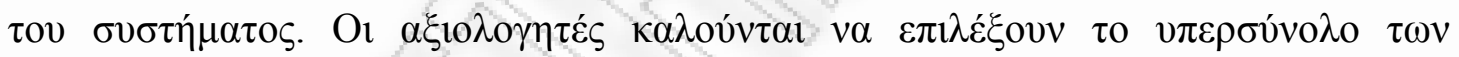

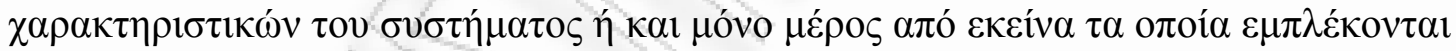

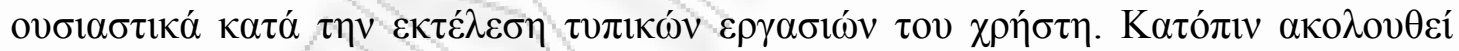

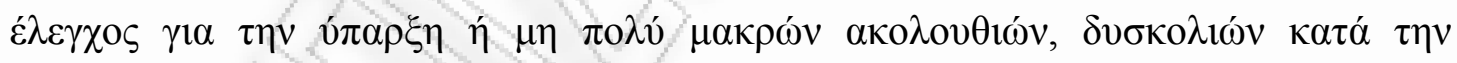

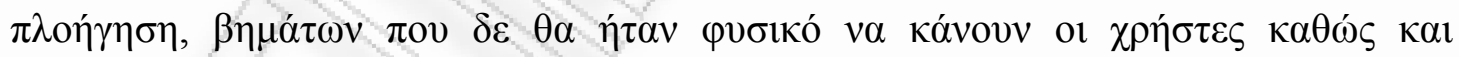

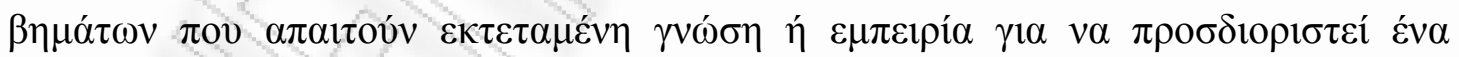

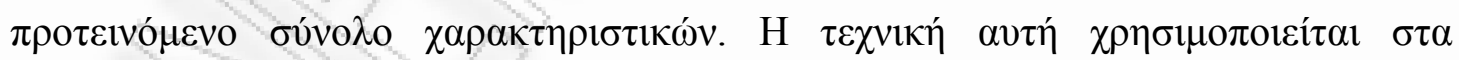

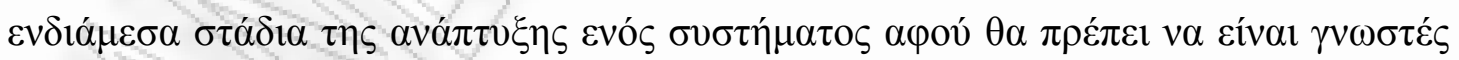

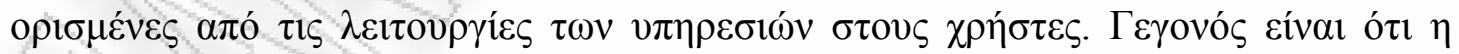

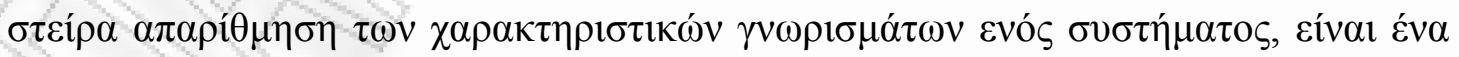

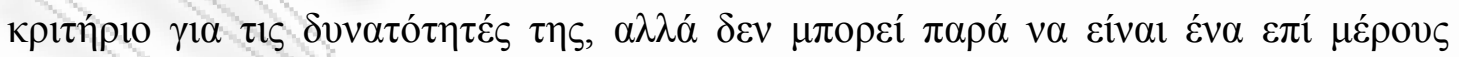

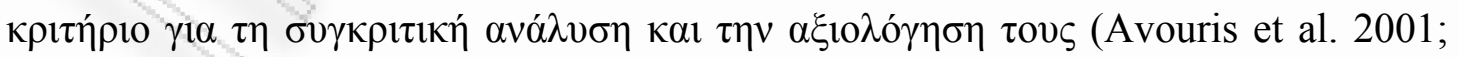
Grigoriadou and Papanikolaou 2000). 


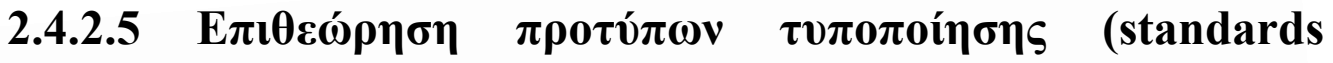 inspection)}

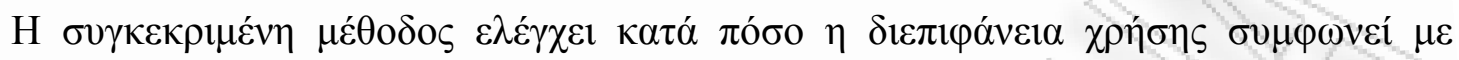

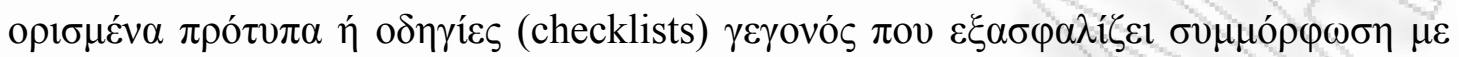

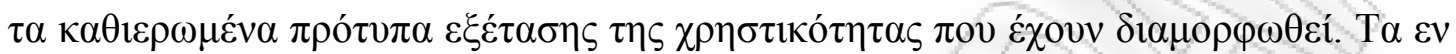

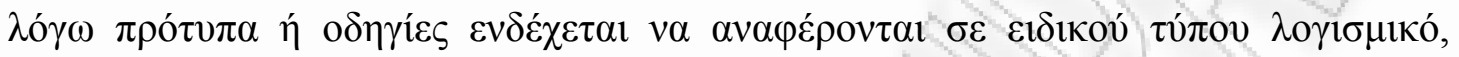

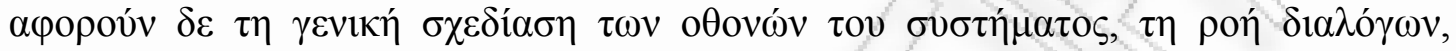

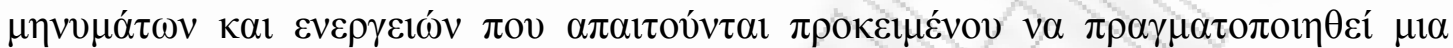

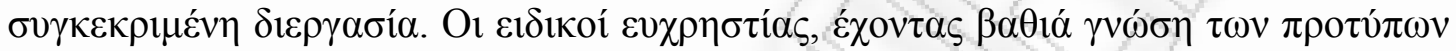

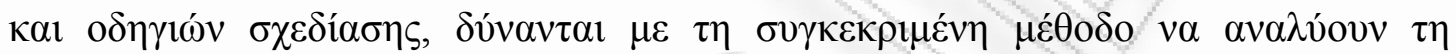

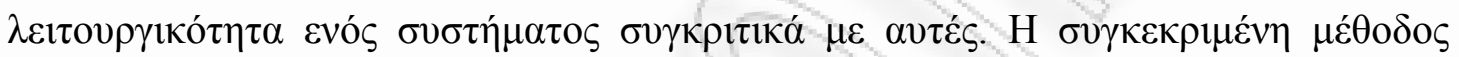

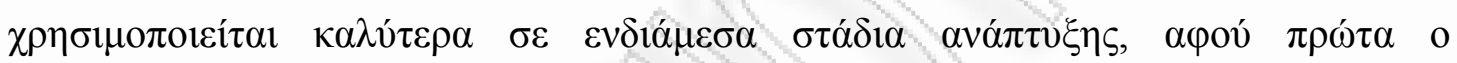

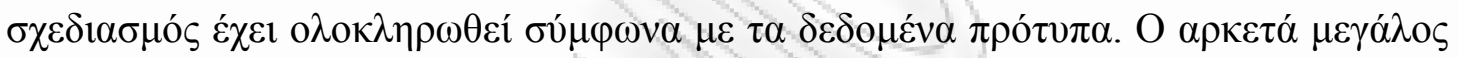

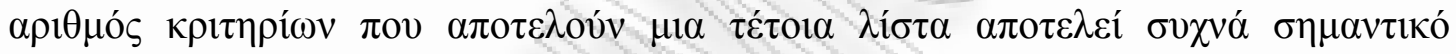

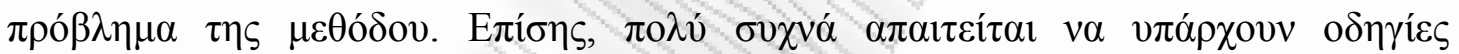

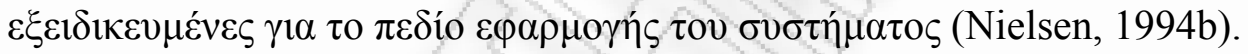

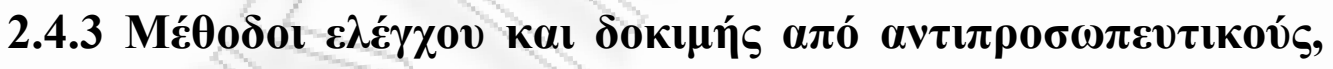 $\chi \rho \eta ́ \sigma \tau \varepsilon \varsigma$ (user testing methods)}

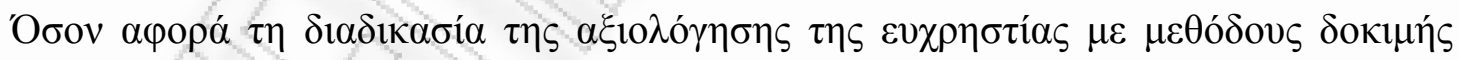

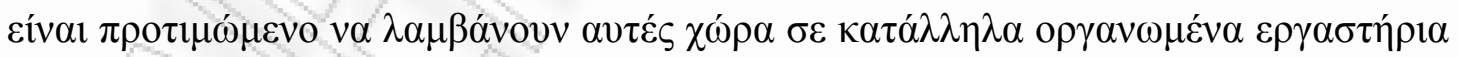

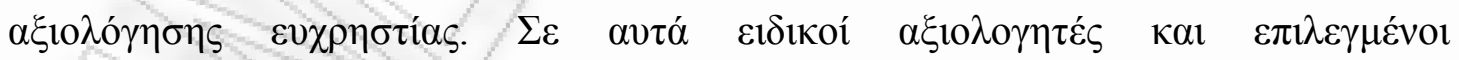

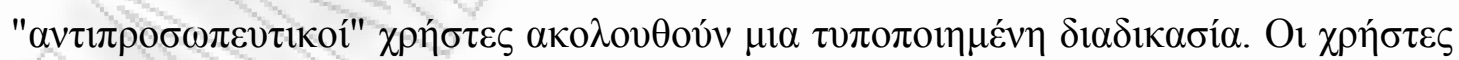

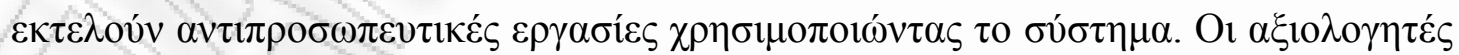

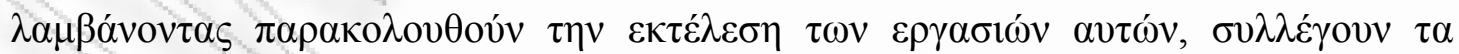

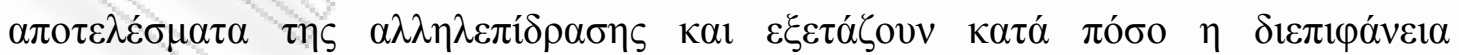

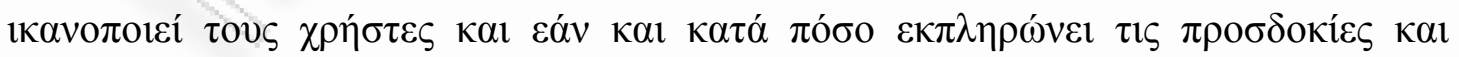

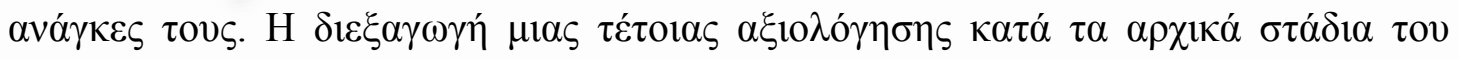

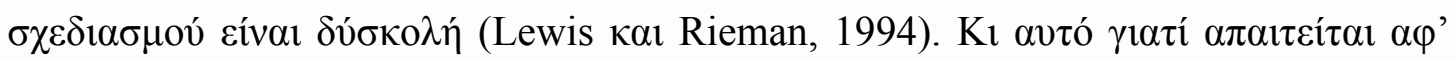

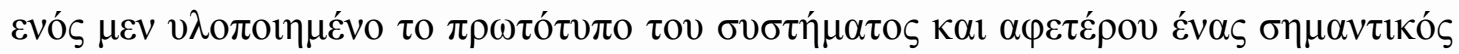

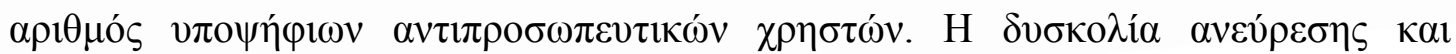




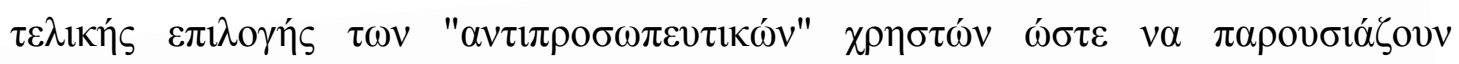

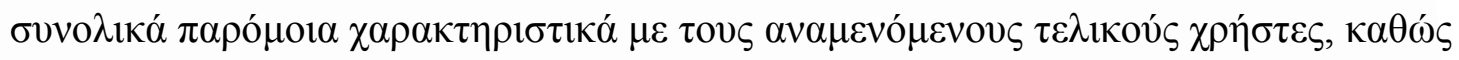

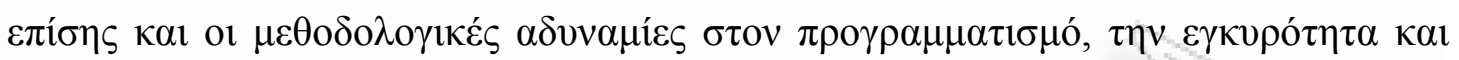

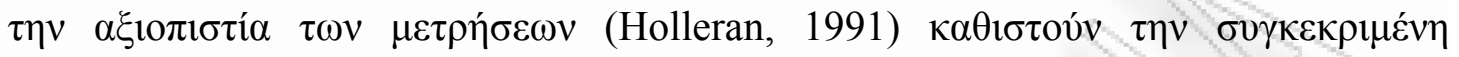

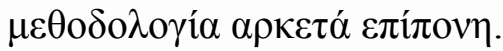

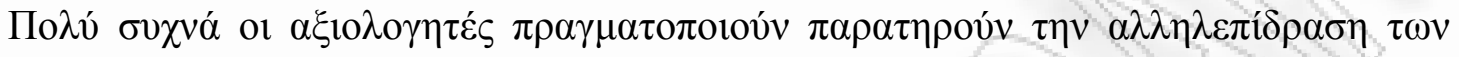

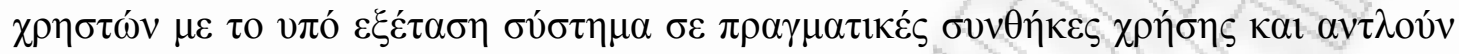

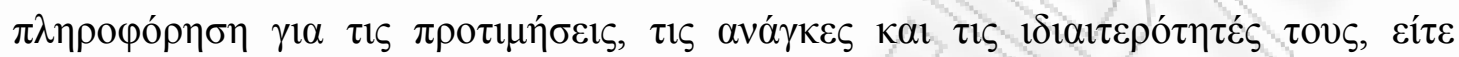

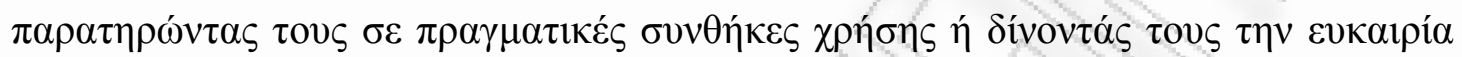

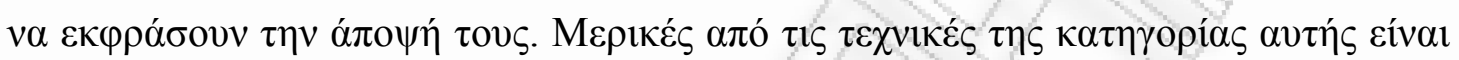
ol $\alpha \kappa o ́ \lambda o v \theta \varepsilon \varsigma$ :

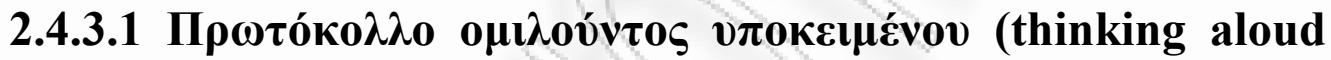 protocol)}

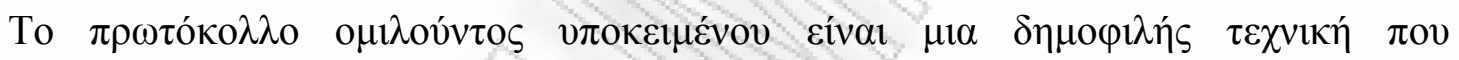

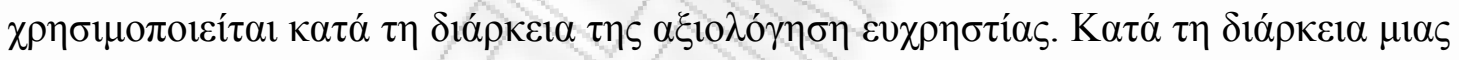

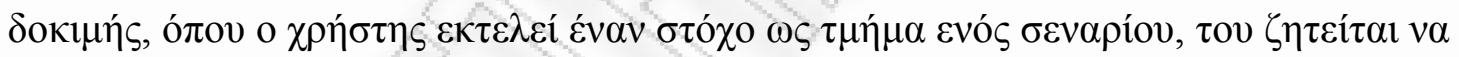

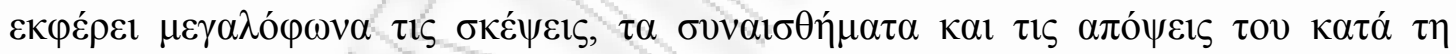

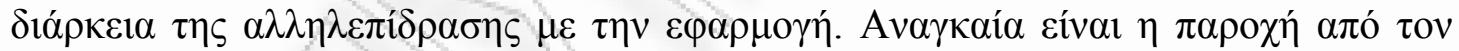

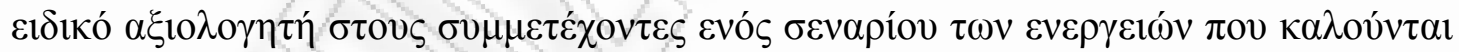

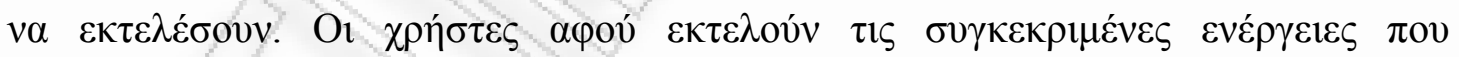

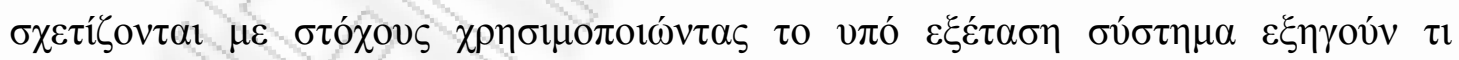

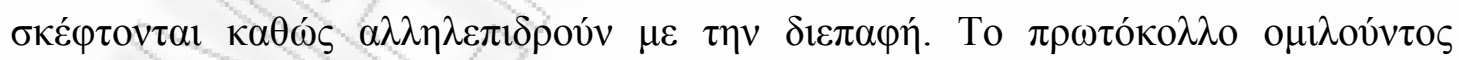

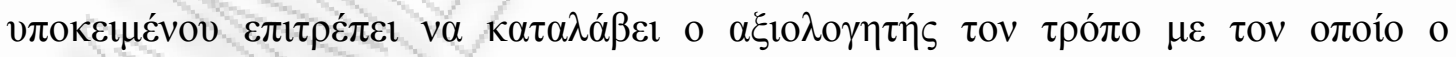

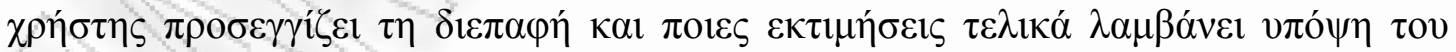

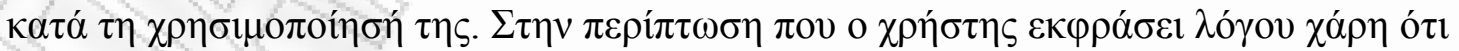

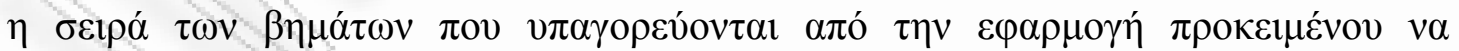

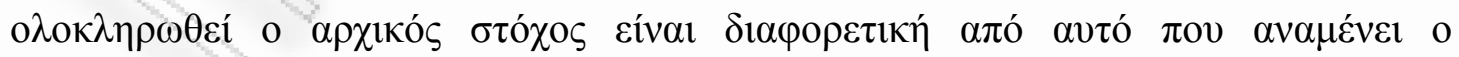

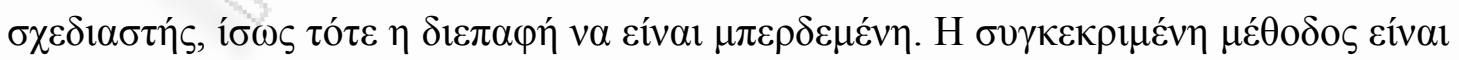

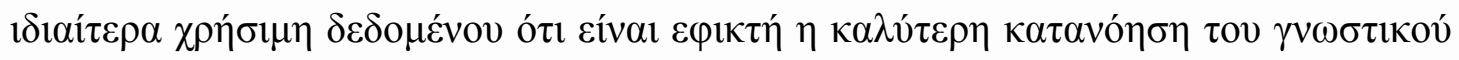

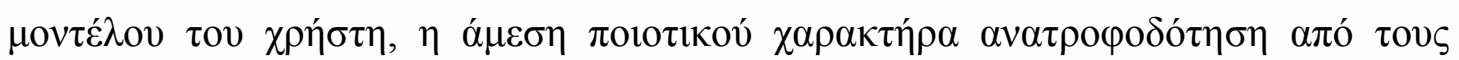

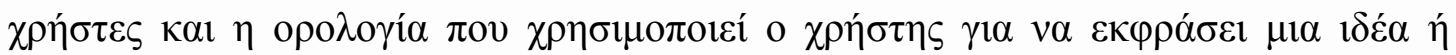




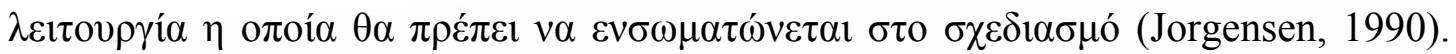

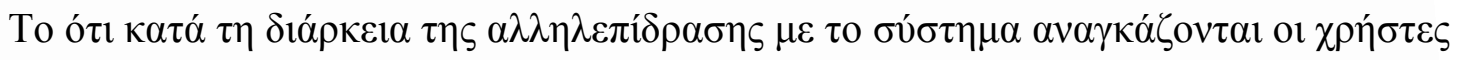

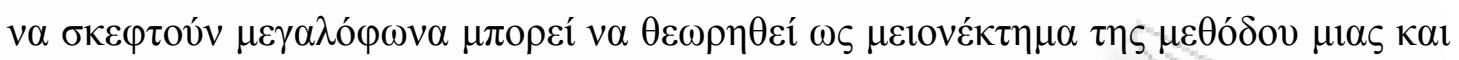

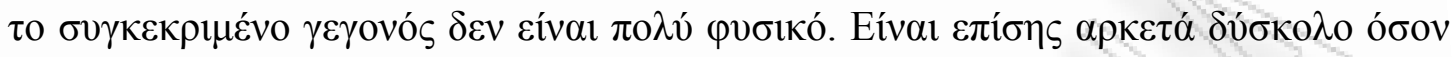

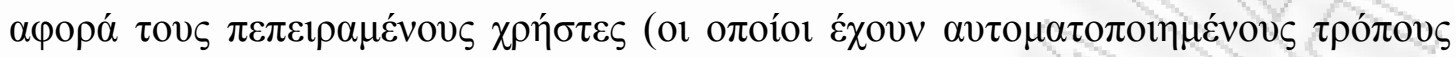

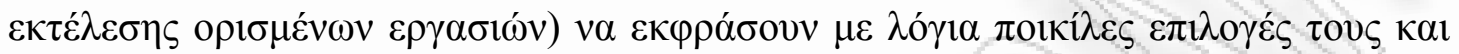

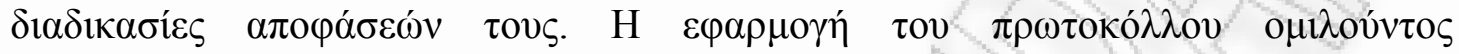

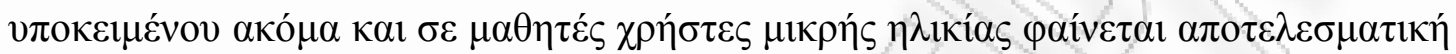

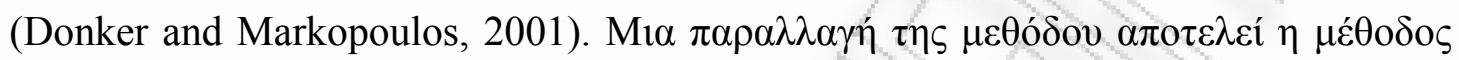

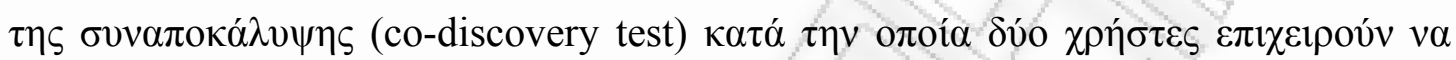

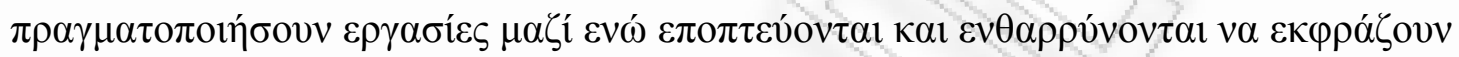

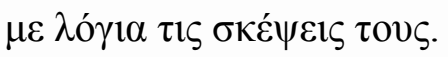

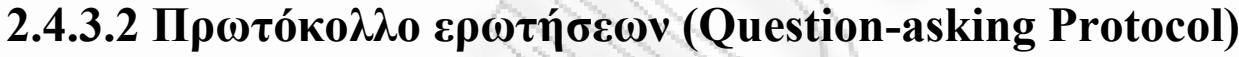

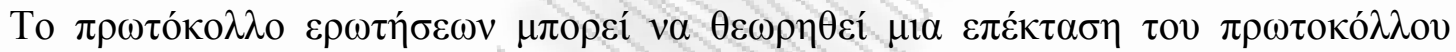

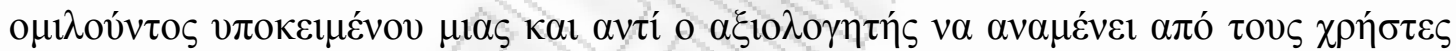

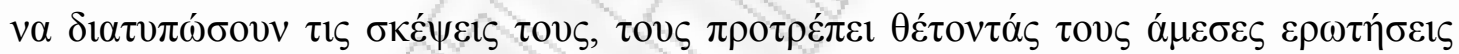

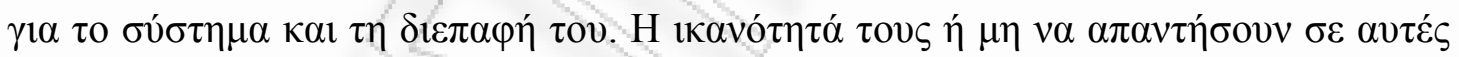

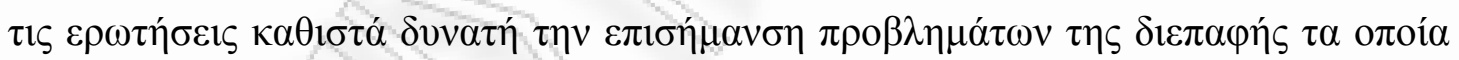

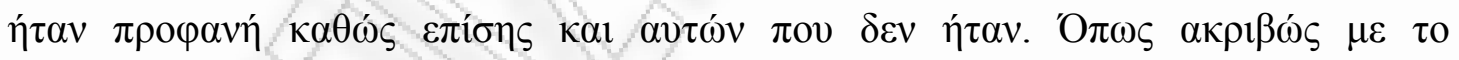

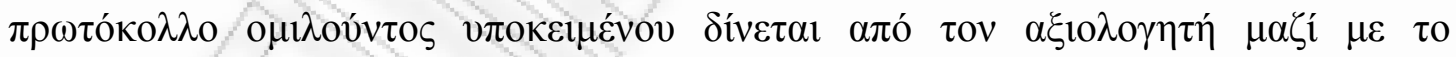

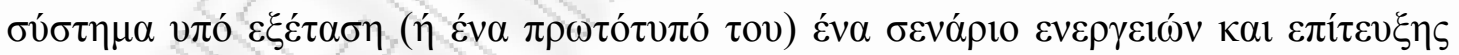

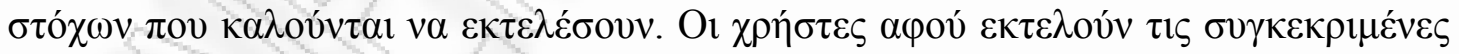

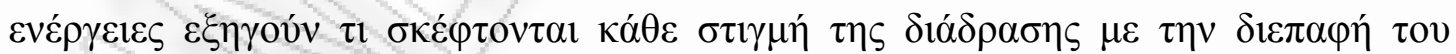

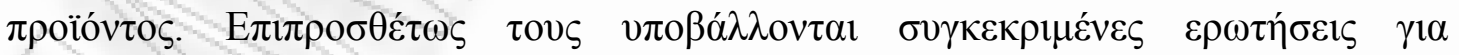

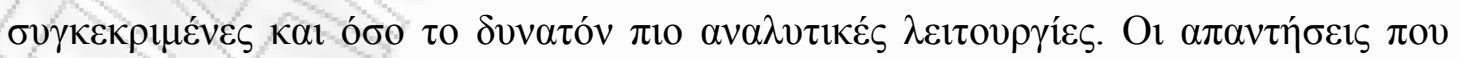

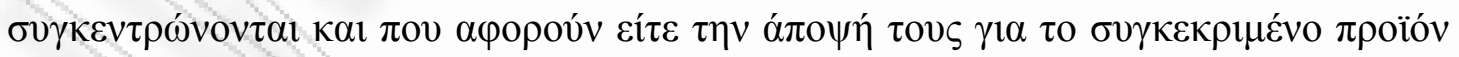

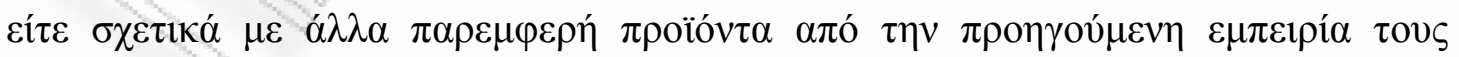

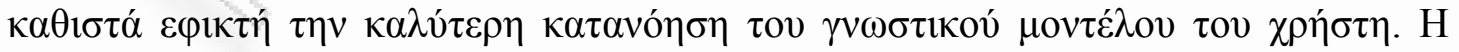

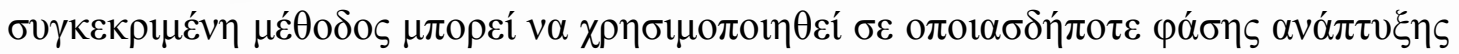

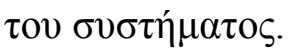




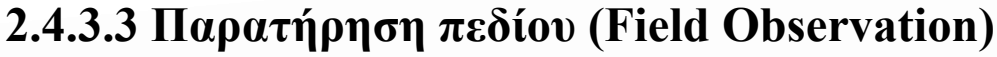

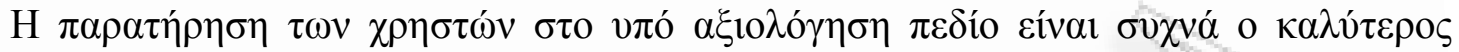

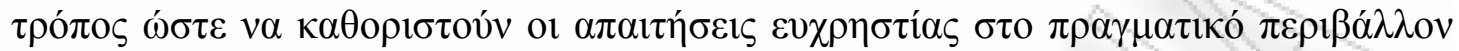

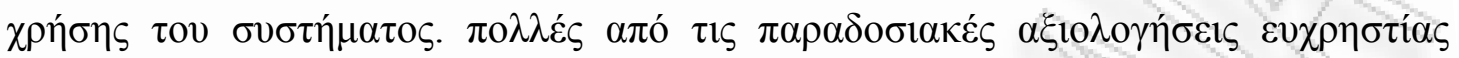

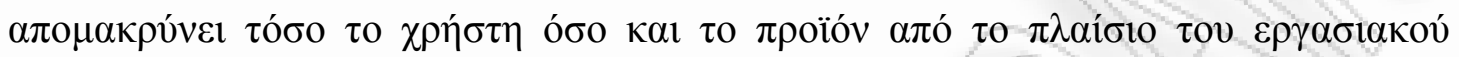

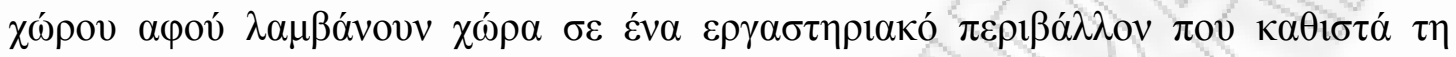

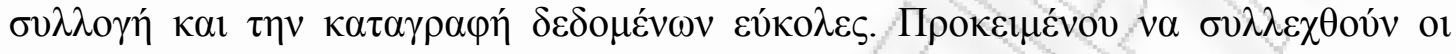

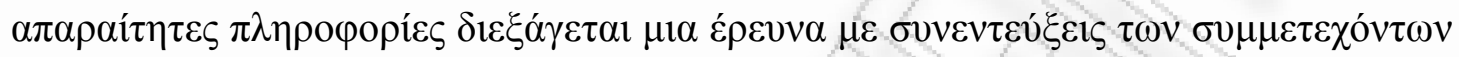

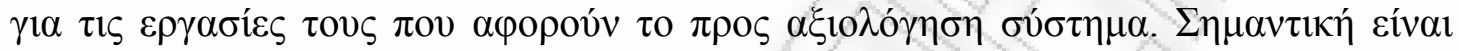

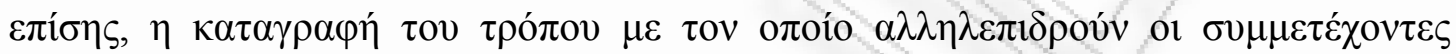

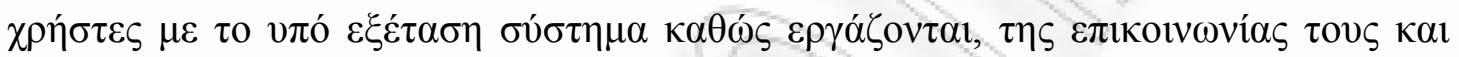

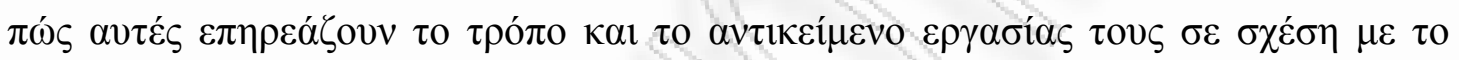

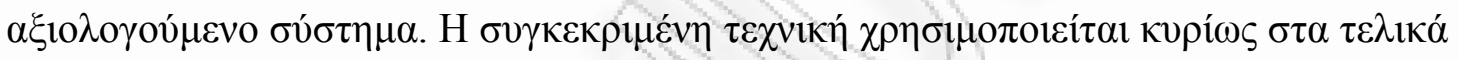

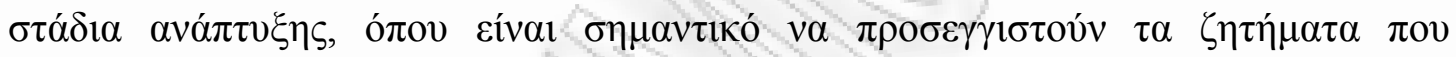

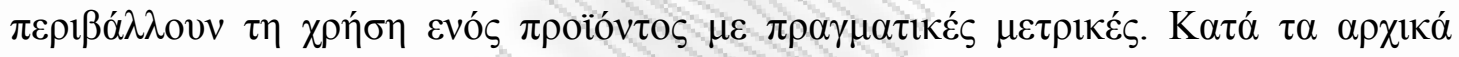

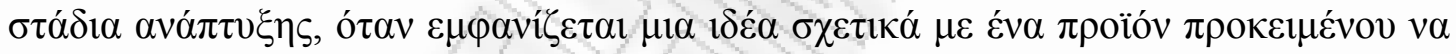

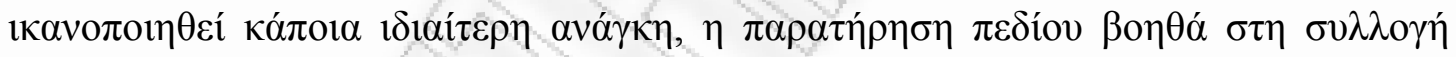

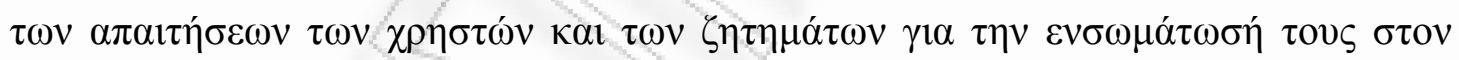

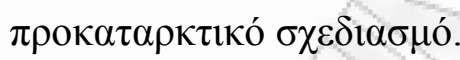

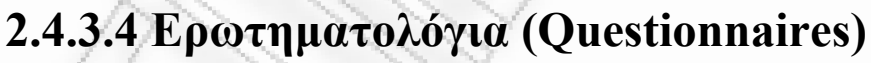

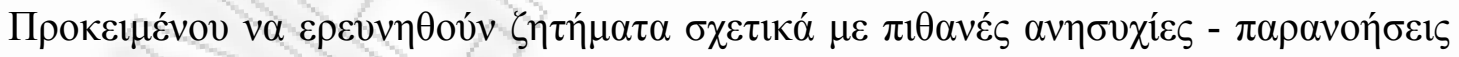

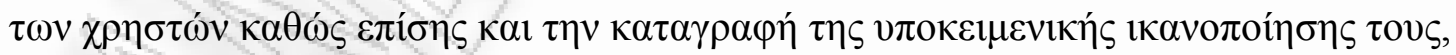
$\tau \alpha \varepsilon \rho \omega \tau \eta \mu \alpha \tau o \lambda o ́ \gamma 1 \alpha$ a

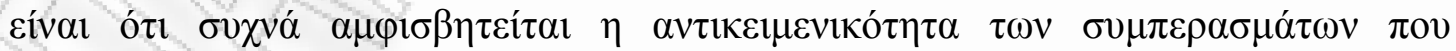

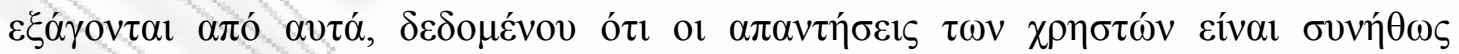

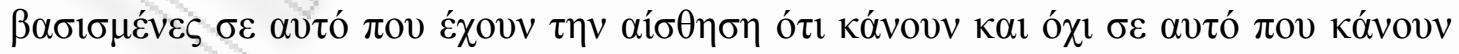

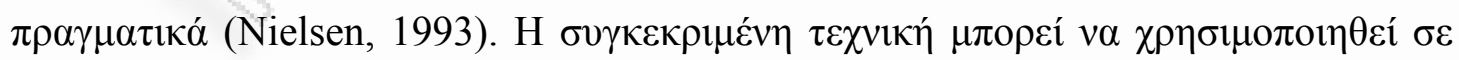

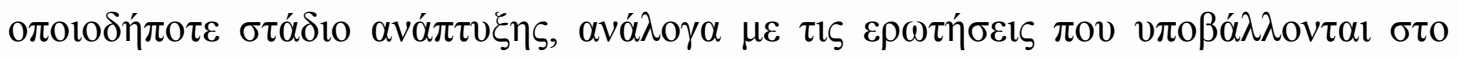

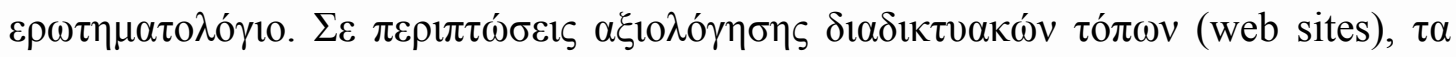

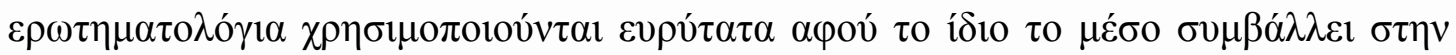




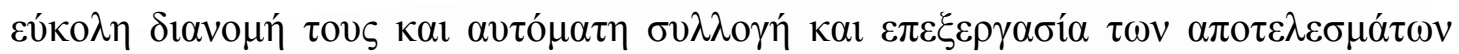

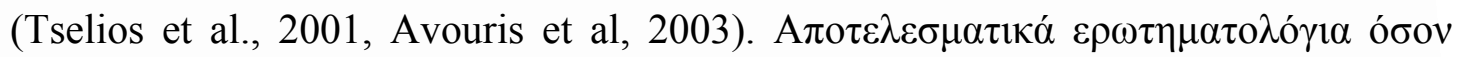

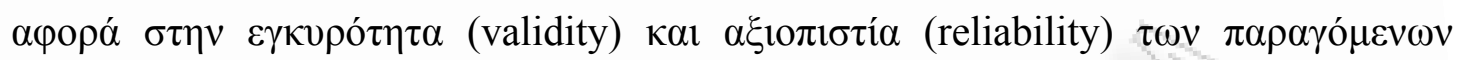

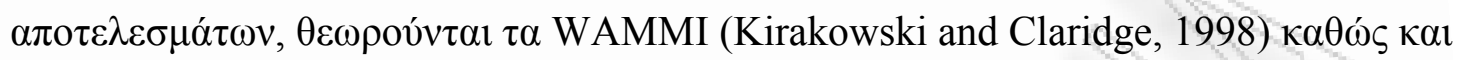
QUIS кal SUMI (Schneiderman, 1998).

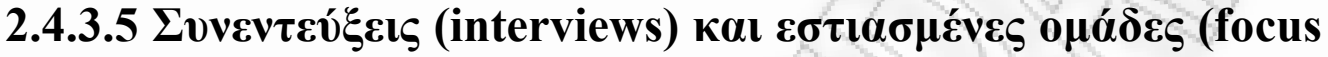 groups)}

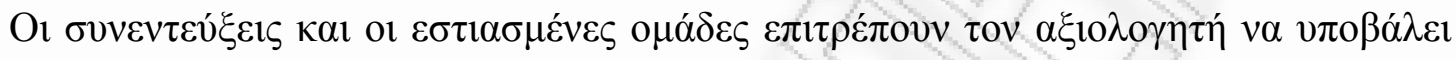

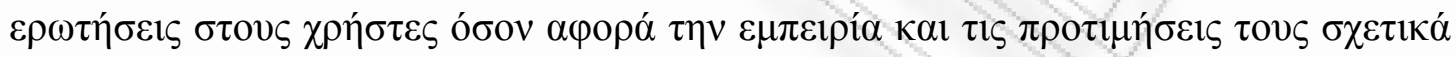

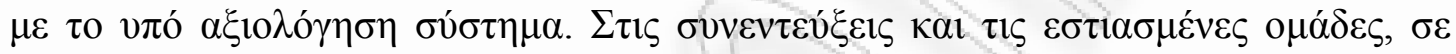

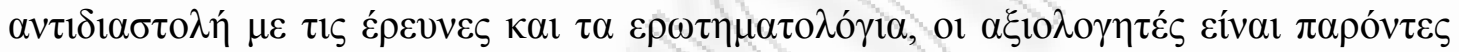

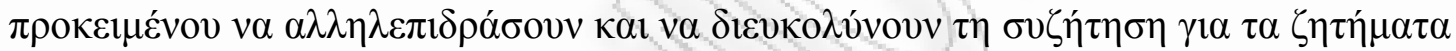

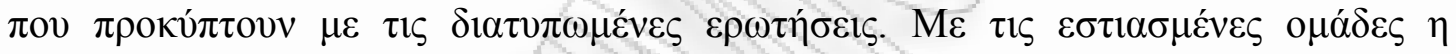

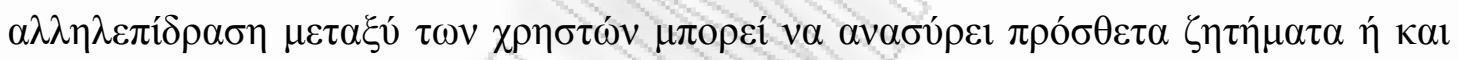

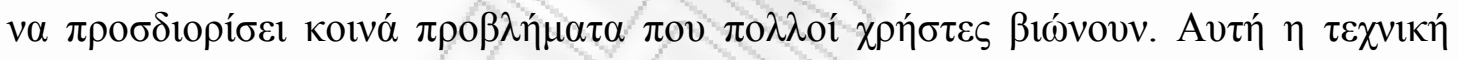

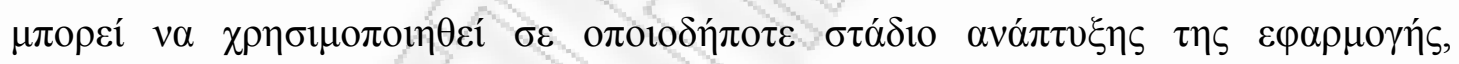

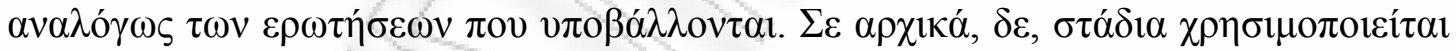

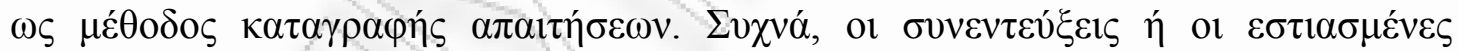

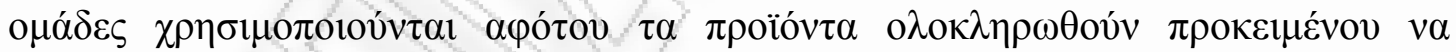

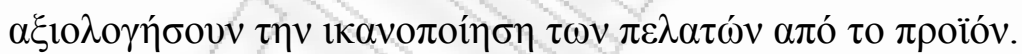

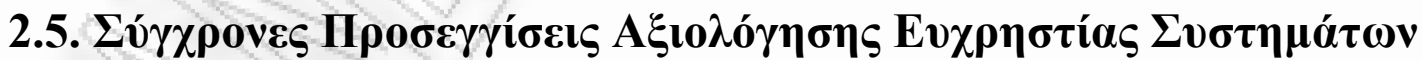

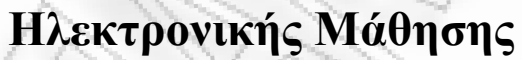

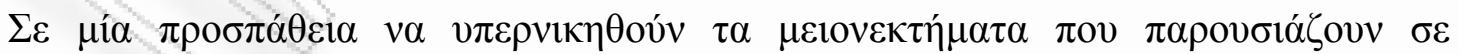

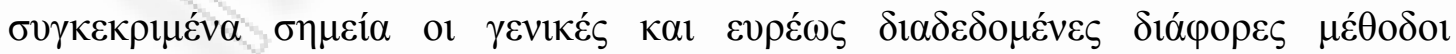

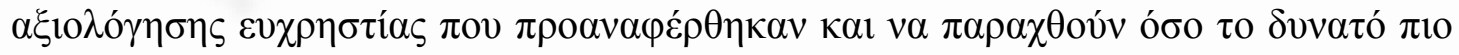

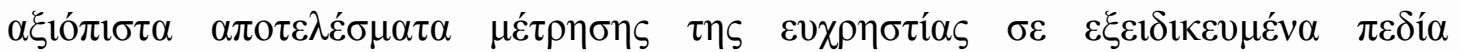

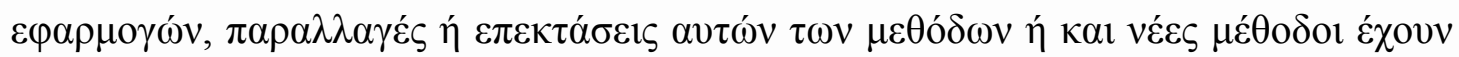

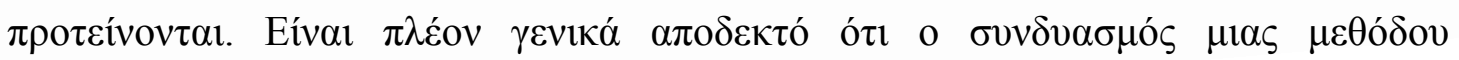




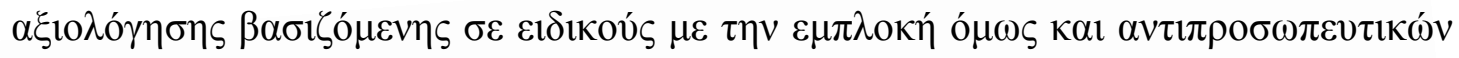

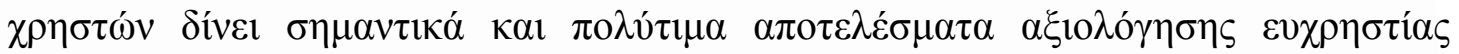
(Tselios et. al., 2008).

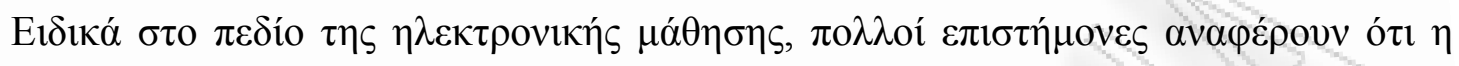

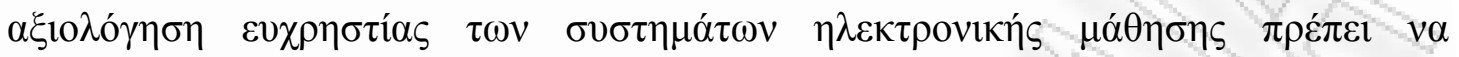

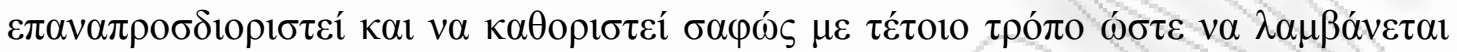

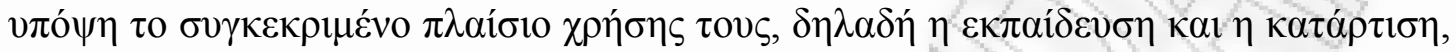

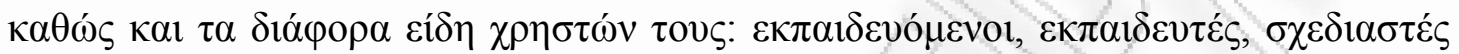

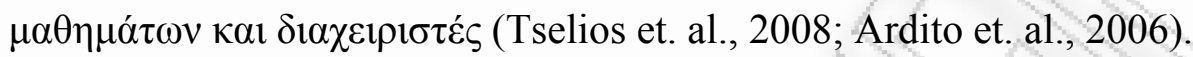

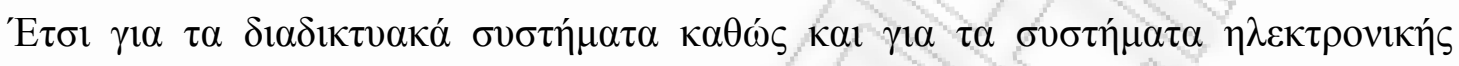

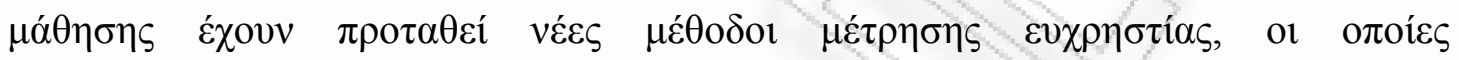

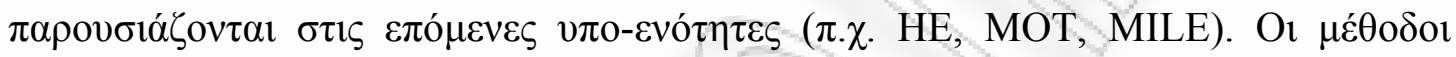

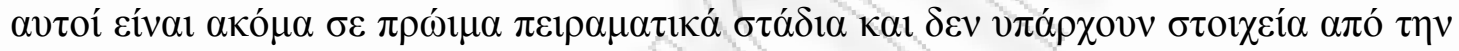

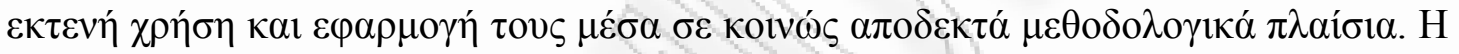

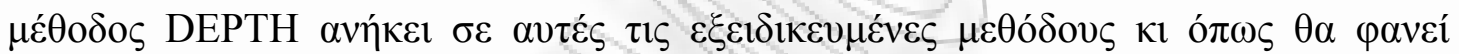

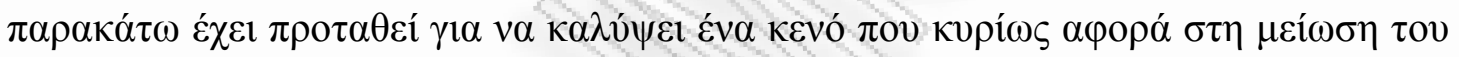

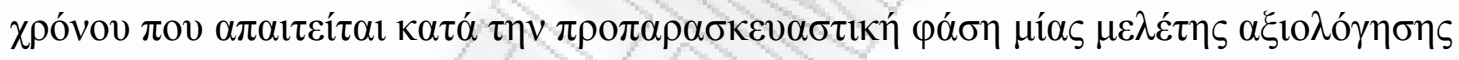

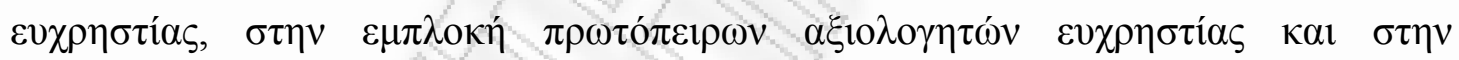

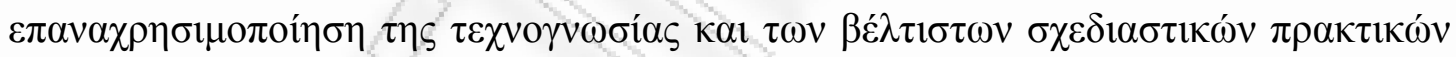

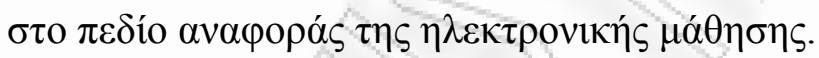

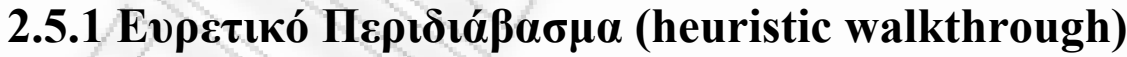

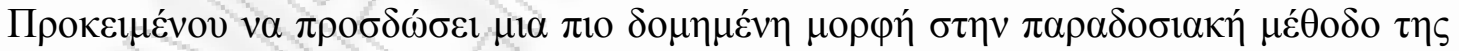

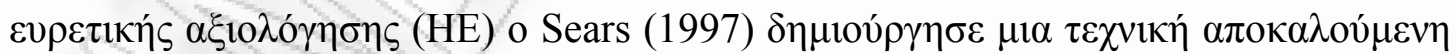

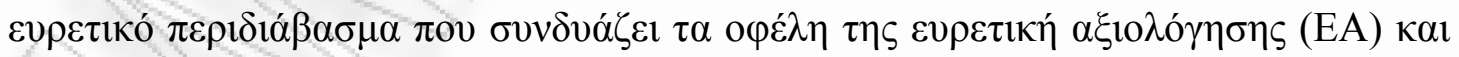

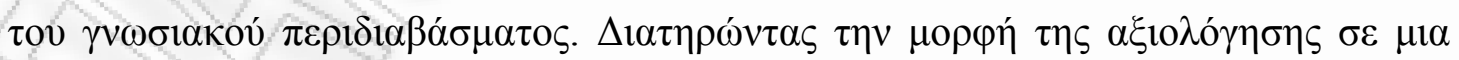

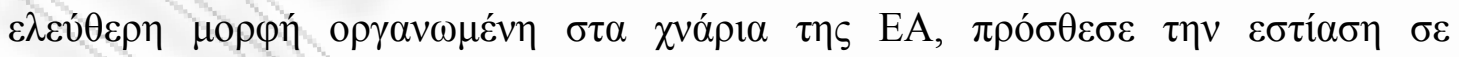

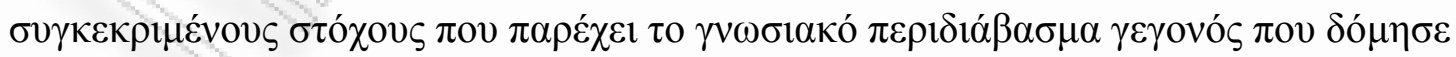

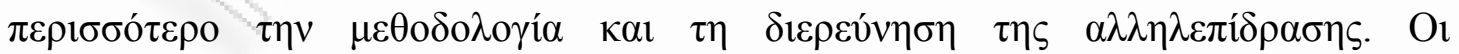

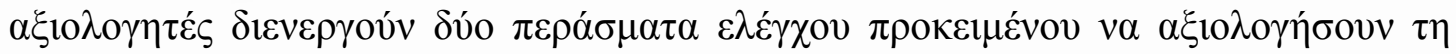

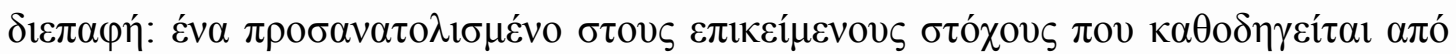

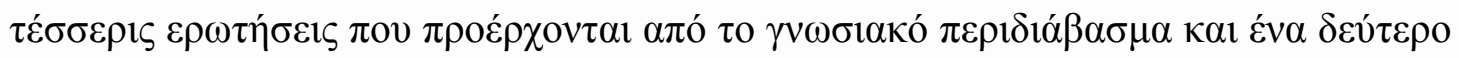




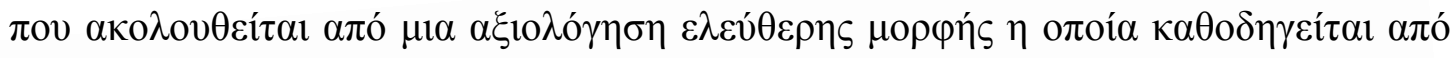

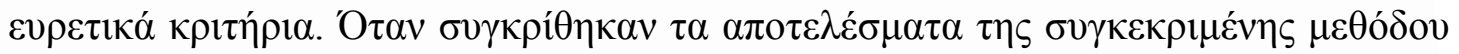

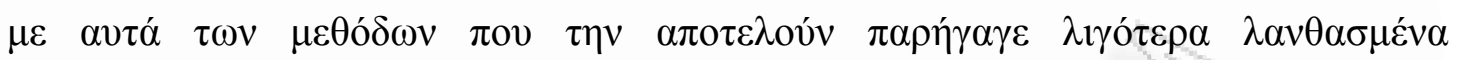

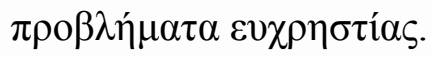

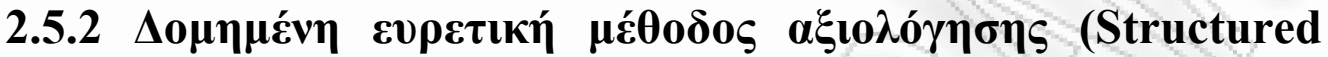 Heuristic Evaluation Method).}

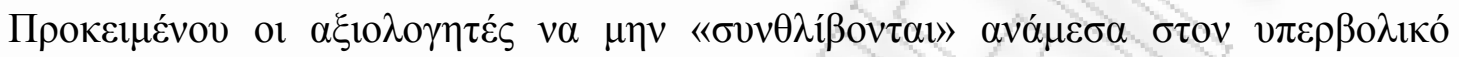

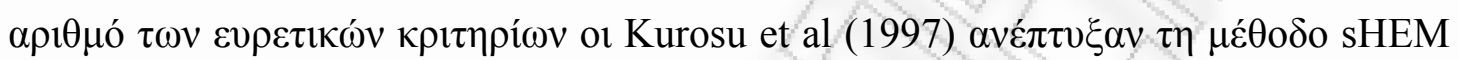

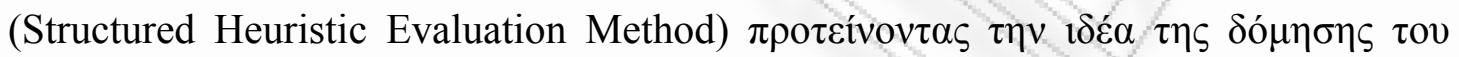

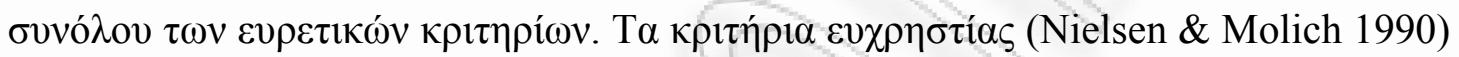

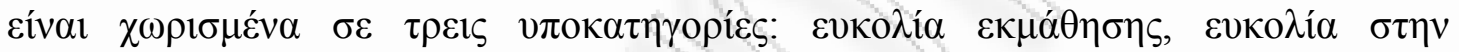

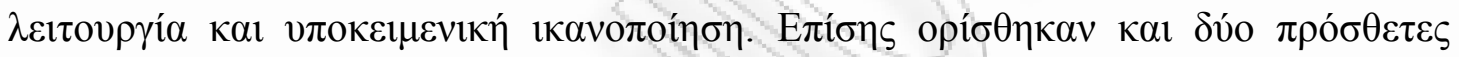

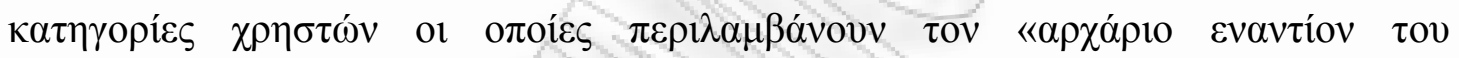

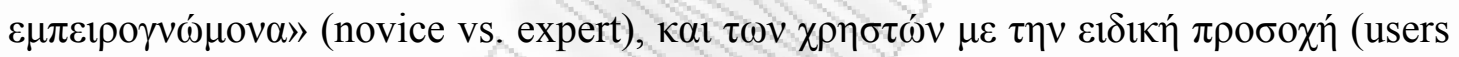

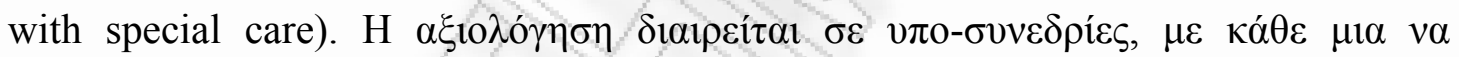

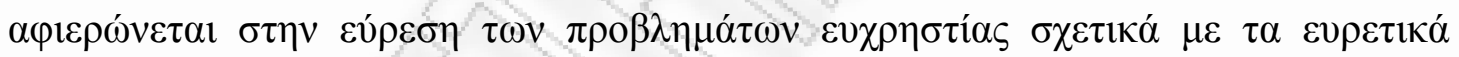

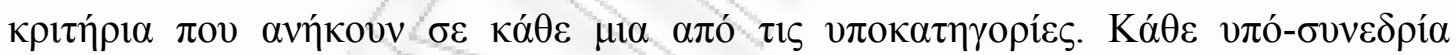

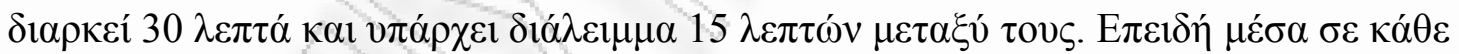

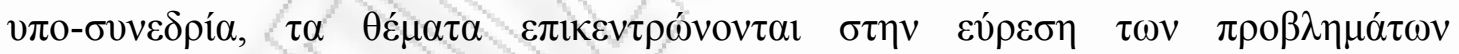

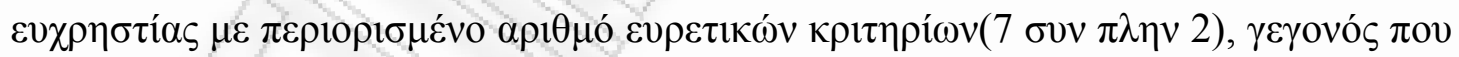

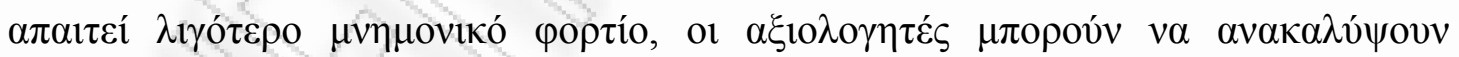

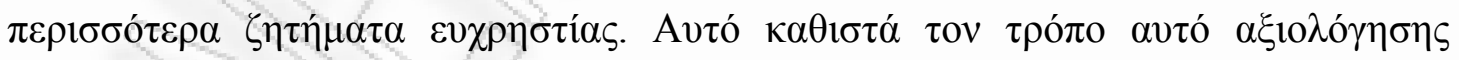

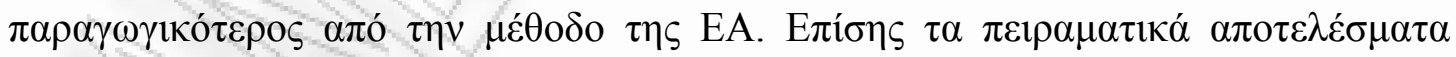

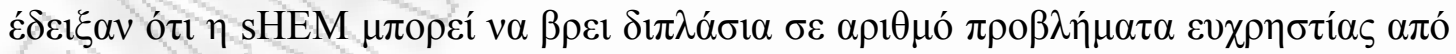

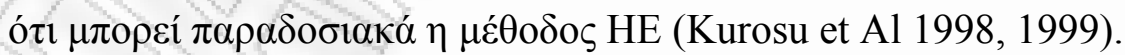

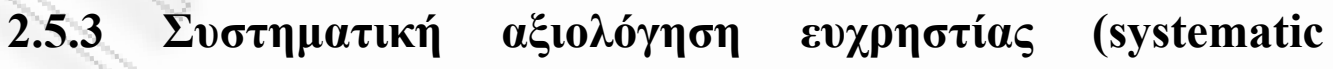 usability evaluation (SUE))}

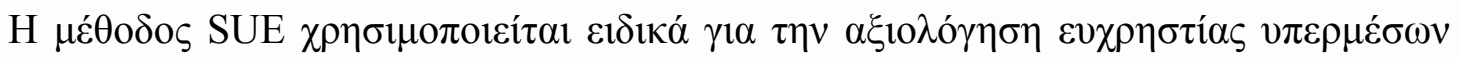

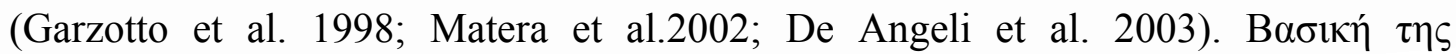




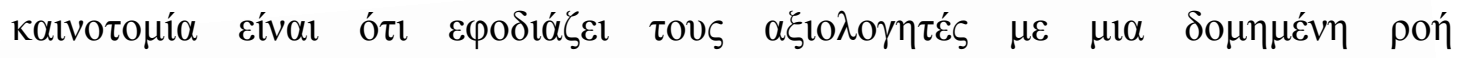

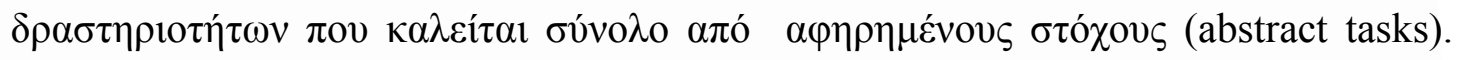

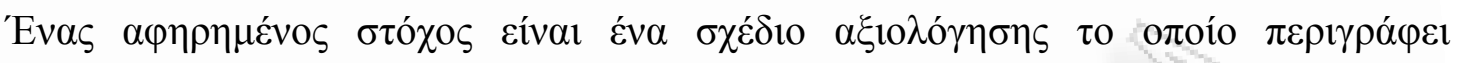

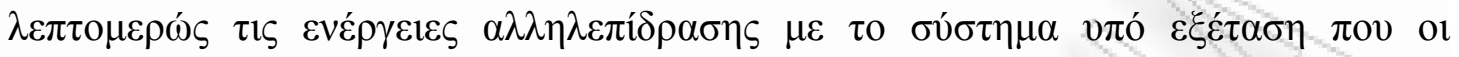

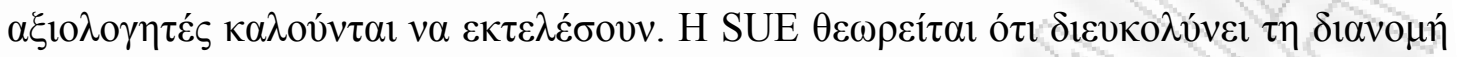

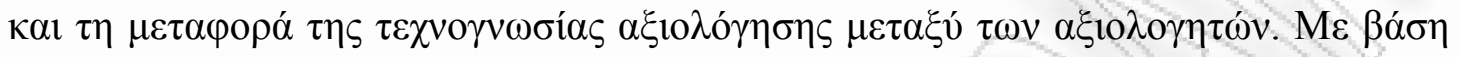

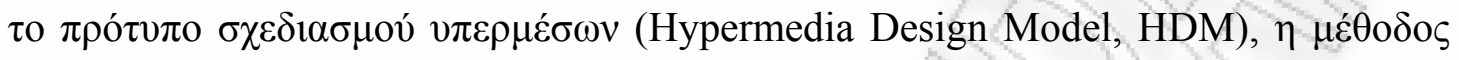

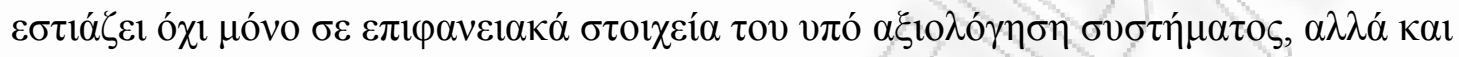

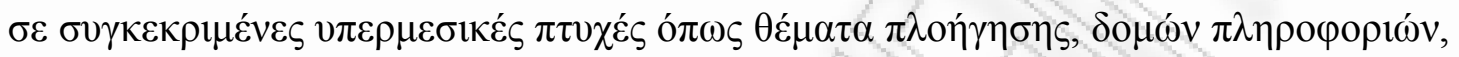

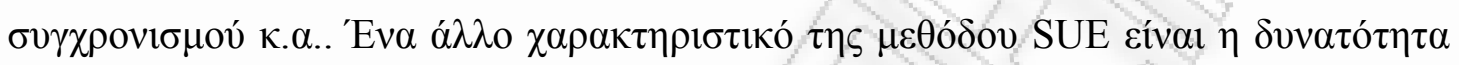

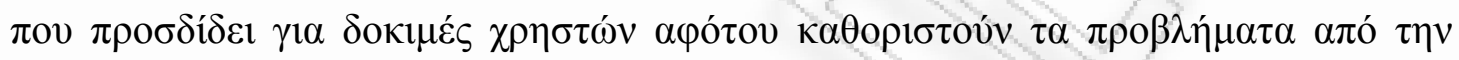

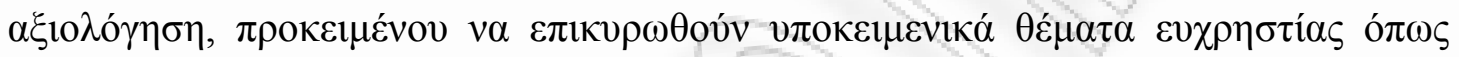

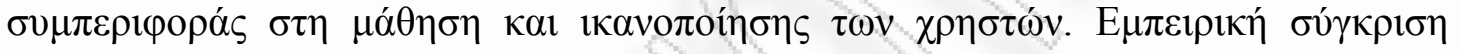

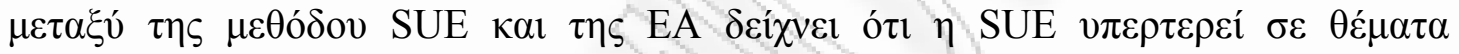

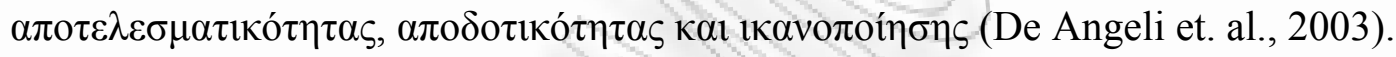

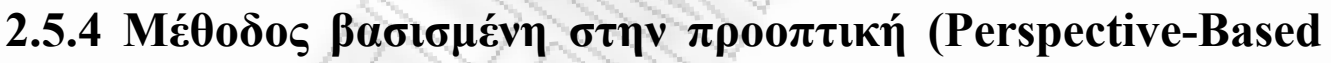 Method)}

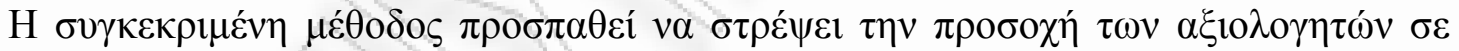

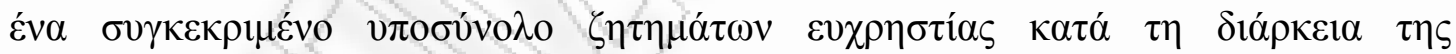

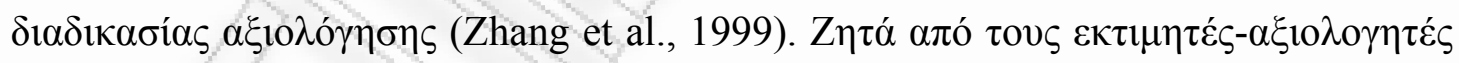

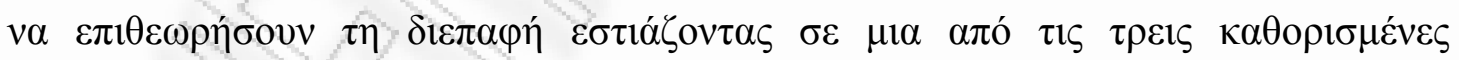

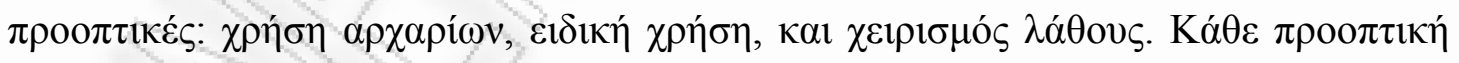

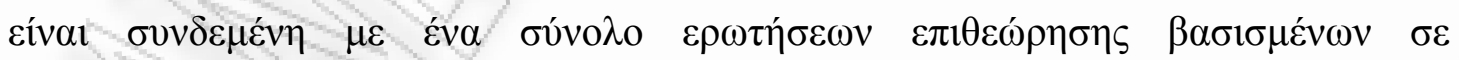

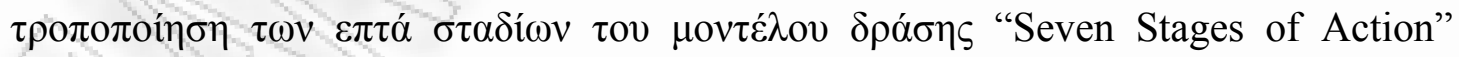

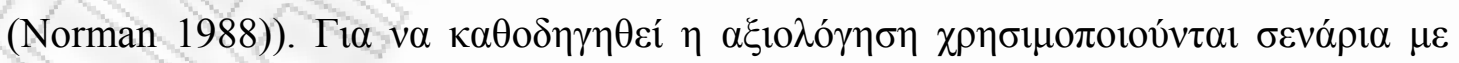

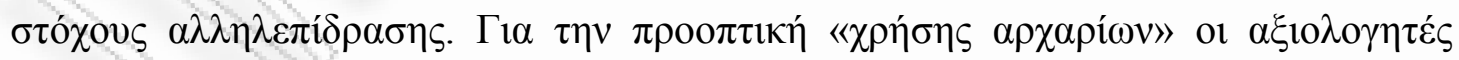

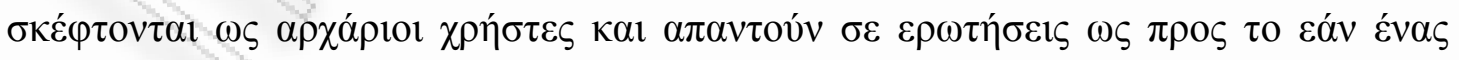

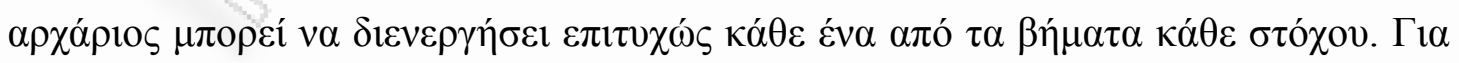

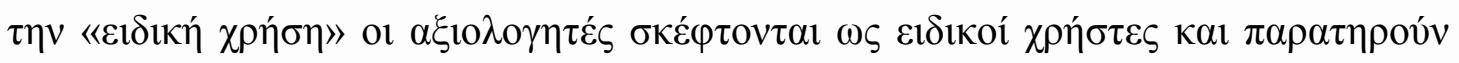

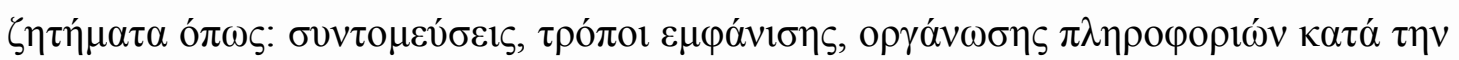

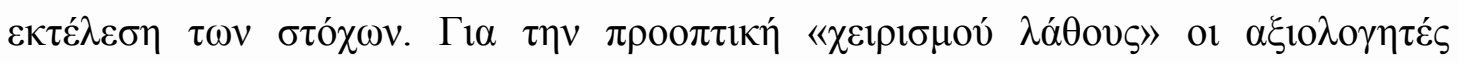




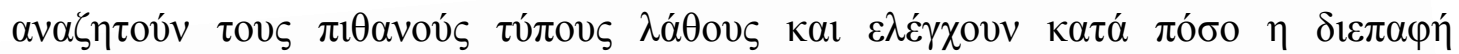

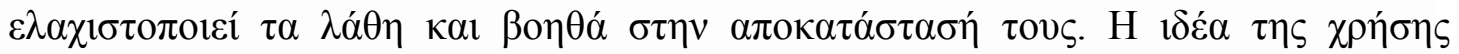

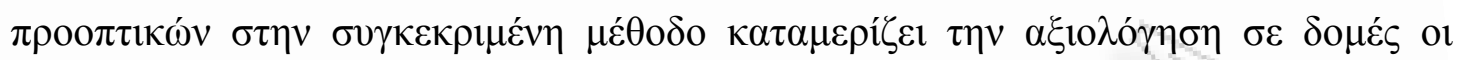

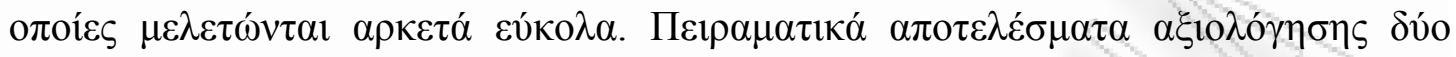

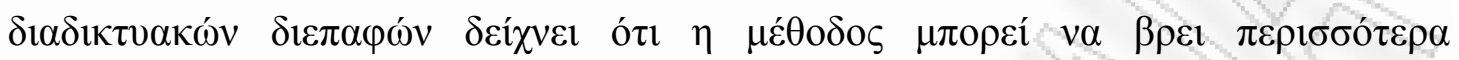

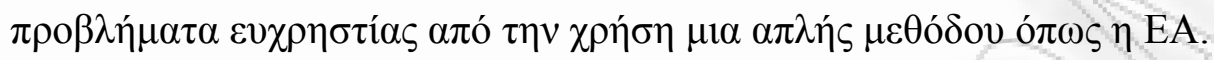

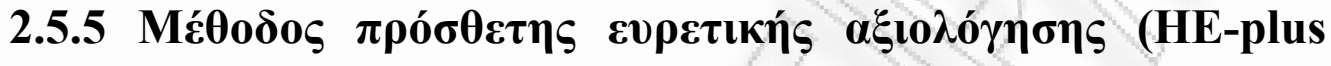 method)}

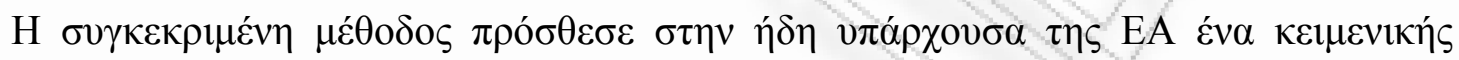

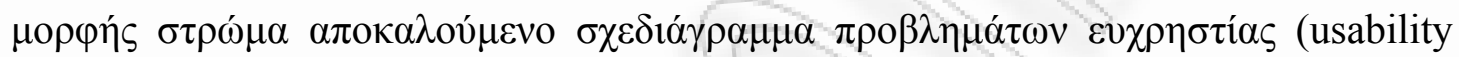

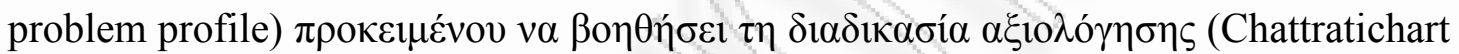

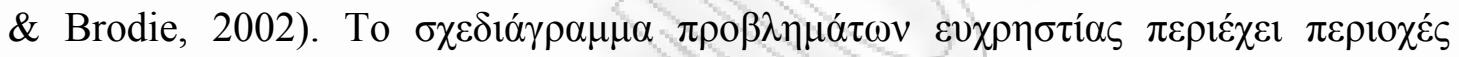

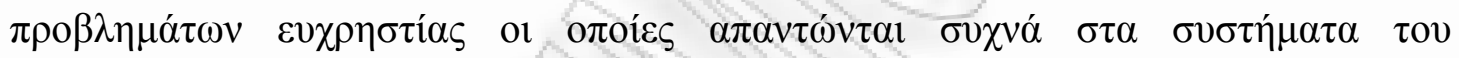

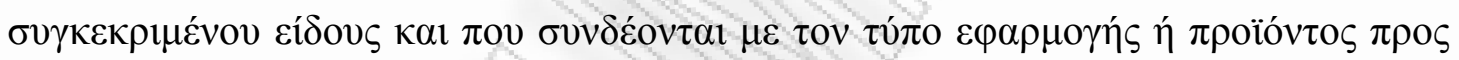

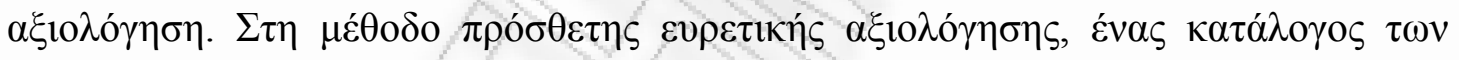

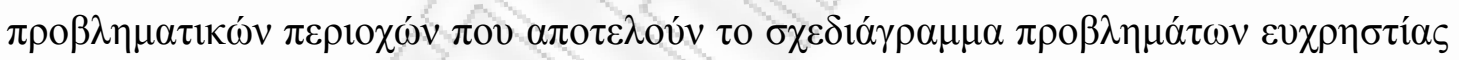

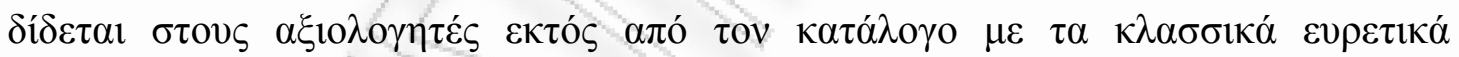

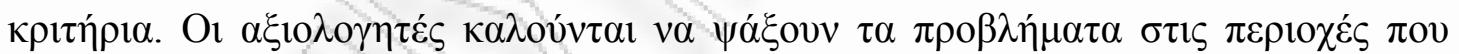

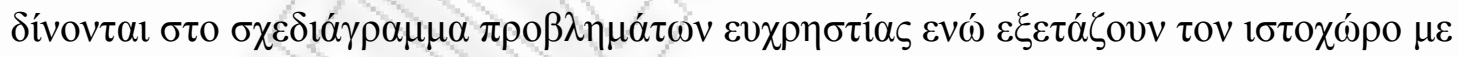

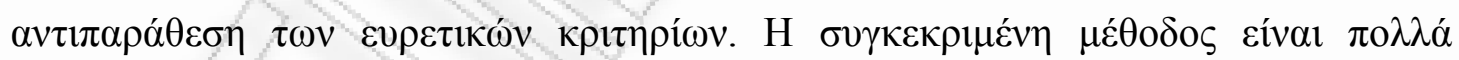

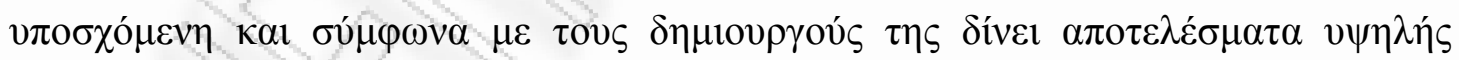

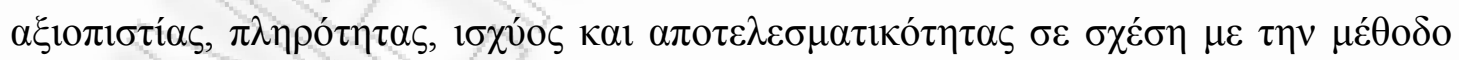

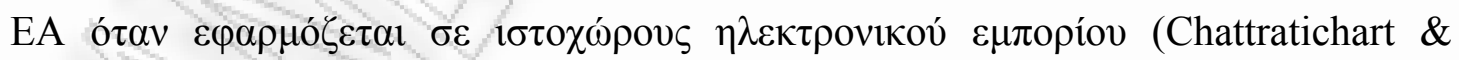

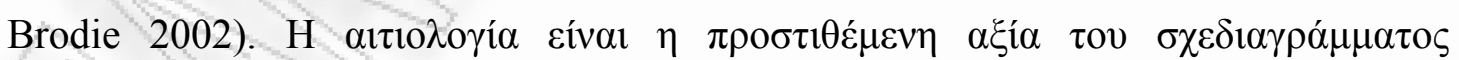

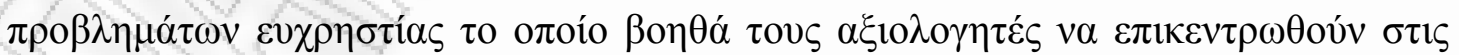

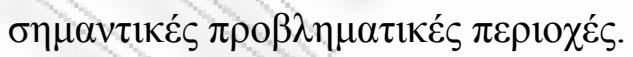

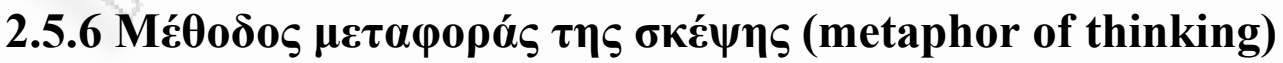

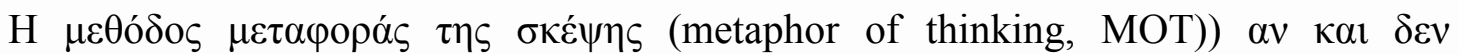

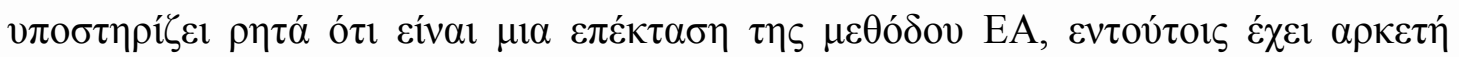




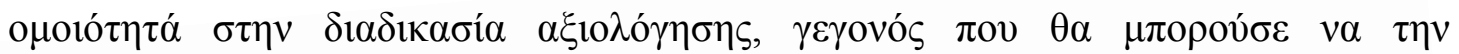

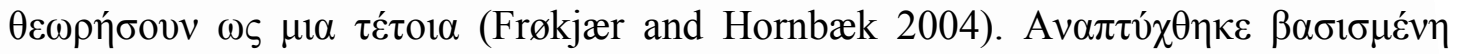

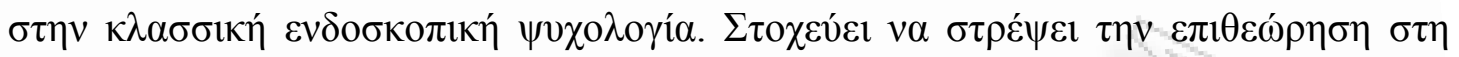

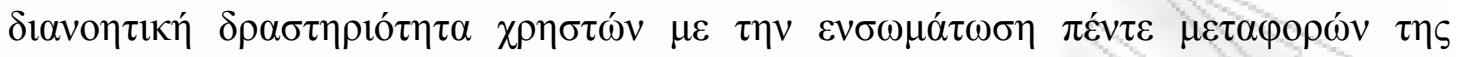

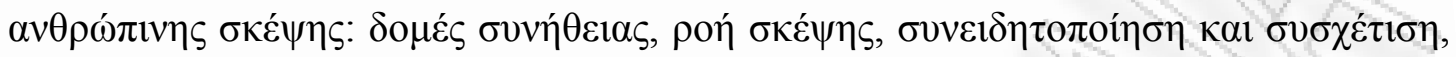

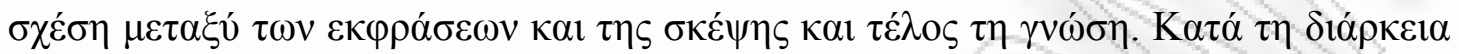

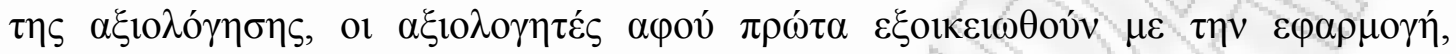

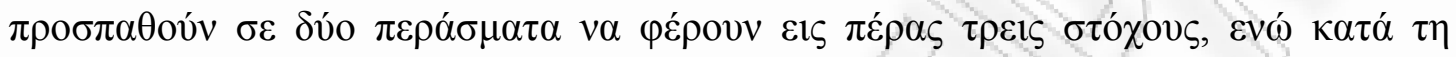

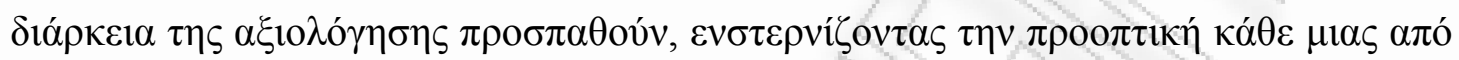

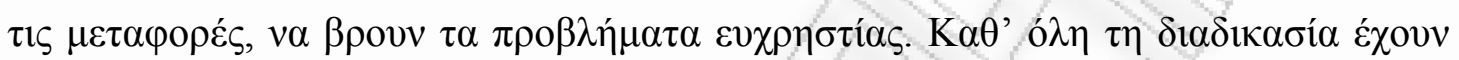

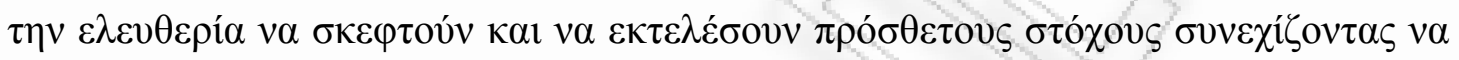

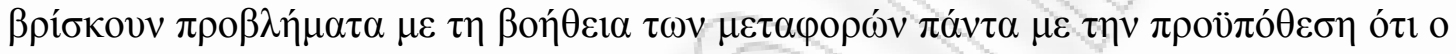

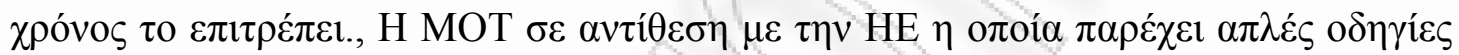

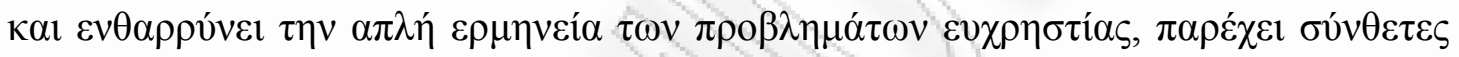

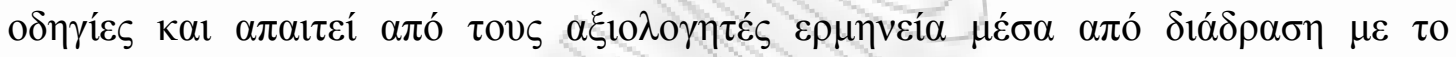

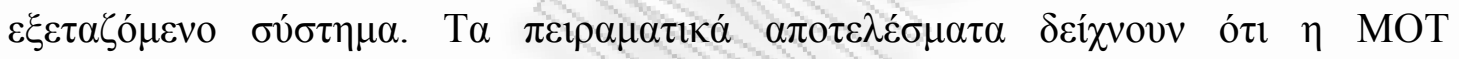

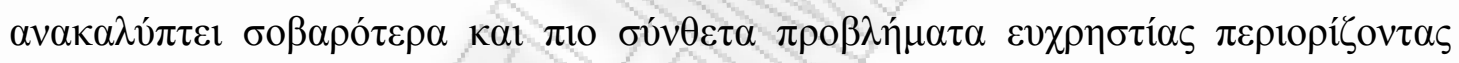

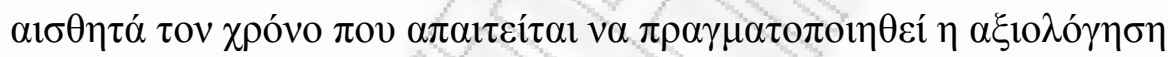

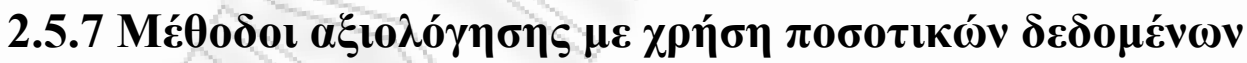

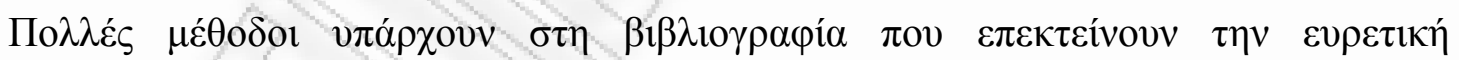

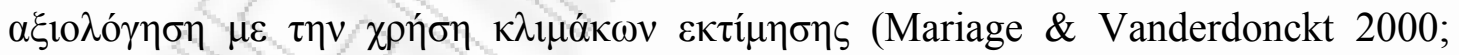
Avouris et. al., 2001; Sutcliffe 2001; Agarwal \& Venkatech 2002; Berry 2003;

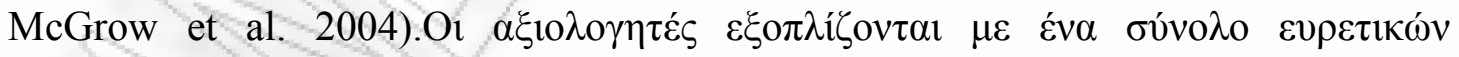

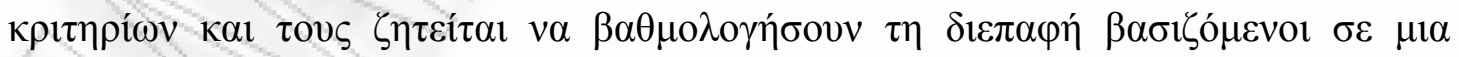

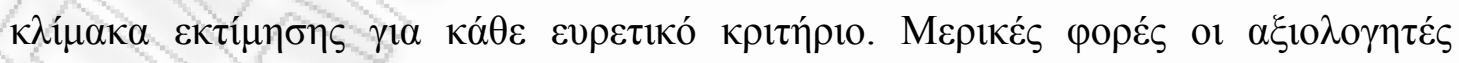

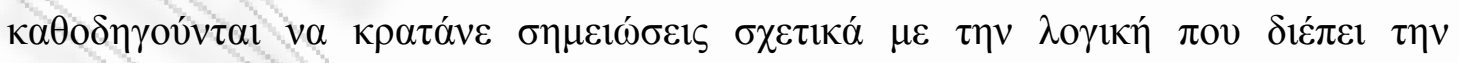

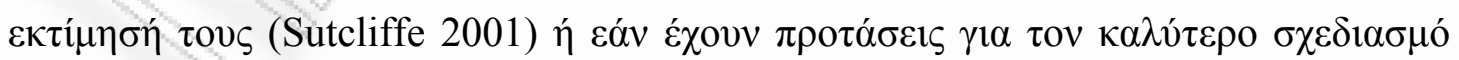

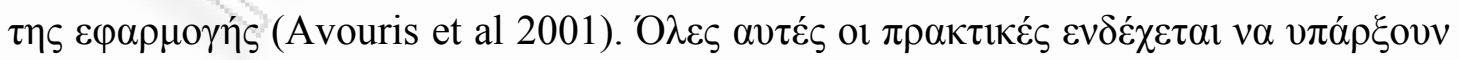

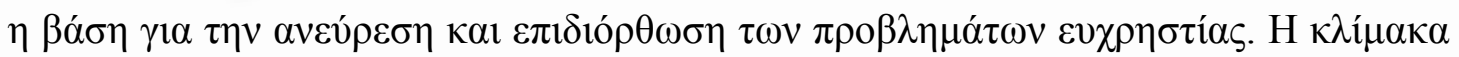

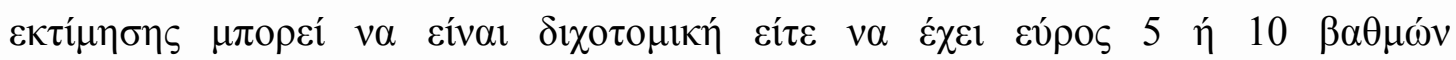

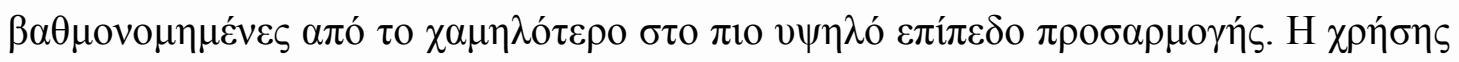




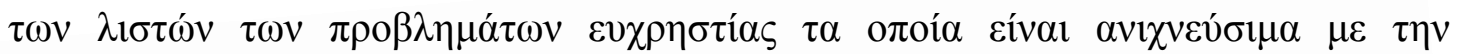

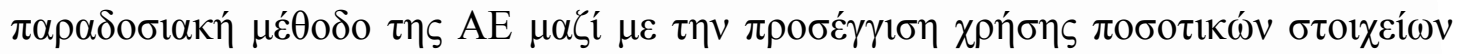

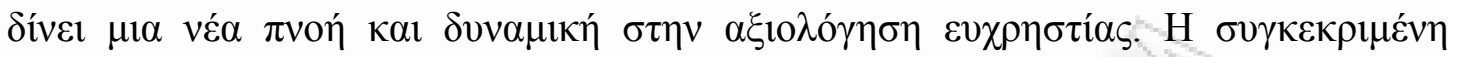

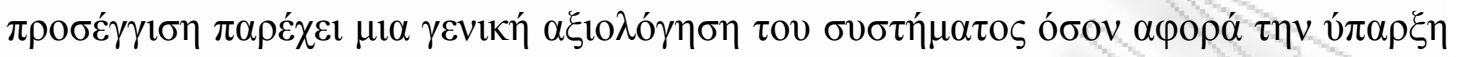

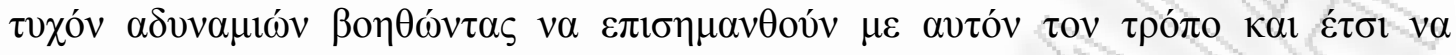

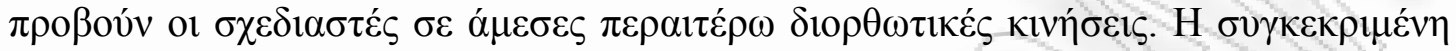

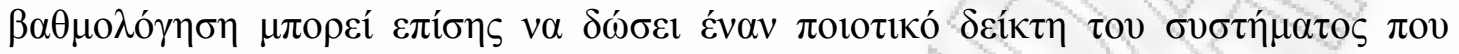

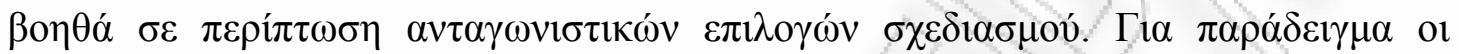

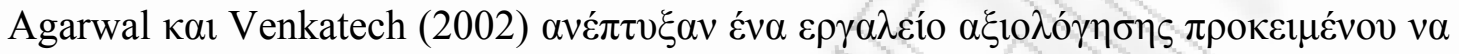

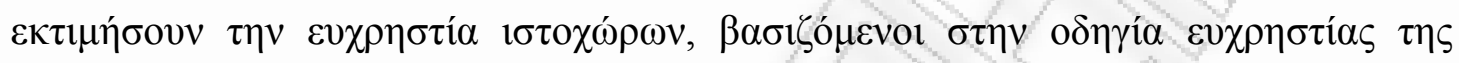

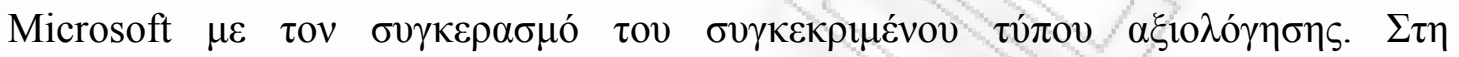

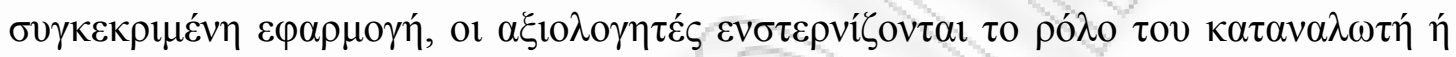

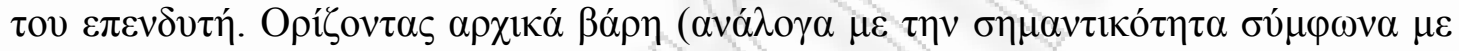

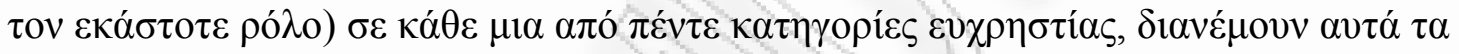

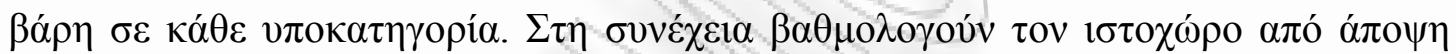

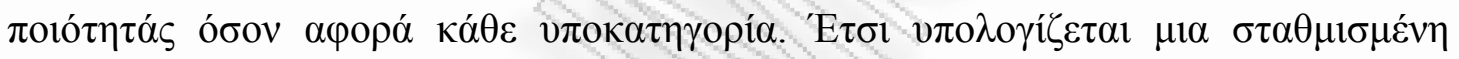

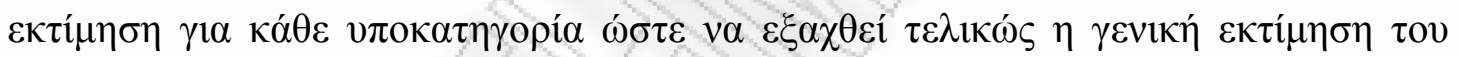
$1 \sigma \tau$ $\chi \omega ́ \rho о v$.

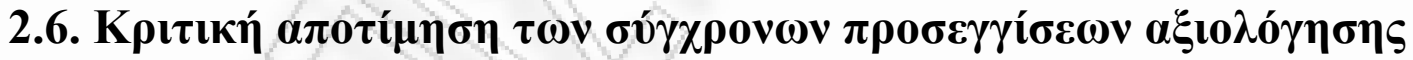

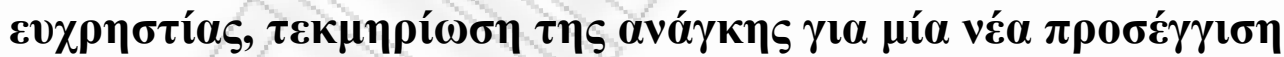

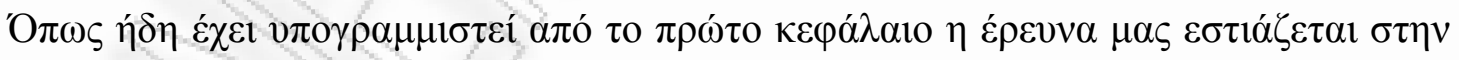

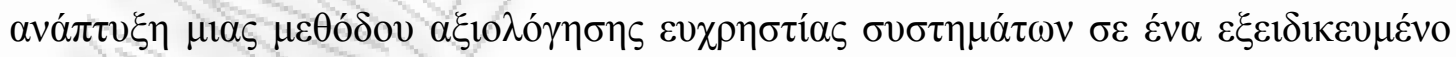

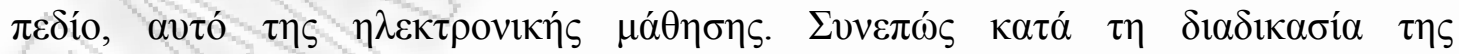

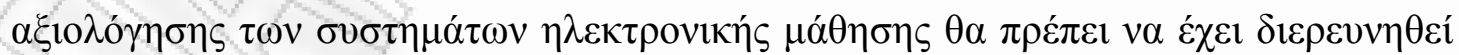

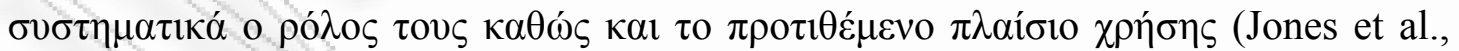

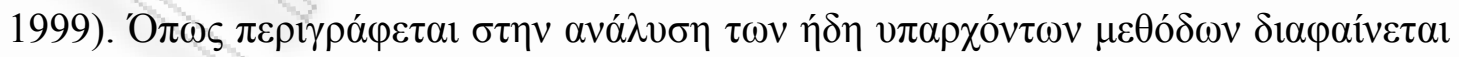

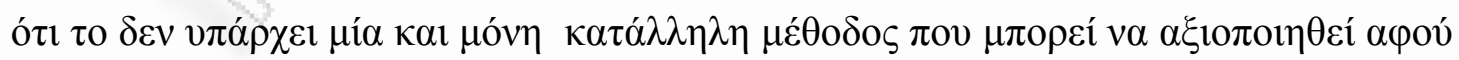

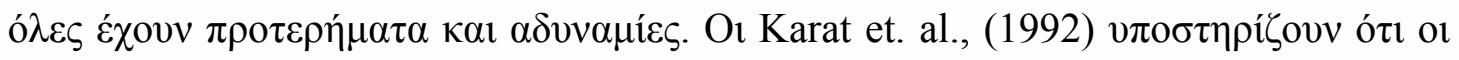

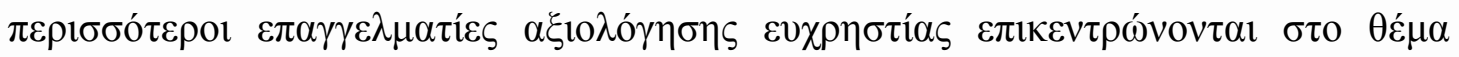

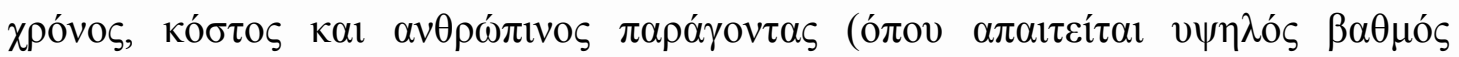




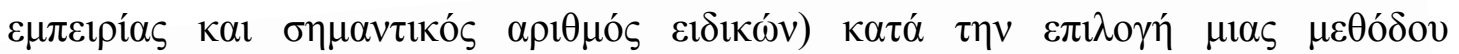

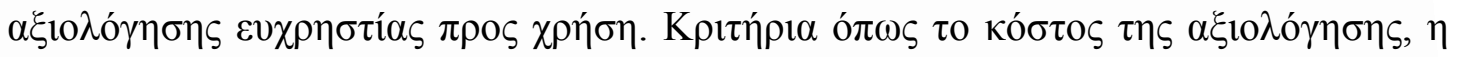

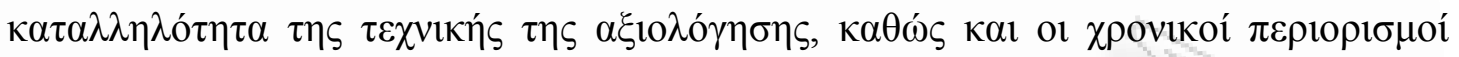

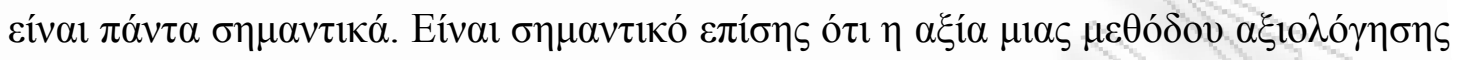

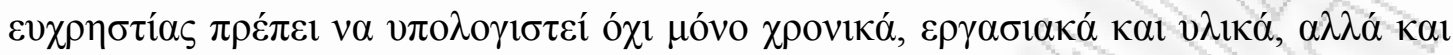

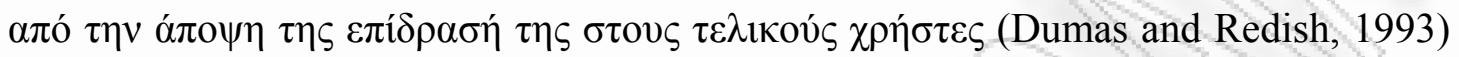

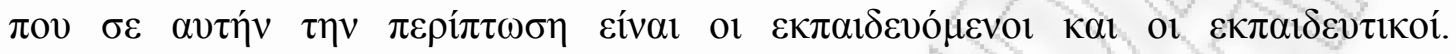

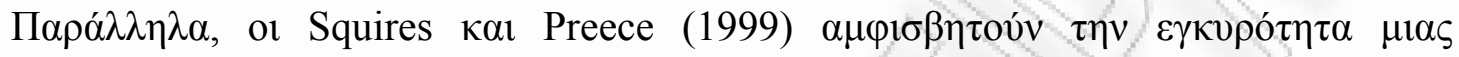

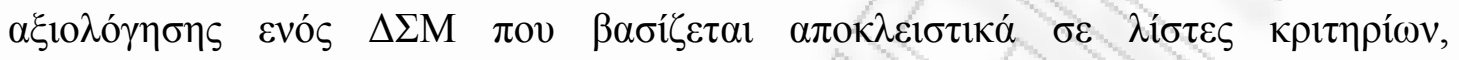

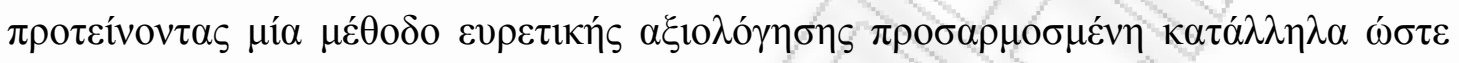

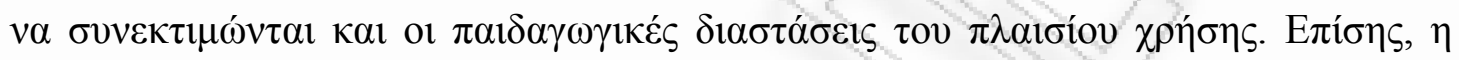

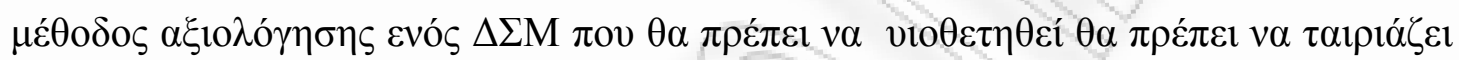

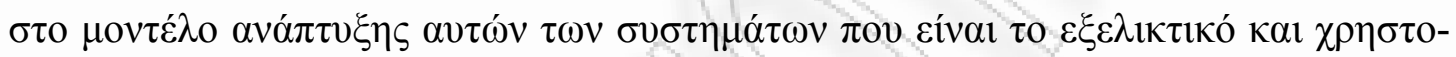

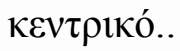

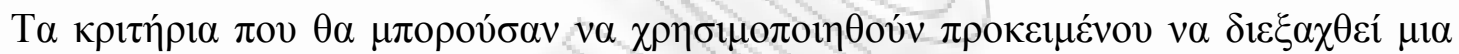

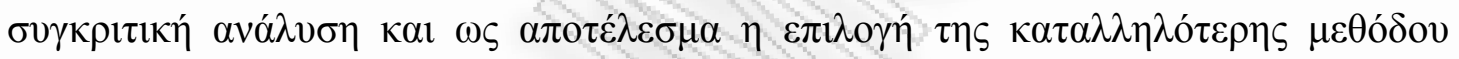

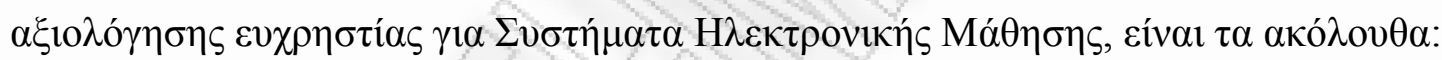

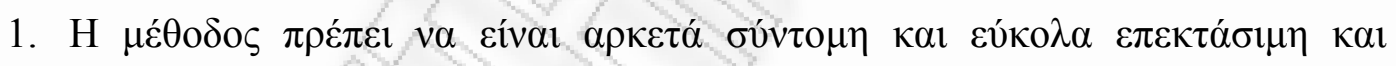

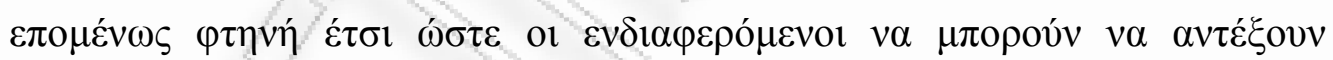

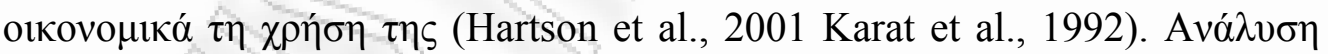

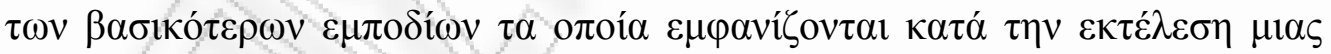

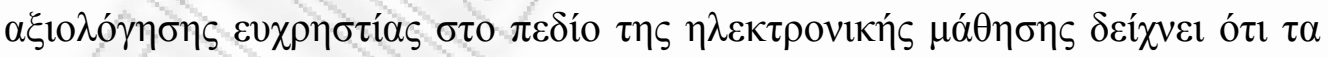

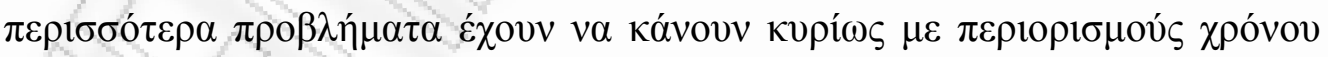

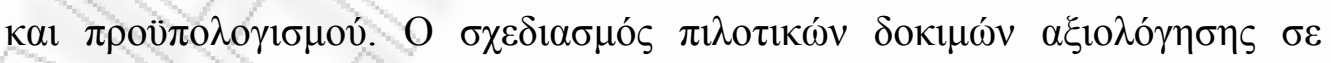

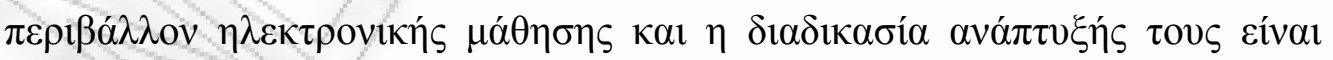

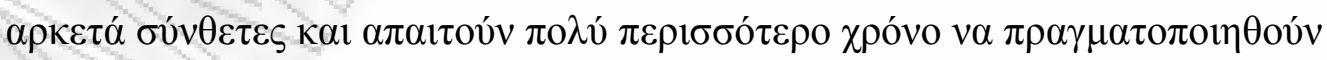

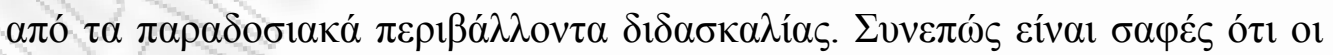

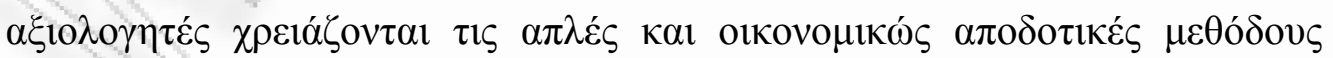
$\alpha \xi 10 \lambda o ́ \gamma \eta \sigma \eta s$.

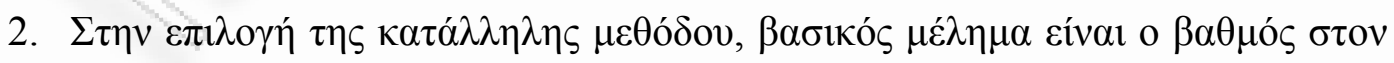

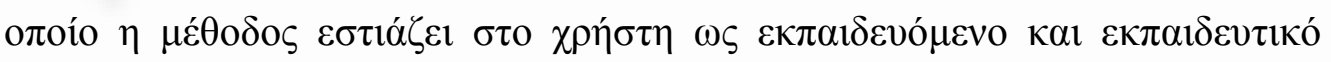

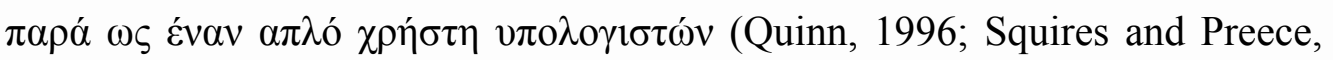

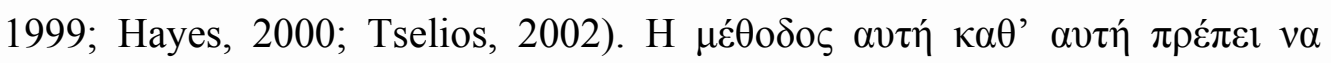

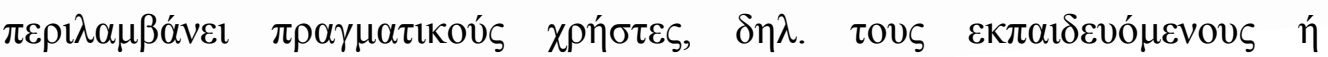




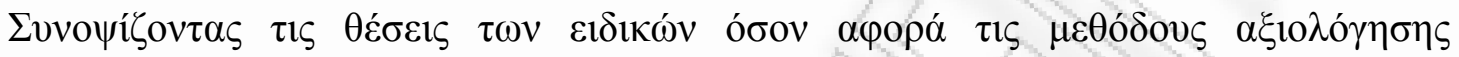

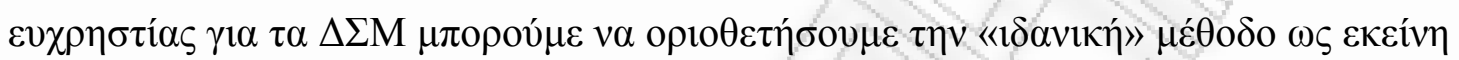

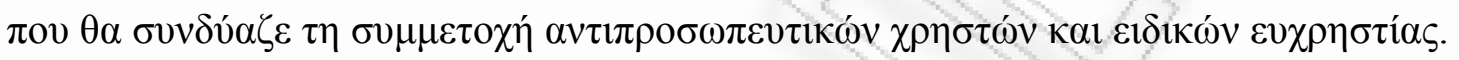

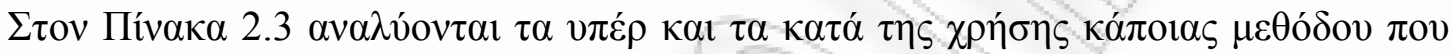

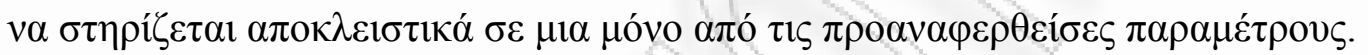

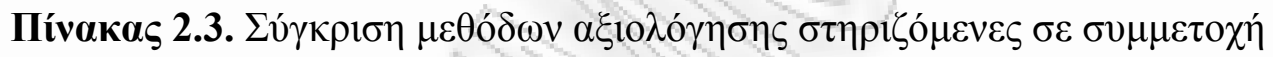

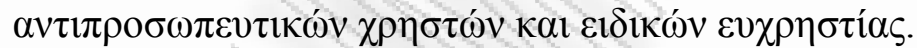

\begin{tabular}{|c|c|c|}
\hline & 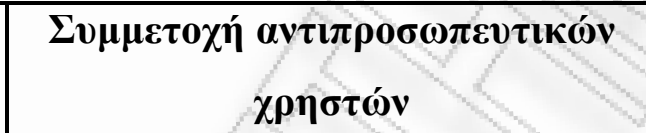 & $\Sigma v \mu \mu \varepsilon \tau 0 \chi \eta ́ ~ \varepsilon เ \delta เ \kappa \omega ́ v ~ \varepsilon v \chi \rho \eta \sigma \tau i ́ \alpha \varsigma$ \\
\hline Y $\pi^{\varepsilon} \rho$ & 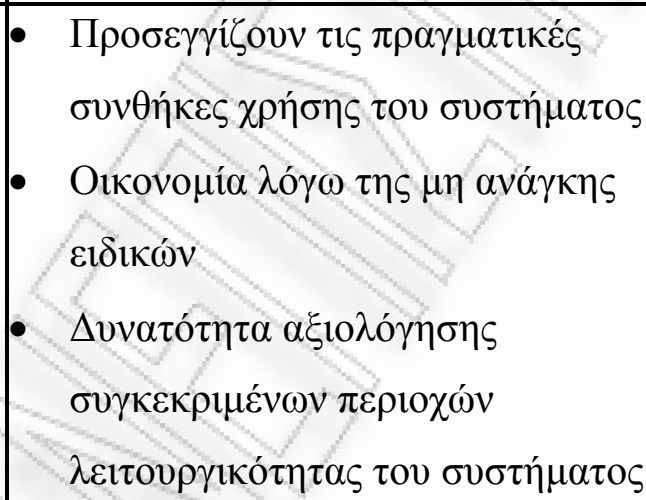 & 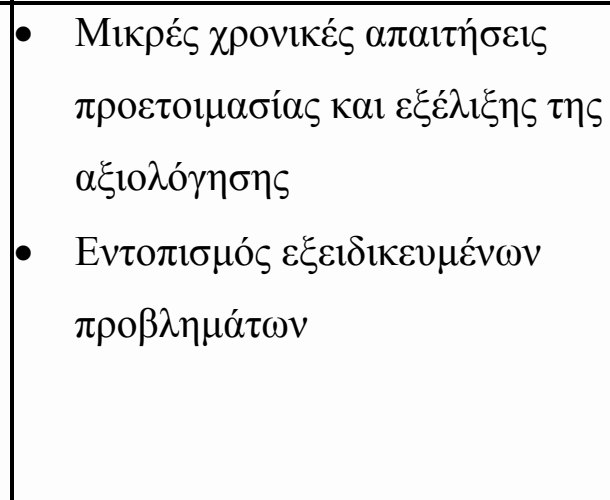 \\
\hline Kató & 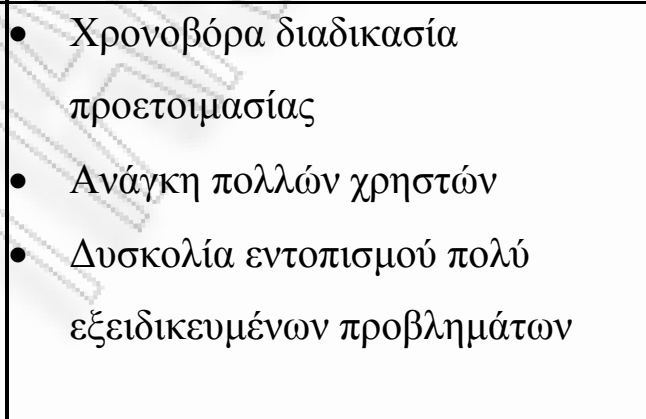 & 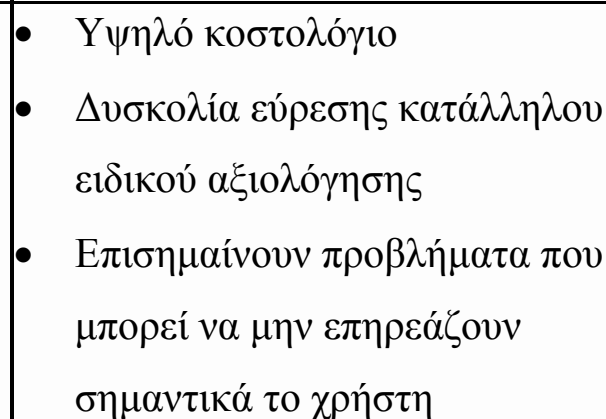 \\
\hline
\end{tabular}




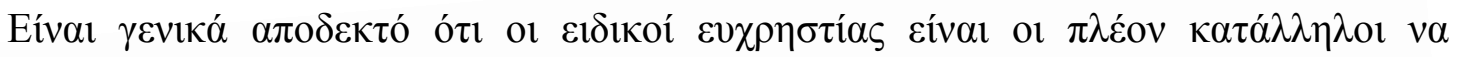

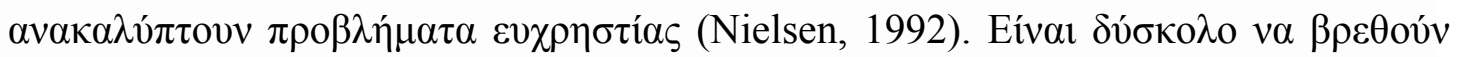

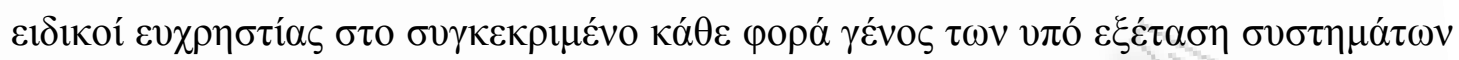

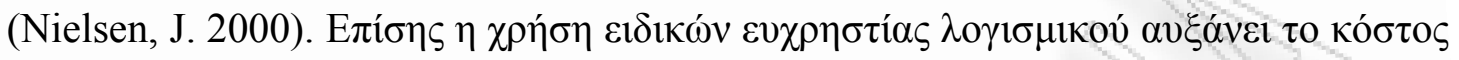

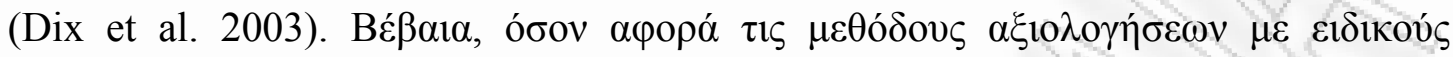

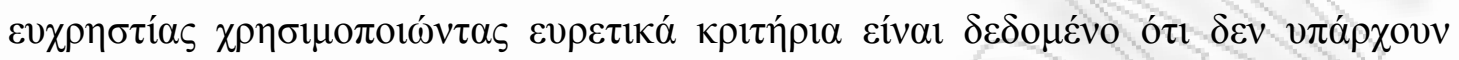

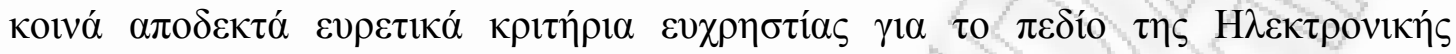

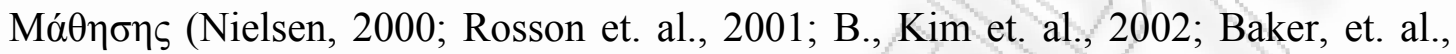

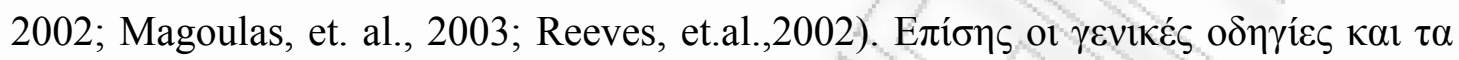

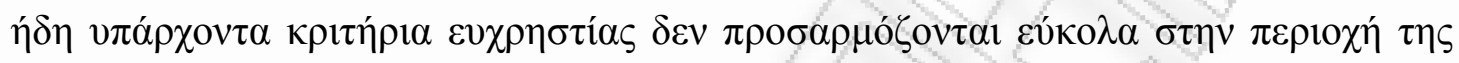

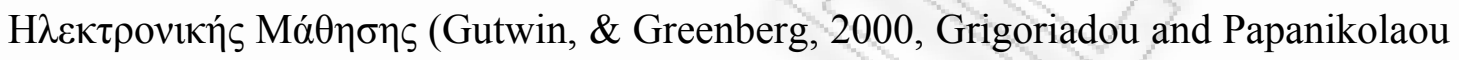

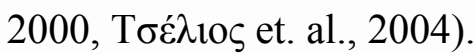

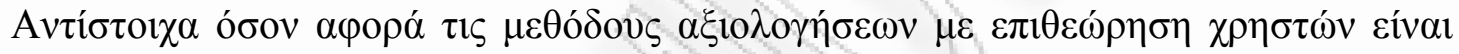

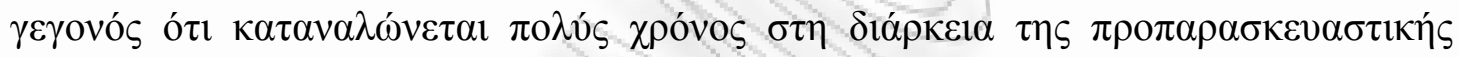

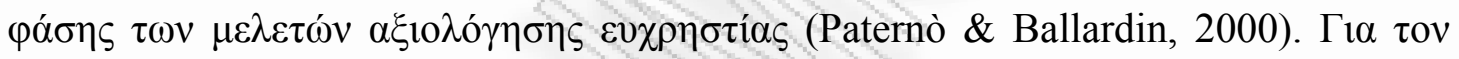

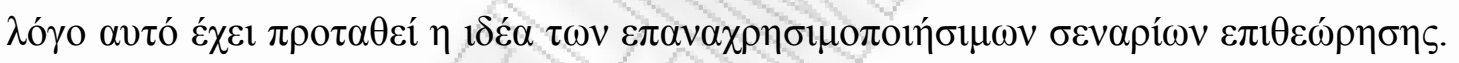

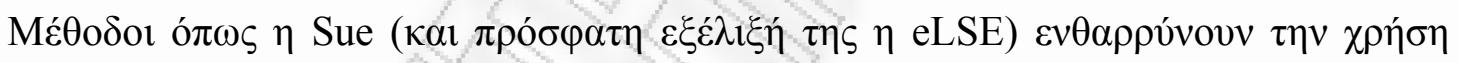

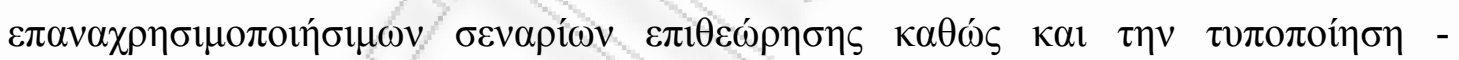

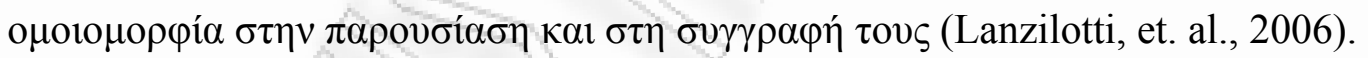

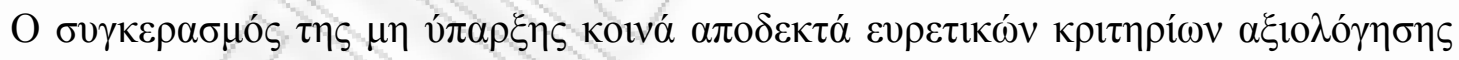

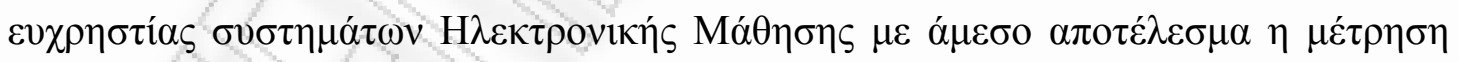

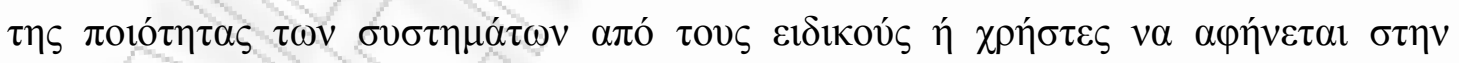

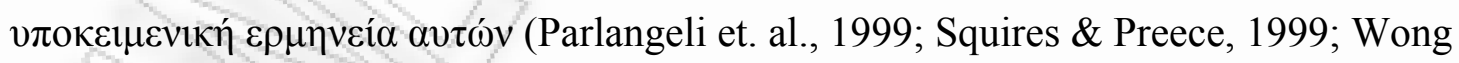

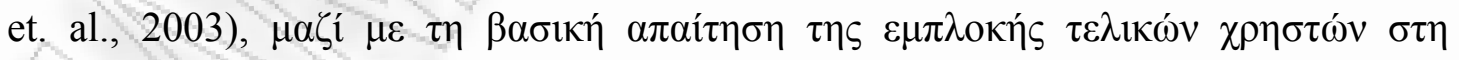

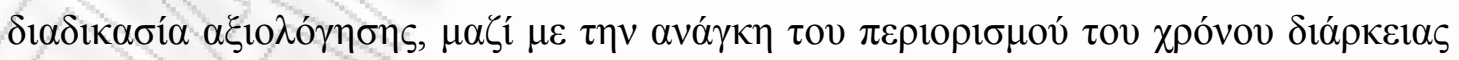

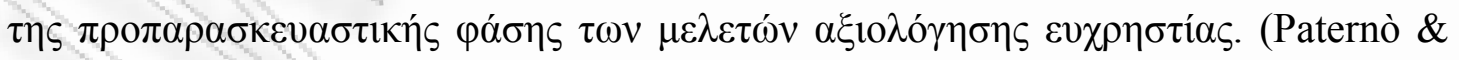

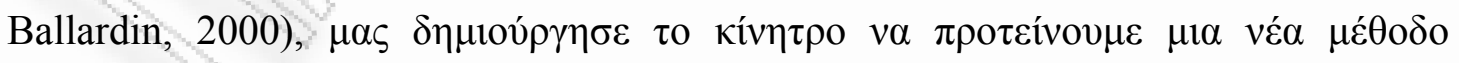

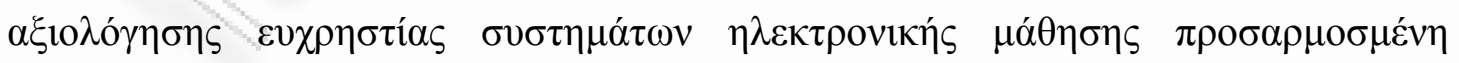

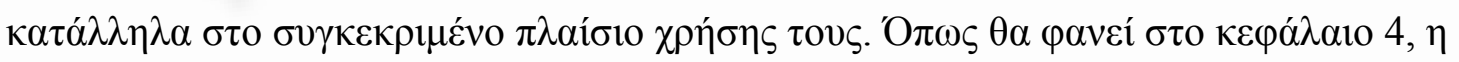

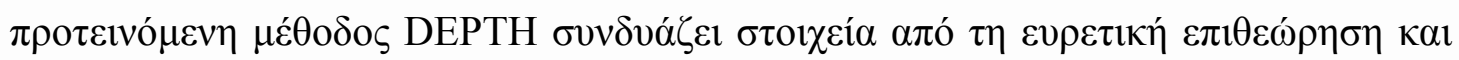

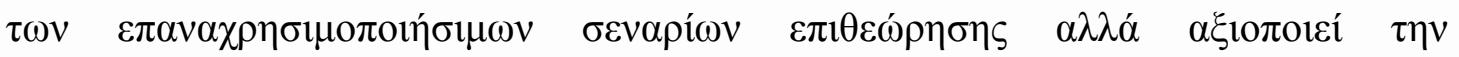




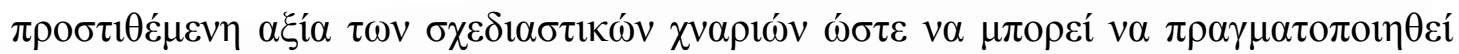

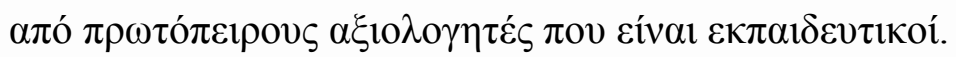

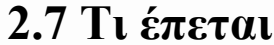

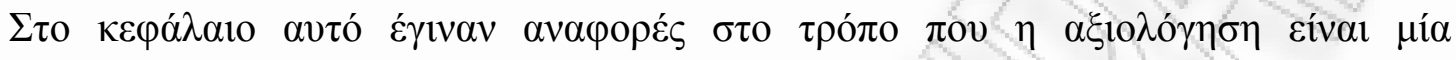

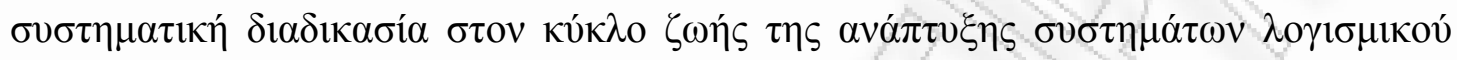

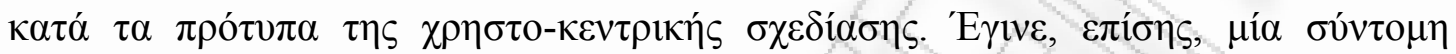

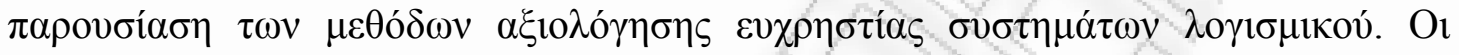

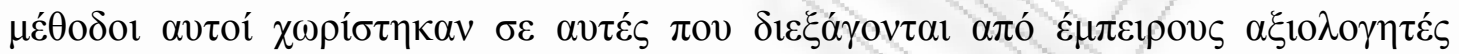

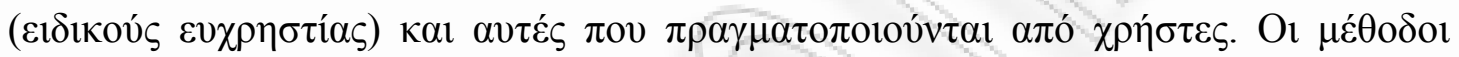

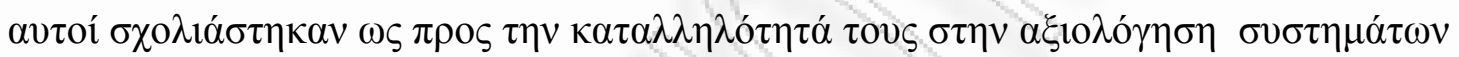

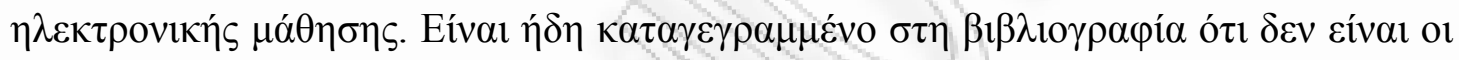

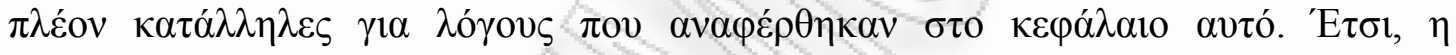

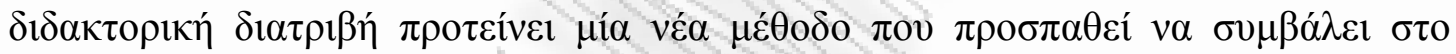

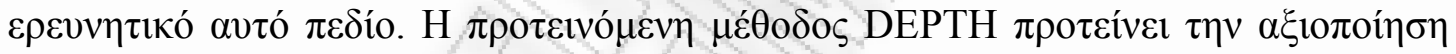

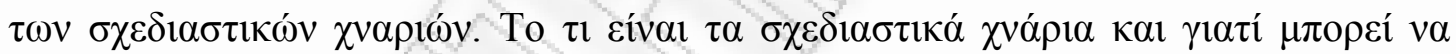

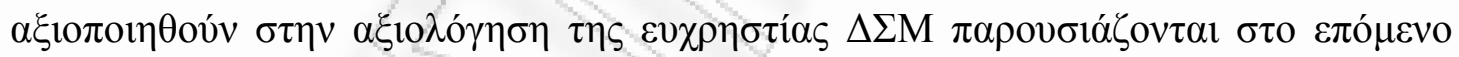

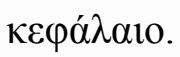




\section{КЕФАААIO 3}

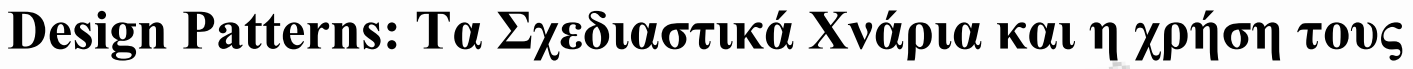

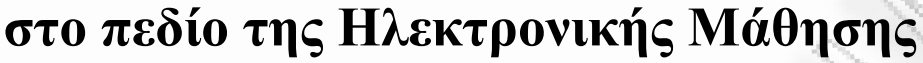

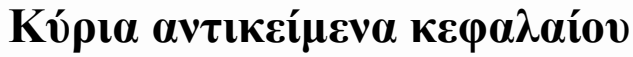

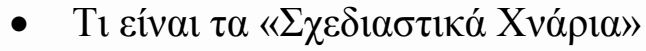

- Поц

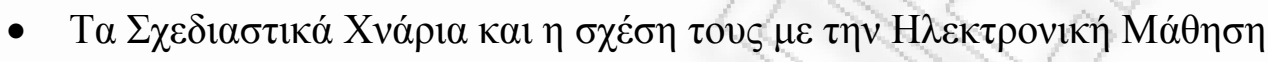

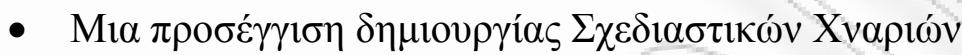

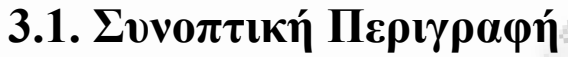

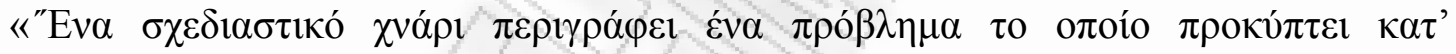

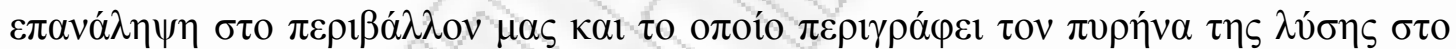

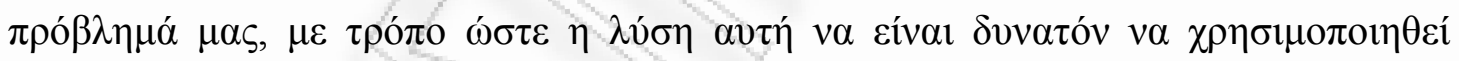

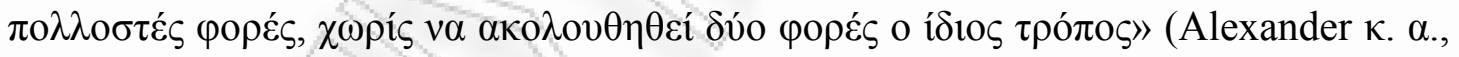

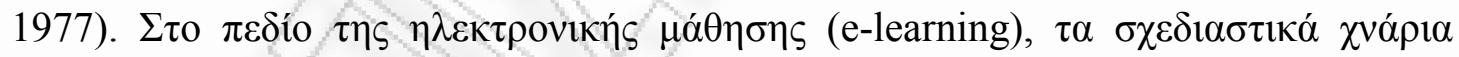

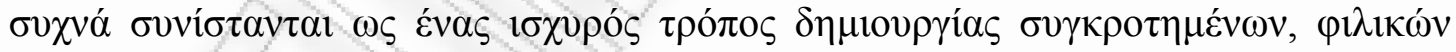

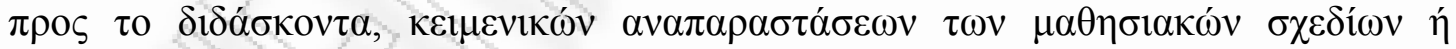

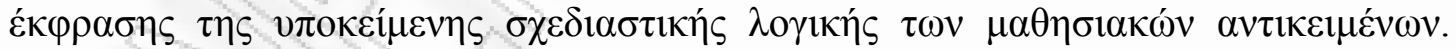

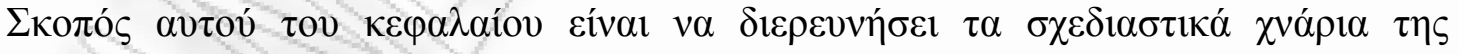

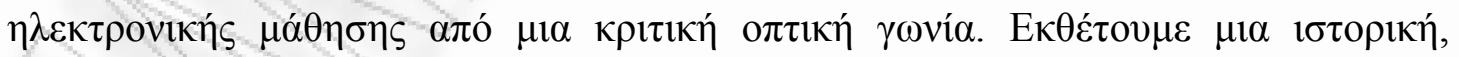

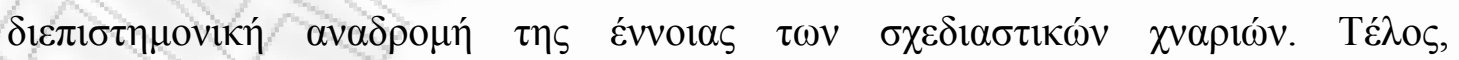

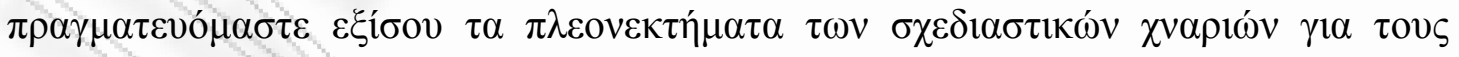

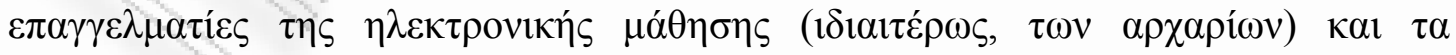

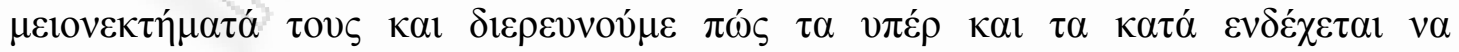

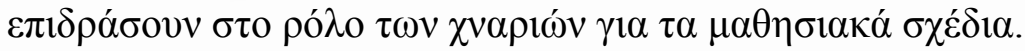




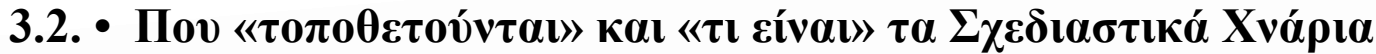

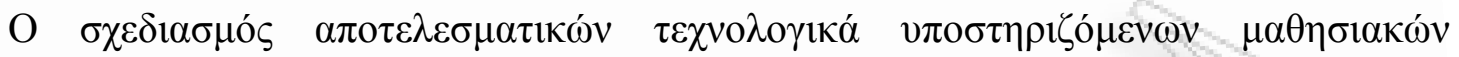

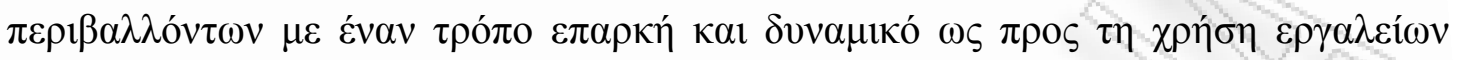

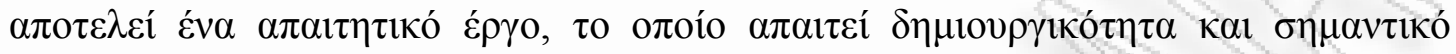

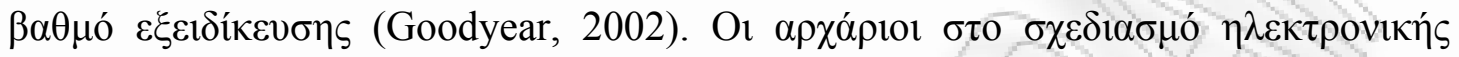

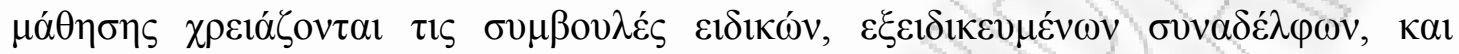

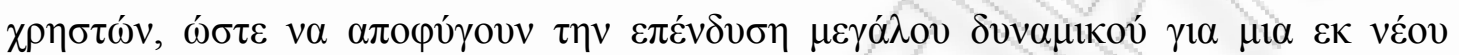

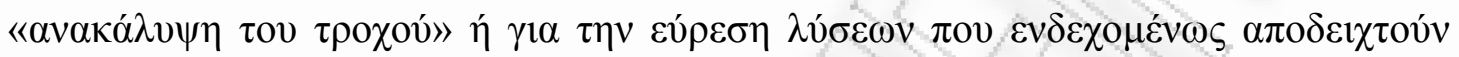

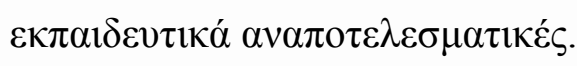

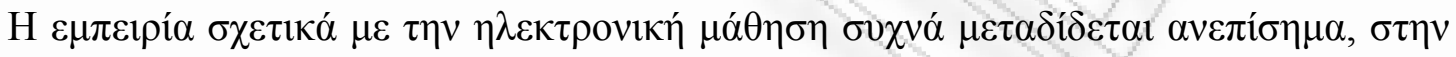

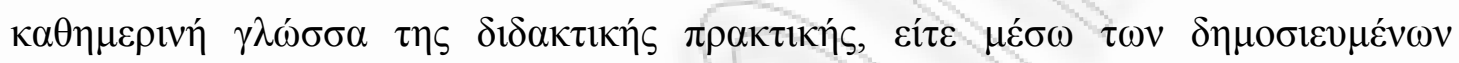

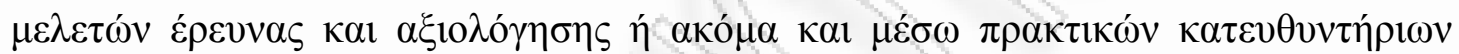

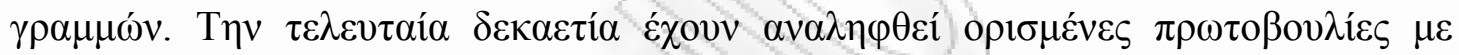

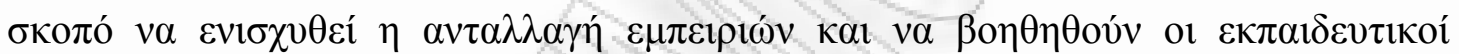

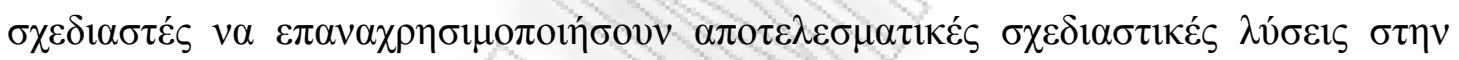

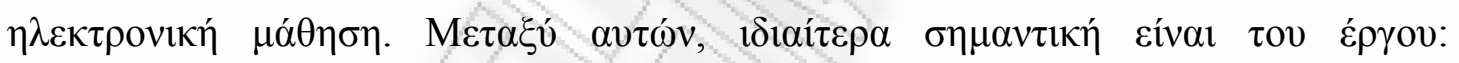
Australian University Teaching Committee (AUTC) Project. H $\pi \rho \omega \tau$ oßoviía

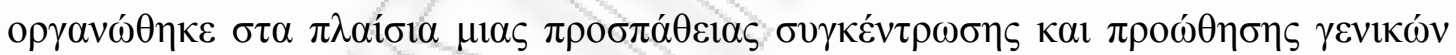

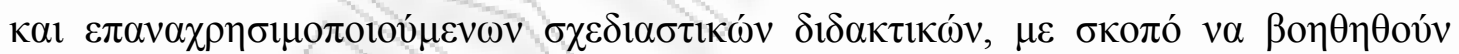

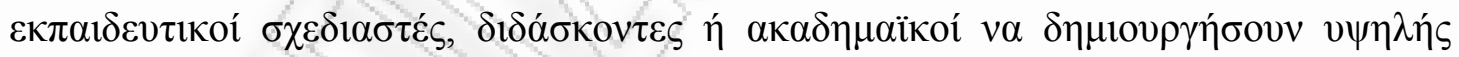

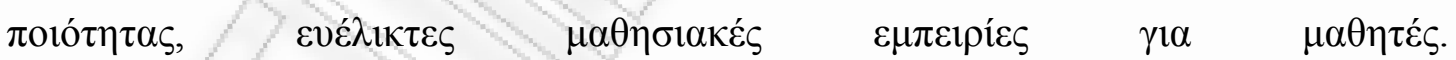
[http://www.learningdesigns.uow.edu.au/]

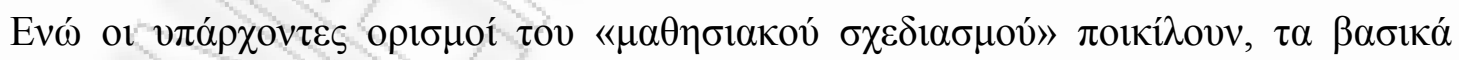

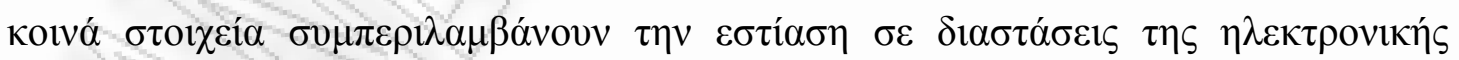

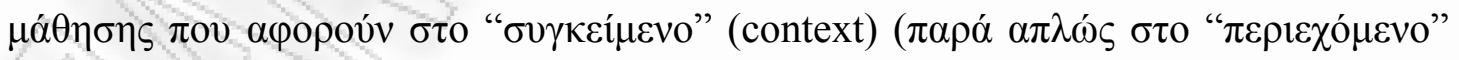

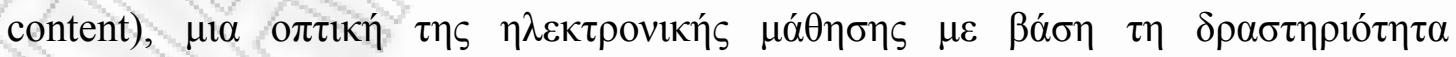

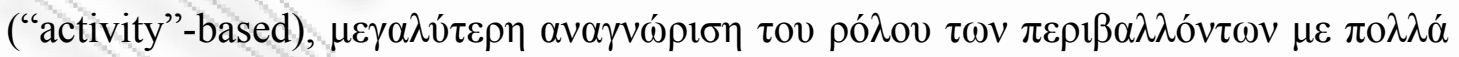

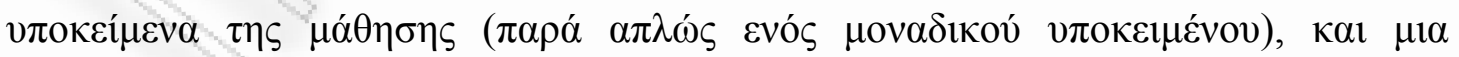

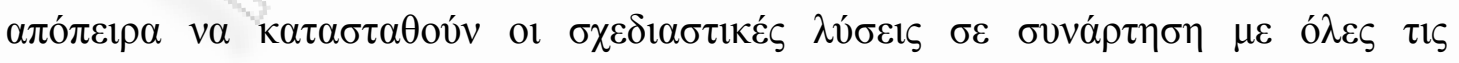

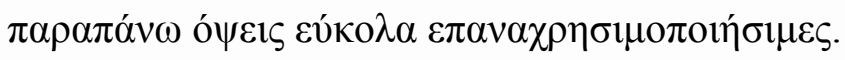

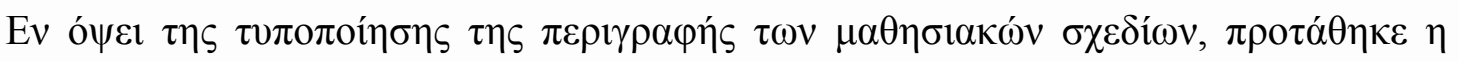

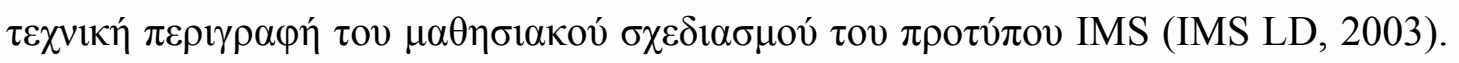




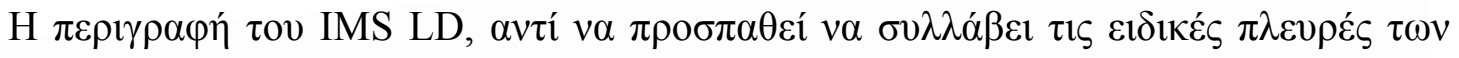

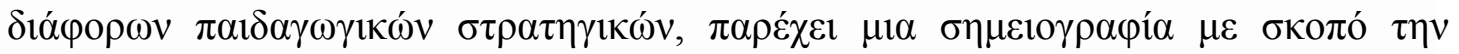

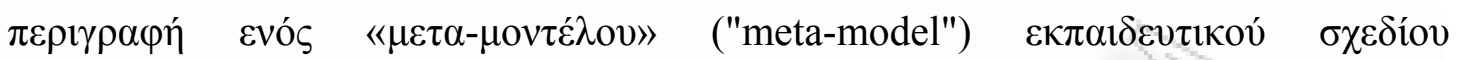

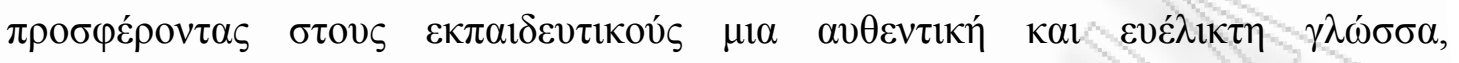

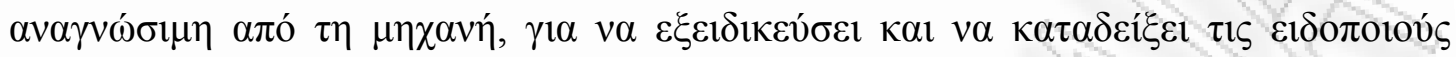

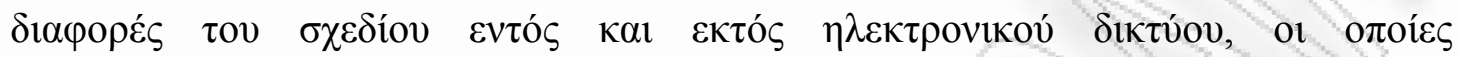

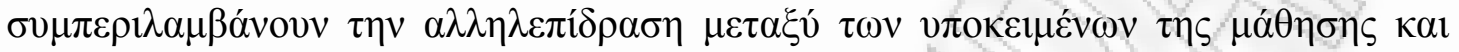

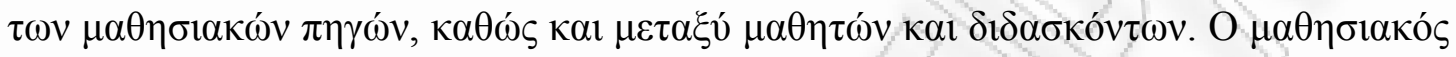

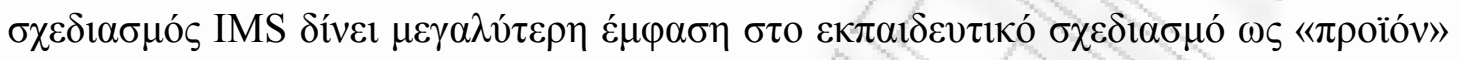

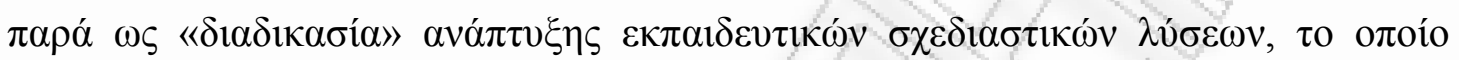

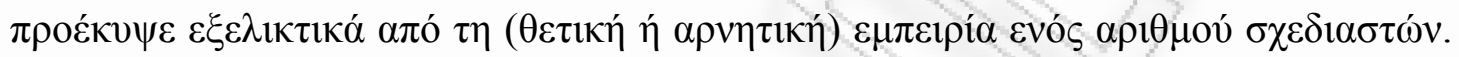

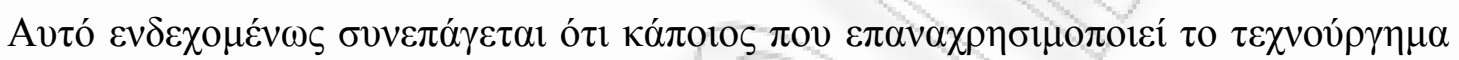

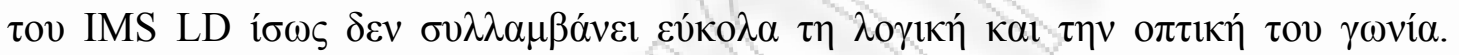

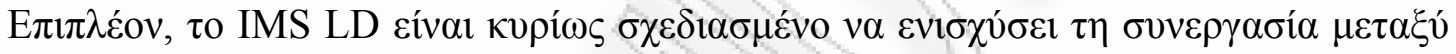

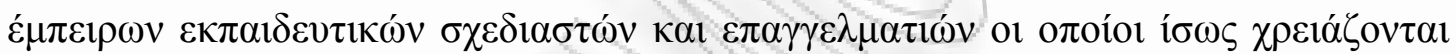

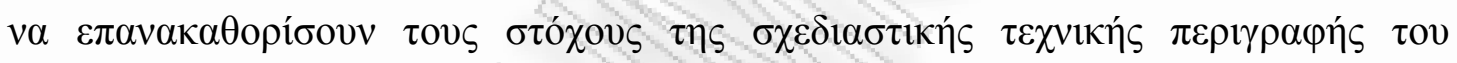

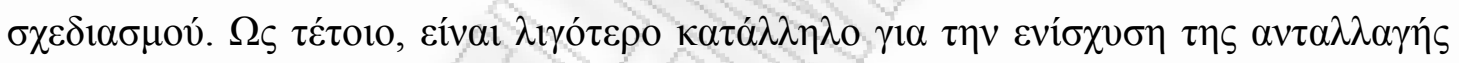

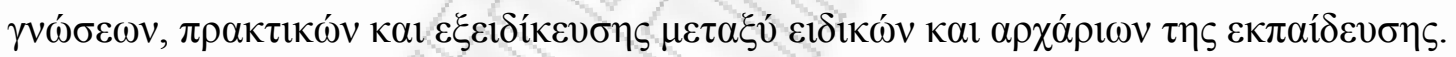

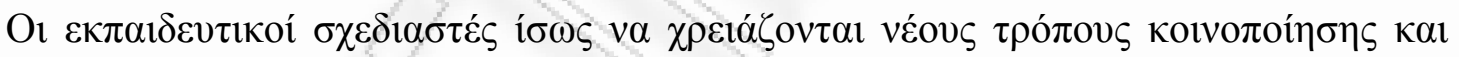

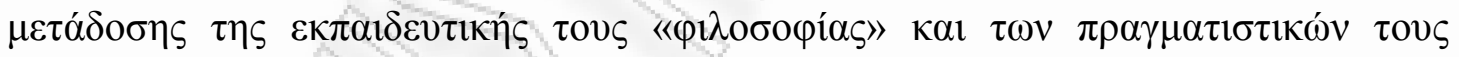

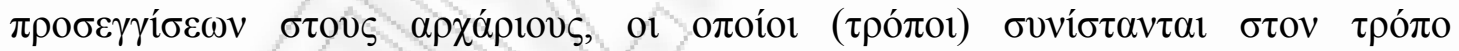

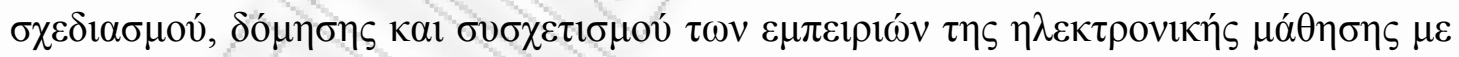

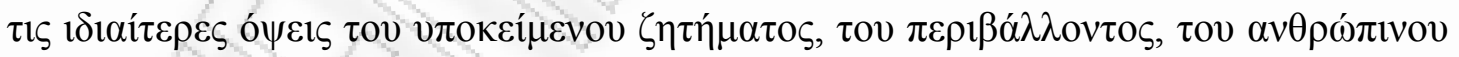

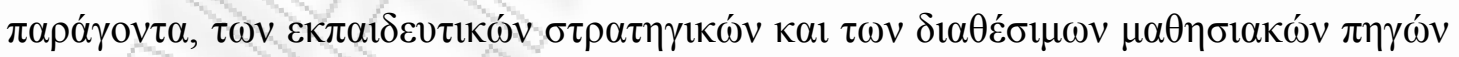

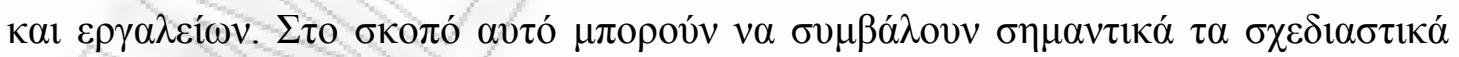

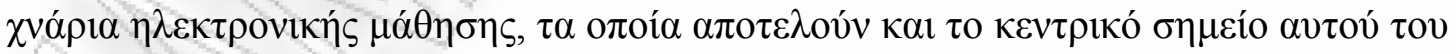

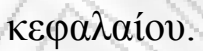

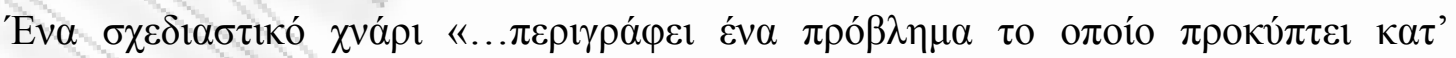

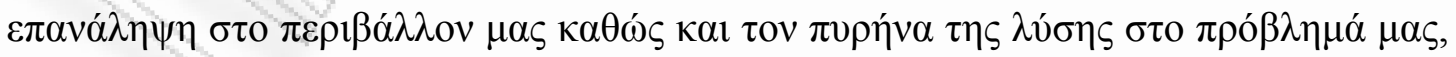

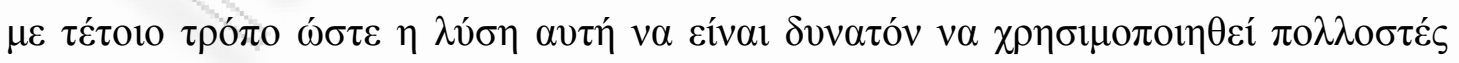

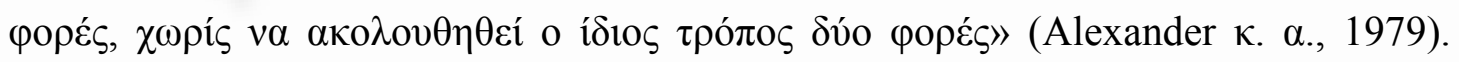

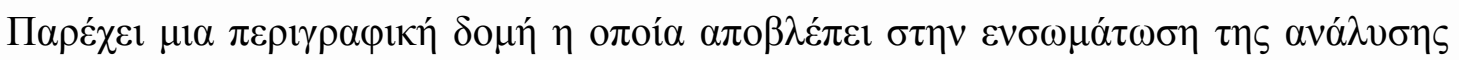

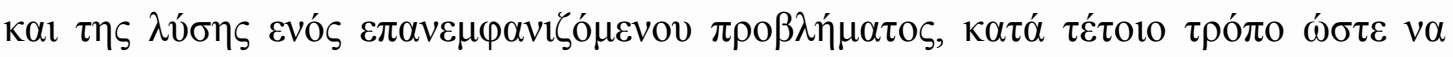

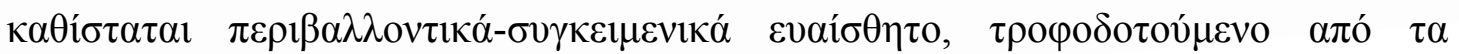




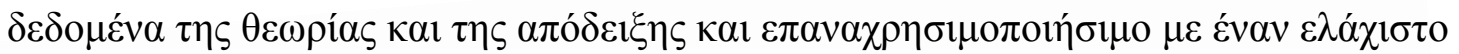

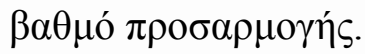

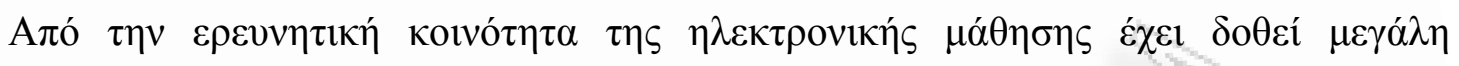

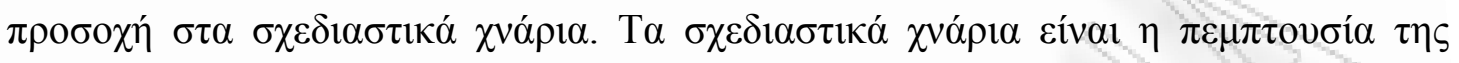

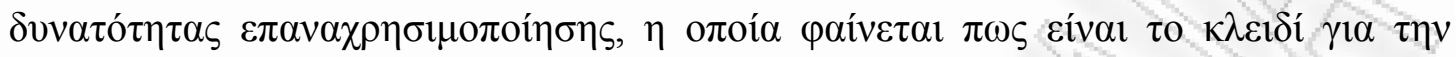

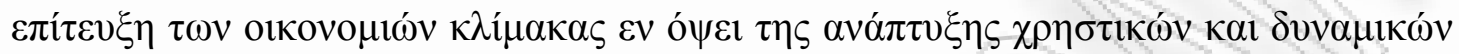

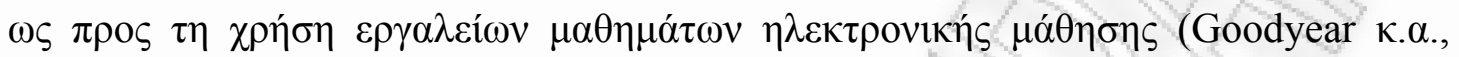

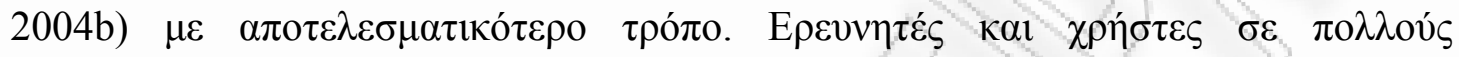

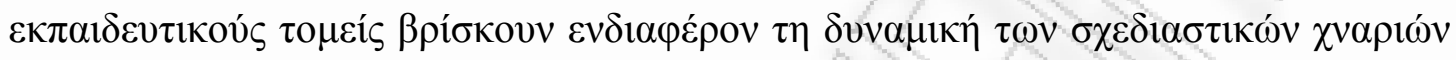

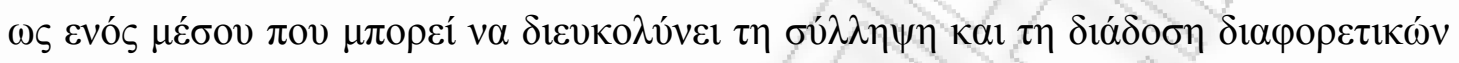

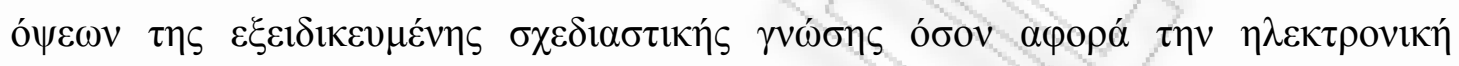

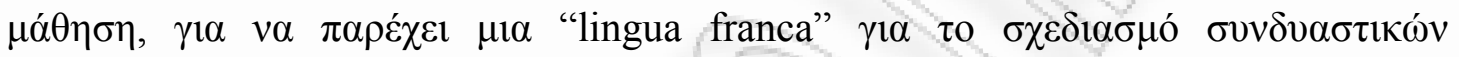

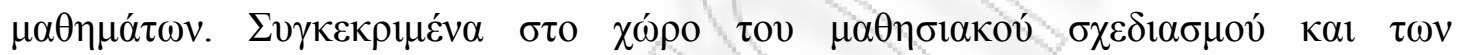

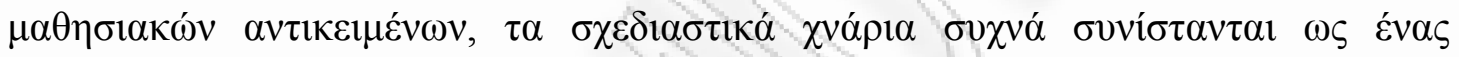

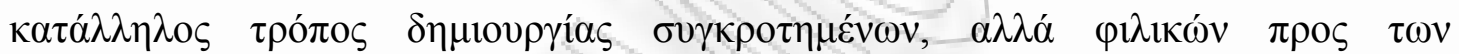

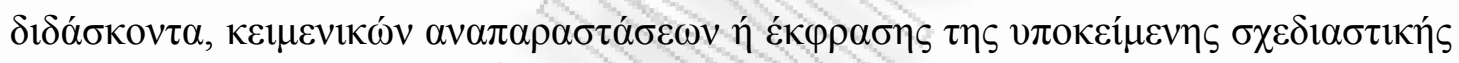

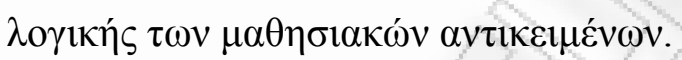

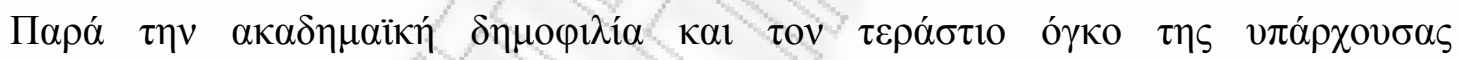

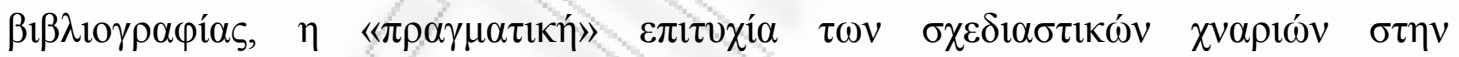

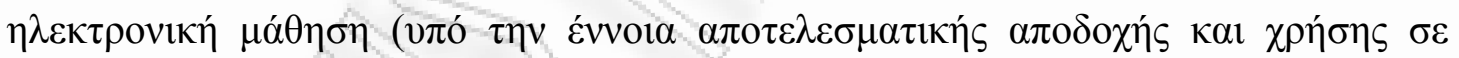

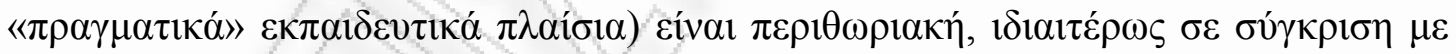

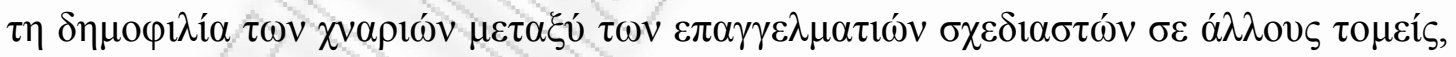

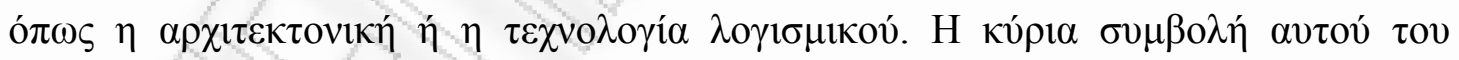

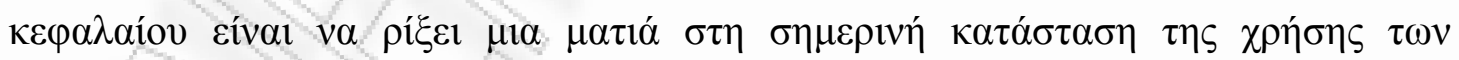

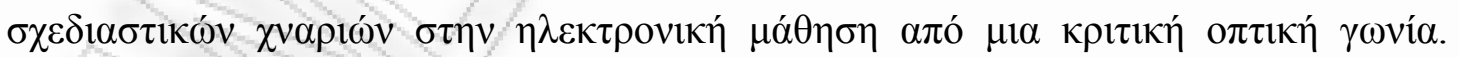

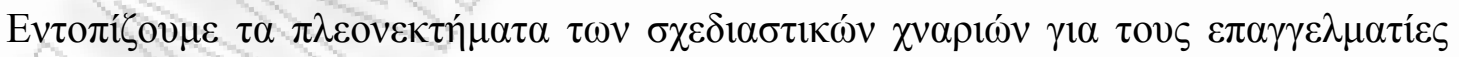

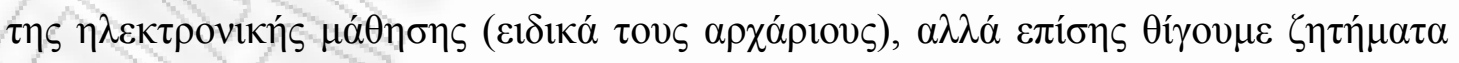

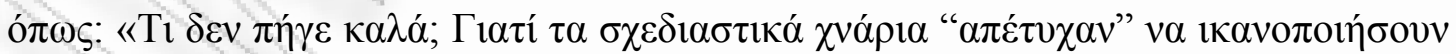

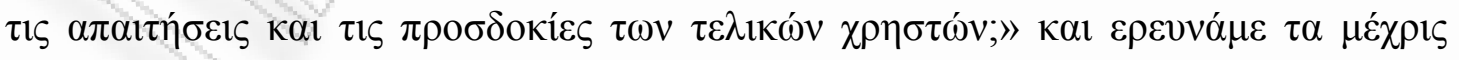

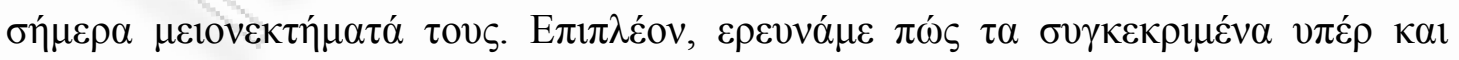

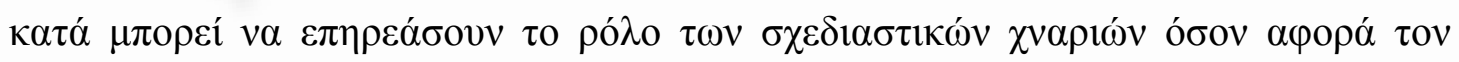

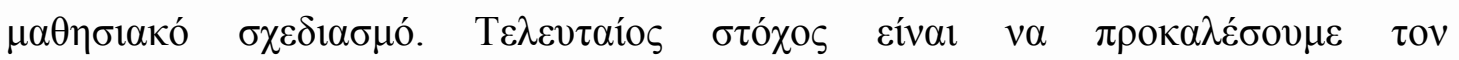

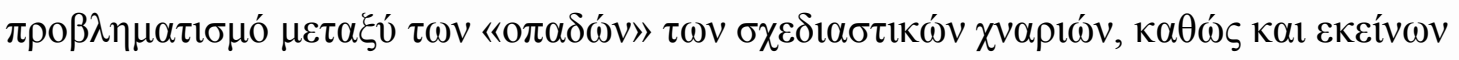

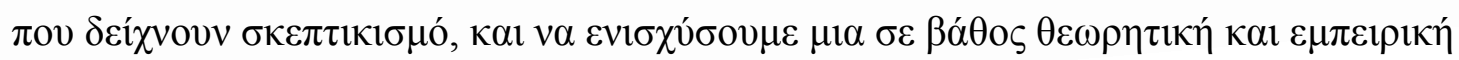




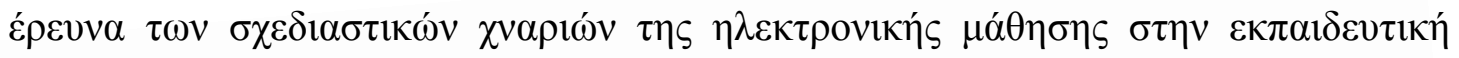

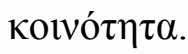

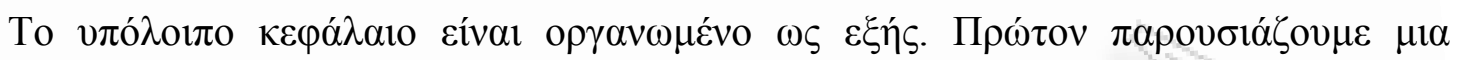

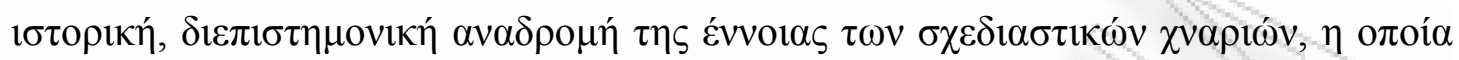

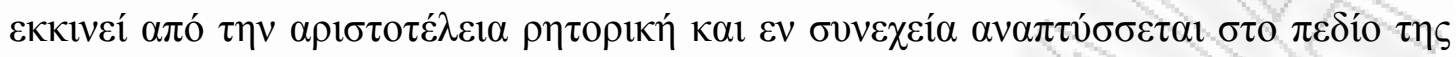

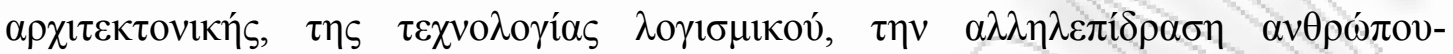

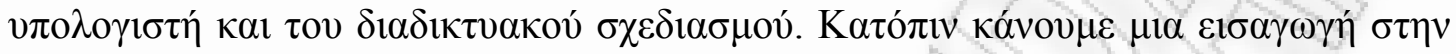

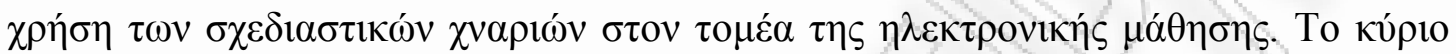

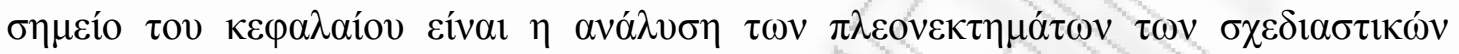

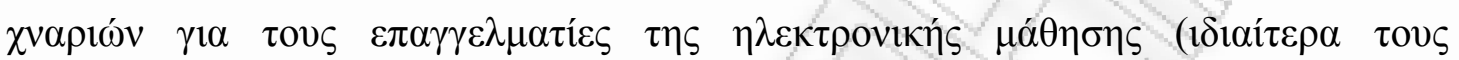

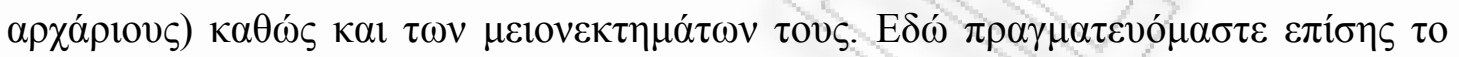

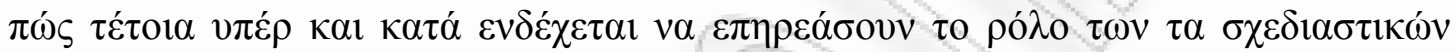

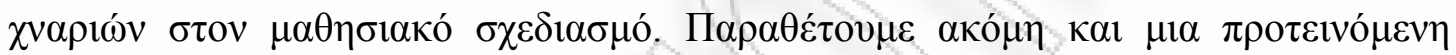

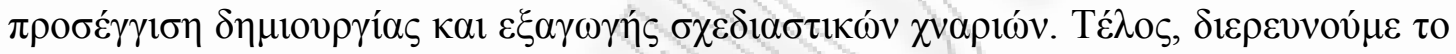

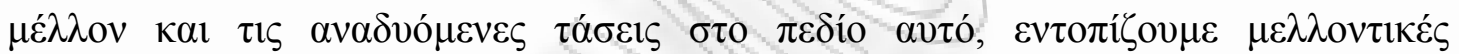

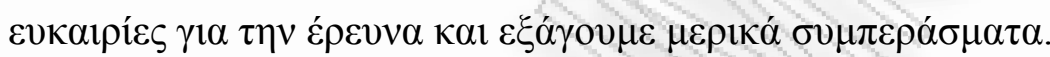

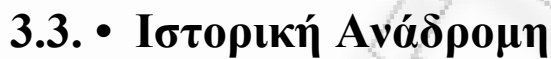

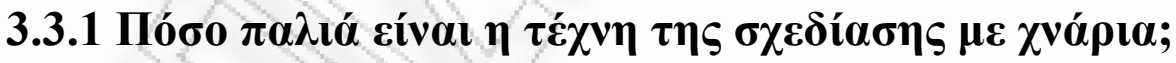

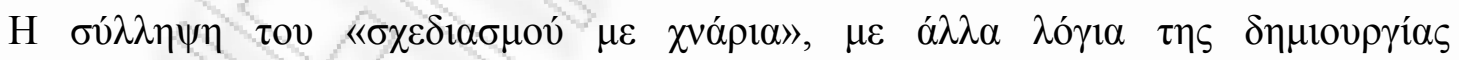

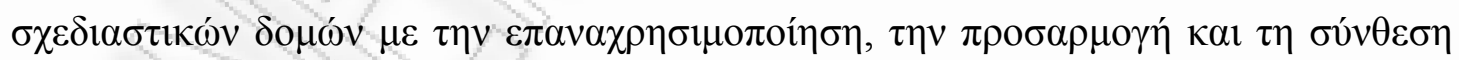

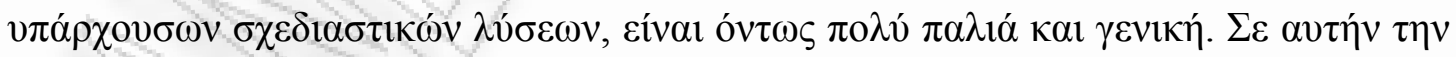

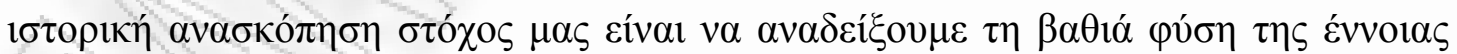

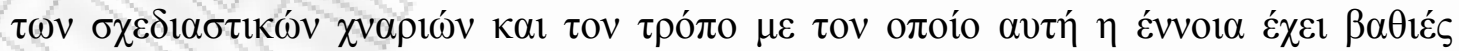

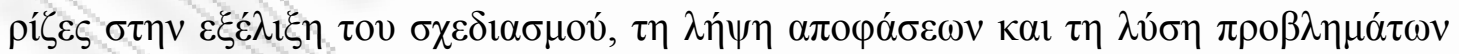

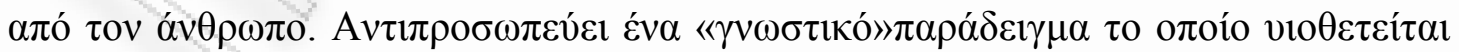

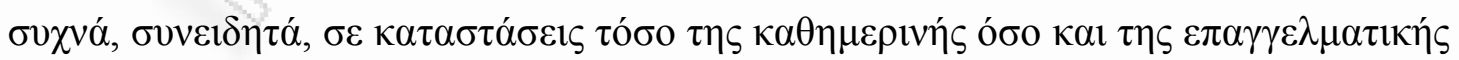

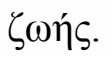

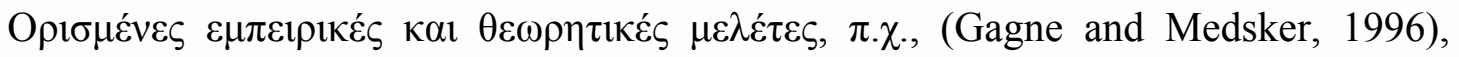

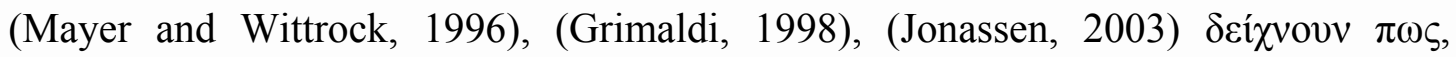




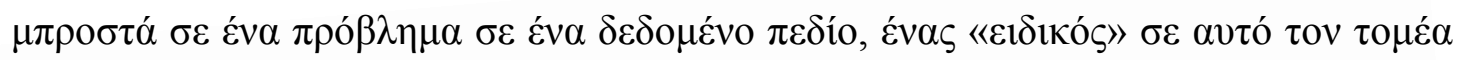

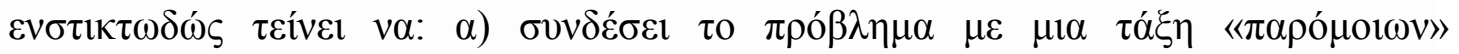

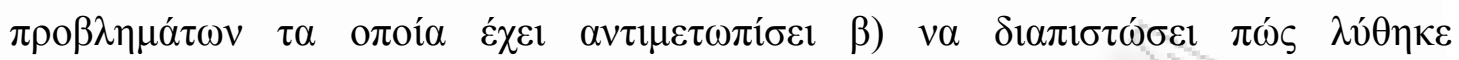

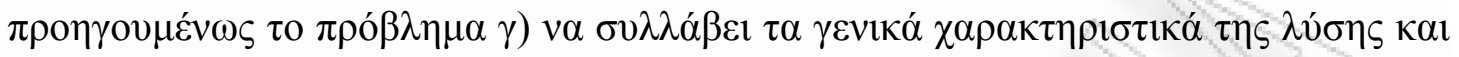

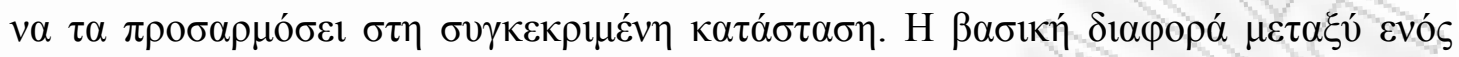

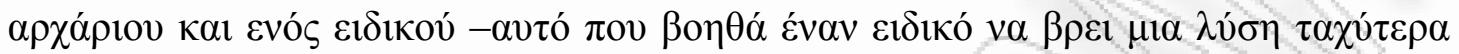

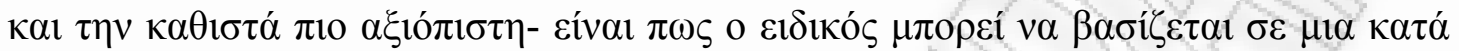

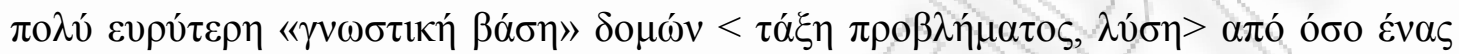

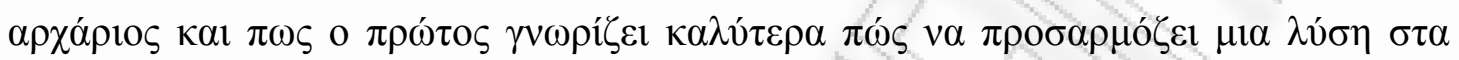
$\tau \rho \varepsilon ́ \chi 0 \nu \tau \alpha \delta \varepsilon \delta o \mu \varepsilon ́ v \alpha$.

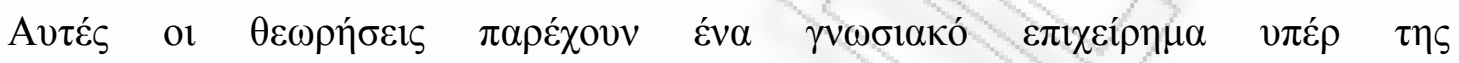

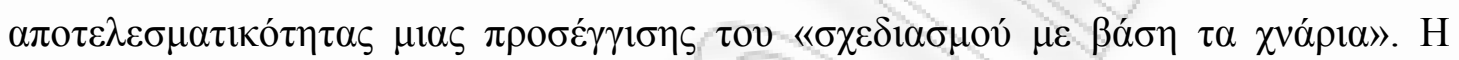

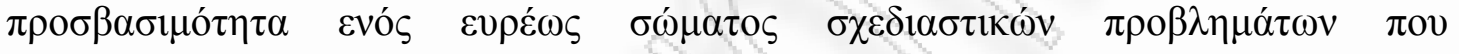

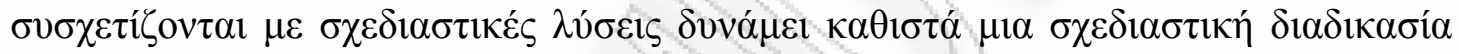

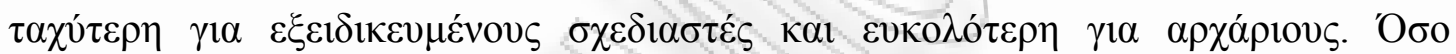

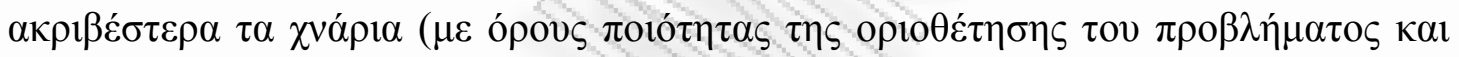

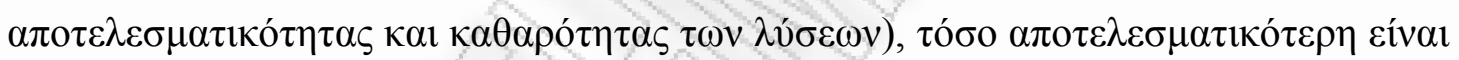

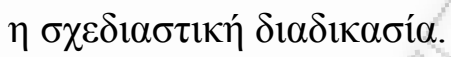

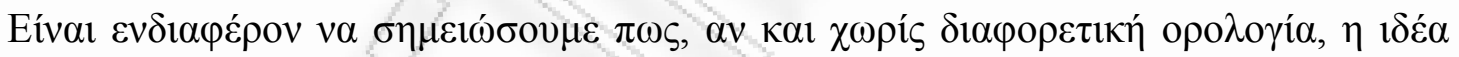

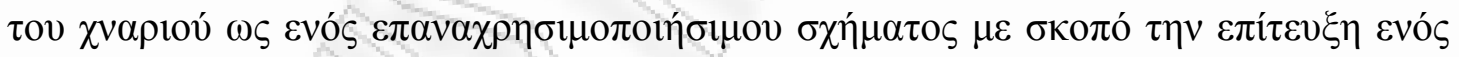

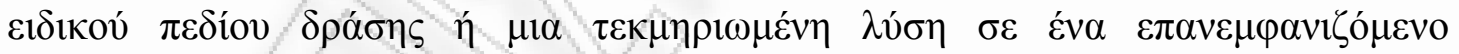

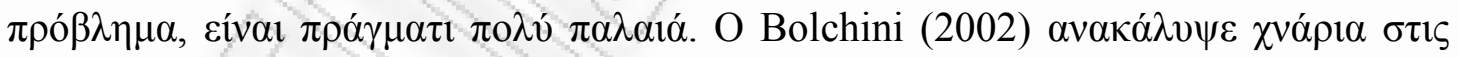

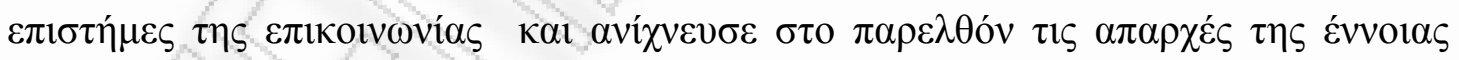

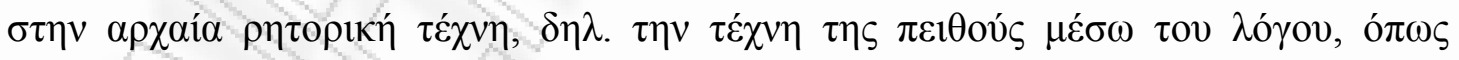

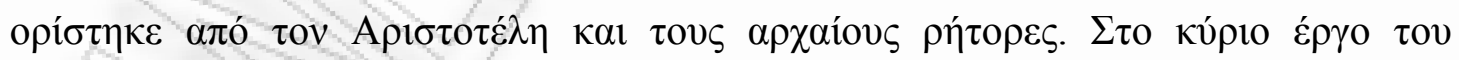

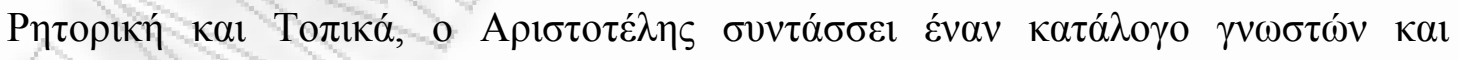

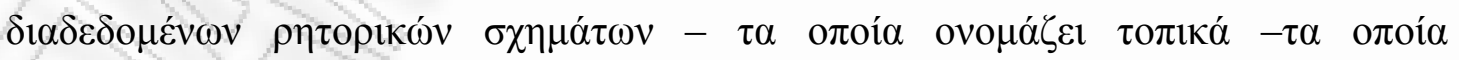

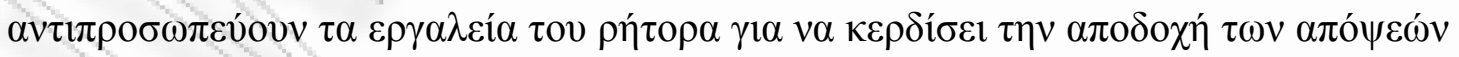

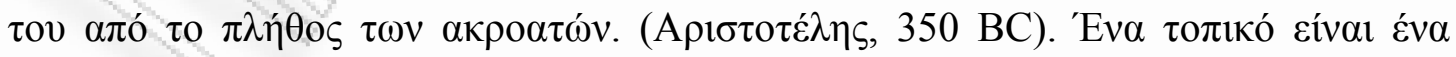

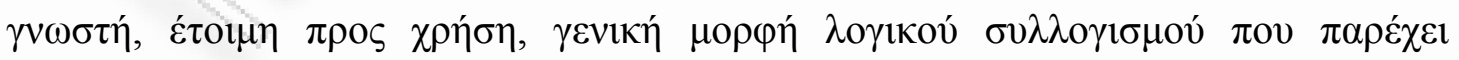

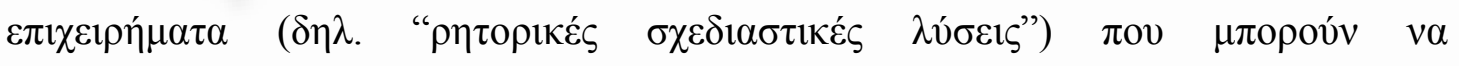

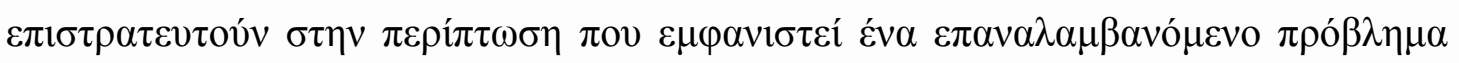

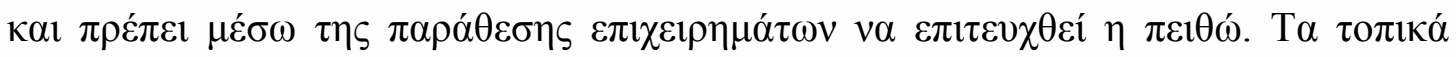

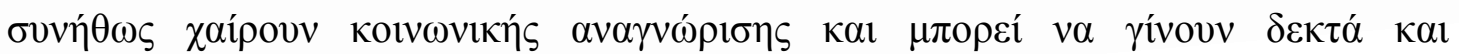




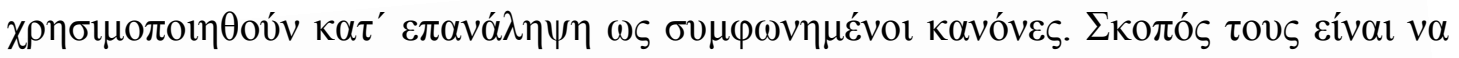

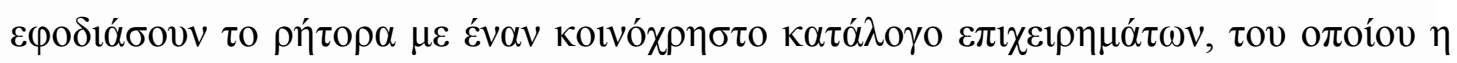

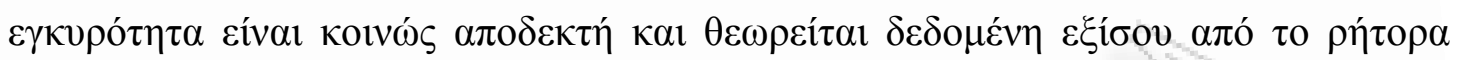

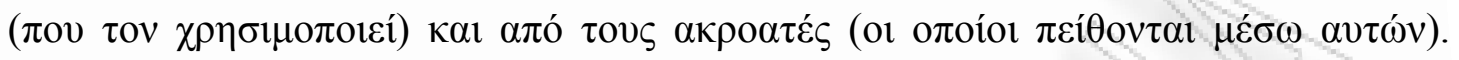

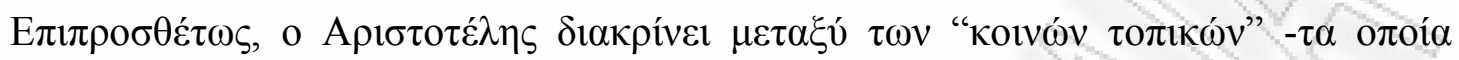

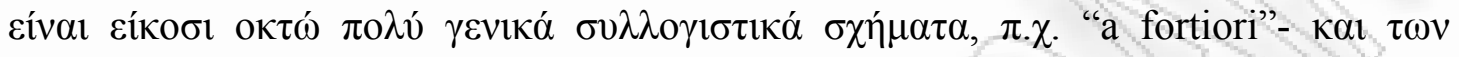

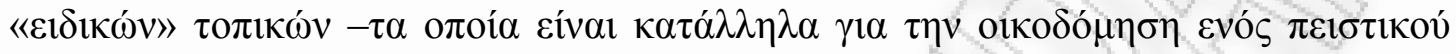

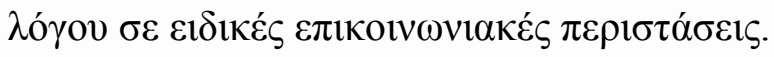

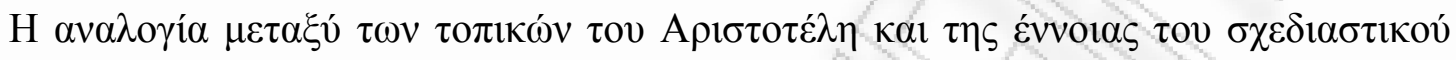

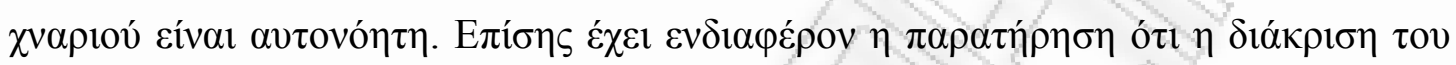

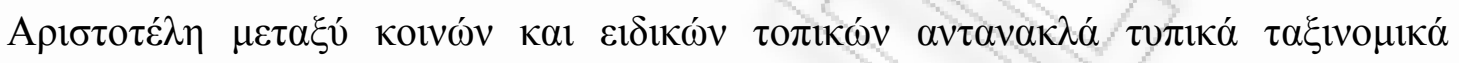

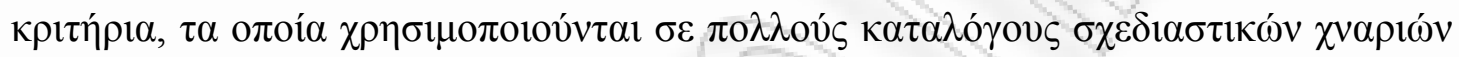

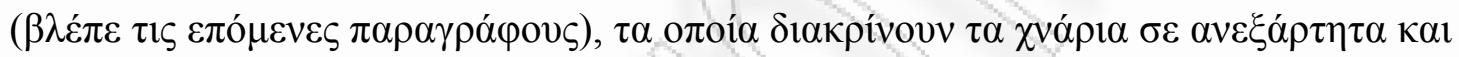

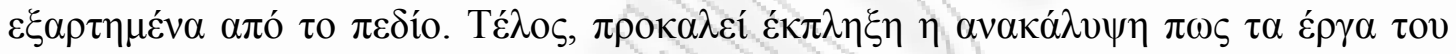

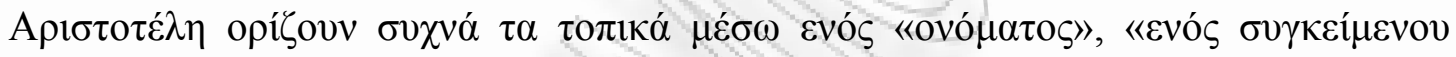

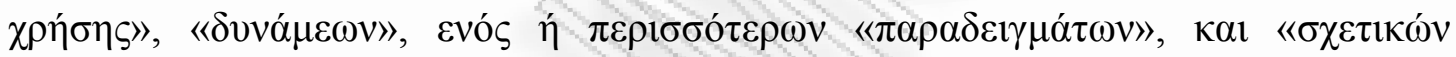

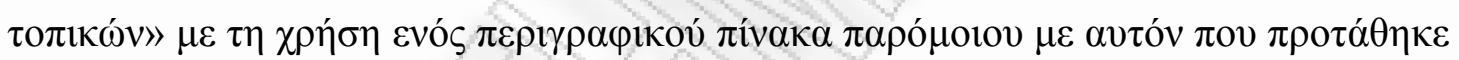

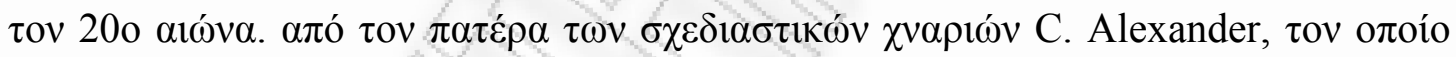
$\theta \alpha \pi \alpha \rho o v \sigma i \alpha ́ \sigma o v \mu \varepsilon \sigma \tau \eta v \varepsilon \pi o ́ \mu \varepsilon v \eta \varepsilon v o ́ \tau \eta \tau \alpha$.

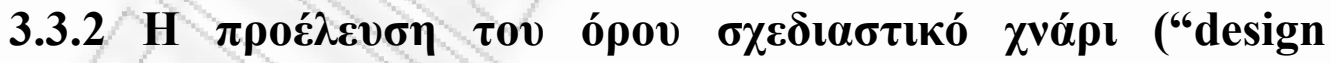 pattern")}

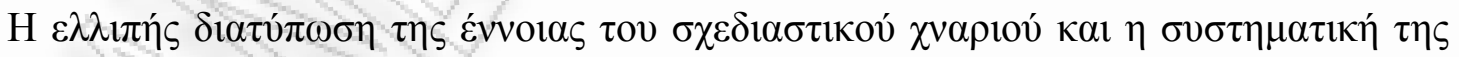

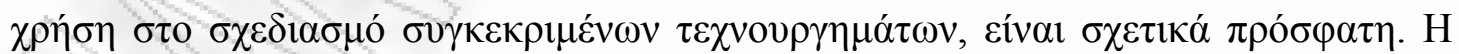

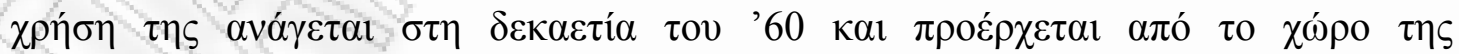

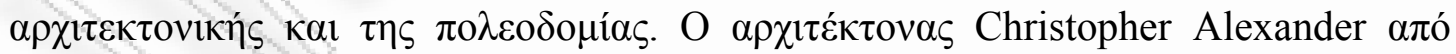

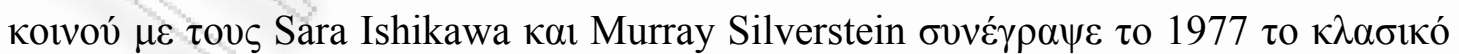

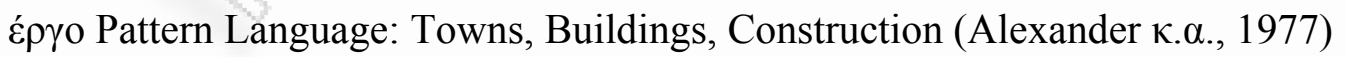

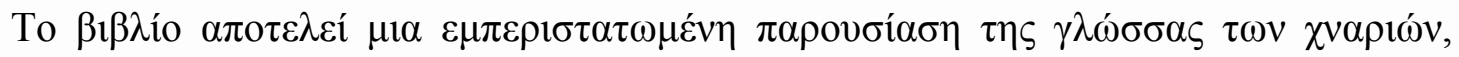

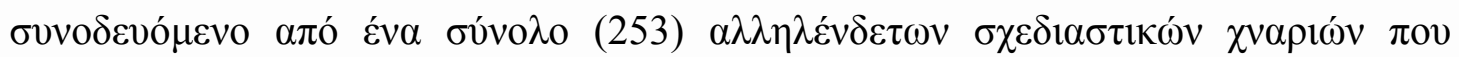

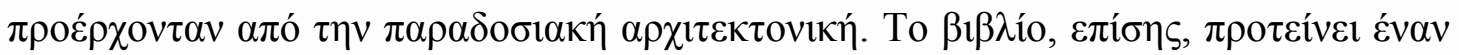




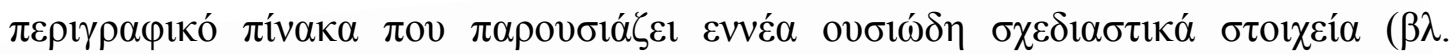

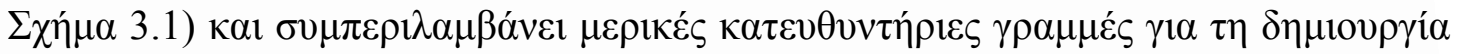

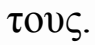

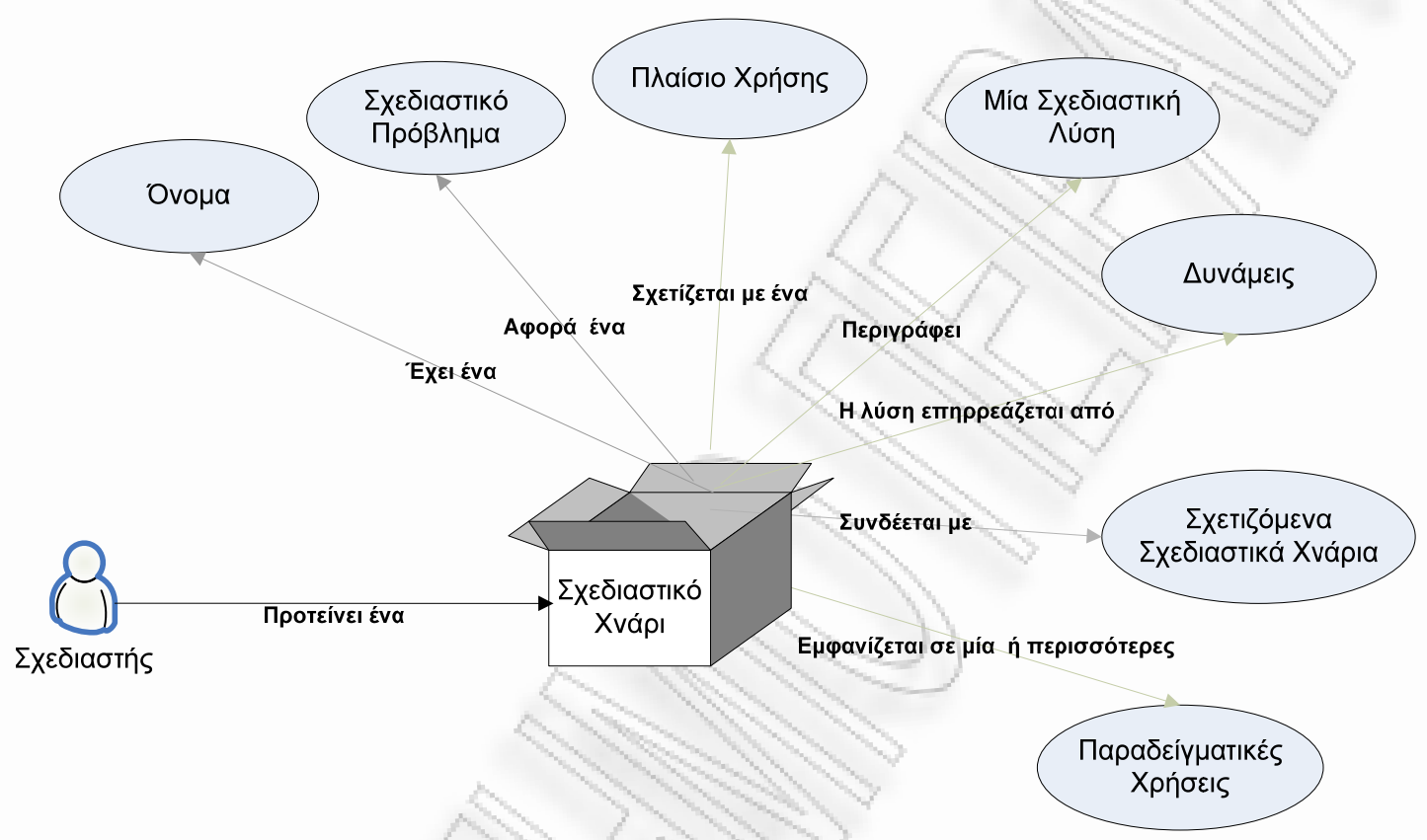

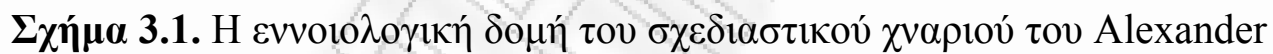

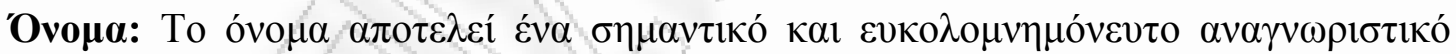

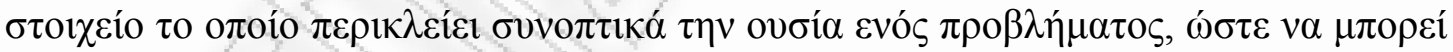

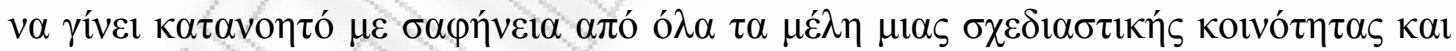

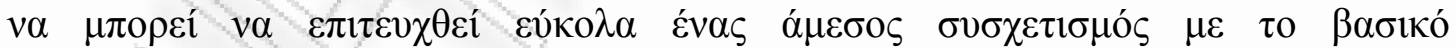

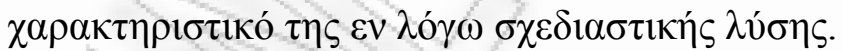

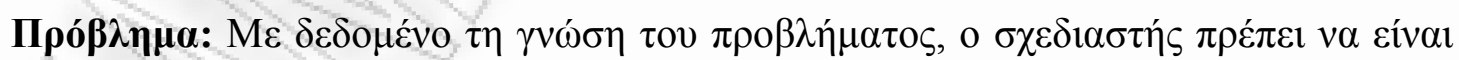

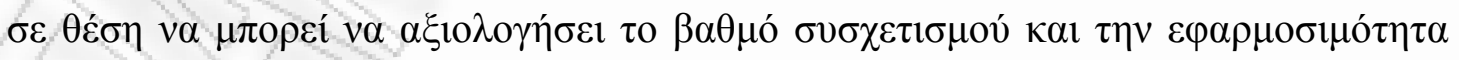

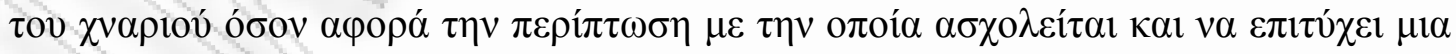

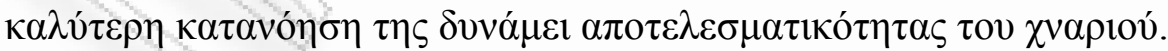

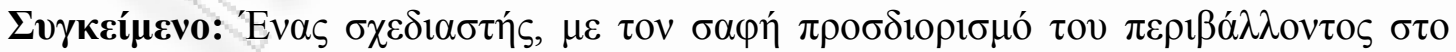

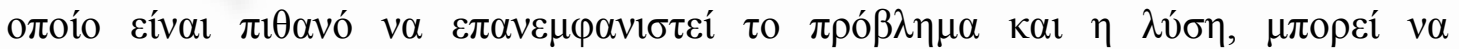

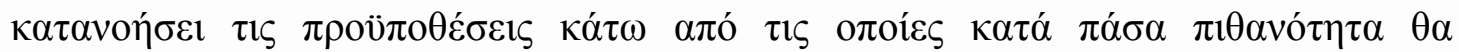

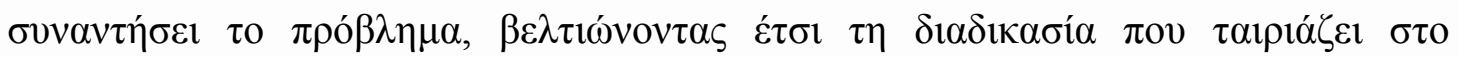
$\pi \rho o ́ \beta \lambda \eta \mu \alpha$. 


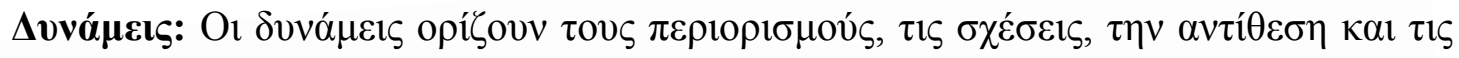

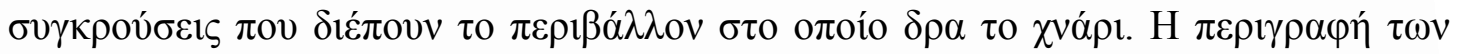

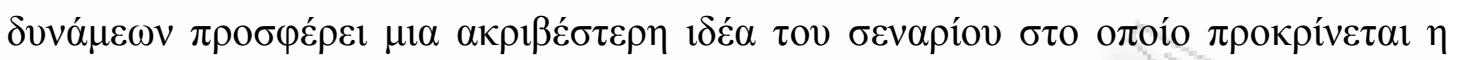

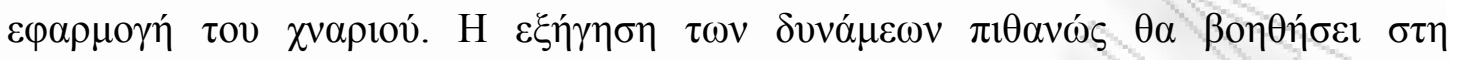

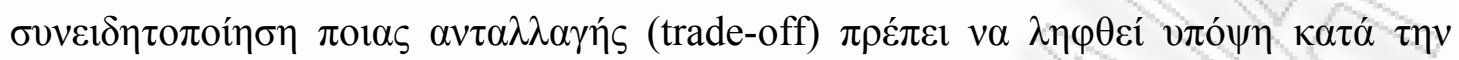

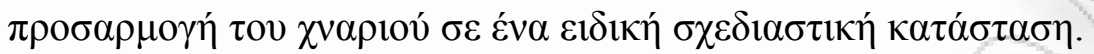

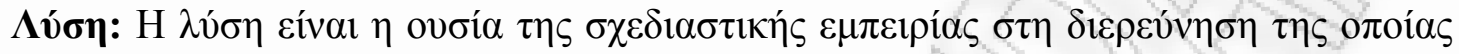

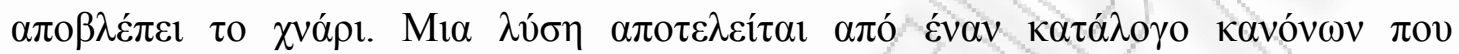

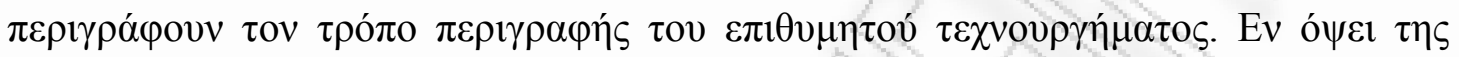

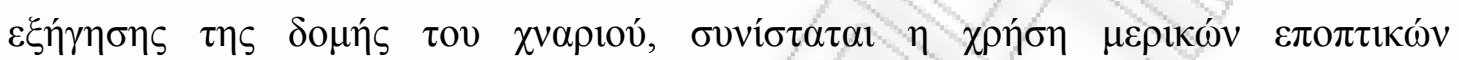

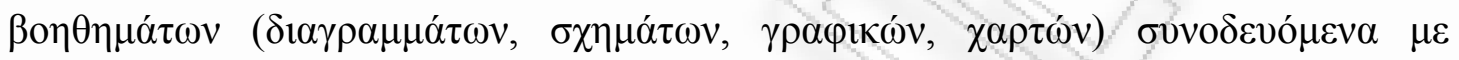

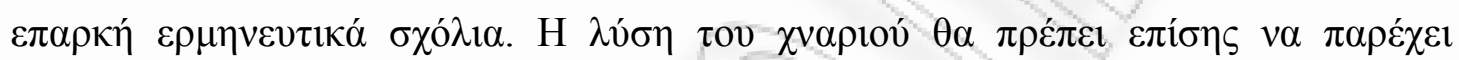

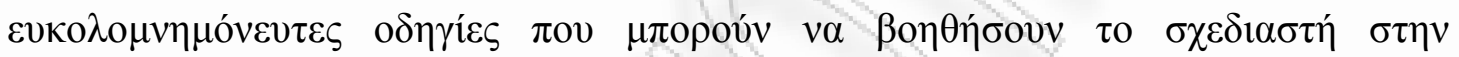

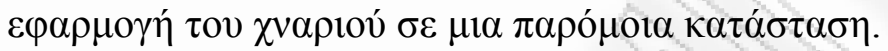

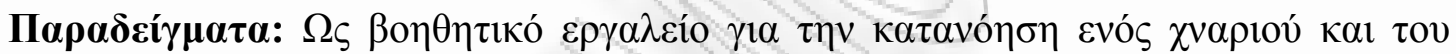

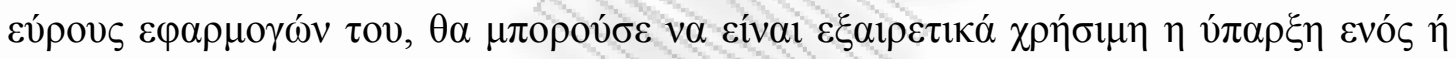

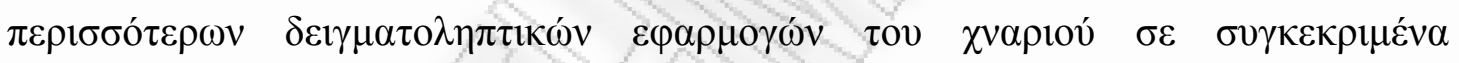
$\sigma v \gamma \kappa \varepsilon i ́ \mu \varepsilon v \alpha$.

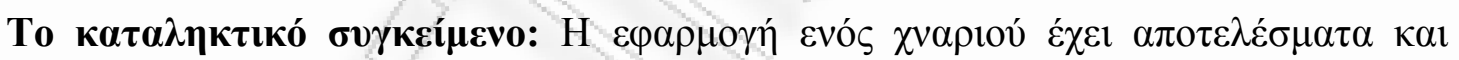

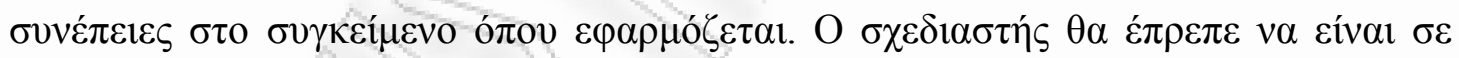

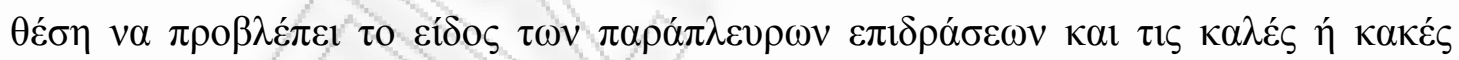

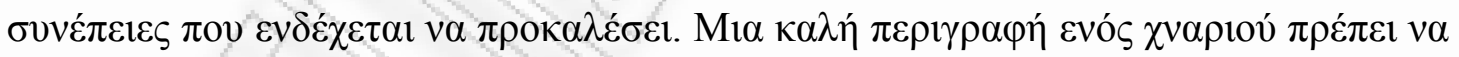

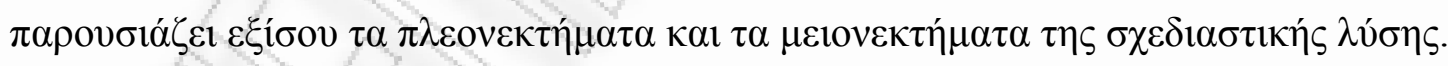

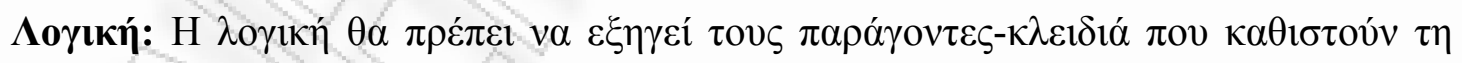

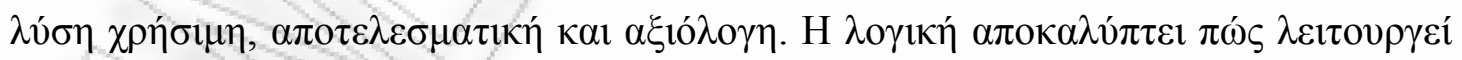

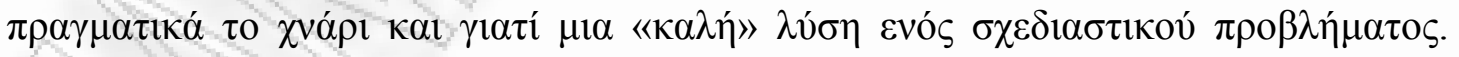

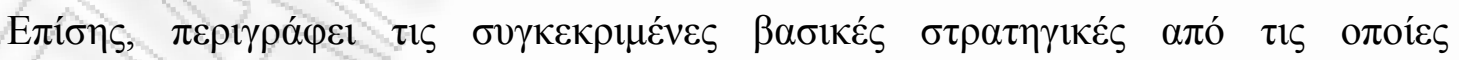

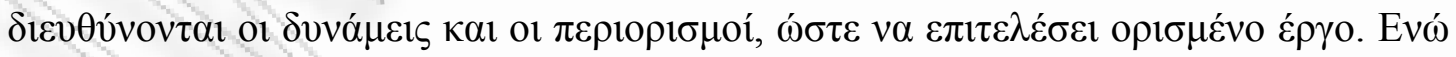

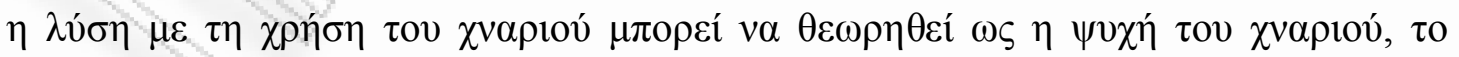

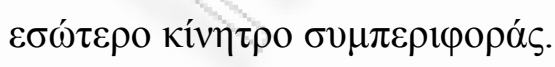

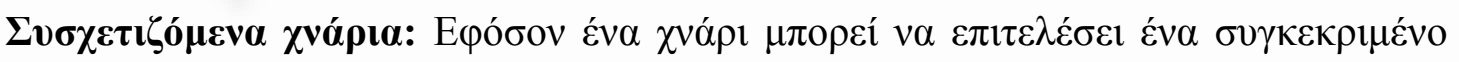

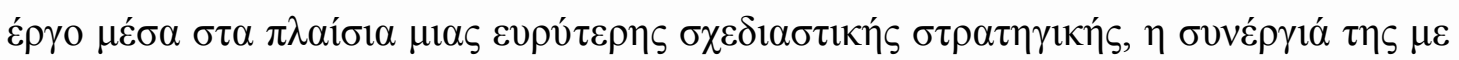

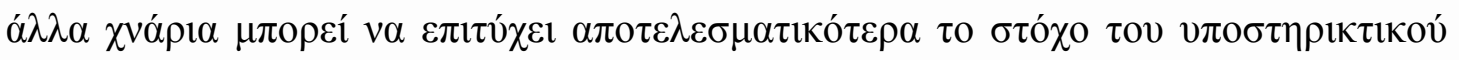

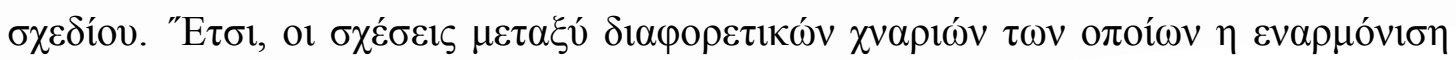




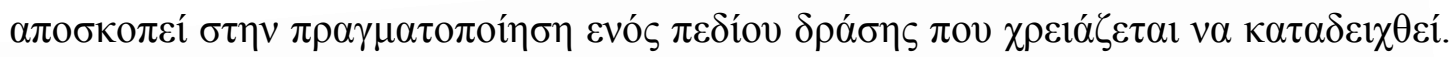

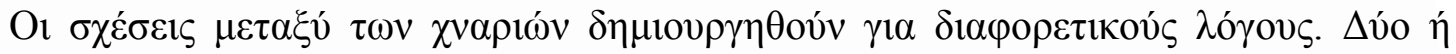

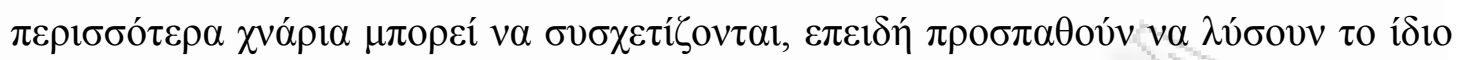

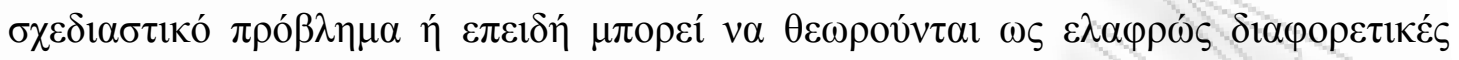

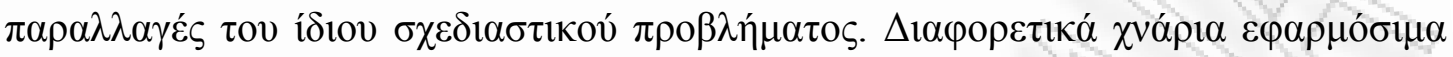

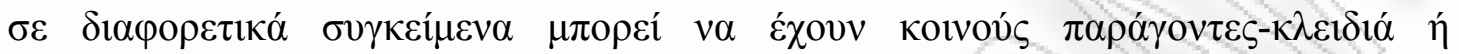

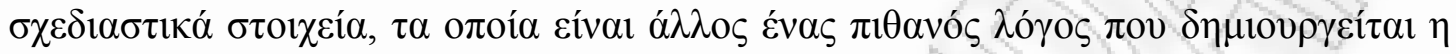

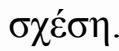

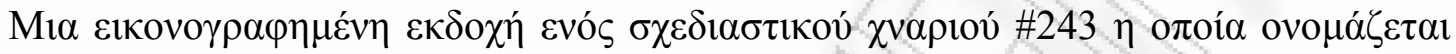

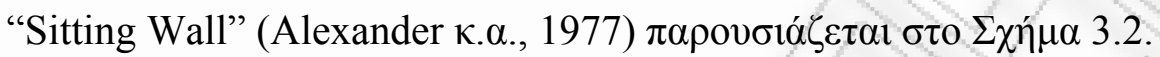

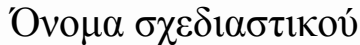

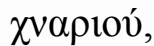

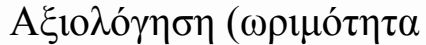
$\beta \alpha \theta \mu \mathrm{o} \lambda о \gamma \eta \mu \varepsilon ́ v \eta \alpha \pi$ ó 1 $\varepsilon \dot{\omega} \omega \varsigma 3 \alpha \sigma \tau \dot{\varepsilon} \rho t \alpha(*))$,

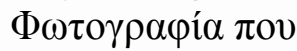

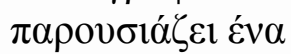
$\alpha \rho \chi \varepsilon ́ \tau v \pi \mathrm{\varepsilon} \pi \alpha \rho \alpha ́ \delta \varepsilon \imath \gamma \mu \alpha$

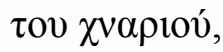

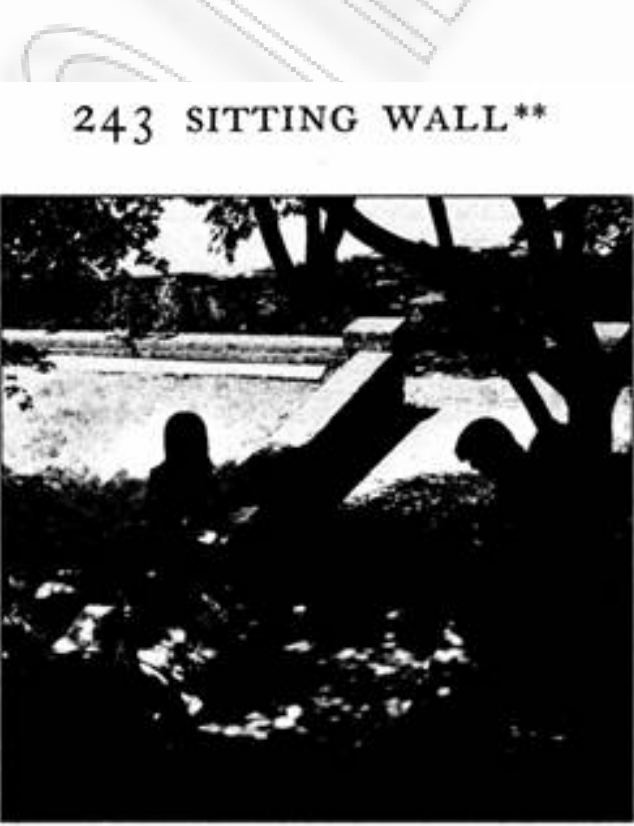

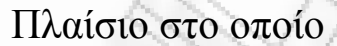
$\varepsilon v \tau \alpha ́ \sigma \sigma \varepsilon \tau \alpha i$ to $\sigma \chi \varepsilon \delta 1 \alpha \sigma \tau \iota \kappa o ́ ~ \chi v \alpha ́ \rho ı$ ( $\varepsilon \pi \varepsilon \xi \eta \gamma \omega \dot{*} \tau \alpha \varsigma \pi \omega \varsigma \alpha v \tau o ́$

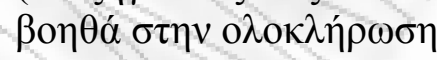

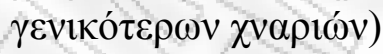

... if all is well, the outdoor areas are largely made up of positive spaces-Posirive outdoor spaces (106); in some fashion you have marked boundaries between gardens and streets, between terraces and gardens, between outdoor rooms and terraces, between play areas and gardens-GREEN STREETS (51), PEDESTRIAN STREET ( 100 ), HALF-HIDDEN GARDEN (III), HIERARCHY OF OPEN SPACE (114), PATH SHAPE (121), ACTIVITY POCKETS (124), PRIVATE TERRACE ON THE STREET (140), OUTDOOR ROOM (163), OPENING TO THE STREET (165), GALLERY SURROUND (166), GARDEN GROWING wiLD (172). With this pattern, you can help these natural boundaries take on their proper character, by building walls, just low enough to sit on, and high enough to mark the boundaries.

If you have also marked the places where it makes sense to build seats-SEAT SPOTS (241), FRONT DOOR BENCH (242) - you can kill two birds with one stone by using the walls as seats which help enclose the outdoor space wherever its positive character is weakest. 


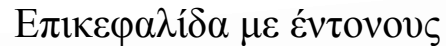

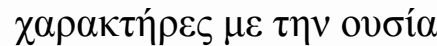

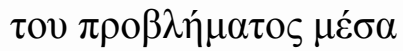

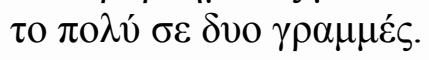

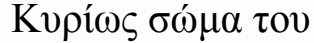
$\pi \rho \circ \beta \lambda \eta ́ \mu \alpha \tau о \varsigma \mu \varepsilon$

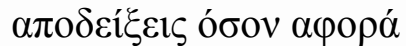
$\tau \eta v \varepsilon \gamma \kappa v \rho o ́ \tau \eta \tau \dot{~} \tau$ ov, $\varepsilon \mu \pi \varepsilon 1 \rho i ́ \varepsilon \varsigma \pi \mathrm{ov}$ $\alpha v \alpha \delta \varepsilon เ \kappa v v ́ \mathrm{ovv} \tau \eta \nu$ v́ $\alpha \rho \xi \eta \dot{~ \tau o v, ~}$

\section{Поюкí $\lambda \alpha \pi \alpha \rho \alpha \delta \varepsilon i ́ \gamma \mu \alpha \tau \alpha$ $\pi$ ov $\varphi \alpha v \varepsilon \rho \omega ́ v o v v \tau \eta v$

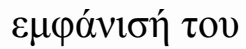$$
* * *
$$

In many places walls and fences between outdoor spaces are too high; but no boundary at all does injustice to the subtlety of the divisions between the spaces.
\end{abstract}

Consider, for example, a garden on a quiet street. At least somewhere along the edge between the two there is a need for a seam, a place which unites the two, but does so without breaking down the fact that they are separate places. If there is a high wall or a hedge, then the people in the garden have no way of being connected to the street; the people in the street have no way of being connected to the garden. But if there is no barrier at all-then the division between the two is hard to maintain. Stray dogs can wander in and out at will; it is even uncomfortable to sit in the garden, because it is essentially like sitting in the street.

The problem can only be solved by a kind of barrier which functions as a barrier which separates, and as a seam which joins, at the same time.

A low wall or balustrade, just at the right height for sitting, is perfect. It creates a barrier which separates. But because it invites people to sit on it-invites them to sit first with their legs on one side, then with their legs on top, then to swivel round still further to the other side, or to sit astride it-it also functions as a seam, which makes a positive connection between the two places.

Examples: A low wall with the children's sandbox on one side, circulation path on the other; low wall at the front of the garden, connecting the house to the public path; a sitting wall that is a retaining wall, with plants on one side, where people can sit close to the flowers and eat their lunch.

Ruskin describes a sitting wall he experienced:

Last summer I was lodging for a little while in a cottage in the country, and in front of my low window there were, first, some beds of daisies, then a row of gooseberry and currant bushes, and then a low wall about three feet above the ground, covered with stonecress. Outside, a corn-field, with its green ears glistening in the sun, and a field path through it, just past the garden gate. From my window I could see every peasant of the village who passed that way, with basket on arm for market, or spade on shoulder for field. When I was inclined for society, I could lean over my wall, and talk fo anybody; when I was inclined for science, I could botanize all along the top of my wall-there were four species of stone-cress alone growing on it; and when I was inclined for exercise, I could jump over my wall, backwards and forwards. That's the sort of fence to have in a Christian country; not a thing which you can't walk inside of without making yourself look like a wild beast, nor look at out of your window in the morning without expecting to see somebody impaled upon it in the night. (John Ruskin, The Two Paths, New York: Everyman's Library, 1907, p. 203.)

Therefore:

Surround any natural outdoor area, and make minor boundaries between outdoor areas with low walls, about 16 inches high, and wide enough to sit on, at least 12 inches wide. 


\author{
$\Delta 1 \alpha \gamma \rho \alpha \mu \mu \alpha \tau 1 \kappa \eta ́$ \\ $\alpha v \alpha \pi \alpha \rho \alpha ́ \sigma \tau \alpha \sigma \eta \tau \eta \varsigma$ \\ $\lambda$ víns,

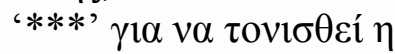 \\ $\kappa \alpha \tau \alpha \dot{\lambda} \eta \xi \eta \tau$ \\ $\sigma \omega ́ \mu \alpha \tau \circ \varsigma \tau 0 v$ \\ $\sigma \chi \varepsilon \delta 1 \alpha \sigma \tau \iota \kappa o v ́ ~ \chi v \alpha \rho ı v^{\prime}$
}

\section{SITTING WALL}
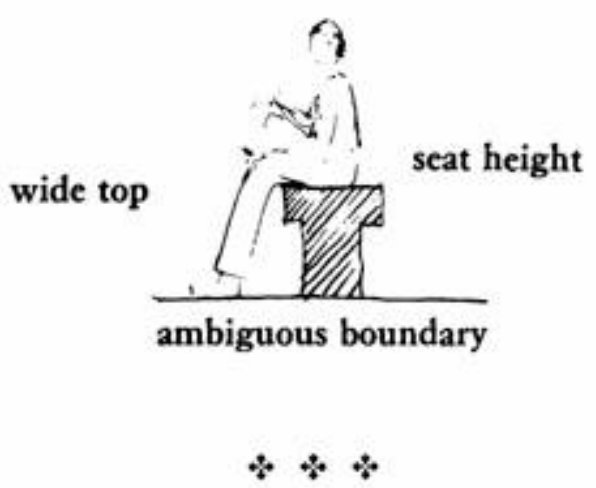

Place the walls to coincide with natural seat spots, so that extra benches are not necessary- SEAT sPoTs (241); make them of brick or tile, if possible-sOPT TILE AND BRICK (248); if they separate two areas of slightly different height, pierce them with holes to make them balustrades-ORNAMENT (249). Where they are in the sun, and can be large enough, plant flowers in them or against them-RAISED FLoWERS (245). . . .

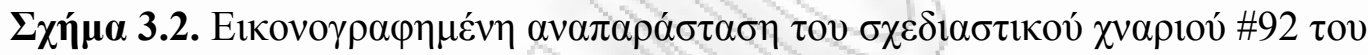

Alexander: $\Sigma \tau \alpha ́ \sigma \eta \eta \lambda \varepsilon \omega \varphi \circ \rho \varepsilon i ́ o v$.

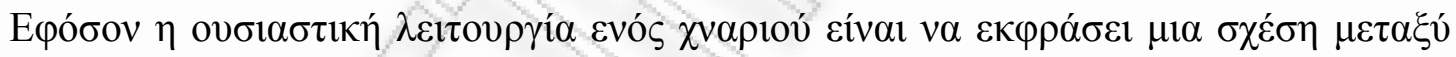

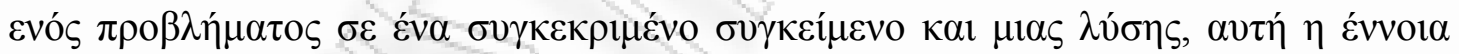

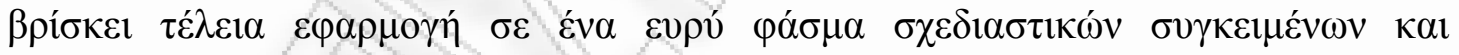
$\varepsilon \pi \imath \sigma \tau \eta \mu$ ovıкஸ́v $\pi \varepsilon \delta i ́ \omega v$.

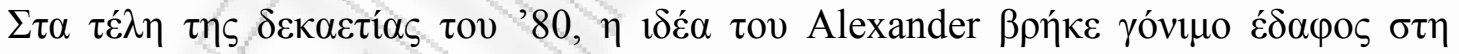

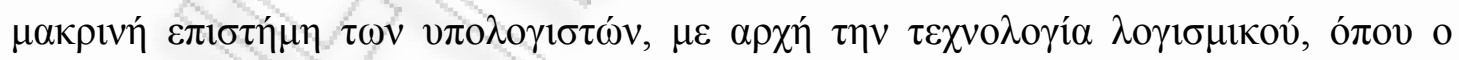

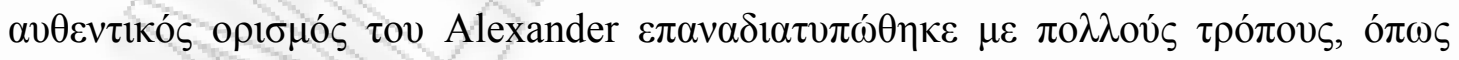

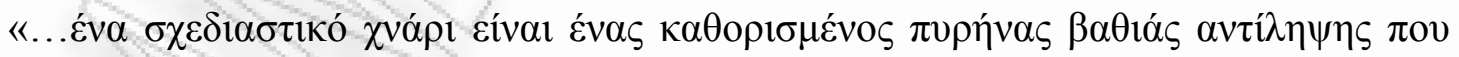

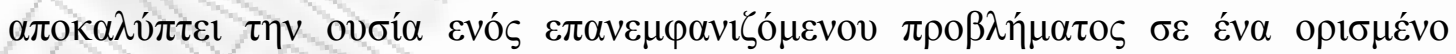

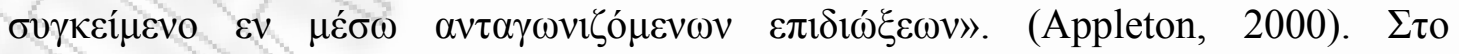

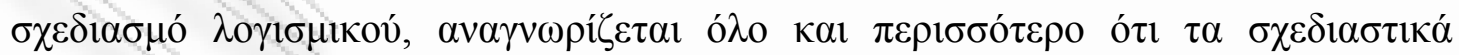

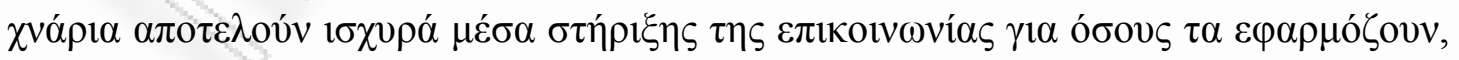

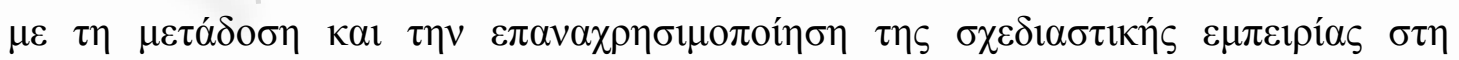

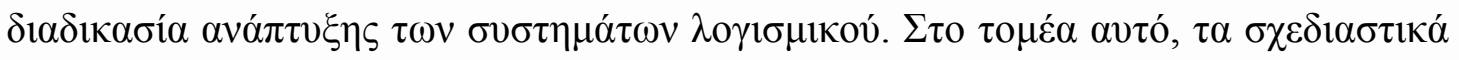

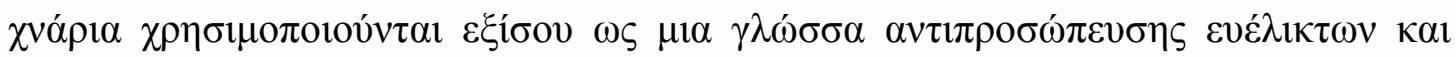

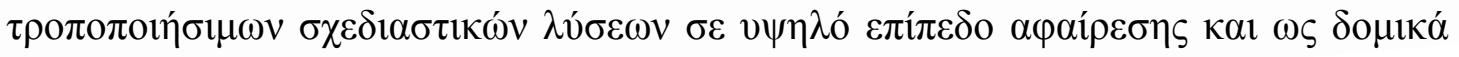




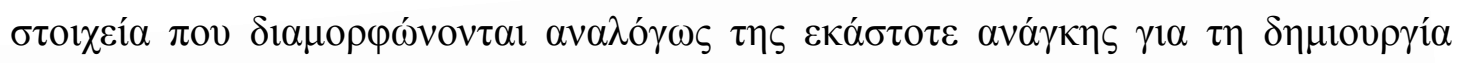

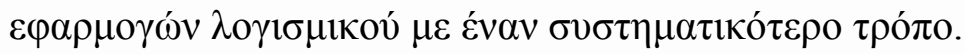

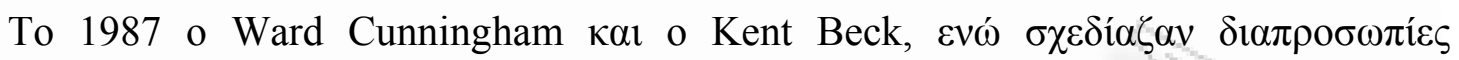

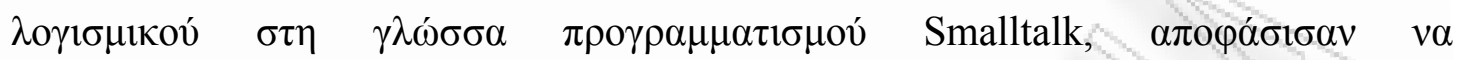

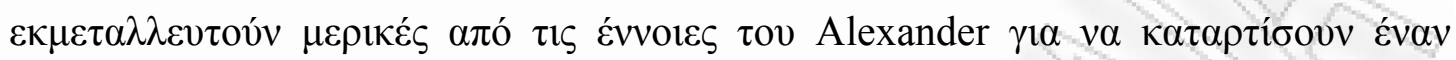

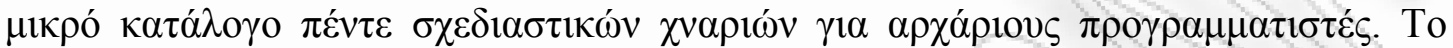

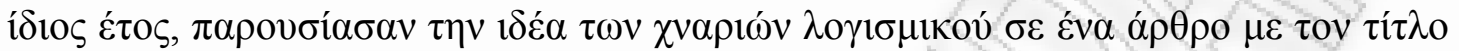
Using Pattern Languages for Object Oriented Programmes (H $\chi \rho \eta \dot{\sigma} \gamma \lambda \lambda \omega \sigma \sigma \omega ́ v$

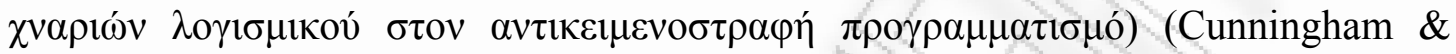

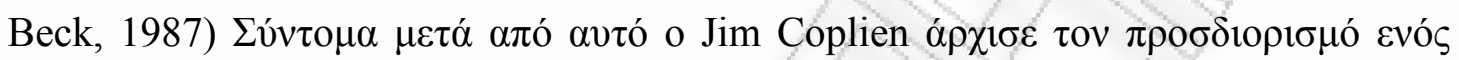

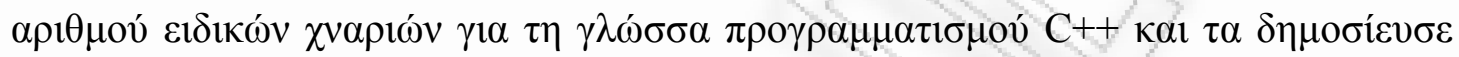

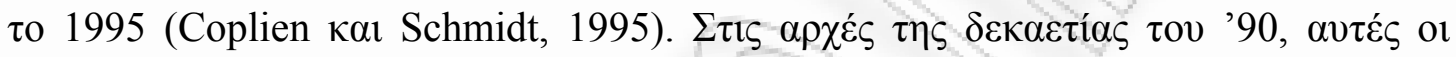

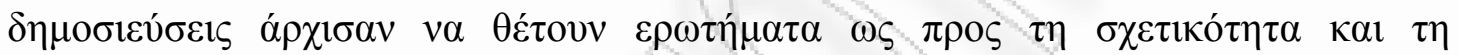

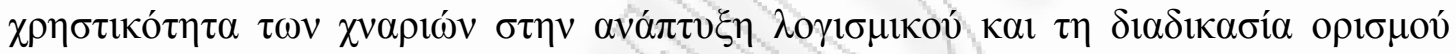

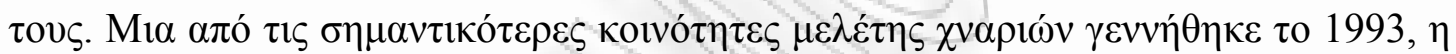

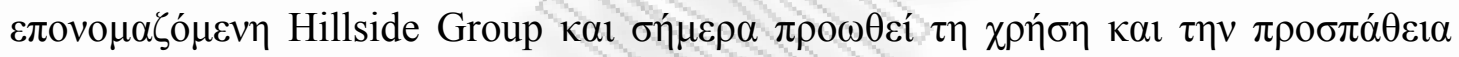

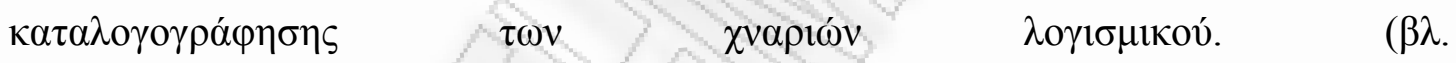
[http://www.hillside.net/patterns/onlinepatterncatalog.htm])

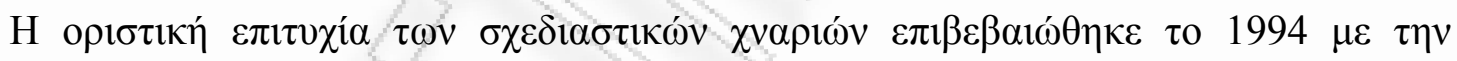

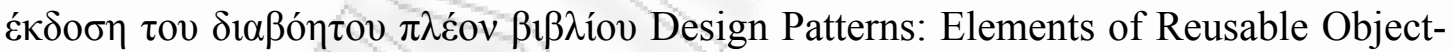

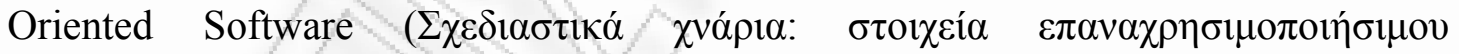

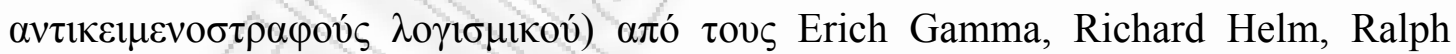

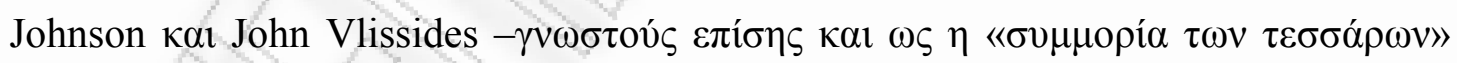

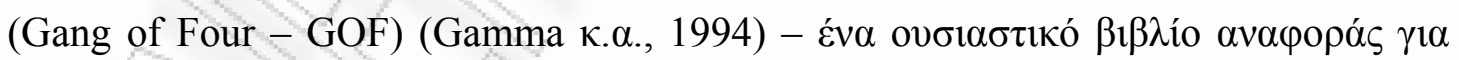

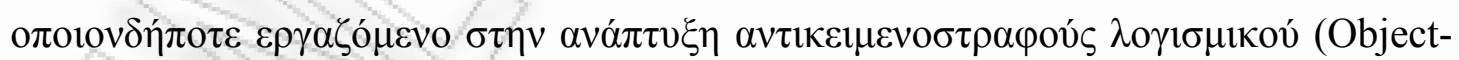

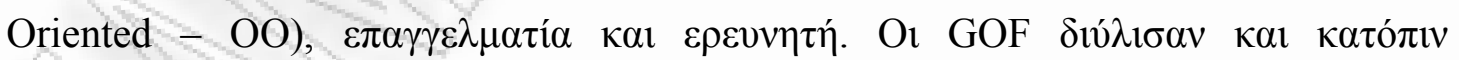

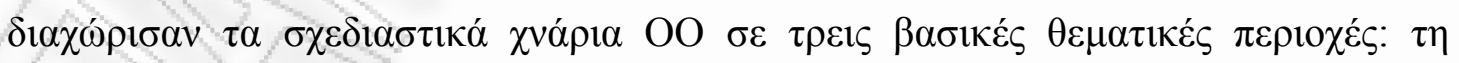

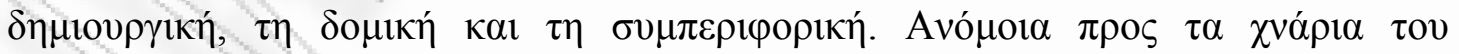

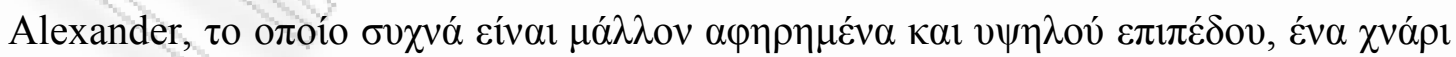

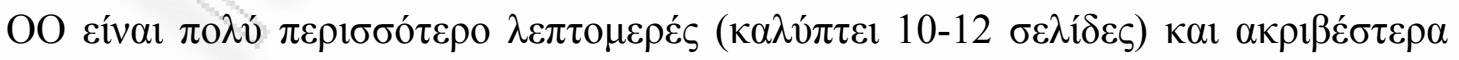

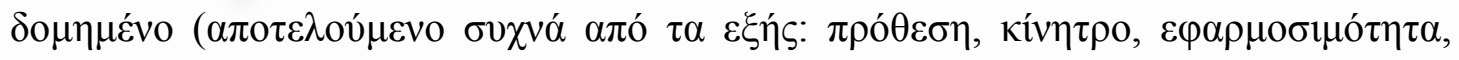

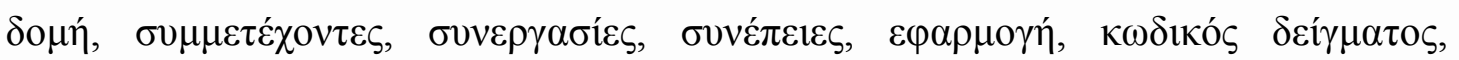

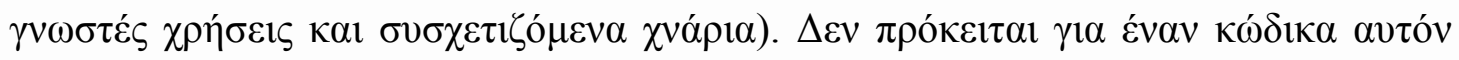

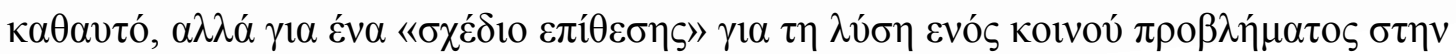




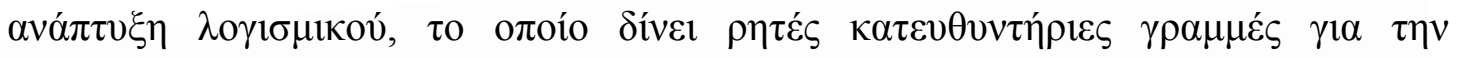

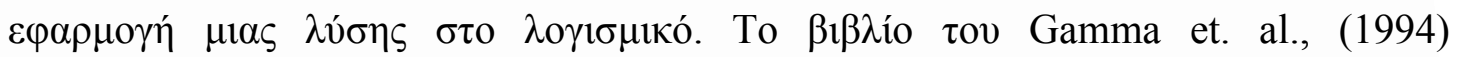
$\alpha \kappa o \lambda o v ́ \theta \eta \sigma \varepsilon \eta \eta$

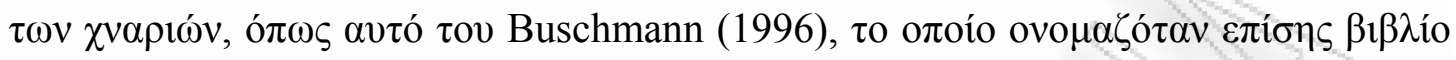

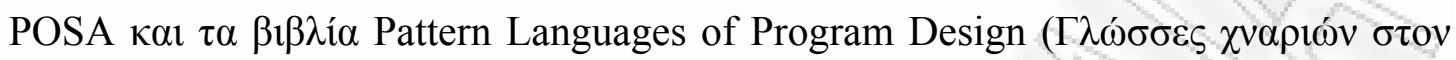

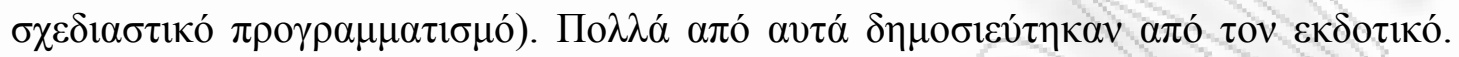

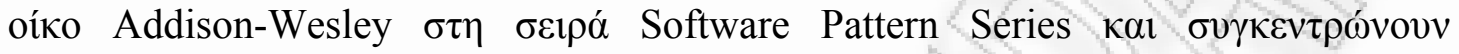

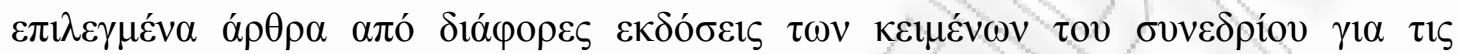

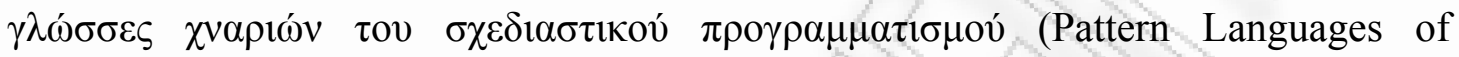
Program Design - PloP $\eta$ PloPd).

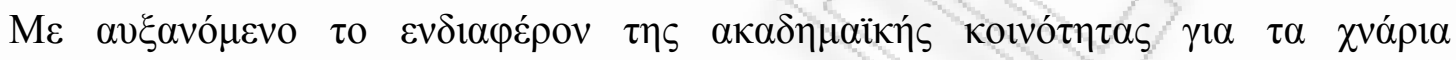

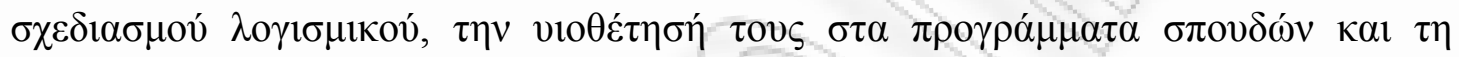

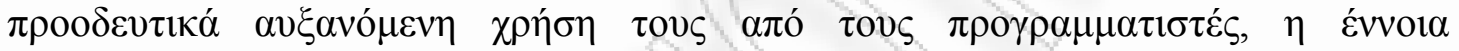

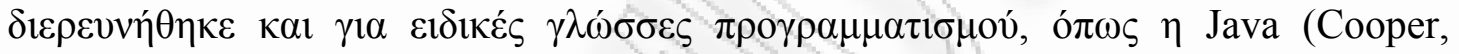

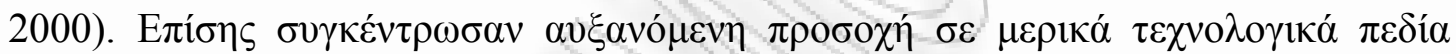

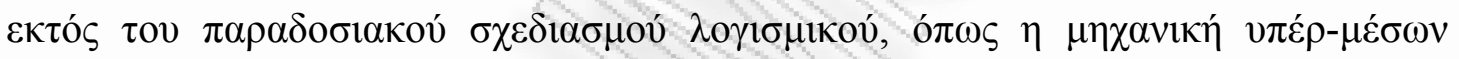

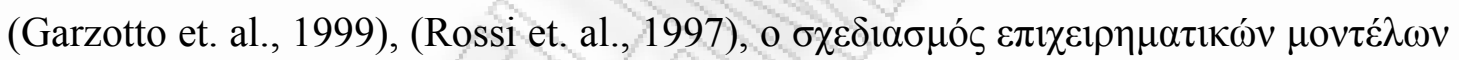

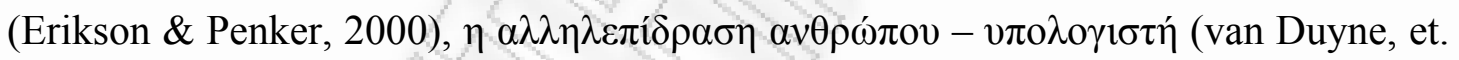

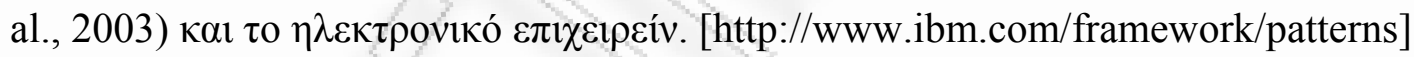

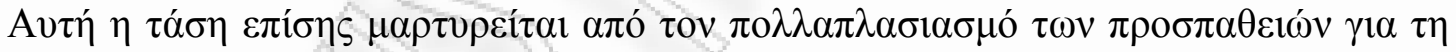

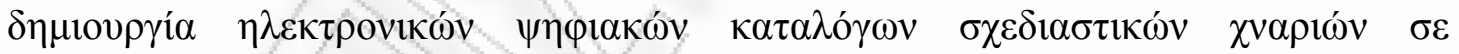

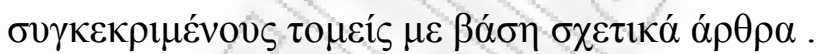

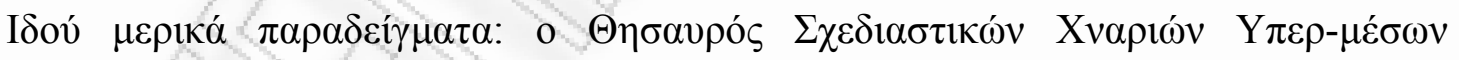
[http://www.designpattern.lu.unisi.ch/PatternsRepository.htm] $\tau \alpha \Sigma \chi \varepsilon \delta 1 \alpha \sigma \tau \imath \kappa \alpha ́$ Xvópı $\alpha$

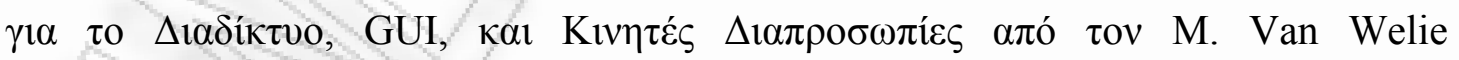
[http://www.welie.com] o

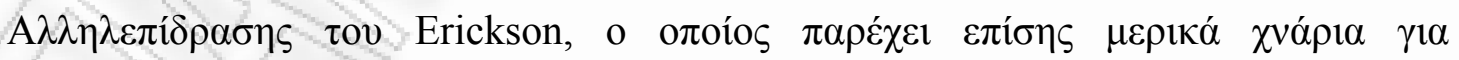

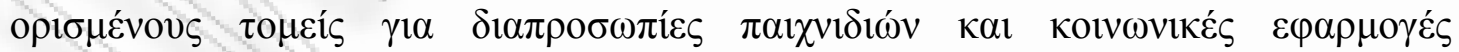
[http://www.pliant.org/personal/Tom_Erickson/InteractionPatterns.html] $\tau \alpha \varepsilon v \varepsilon v \eta ́ v \tau \alpha$

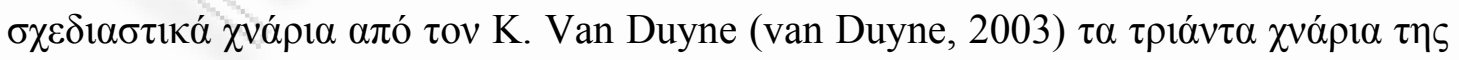

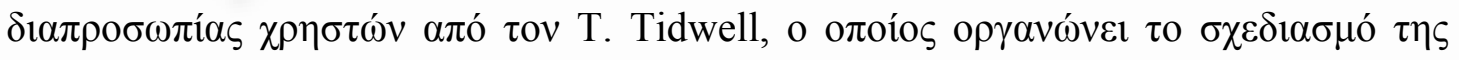

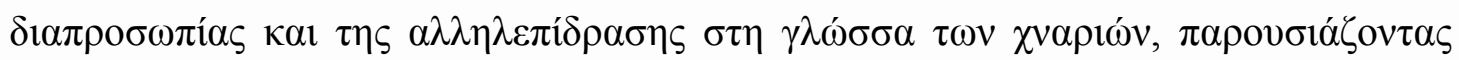

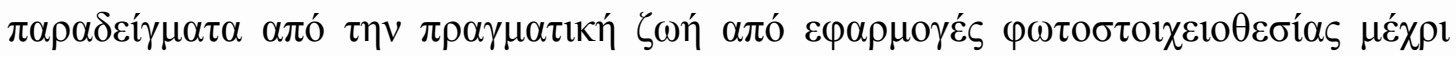




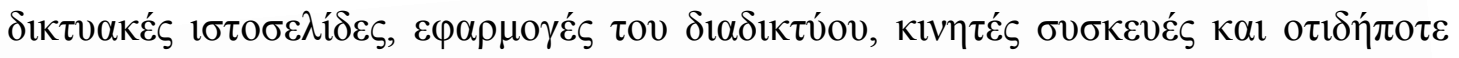

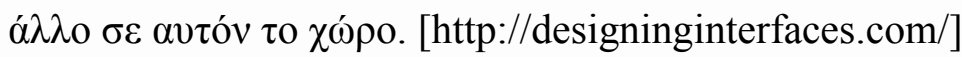

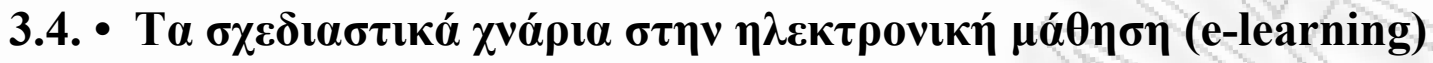

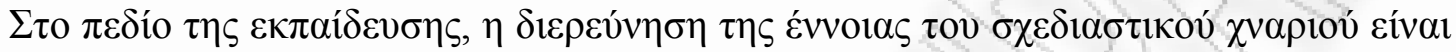

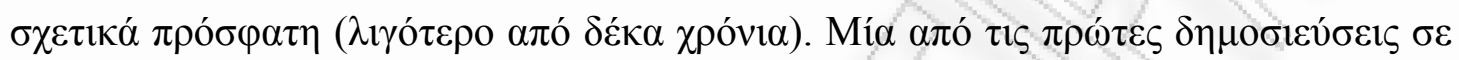

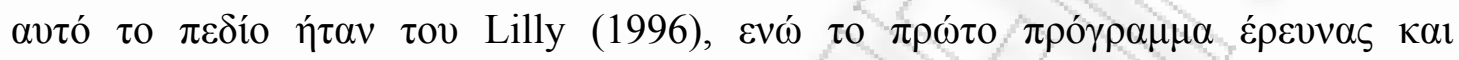

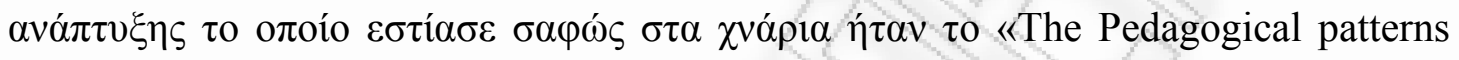

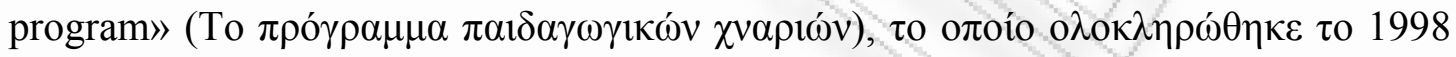

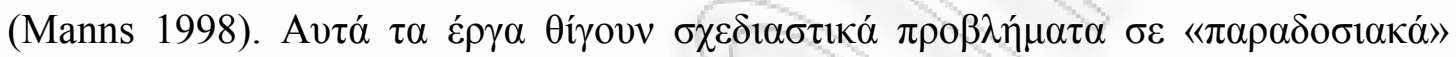

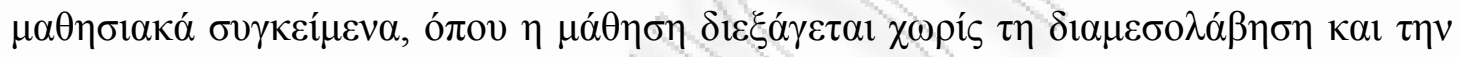

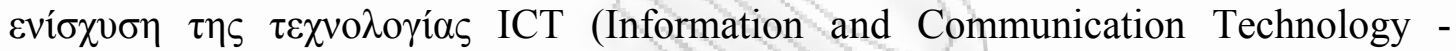

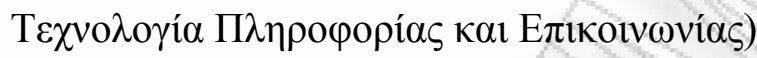

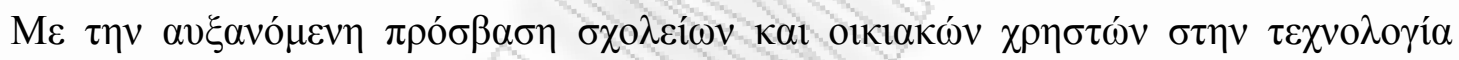

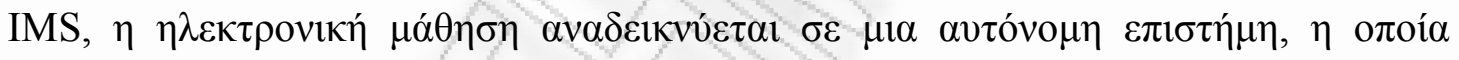

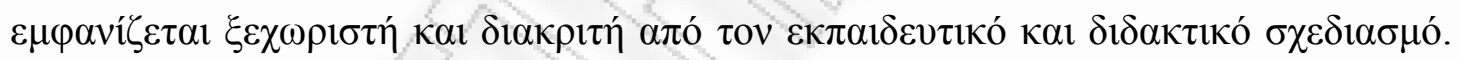

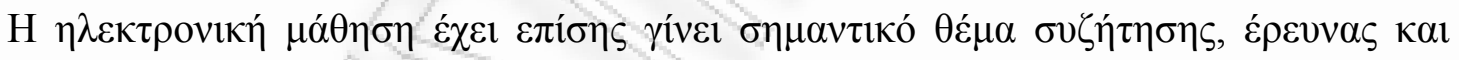

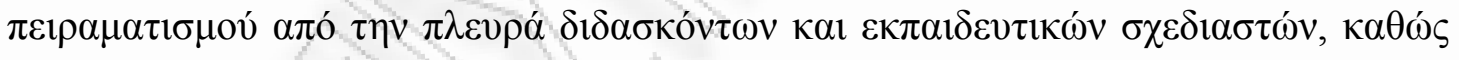

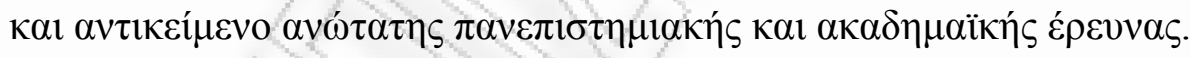

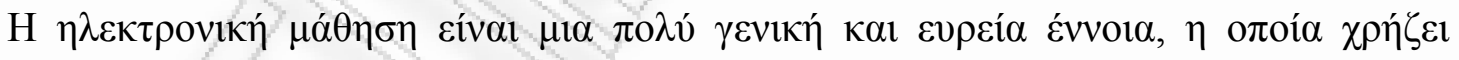

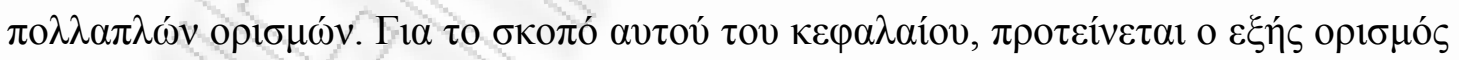

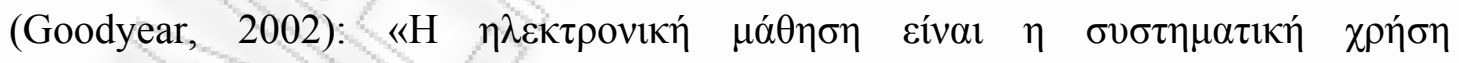

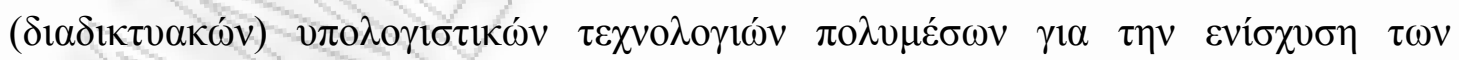

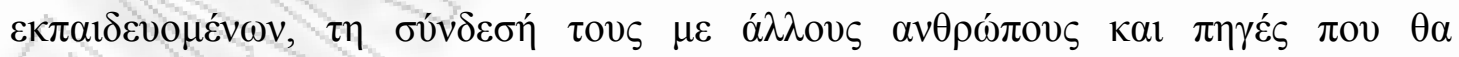

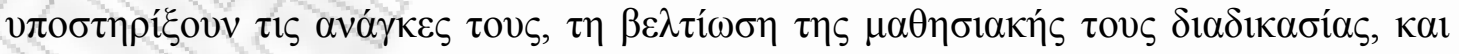

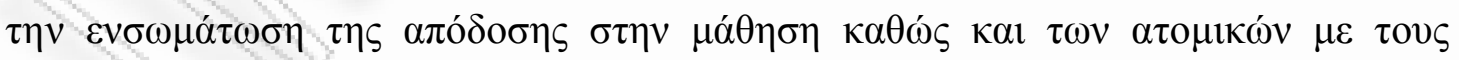

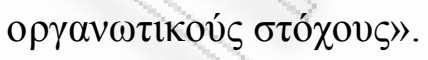

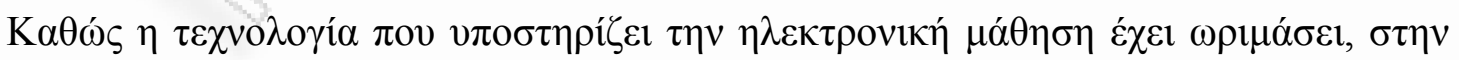

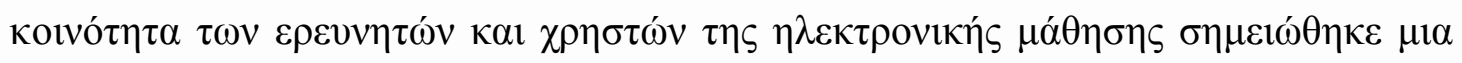

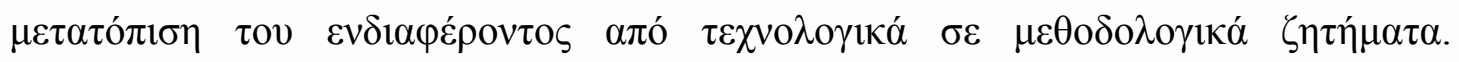

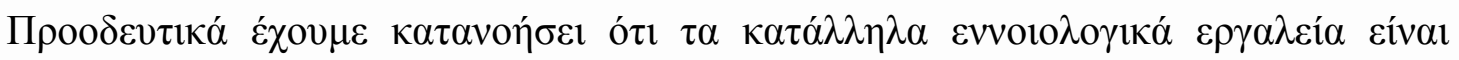




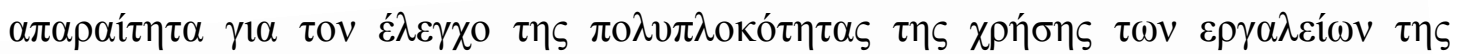

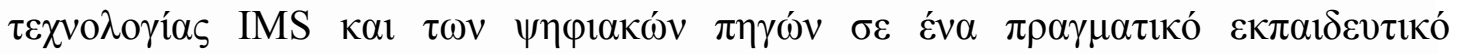

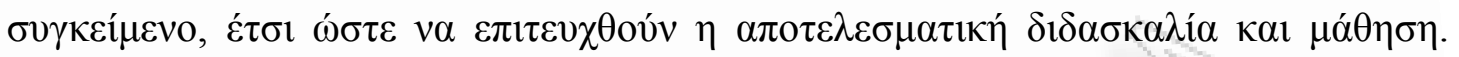

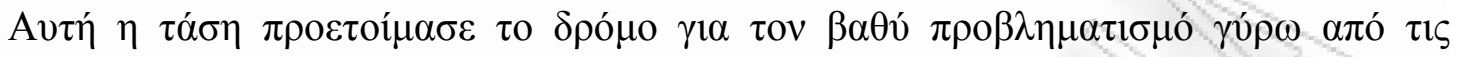

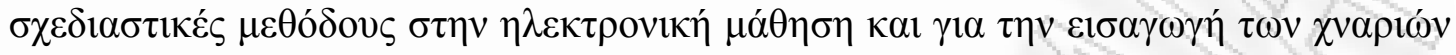

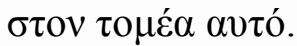

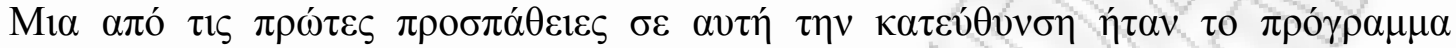
PoInter (PoInter project).

[http://www.comp.lancs.ac.uk/computing/research/cseg/projects/pointer/pointer.html]

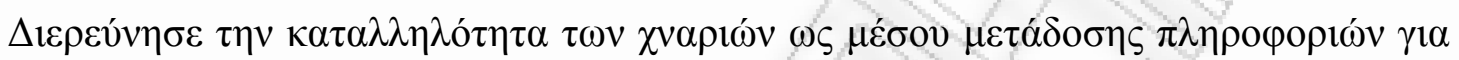

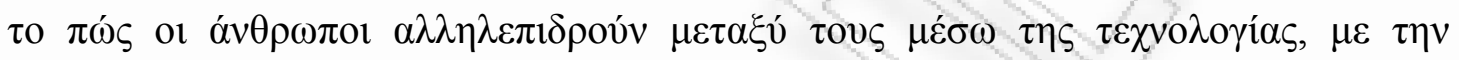

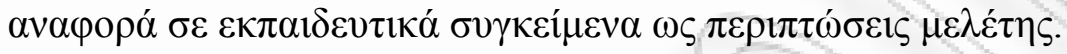

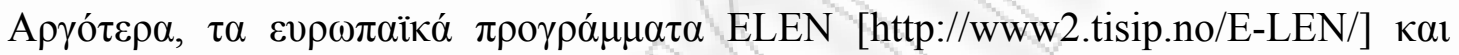

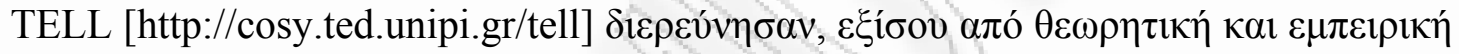

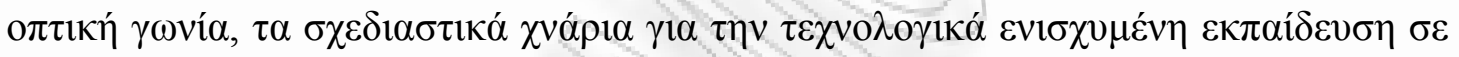

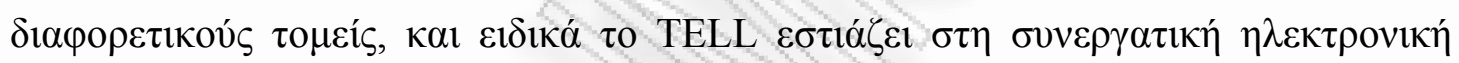
$\mu \alpha ́ \theta \eta \sigma \eta$.

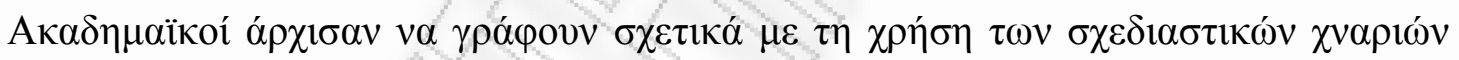

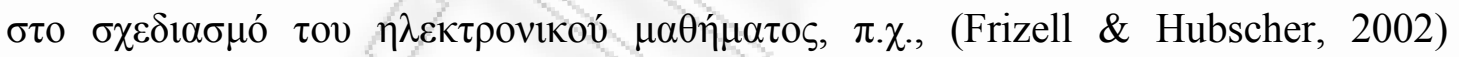
(Goodyear et. al., 2004) (Garzotto \& Retalis, 2004), (Goodyear, 2005), (Hernandez-

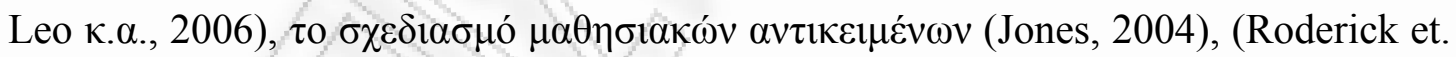

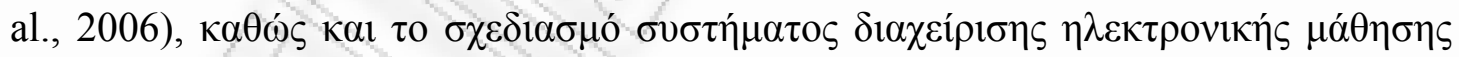
(Avgeriou et. al., 2001), (Schuemmer, 2003), (Georgiakakis et. al., 2005).

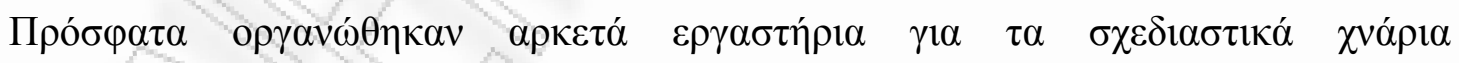

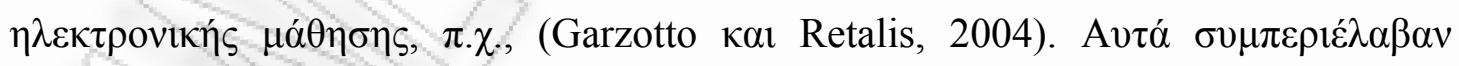

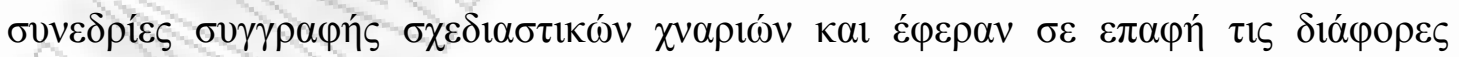

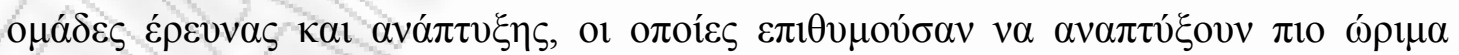

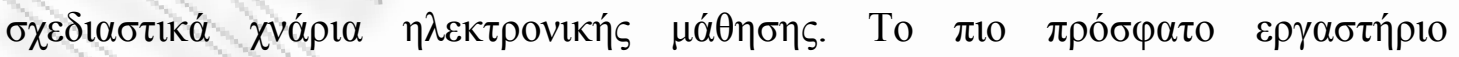

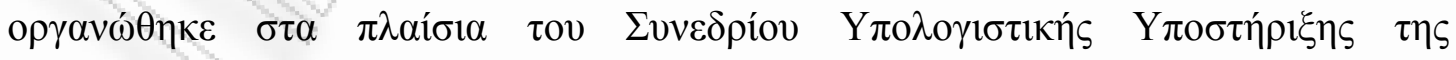

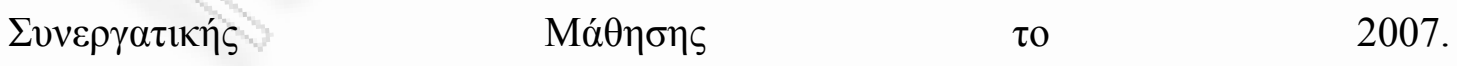
[http://cosy.ted.unipi.gr/CSCL_DPatterns_workshop.htm]

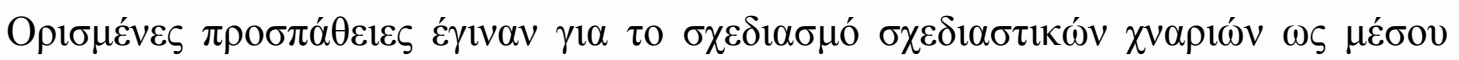

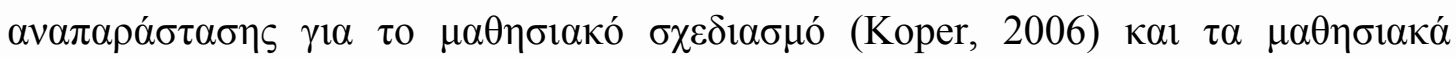

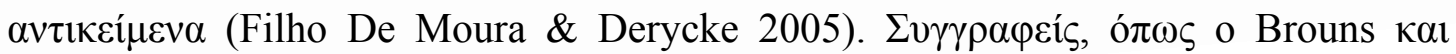




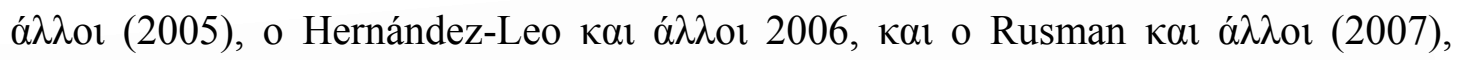

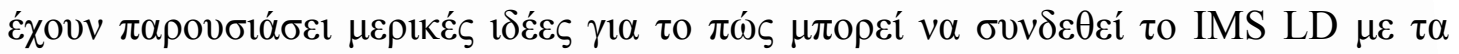

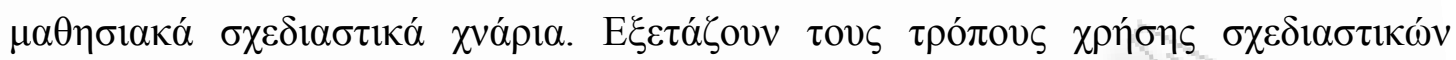

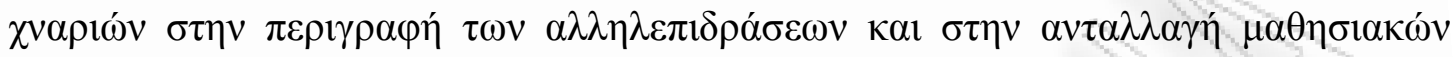

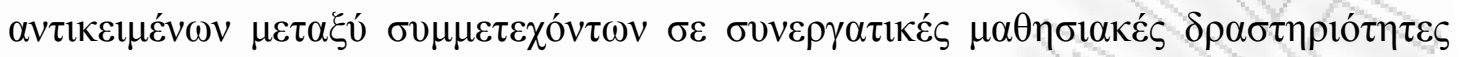

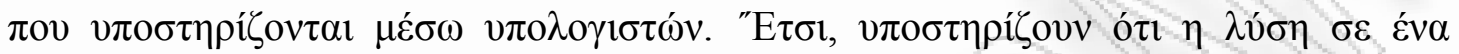

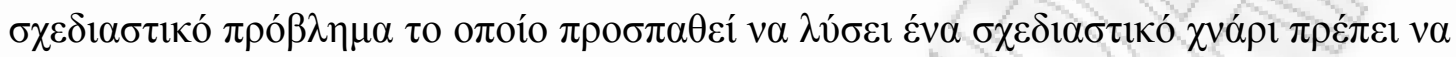

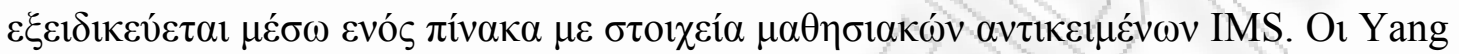

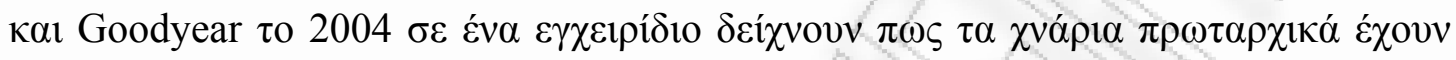

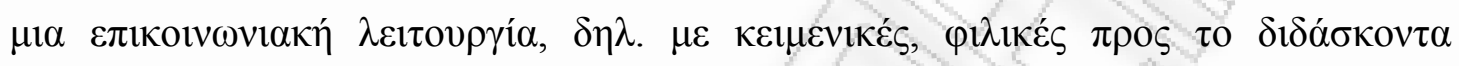

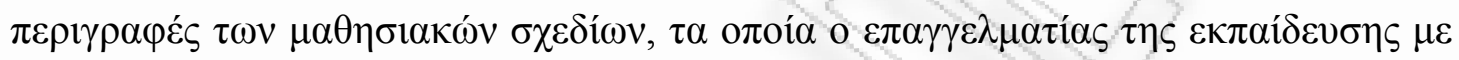

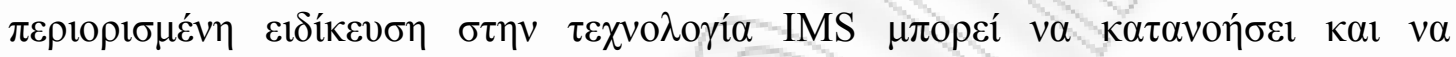

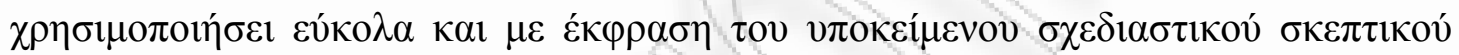

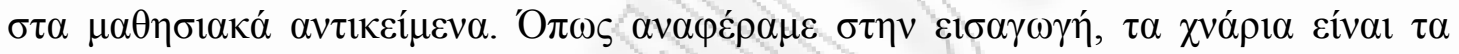

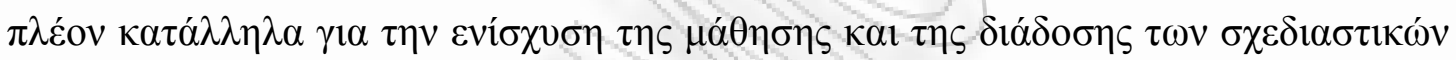

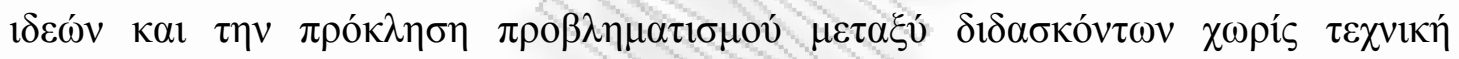

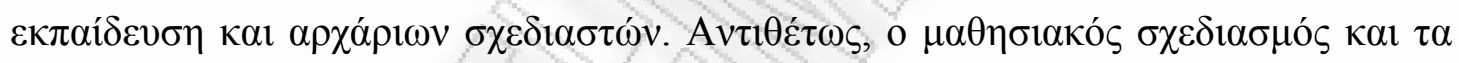

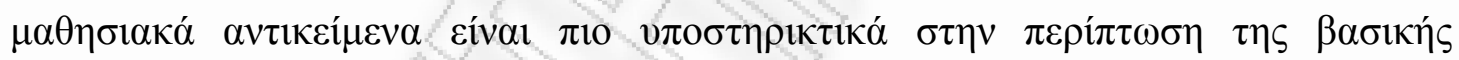

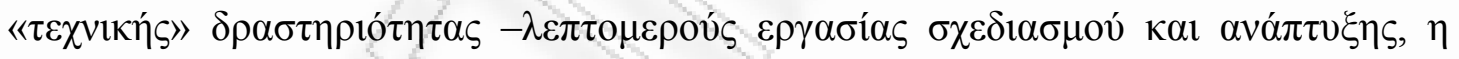

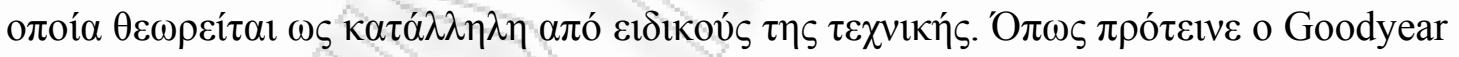

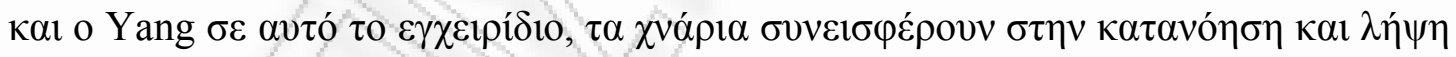

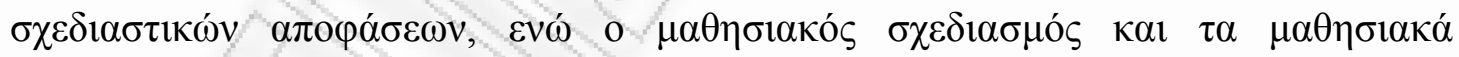

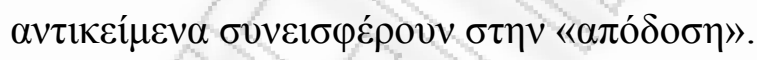

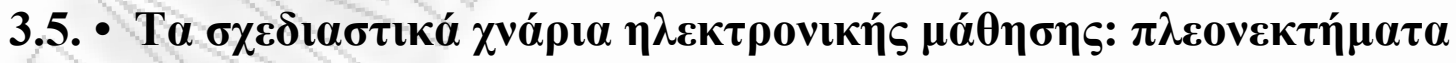

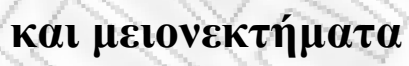

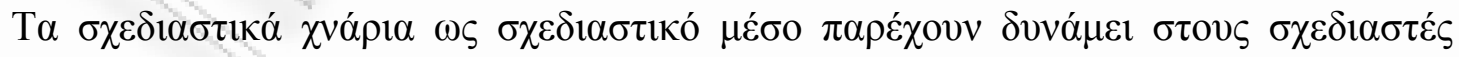

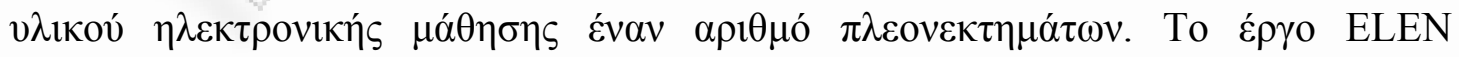

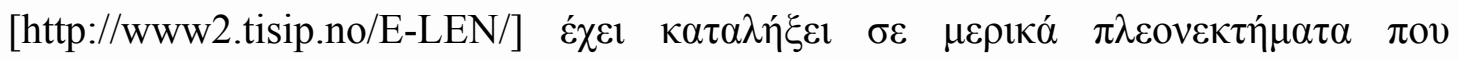

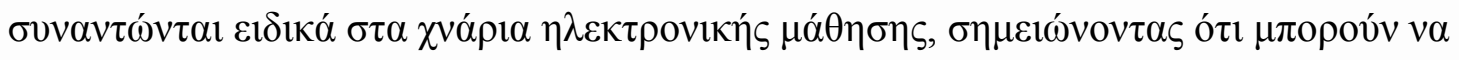

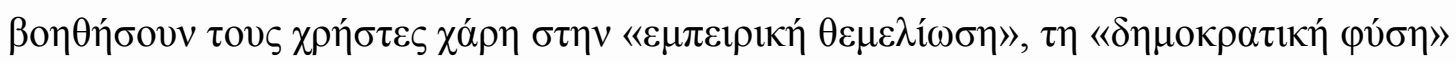




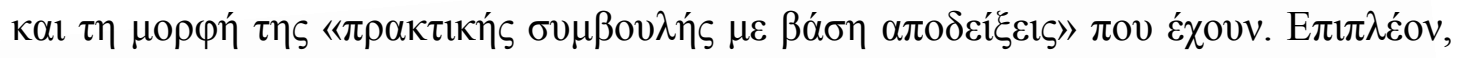

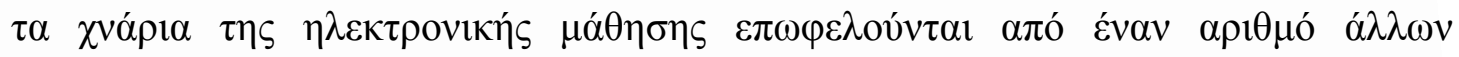

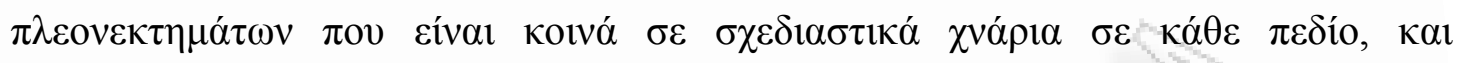

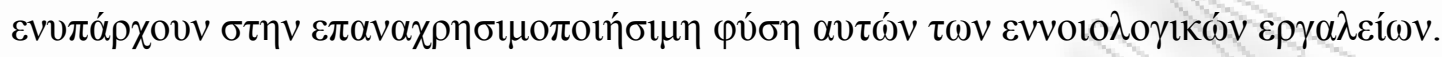

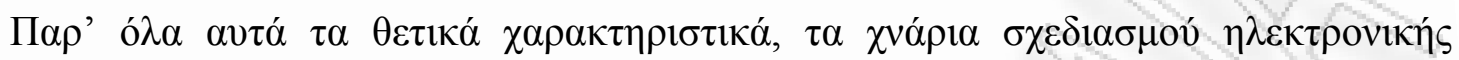

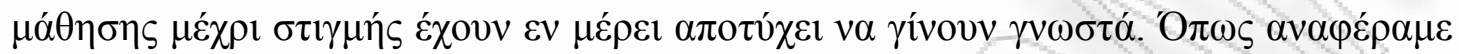

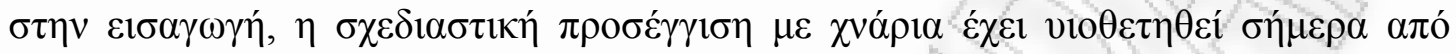

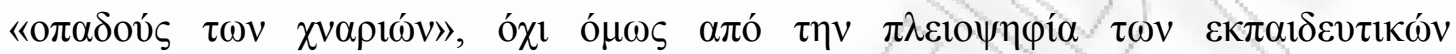

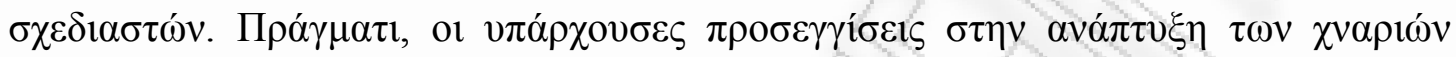

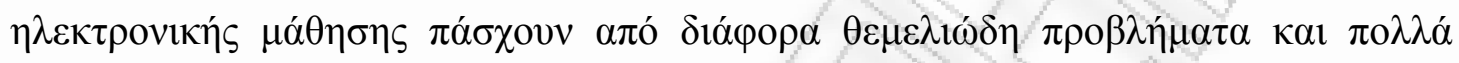

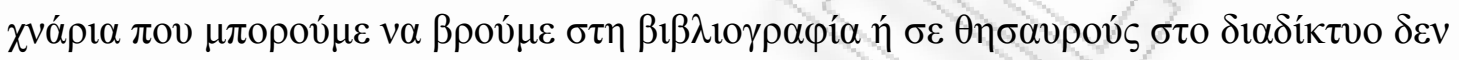

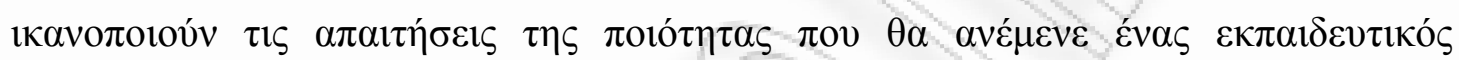
$\sigma \chi \varepsilon \delta 1 \alpha \sigma \tau \eta ́ s$.

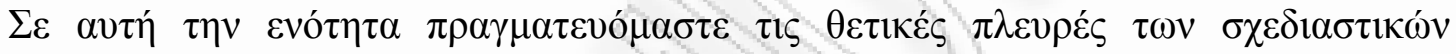

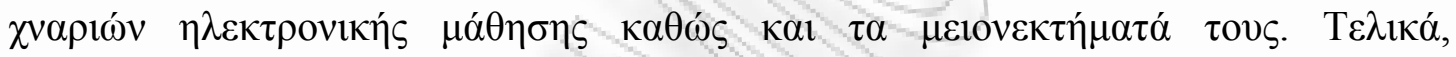

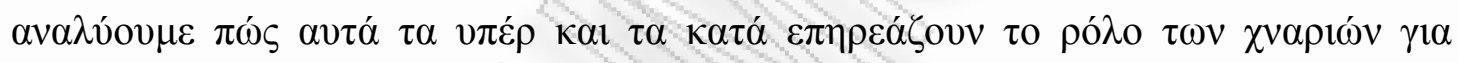

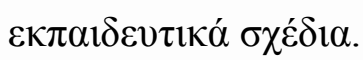

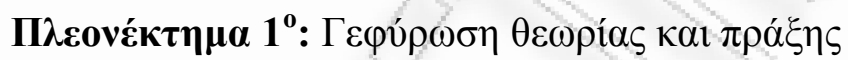

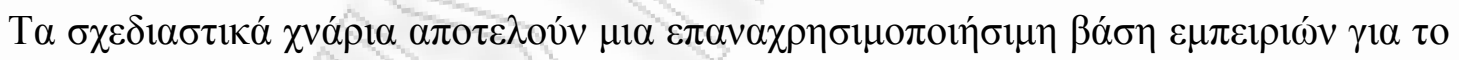

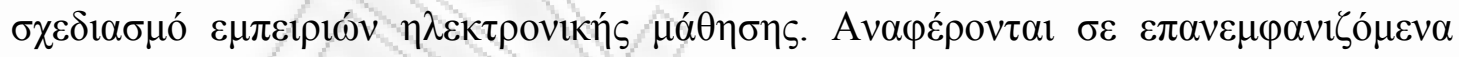

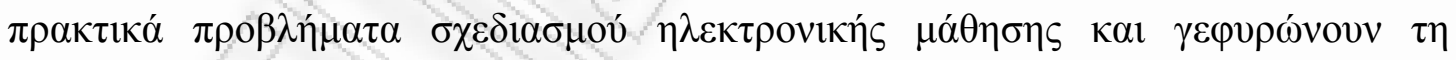

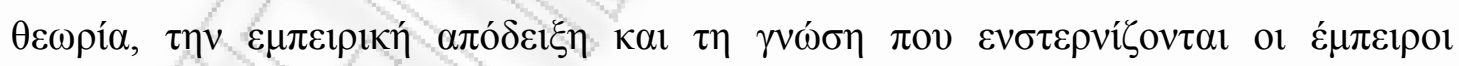
$\varepsilon \pi \alpha \gamma \gamma \varepsilon \lambda \mu \alpha \tau i \varepsilon \varsigma \sigma \chi \varepsilon \delta 1 \alpha \sigma \tau \varepsilon \dot{\zeta}$.

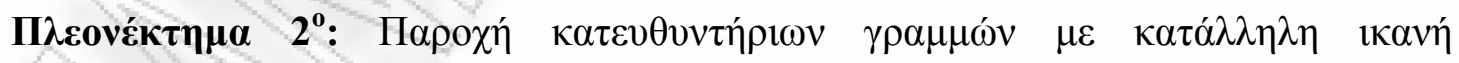

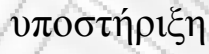

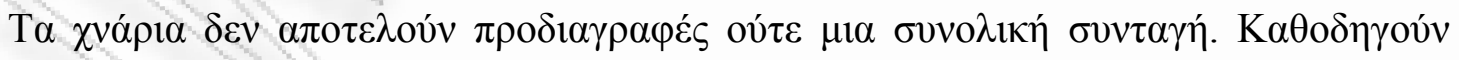

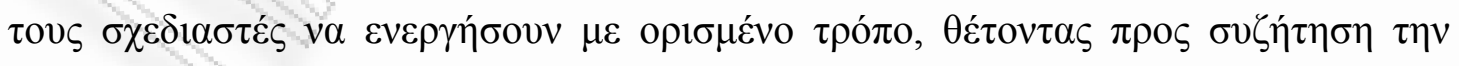

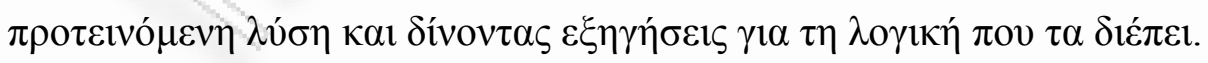

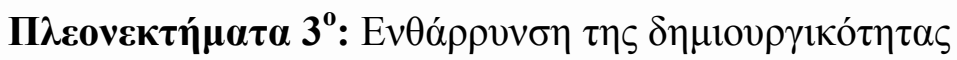

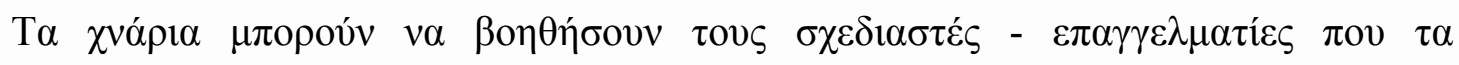

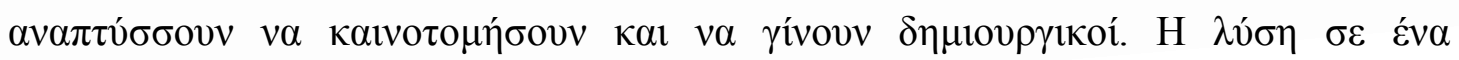




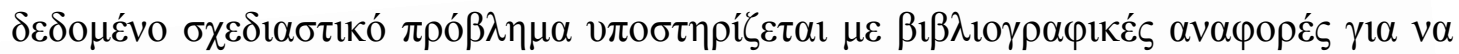

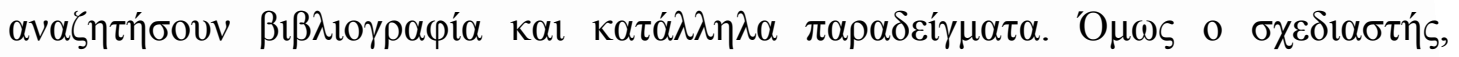

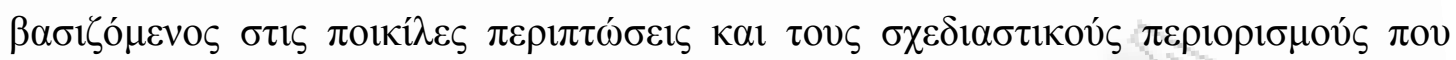

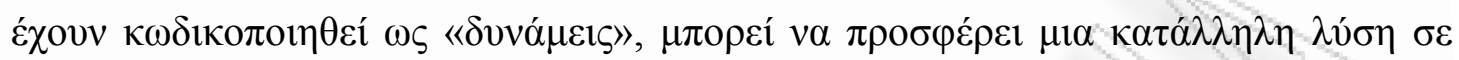

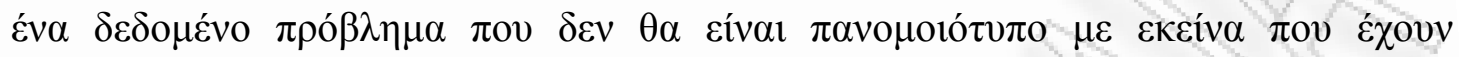

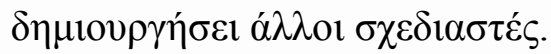

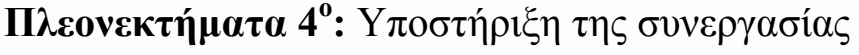

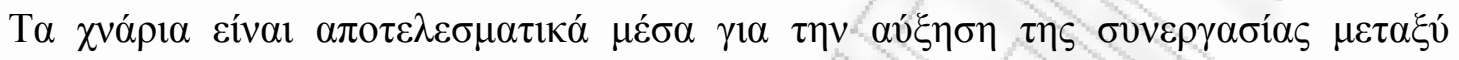

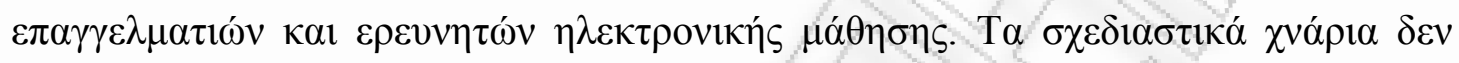

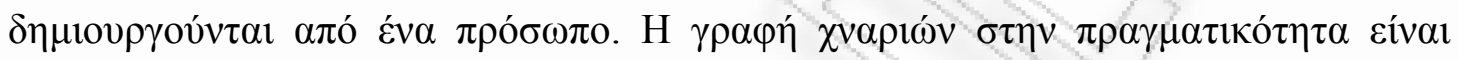

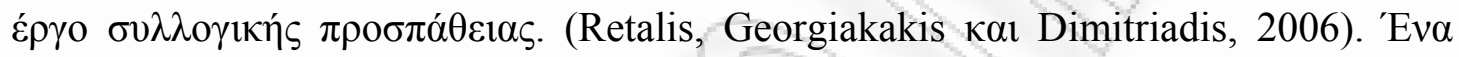

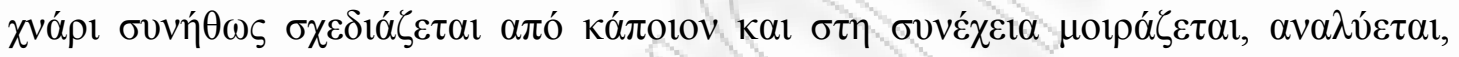

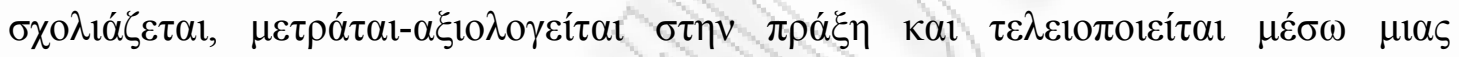

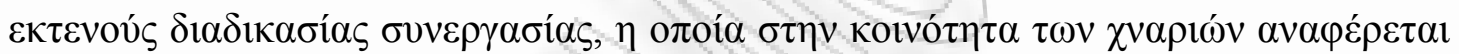

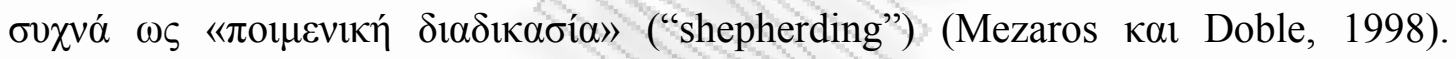

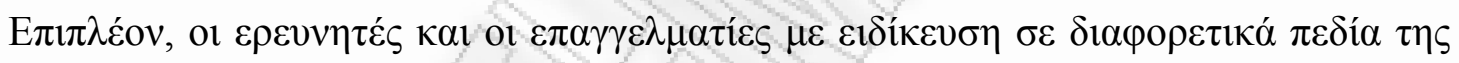

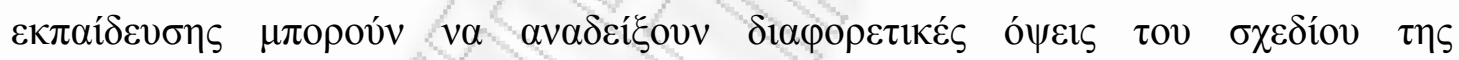

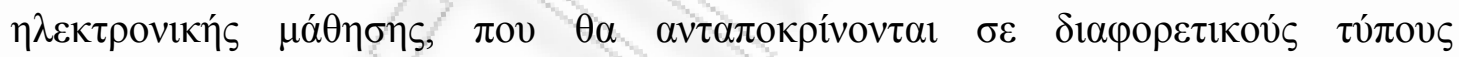

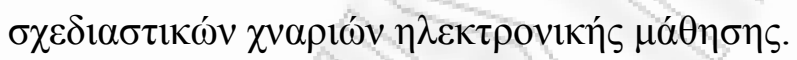

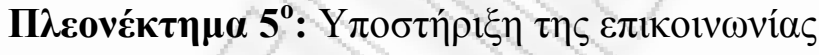

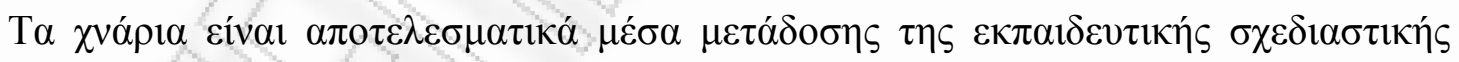

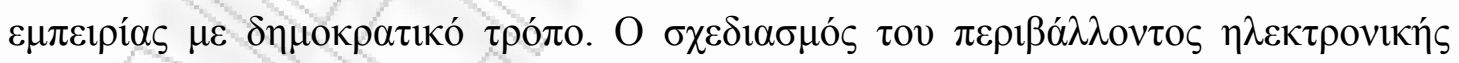

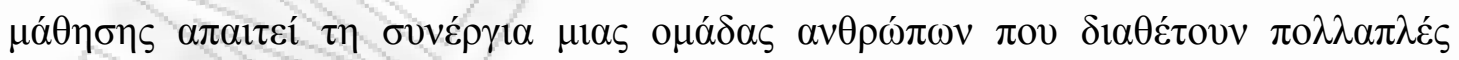

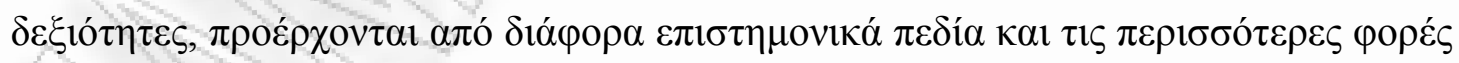

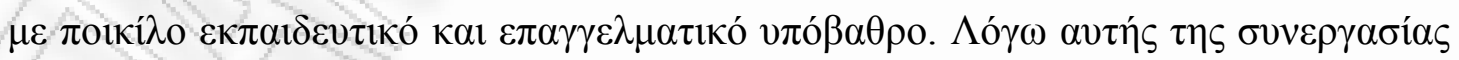

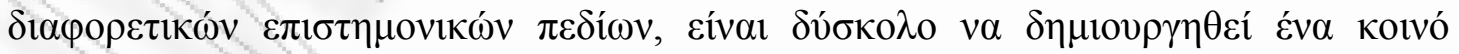

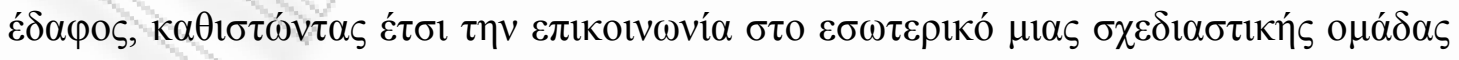

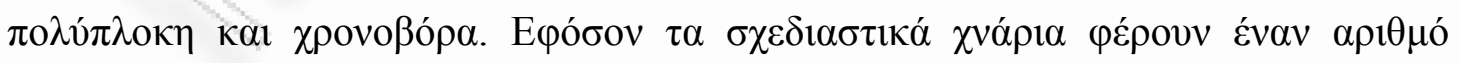

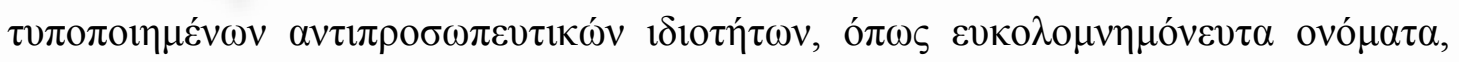

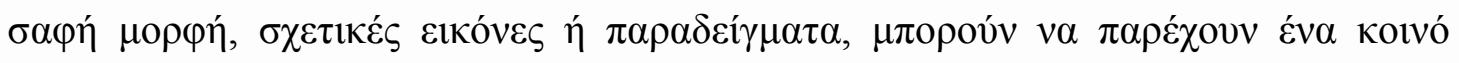

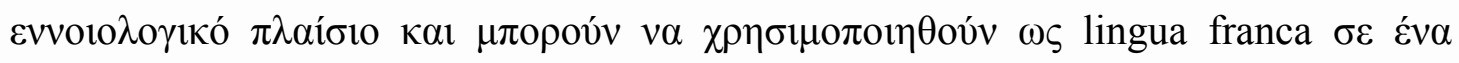

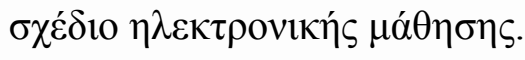




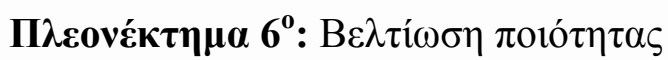

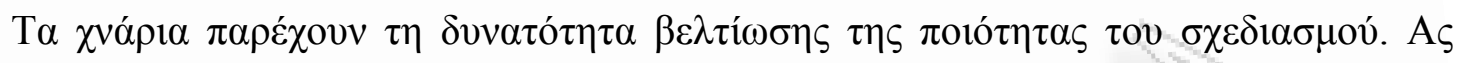

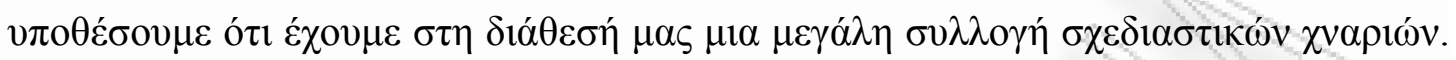

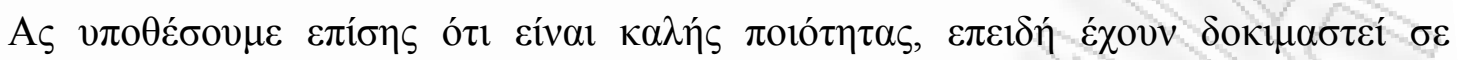

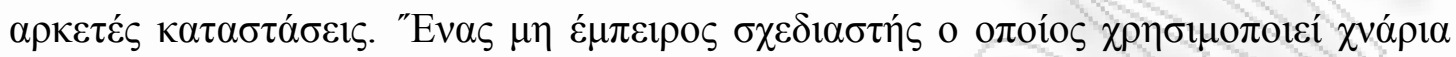

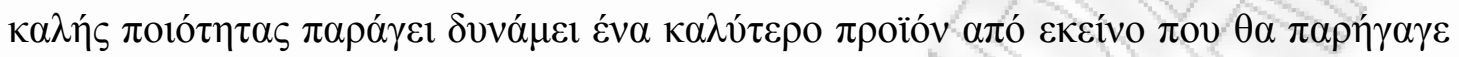

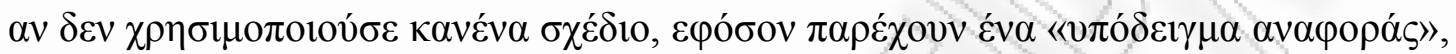

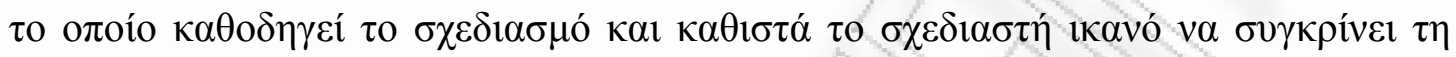

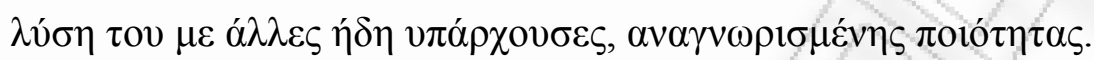

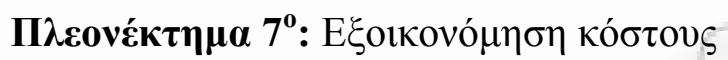

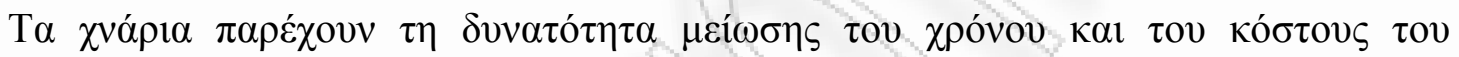

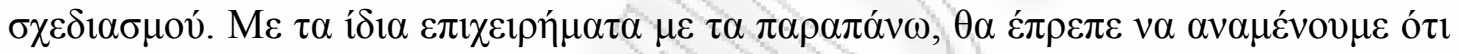

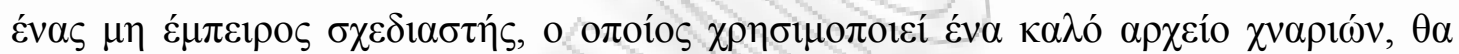

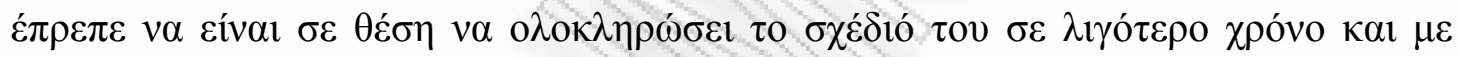

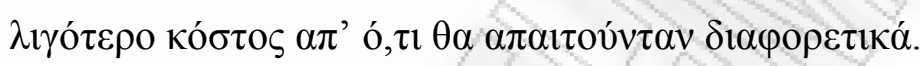

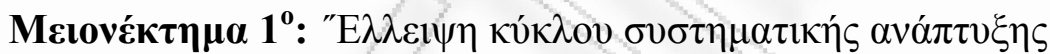

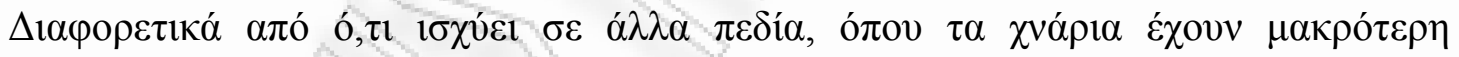

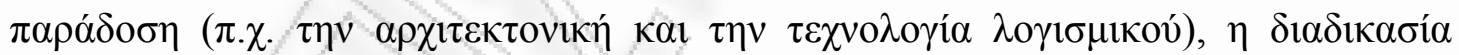

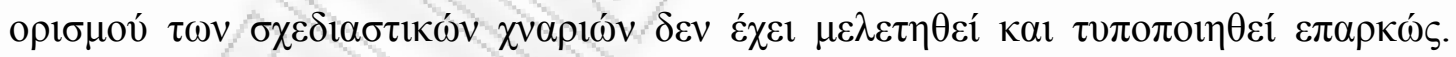

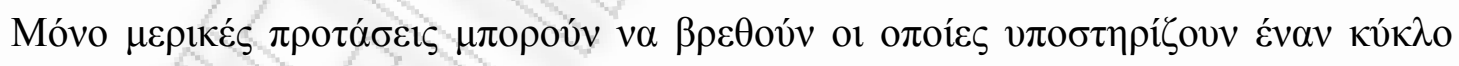

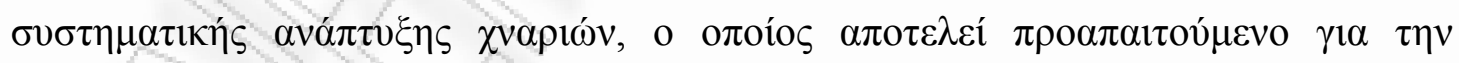

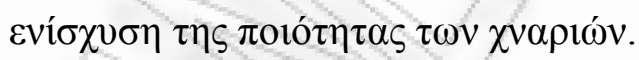

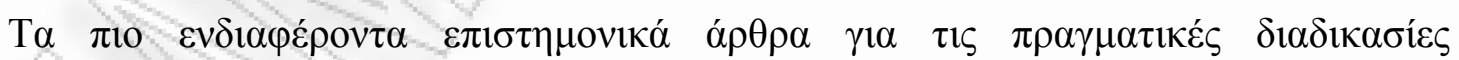

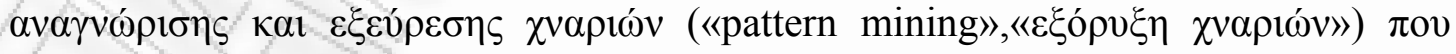

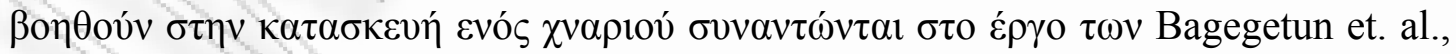

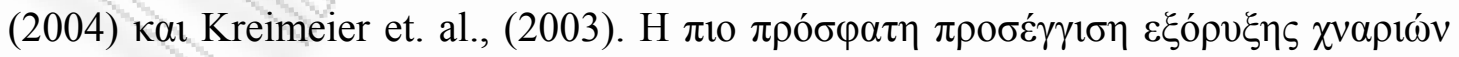

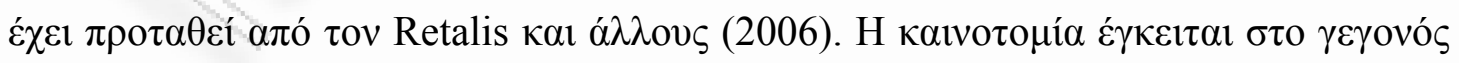

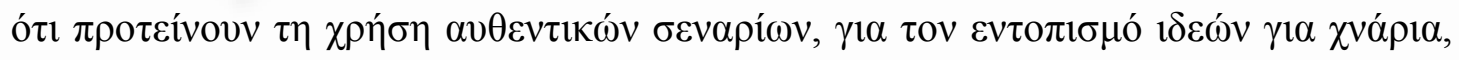

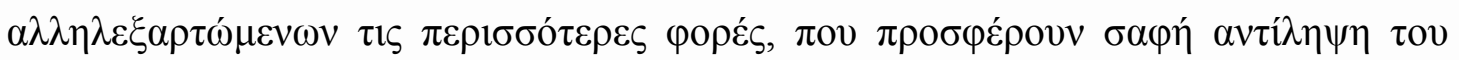
$\sigma \chi \varepsilon \delta i ́ o v$. 


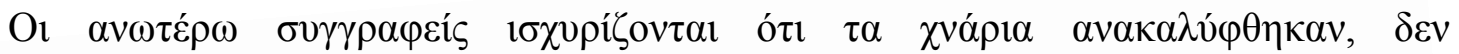

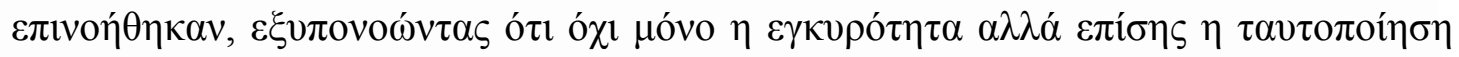

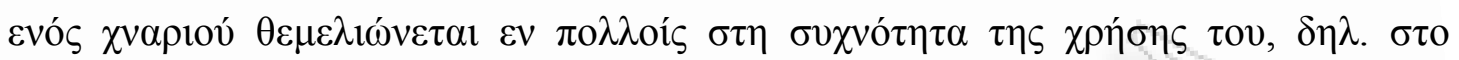

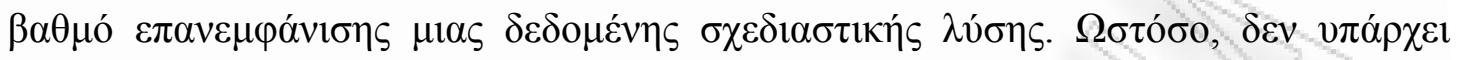

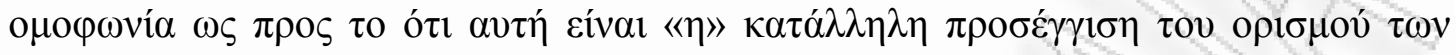

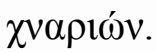

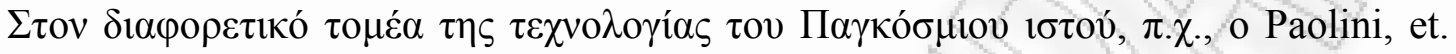

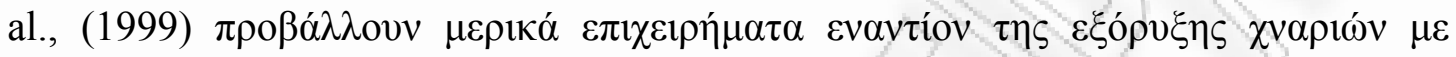

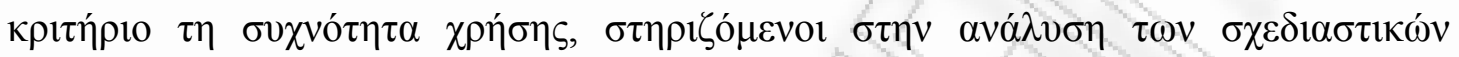

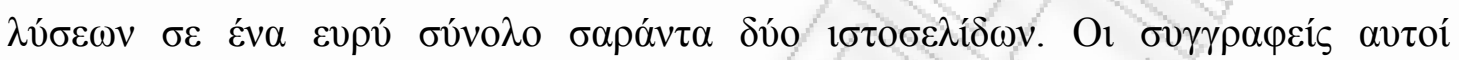

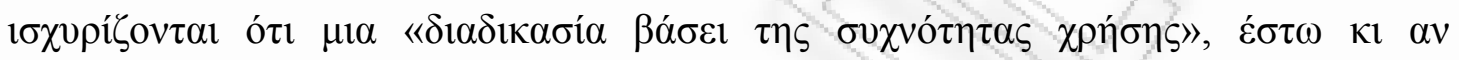

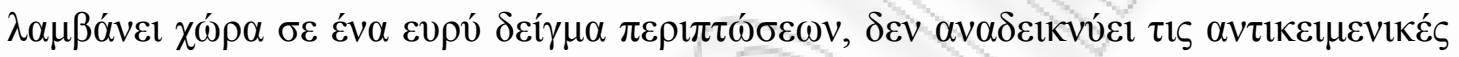

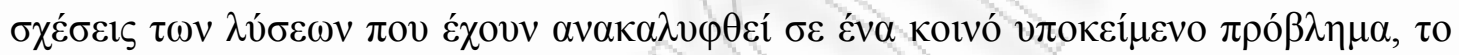

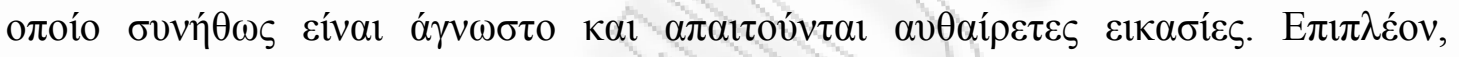

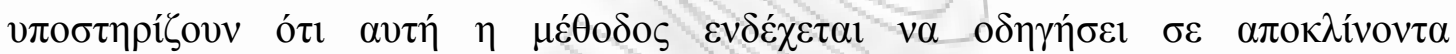

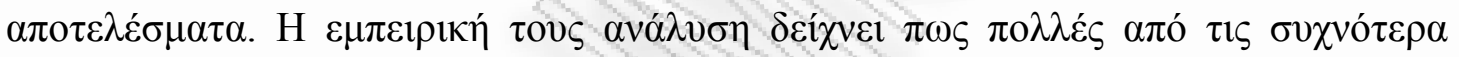

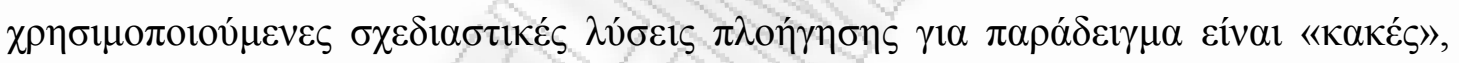

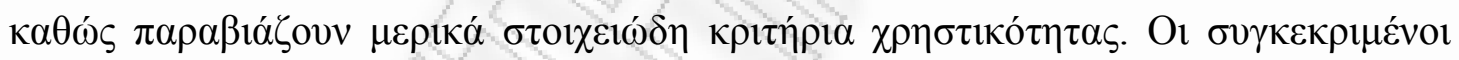

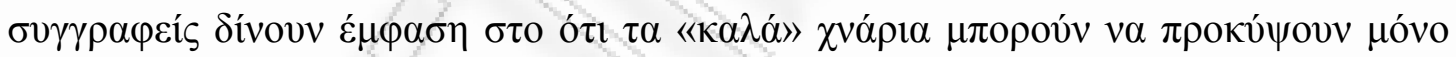

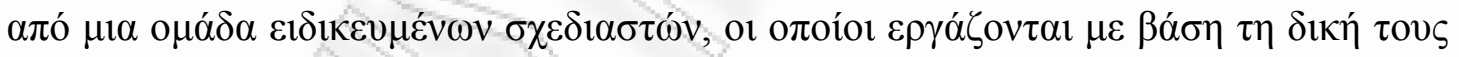

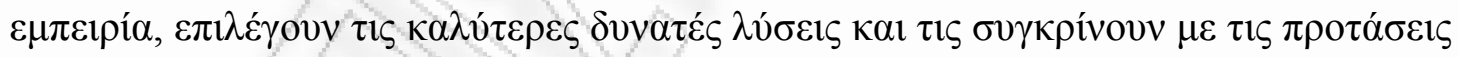

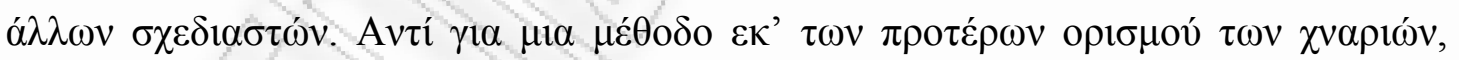

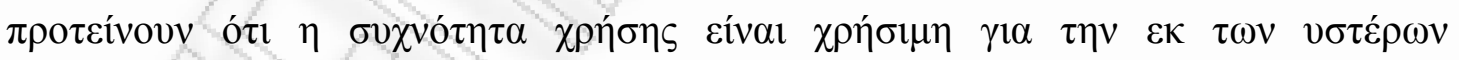

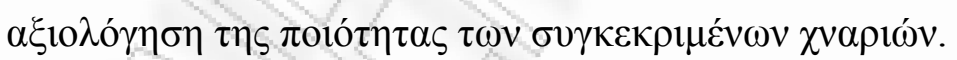

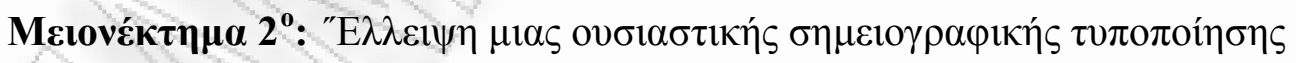

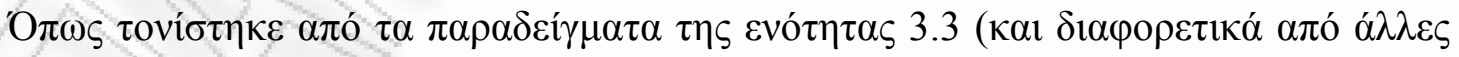

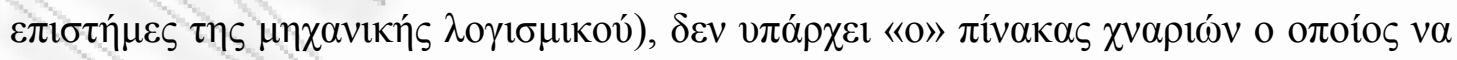

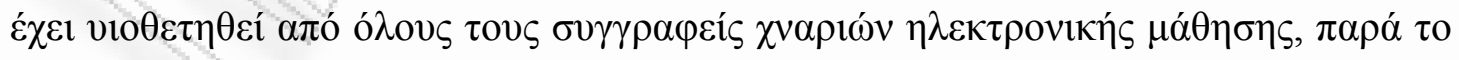

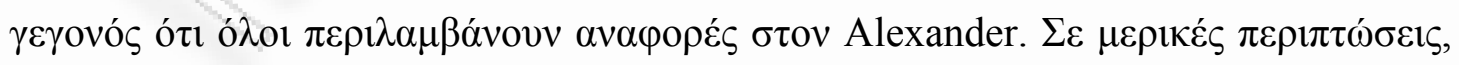

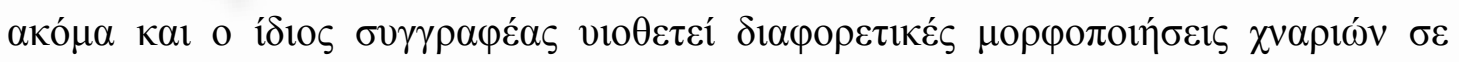

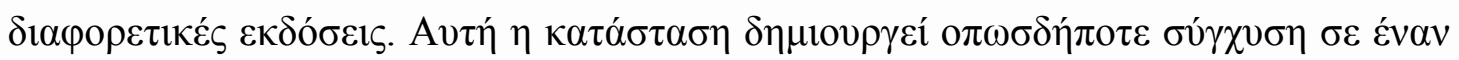

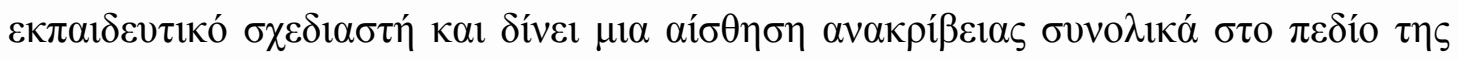

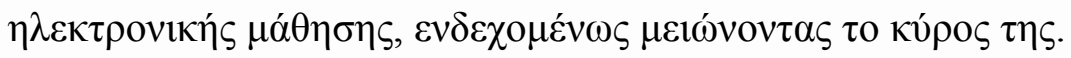




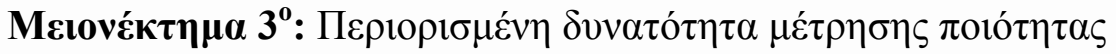

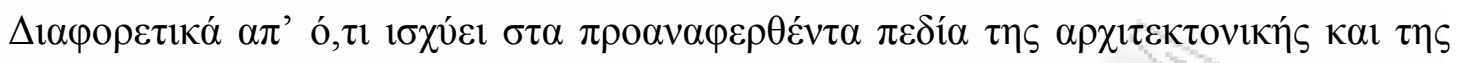

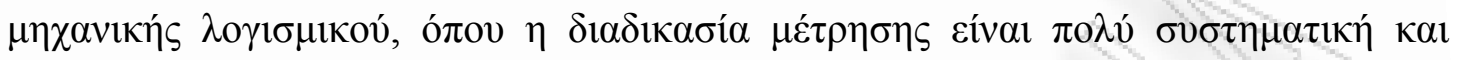

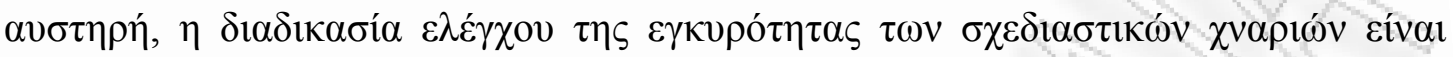

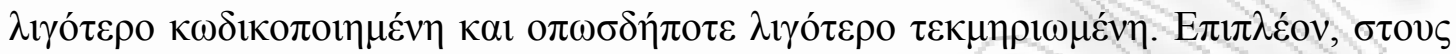

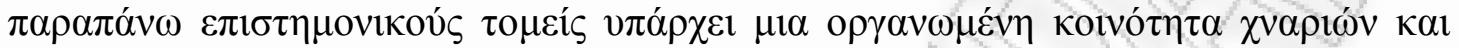

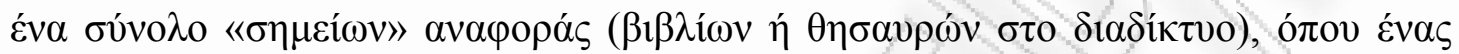

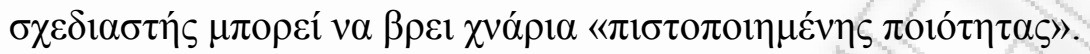

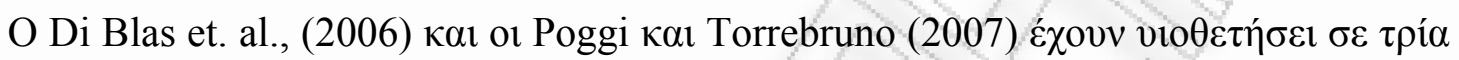

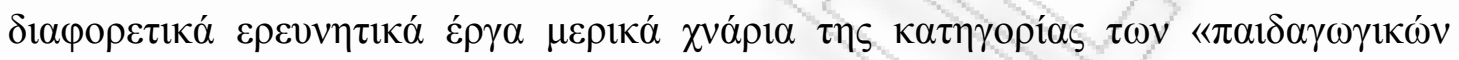

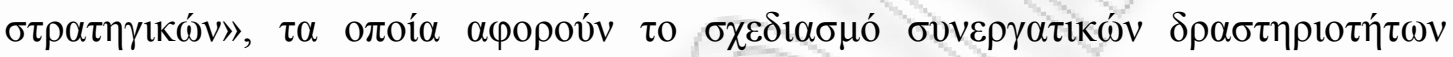

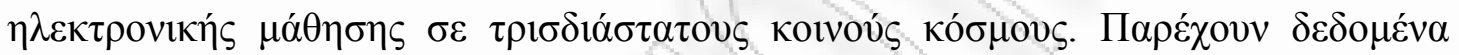

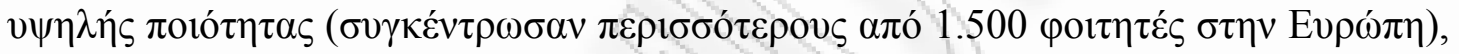

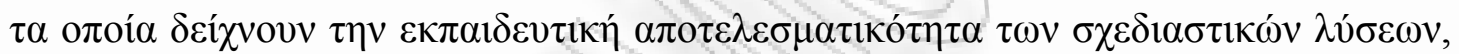

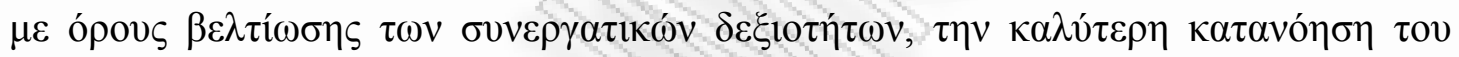

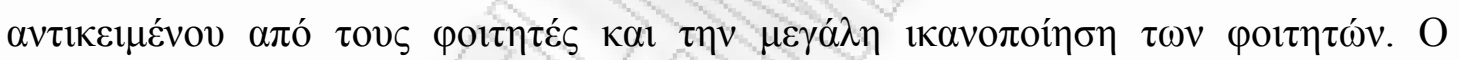

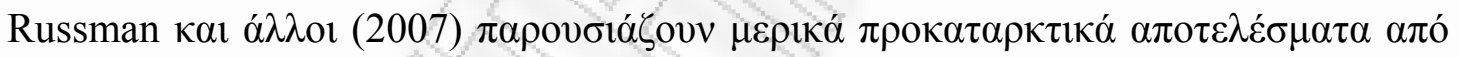

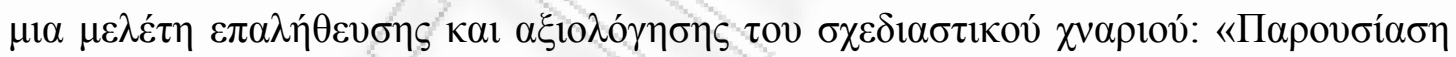

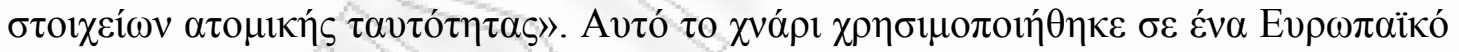

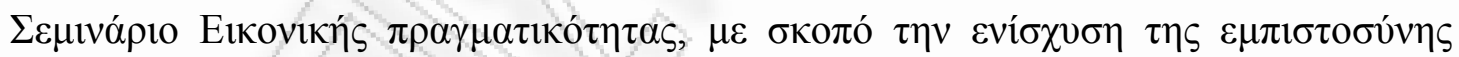

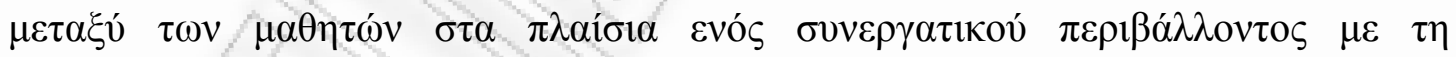

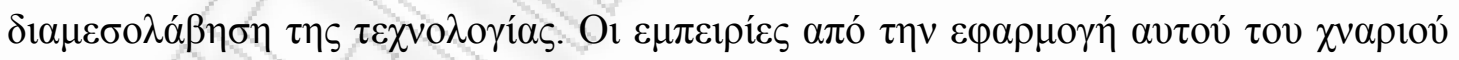

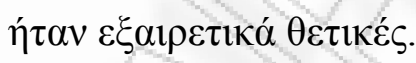

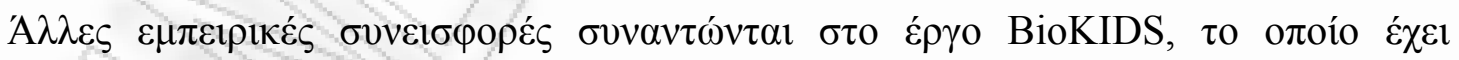

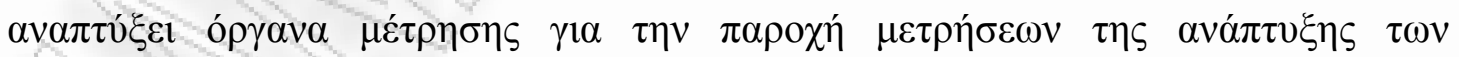

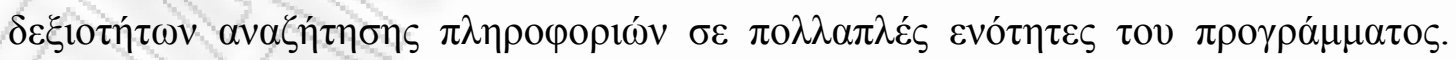

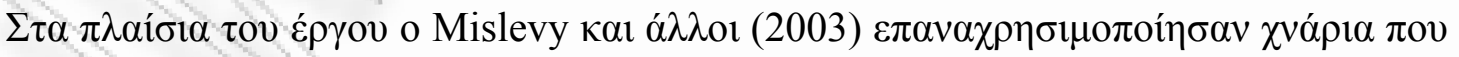

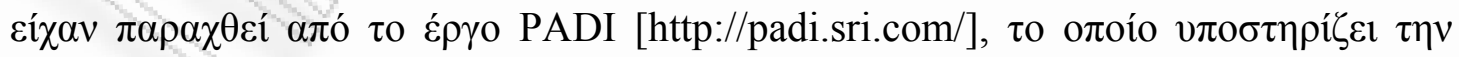
$\alpha \pi \circ \delta \varepsilon \delta \varepsilon \imath \gamma \mu \varepsilon \dot{v} \eta \mu \varepsilon \lambda \varepsilon \dot{\tau} \eta \eta$

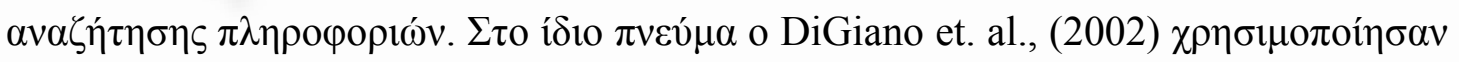

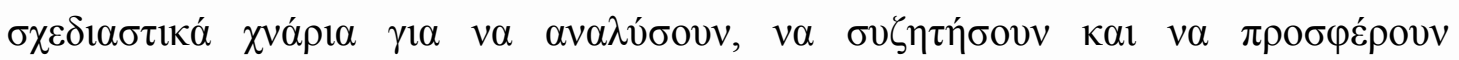

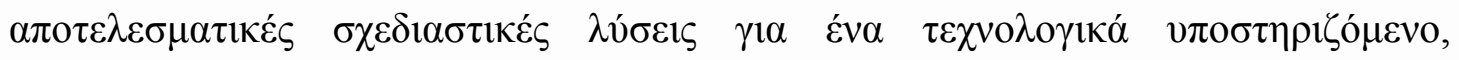

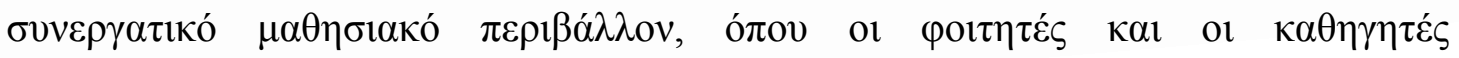




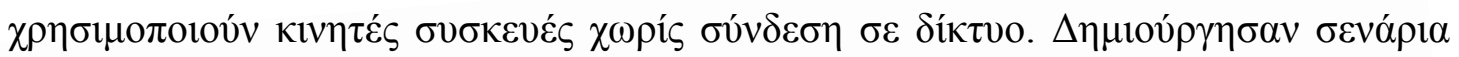

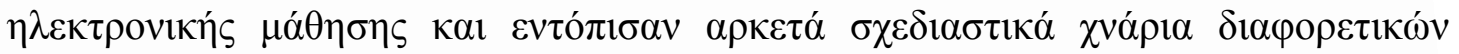

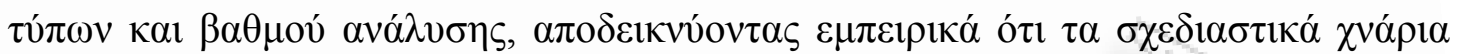

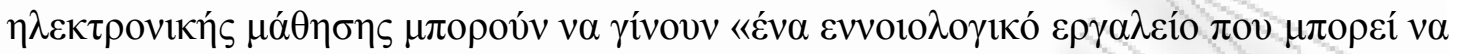

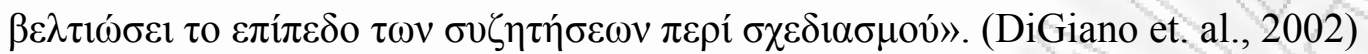

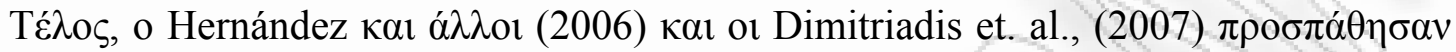

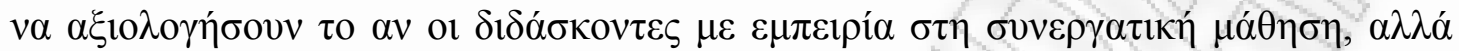

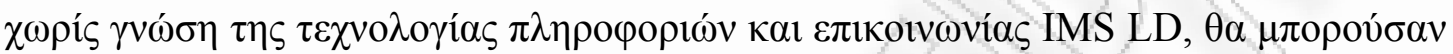

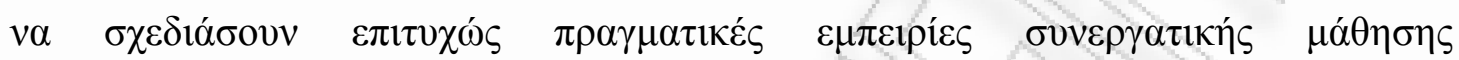

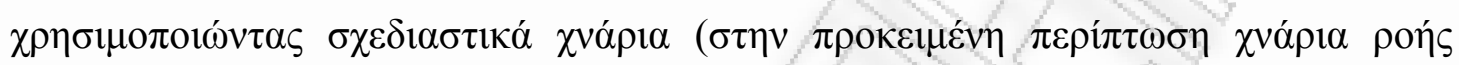

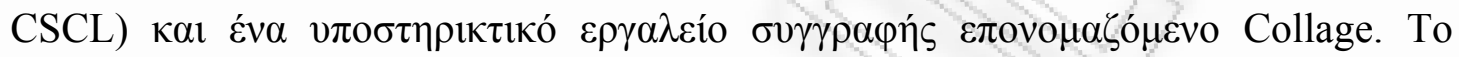

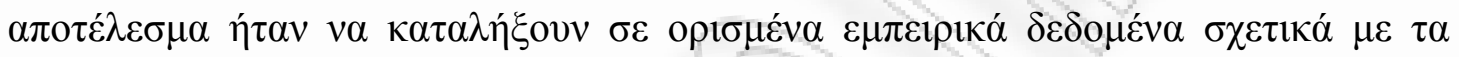

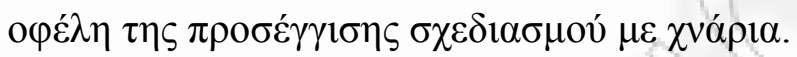

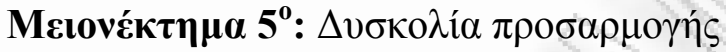

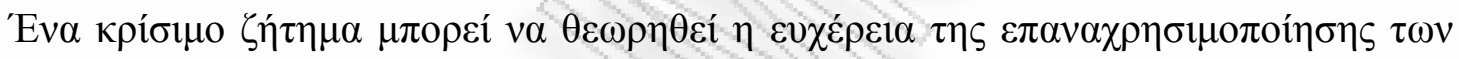

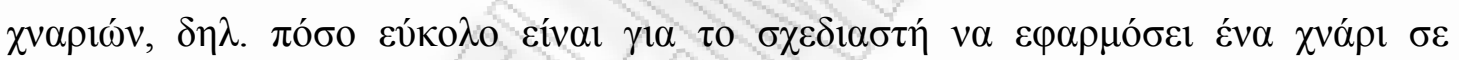

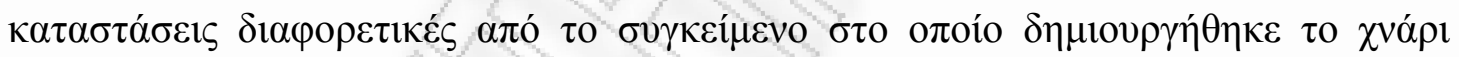

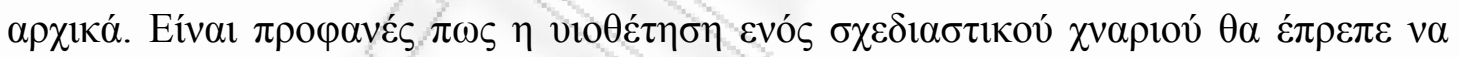

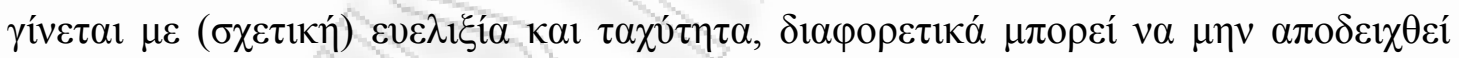

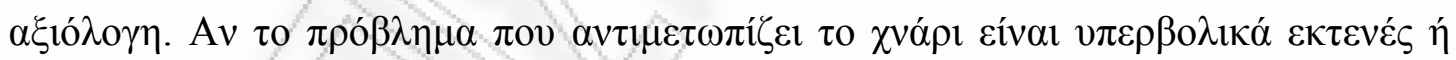

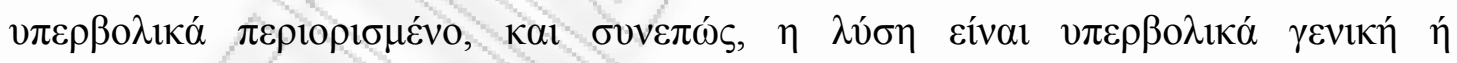

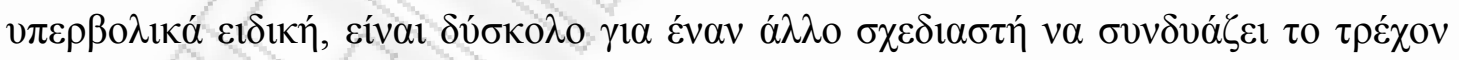

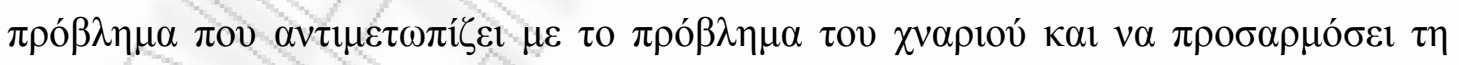

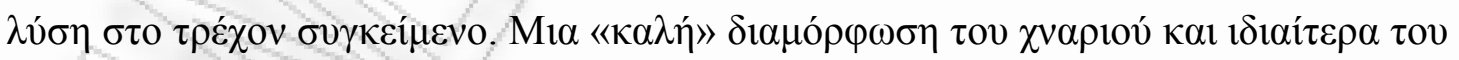

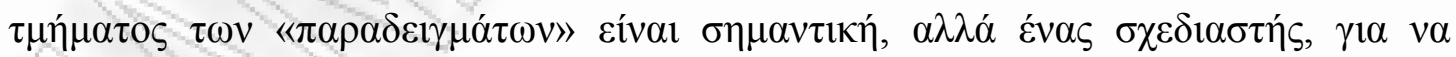

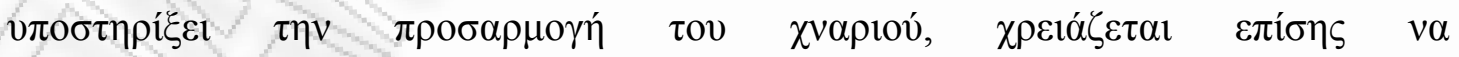

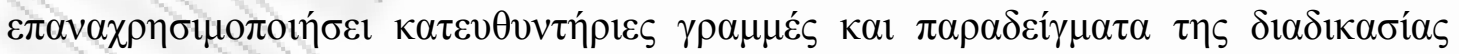

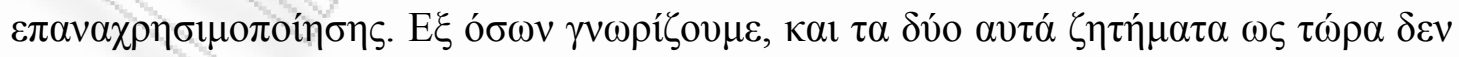

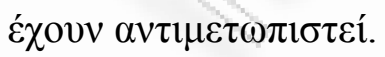

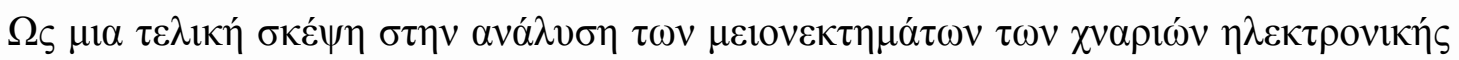

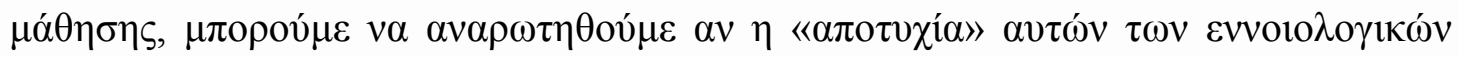

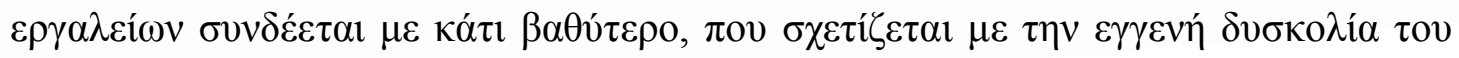




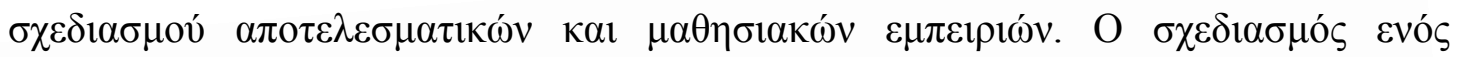

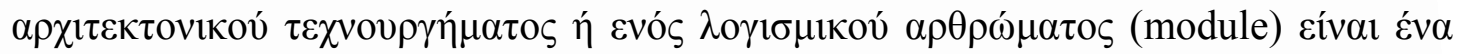

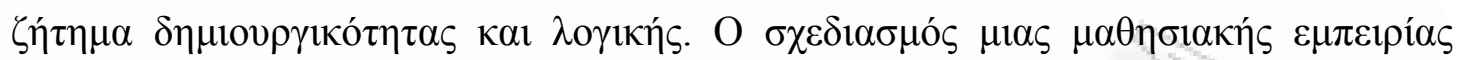

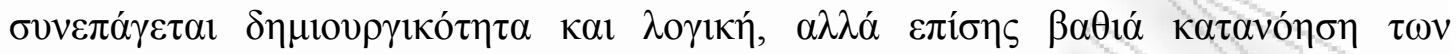

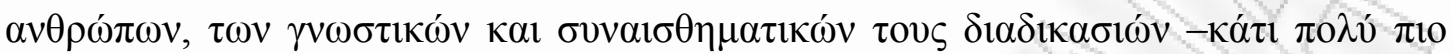

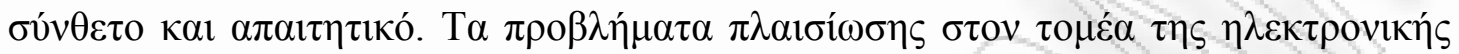

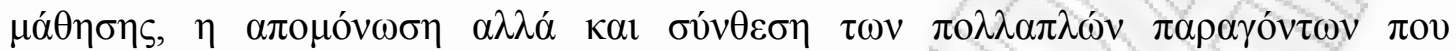

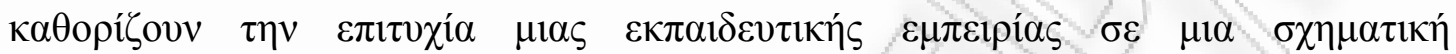

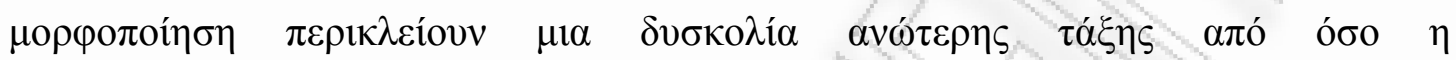

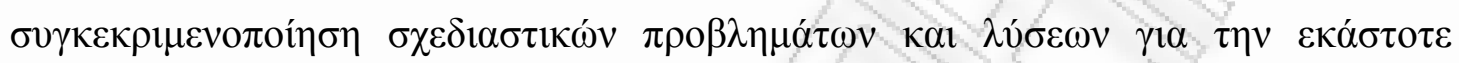

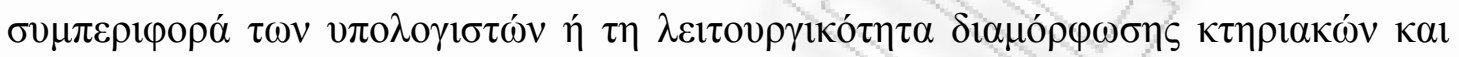

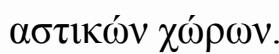

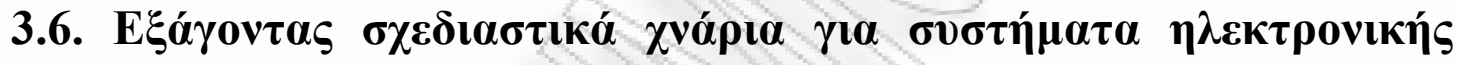 $\mu \alpha ́ \theta \eta \sigma \eta \varsigma$}

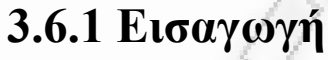

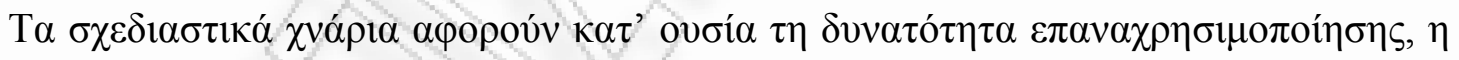

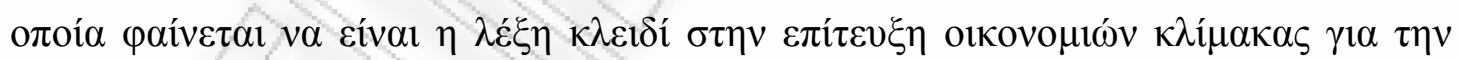

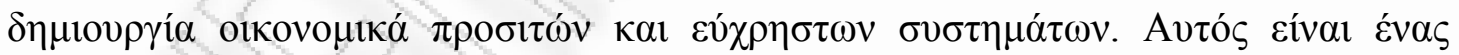

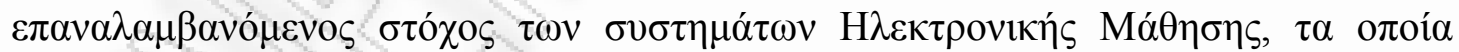

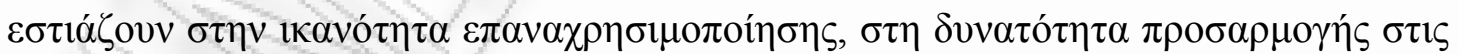

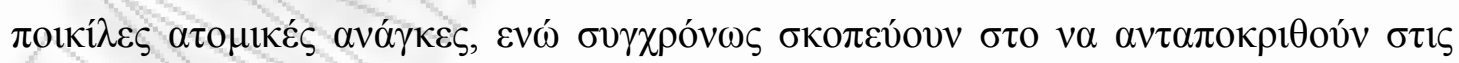

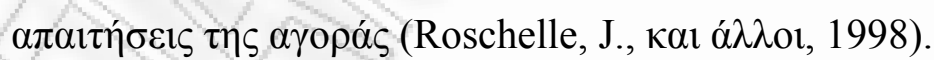

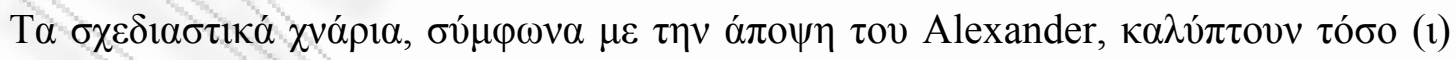

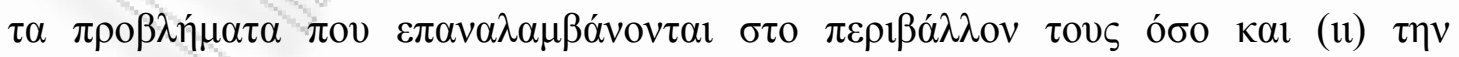

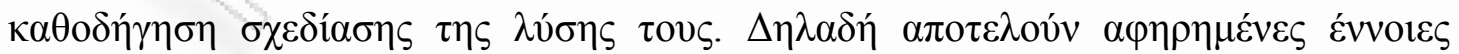

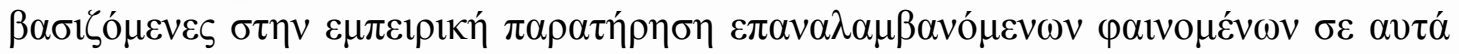

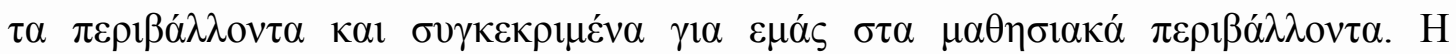




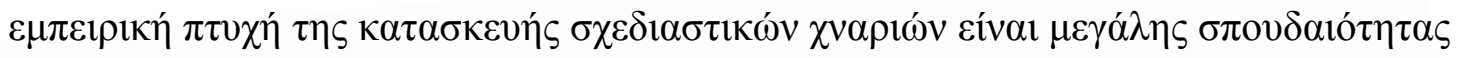

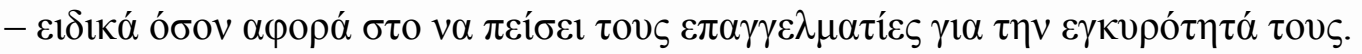

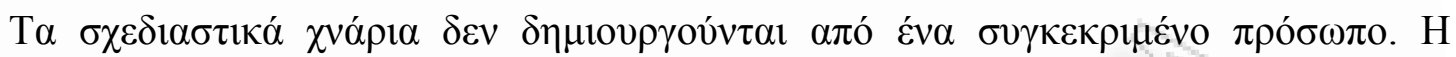

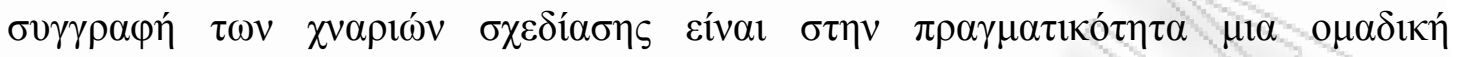

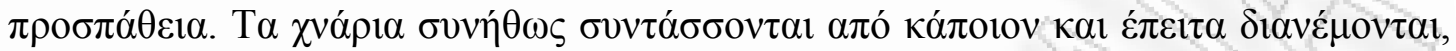

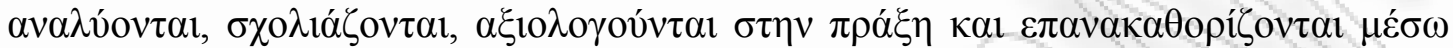

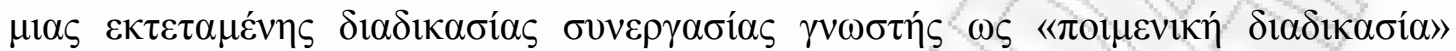
(shepherding) (Mezaros \& Doble, 1998).

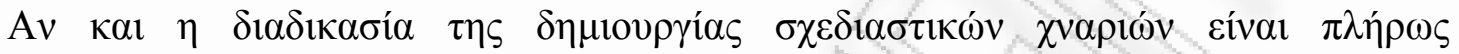

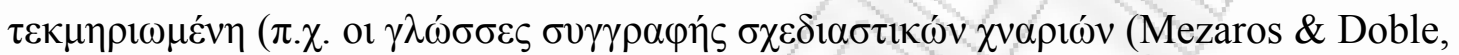

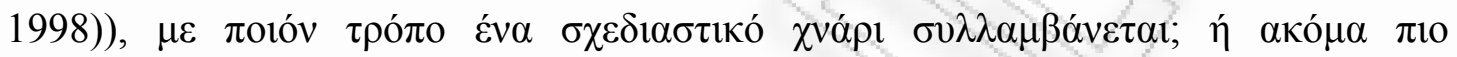

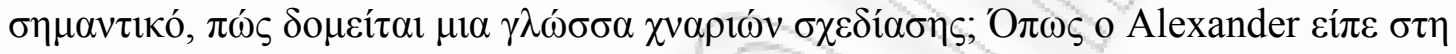

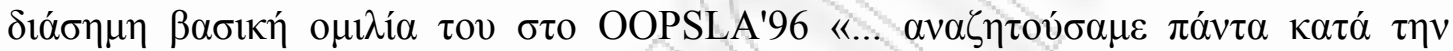

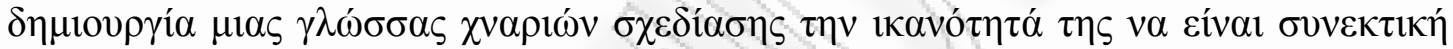

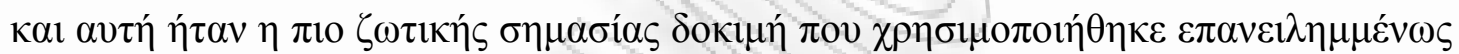

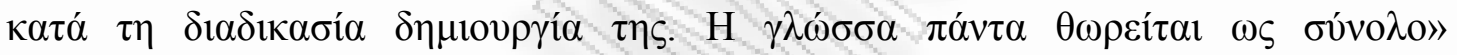

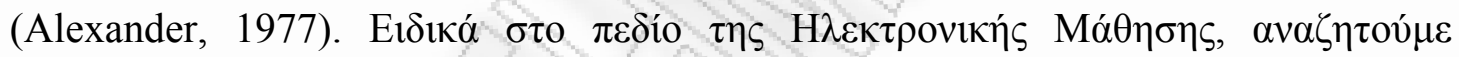

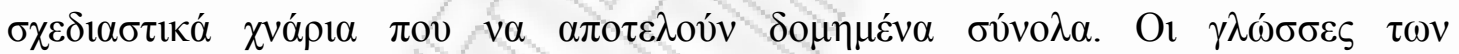

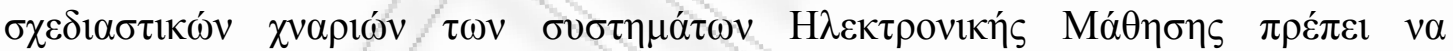

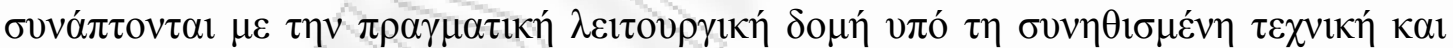

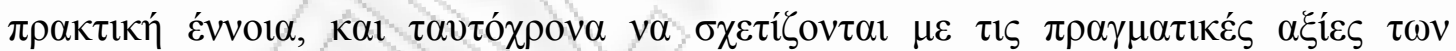

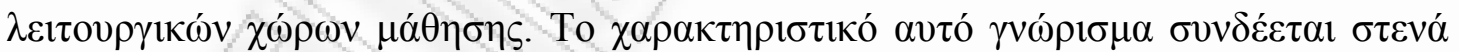

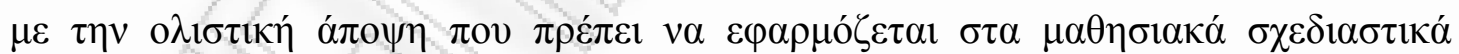
$\chi v \alpha ́ \rho 1 \alpha$.

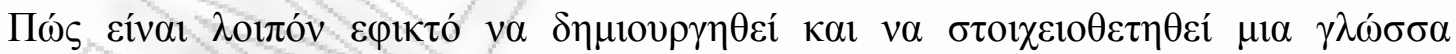

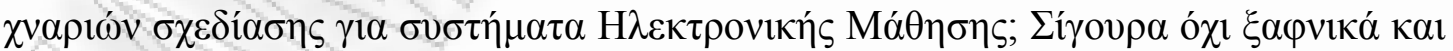

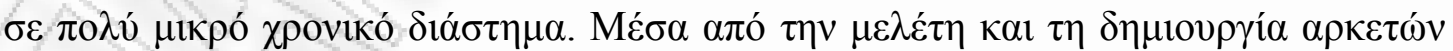

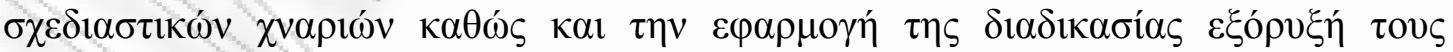

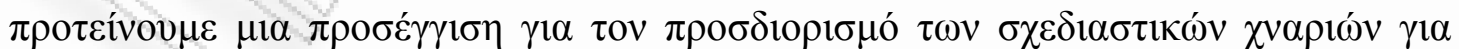

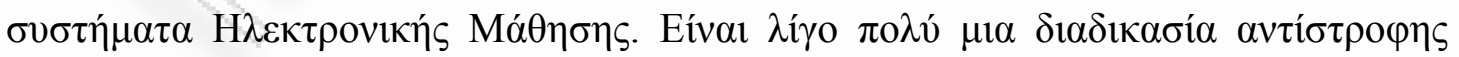

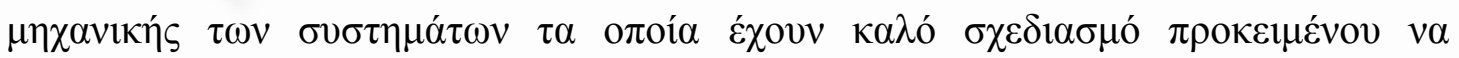

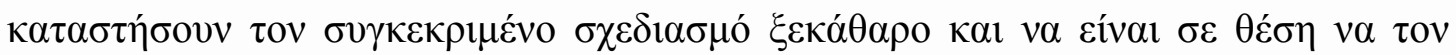

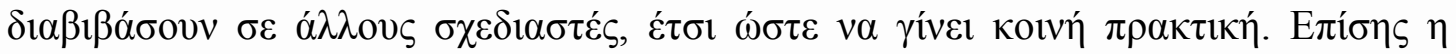

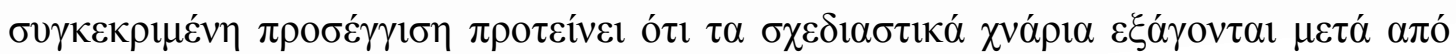




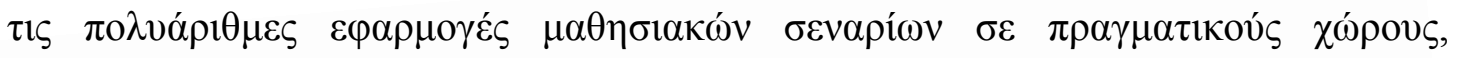

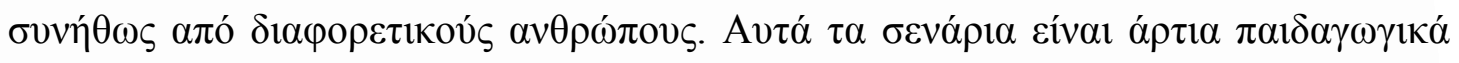

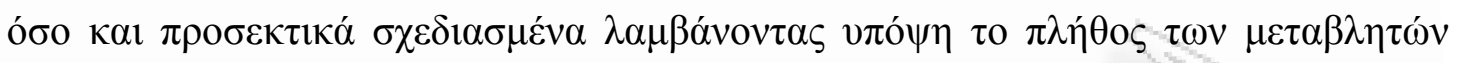

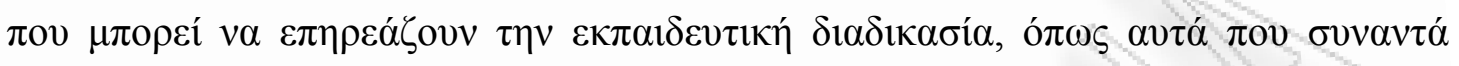

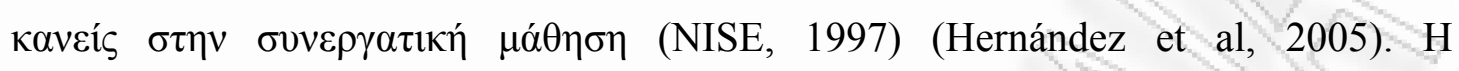

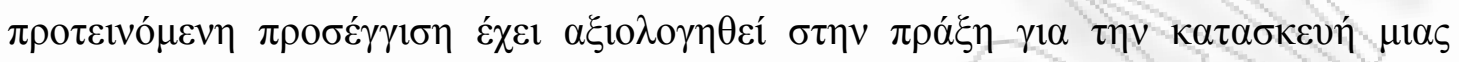

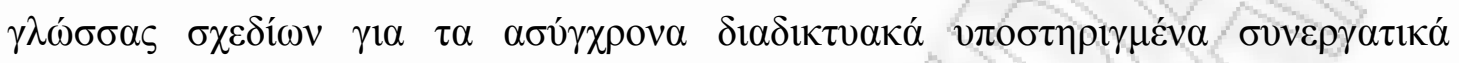

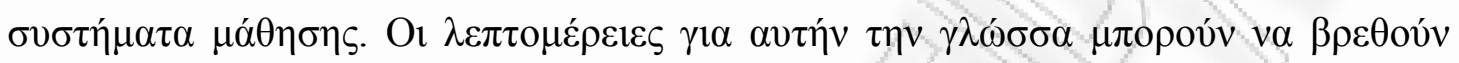

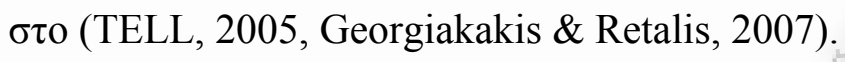

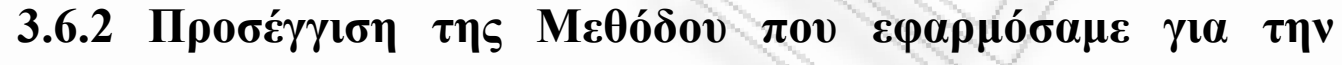

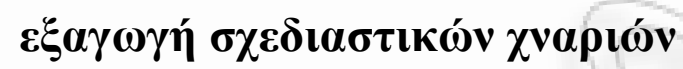

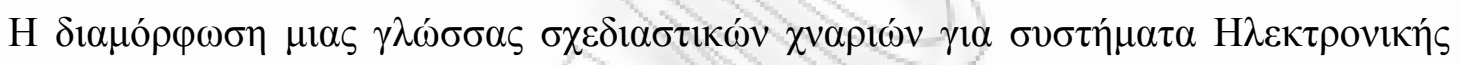

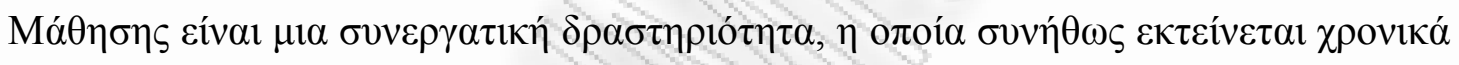

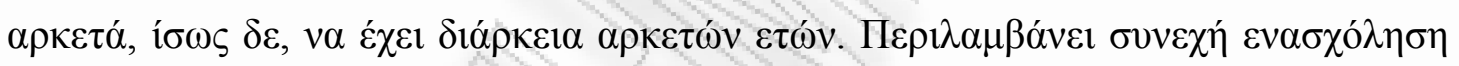

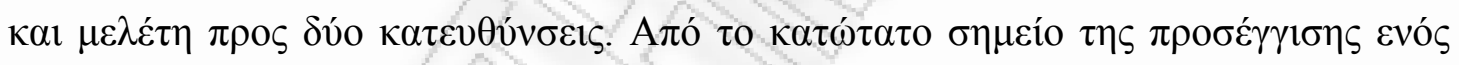

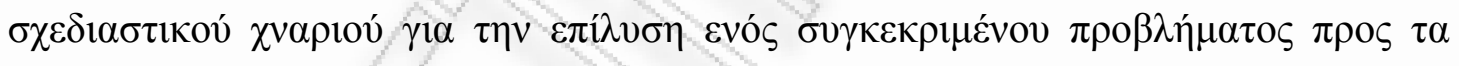

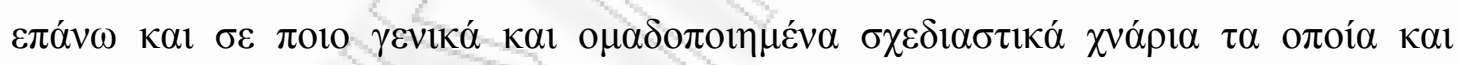

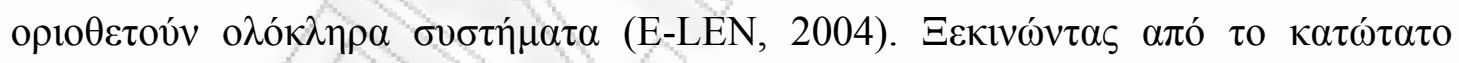

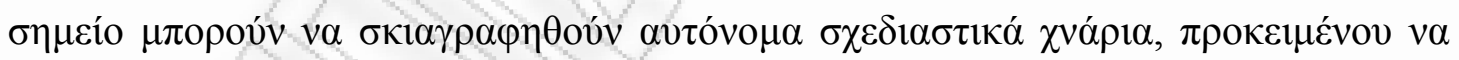

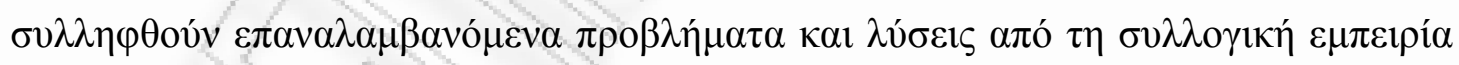

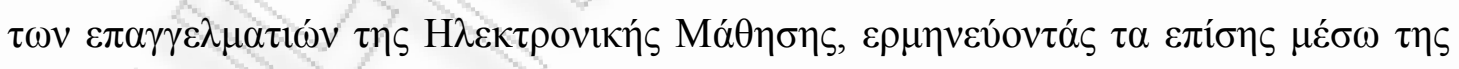

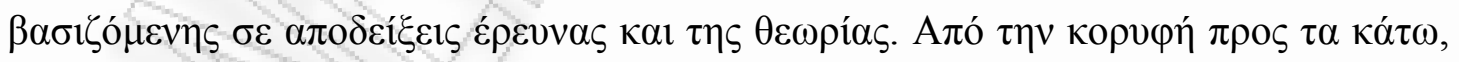

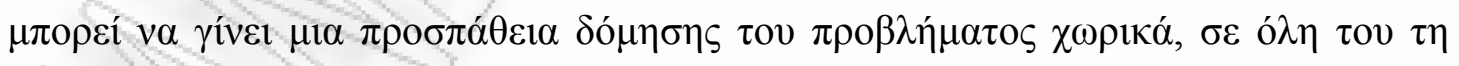

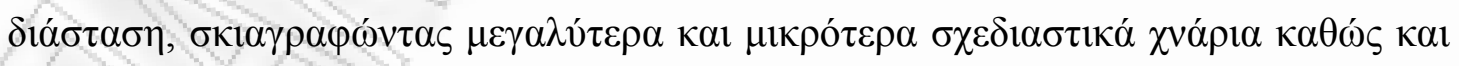

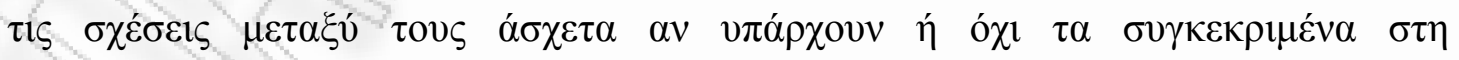
$\beta 1 \beta \lambda$ io $\rho \alpha \varphi i ́ \alpha$.

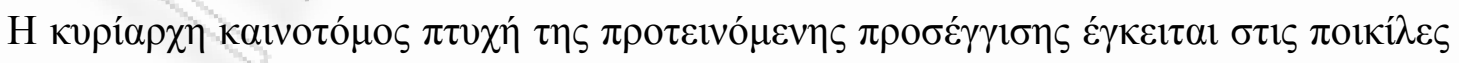

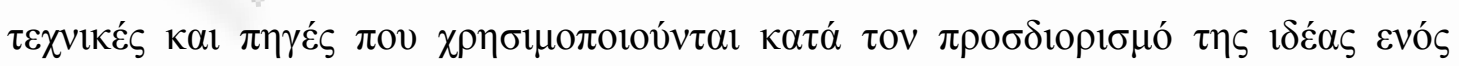

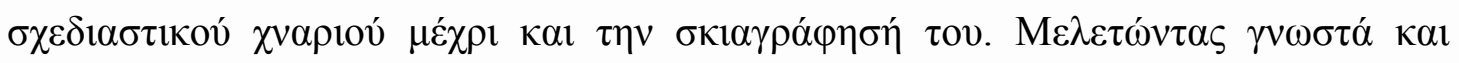

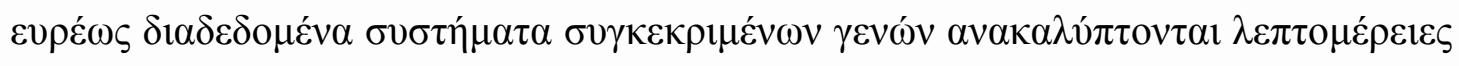

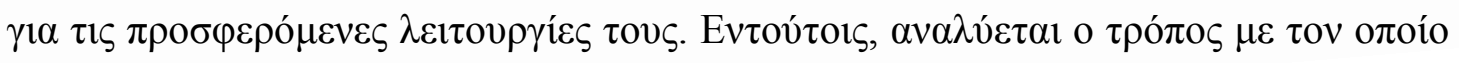




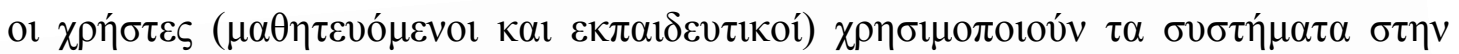

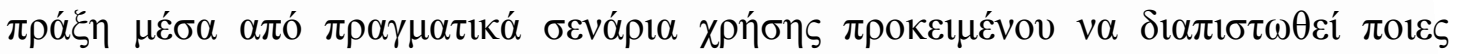

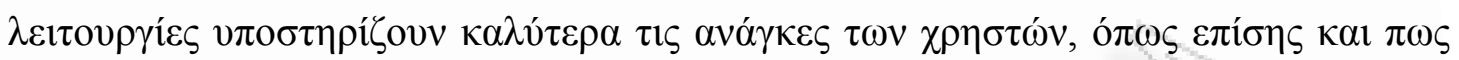

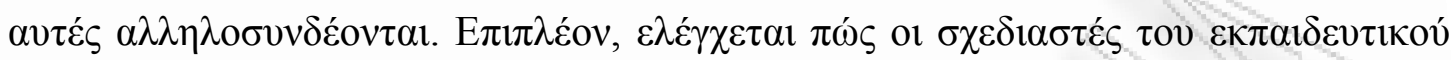

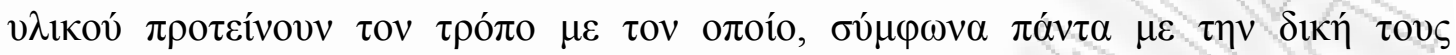

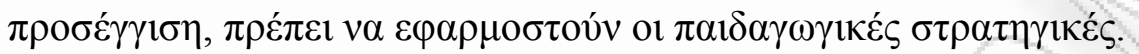

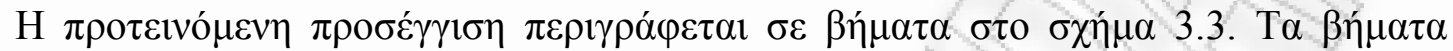
Eíval $\tau \alpha \alpha \kappa o ́ \lambda o v \theta \alpha$ :

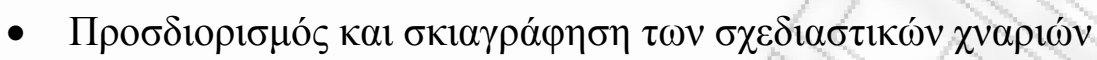

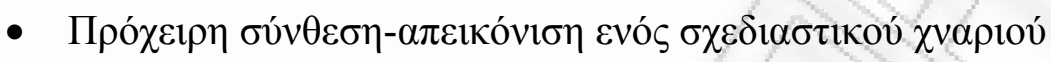

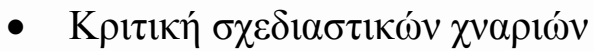

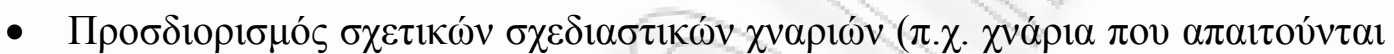

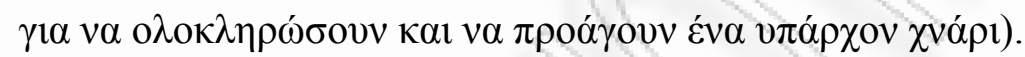

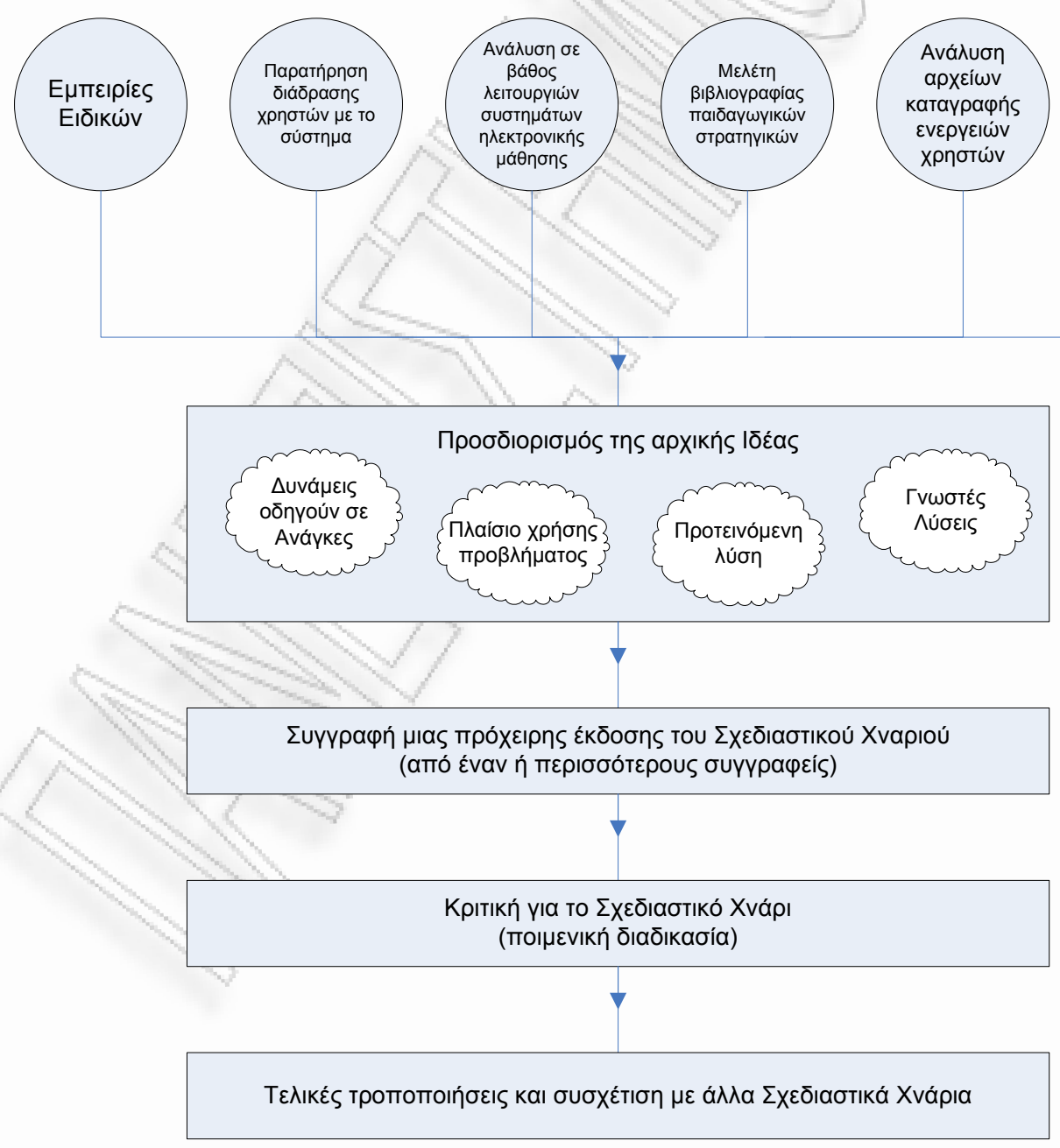

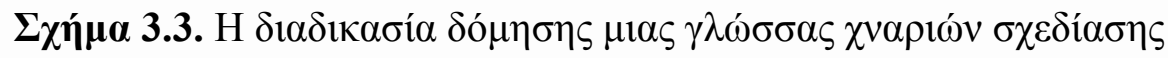




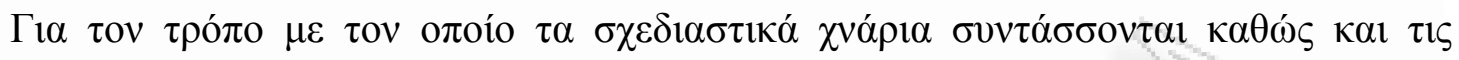

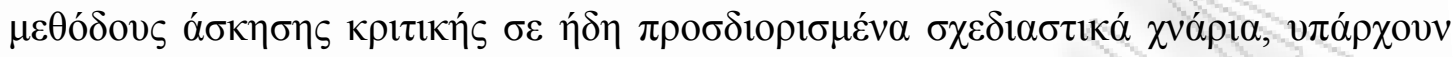

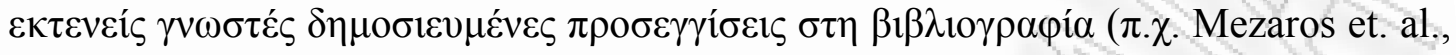
1998).

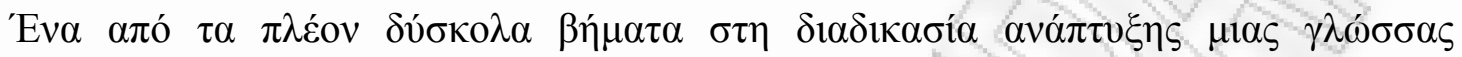

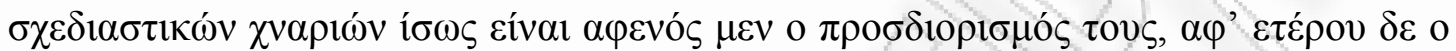

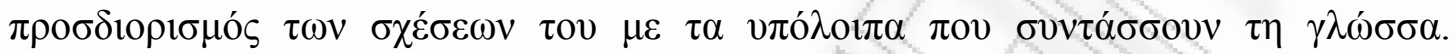

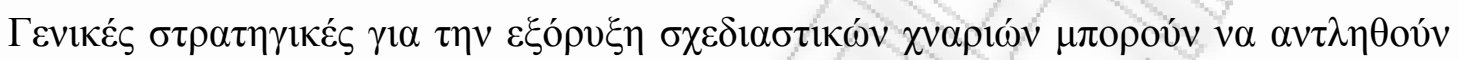
$\alpha \pi$ á $^{-1}$

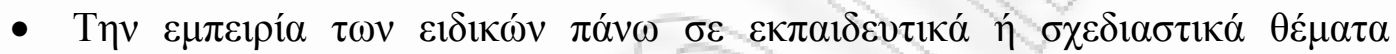

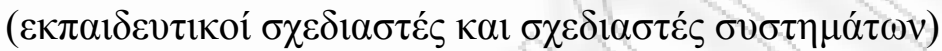

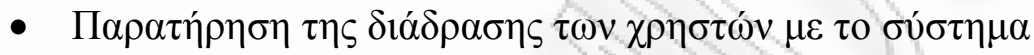

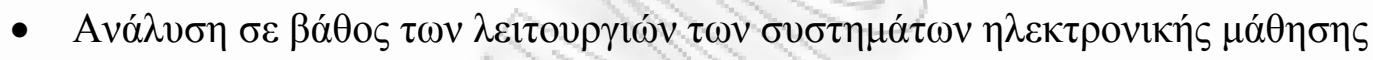

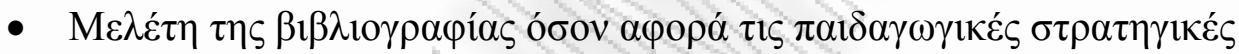

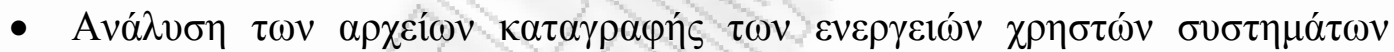

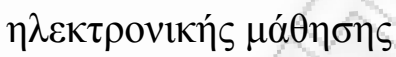

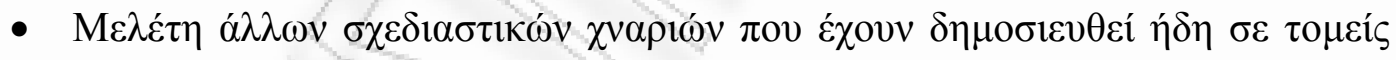

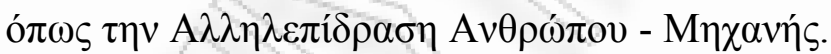

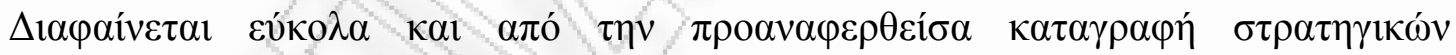

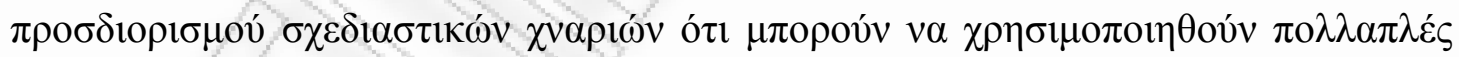

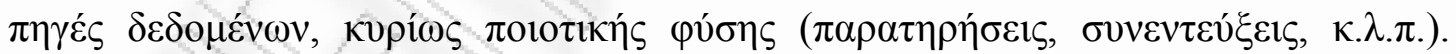

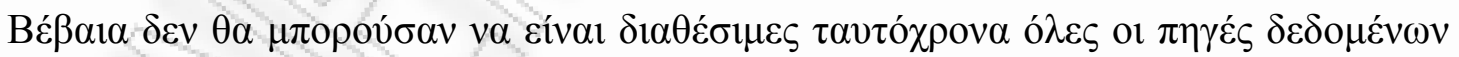

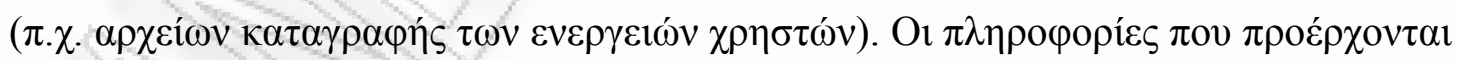

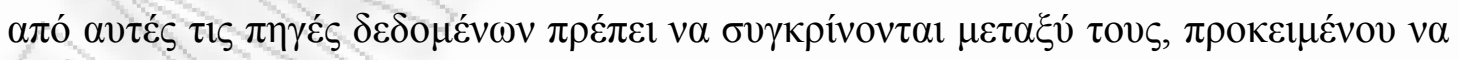

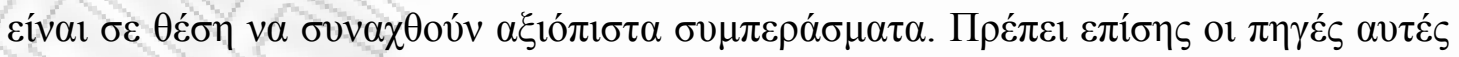

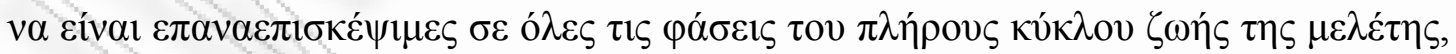

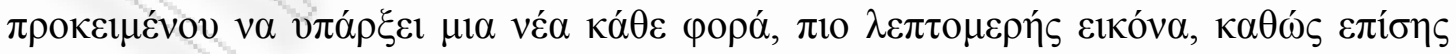

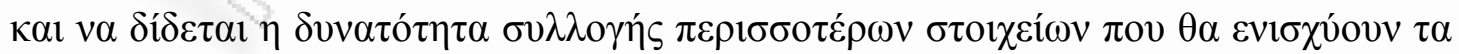

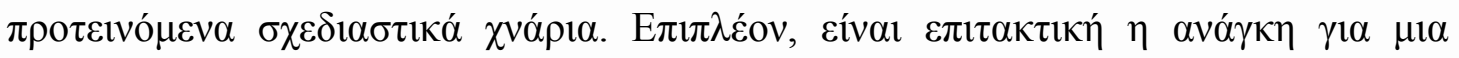

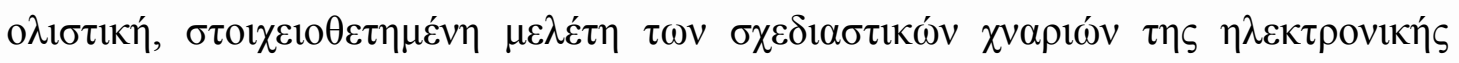




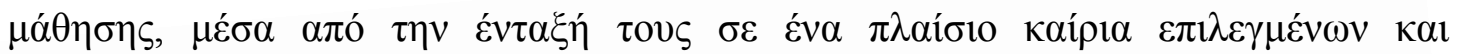

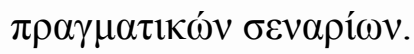

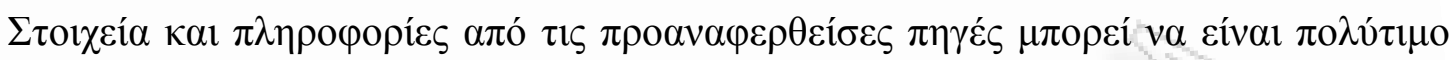

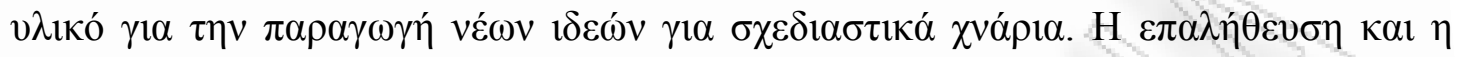

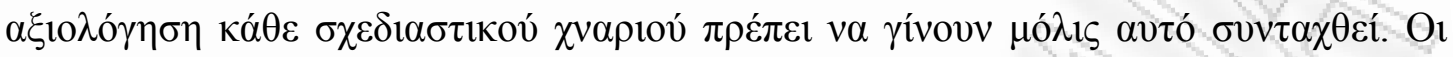

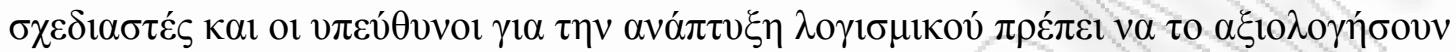

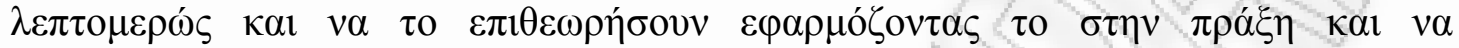

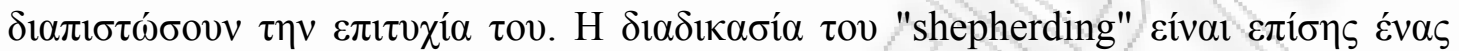

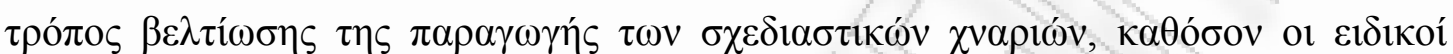

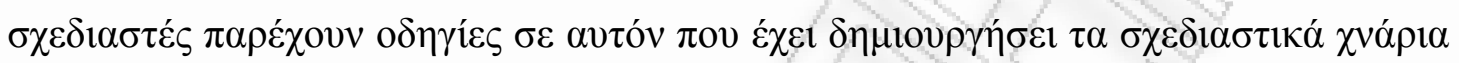

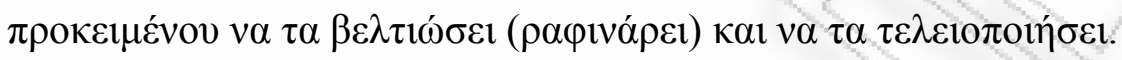

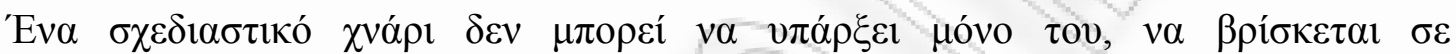

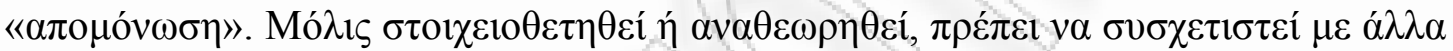

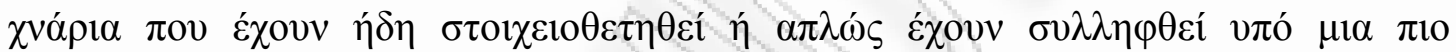

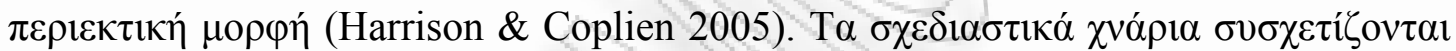

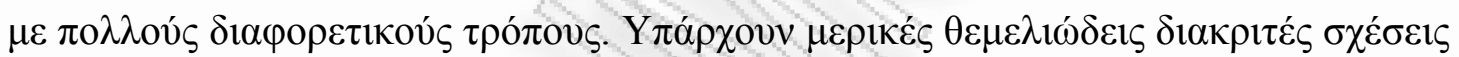
(van Welie, et. al., 2003):

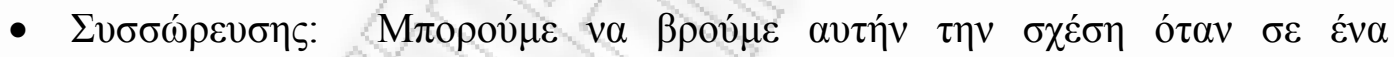

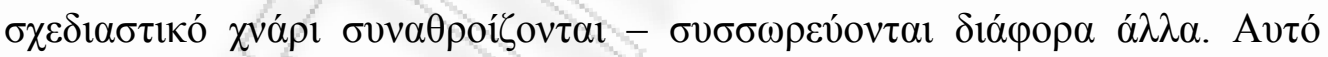

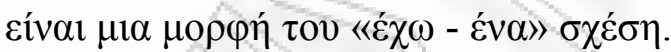

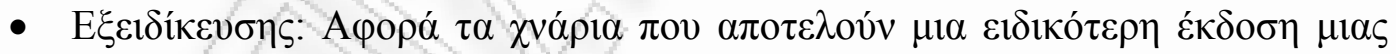

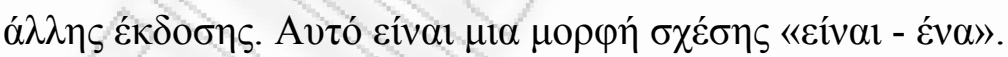

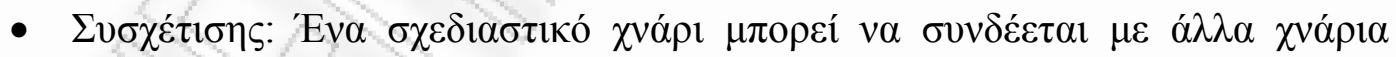

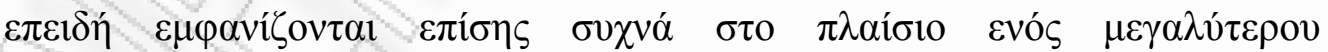

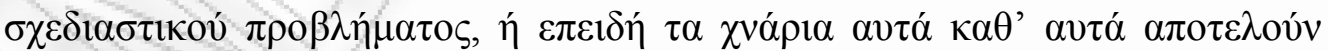

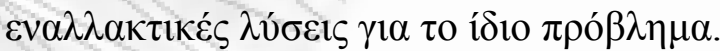

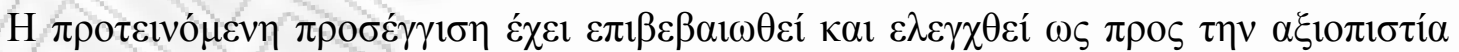

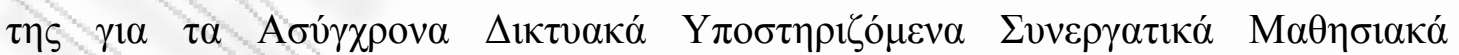
$\Sigma v \sigma \tau \eta ́ \mu \alpha \tau \alpha$ (Asynchronous Network Supported Collaborative Learning systems

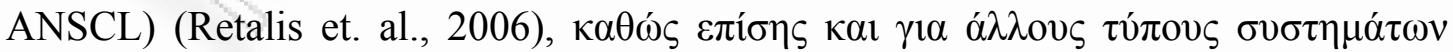

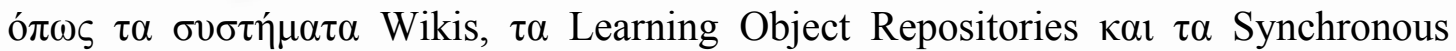

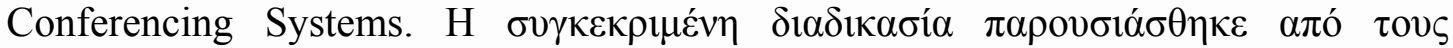

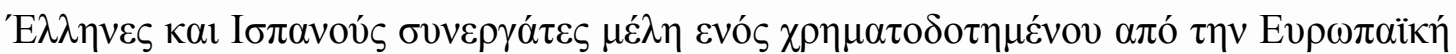




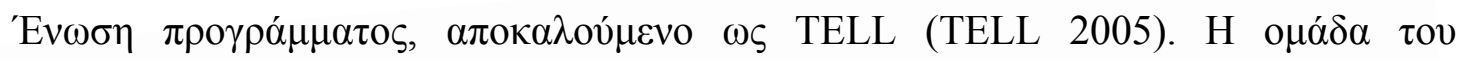

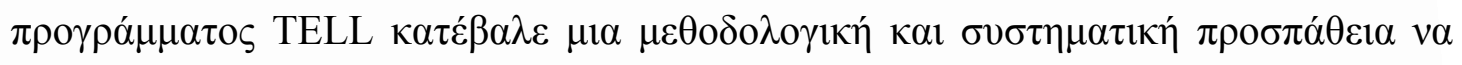

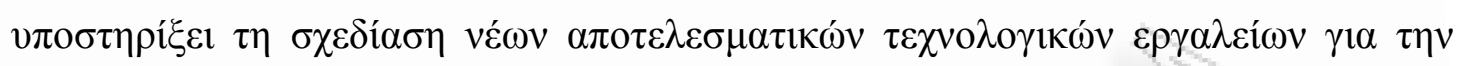

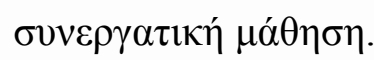

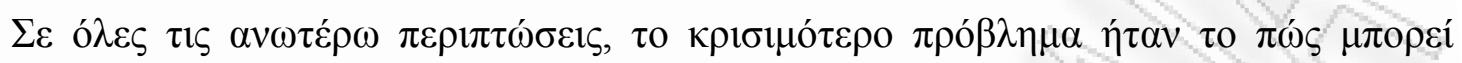

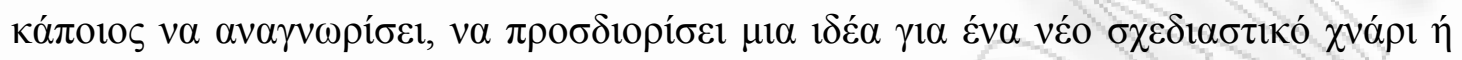

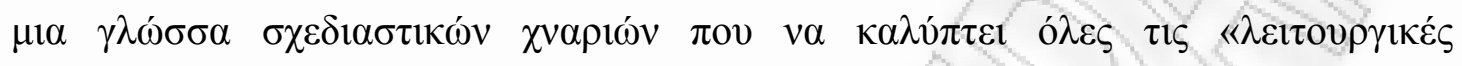

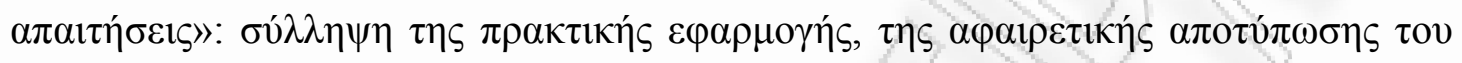

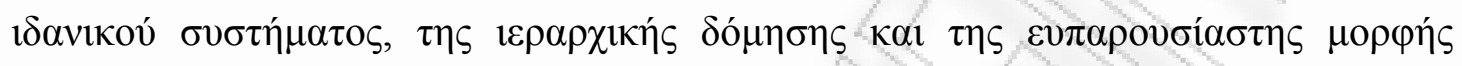
(Fincher \& Utting 2002);

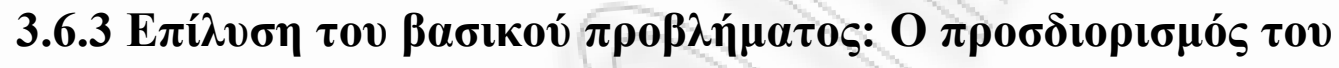

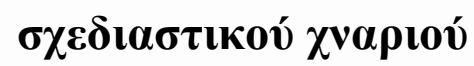

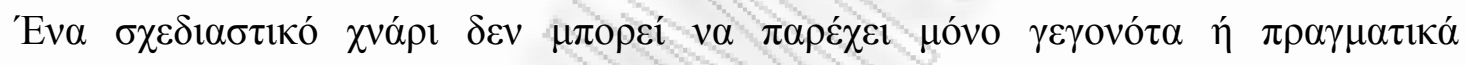

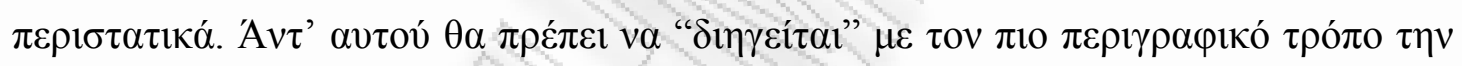

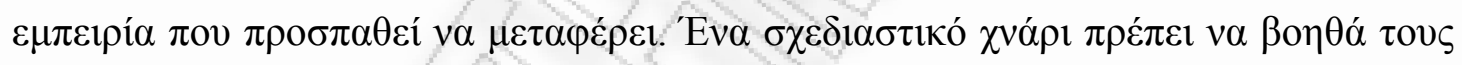
$\varepsilon \mu \pi \lambda \varepsilon \kappa o ́ \mu \varepsilon v o v \varsigma \mu \varepsilon \alpha v \tau o ́$ va:

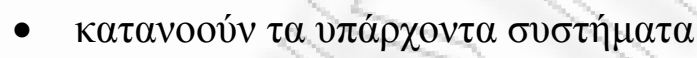

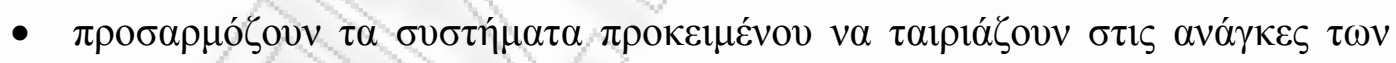
$\chi \rho \eta \sigma \tau \omega \dot{v}$

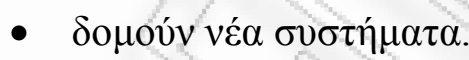

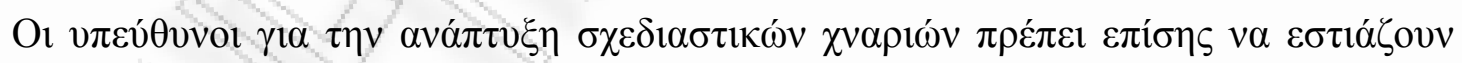

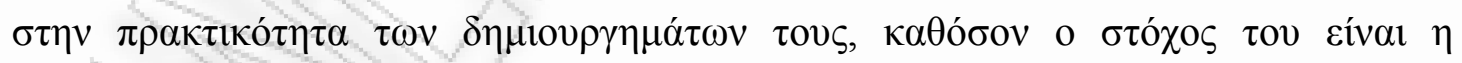

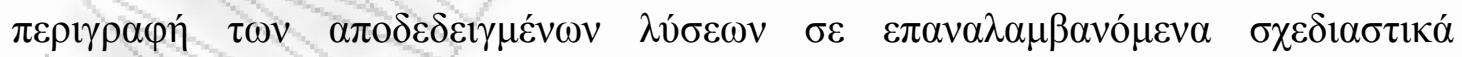
$\pi \rho \circ \beta \lambda \eta \dot{\mu} \alpha \alpha$.

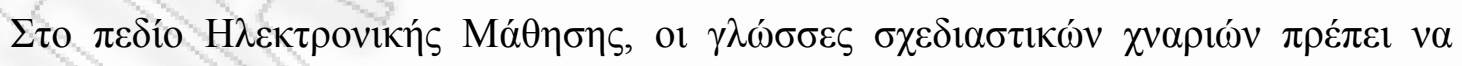

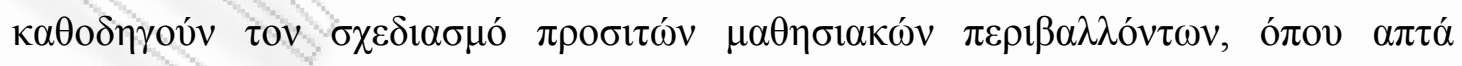

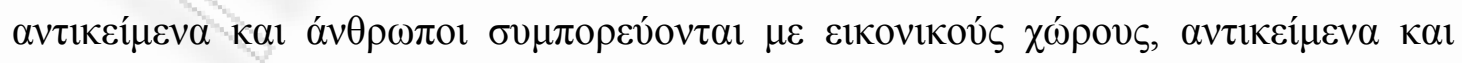

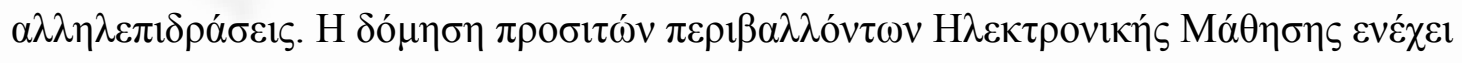

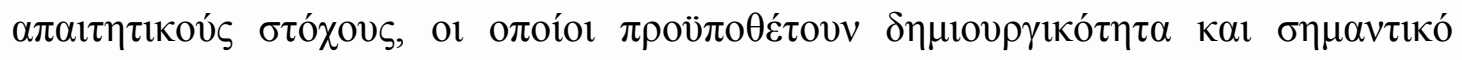

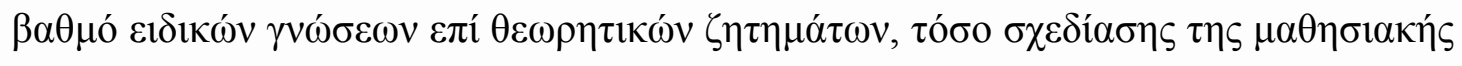

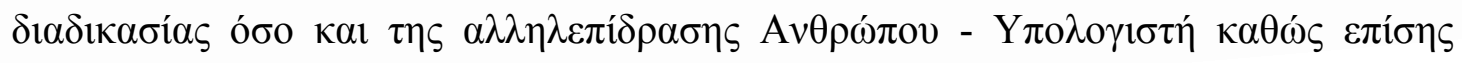




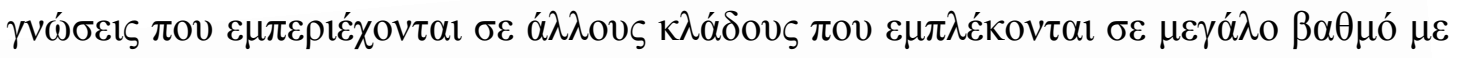

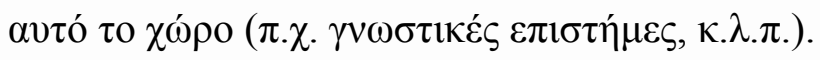

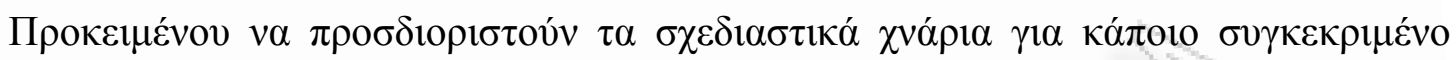

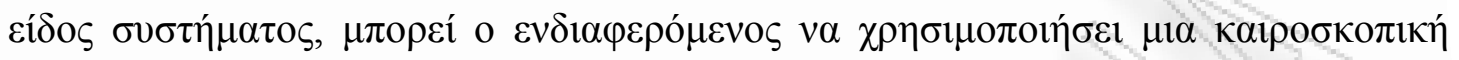

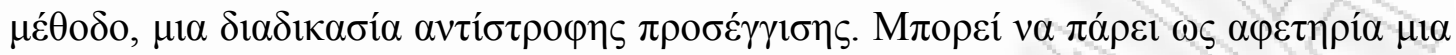

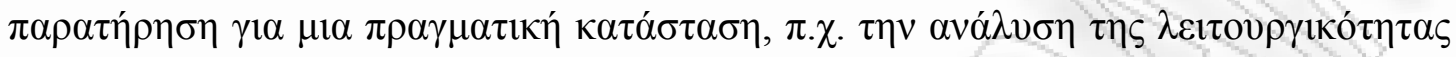

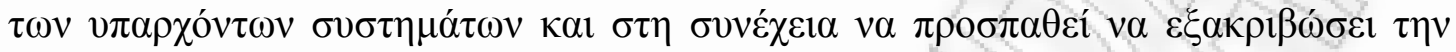

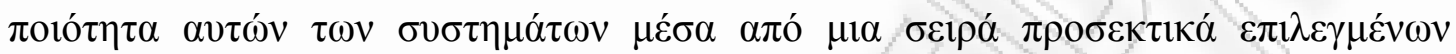

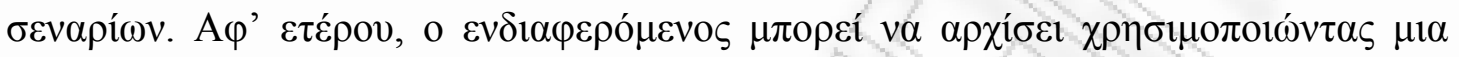

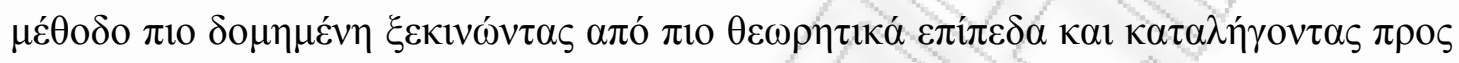

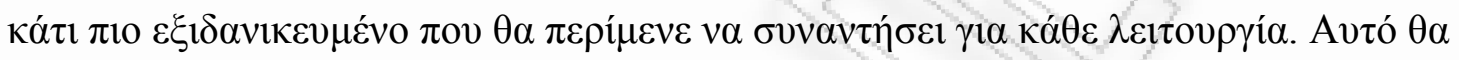

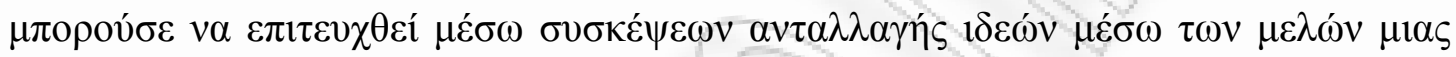

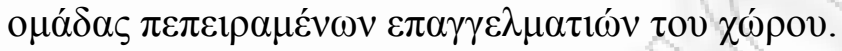

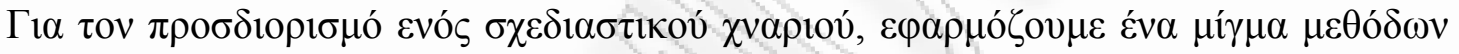

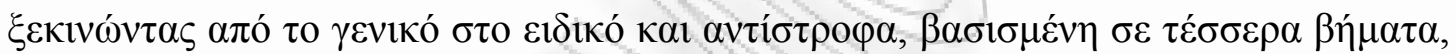

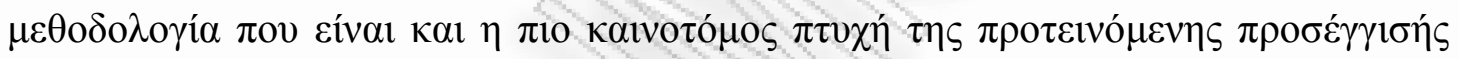
$\mu \alpha \varsigma:$

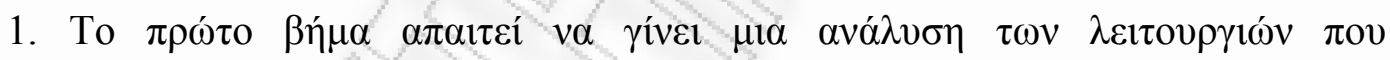

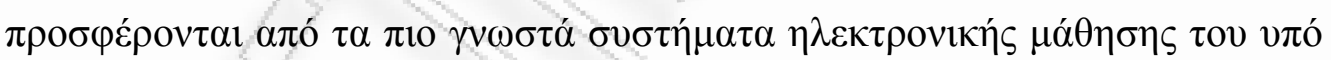

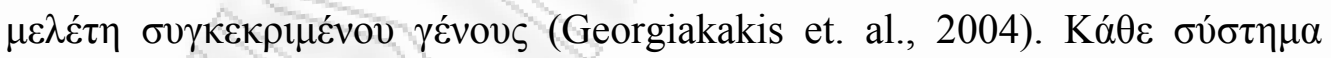

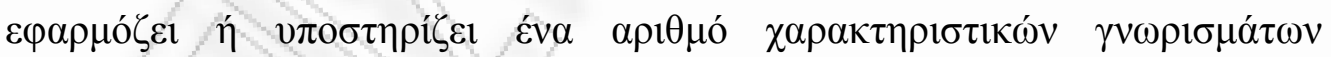

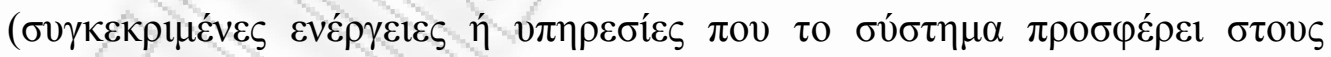

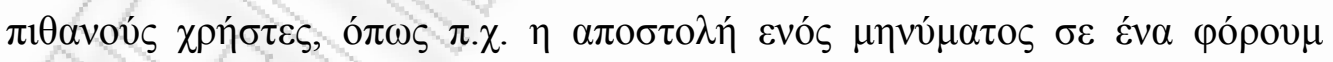

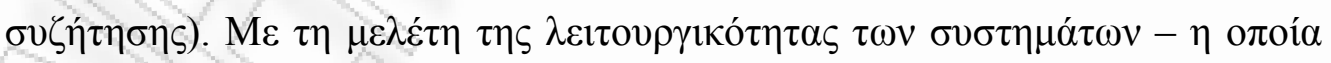

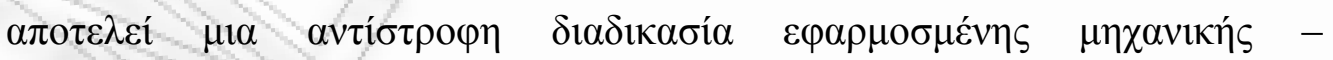

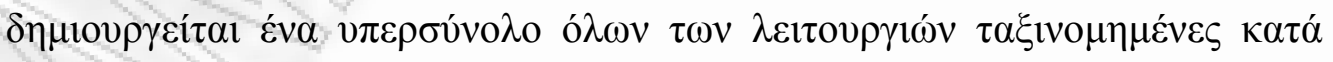

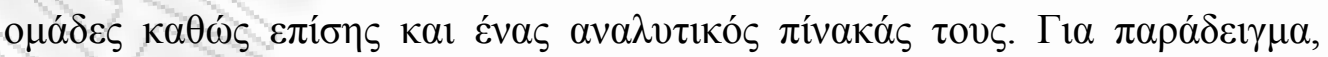

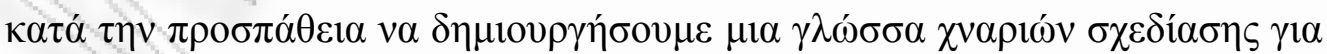

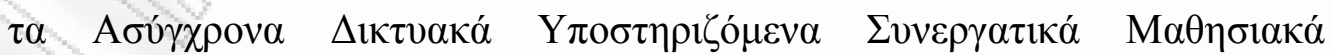

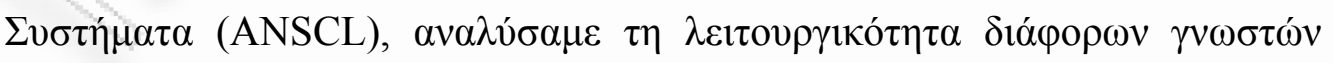

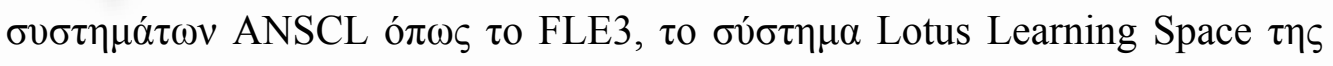

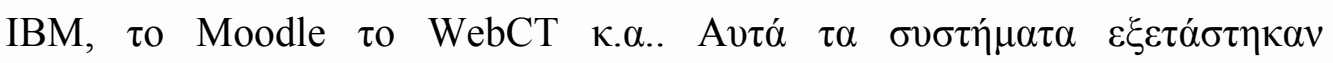

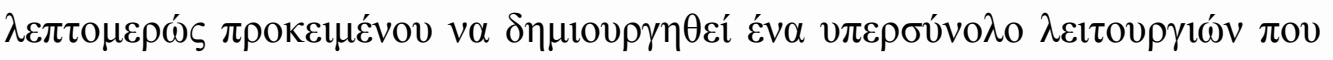

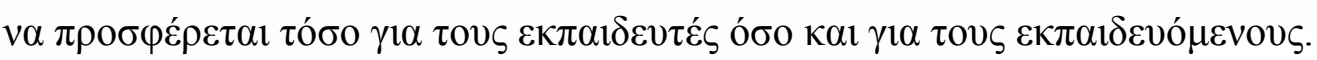




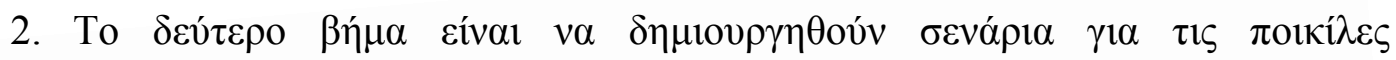

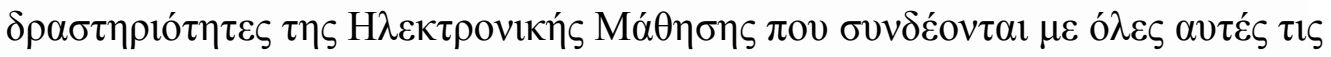

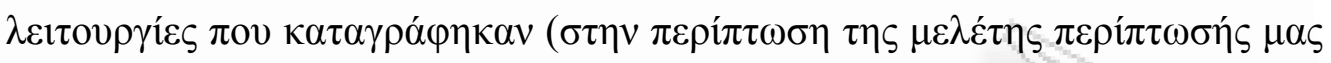

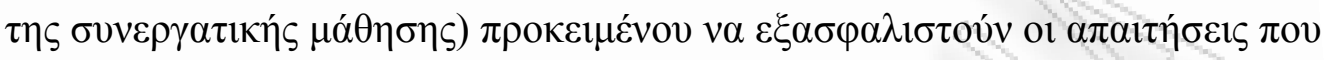

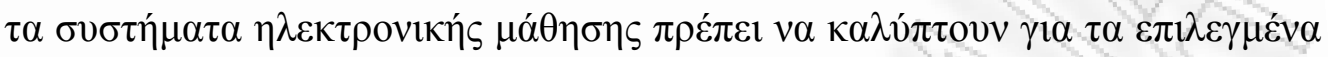

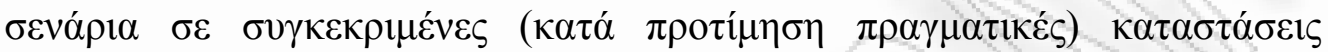

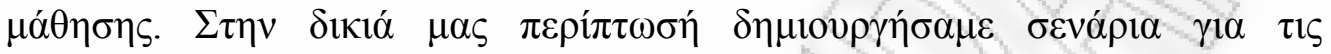

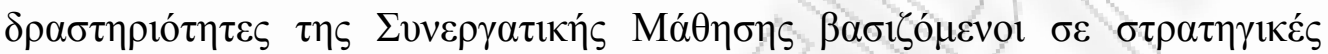

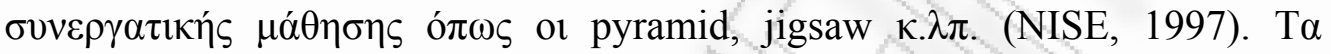

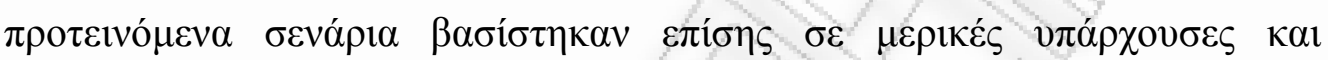

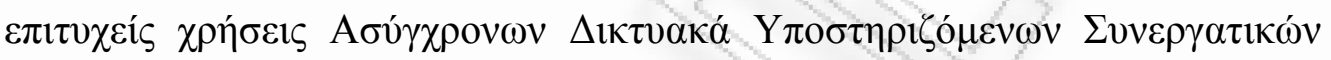

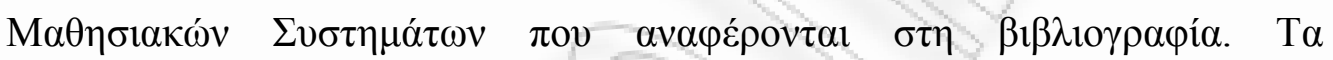

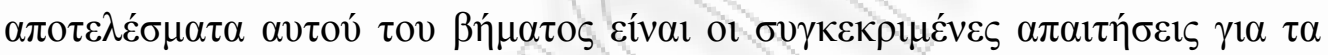

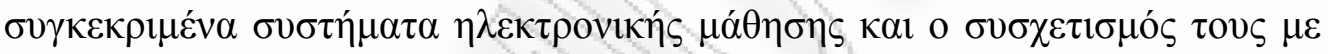

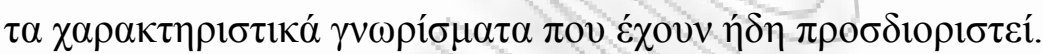

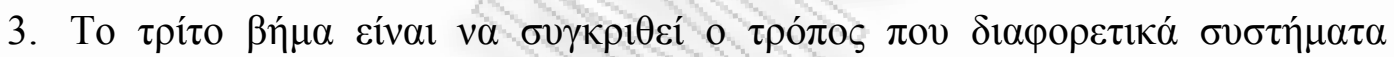

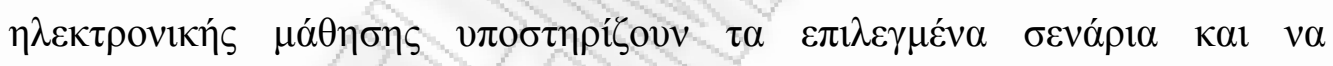

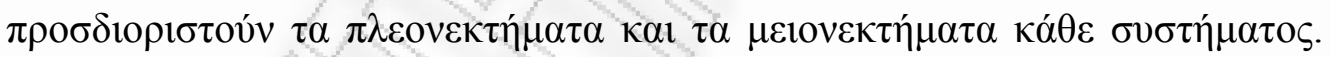

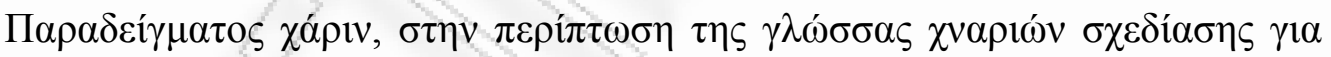

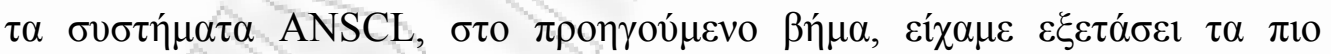

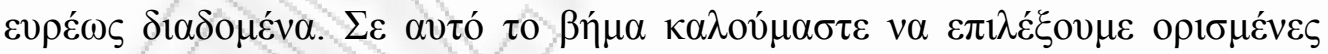

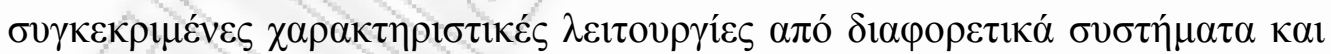

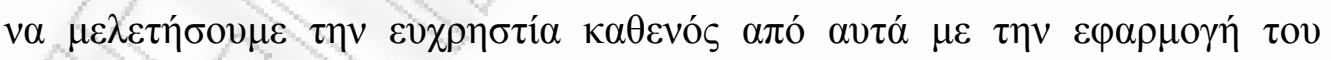

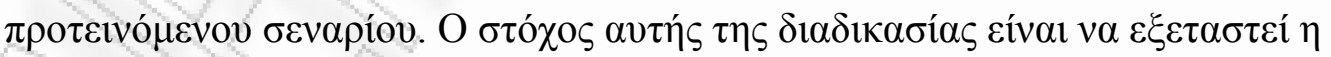

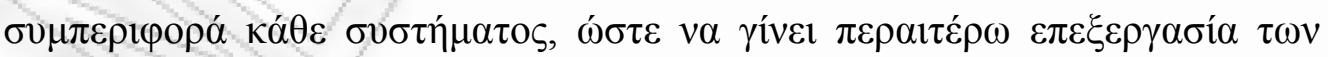

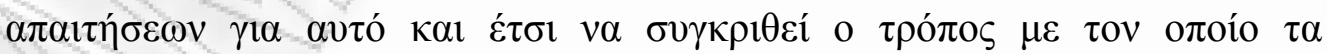

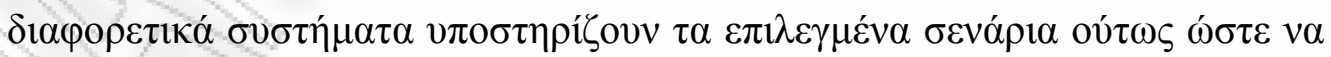

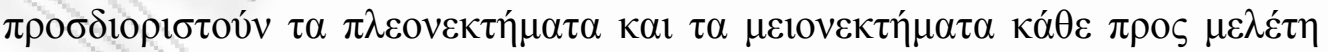

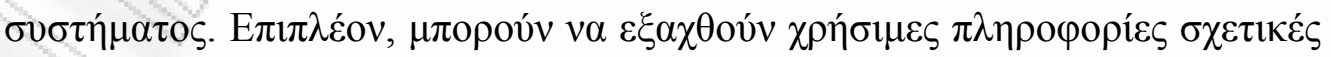

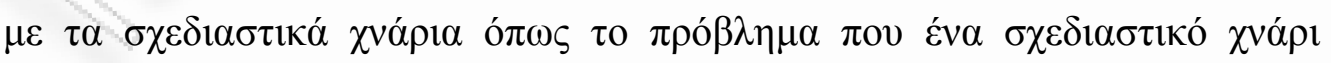

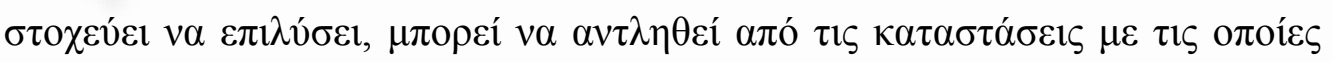

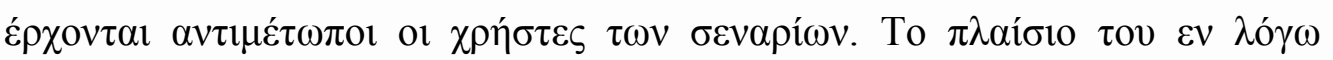

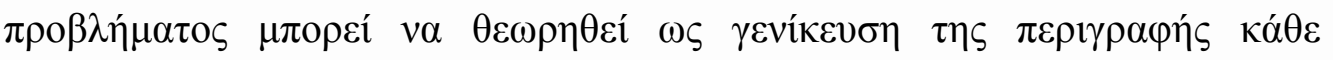

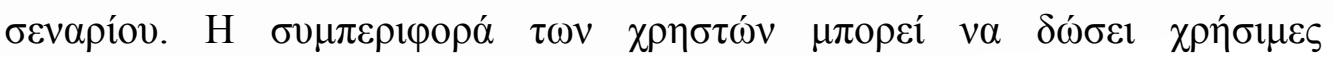




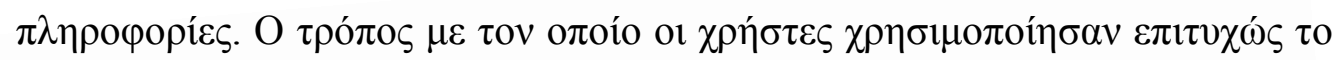

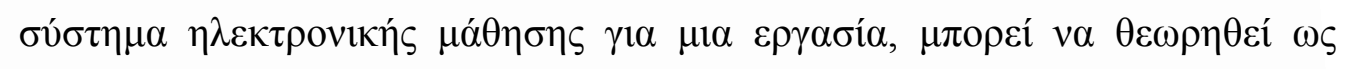

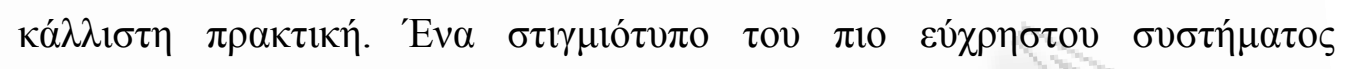

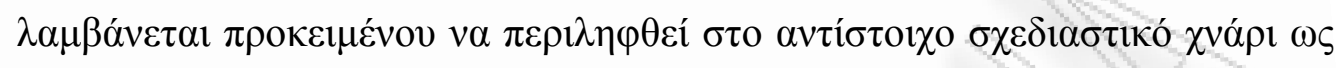

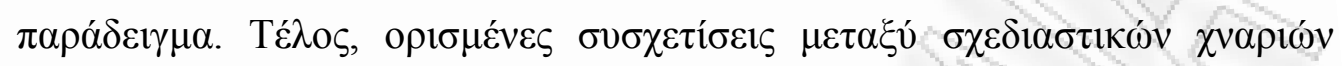

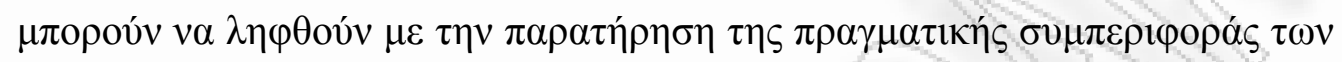

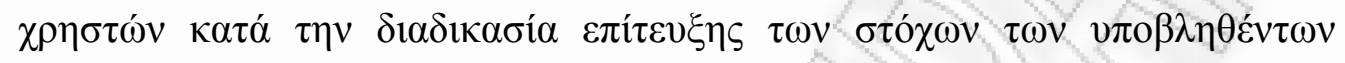

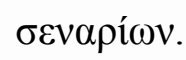

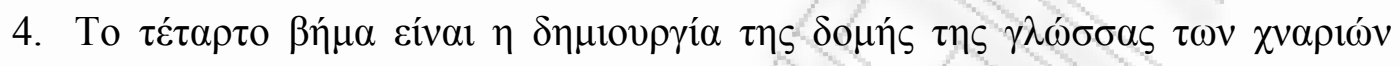

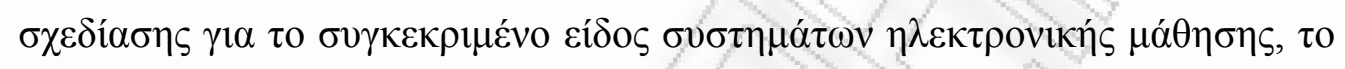

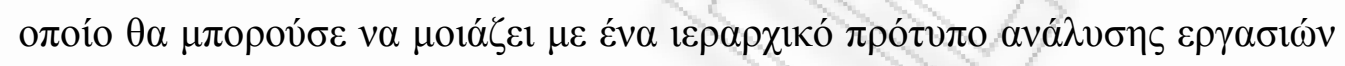
(van Duyne et al, 2003).

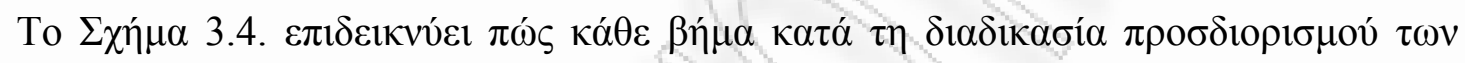

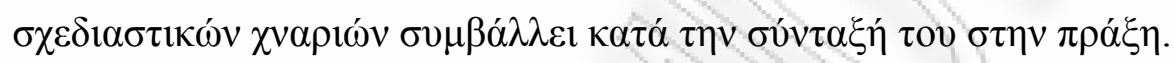

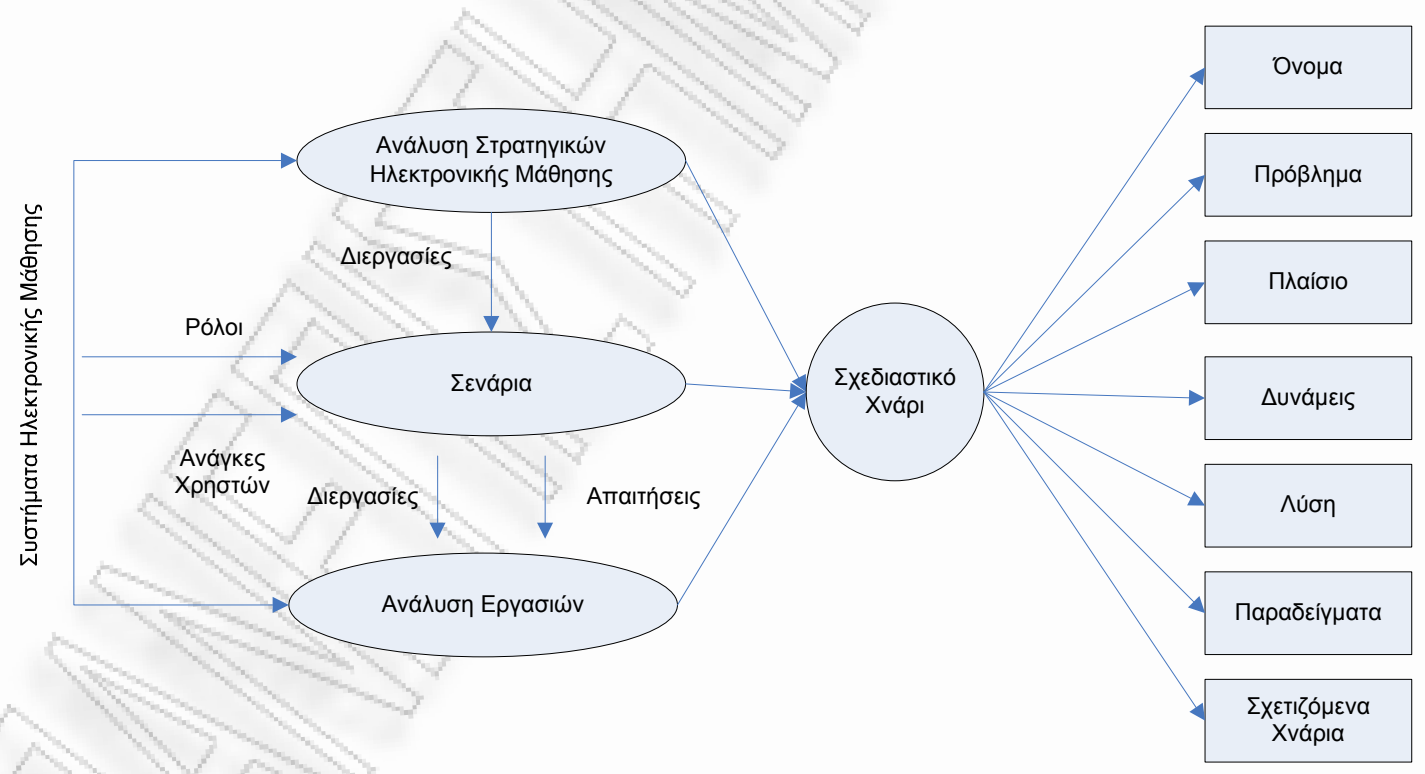

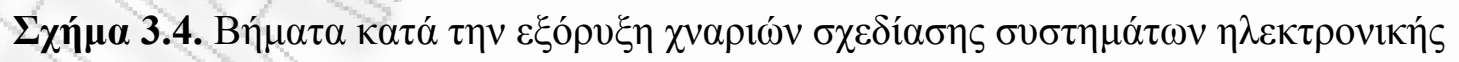
$\mu \alpha ́ \theta \eta \sigma \eta \varsigma$

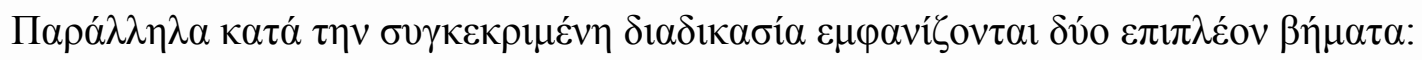

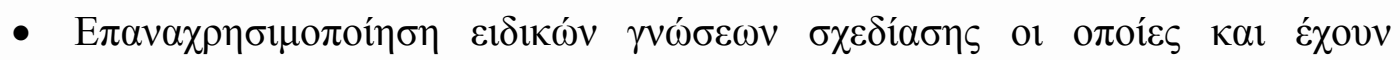

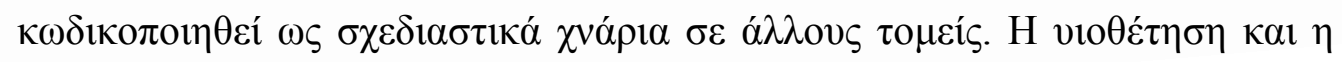




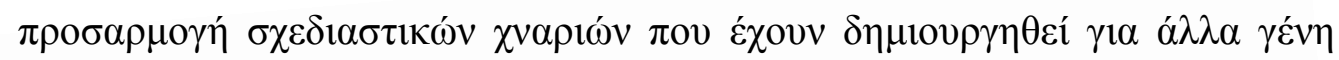

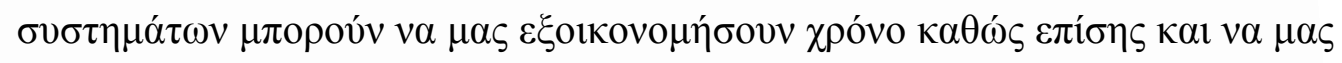

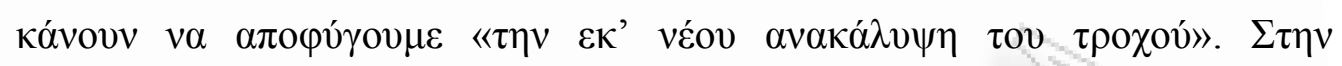

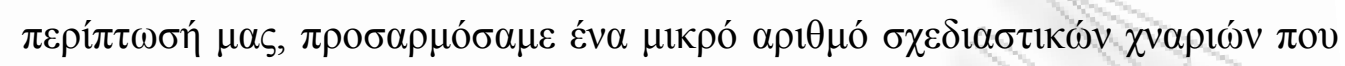

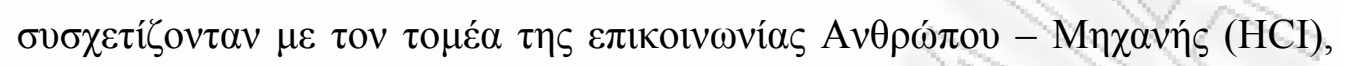

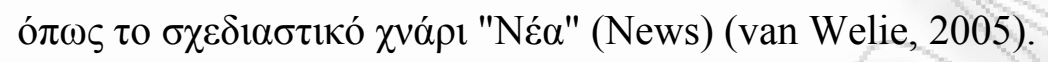

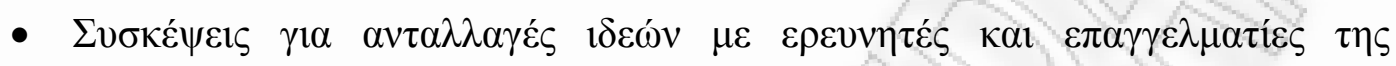

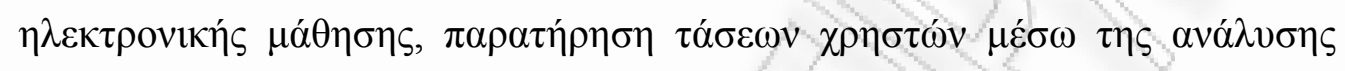

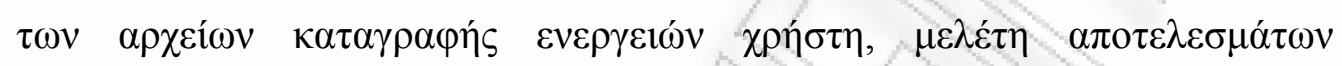

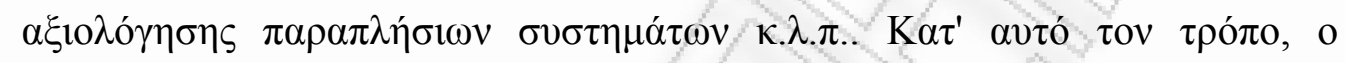

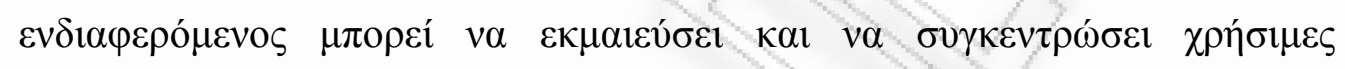

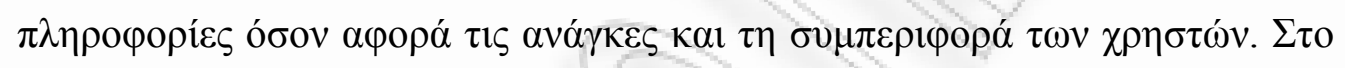

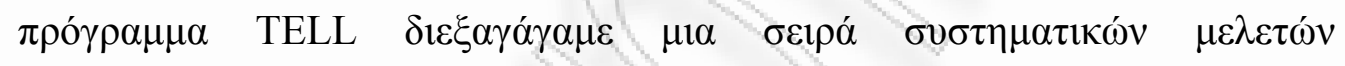

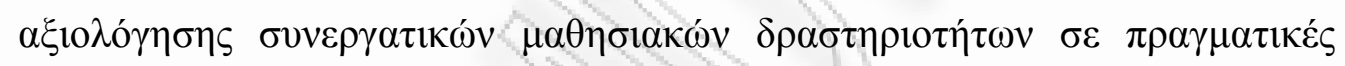

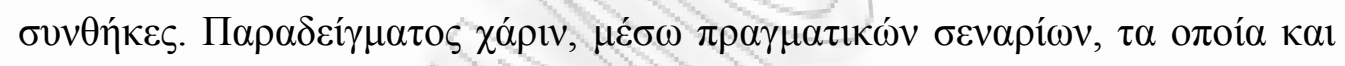

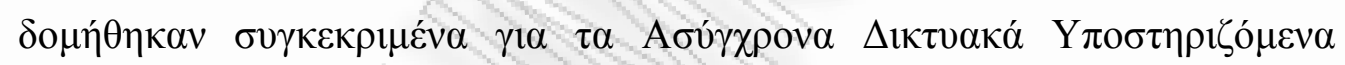

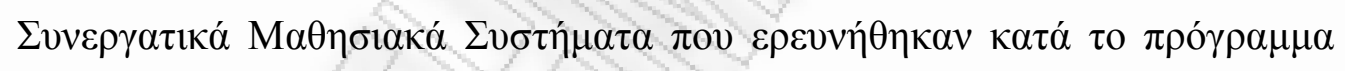

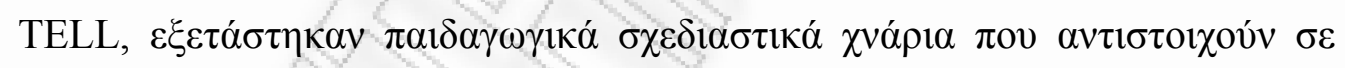

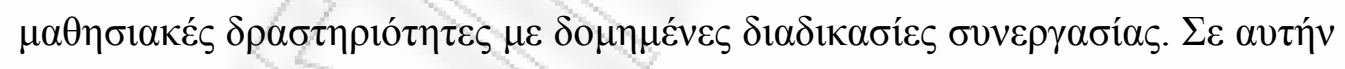

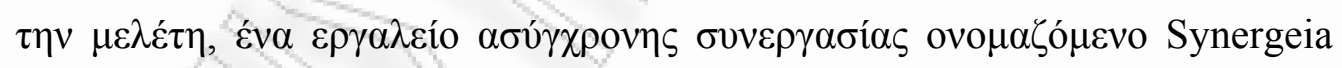

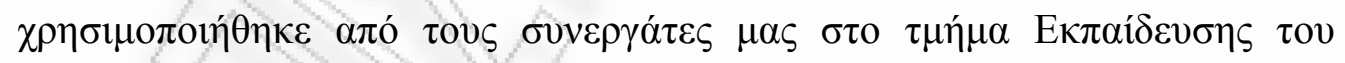

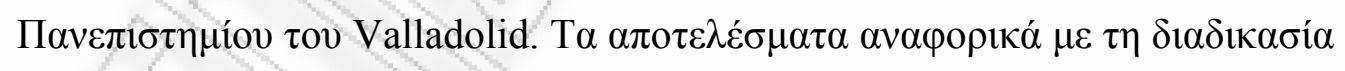

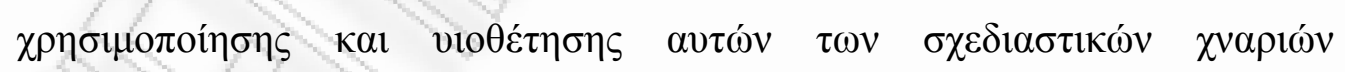

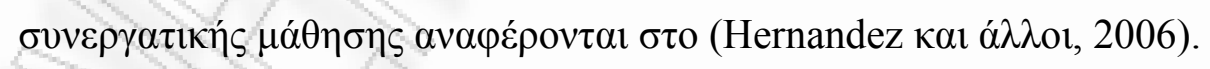

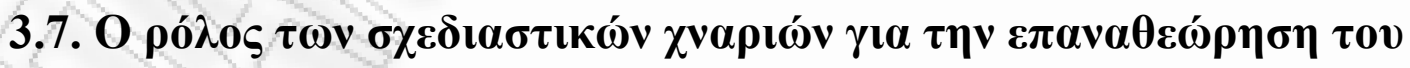 $\mu \alpha \theta \eta \sigma \iota \alpha \sigma_{0} \sigma \chi \varepsilon \delta 1 \alpha \sigma \mu 0 v ́$}

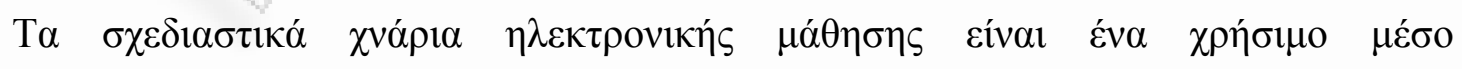

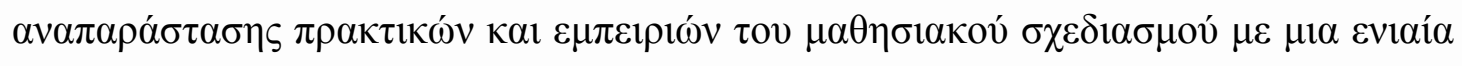

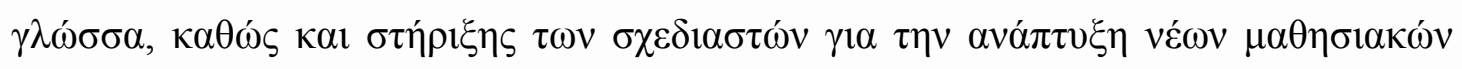

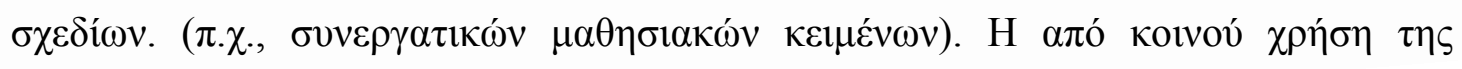




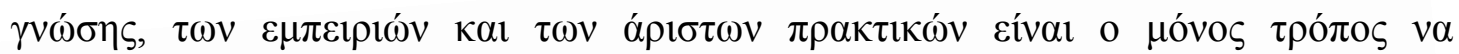

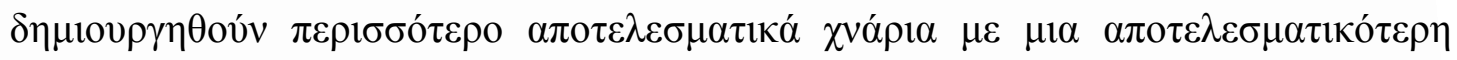

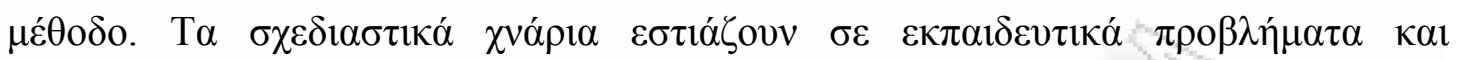

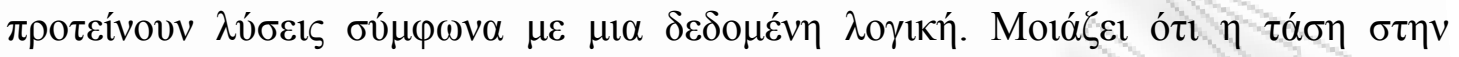

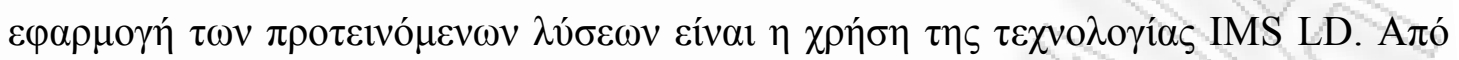

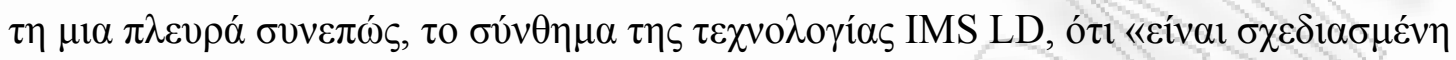

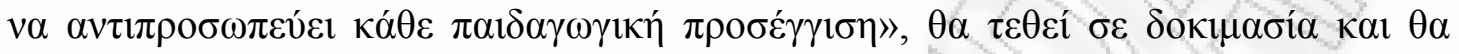
$\varepsilon \lambda \varepsilon \gamma \chi \theta \varepsilon i ́ \eta \eta$

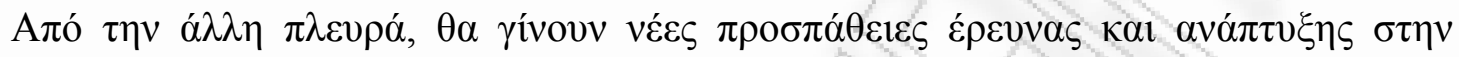

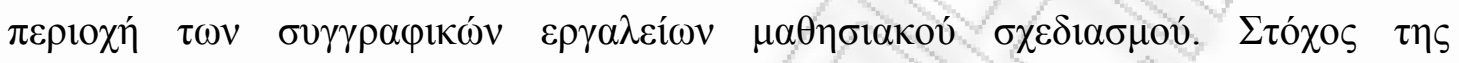

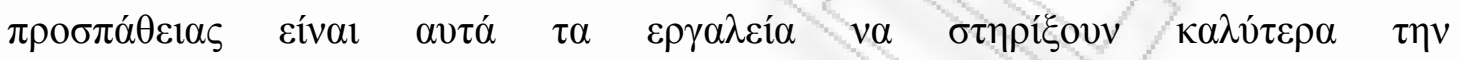

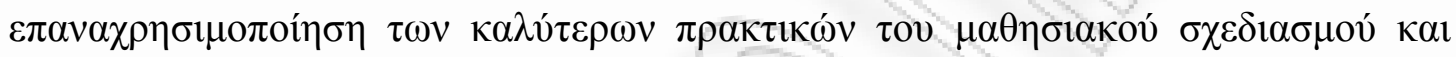

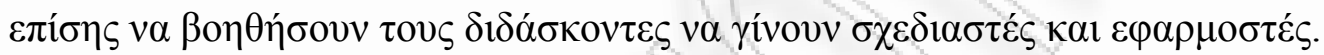

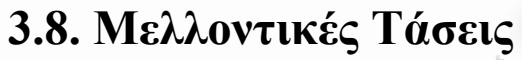

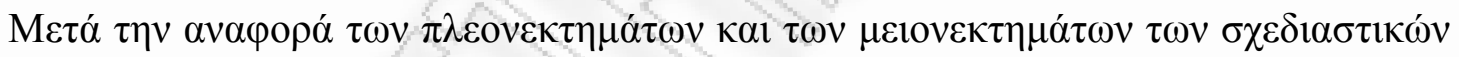

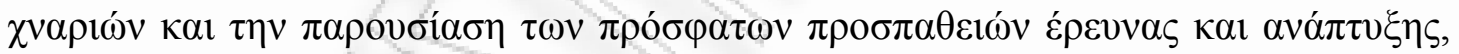

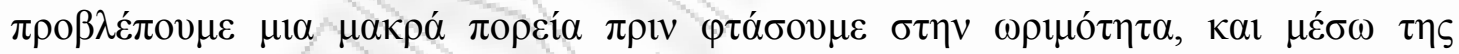

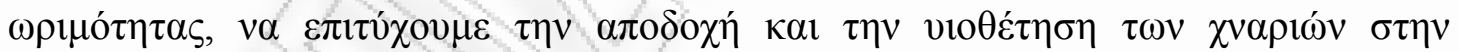

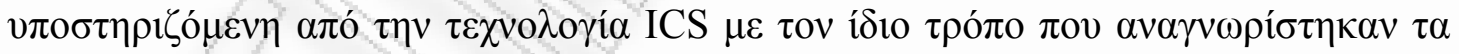

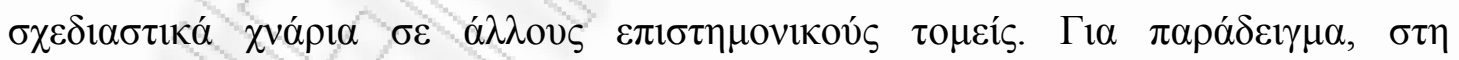

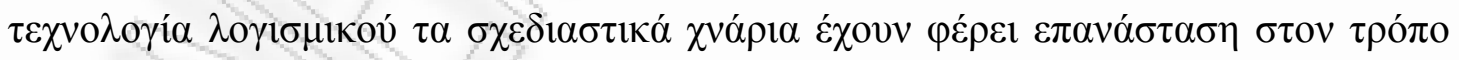

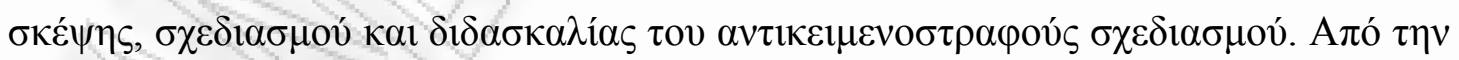

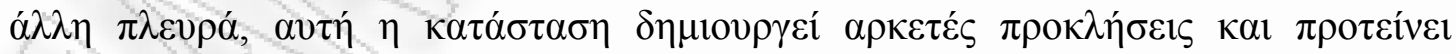

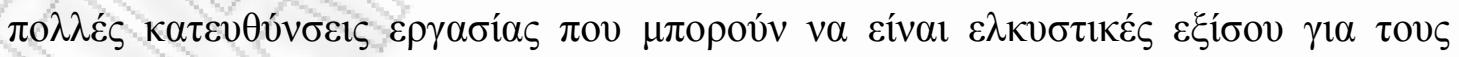

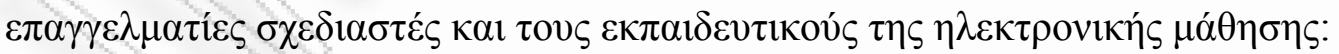

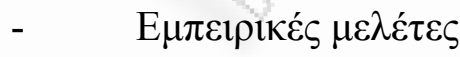

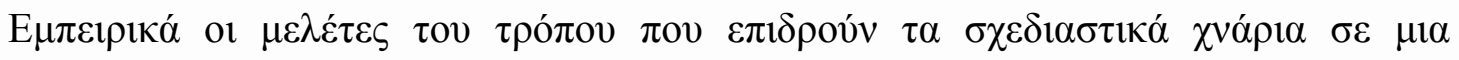

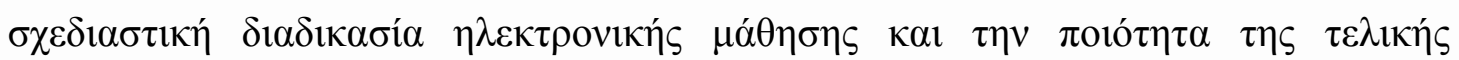




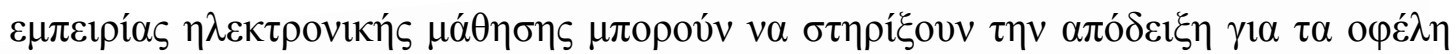
$\tau \omega \nu \chi v \alpha \rho \iota \omega ́ v(\eta ́ v \alpha \tau \eta v \alpha v \tau \imath \kappa \rho o v ́ \sigma o v v)$

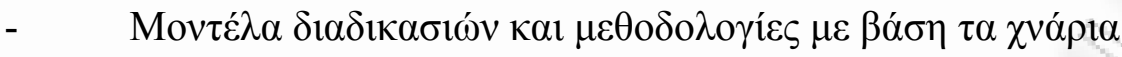

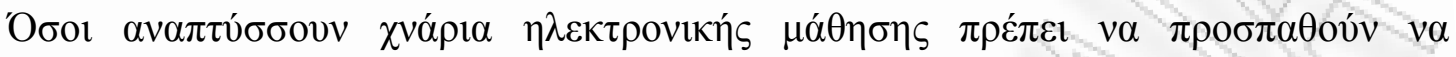

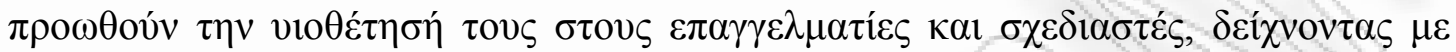

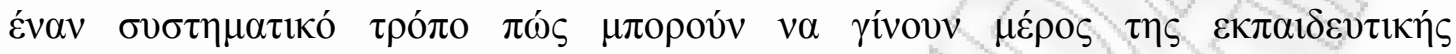

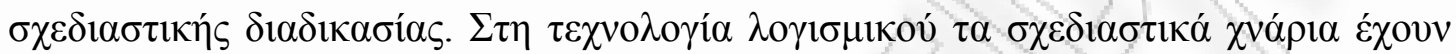

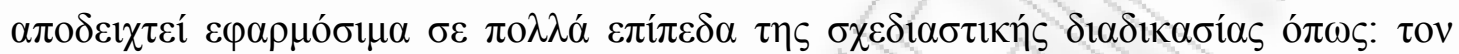

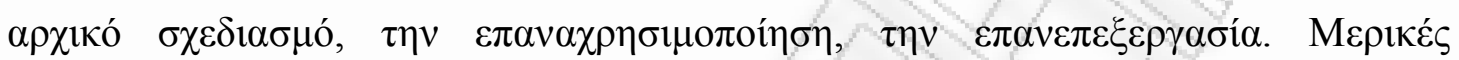

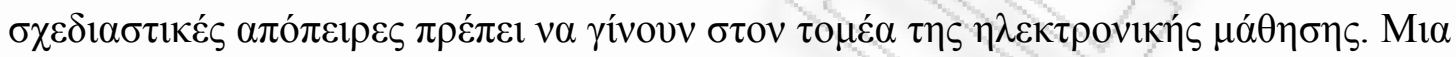

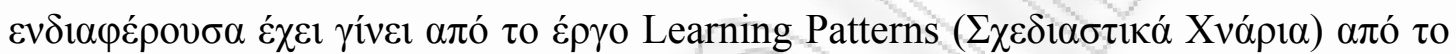

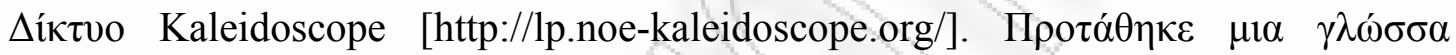

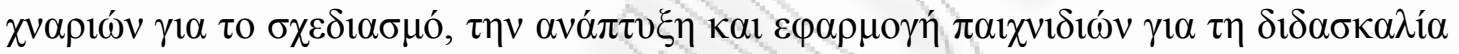

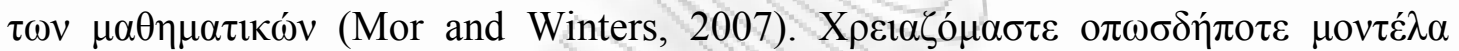

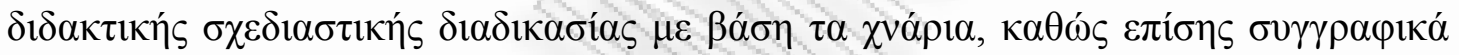

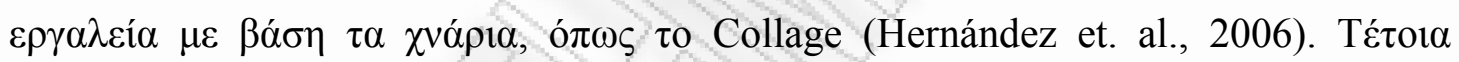

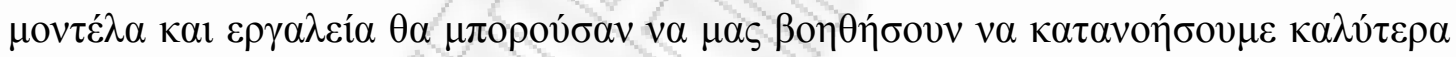

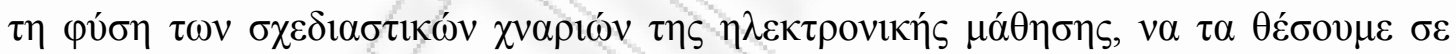

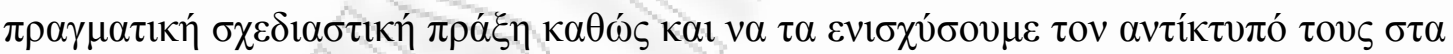

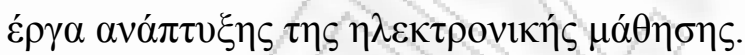

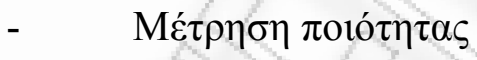

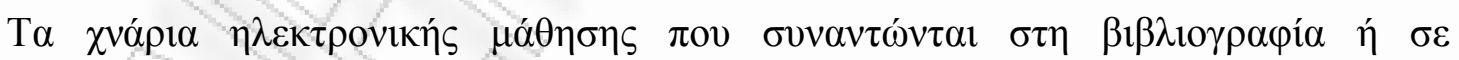

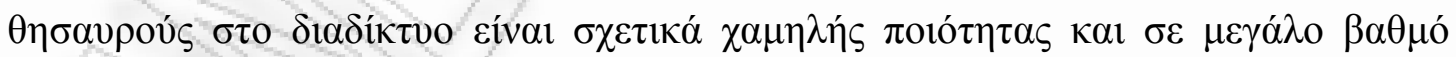

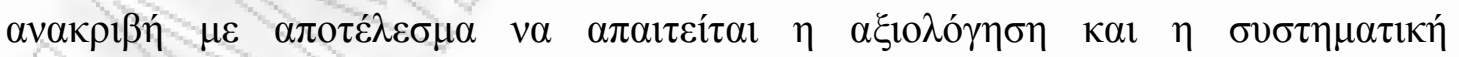

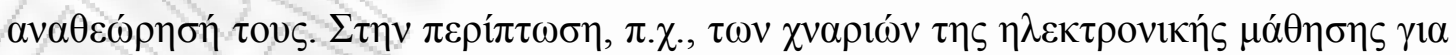

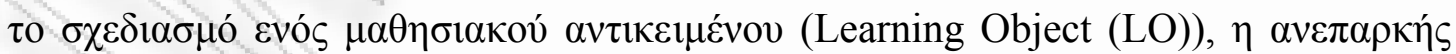

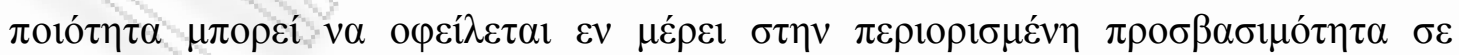

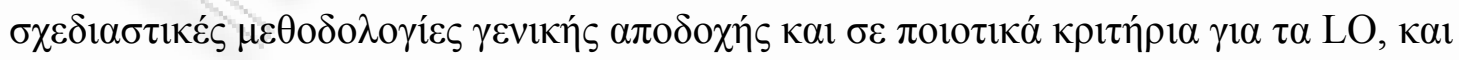

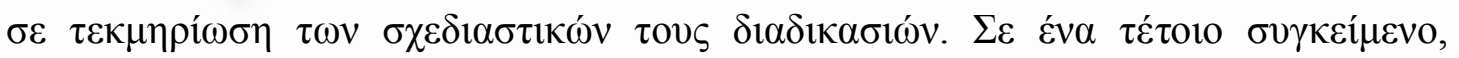

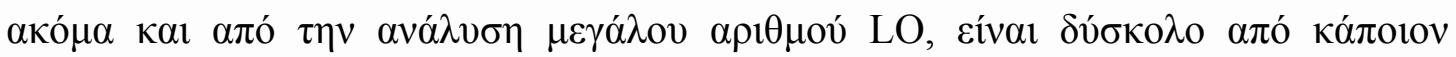

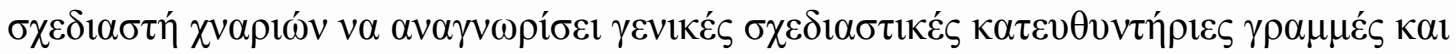

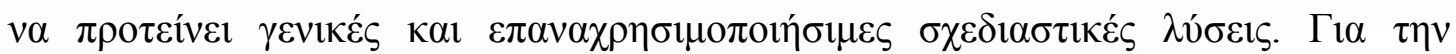




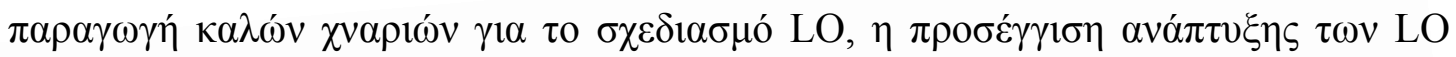

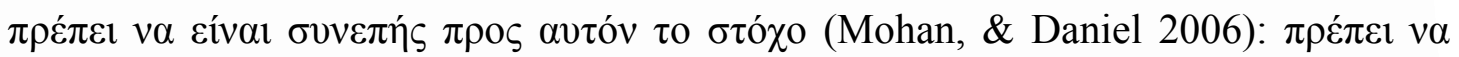

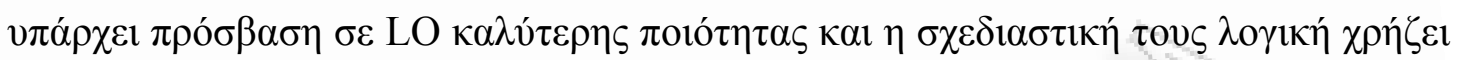

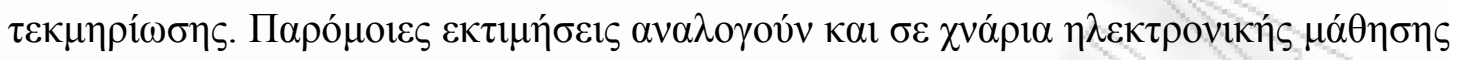

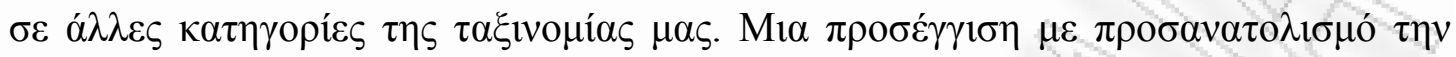

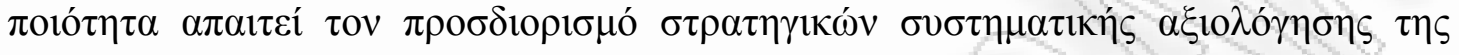

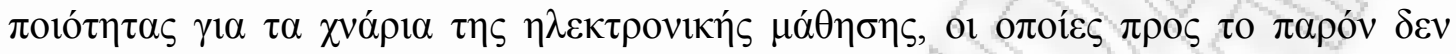

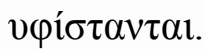

- B B B B B B B

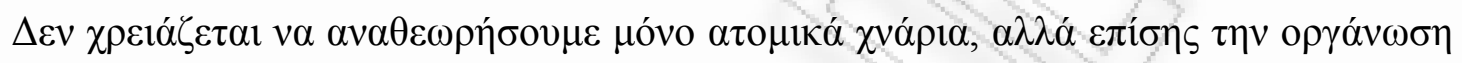

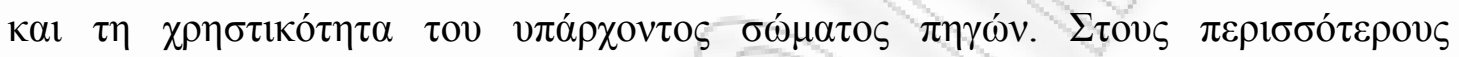

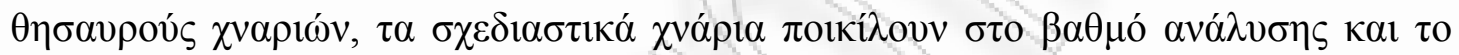

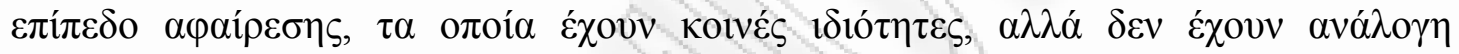

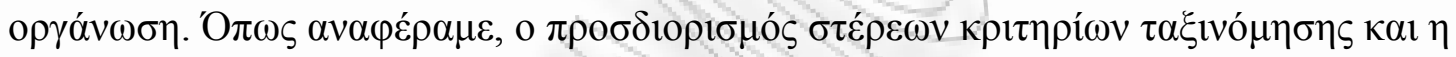

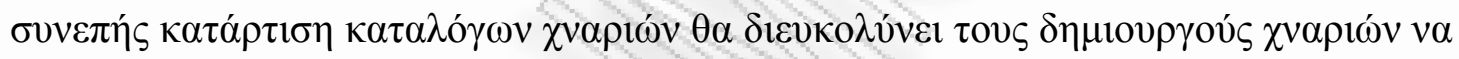

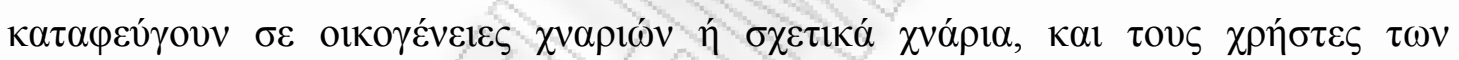

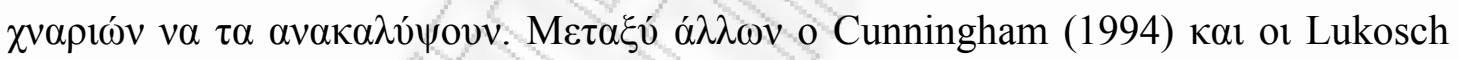

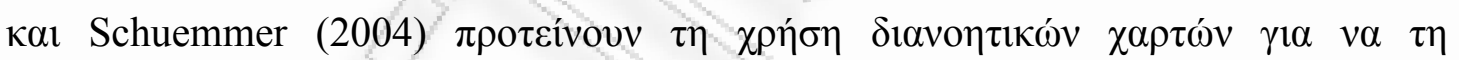

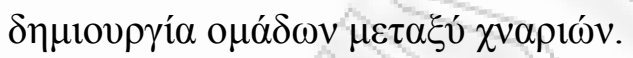

\section{- $\quad H \alpha v \alpha ́ \pi \tau v \xi \eta \alpha v \tau \imath-\chi v \alpha \rho ı \omega v$}

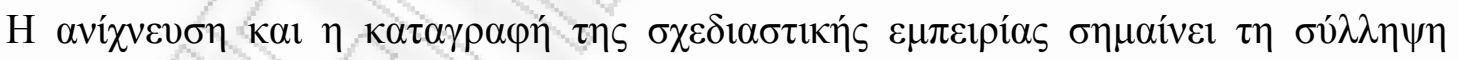

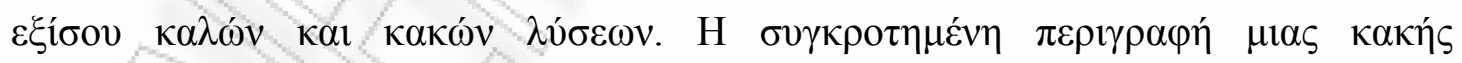

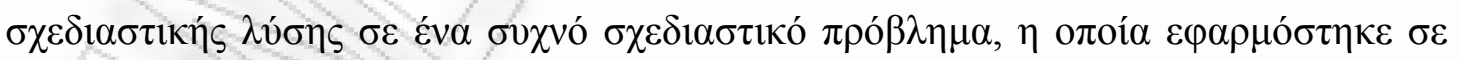

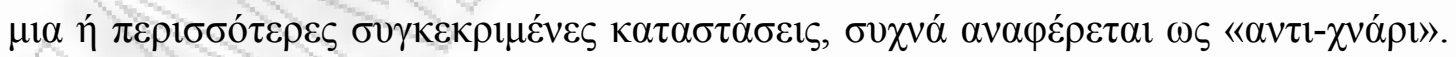

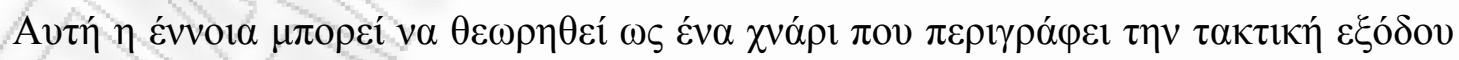

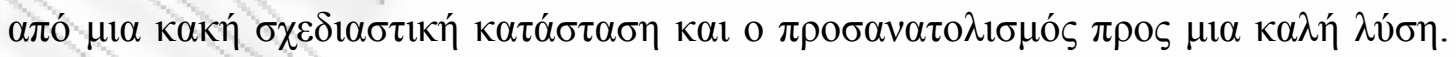

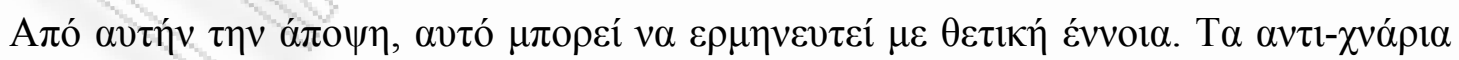

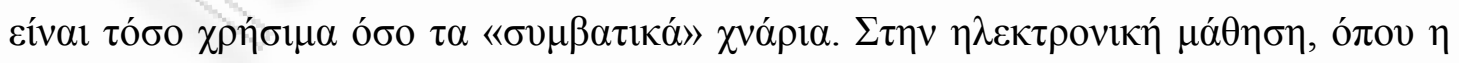

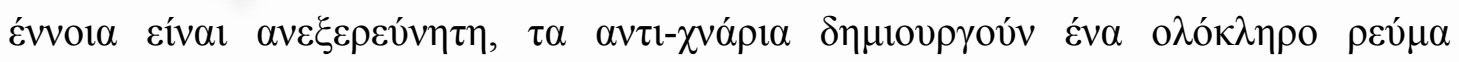
غ́pevvas. 


\section{9. $\Sigma v \mu \pi \varepsilon \rho \alpha ́ \sigma \mu \alpha \tau \alpha$}

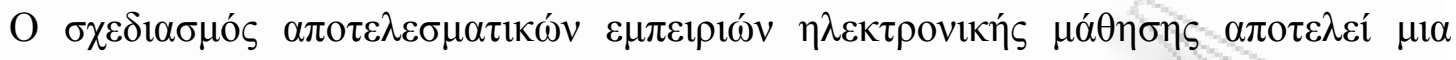

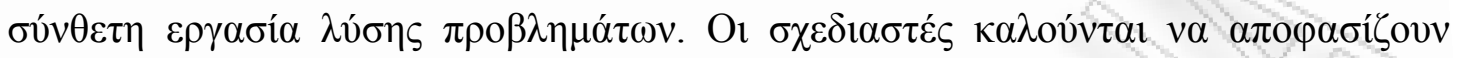

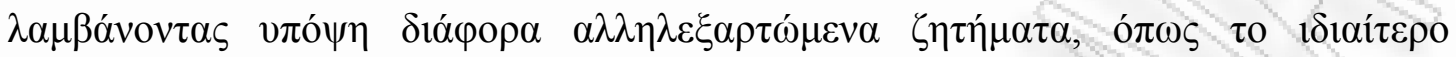

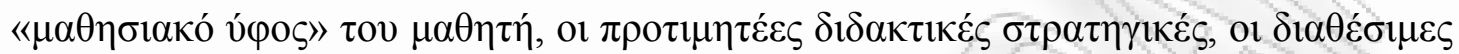

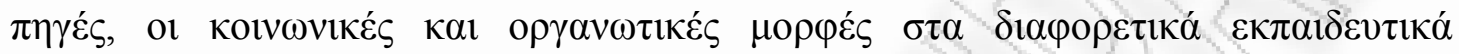

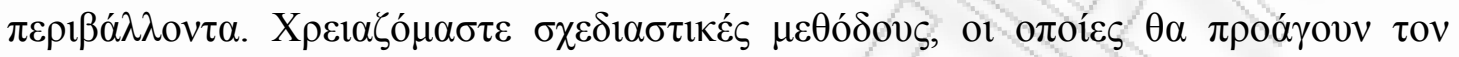

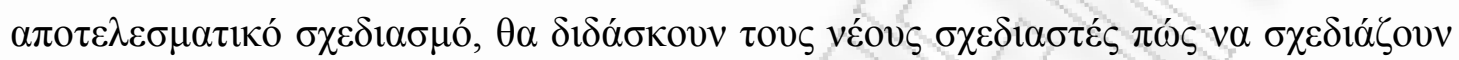

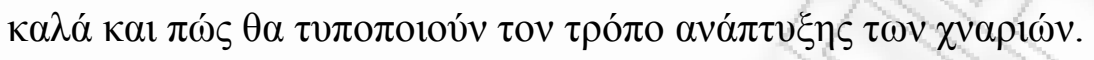

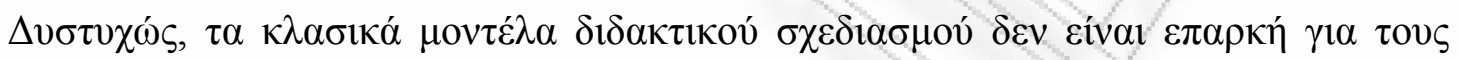

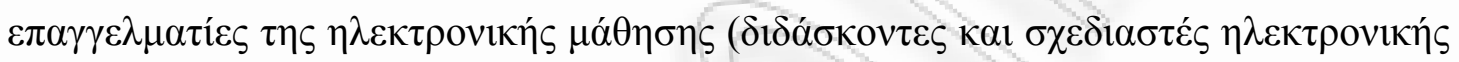

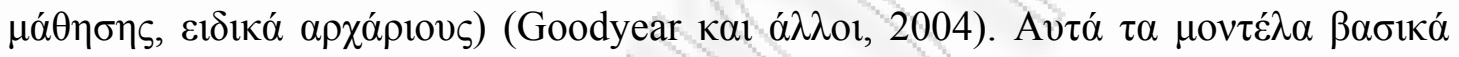

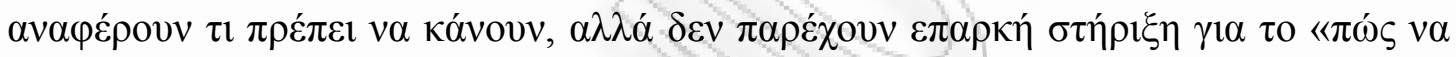

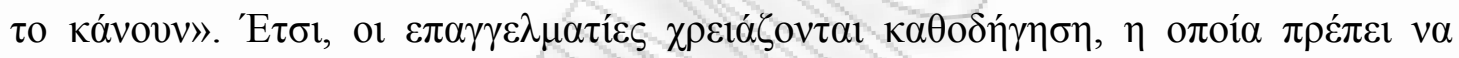

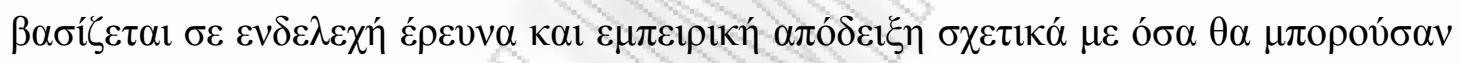

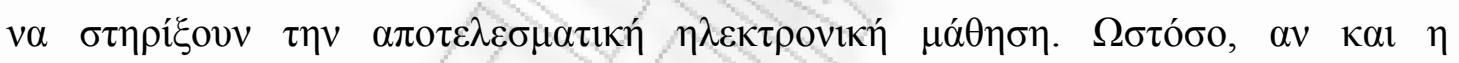

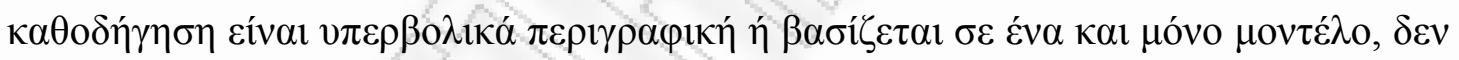

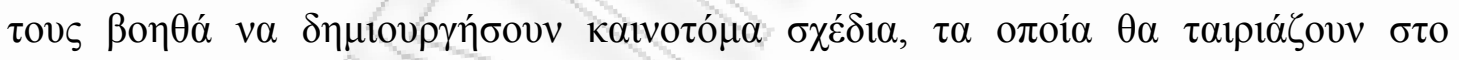

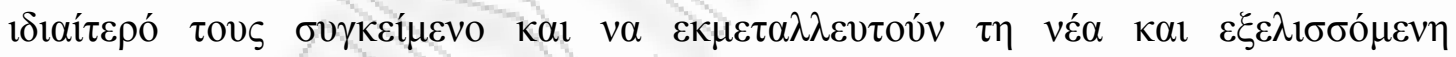

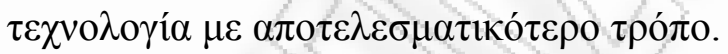

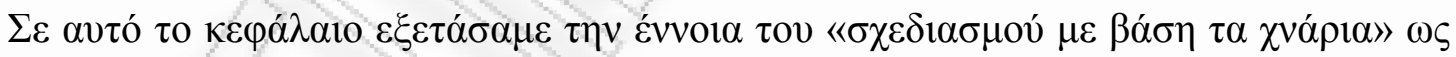

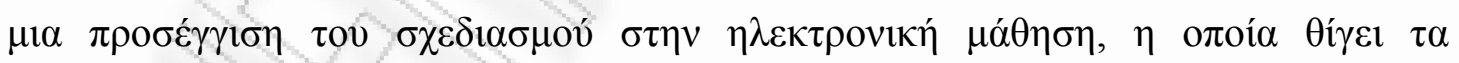

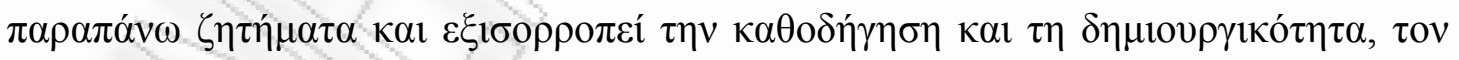

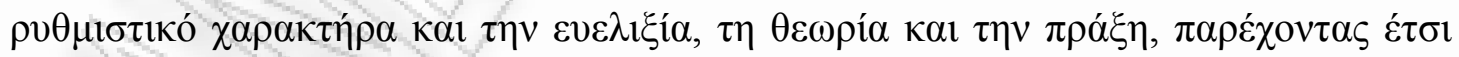

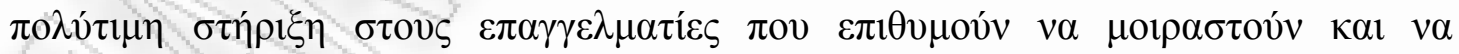

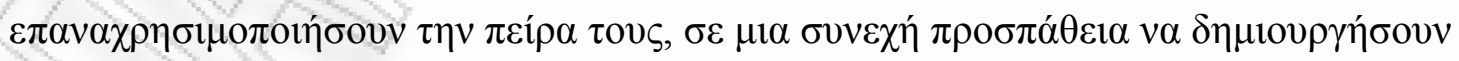

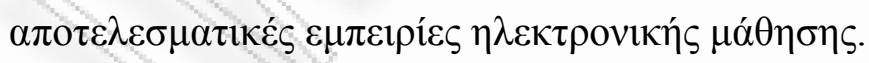

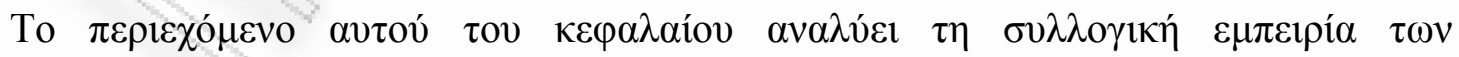

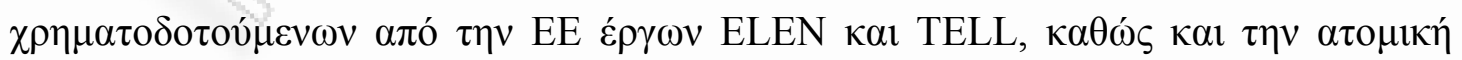

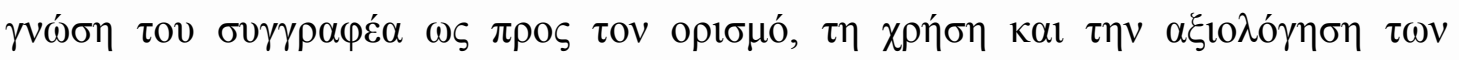

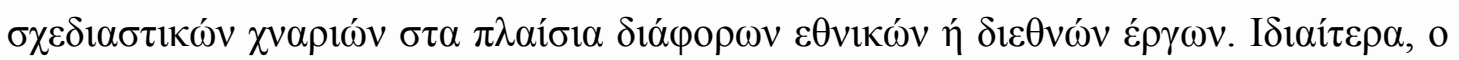

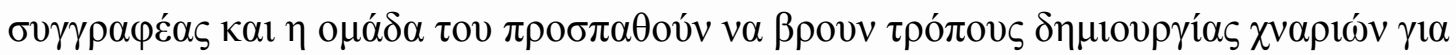




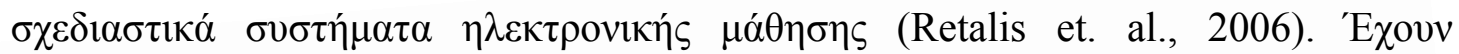

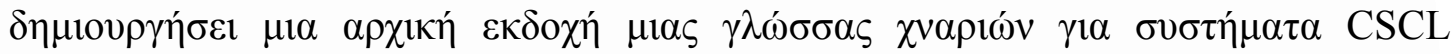

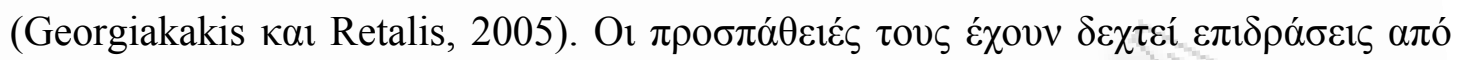

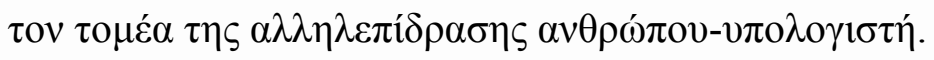

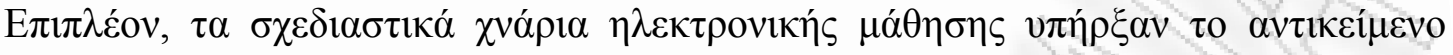

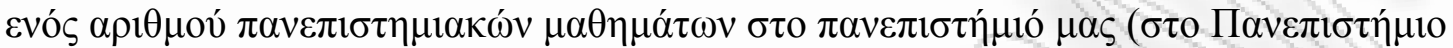

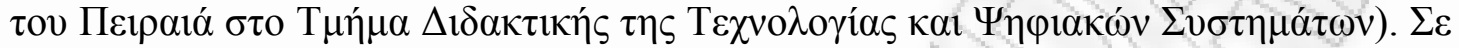

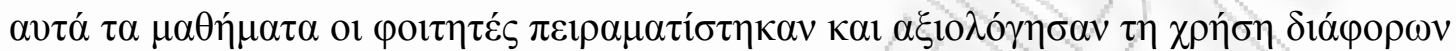

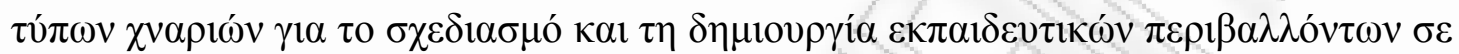

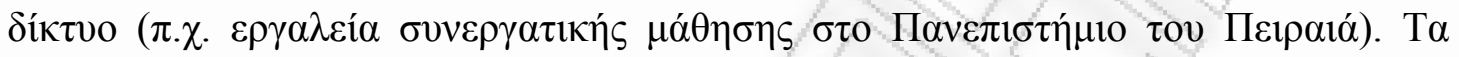

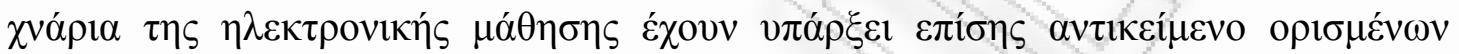

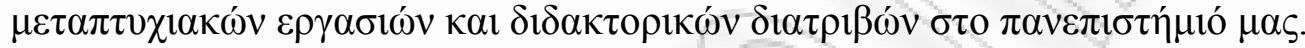

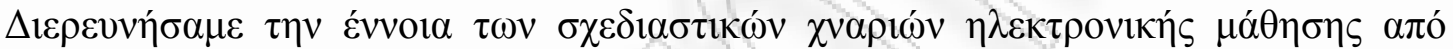

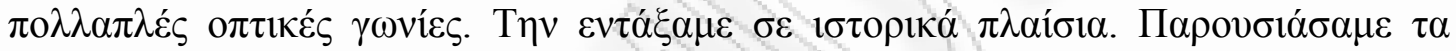

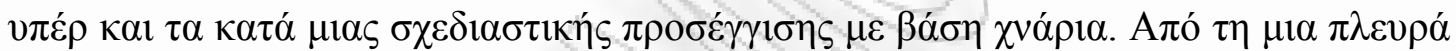

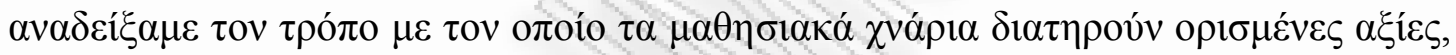

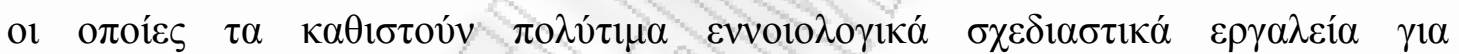

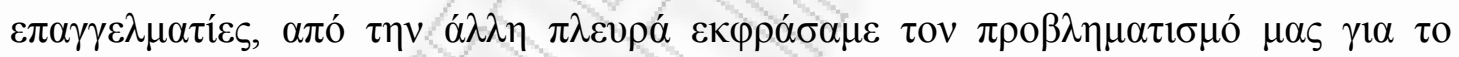

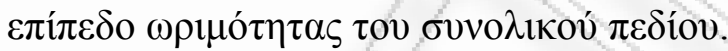

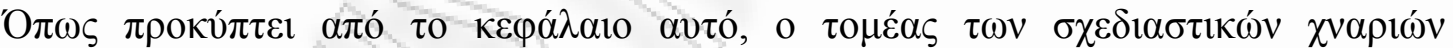

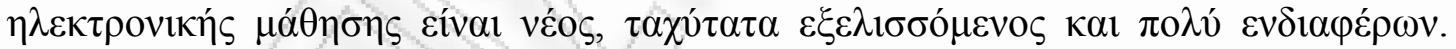

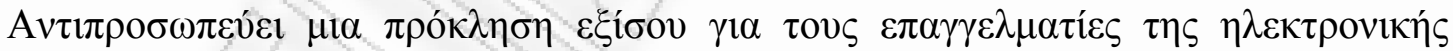

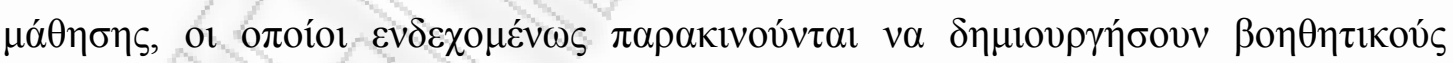

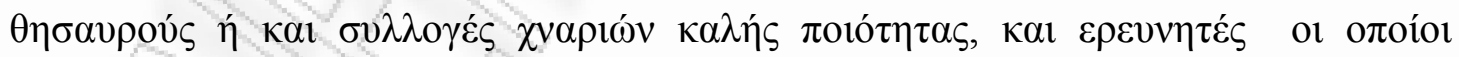

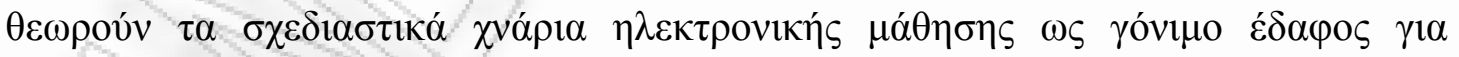

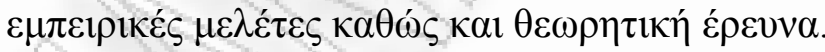




\section{КЕФААAIO 4

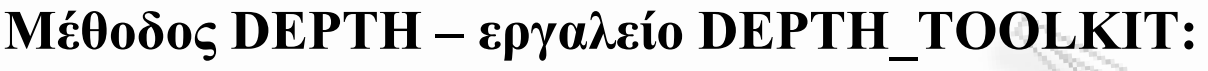

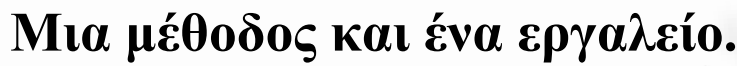

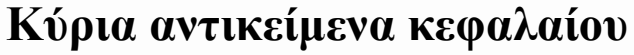

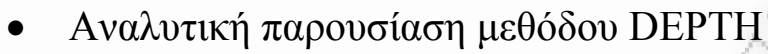

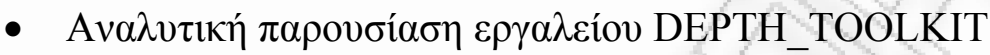

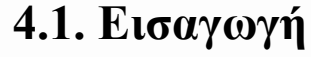

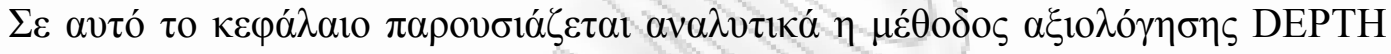

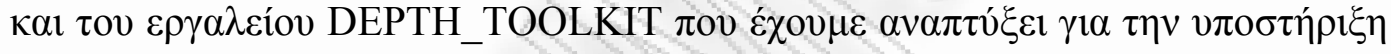

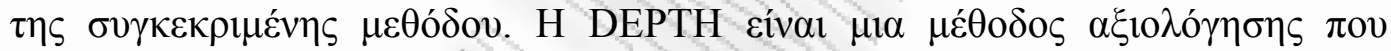
$\alpha \pi 0 \tau \varepsilon \lambda \varepsilon i ́ \tau \alpha 1$ a

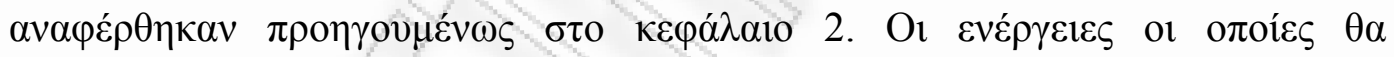

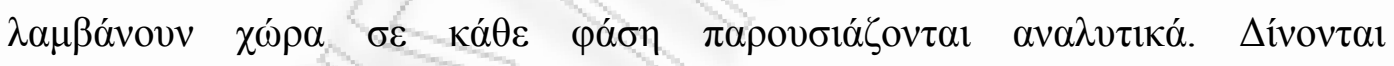

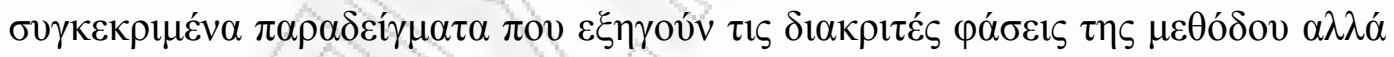

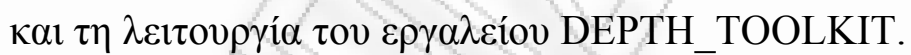

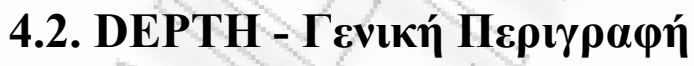

H $\pi \rho 0 \sigma \varepsilon ́ \gamma \gamma \imath \sigma$ DEPTH (DEsign PaTterns and Heuristic Criteria) síval $\mu 1 \alpha$

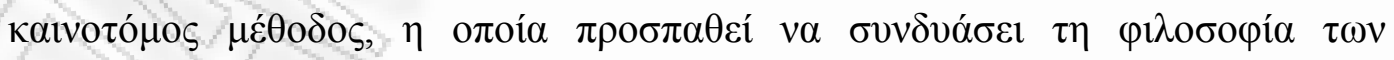

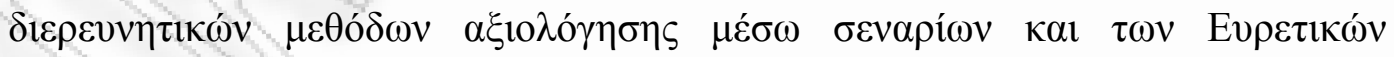

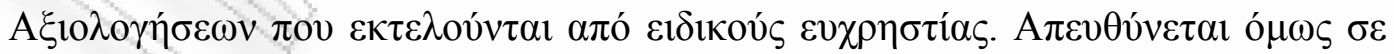

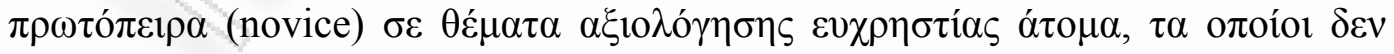

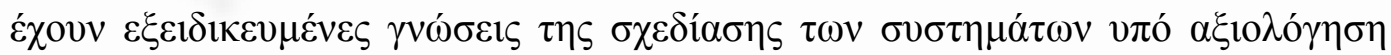

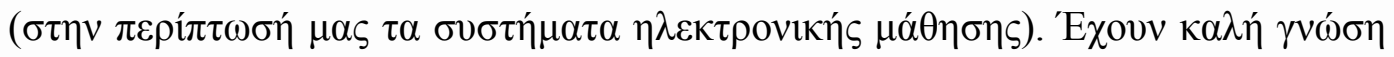

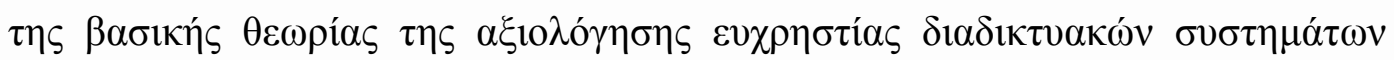




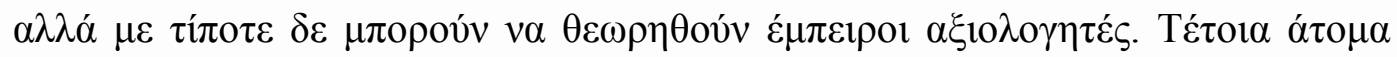

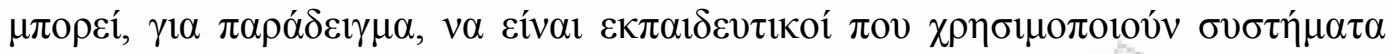

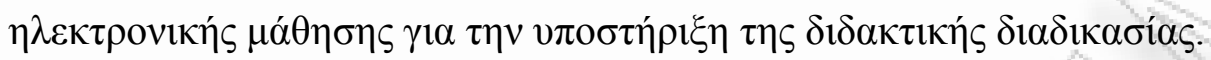

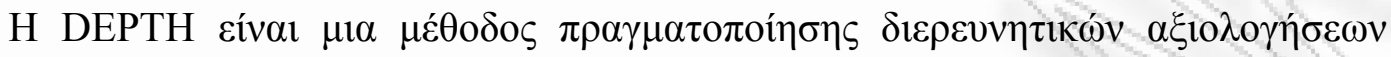

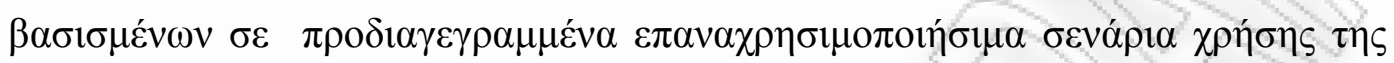

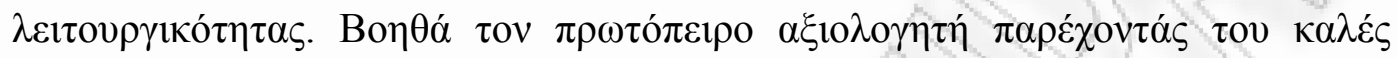

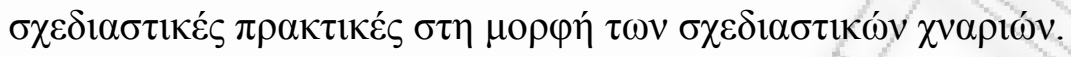

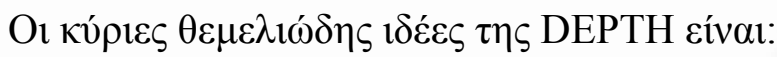

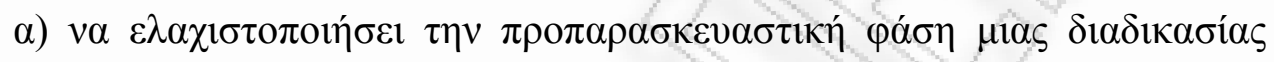

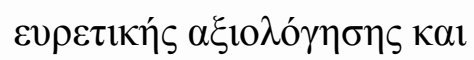

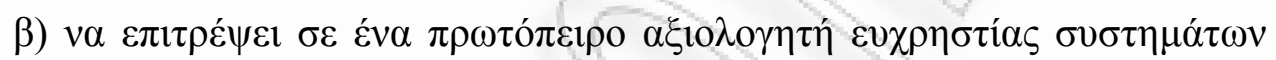

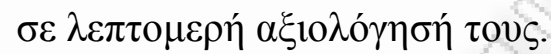

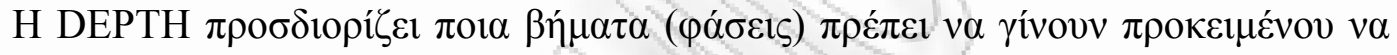

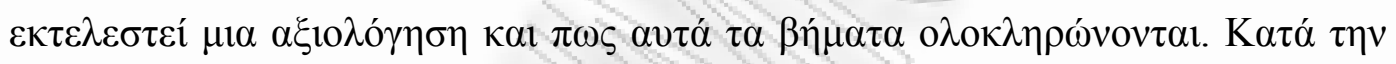

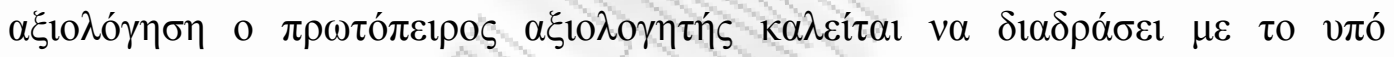

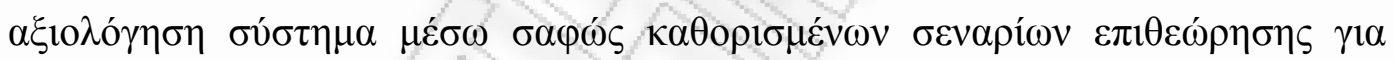

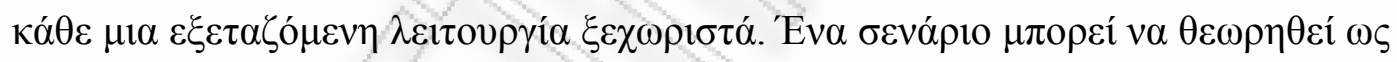

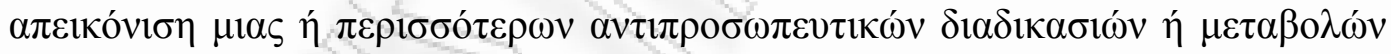

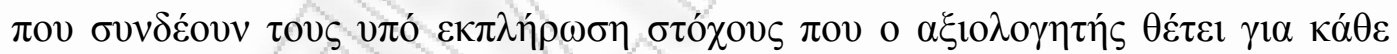

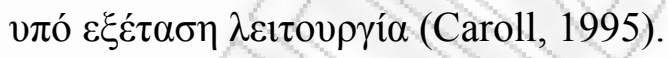

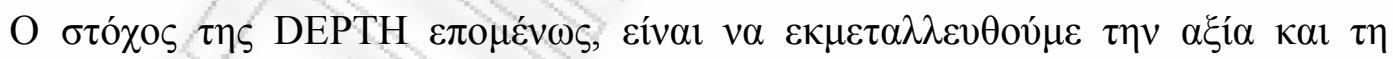

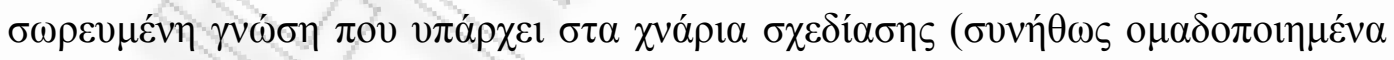

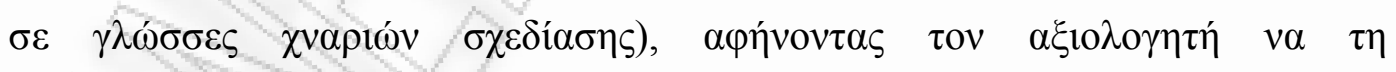

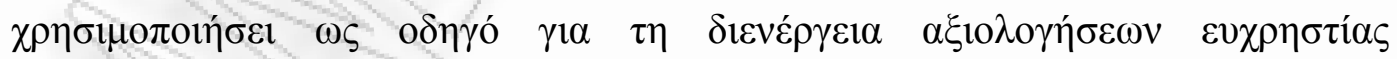

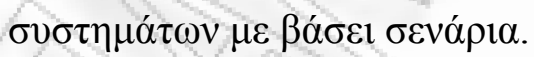

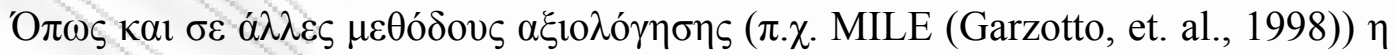

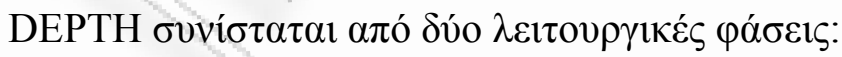

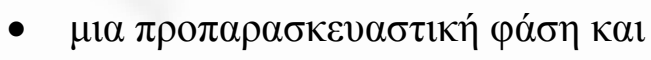

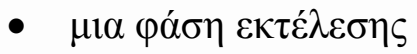




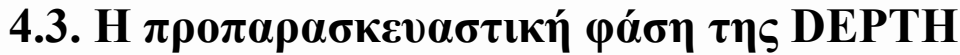

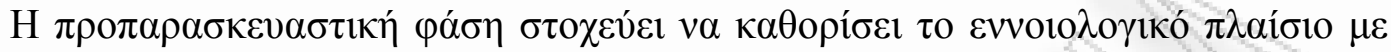

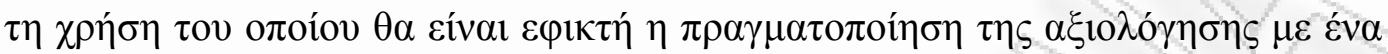

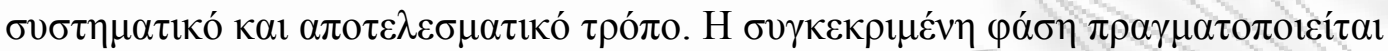

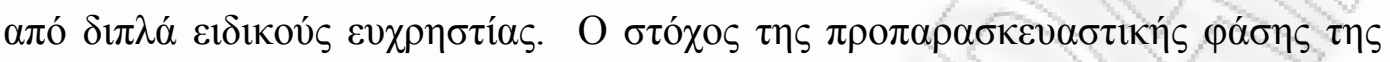

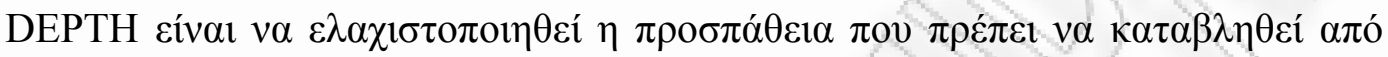

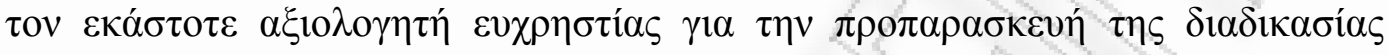

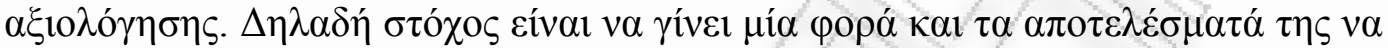

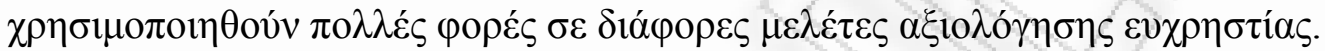

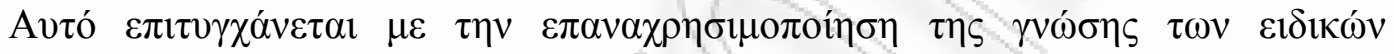

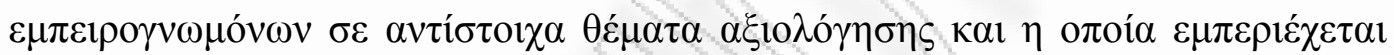

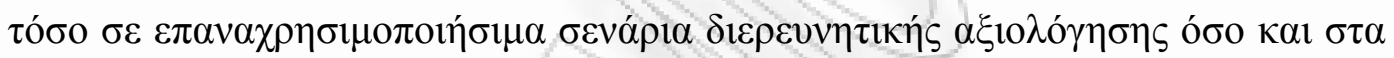

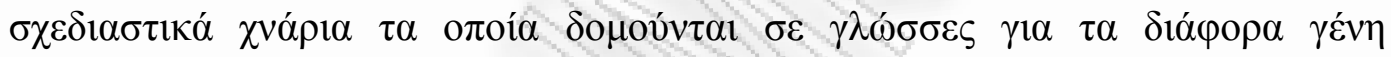

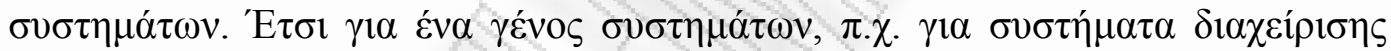

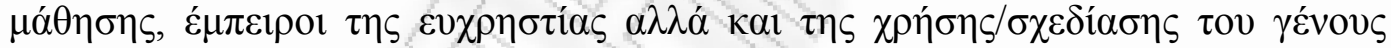

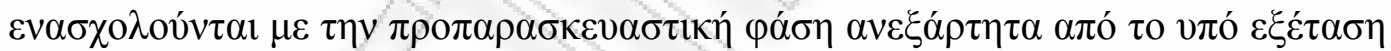

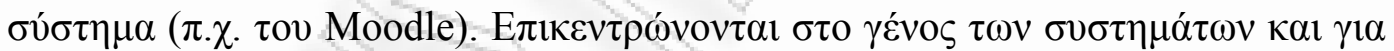

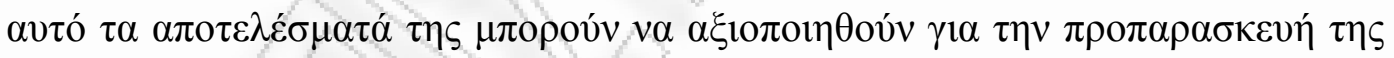

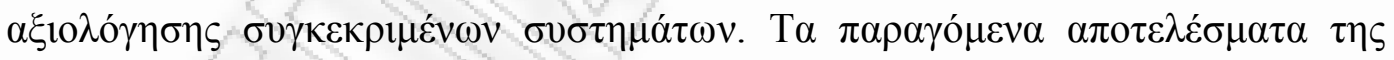

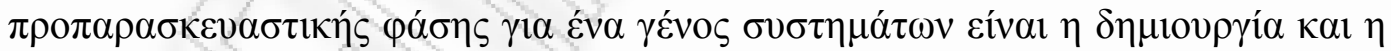

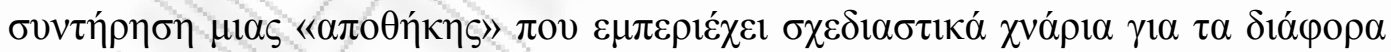

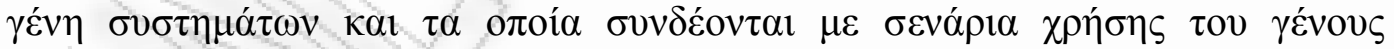
$\sigma v \sigma \tau \eta \mu \alpha ́ \tau \omega v$.

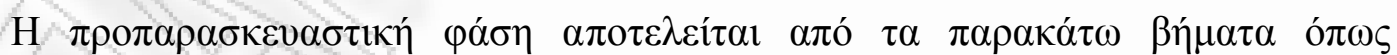

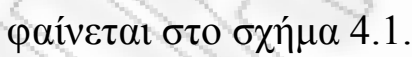

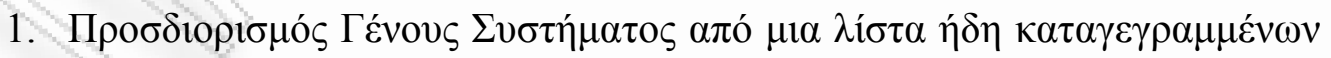

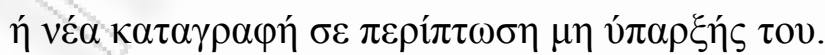

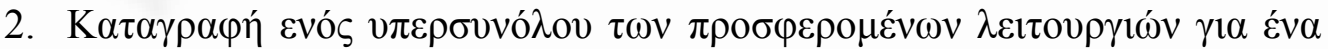

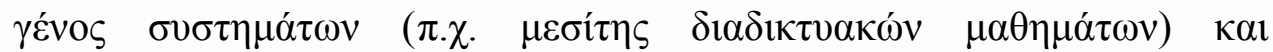

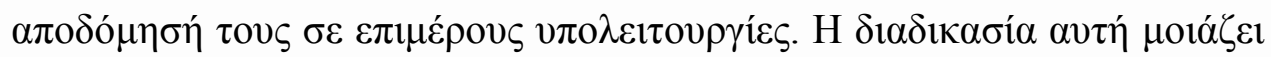




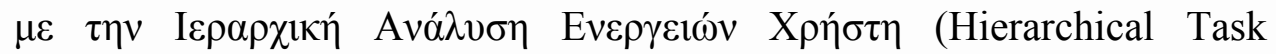
Analysis) (Dix et. al., 2003)

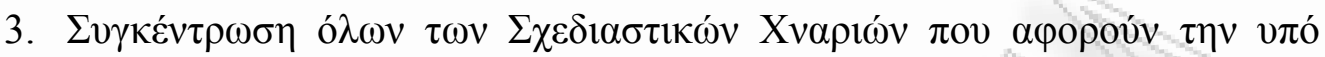

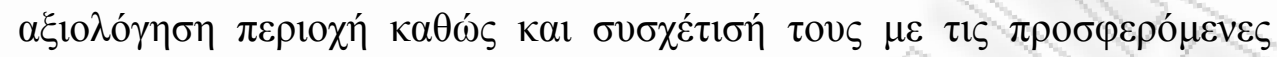

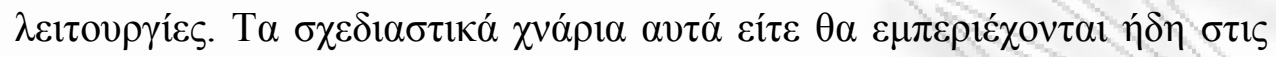

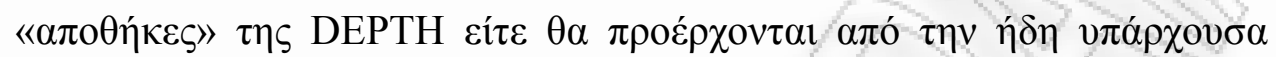

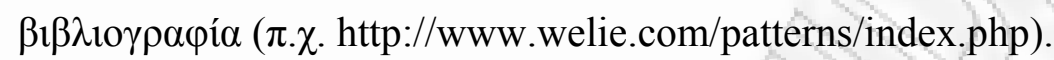

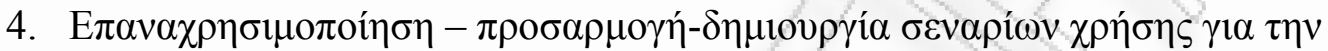

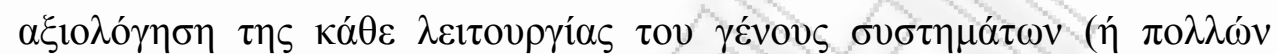

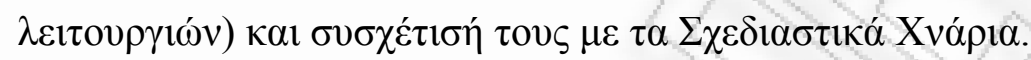

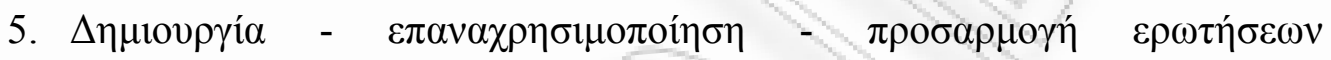

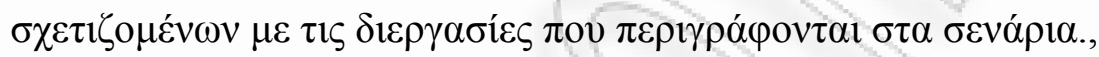

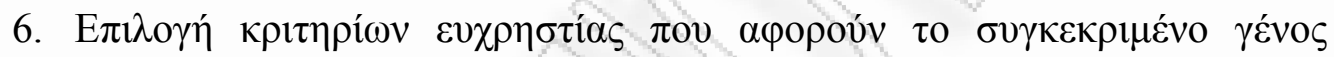

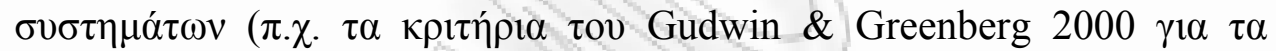

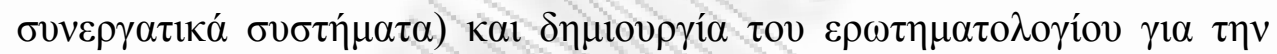

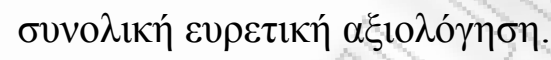

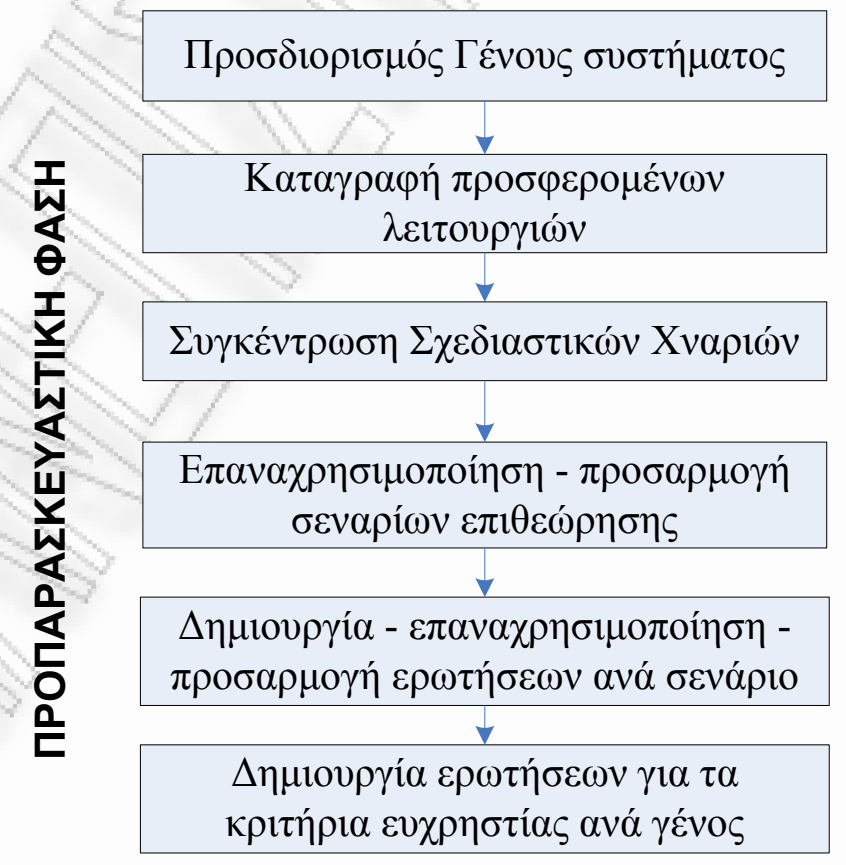

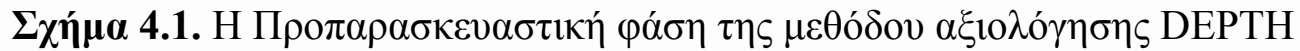




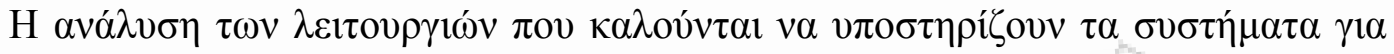

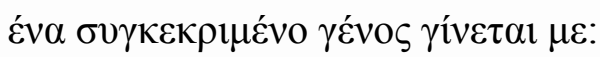

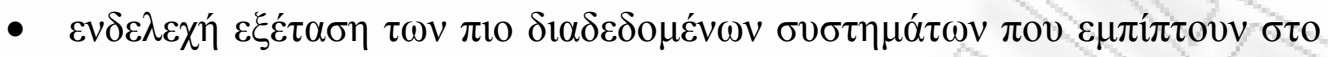

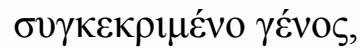

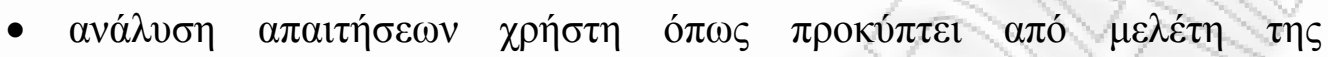

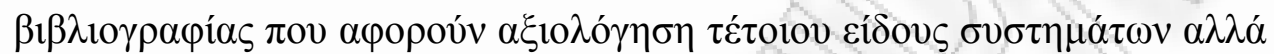

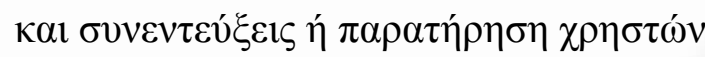

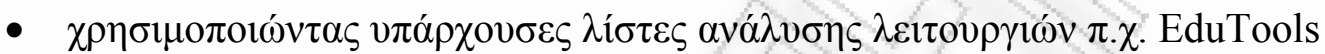
(http://www.edutools.info/static.jsp?pj=8\&page=HOME) $>\gamma l \alpha \quad \tau \alpha$

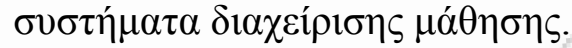

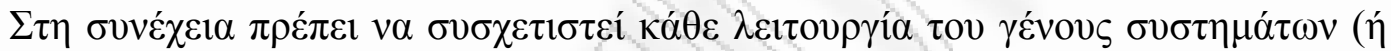

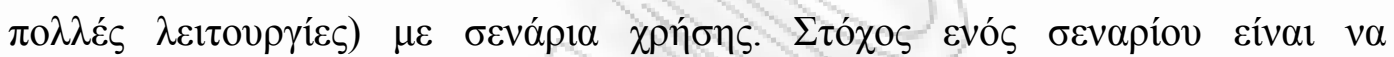

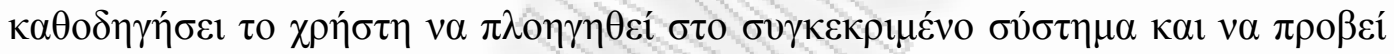

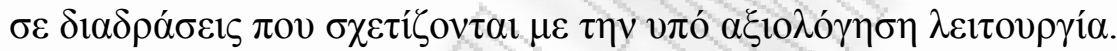

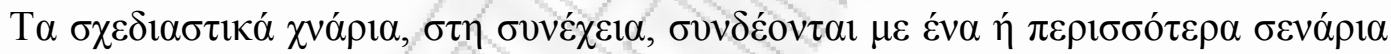

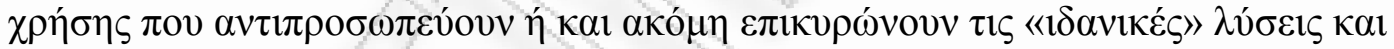

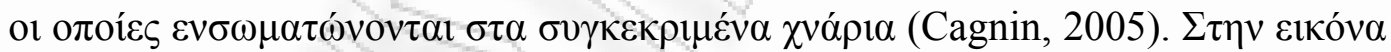

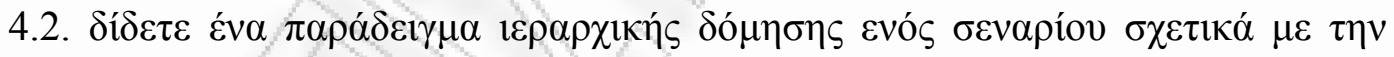

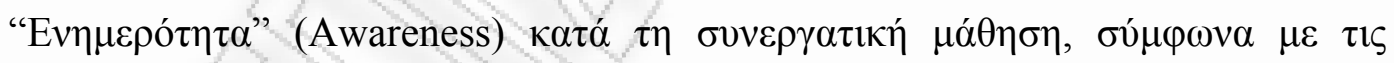

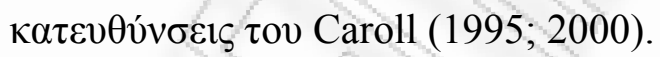




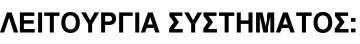

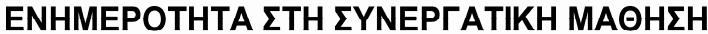

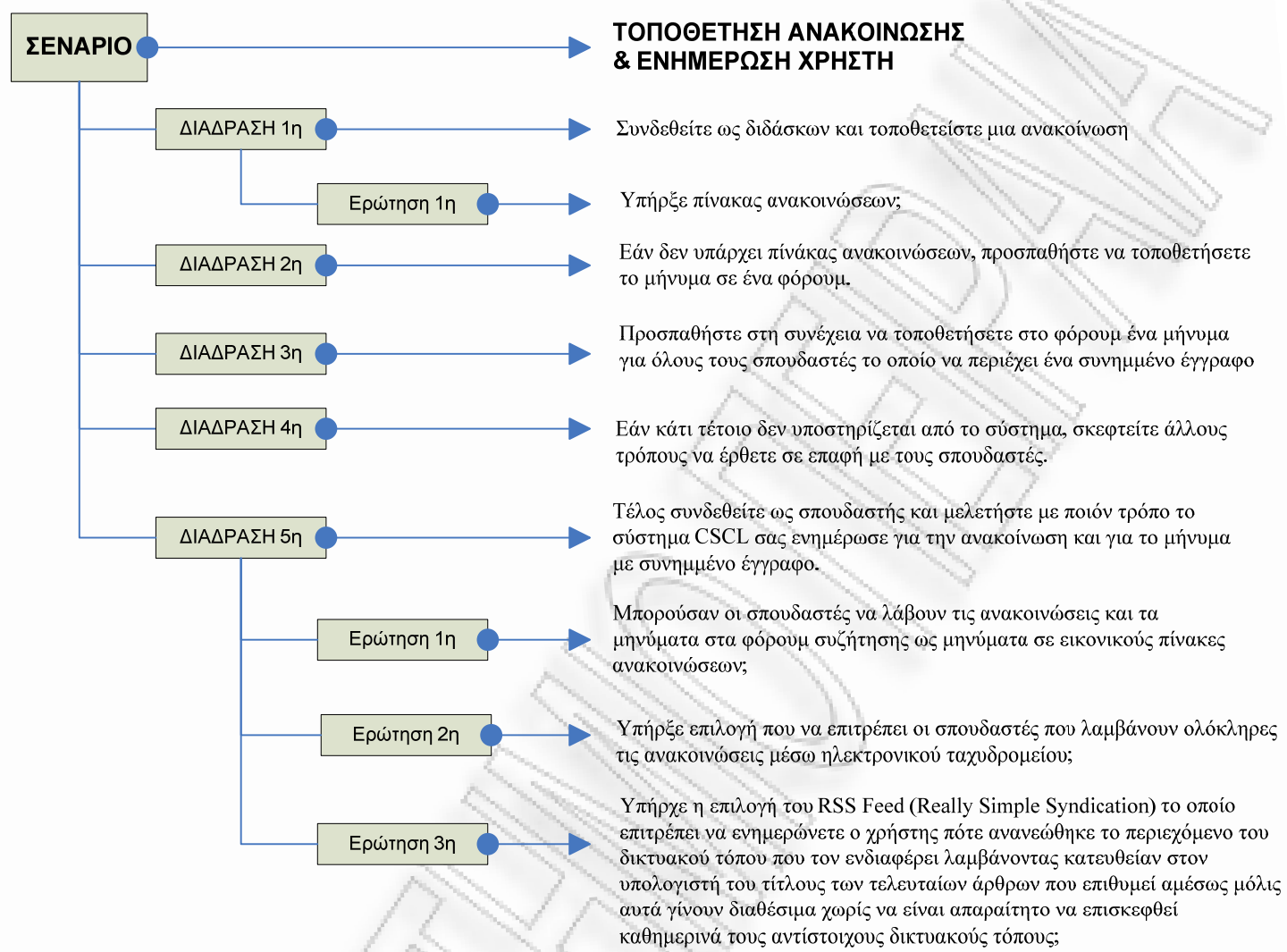

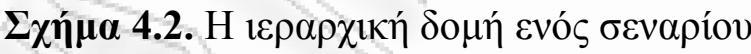

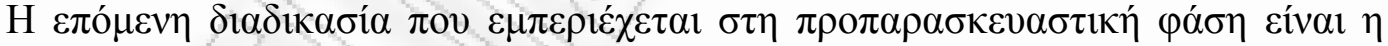

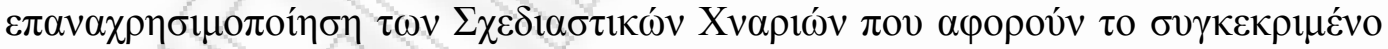

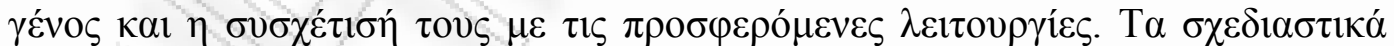

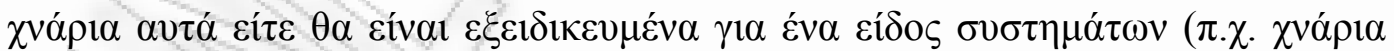

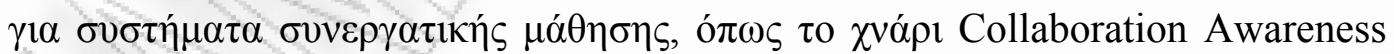

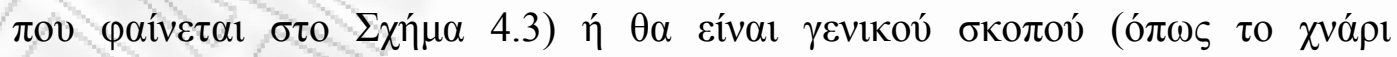
WYSIWYS editor). 


\section{Collaboration Awareness}

Problem Instead of the users looking for news and postings, can the latest news \& messages reach those interested?

Forces - One step message reading

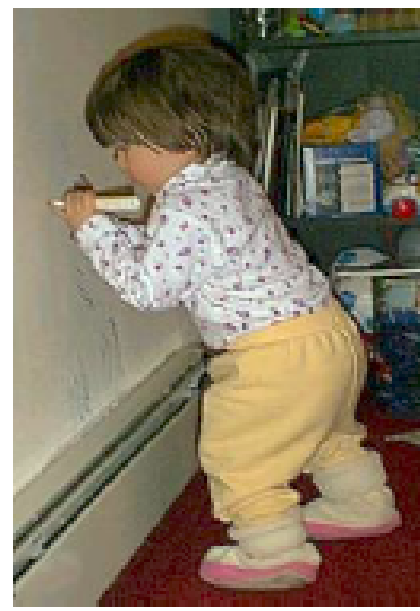

- Users want to avoid wasting time searching through the CSCL every day for new postings.

- Users need to be notified, every time a new posting/message has been posted

- Users should be informed when a teacher sends messages with attachment files with important material for the learners

- Users sometimes need to block some community members so they won't receive specific notification from them

Solution Awareness of activity increases the quality of asynchronous collaboration.

Automatic briefing, for all kinds of newly placed statements, via notification of their choice (email, or SMS), can give the participants the opportunity to be notified about all the events that took or will take place, without them having to spend any time visiting the system. Also the choice of briefing or no briefing, according to subject, sender, or time period, should be an option (in the case the participant doesn't want to get this kind of briefing).

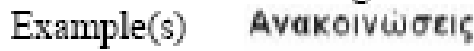

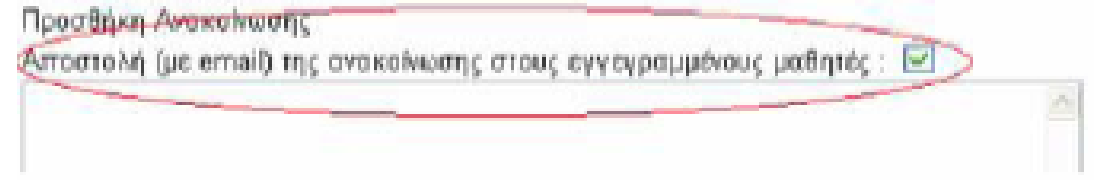

In the LMS E-class, when tutors post messages, they can decide whether they want to send those announcements to the registered students (or to selected ones) via e-mail. It would be nice to offer tutors

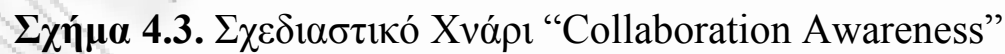

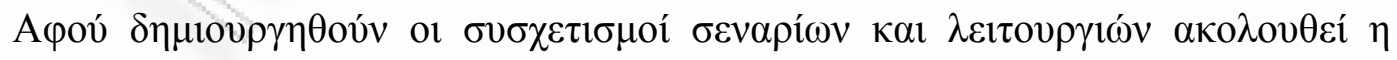

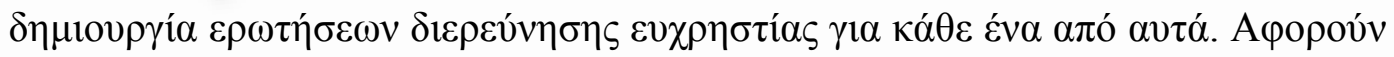

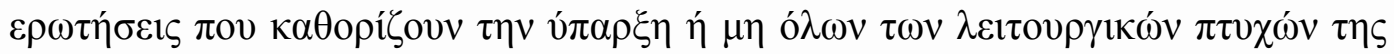

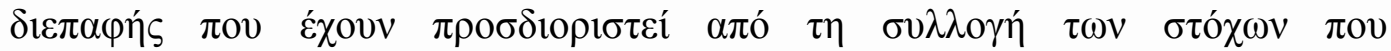




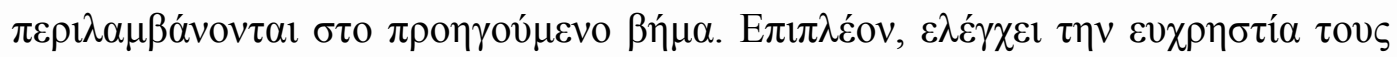

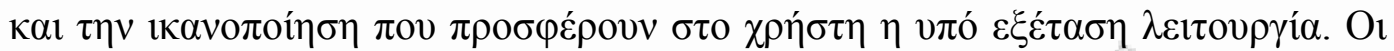

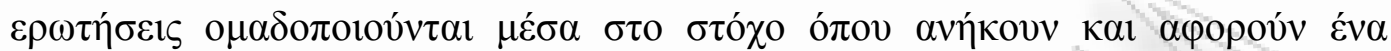

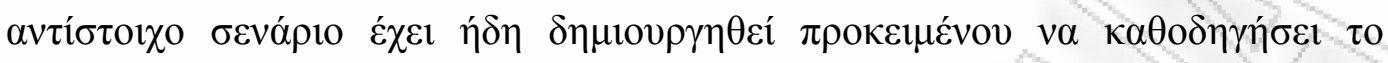

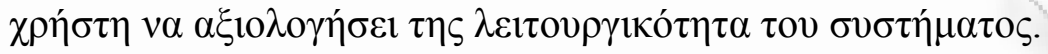

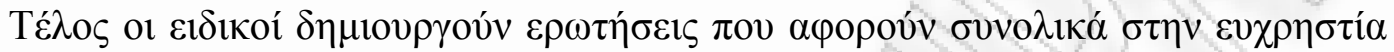

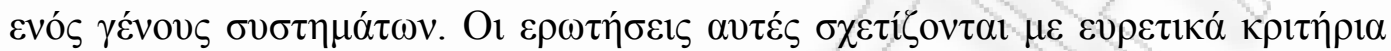

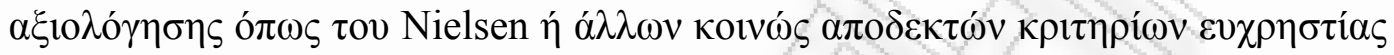

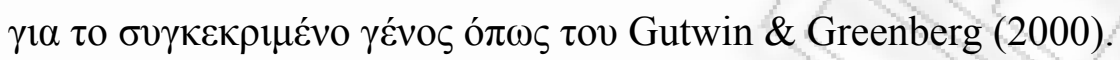

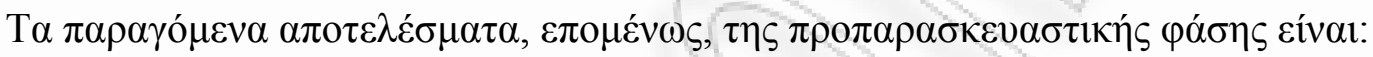

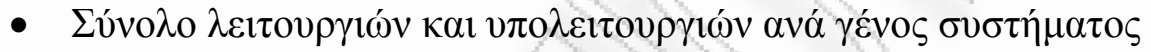

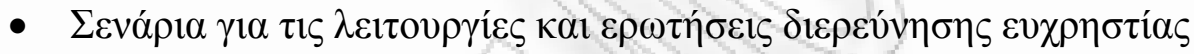

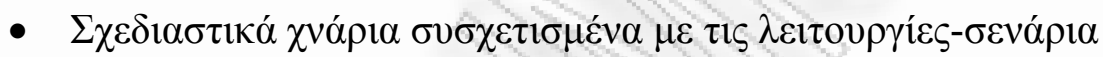

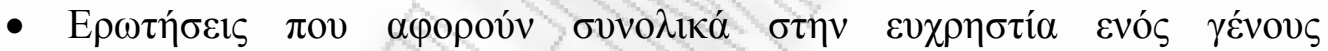
$\sigma v \sigma \tau \eta \mu \alpha ́ \tau \omega v$

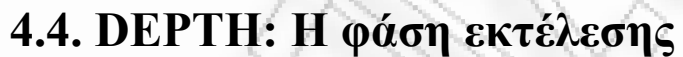

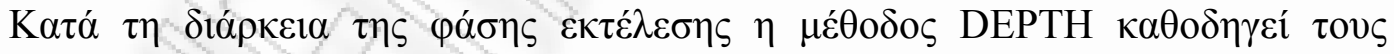

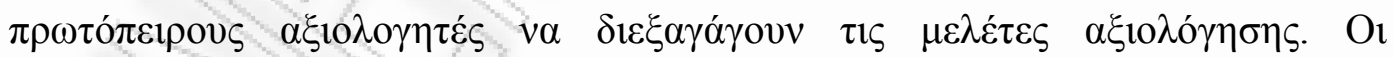

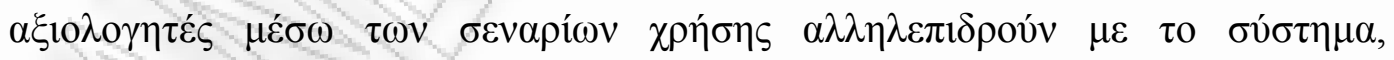

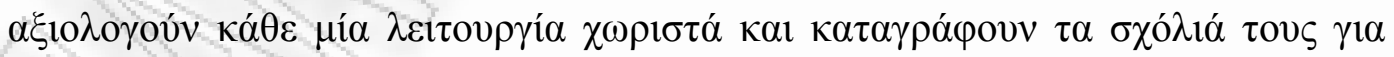

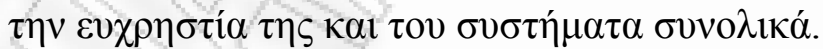

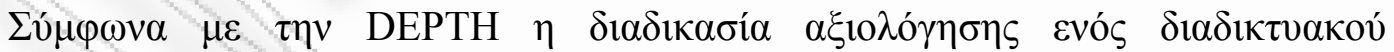

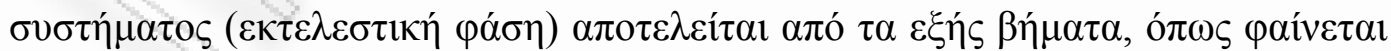

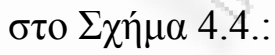

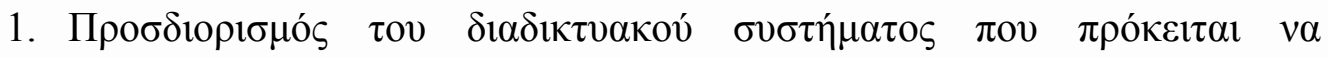

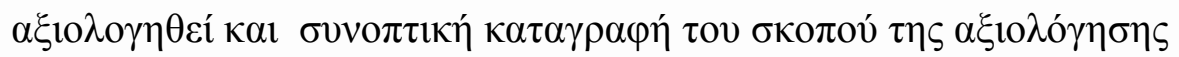




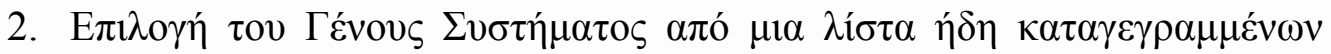

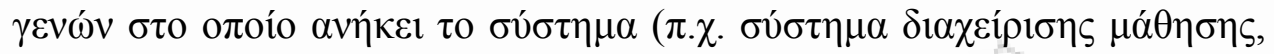

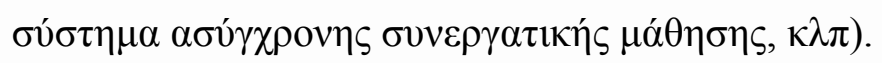

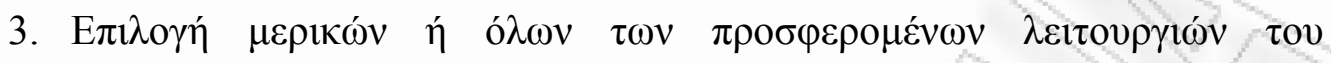

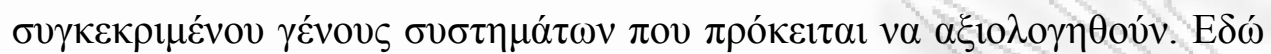

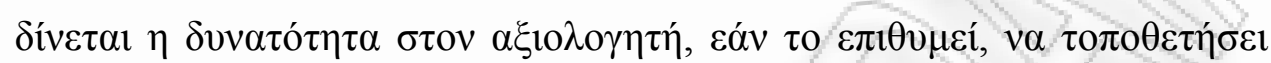

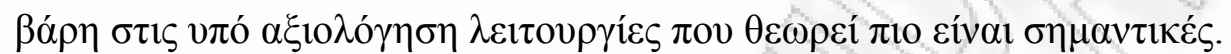

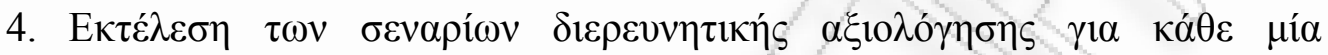

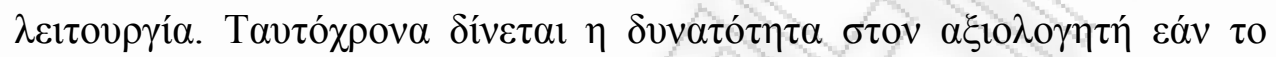

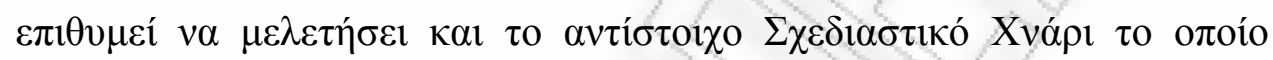

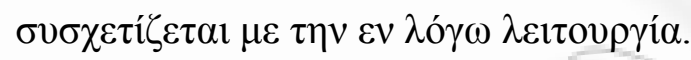

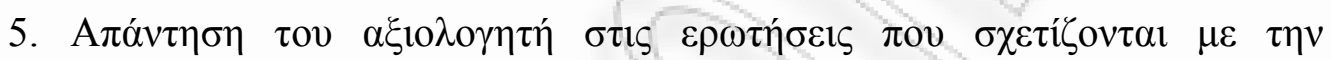

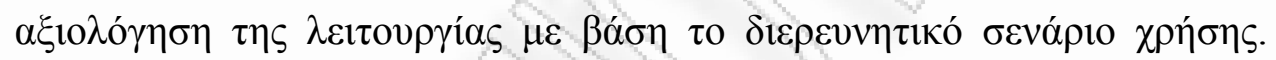

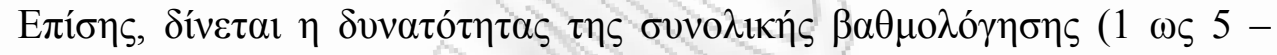

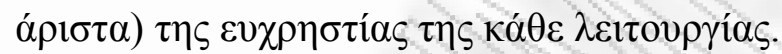

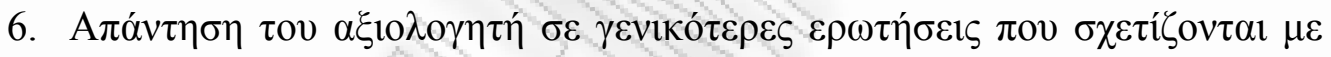

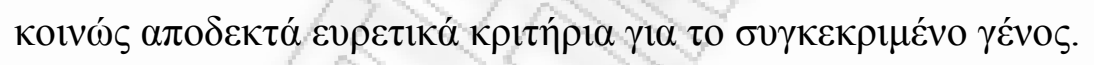

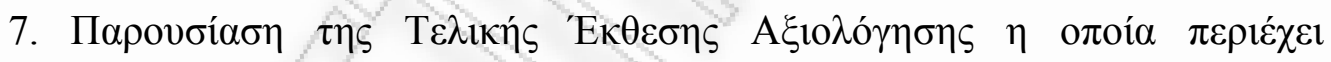

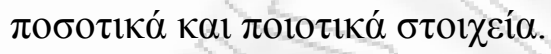




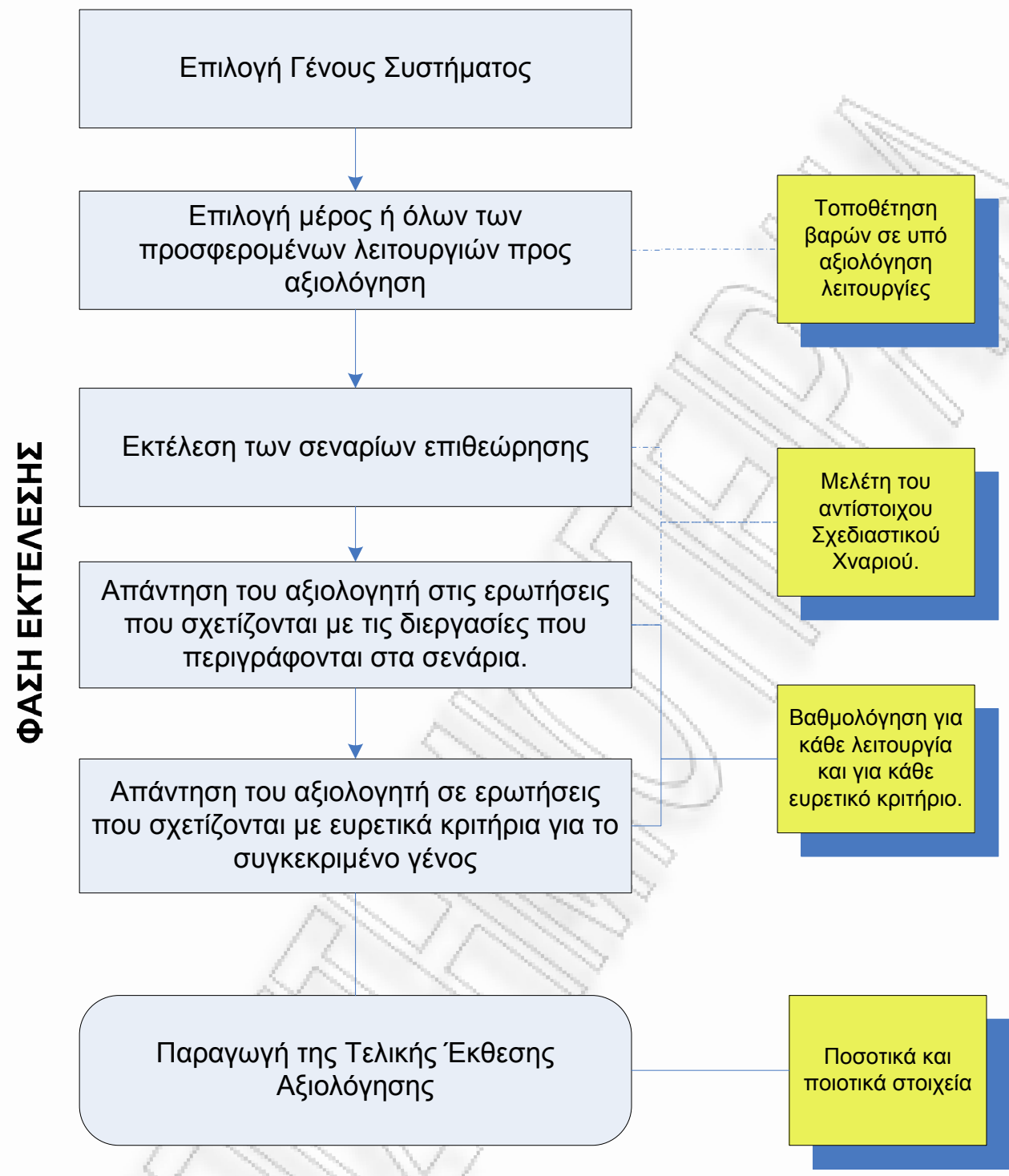

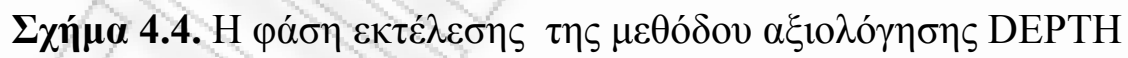

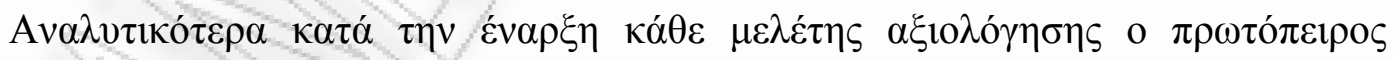

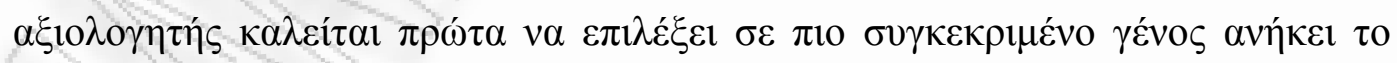

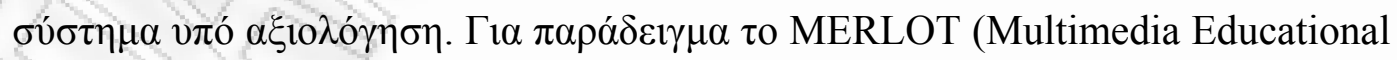
Resource for Online Learning and Teaching, (www.merlot.org)) $\alpha \vee \eta ́ \kappa \varepsilon \iota ~ \sigma \tau o ~ \gamma \varepsilon ́ v o \varsigma$

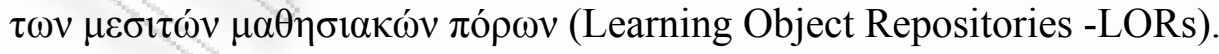

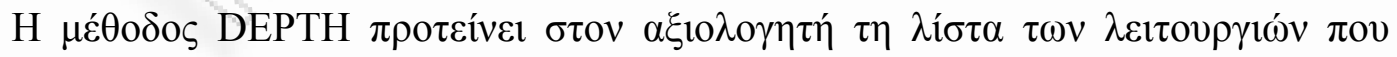

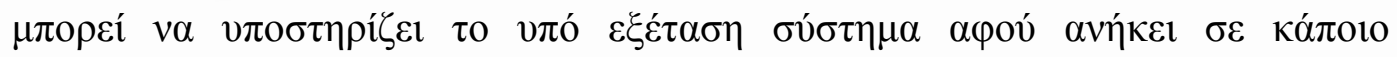

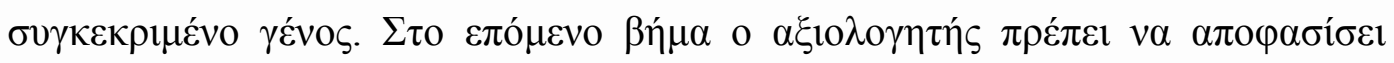

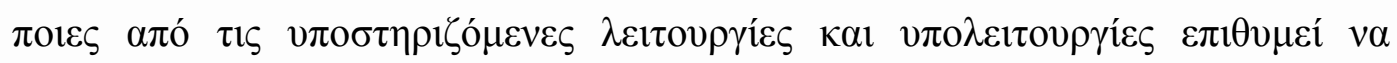


$\alpha \xi$ io

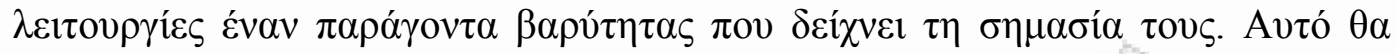

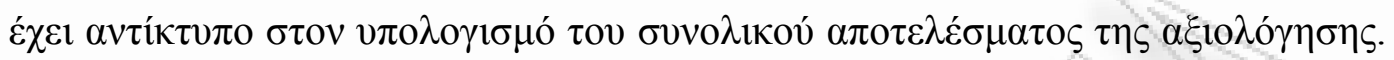

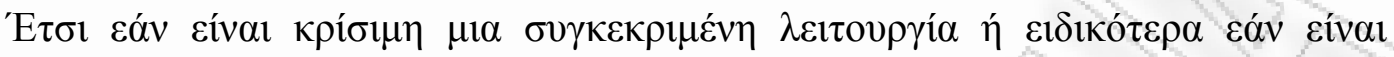

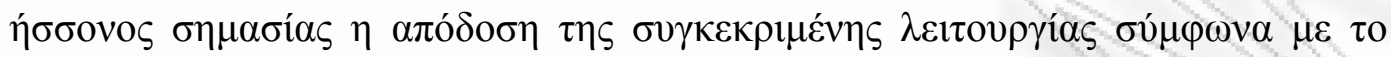

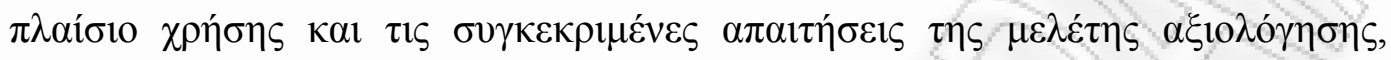

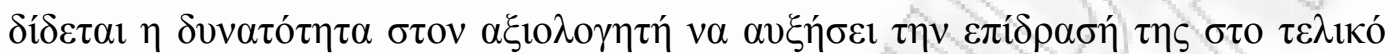

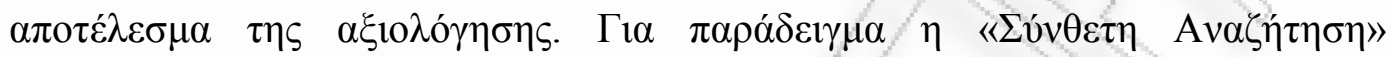

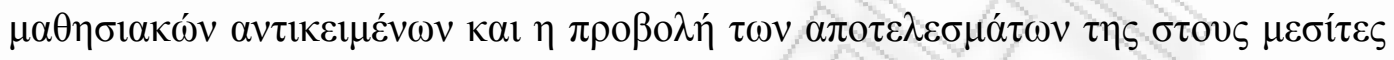

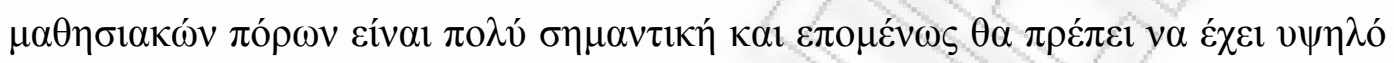

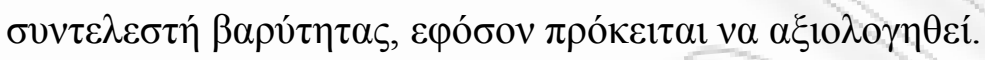

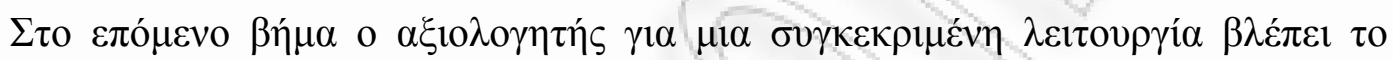

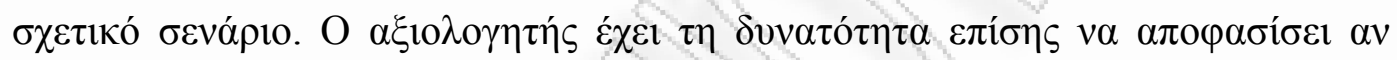

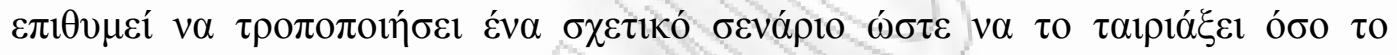

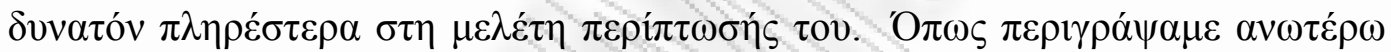

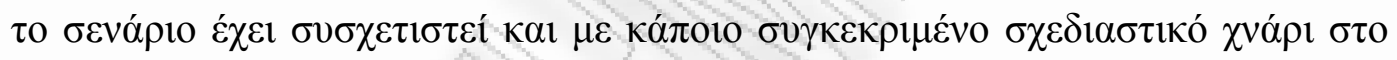

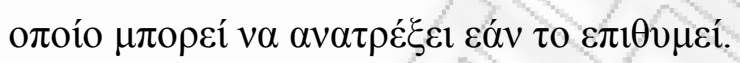

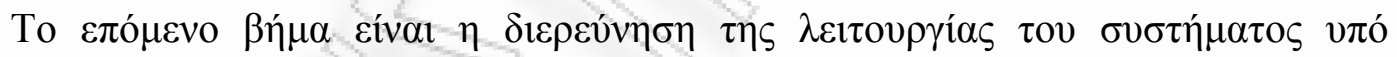

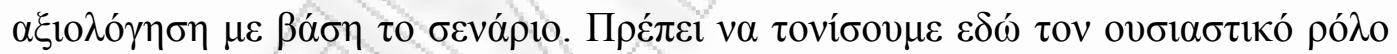

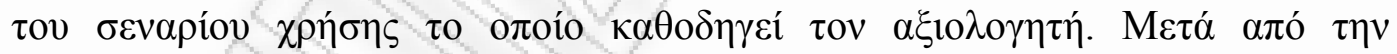

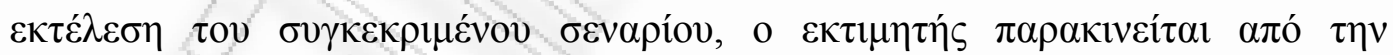

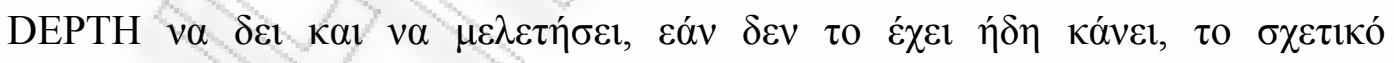

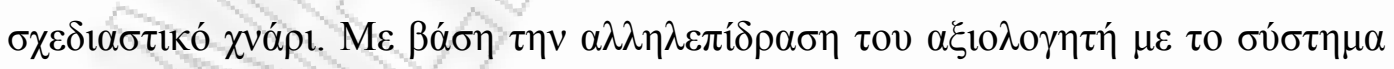

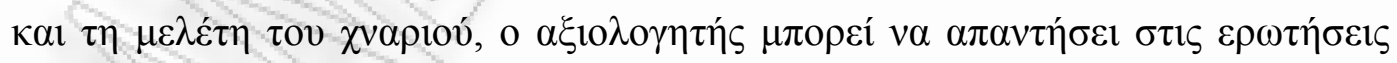

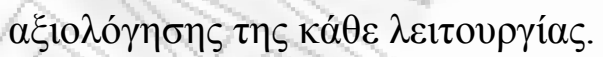

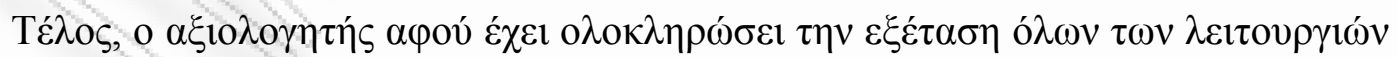

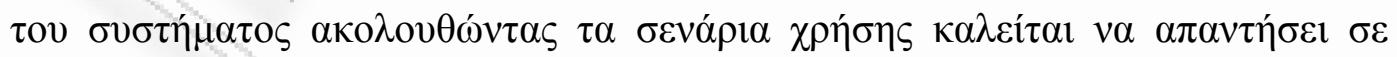

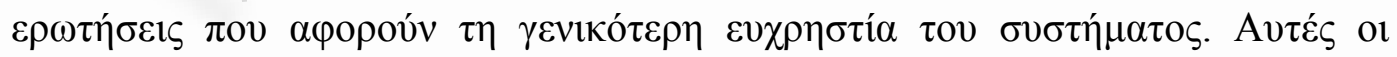

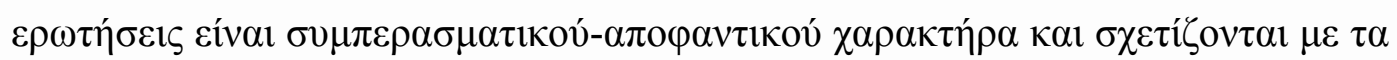

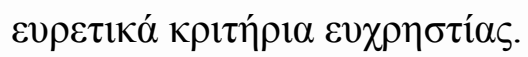




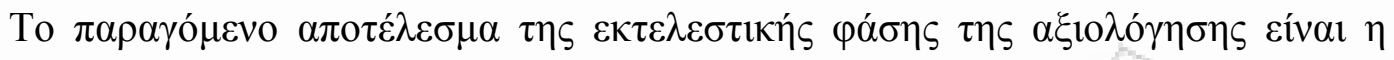

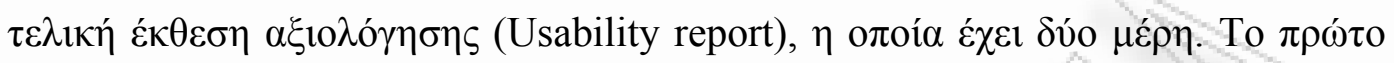

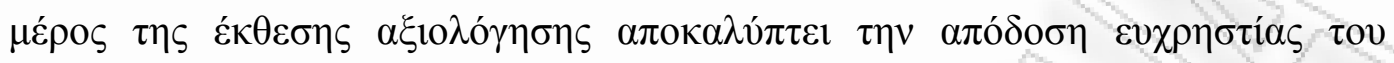

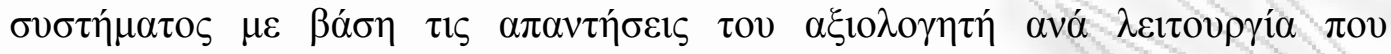

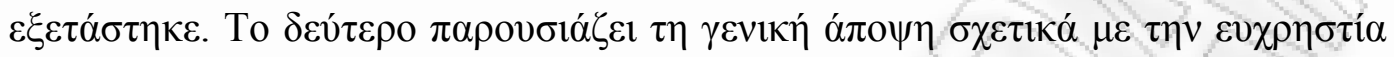

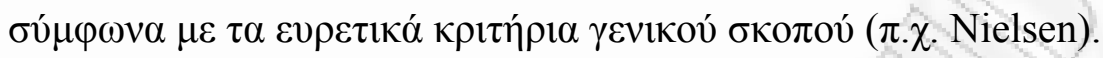

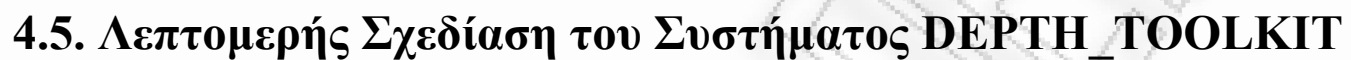

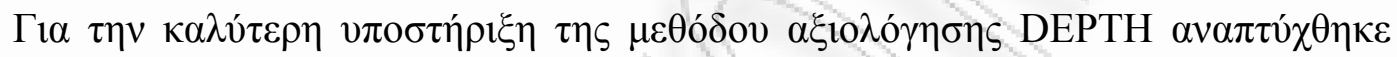

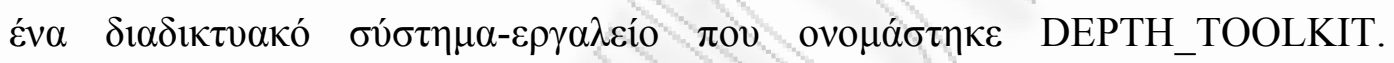

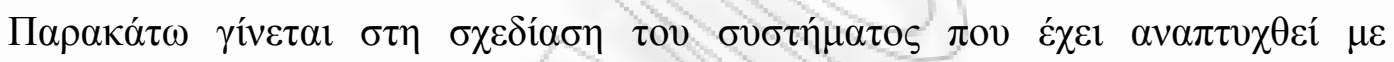

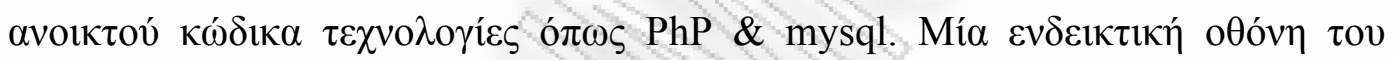

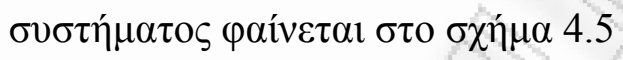

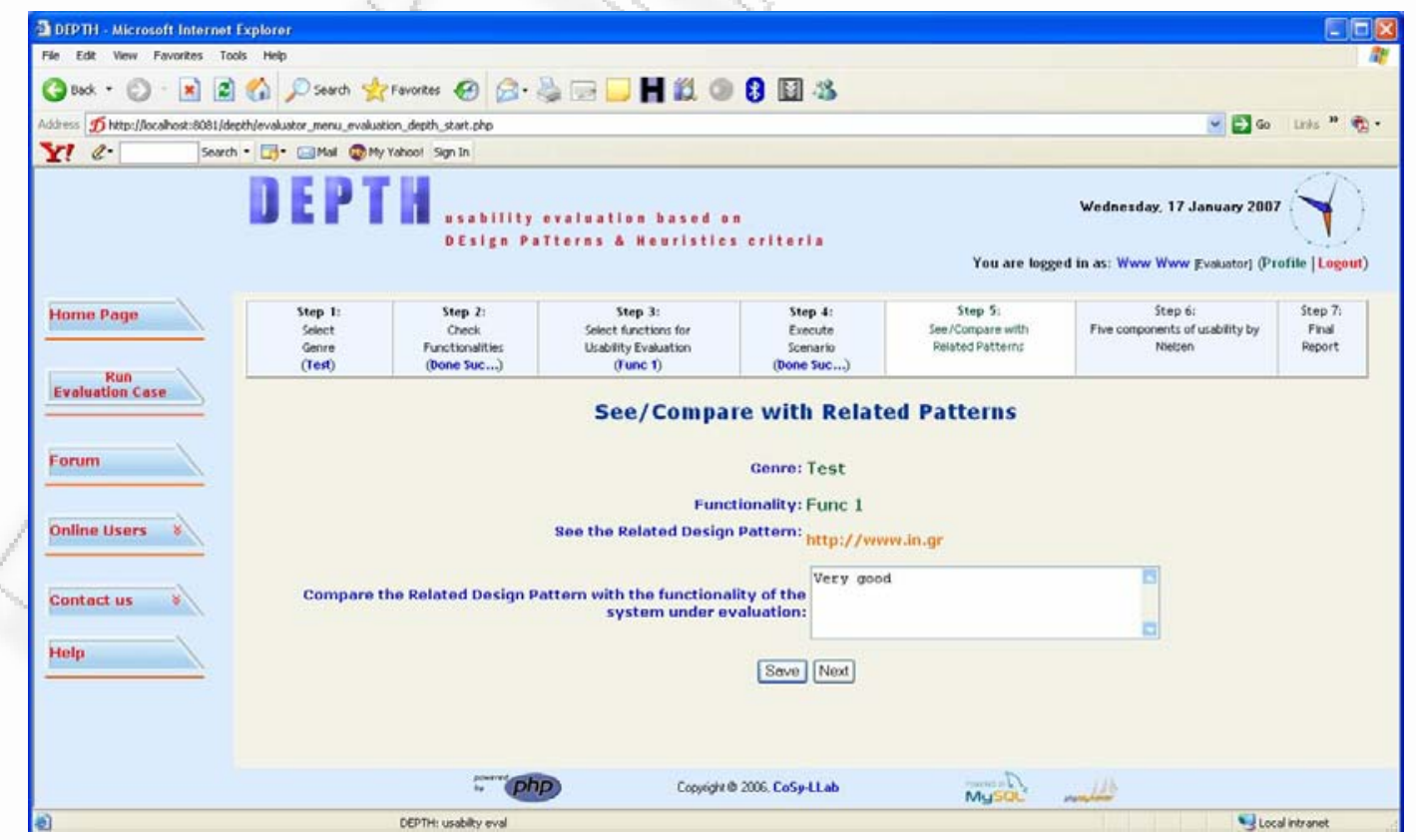

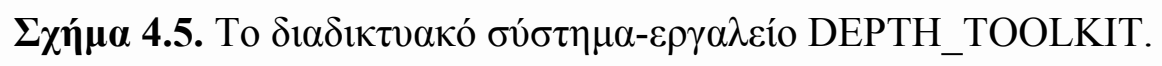




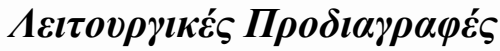

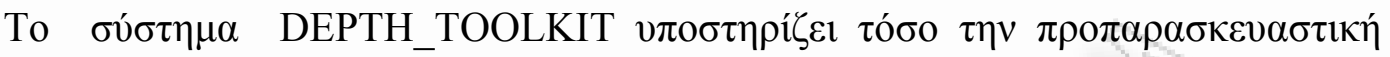

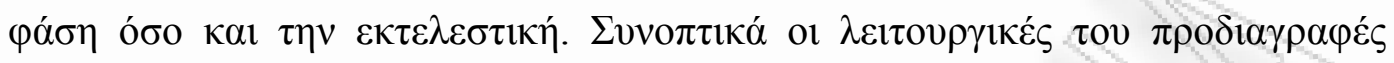
cíval:

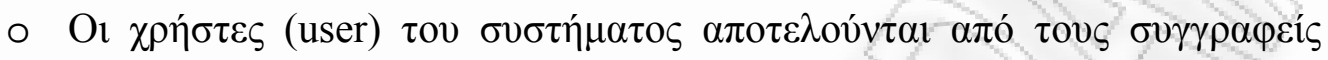

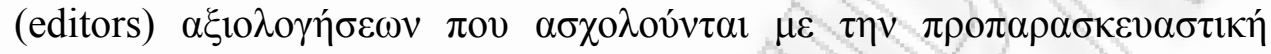

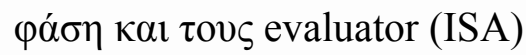

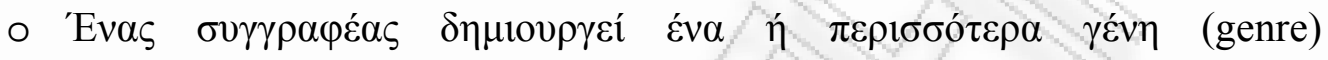
$\sigma v \sigma \tau \eta \mu \alpha ́ \tau \omega \nu$

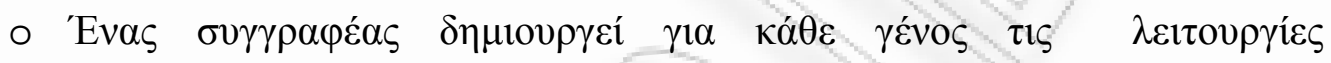
(functionalities) $\tau$ ov

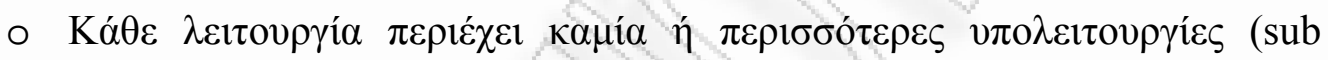
functionality).

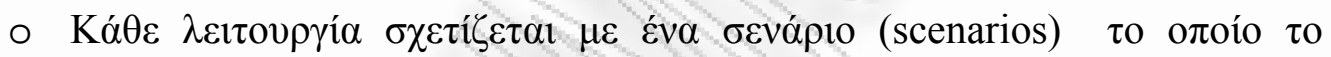
$\pi \rho о \sigma \delta 10 \rho i \zeta \zeta \varepsilon 1 ~ о ~ \sigma v \gamma \gamma \rho \alpha \varphi \varepsilon ́ \alpha \varsigma$

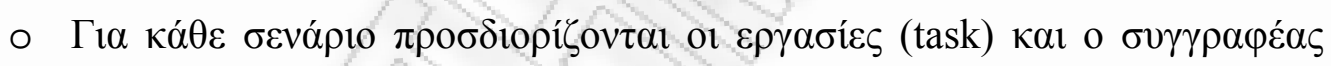

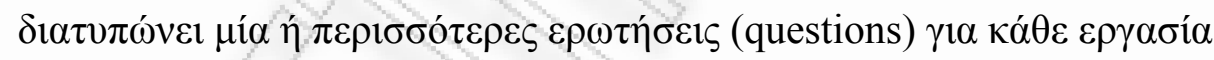

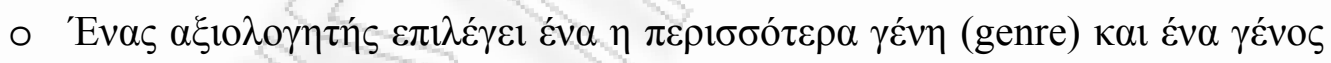

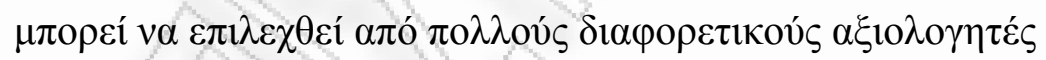

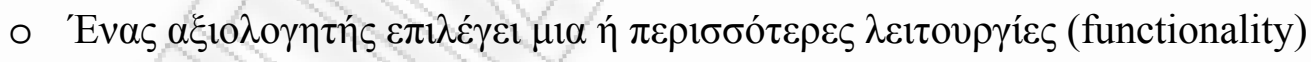

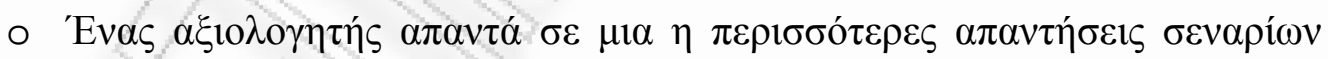
(scenarios_answer)

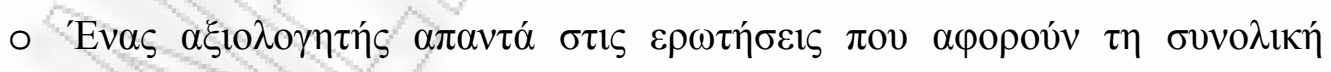

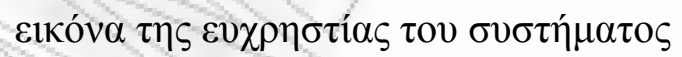

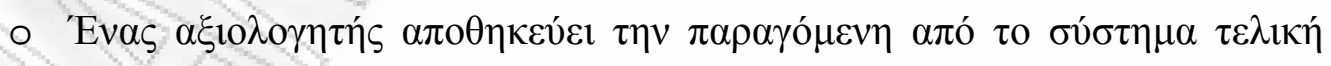

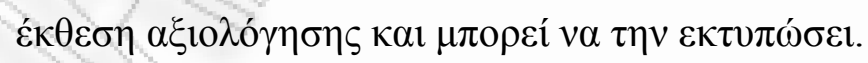

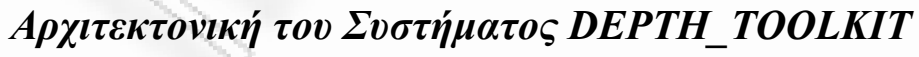

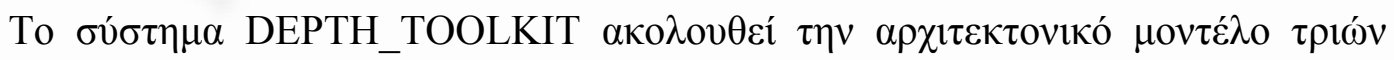

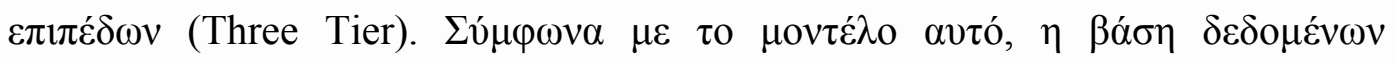

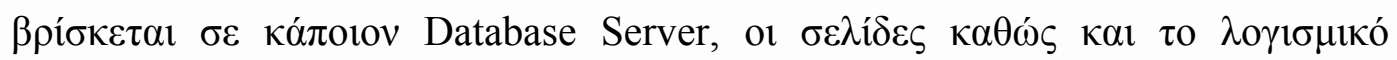




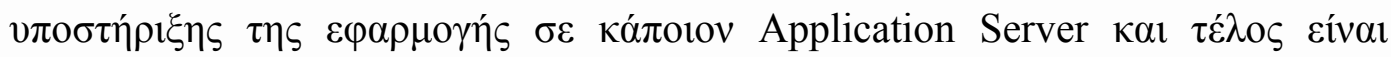

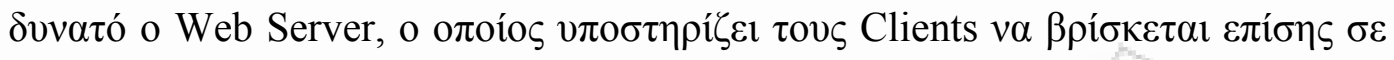

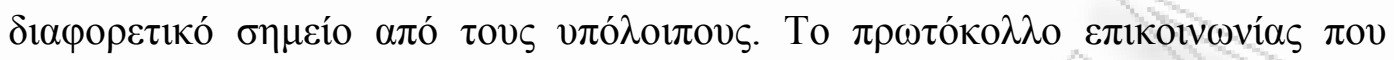

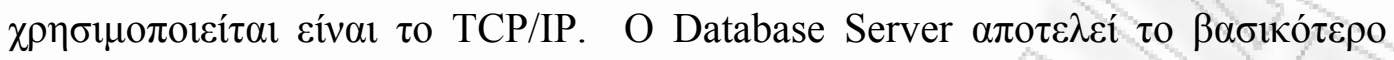

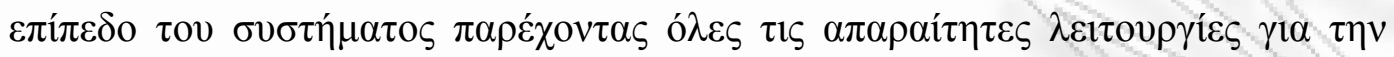

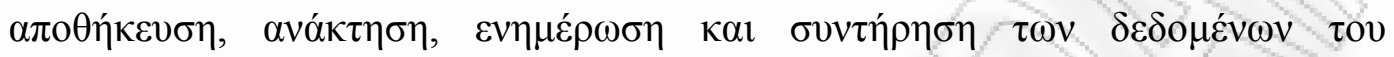

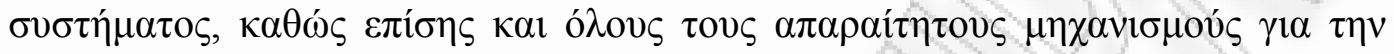

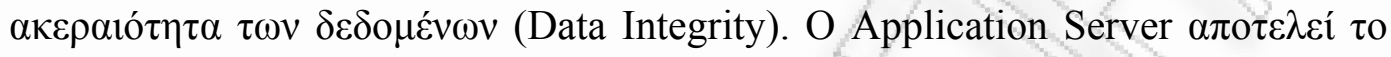

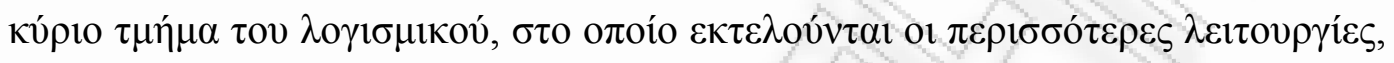

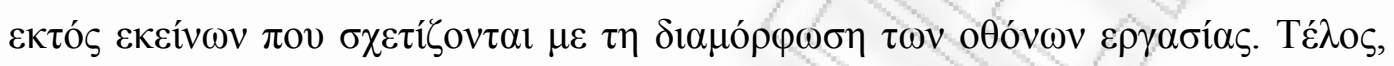

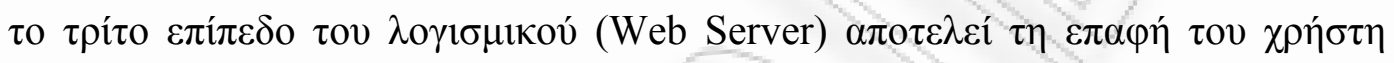

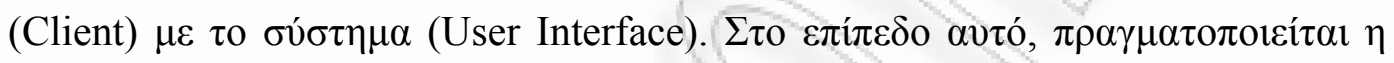

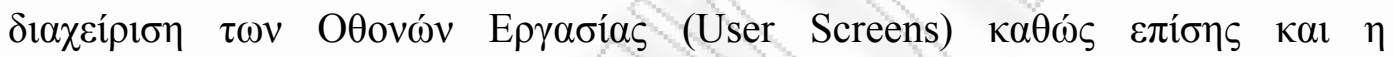

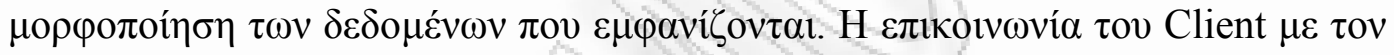

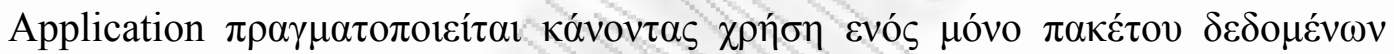

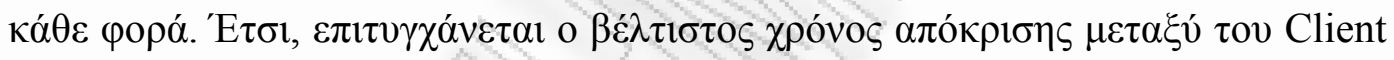

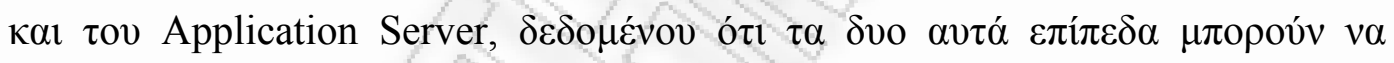

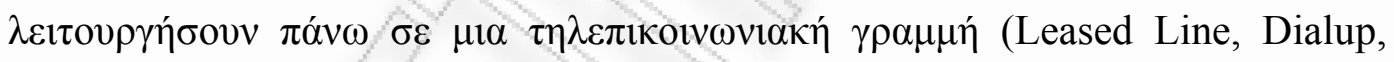

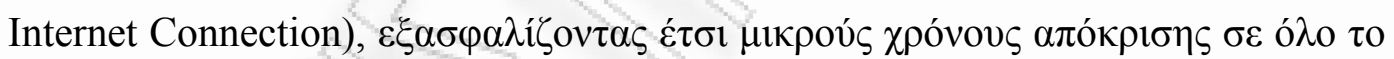

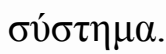

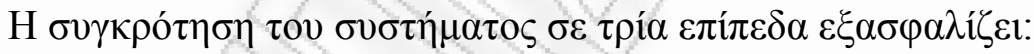

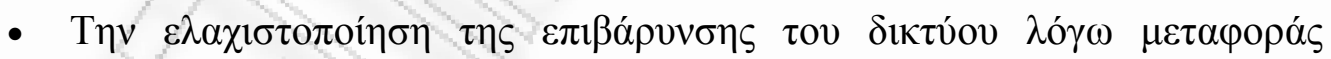

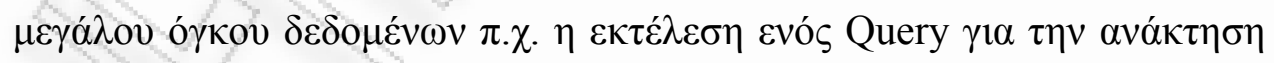

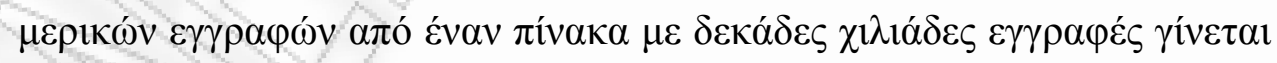

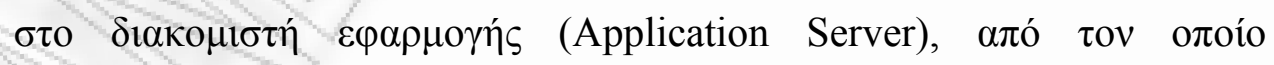

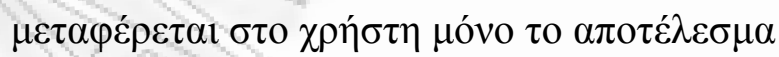

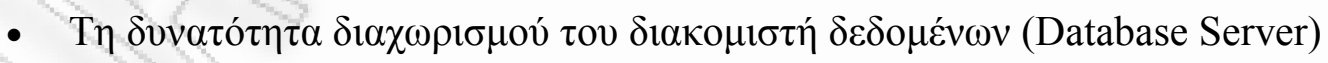

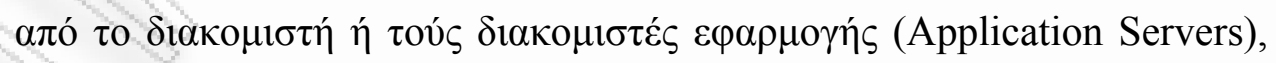

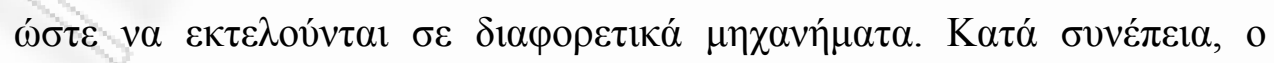

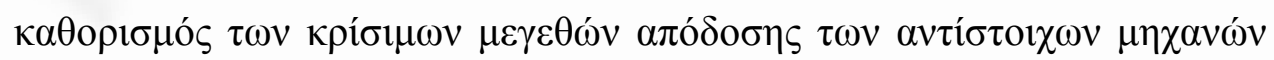

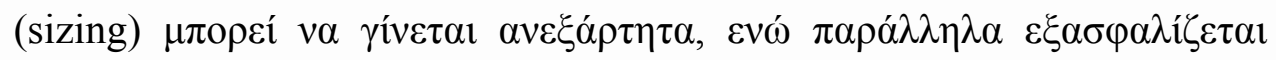

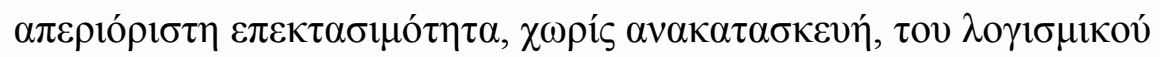




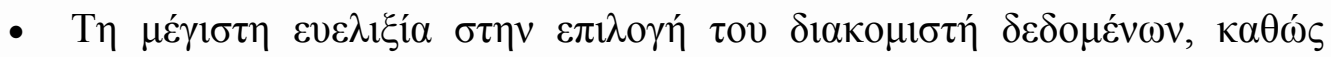

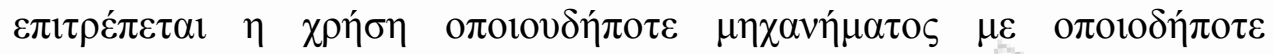

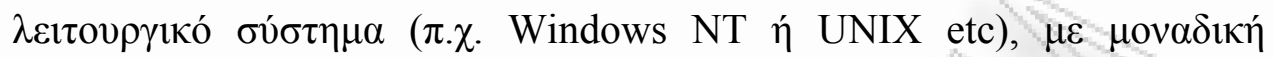

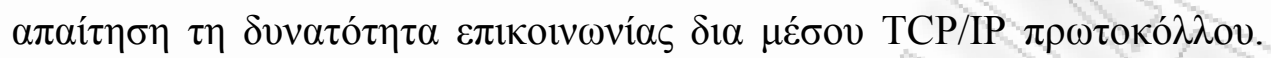

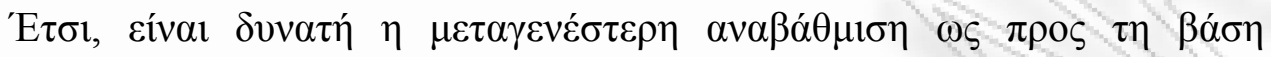

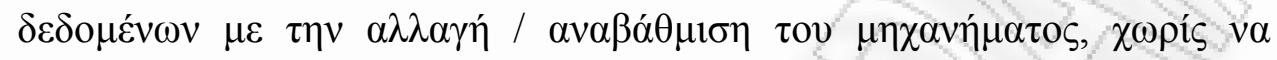

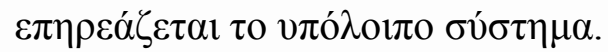

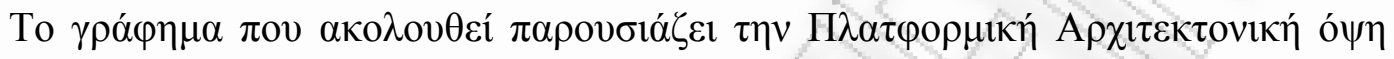

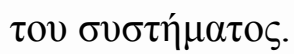

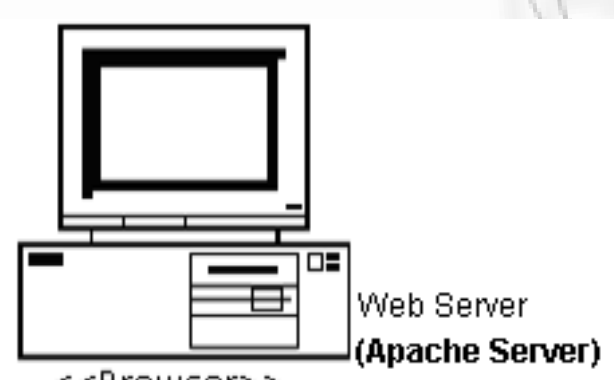

$<<$ Browser $>$

Machine with Internet Browser

HTTP

Internet Protocol

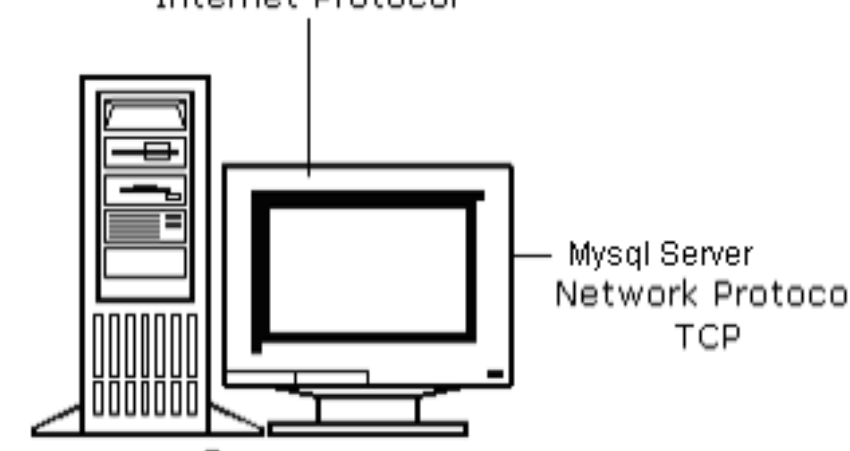

$<<$ Server $>>$

Machine with Application Server

(Web site \& PHP)

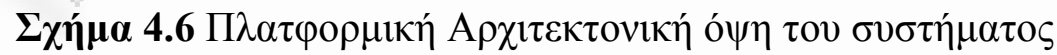

\section{$B a ́ \sigma \eta \Delta \varepsilon \delta \sigma \mu \varepsilon ́ v \omega v$}




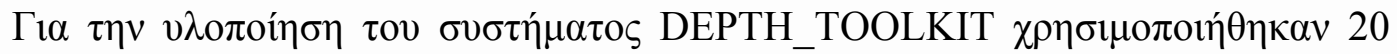

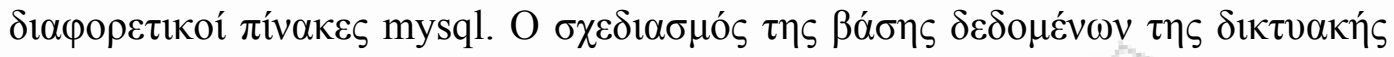

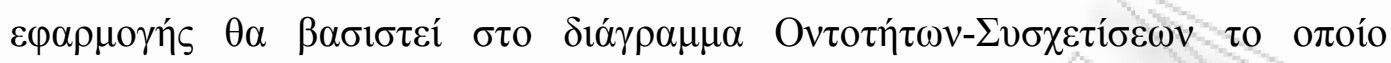

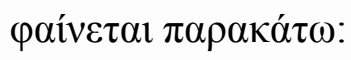




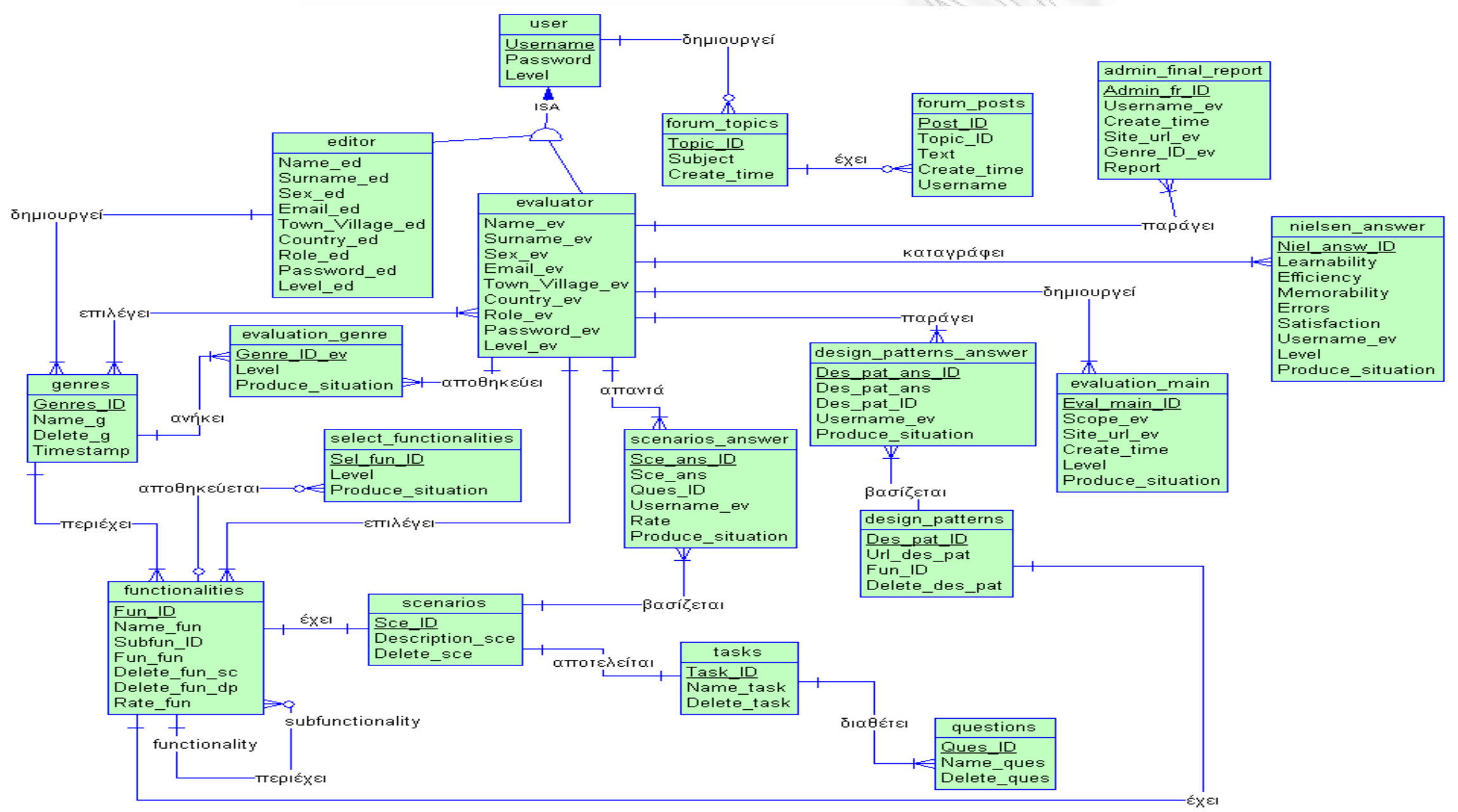

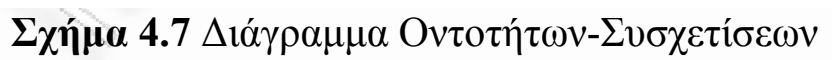




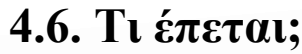

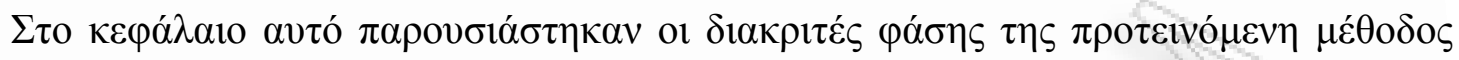

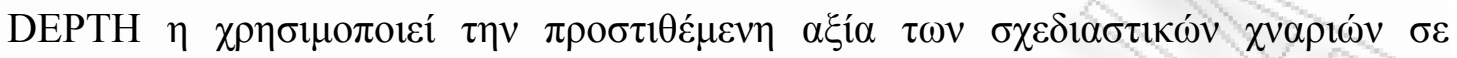

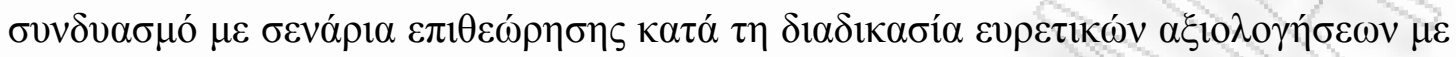

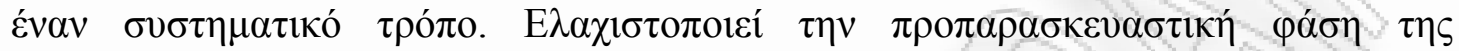

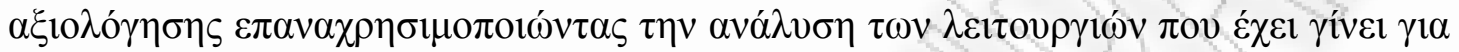

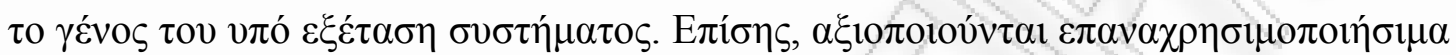

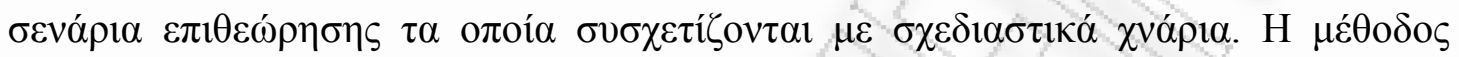

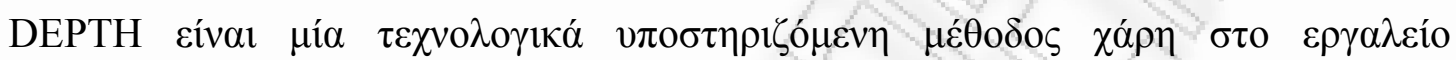
DEPTH_TOOLKIT.

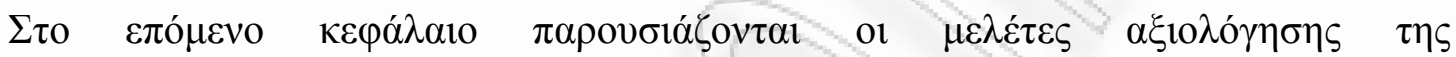

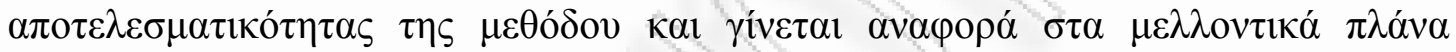

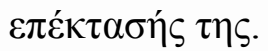




\section{КЕФАМАIO 5

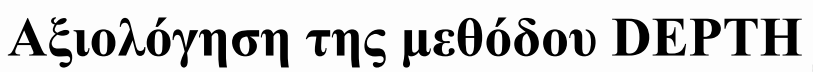

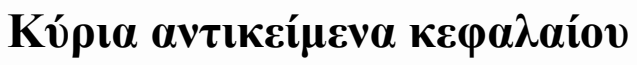

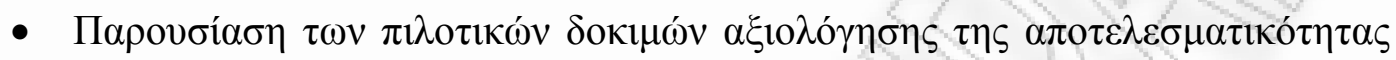
$\tau \eta \varsigma \mu \varepsilon \theta$ ó $\mathrm{ov}$ DEPTH.

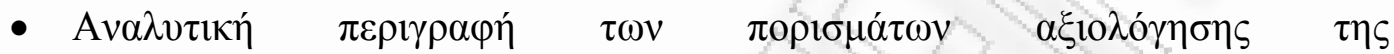

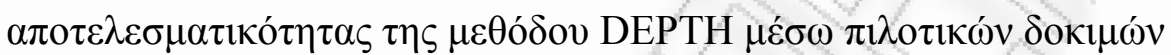

\subsection{A

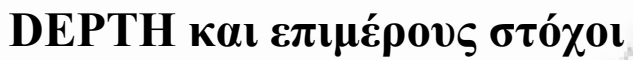

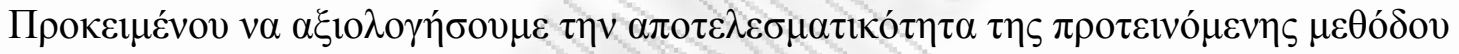
DEPTH

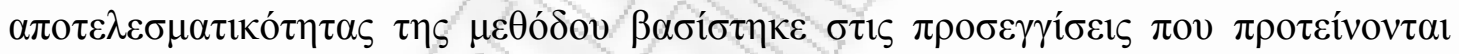

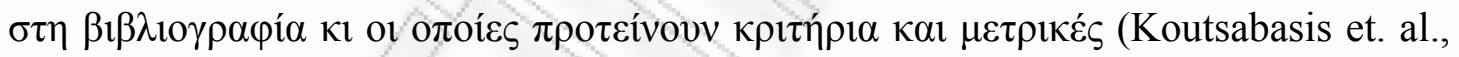
2007) ó $\pi \omega \varsigma:$

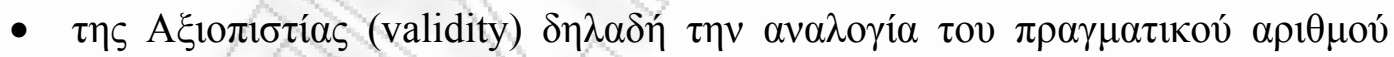

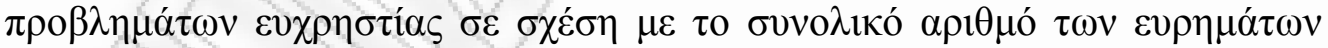

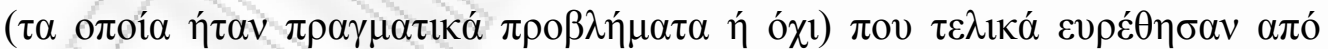

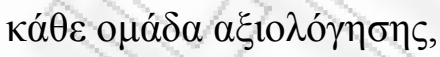

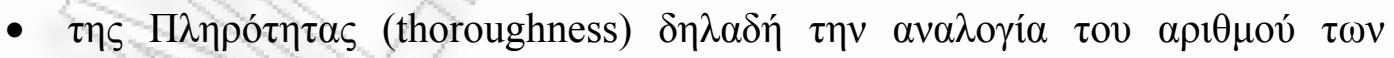

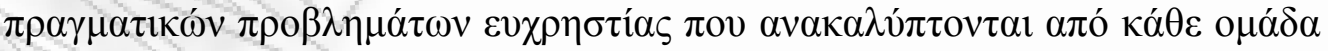

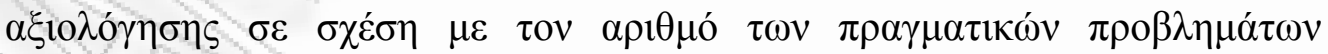

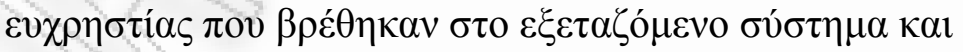

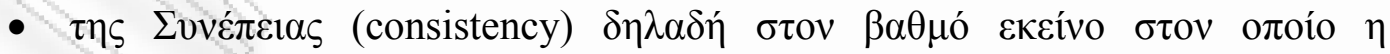

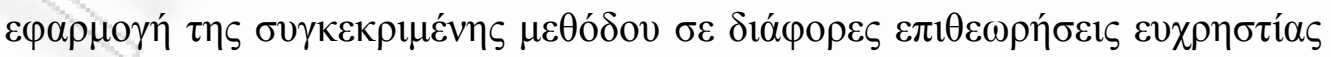

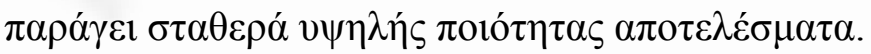

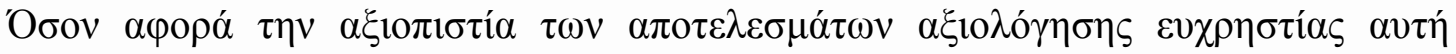

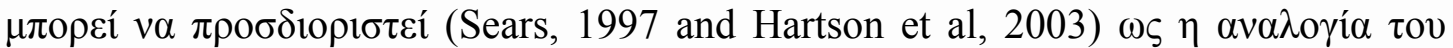

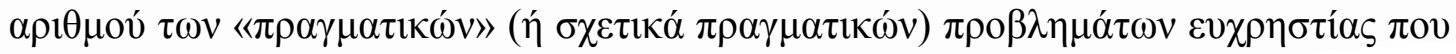




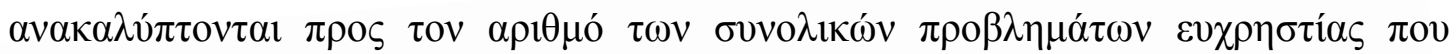

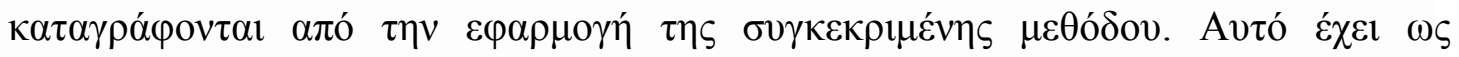

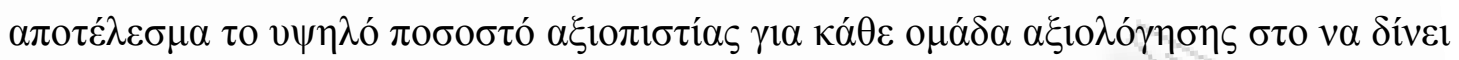

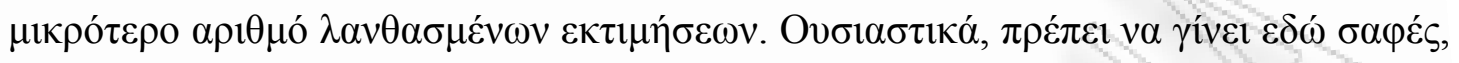

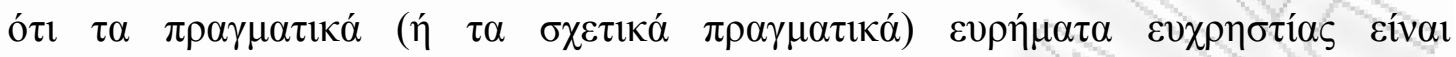

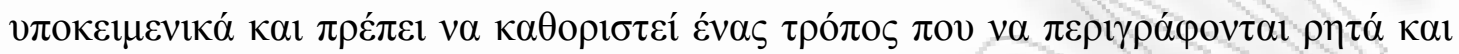

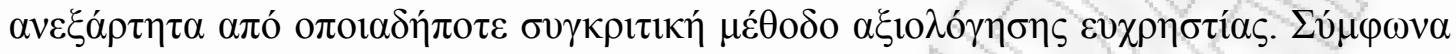

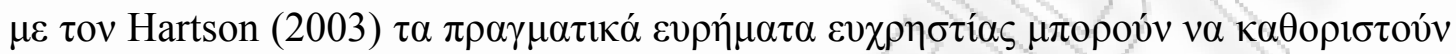

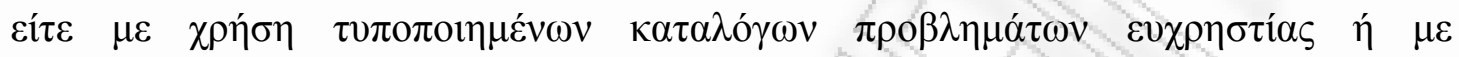

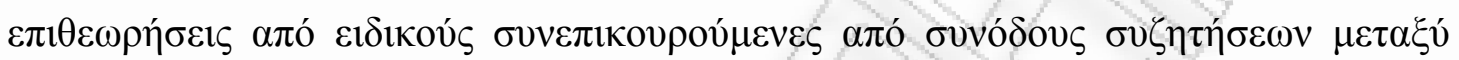

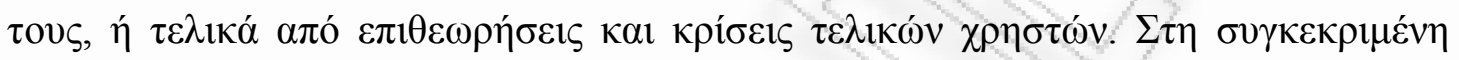

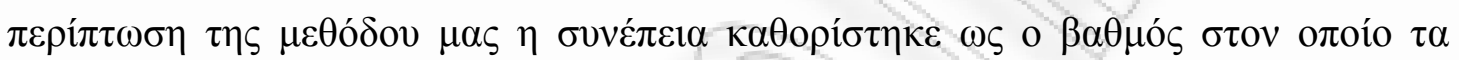

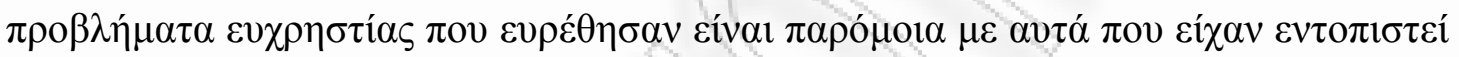

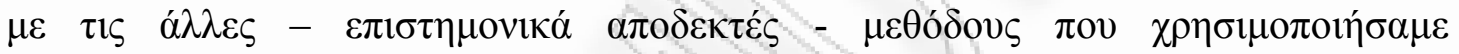

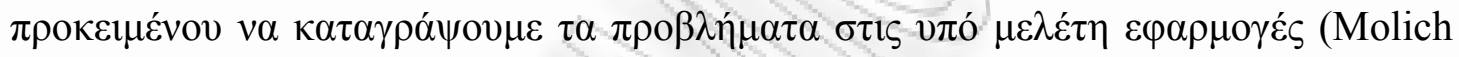
et al (2004).

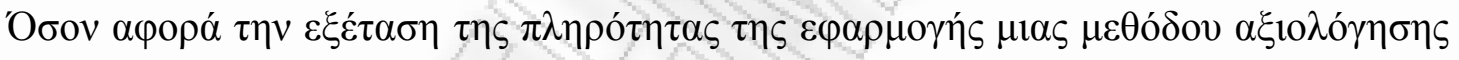

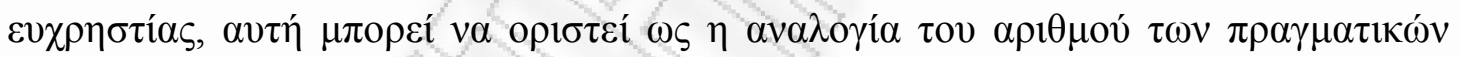

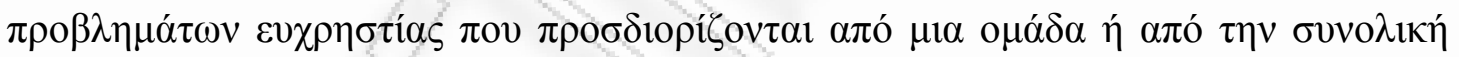

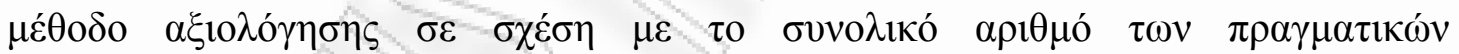

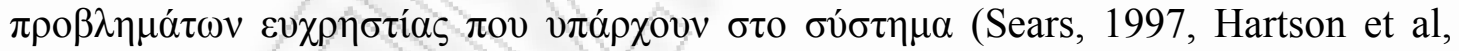

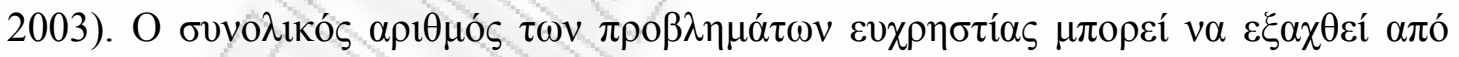

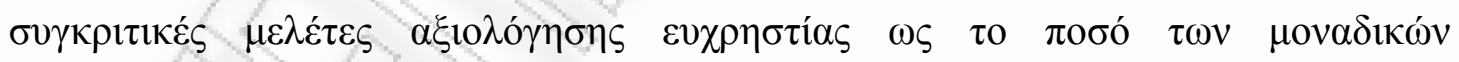

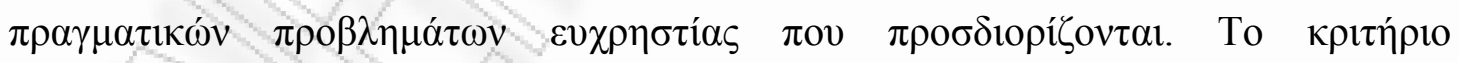

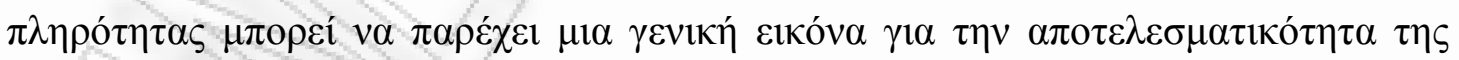

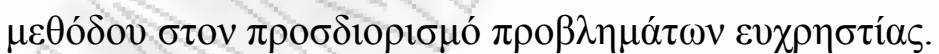

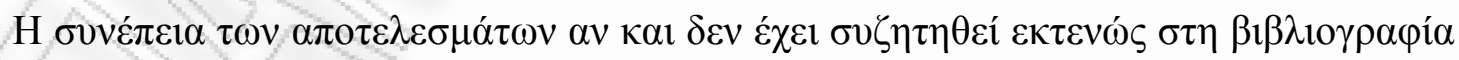

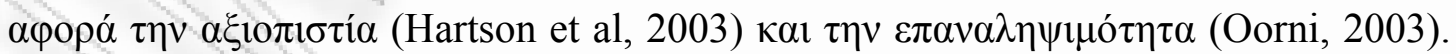

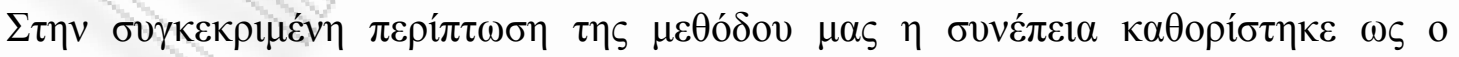

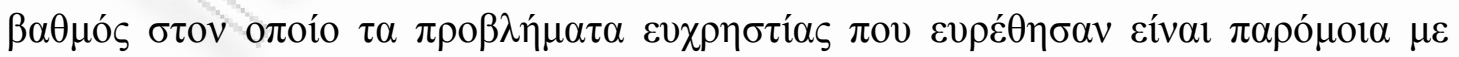

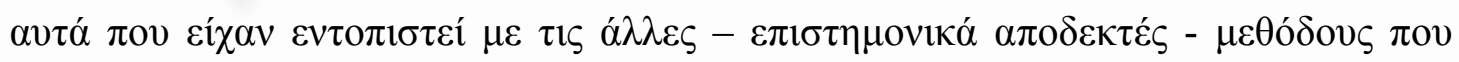

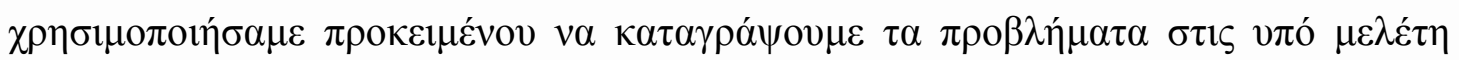
$\varepsilon \varphi \alpha \rho \mu о \gamma \varepsilon \dot{\varsigma}$ (Molich et. al., 2004). 


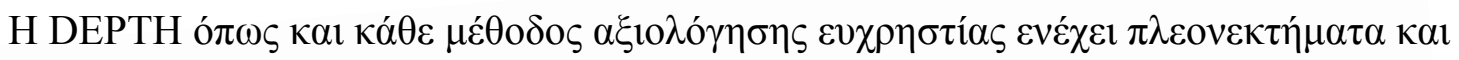

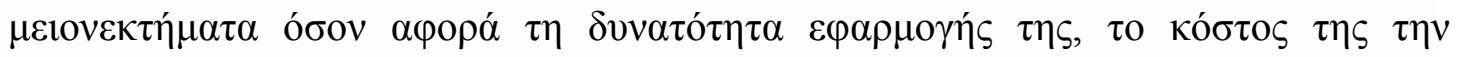

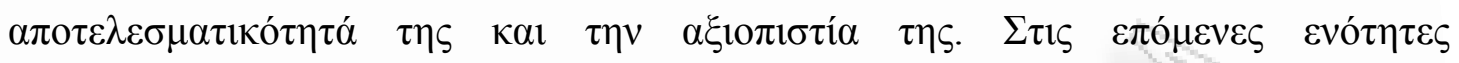

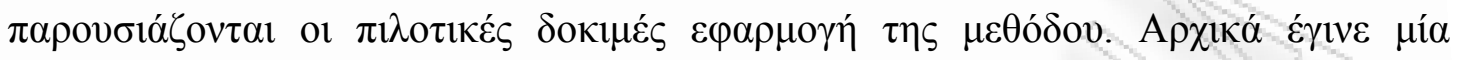

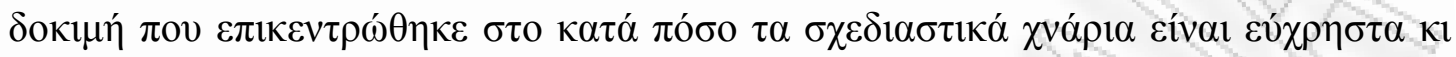

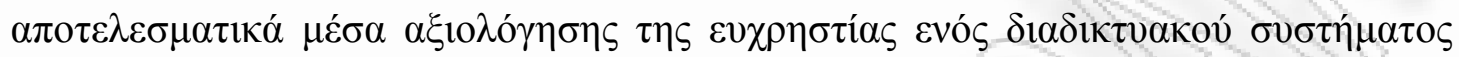

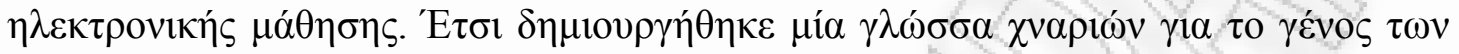

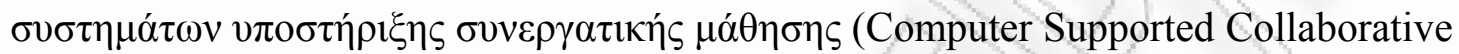

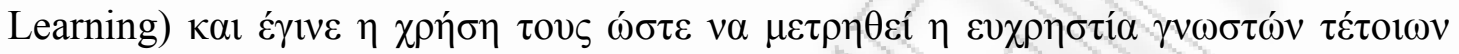

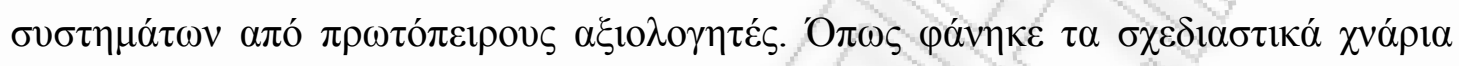

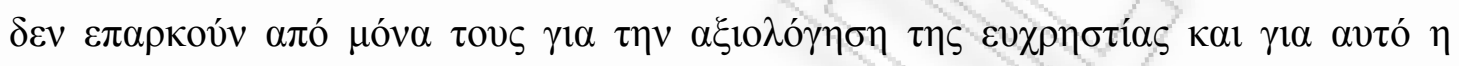

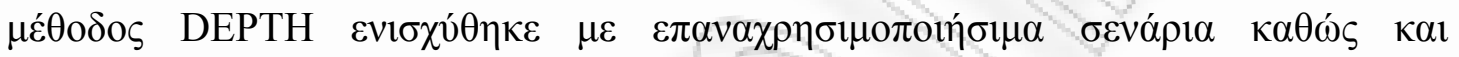

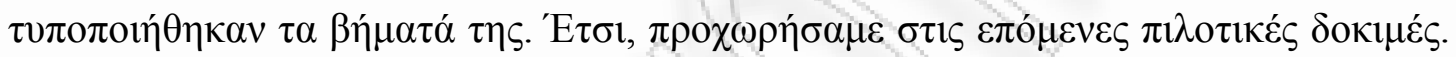

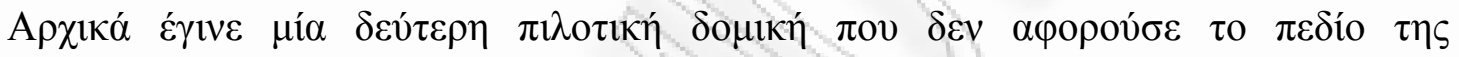

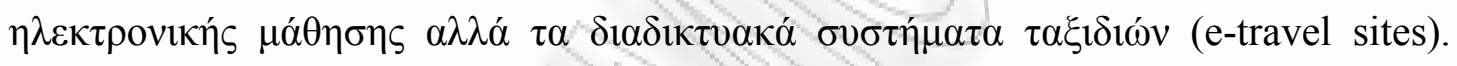

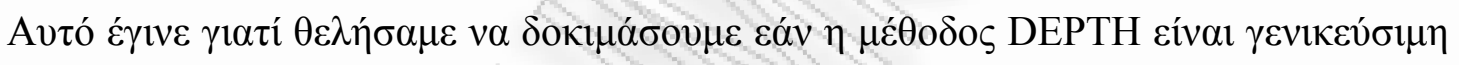

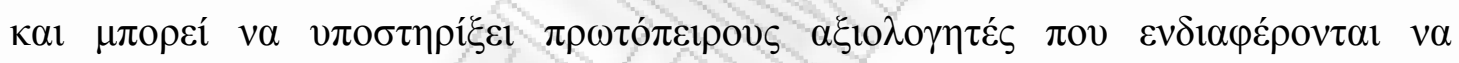

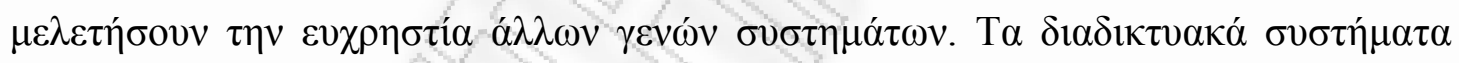

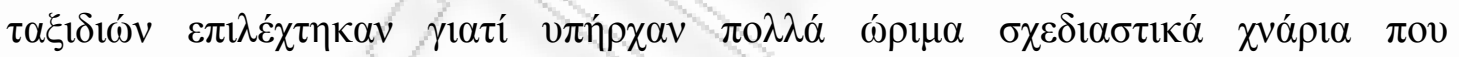

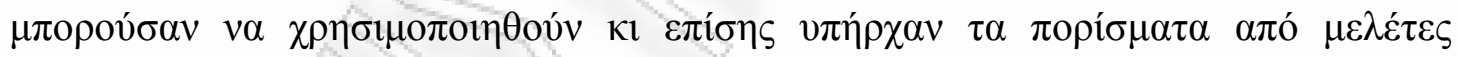

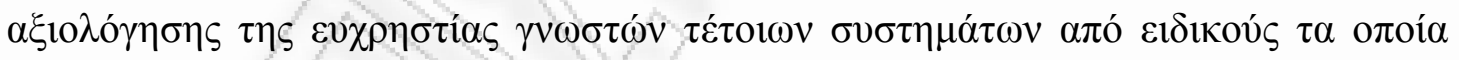

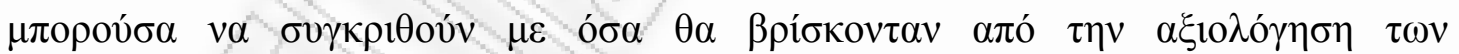

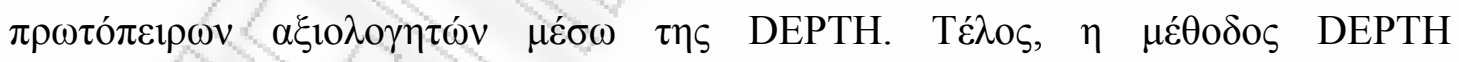

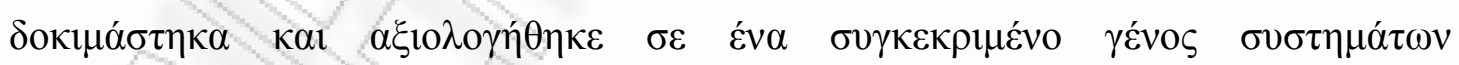

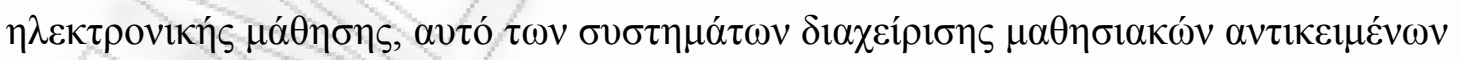

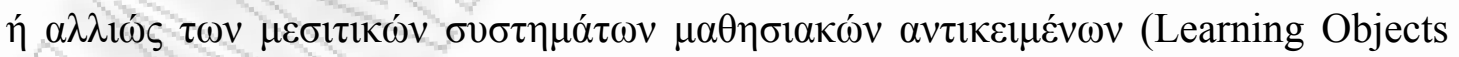

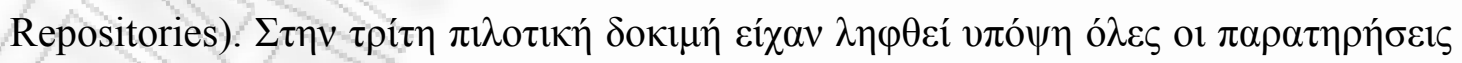

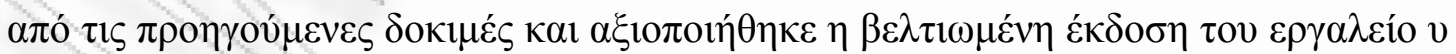

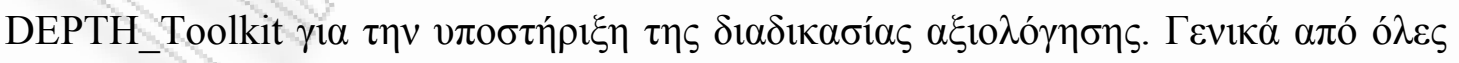

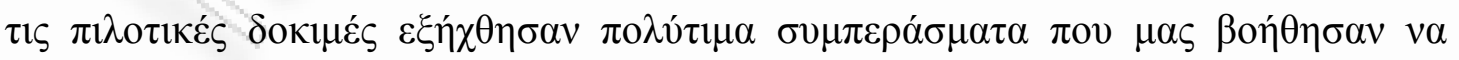

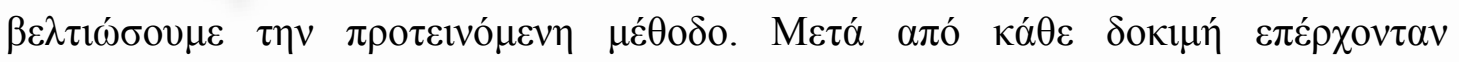

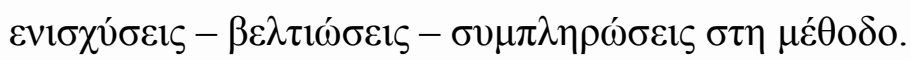




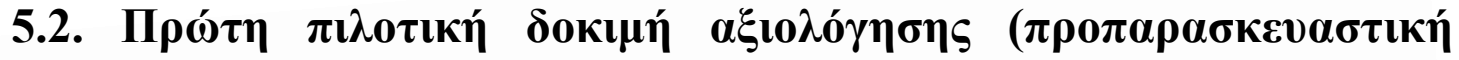

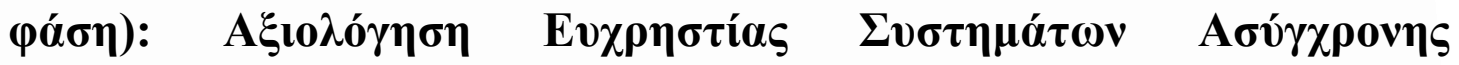

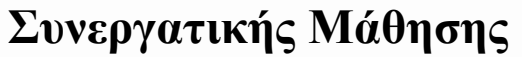

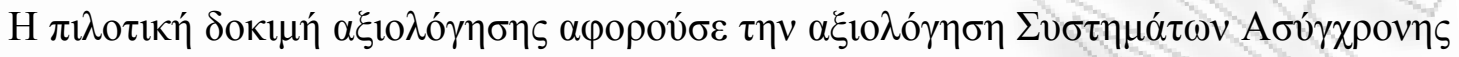

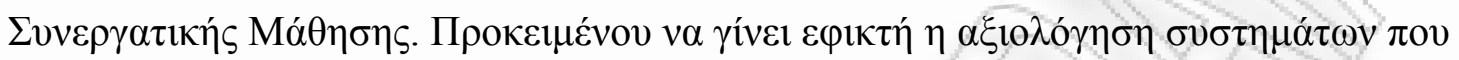

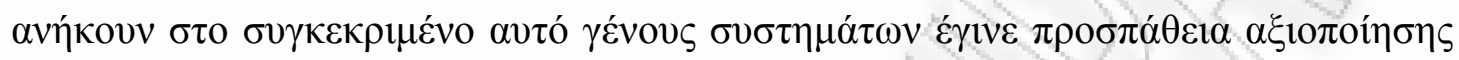

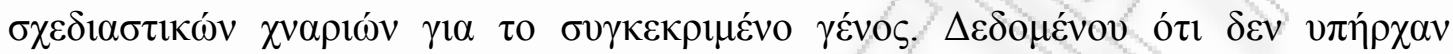

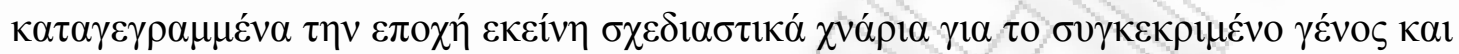

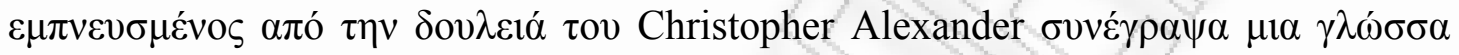

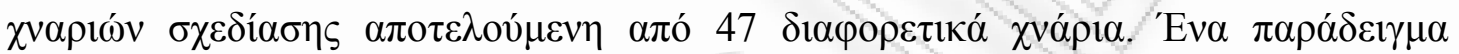

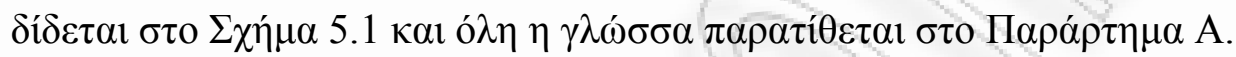

\begin{tabular}{|c|c|}
\hline $\begin{array}{l}\text { Pattern } \\
\text { Name }\end{array}$ & Annotation on posted message \\
\hline Problem & $\begin{array}{l}\text { The large amount of postings in } \\
\text { asynchronous communication } \\
\text { environments is a frequent } \\
\text { inconvenience for the receivers of } \\
\text { these postings. Information overload } \\
\text { in mailboxes wastes a lot of readers' } \\
\text { time. Users need to classify incoming } \\
\text { messages proportionally to their } \\
\text { desires. How should users of } \\
\text { ANSCL annotate their messages? }\end{array}$ \\
\hline Forces & $\begin{array}{l}\text { Users that participate to collaborative learning environments where } \\
\text { there is a large volume of messages exchanges need to have an } \\
\text { effective and powerful tool to uncluttered their incoming messages in } \\
\text { the large ambience of inboxes. Reminders for actions that took place in } \\
\text { the past, especially those opened long time ago, are convenient } \\
\text { functions. Users need to get notification about every alteration they } \\
\text { made in the material or the content of the postings or about their } \\
\text { importance. Users might also need to get informed about messages } \\
\text { posted by specific peers who either belong to the same group or their } \\
\text { opinion matters (e.g. a teacher, or the best student). The annotations } \\
\text { used must be comprehensible in order not to waste time trying to } \\
\text { understand what each annotation mark stands for. Of course, a great } \\
\text { danger arouses; with all that amount of colourful information assigned } \\
\text { to each posting it is probable that the users will be confused instead of } \\
\text { being notified, }\end{array}$ \\
\hline
\end{tabular}




\begin{tabular}{|c|c|}
\hline Solution & $\begin{array}{l}\text { Give users a tool to annotate messages in a way that suits them. } \\
\text { Give users the option each message according to its importance (e.g. } \\
\text { urgent, unread, trash) in order to avoid spending time reading or trying } \\
\text { to remember what were the previous interactions with each posting. An } \\
\text { annotation toolbar can be used so as when users open messages they } \\
\text { can easily assign remarks or even comments that will "escort" it } \\
\text { through out its "life cycle". Annotations should be widely acceptable } \\
\text { and easily recognisable regarding their expedient actions. }\end{array}$ \\
\hline $\begin{array}{l}\text { Example(s } \\
\text { ) }\end{array}$ & 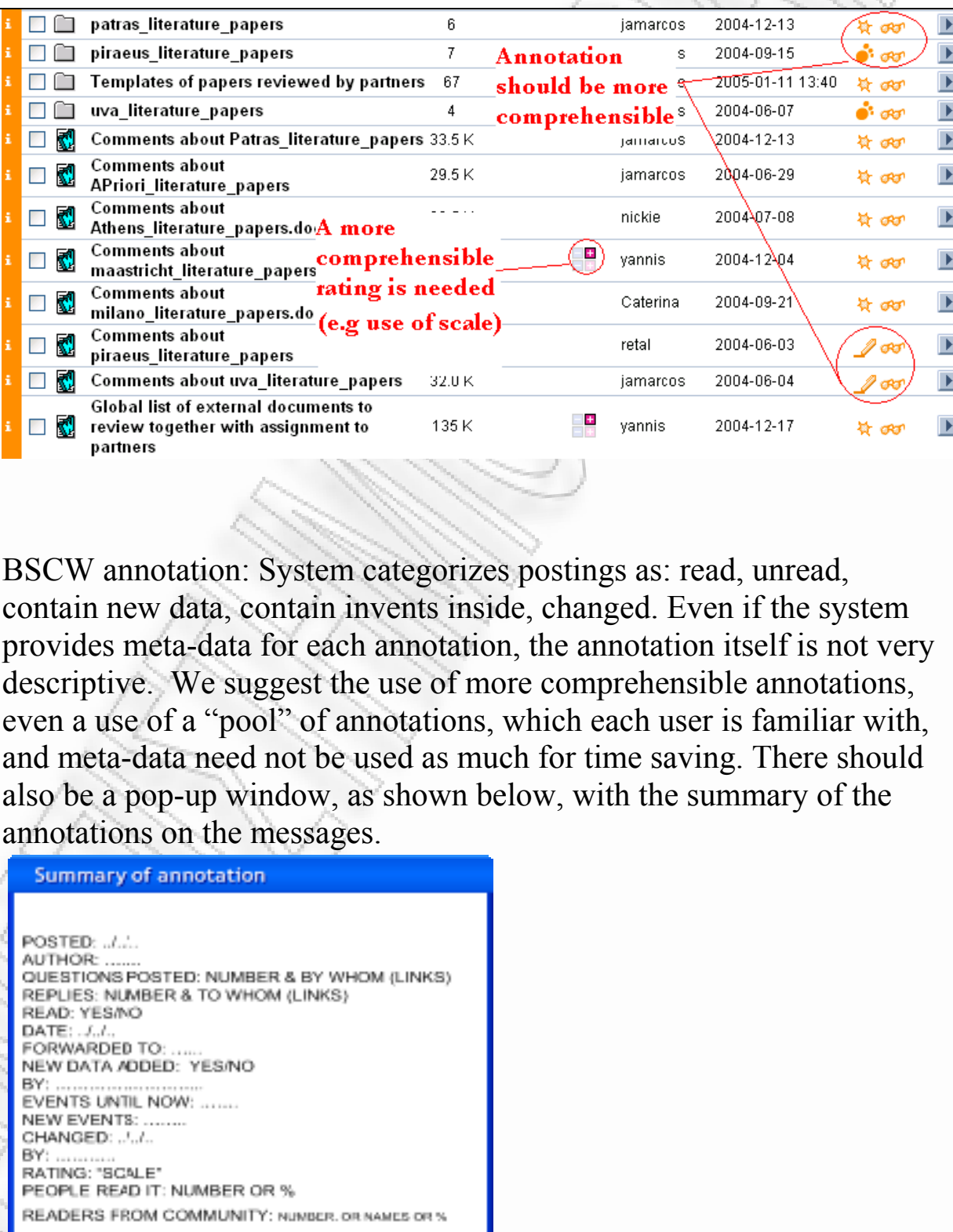 \\
\hline
\end{tabular}

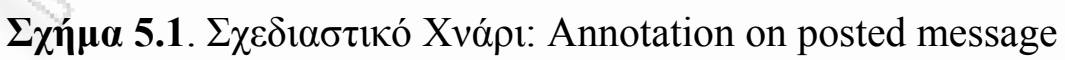




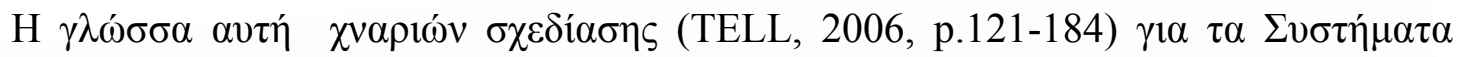

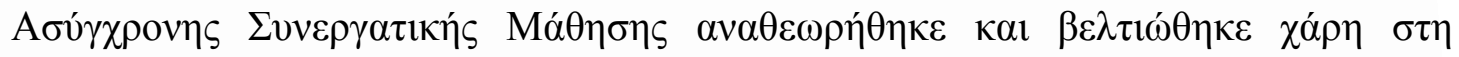

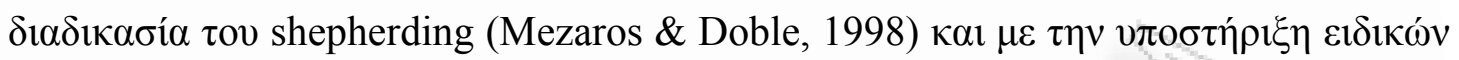

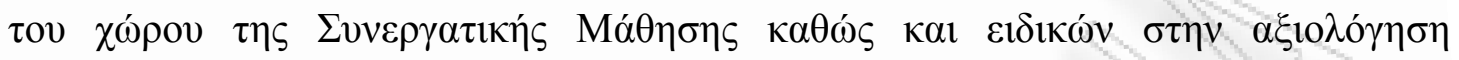

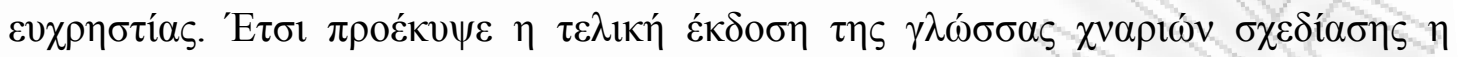

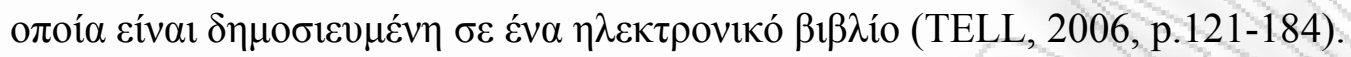

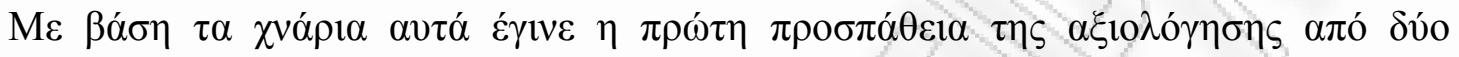

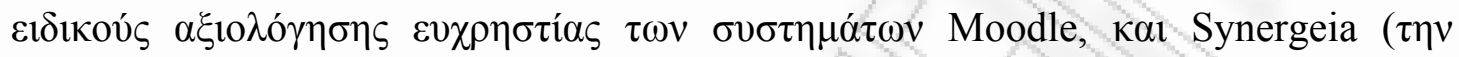

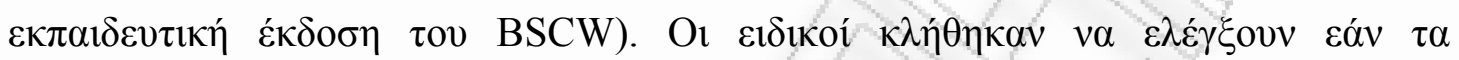

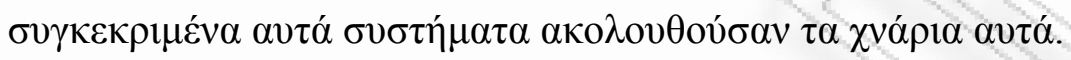

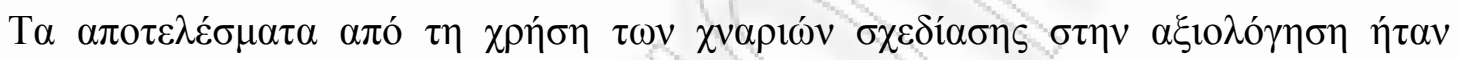

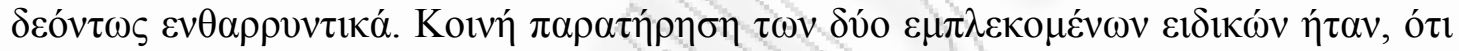

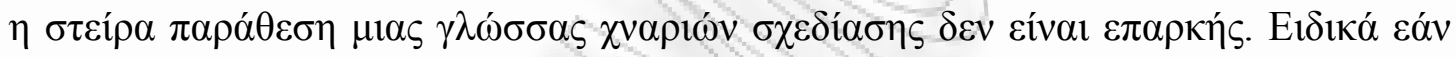

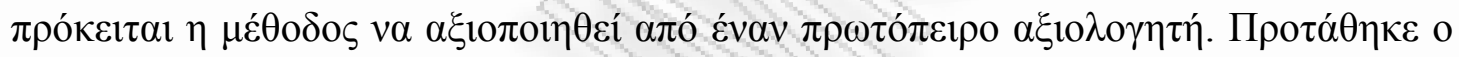

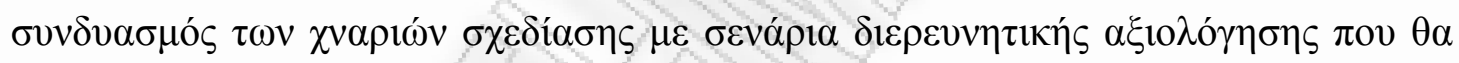

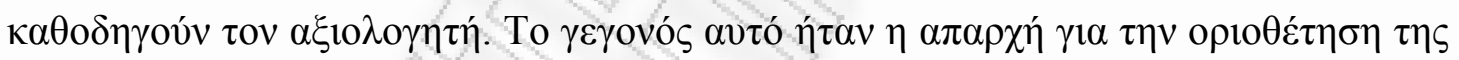
$\pi \rho \circ \sigma \varepsilon ́ \gamma \gamma 1 \sigma \eta_{\varsigma}$ DEPTH,.

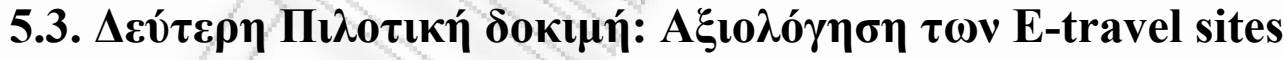

\subsection{1. Гevıкó}

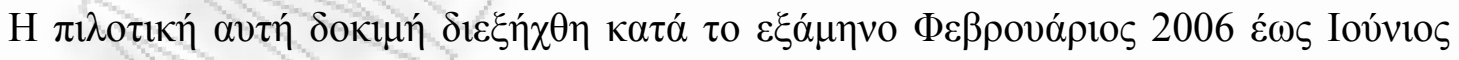

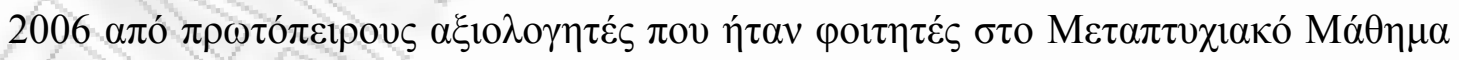

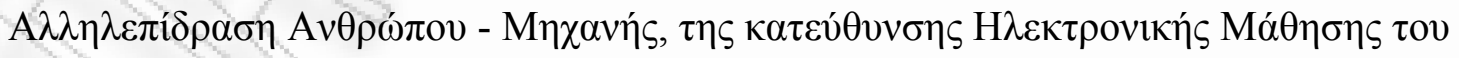

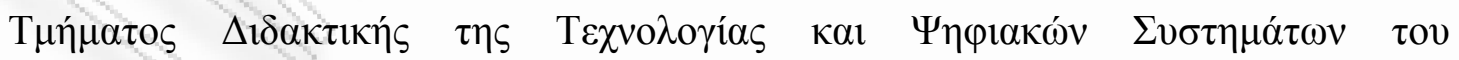

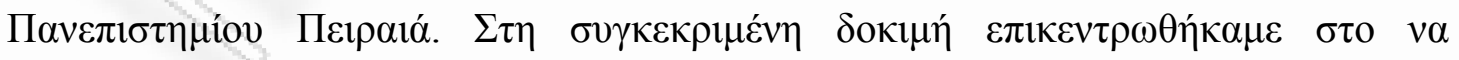

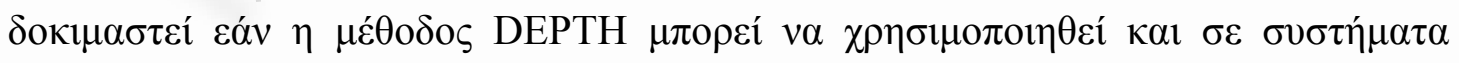

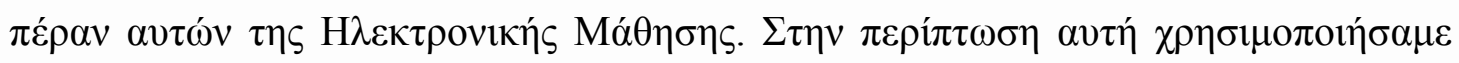

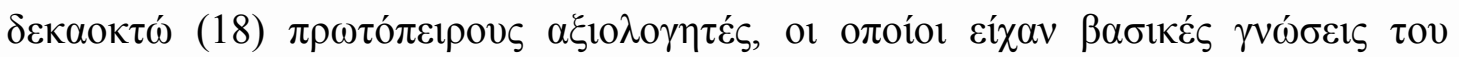

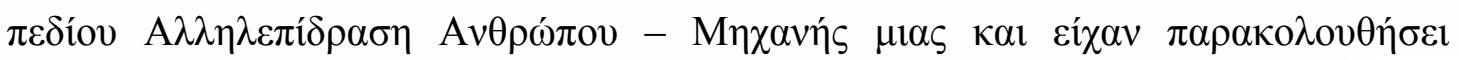




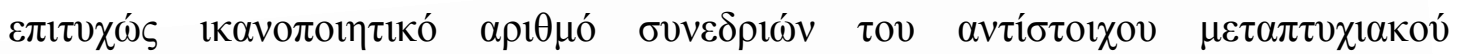

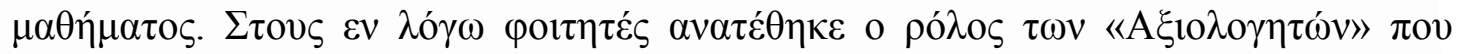

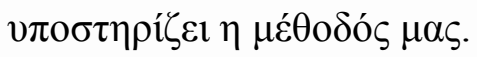

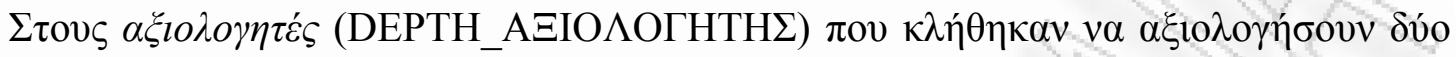

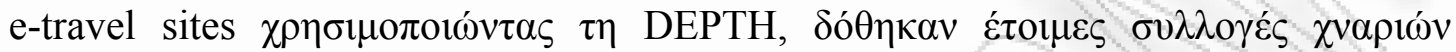

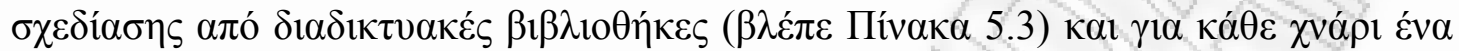

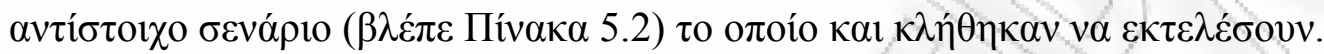

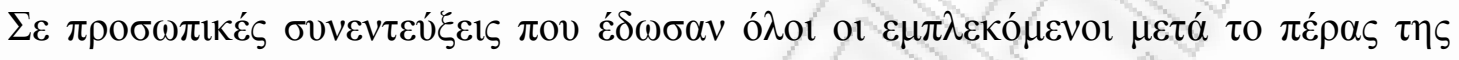

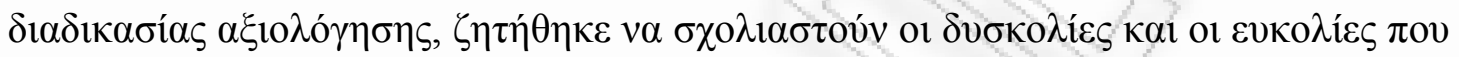

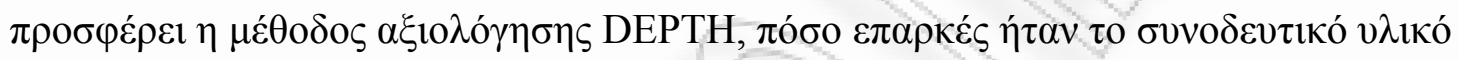

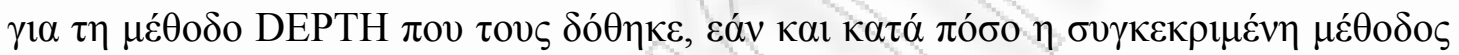

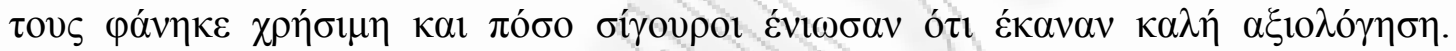

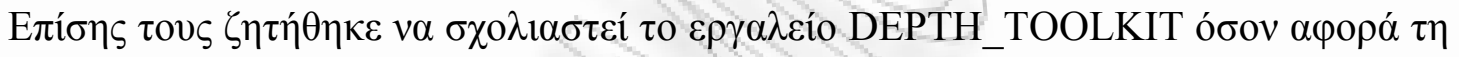

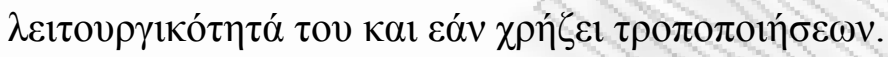

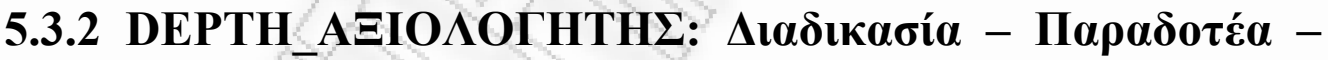 $\Sigma v \mu \pi \varepsilon \rho \alpha ́ \sigma \mu \alpha \tau \alpha \pi 0 v \pi \rho 0 \varepsilon ́ \kappa v \psi \alpha \nu$}

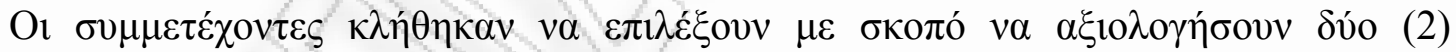

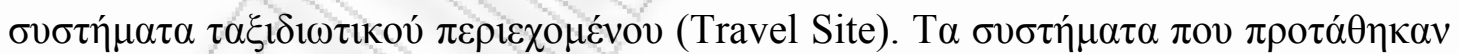

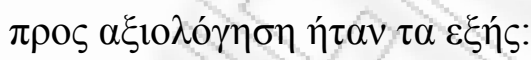

- $\tau o$ Airtickets (http://www.airtickets.gr/)

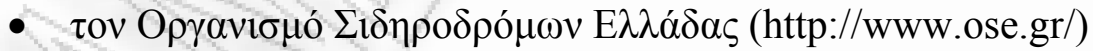

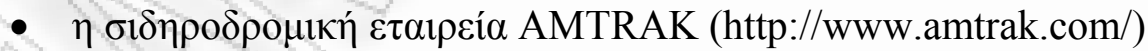

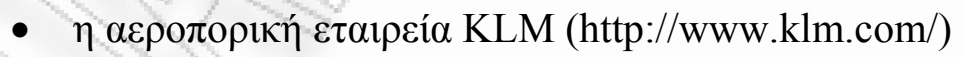

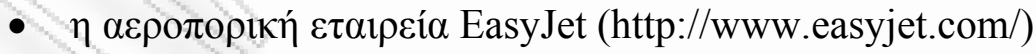

- $\tau_{0}$ Cheapflights (http://www.cheapflights.com/)

- $\tau$ o Flightsdirect (http://www.flightsdirect.com/)

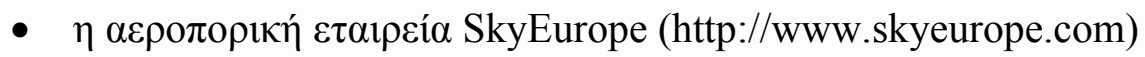

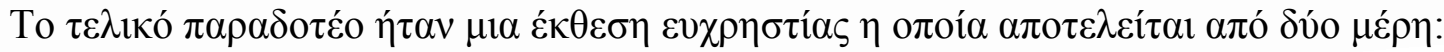




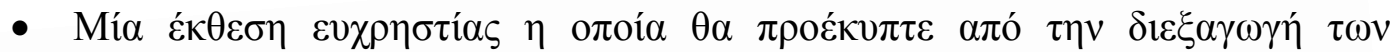

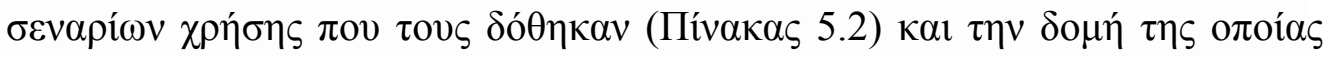
$\tau \eta v \varepsilon \varepsilon^{\prime} \alpha \mu \varepsilon \varepsilon \pi \rho \alpha \theta \varepsilon \dot{\sigma \varepsilon}$

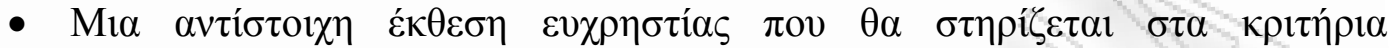

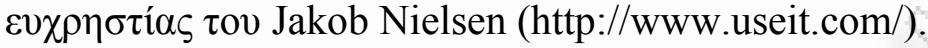

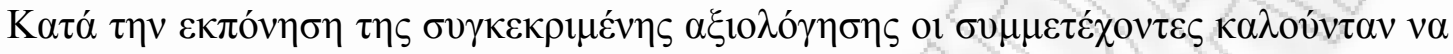

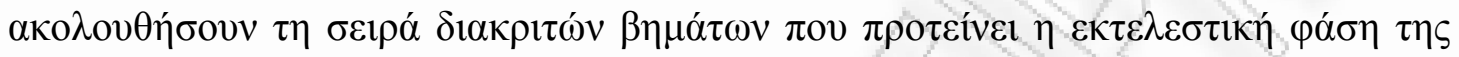

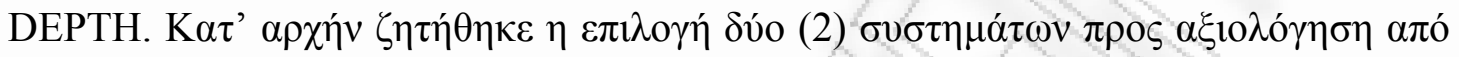

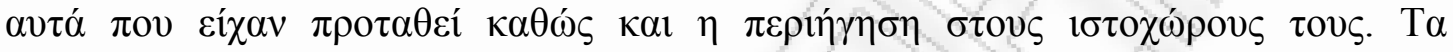

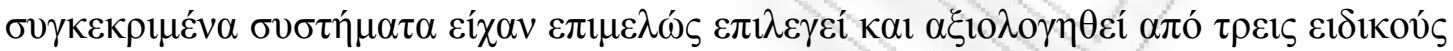

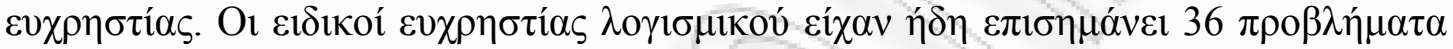

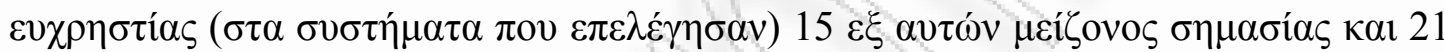

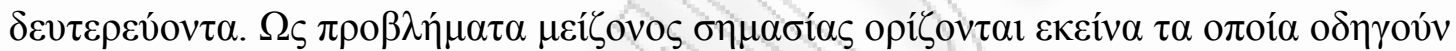

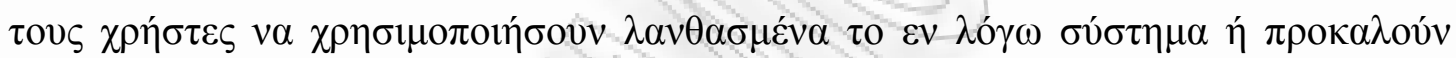

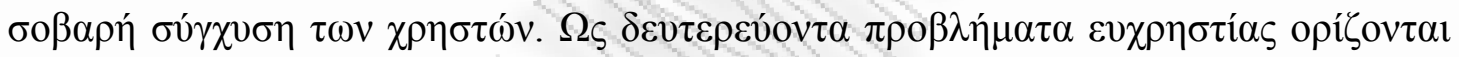

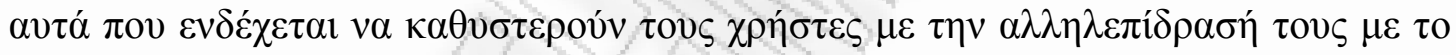

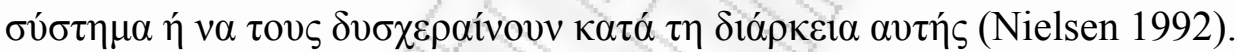

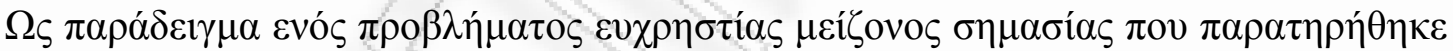

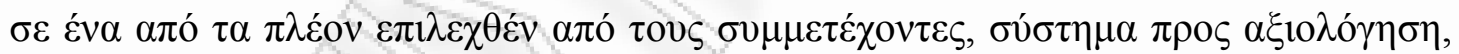

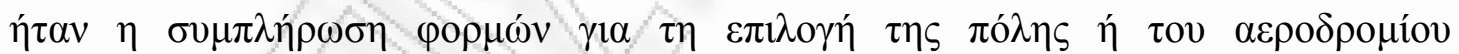

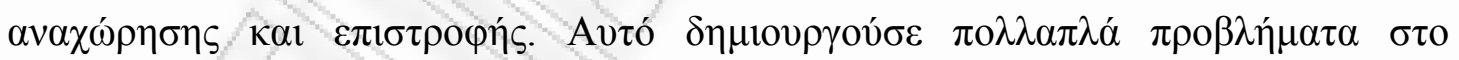

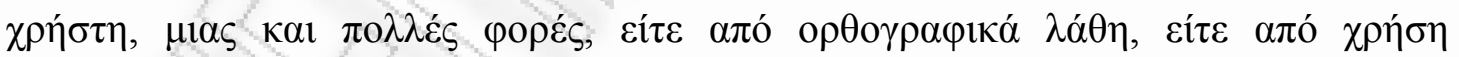

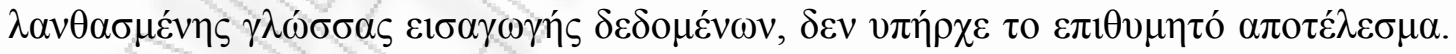

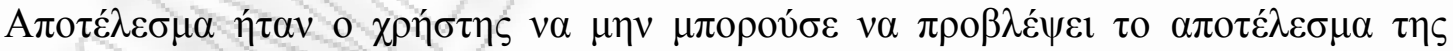

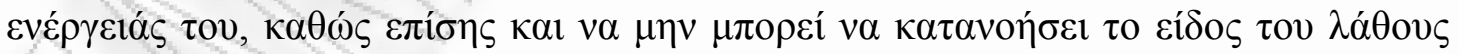

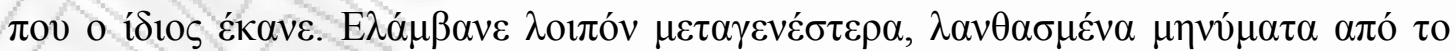

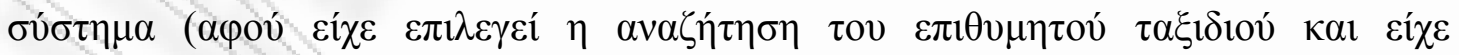

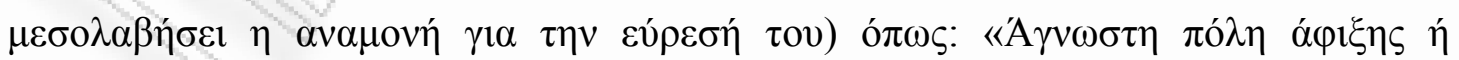

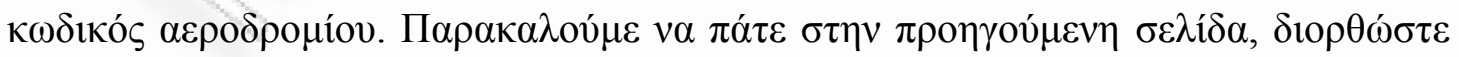

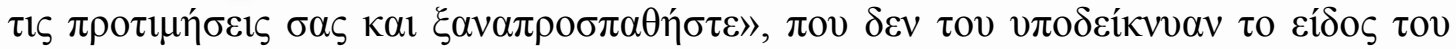

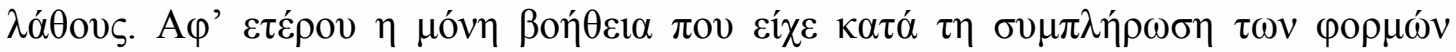




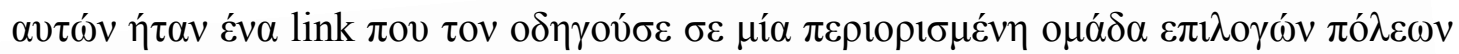
$\kappa \alpha \imath \alpha \varepsilon \rho \delta \delta \rho \mu \mu i ́ \omega v$.

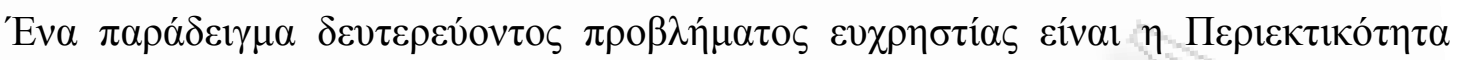

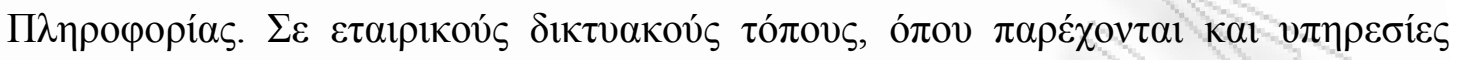

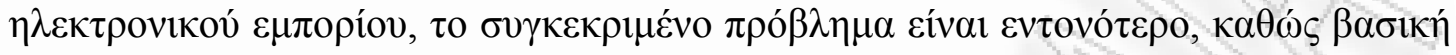

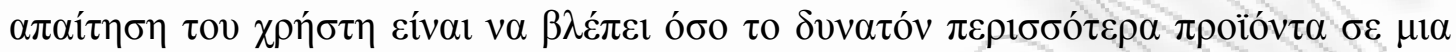

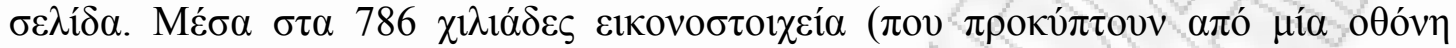

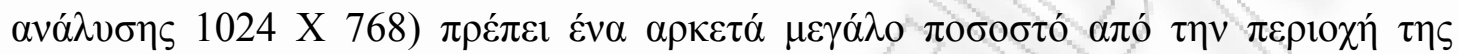

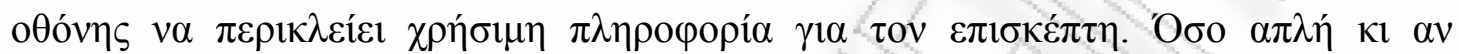

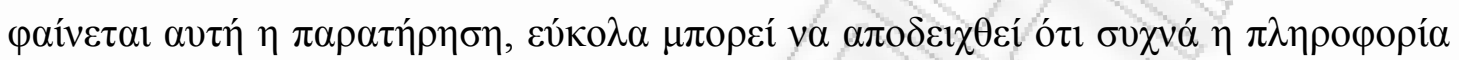

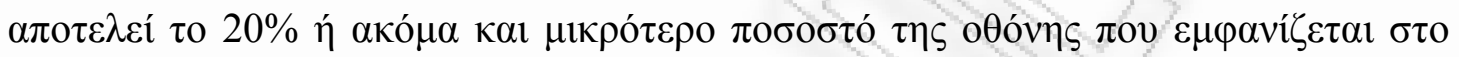

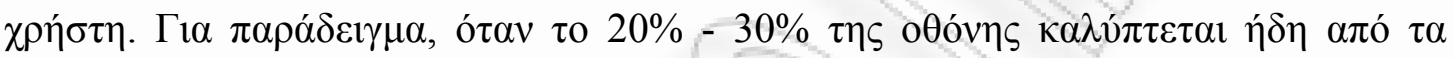

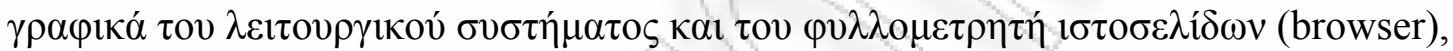

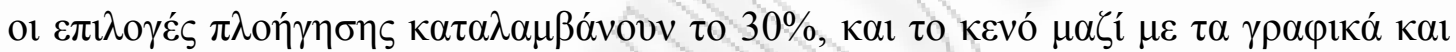

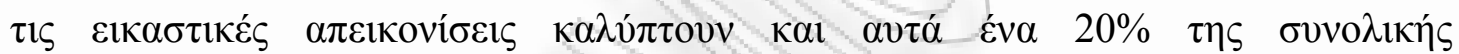

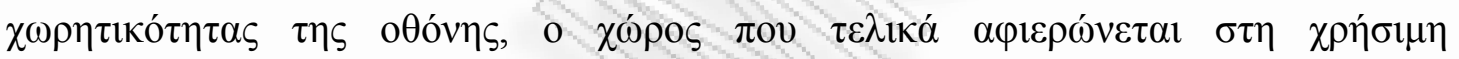

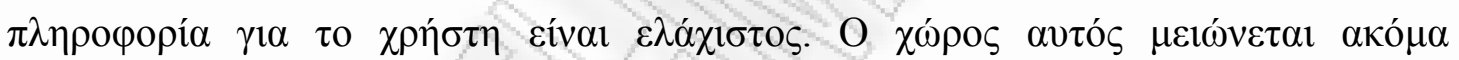

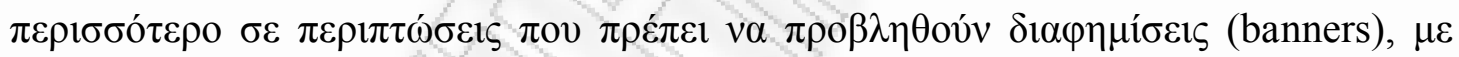

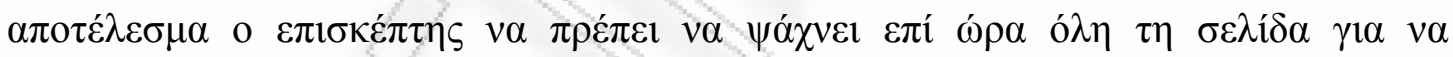

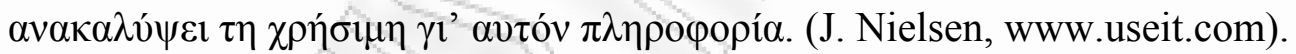

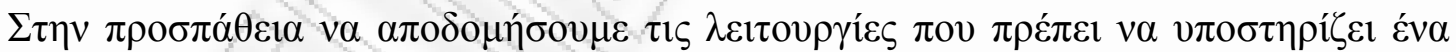

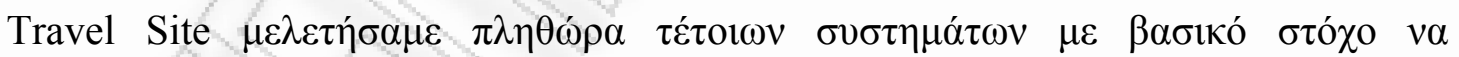

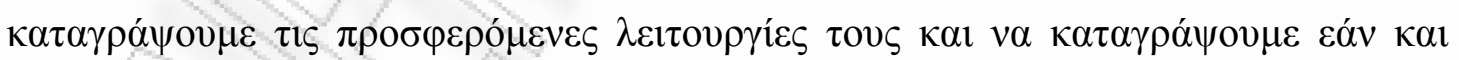

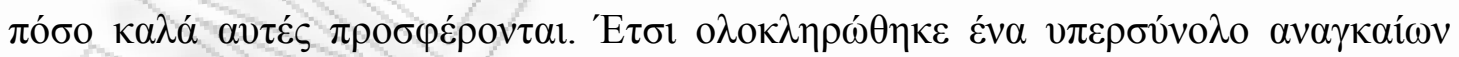

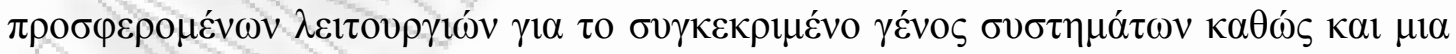

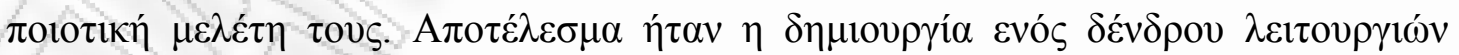

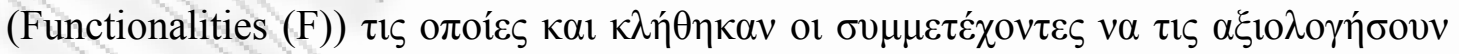

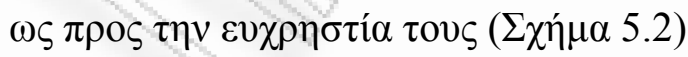




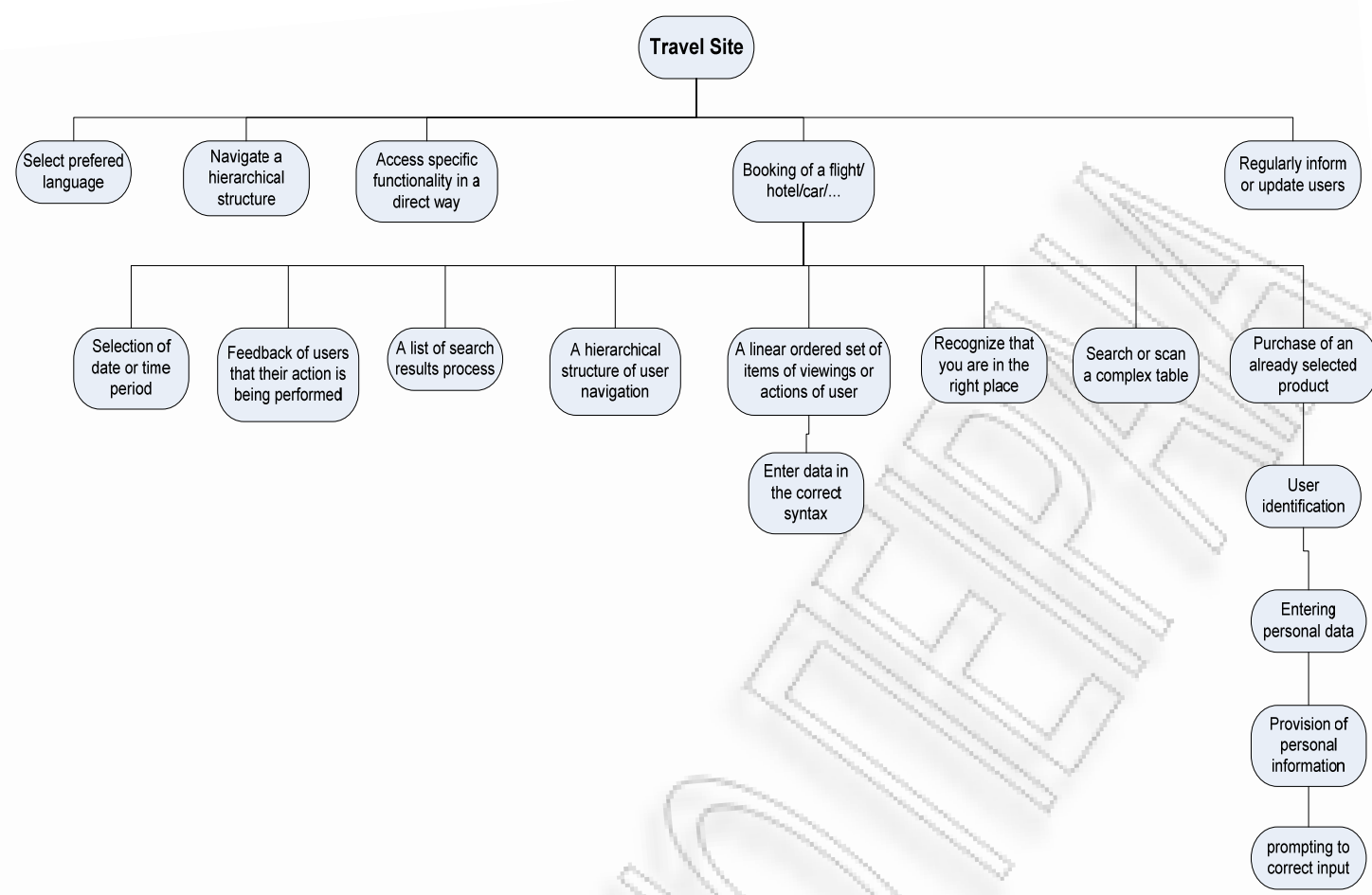

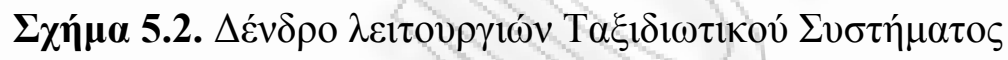

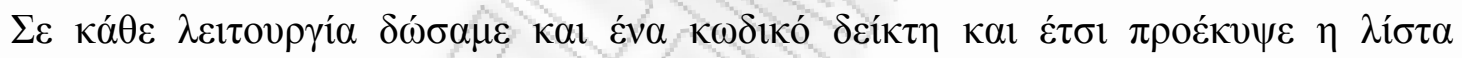

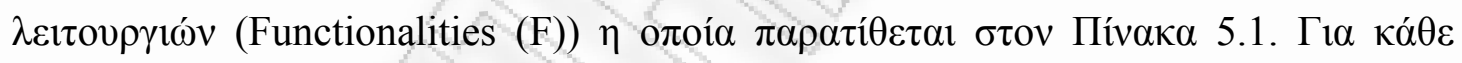

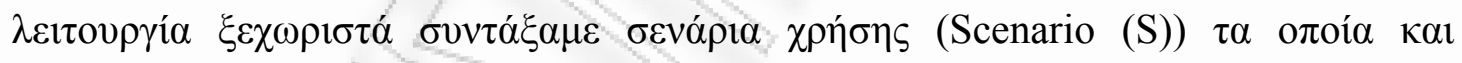

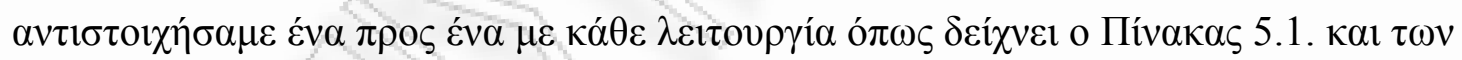

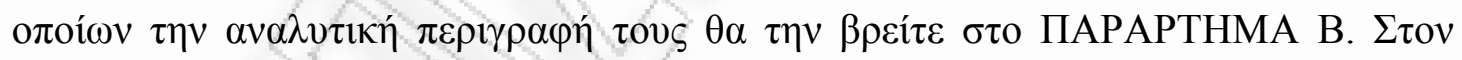

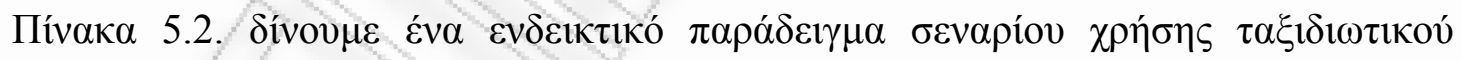

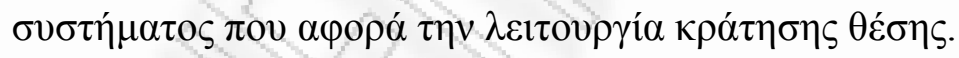

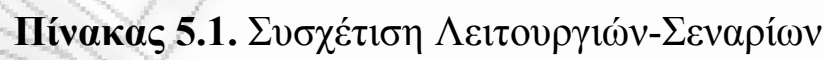

\begin{tabular}{|c|c|}
\hline 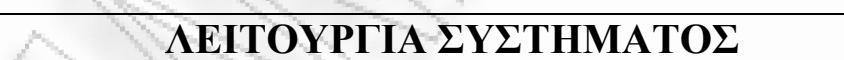 & 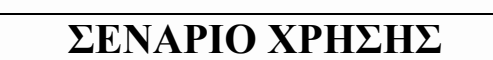 \\
\hline F1. Booking of a flight/hotel/car/ & S1. Booking \\
\hline Navigate a hierarchical structure & S2. Double Tab Navigation \\
\hline Access specific functionality in a direct way & S3. Shortcut Box \\
\hline F4. $\quad$ Select preferred language & S4. Language Selector \\
\hline F5. $\quad$ Regularly inform or update users & S5. Newsletter \\
\hline F6. Purchase of an already selected product & S6. Purchase Process \\
\hline User identification & S7. Login \\
\hline F8. A list of search results process & S8. Search Results \\
\hline F9. A hierarchical structure of user navigation & S9. Breadcrumbs \\
\hline $\begin{array}{l}\text { F10. A linear ordered set of items of viewings or } \\
\text { actions of user }\end{array}$ & S10. Stepping \\
\hline
\end{tabular}




\begin{tabular}{|l|l|}
\hline $\begin{array}{l}\text { F11. Feedback of users that their action is being } \\
\text { performed }\end{array}$ & S11. Processing Page \\
\hline F12. Search or scan a complex table & S12. Table Sorter \\
\hline F13. Recognize that you are in the right place & S13. Color Coded Section \\
\hline F14. Selection of date or time period & S14. Date Selector \\
\hline F15. Enter data in the correct syntax & S15. Constraint Input \\
\hline F16. Entering personal data & S16. Registration \\
\hline F17. Provision of personal information & S17. Form \\
\hline F18. Prompting to correct input & S18. Input Error Message \\
\hline F19. Print content of a page & S19. Printer-friendly Page \\
\hline
\end{tabular}

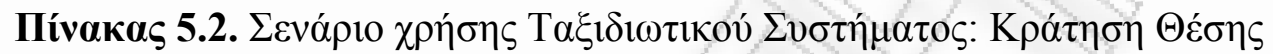

\begin{tabular}{|c|c|}
\hline (S1)Description: & Booking \\
\hline Task: & $\begin{array}{l}\text { You are looking for the price of a ticket in association with date } \\
\text { and time factors. Determine the item you wish to book or buy } \\
\text { among probably some other options given. Select town or even } \\
\text { airport you wish to leave from and going to. If you don't know the } \\
\text { names or the abbreviations find out from information given from } \\
\text { the site. Choose the departing date, the time, the booking class, } \\
\text { and the people traveling with you. Because you are looking for the } \\
\text { best available price and you don't mind to be precise to the dates } \\
\text { you've given choose these dates to be flexible but near to what } \\
\text { you want. Submit tour choices. }\end{array}$ \\
\hline Questions: & $\begin{array}{l}\text { - Have you easily found the searching area where you can place } \\
\text { - In the area where you can choose the departing and the arriving } \\
\text { airport or town did you had to write down to the form the name } \\
\text { or the abbreviation or you could select from a list given? } \\
\text { - If you should write down was there any help to find out the } \\
\text { correct abbreviation or spelling? } \\
\text { - Were there any error messages in case something wrong has } \\
\text { been typed? } \\
\text { - In the departing and returning date forms was there the help of } \\
\text { - Could you select flexible dates for example } \pm 3 \text { days from what } \\
\text { - you've chosen? } \\
\text { - When you select the persons traveling was it obvious what they } \\
\text { meant with the term Adult or Senior, or Children? } \\
\text { - Could you search for a one way trip if you wised? } \\
\text { - When you submitted your choices and a search in their } \\
\text { databases was performed were you informed that some action } \\
\text { was taken place? }\end{array}$ \\
\hline
\end{tabular}




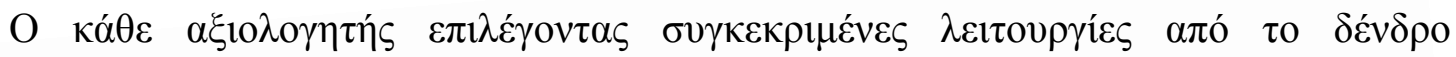

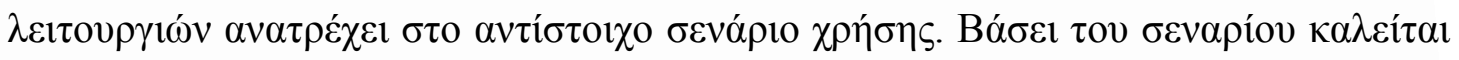

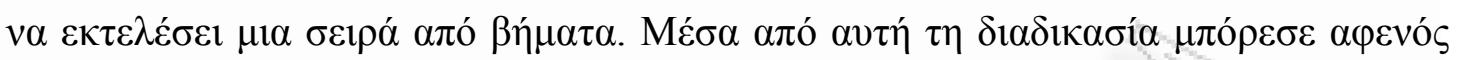

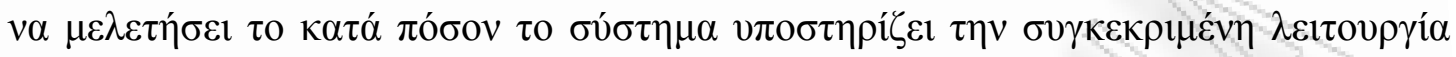

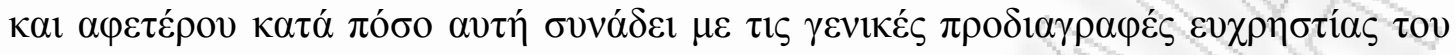

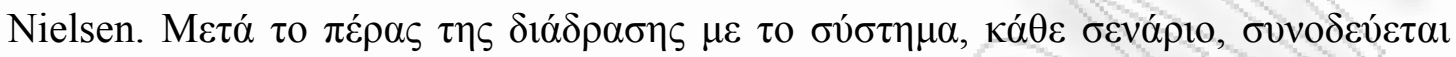

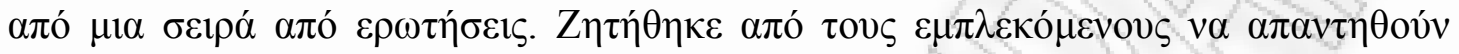

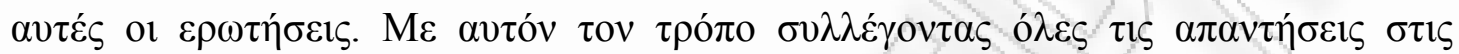

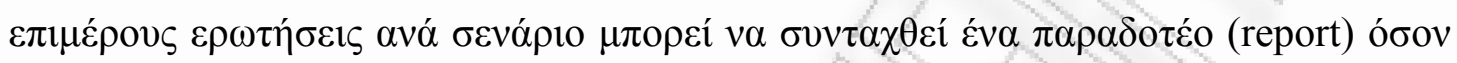

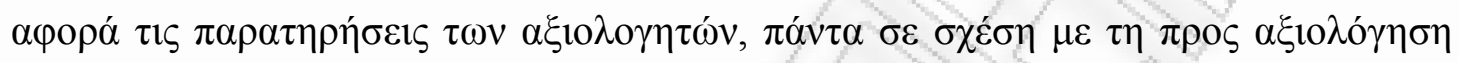

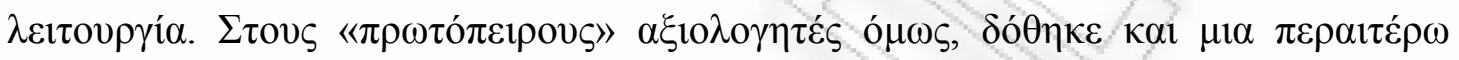

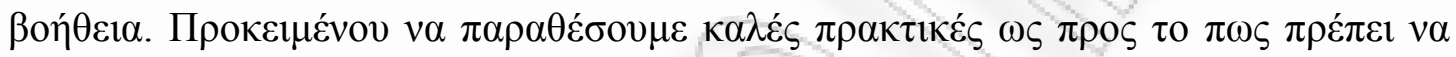

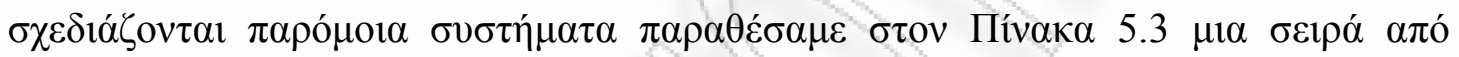

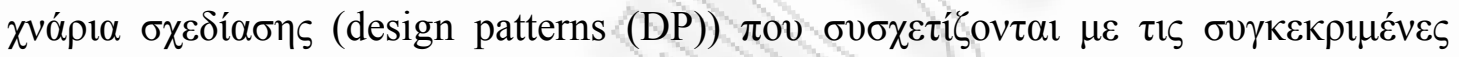

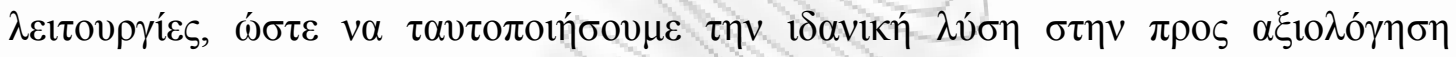

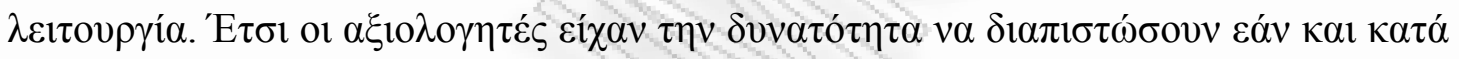

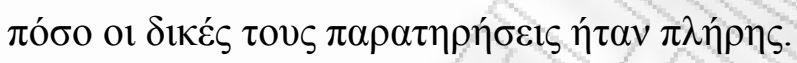

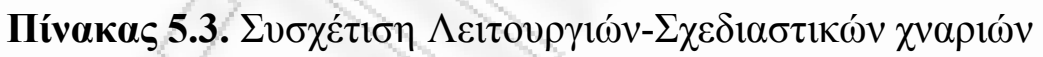

\begin{tabular}{|c|c|}
\hline АЕІТОУРГІА ЕY & 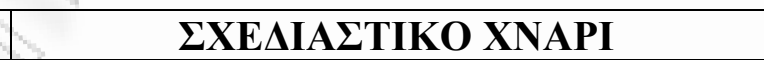 \\
\hline F1. Booking of a flight/hotel/car/ & DP1. $\frac{\text { http://www.welie.com/patterns/show }}{\text { Pattern.php?patternID=booking }}$ \\
\hline F2. Navigate a hierarchical structure & $\begin{array}{l}\text { DP2. http://www.welie.com/patterns/show } \\
\text { Pattern.php?patternID=doubletab }\end{array}$ \\
\hline $\begin{array}{l}\text { F3. Access specific functionality in a } \\
\text { direct way }\end{array}$ & $\begin{array}{ll}\text { DP3. http://www.welie.com/patterns/show } \\
\text { Pattern.php?patternID=shortcut-box }\end{array}$ \\
\hline F4. Select preferred language & \begin{tabular}{|l} 
DP4. \\
$\begin{array}{l}\text { http://www.welie.com/patterns/show } \\
\text { Pattern.php?patternID=language- } \\
\text { selector }\end{array}$
\end{tabular} \\
\hline F5. Regularly inform or update users & $\begin{array}{ll}\text { DP5. http://www.welie.com/patterns/show } \\
\text { Pattern.php?patternID=newsletter }\end{array}$ \\
\hline $\begin{array}{l}\text { F6. Purchase of an already selected } \\
\text { product }\end{array}$ & $\begin{array}{ll}\text { DP6. } & \text { http://www.welie.com/patterns/show } \\
& \text { Pattern.php?patternID=purchase- } \\
& \text { process }\end{array}$ \\
\hline F7. User identification & $\begin{array}{l}\text { DP7. http://www.welie.com/patterns/show } \\
\text { Pattern.php?patternID=login }\end{array}$ \\
\hline F8. A list of search results process & $\begin{array}{ll}\text { DP8. } & \text { http://www.welie.com/patterns/show } \\
& \text { Pattern.php?patternID=search-results }\end{array}$ \\
\hline $\begin{array}{l}\text { F9. A hierarchical structure of user } \\
\text { navigation }\end{array}$ & $\begin{array}{l}\text { DP9. http://developer.yahoo.com/ypatterns } \\
\text { /pattern_breadcrumbs.php or }\end{array}$ \\
\hline
\end{tabular}




\begin{tabular}{|c|c|}
\hline & $\begin{array}{l}\text { http://www.welie.com/patterns/show } \\
\text { Pattern.php?patternID=crumbs }\end{array}$ \\
\hline $\begin{array}{l}\text { F10. A linear ordered set of items of } \\
\text { viewings or actions of user }\end{array}$ & $\begin{array}{l}\text { DP10. http://www.welie.com/patterns/show } \\
\text { Pattern.php?patternID=stepping }\end{array}$ \\
\hline $\begin{array}{l}\text { F11. Feedback of users that their } \\
\text { action is being performed }\end{array}$ & $\begin{array}{l}\text { DP11. http://www.welie.com/patterns/show } \\
\text { Pattern.php?patternID=processing- } \\
\text { page }\end{array}$ \\
\hline F12. Search or scan a complex table & $\begin{array}{l}\text { DP12. http://www.welie.com/patterns/show } \\
\text { Pattern.php?patternID=table-sorter }\end{array}$ \\
\hline $\begin{array}{l}\text { F13. Recognize that you are in the } \\
\text { right place }\end{array}$ & $\begin{array}{l}\text { DP13. http://www.welie.com/patterns/show } \\
\text { Pattern.php?patternID=color-coded- } \\
\text { section }\end{array}$ \\
\hline $\begin{array}{l}\text { F14. Selection of date or time } \\
\text { period }\end{array}$ & $\begin{array}{l}\text { DP14. http://www.welie.com/patterns/show } \\
\text { Pattern.php?patternID=date-selector }\end{array}$ \\
\hline F15. Enter data in the correct syntax & $\begin{array}{l}\text { DP15. http://www.welie.com/patterns/show } \\
\text { Pattern.php?patternID=format }\end{array}$ \\
\hline F16. Entering personal data & $\begin{array}{l}\text { DP16. http://www.welie.com/patterns/show } \\
\text { Pattern.php?patternID=registration }\end{array}$ \\
\hline $\begin{array}{l}\text { F17. Provision of personal } \\
\text { information }\end{array}$ & $\begin{array}{l}\text { DP17. http://www.welie.com/patterns/show } \\
\text { Pattern.php?patternID=forms }\end{array}$ \\
\hline F18. Prompting to correct input & $\begin{array}{l}\text { DP18. http://www.welie.com/patterns/show } \\
\text { Pattern.php?patternID=input-error }\end{array}$ \\
\hline F19. Print content of a page & $\begin{array}{l}\text { DP19. http://www.welie.com/patterns/show } \\
\text { Pattern.php?patternID=printable- } \\
\text { pages }\end{array}$ \\
\hline
\end{tabular}

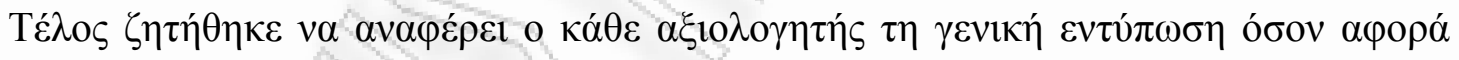

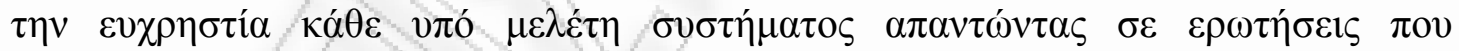

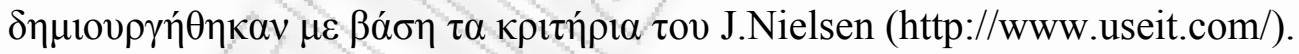

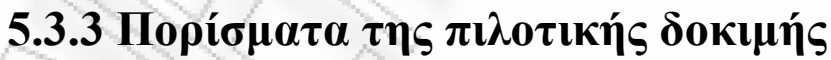

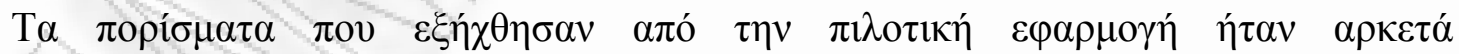

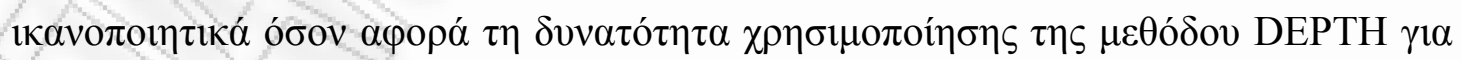

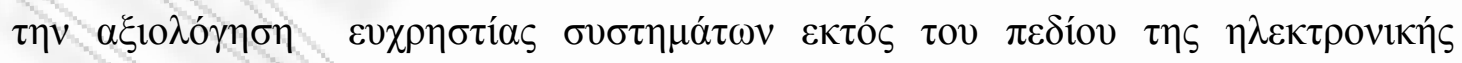
$\mu \alpha ́ \theta \eta \sigma \eta \varsigma$.

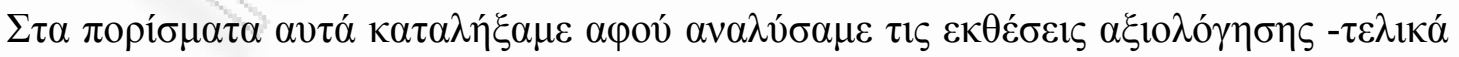

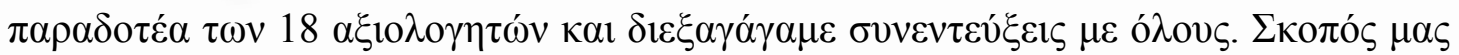

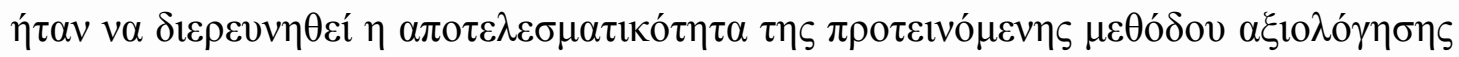

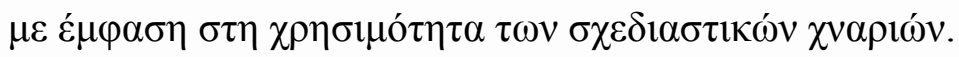




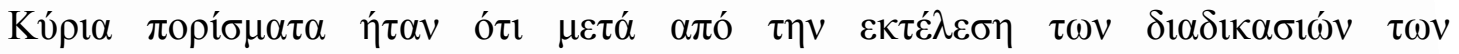

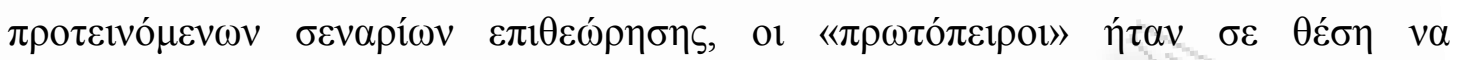

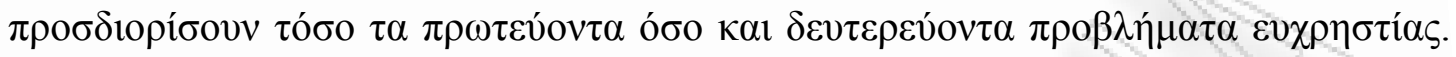

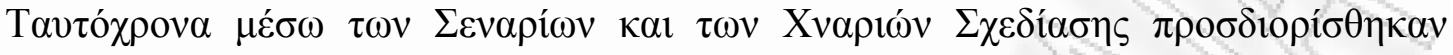

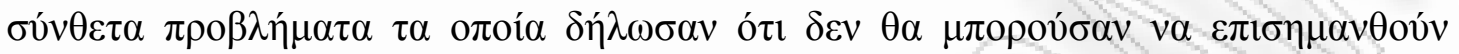

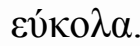

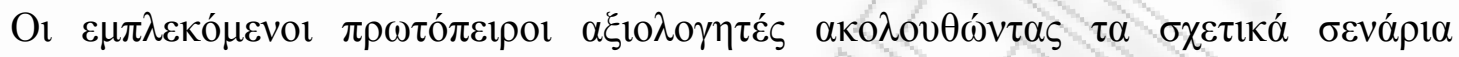

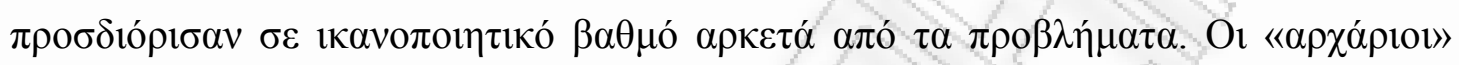

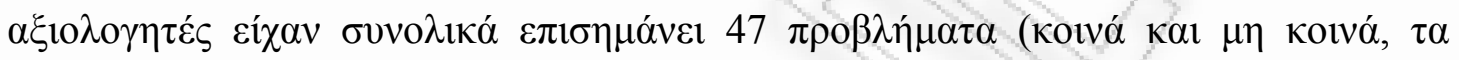

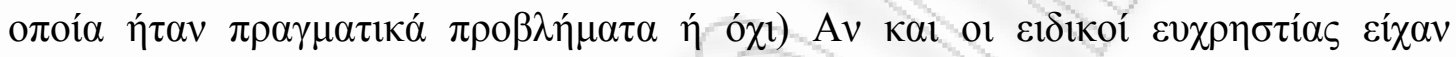

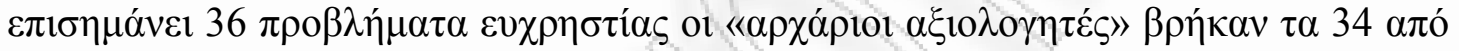

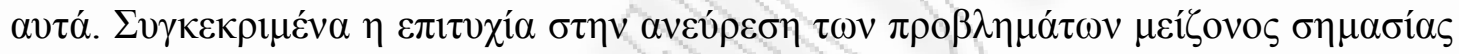

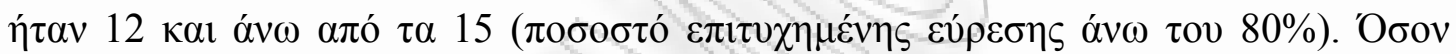

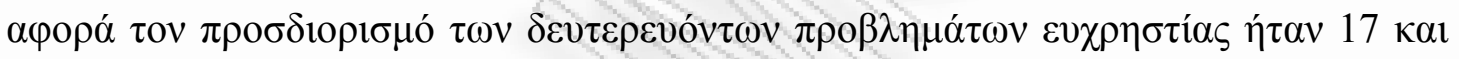

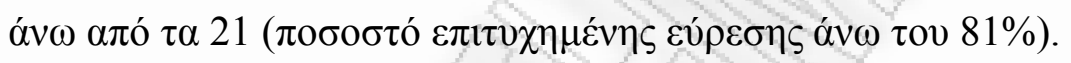

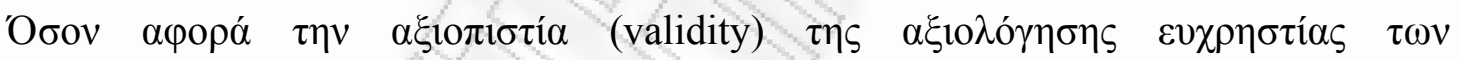

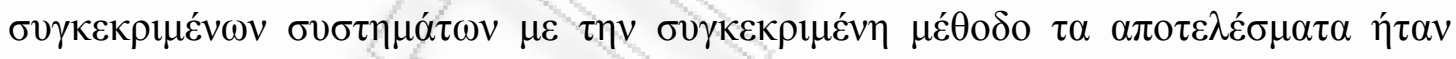

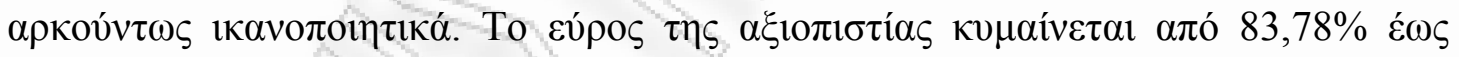

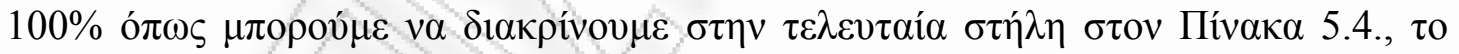

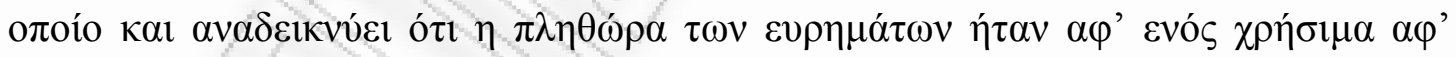

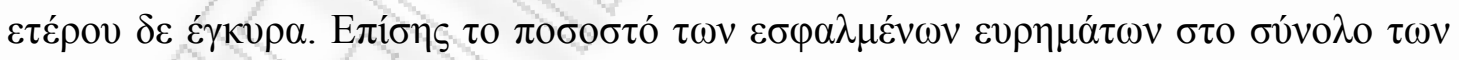

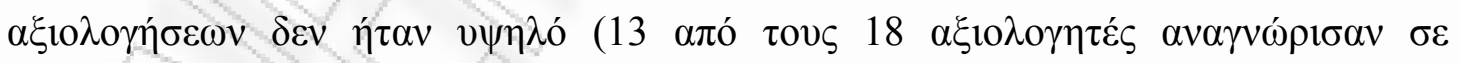

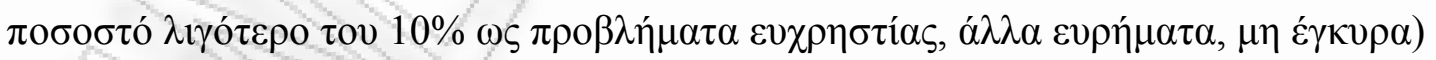

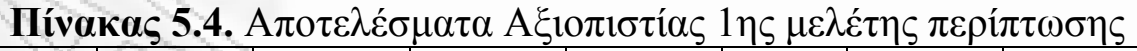

\begin{tabular}{|c|c|c|c|c|c|c|c|c|c|}
\hline $\begin{array}{c}\mathrm{A} / \mathrm{A} \\
\mathrm{A} \xi \xi 10 \lambda \circ \gamma \eta \tau \dot{m}\end{array}$ & 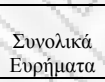 & $\begin{array}{l}M \eta \dot{\varepsilon} \gamma \kappa \nu \rho \alpha \\
\pi \rho \circ \beta \lambda \eta \mu \alpha \tau \alpha\end{array}$ & 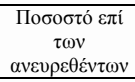 & 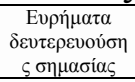 & $\begin{array}{c}\text { Побобтó } \varepsilon \pi i \\
\tau \omega v \\
\alpha v \varepsilon v \rho \varepsilon \theta \dot{\varepsilon} v \tau \omega v \\
\end{array}$ & 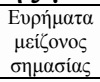 & $\begin{array}{c}\text { Побобт́́ } \varepsilon \pi \dot{1} \\
\tau \omega v \\
\alpha v \varepsilon v \rho \varepsilon \theta \dot{\varepsilon} \tau \tau \omega v \\
\end{array}$ & \multicolumn{2}{|c|}{$\mathrm{A} \xi \xi \iota \pi ı \sigma \tau i \alpha$} \\
\hline & & & & & & & & 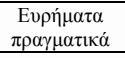 & $\%$ \\
\hline 1 & 35 & 4 & 5,71 & 20 & 57,14 & 13 & 37,14 & 33 & 94,29 \\
\hline 2 & 36 & 4 & 11,11 & 18 & 50,00 & 14 & 38,89 & 32 & 88,89 \\
\hline 3 & 32 & 3 & 9,38 & 17 & 53,13 & 12 & 37,50 & 29 & 90,63 \\
\hline 4 & 35 & 1 & 2,86 & 20 & 57,14 & 14 & 40,00 & 34 & 97,14 \\
\hline 5 & 32 & 0 & 0,00 & 20 & 62,50 & 12 & 37,50 & 32 & 100,00 \\
\hline 6 & 34 & 3 & 8,82 & 19 & 55,88 & 12 & 35,29 & 31 & 91,18 \\
\hline 7 & 30 & 1 & 3,33 & 17 & 56,67 & 12 & 40,00 & 29 & 96,67 \\
\hline 8 & 33 & 1 & 3,03 & 20 & 60,61 & 12 & 36,36 & 32 & 96,97 \\
\hline
\end{tabular}




\begin{tabular}{|r|r|r|r|r|r|r|r|r|r|}
\hline 9 & 34 & 3 & 8,82 & 17 & 50,00 & 14 & 41,18 & 31 & 91,18 \\
\hline 10 & 35 & 5 & 14,29 & 18 & 51,43 & 12 & 34,29 & 30 & 85,71 \\
\hline 11 & 32 & 1 & 3,13 & 19 & 59,38 & 12 & 37,50 & 31 & 96,88 \\
\hline 12 & 36 & 2 & 5,56 & 20 & 55,56 & 14 & 38,89 & 34 & 94,44 \\
\hline 13 & 34 & 2 & 5,88 & 18 & 52,94 & 14 & 41,18 & 32 & 94,12 \\
\hline 14 & 36 & 5 & 13,89 & 18 & 50,00 & 13 & 36,11 & 31 & 86,11 \\
\hline 15 & 37 & 6 & 16,22 & 19 & 51,35 & 12 & 32,43 & 31 & 83,78 \\
\hline 16 & 34 & 4 & 11,76 & 18 & 52,94 & 12 & 35,29 & 30 & 88,24 \\
\hline 17 & 32 & 1 & 3,13 & 17 & 53,13 & 14 & 43,75 & 31 & 96,88 \\
\hline 18 & 34 & 3 & 8,82 & 17 & 50,00 & 14 & 41,18 & 31 & 91,18 \\
\hline
\end{tabular}

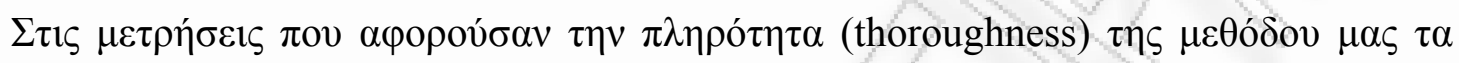

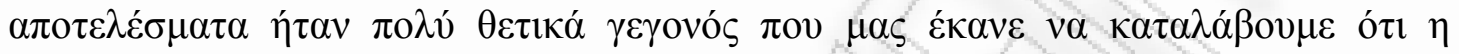

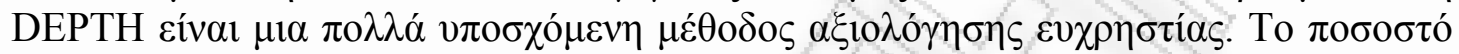

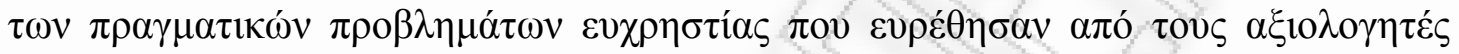

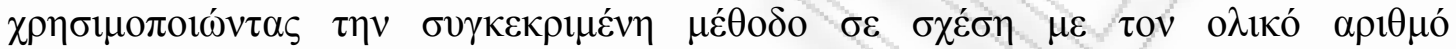

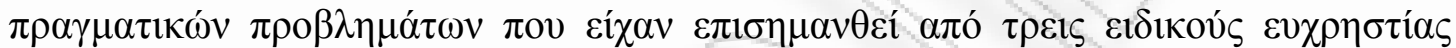

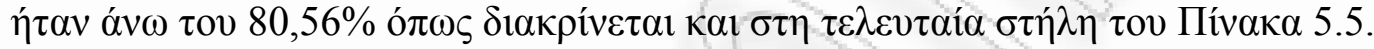

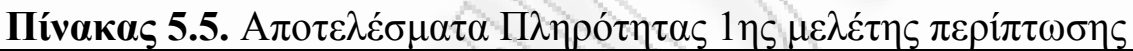

\begin{tabular}{|c|c|c|c|}
\hline $\begin{array}{l}\mathrm{A} / \mathrm{A} \\
\mathrm{A} \xi 10 \lambda \mathrm{o} \gamma \eta \tau \eta \dot{~}\end{array}$ & 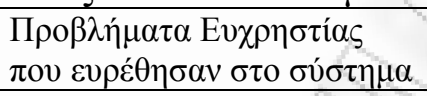 & 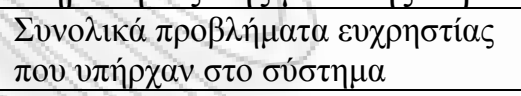 & $\Pi \lambda \eta \rho{ }^{\tau} \tau \tau \alpha$ \% \\
\hline 1 & 33 & $\begin{array}{ll} & 36\end{array}$ & 91,67 \\
\hline 2 & 32 & Whenty & 88,89 \\
\hline 3 & 29 & 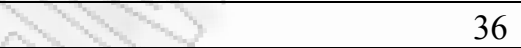 & 80,56 \\
\hline 4 & 34 & why & 94,44 \\
\hline 5 & 32 & 36 & 88,89 \\
\hline 6 & 31 & 36 & 86,11 \\
\hline 7 & 29 & 36 & 80,56 \\
\hline 8 & 32 & 36 & 88,89 \\
\hline 9 & 31 & 36 & 86,11 \\
\hline 10 & 30 & 36 & 83,33 \\
\hline 11 & 31 & 36 & 86,11 \\
\hline 12 & 34 & 36 & 94,44 \\
\hline 13 & 32 & 36 & 88,89 \\
\hline 14 & 31 & 36 & 86,11 \\
\hline 15 & 31 & 36 & 86,11 \\
\hline 16 & 30 & 36 & 83,33 \\
\hline 17 & 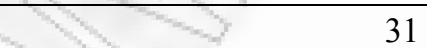 & 36 & 86,11 \\
\hline 18 & 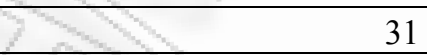 & 36 & 86,11 \\
\hline
\end{tabular}

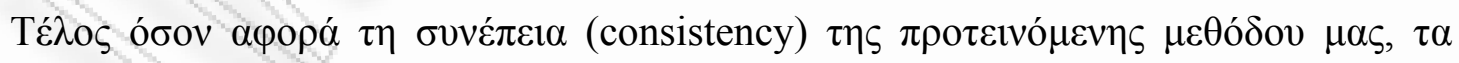

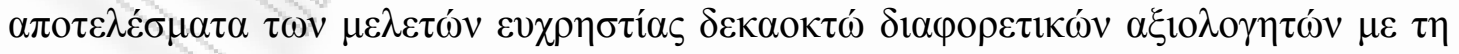

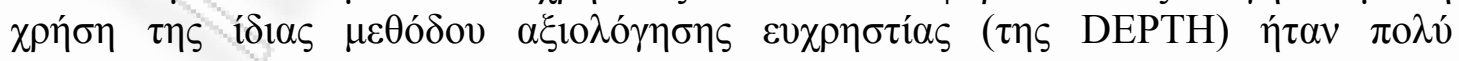

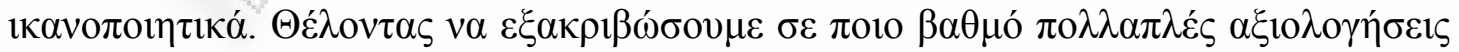

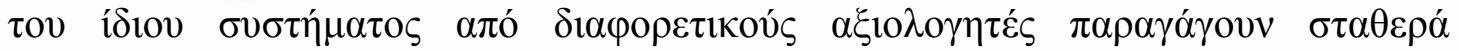
$\pi \alpha \rho$ ó

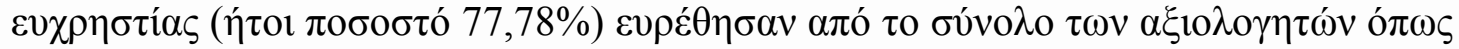

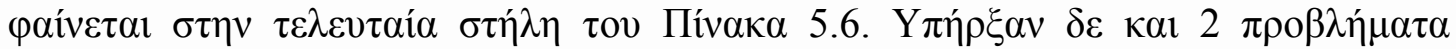

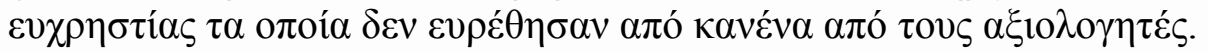




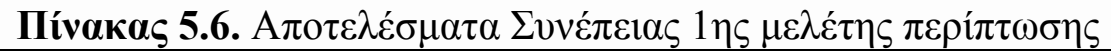

\begin{tabular}{|c|c|c|}
\hline 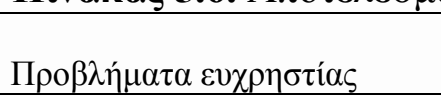 & 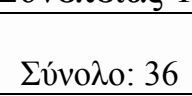 & 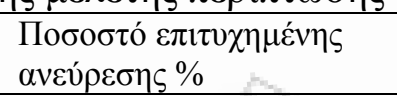 \\
\hline 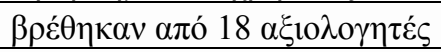 & 28 & 77,78 \\
\hline 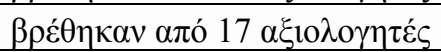 & 29 & 80,56 \\
\hline 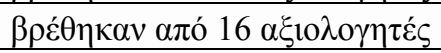 & 29 & 80,56 \\
\hline 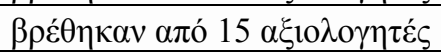 & 29 & 80,56 \\
\hline 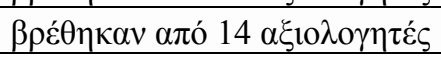 & 30 & 83,33 \\
\hline 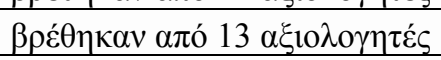 & 30 & 83,33 \\
\hline 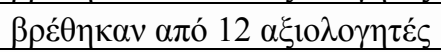 & 30 & 83,33 \\
\hline 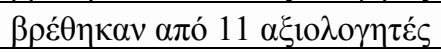 & 30 & 83,33 \\
\hline 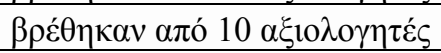 & 30 & 83,33 \\
\hline 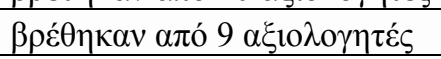 & 31 & 86,11 \\
\hline 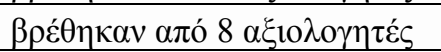 & 31 & 86,11 \\
\hline 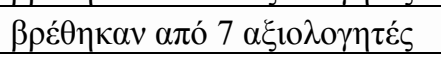 & 31 & 86,11 \\
\hline 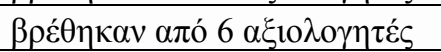 & 31 & 86,11 \\
\hline 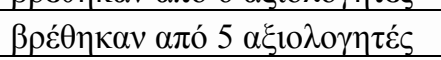 & 32 & 88,89 \\
\hline 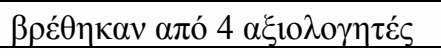 & 32 & 88,89 \\
\hline 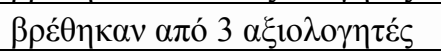 & 32 & 88,89 \\
\hline 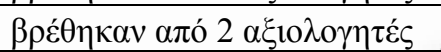 & 33 & 91,67 \\
\hline 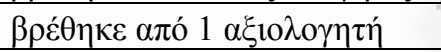 & 34 & 94,44 \\
\hline
\end{tabular}

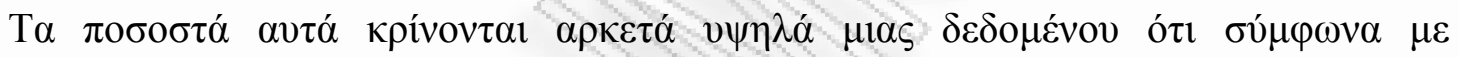

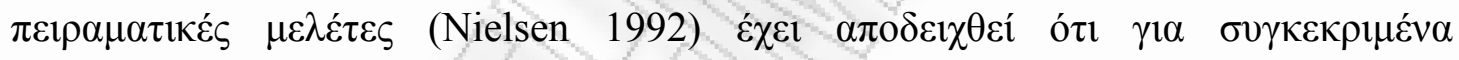

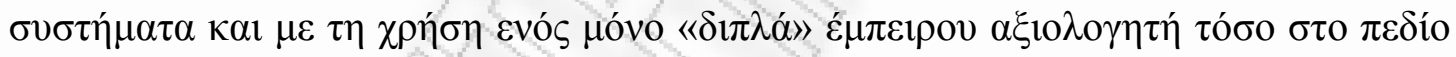

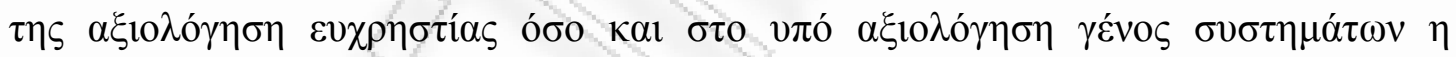

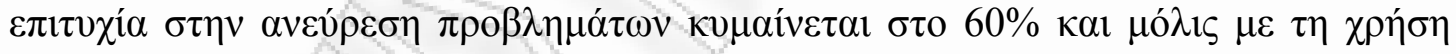

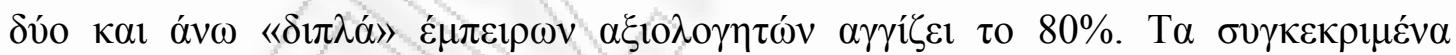

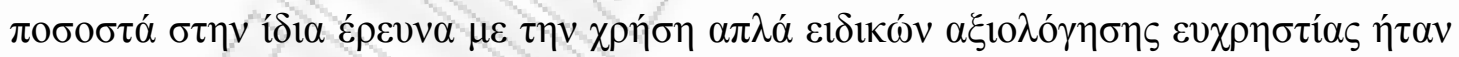

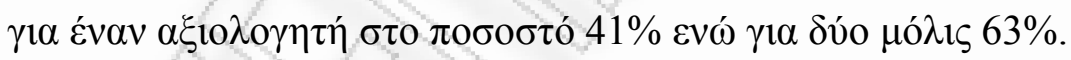

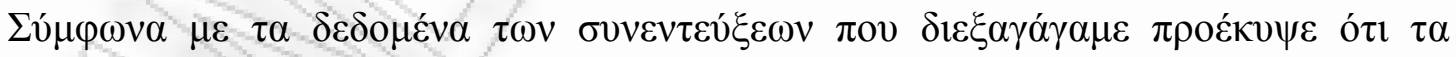

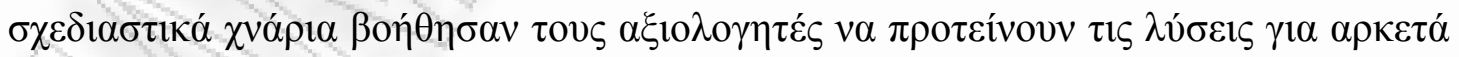

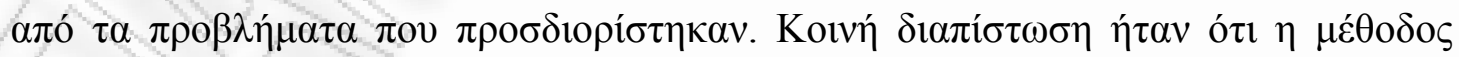

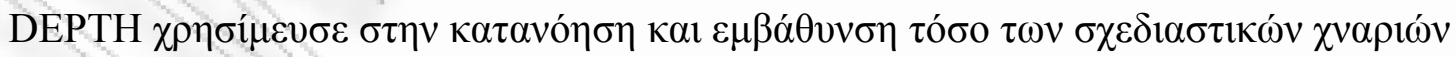

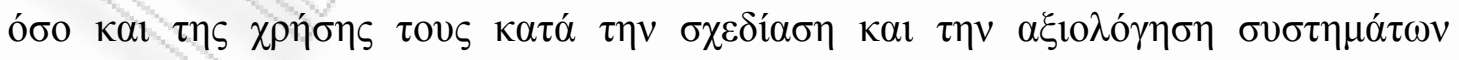

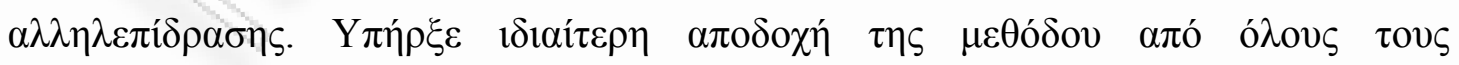

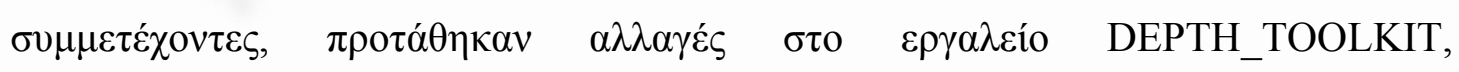

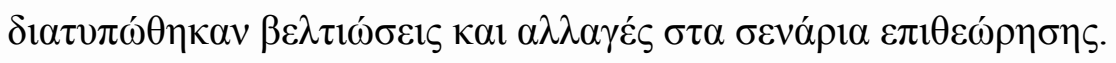




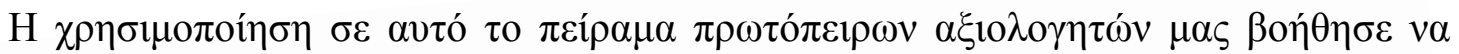

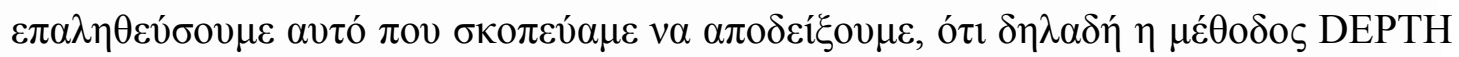

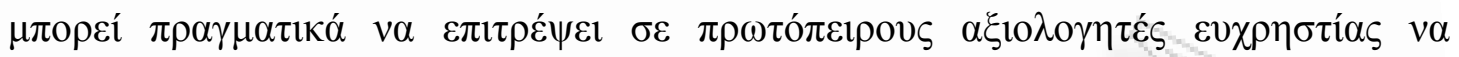

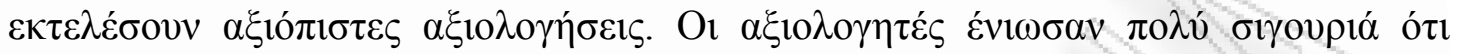

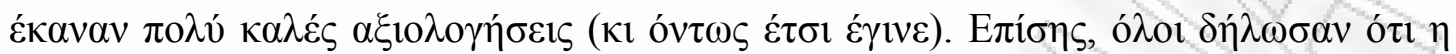

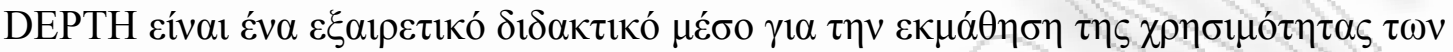

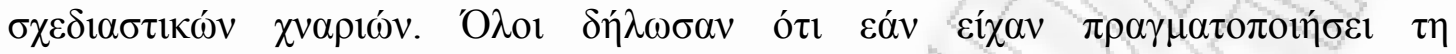

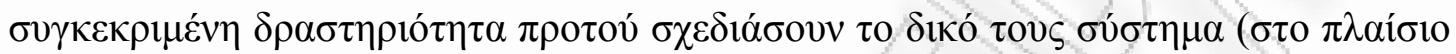

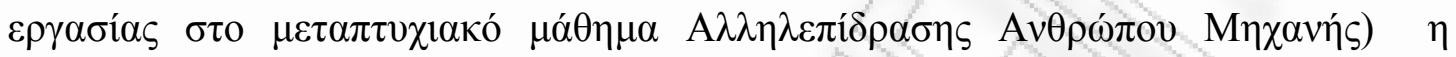

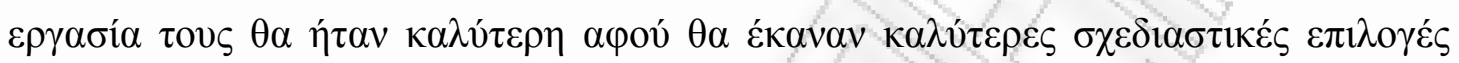

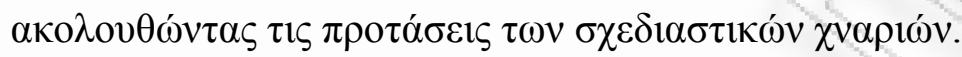

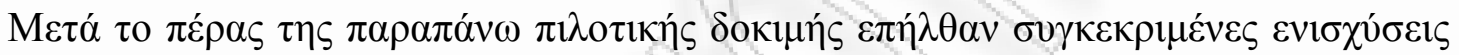

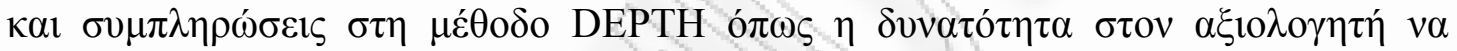

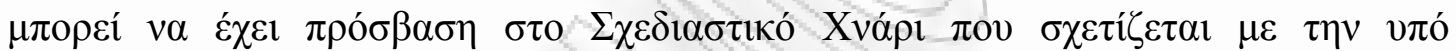

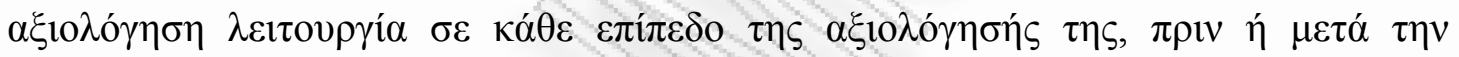

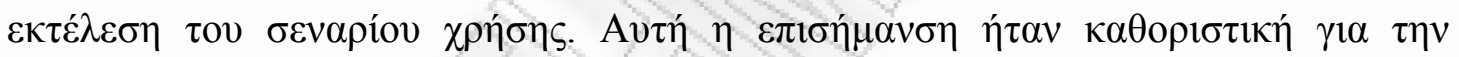

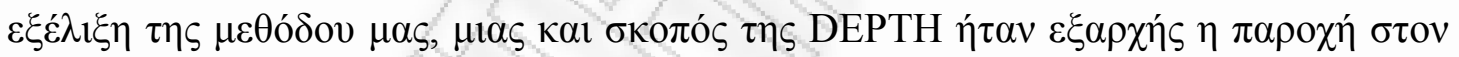

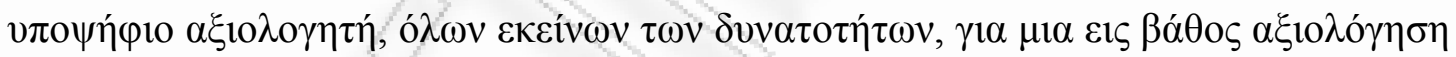

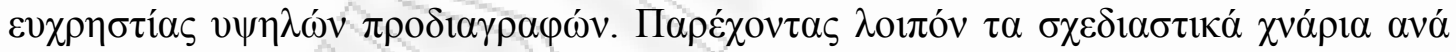

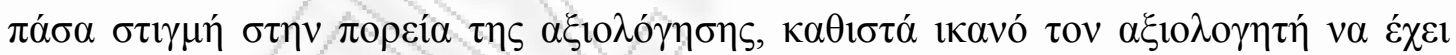

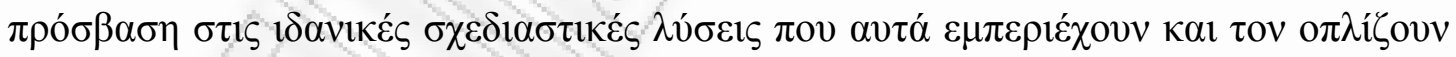

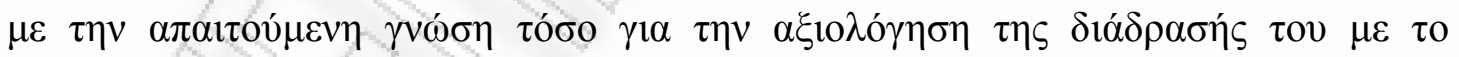

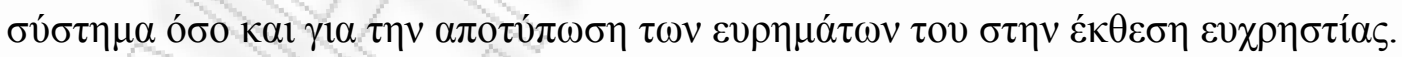

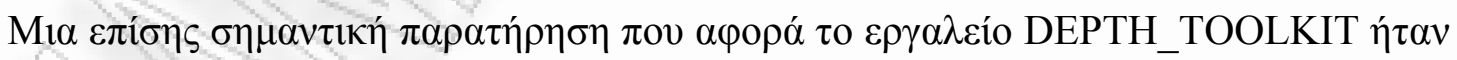

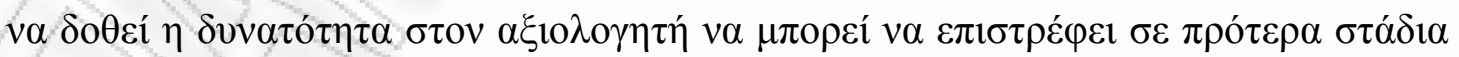

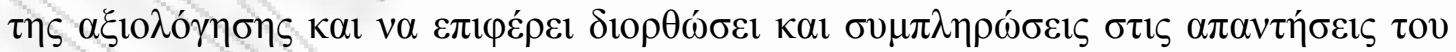

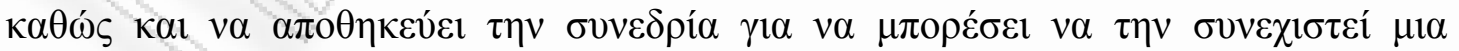
$\mu \varepsilon \tau \alpha \gamma \varepsilon v \varepsilon \dot{\sigma} \tau \varepsilon \rho \eta \sigma \tau \imath \gamma \mu \eta ́$.

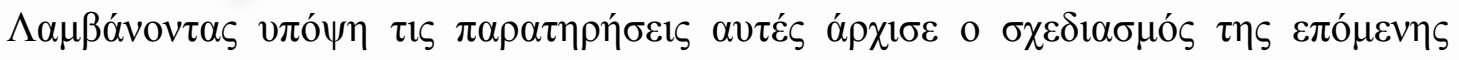

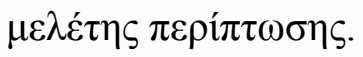




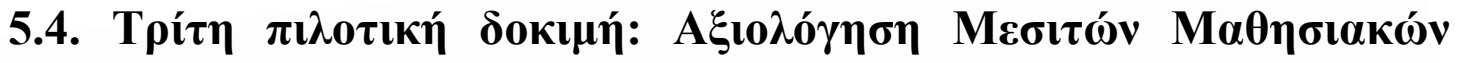

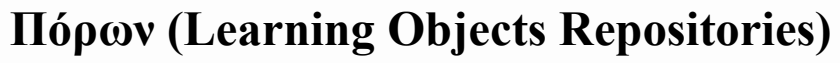

\subsection{1. Геvıкó}

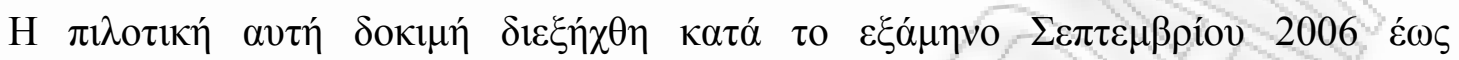

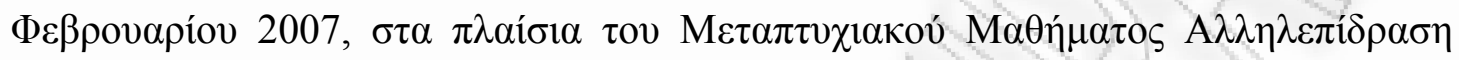

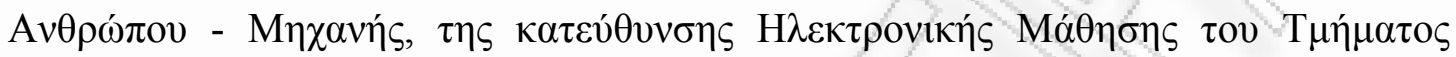

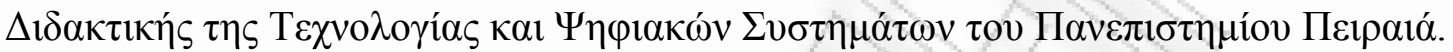

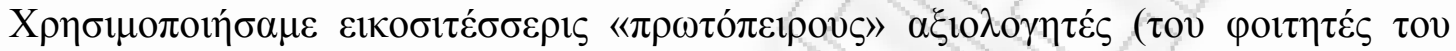

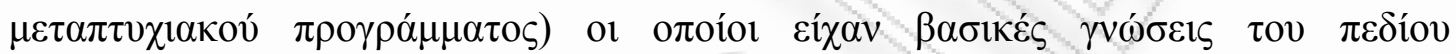

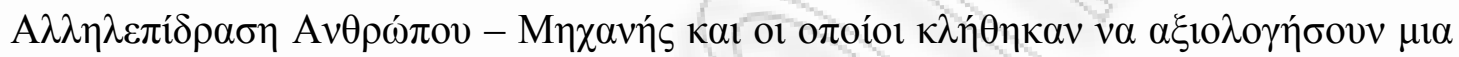

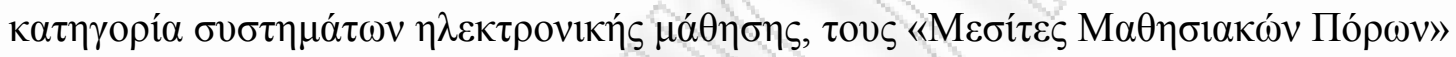

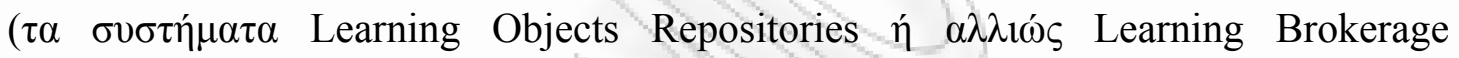

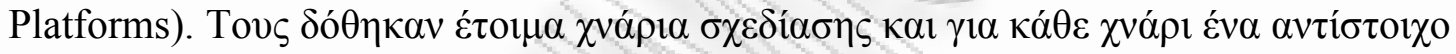

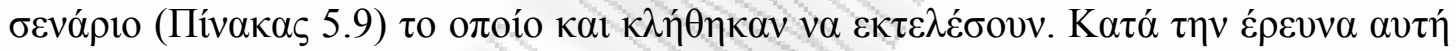

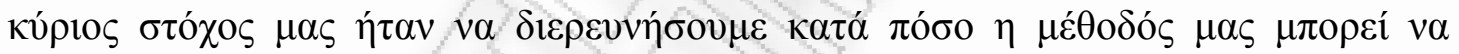

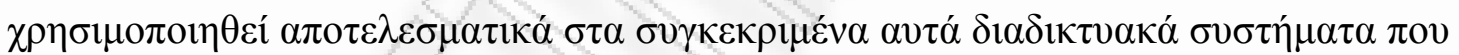

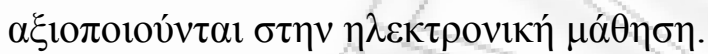

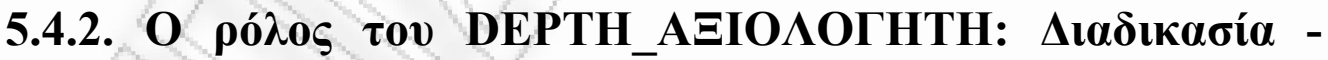

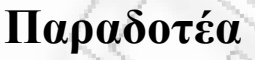

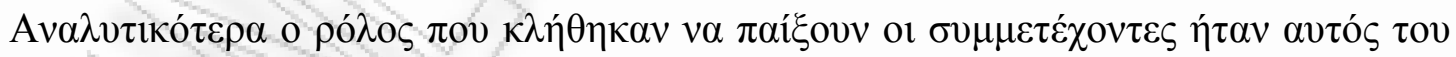

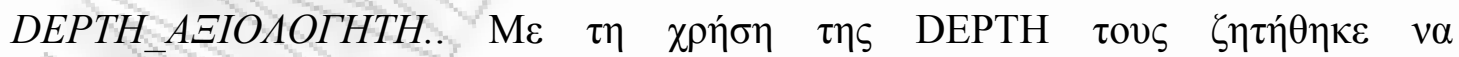

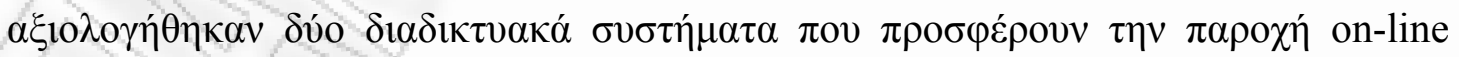

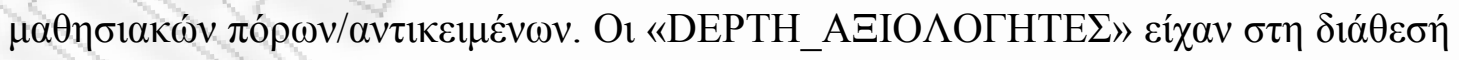

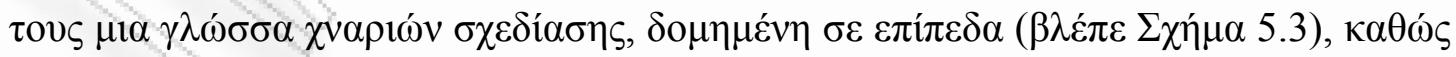

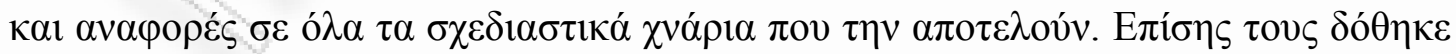

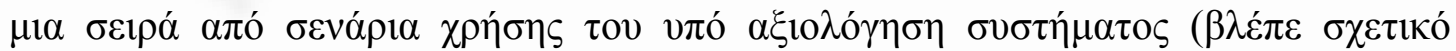

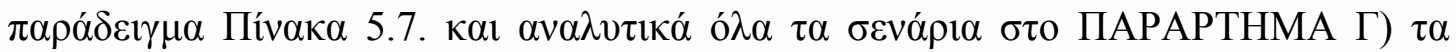

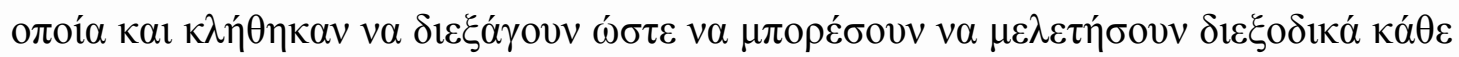

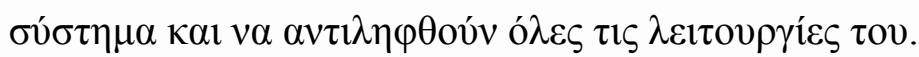




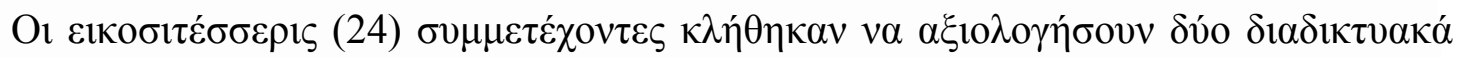
$\sigma v \sigma \tau \eta ́ j \alpha \tau \alpha \mu \varepsilon \sigma \iota \omega_{v}:$

- Adobe Store - North America

- Premier Training on line

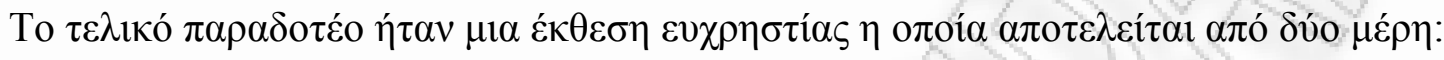

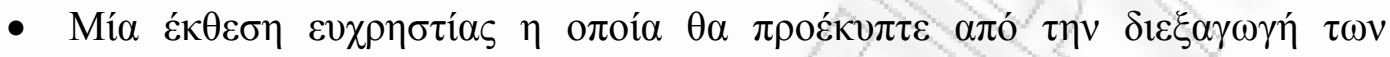

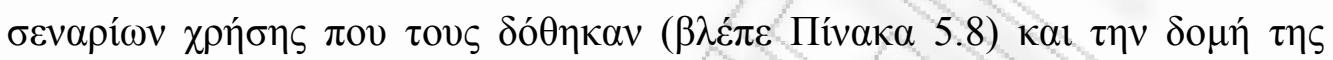

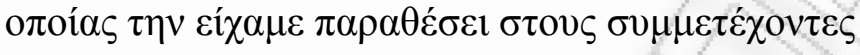

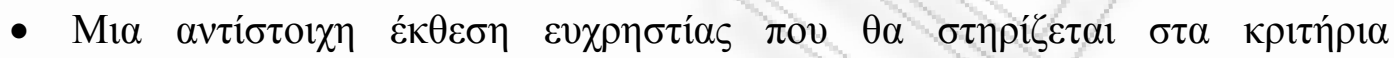

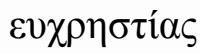

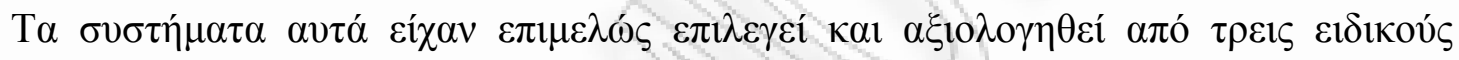

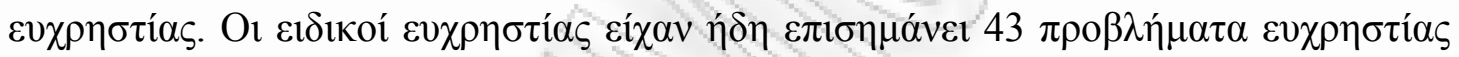

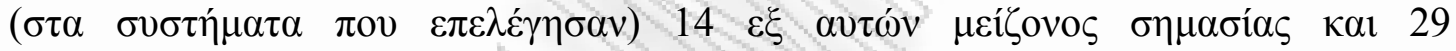

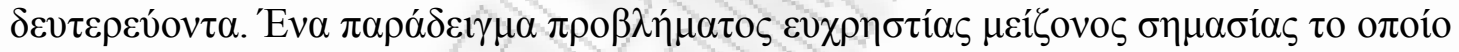

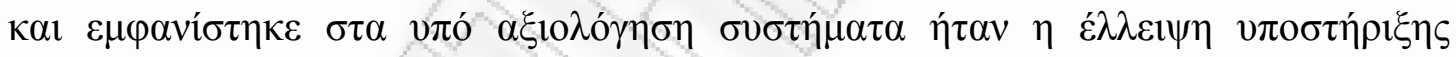

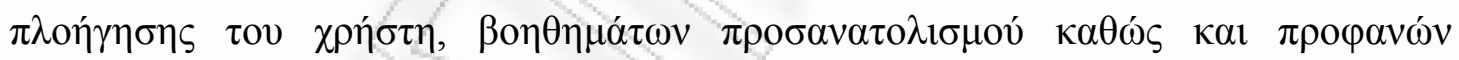

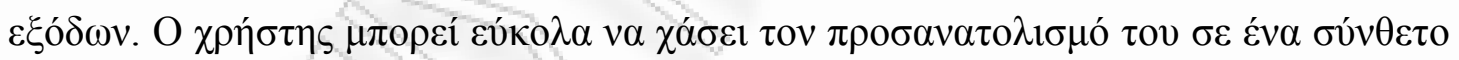

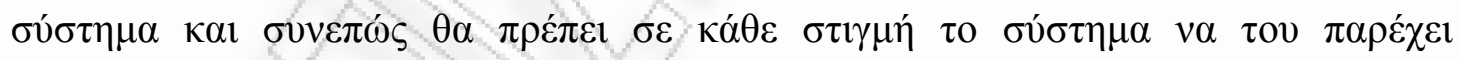

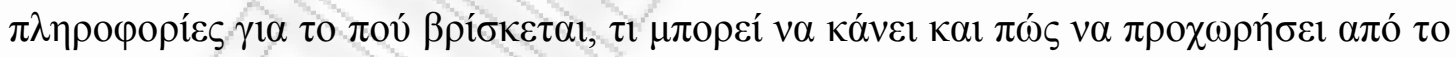

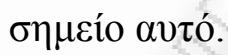

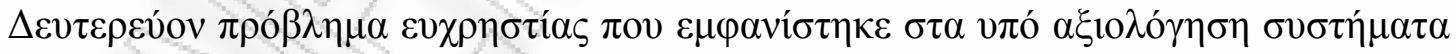

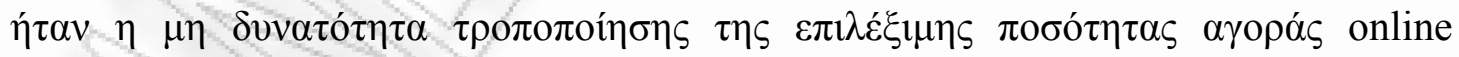

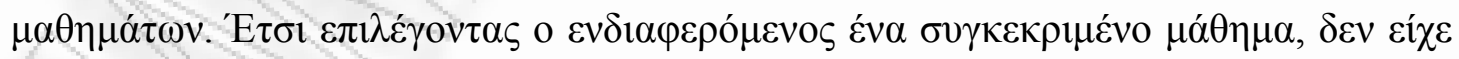

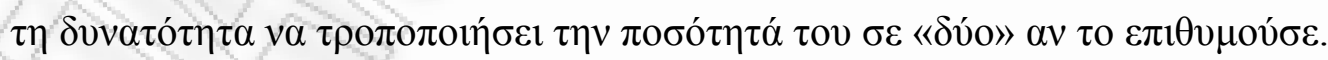

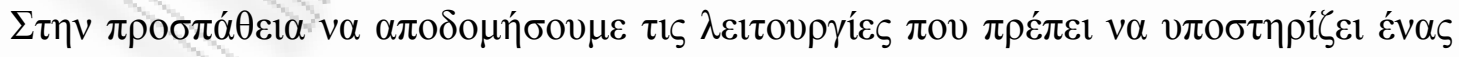

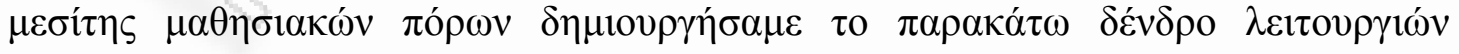

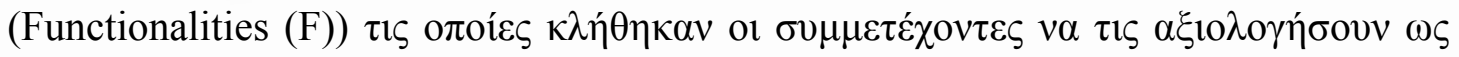

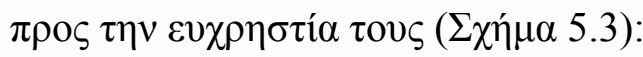




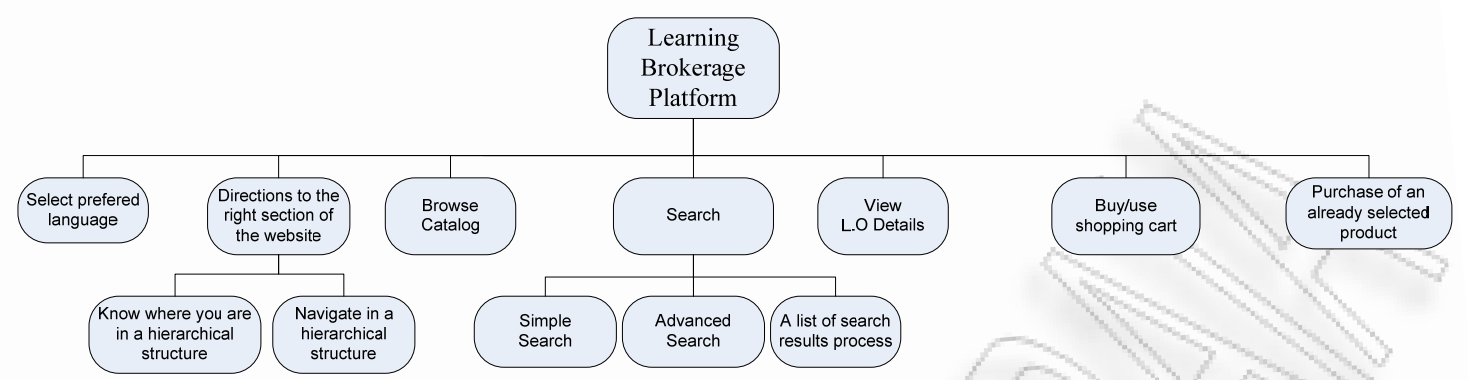

$\Sigma \chi \eta ́ p \alpha$ 5.3. $\Delta \varepsilon \dot{v} \delta \rho o ~ \lambda \varepsilon ı \tau o v \rho \gamma \iota \omega ́ v$ Learning Brokerage Platforms

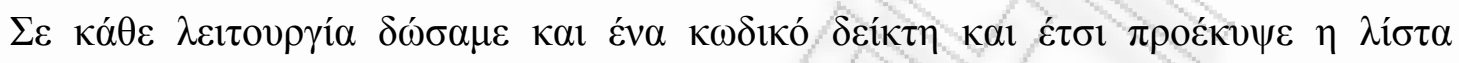

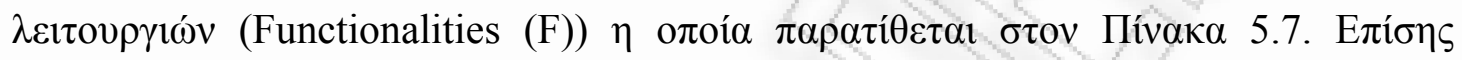

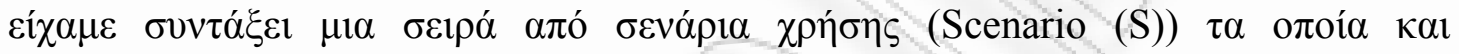

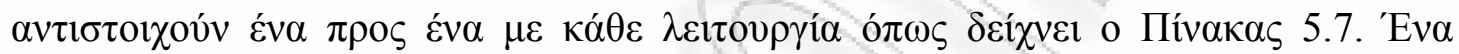

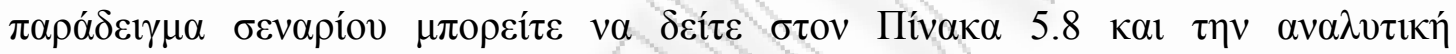

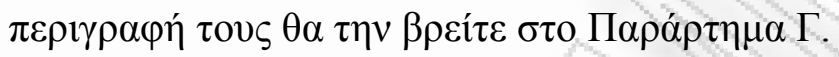

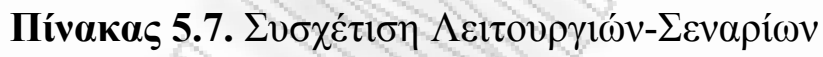

\begin{tabular}{|c|c|}
\hline 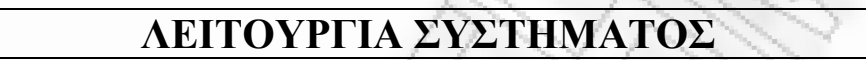 & $\Sigma E N A P I O ~ X P H \Sigma H \Sigma$ \\
\hline F20. Select preferred language & S1. Language Selector \\
\hline F21. Directions to the right section of the website & S2. Doormat Navigation \\
\hline $\begin{array}{l}\text { F22. Know where you are in a hierarchical } \\
\text { structure }\end{array}$ & S3. Breadcrumbs \\
\hline F23. Navigate a hierarchical structure & S4. Double Tab Navigation \\
\hline F24. Browse Catalog $\quad \times$ & S5. Main Navigation \\
\hline F25. Search $\quad$ & S6. Search Area \\
\hline F26. Simple Search & S7. Search Box \\
\hline F27. Advanced Search & S8. Advanced Search \\
\hline F28. A list of search results & S9. Search Results \\
\hline F29. View LO Details & S10. Overview by Detail \\
\hline F30. Buy/use shopping basket & S11. Shopping Cart \\
\hline F31. Purchase of an already selected product & S12. Purchase Process \\
\hline
\end{tabular}

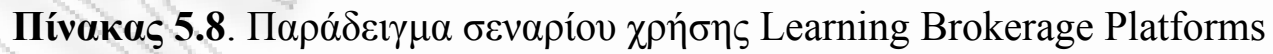

\begin{tabular}{|l|l|}
\hline (S8)Description: & Advanced Search \\
\hline Task: & $\begin{array}{l}\text { Try to put in your searching something very common so a large } \\
\text { collection of items can arouse. Try to find a specific item in this } \\
\text { collection by using full control over the search engine so that you } \\
\text { can be more successful in achieving your goals. Keep in mind that } \\
\text { you have already an additional knowledge of the data that is } \\
\text { available and it is hence only for experienced users like you. }\end{array}$ \\
\hline Questions: & $\bullet$ Was there an advanced search button or option that leads you in \\
\hline
\end{tabular}




\begin{tabular}{|l|l|}
\hline - Was in these options included AND/OR functionality together \\
with exclusion functionality? If so could you use with the same \\
convenience the functionalities "match all terms" and "match \\
any term" instead of AND and OR operators in the text field? \\
- Could you limit the possible Search Results by defining the \\
conditions that a result must meet (for example, results from a \\
particular subsite, item types (articles, video, audio...), or item \\
properties (title, date, location, size, author...)?
\end{tabular}

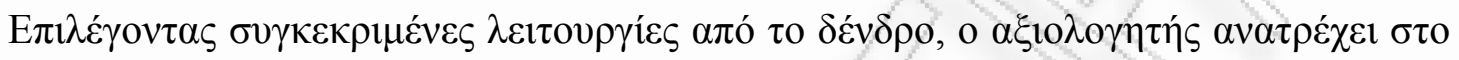

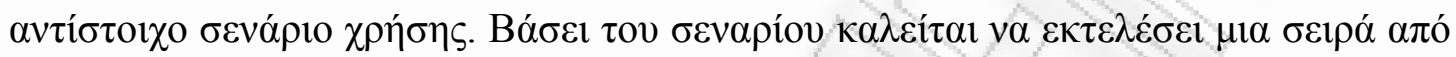

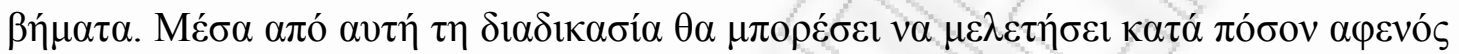

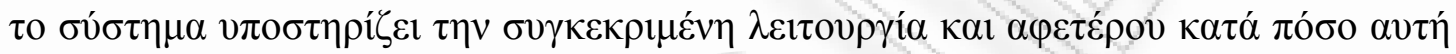

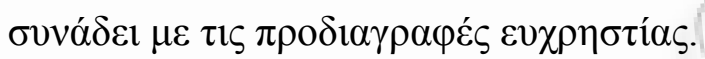

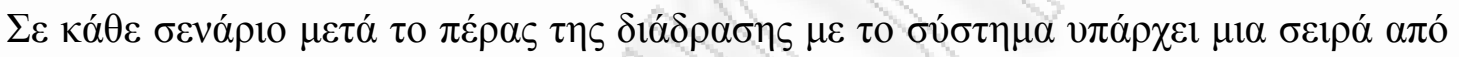

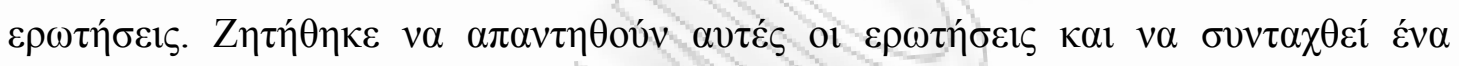

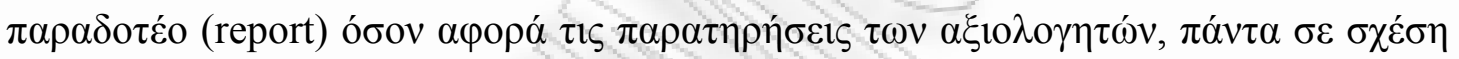

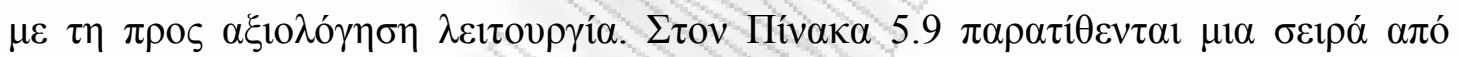

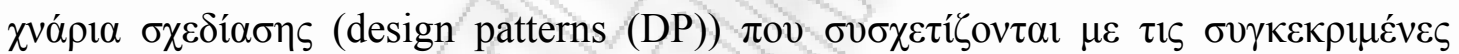

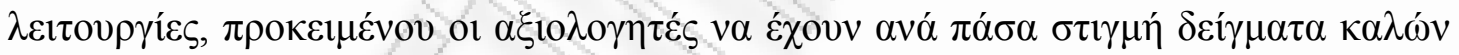

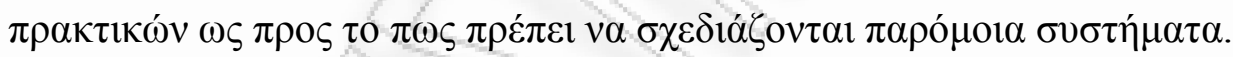

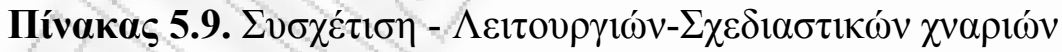

\begin{tabular}{|c|c|}
\hline АЕITOYРГІА ГY & 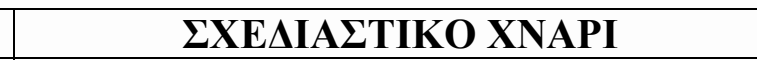 \\
\hline F1. Select preferred language & $\begin{array}{c}\text { http://www.welie.com/patterns/showPattern. } \\
\text { php?patternID=language-selector }\end{array}$ \\
\hline $\begin{array}{l}\text { F2. Directions to the right section } \\
\text { of the website }\end{array}$ & $\begin{array}{c}\text { http://www.welie.com/patterns/showPattern. } \\
\text { php?patternID=doormat }\end{array}$ \\
\hline $\begin{array}{l}\text { F3. Know where you are in a } \\
\text { hierarchical structure }\end{array}$ & $\begin{array}{c}\text { http://www.welie.com/patterns/showPattern. } \\
\text { php?patternID=crumbs }\end{array}$ \\
\hline $\begin{array}{l}\text { F4. Navigate a hierarchical } \\
\text { structure }\end{array}$ & $\begin{array}{l}\text { http://www.welie.com/patterns/showPattern. } \\
\text { php?patternID=doubletab }\end{array}$ \\
\hline F5. Browse Catalog & $\begin{array}{c}\text { http://www.welie.com/patterns/showPattern. } \\
\text { php?patternID=main-navigation }\end{array}$ \\
\hline F6. Search & $\begin{array}{l}\text { http://www.welie.com/patterns/showPattern. } \\
\text { php?patternID=searcharea }\end{array}$ \\
\hline F7. Simple Search & $\begin{array}{c}\text { http://www.welie.com/patterns/showPattern. } \\
\text { php?patternID }=\text { search }\end{array}$ \\
\hline F8. Advanced Search & $\begin{array}{c}\text { http://www.welie.com/patterns/showPattern. } \\
\text { php?patternID=advanced-search }\end{array}$ \\
\hline
\end{tabular}




\begin{tabular}{|l|l|}
\hline F9. A list of search results & $\begin{array}{r}\text { http://www.welie.com/patterns/showPattern. } \\
\text { php?patternID=search-results }\end{array}$ \\
\hline F10. View LO Details & $\begin{array}{r}\text { http://www.welie.com/patterns/showPattern. } \\
\text { php?patternID=overview-detail }\end{array}$ \\
\hline F11. Buy/use shopping basket & $\begin{array}{r}\text { http://www.welie.com/patterns/showPattern. } \\
\text { php?patternID=shopping-cart }\end{array}$ \\
\hline $\begin{array}{c}\text { F12. Purchase of an already } \\
\text { selected product }\end{array}$ & $\begin{array}{r}\text { http://www.welie.com/patterns/showPattern. } \\
\text { php?patternID=purchase-process }\end{array}$ \\
\hline
\end{tabular}

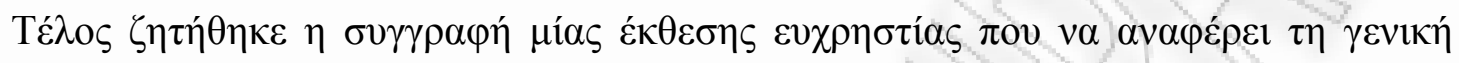

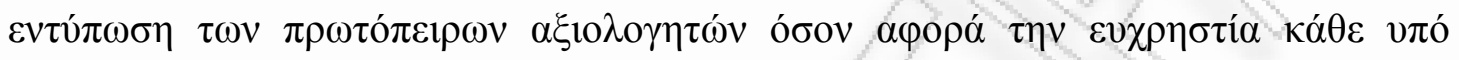

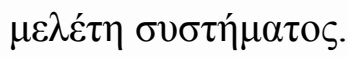

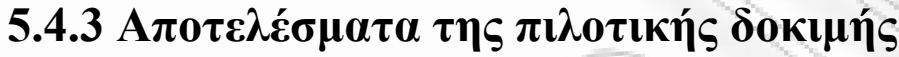

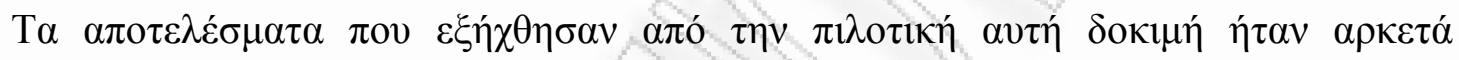

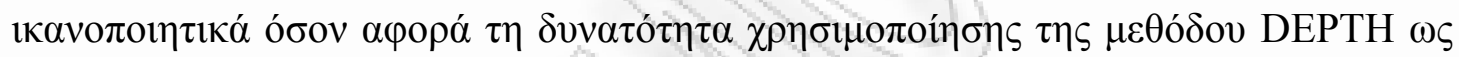

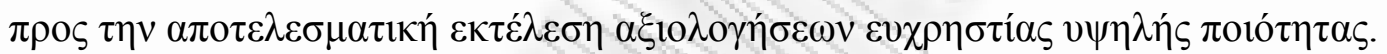

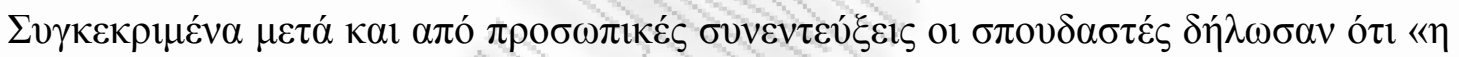

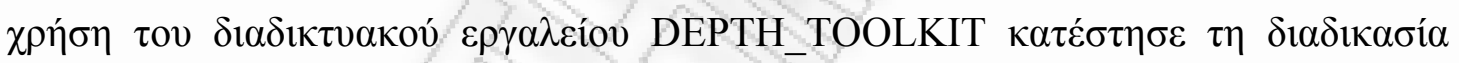

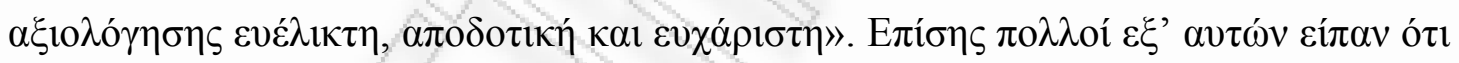

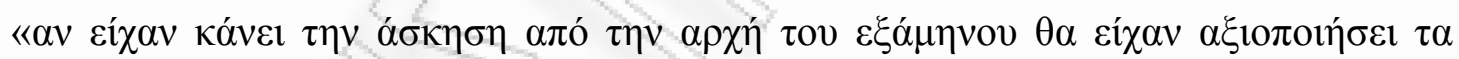

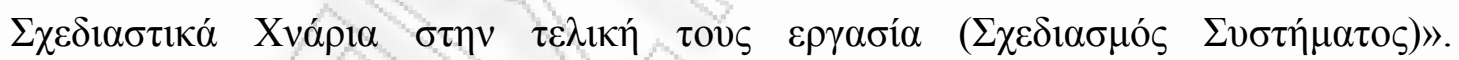

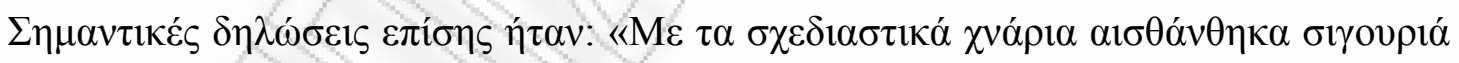

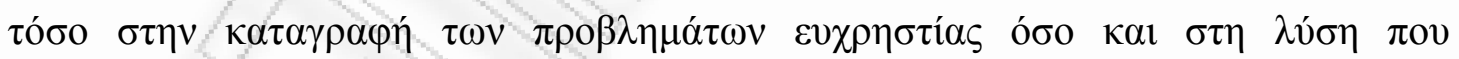
$\pi \rho o ́ \tau \varepsilon เ v \alpha »$.

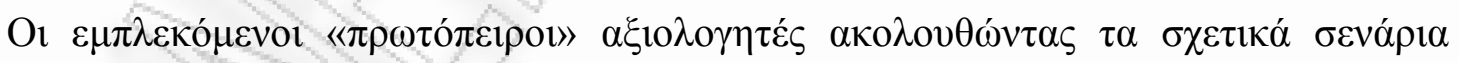

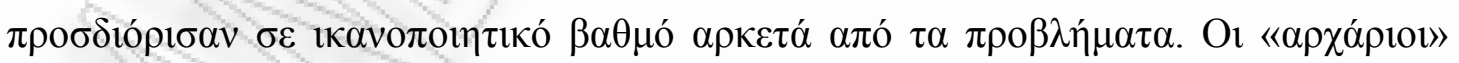

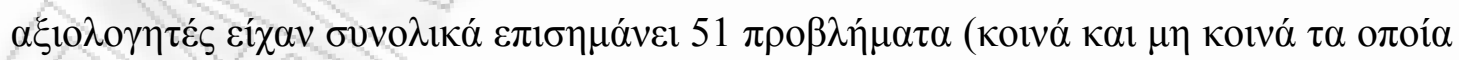

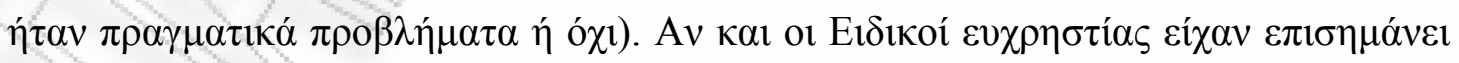

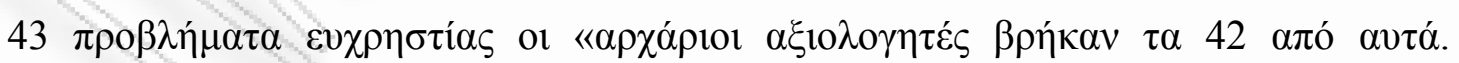

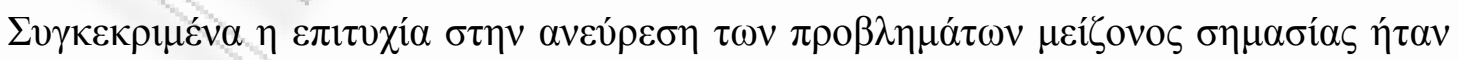

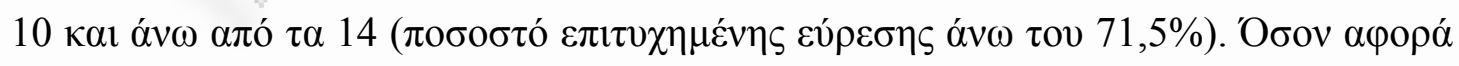

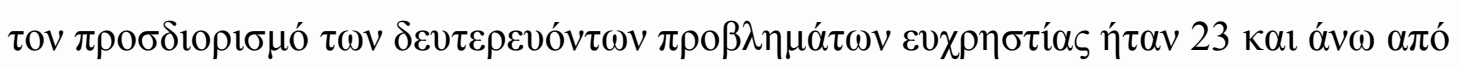

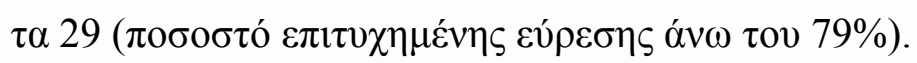




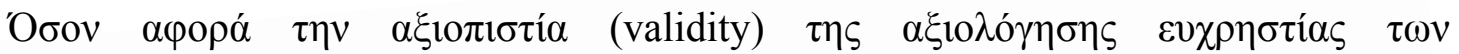

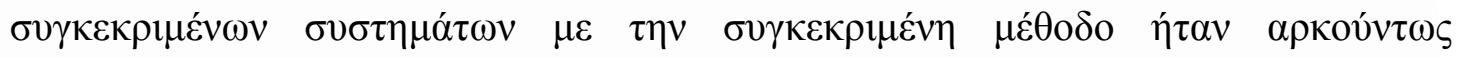

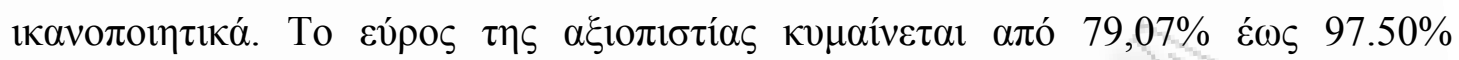

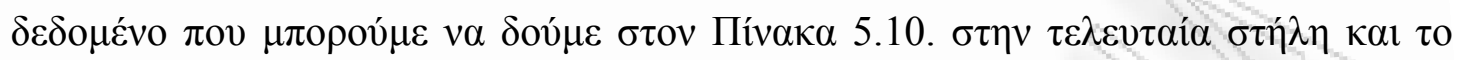

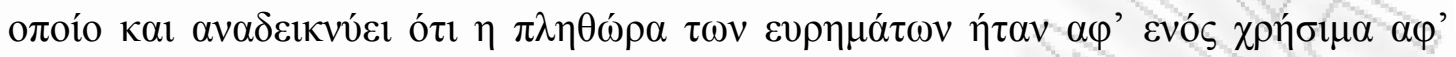

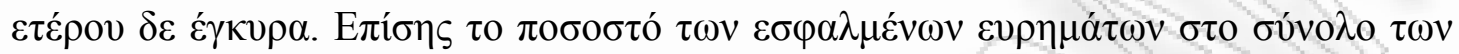

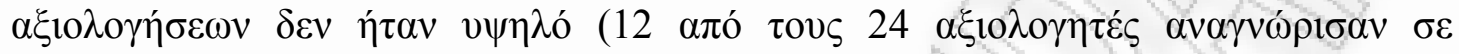

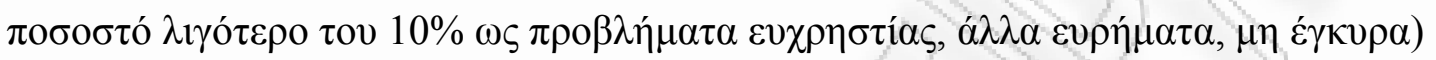

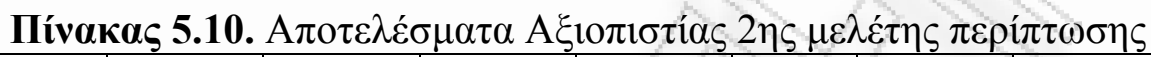

\begin{tabular}{|c|c|c|c|c|c|c|c|c|c|}
\hline $\begin{array}{c}\mathrm{A} / \mathrm{A} \\
\mathrm{A} \xi 10 \lambda \text { orn }{ }^{\prime}\end{array}$ & 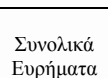 & $\begin{array}{c}\mathrm{M \eta} \dot{\varepsilon} \gamma \kappa v \rho \alpha \\
\tau \rho \rho \beta \lambda \eta \alpha \alpha \tau \alpha\end{array}$ & 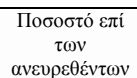 & 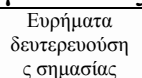 & $\begin{array}{c}\text { Побобто́ } \varepsilon \tilde{i} \\
\tau \omega v \\
\alpha \nu \varepsilon \rho \rho \varepsilon \dot{\varepsilon} v \tau \omega v\end{array}$ & 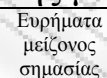 & $\begin{array}{c}\text { Побобто́ } \varepsilon \pi \dot{1} \\
\tau \omega v \\
\alpha v \varepsilon v \rho \varepsilon \varepsilon \dot{v} v \tau \omega v\end{array}$ & \multicolumn{2}{|c|}{ A $\xi_{1} \circ \pi \mid \sigma \tau i \alpha$} \\
\hline & & & & & & & $4=$ & 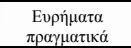 & $\%$ \\
\hline 1 & 38 & 3 & 7,89 & 25 & $\quad 65,79$ & 10 & 26,32 & 35 & 92,11 \\
\hline 2 & 40 & 3 & 7,50 & 26 & 65,00 & 11 & 27,50 & 37 & 92,50 \\
\hline 3 & 39 & 2 & 5,13 & 26 & 66,67 & 11 & 28,21 & 37 & 94,87 \\
\hline 4 & 41 & 6 & 14,63 & 25 & 60,98 & 10 & 24,39 & 35 & 85,37 \\
\hline 5 & 40 & 1 & 2,50 & 29 & 72,50 & 10 & 25,00 & 39 & 97,50 \\
\hline 6 & 39 & 3 & 7,69 & 26 & 66,67 & 10 & 25,64 & 36 & 92,31 \\
\hline 7 & 39 & 4 & 10,26 & 24 & 61,54 & $x_{n}$ & 28,21 & 35 & 89,74 \\
\hline 8 & 43 & 6 & 13,95 & 25 & 58,14 & 12 & 27,91 & 37 & 86,05 \\
\hline 9 & 45 & 3 & 6,67 & 28 & 62,22 & 14 & 31,11 & 42 & 93,33 \\
\hline 10 & 40 & 4 & 10,00 & 25 & 62,50 & 11 & 27,50 & 36 & 90,00 \\
\hline 11 & 38 & 4 & 10,53 & 23 & 60,53 & 11 & 28,95 & 34 & 89,47 \\
\hline 12 & 42 & 7 & 16,67 & 25 & 59,52 & 10 & 23,81 & 35 & 83,33 \\
\hline 13 & 41 & 4 & $\quad 9,76$ & 26 & 63,41 & 11 & 26,83 & 37 & 90,24 \\
\hline 14 & 43 & 9 & 20,93 & 24 & 55,81 & 10 & 23,26 & 34 & 79,07 \\
\hline 15 & 39 & 2 & 5,13 & 25 & 64,10 & 12 & 30,77 & 37 & 94,87 \\
\hline 16 & 43 & 6 & 13,95 & 25 & 58,14 & 12 & 27,91 & 37 & 86,05 \\
\hline 17 & 39 & 3 & 7,69 & 25 & 64,10 & 11 & 28,21 & 36 & 92,31 \\
\hline 18 & 44 & 7 & 15,91 & 26 & 59,09 & 11 & 25,00 & 37 & 84,09 \\
\hline 19 & 40 & 5 & 12,50 & 23 & 57,50 & 12 & 30,00 & 35 & 87,50 \\
\hline 20 & 44 & 4 & 9,09 & 27 & 61,36 & 13 & 29,55 & 40 & 90,91 \\
\hline 21 & 42 & 5 & 11,90 & 25 & 59,52 & 12 & 28,57 & 37 & 88,10 \\
\hline 22 & 43 & 4 & $\quad 9,30$ & 26 & 60,47 & 13 & 30,23 & 39 & 90,70 \\
\hline 23 & 41 & 5 & 12,20 & 25 & 60,98 & 11 & 26,83 & 36 & 87,80 \\
\hline 24 & 39 & & 7,69 & 26 & 66,67 & 10 & 25,64 & 36 & 92,31 \\
\hline
\end{tabular}

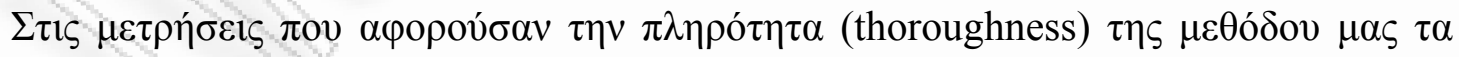

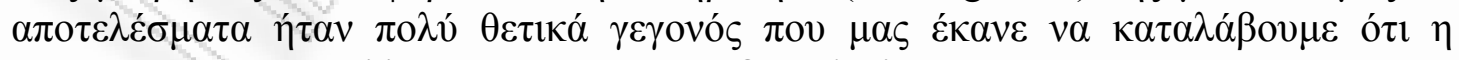

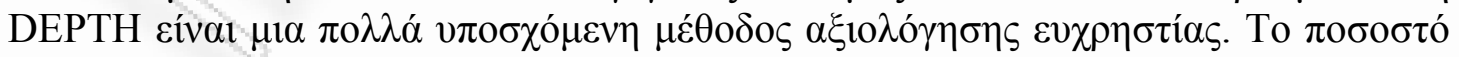

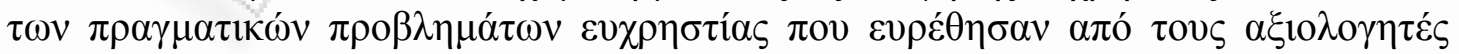

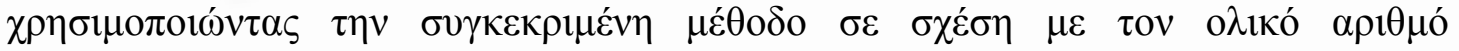

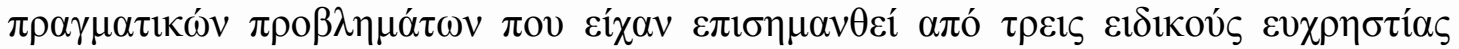

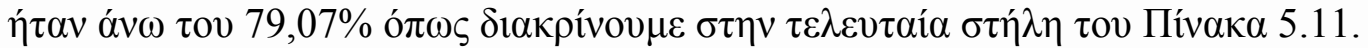




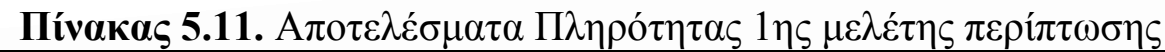

\begin{tabular}{|c|c|c|c|}
\hline $\begin{array}{l}\mathrm{A} / \mathrm{A} \\
\mathrm{A} \xi 10 \lambda \circ \gamma \eta \tau \dot{\eta}\end{array}$ & 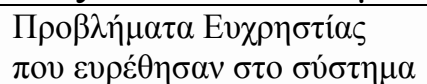 & 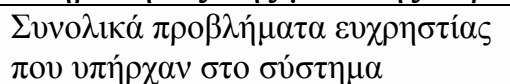 & $\Pi \lambda \eta \rho{ }^{\prime} \tau \eta \tau \alpha \%$ \\
\hline 1 & 35 & 43 & 81,40 \\
\hline 2 & 37 & 43 & 86,05 \\
\hline 3 & 37 & 43 & 86,05 \\
\hline 4 & 35 & 43 & 81,40 \\
\hline 5 & 39 & 43 & 90,70 \\
\hline 6 & 36 & 43 & 83,72 \\
\hline 7 & 35 & 43 & 81,40 \\
\hline 8 & 37 & 43 & 86,05 \\
\hline 9 & 42 & 43 & 97,67 \\
\hline 10 & 36 & 43 & 83,72 \\
\hline 11 & 34 & 43 & 79,07 \\
\hline 12 & 35 & 43 & 81,40 \\
\hline 13 & 37 & 43 & 86,05 \\
\hline 14 & 34 & 43 & 79,07 \\
\hline 15 & 37 & 43 & 86,05 \\
\hline 16 & 37 & 43 & 86,05 \\
\hline 17 & 36 & 43 & 83,72 \\
\hline 18 & 37 & 43 & 86,05 \\
\hline 19 & 35 & 43 & 81,40 \\
\hline 20 & 40 & 43 & 93,02 \\
\hline 21 & $\begin{array}{r}47 \\
\end{array}$ & 43 & 86,05 \\
\hline 22 & 39 & 43 & 90,70 \\
\hline 23 & 36 & 43 & 83,72 \\
\hline 24 & 36 & 43 & 83,72 \\
\hline
\end{tabular}

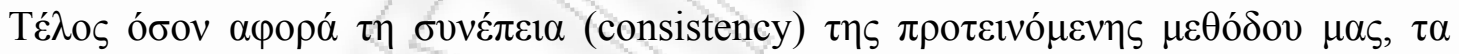

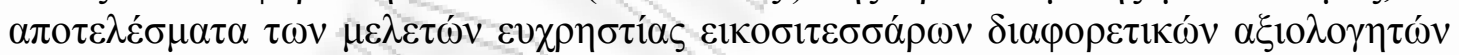

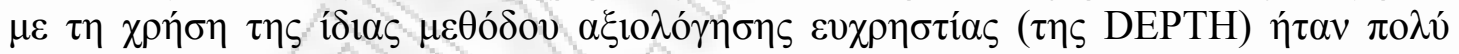

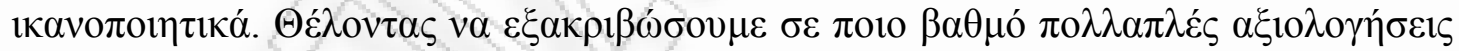

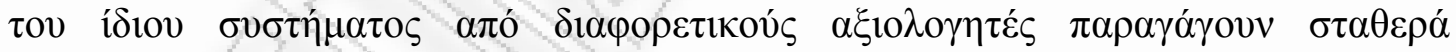

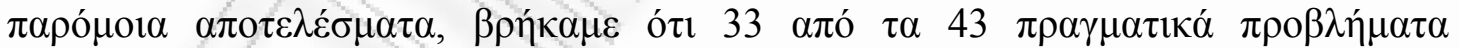

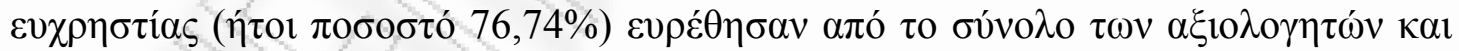

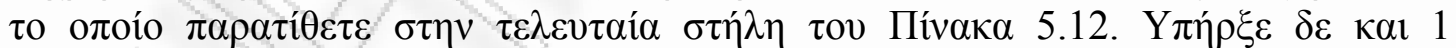

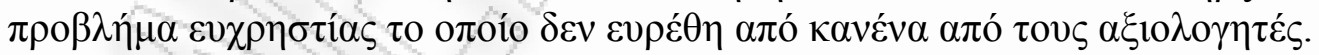

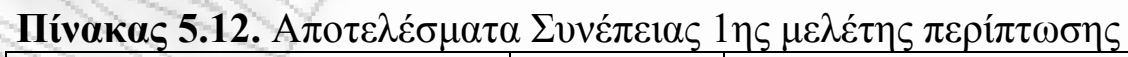

\begin{tabular}{|c|c|c|}
\hline 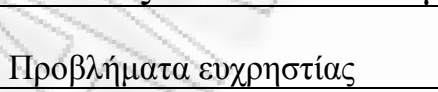 & 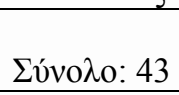 & 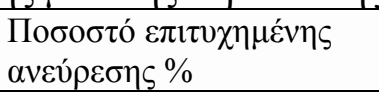 \\
\hline 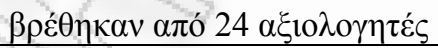 & 33 & 76,74 \\
\hline 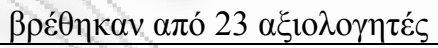 & 34 & 79,07 \\
\hline 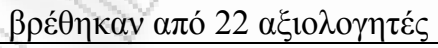 & 34 & 79,07 \\
\hline$\beta \rho \varepsilon \theta^{\prime} \eta \kappa \alpha \nu \alpha \pi$ ó $21 \alpha \xi_{10 \lambda o \gamma \eta \tau} \varepsilon^{\prime} \varsigma$ & 35 & 81,40 \\
\hline 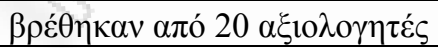 & 35 & 81,40 \\
\hline 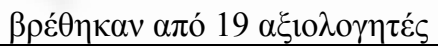 & 36 & 83,72 \\
\hline 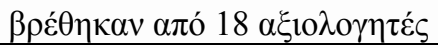 & 35 & 81,40 \\
\hline 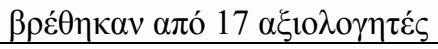 & 36 & 83,72 \\
\hline 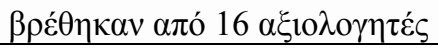 & 36 & 83,72 \\
\hline 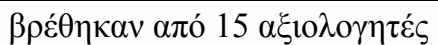 & 37 & 86,05 \\
\hline
\end{tabular}




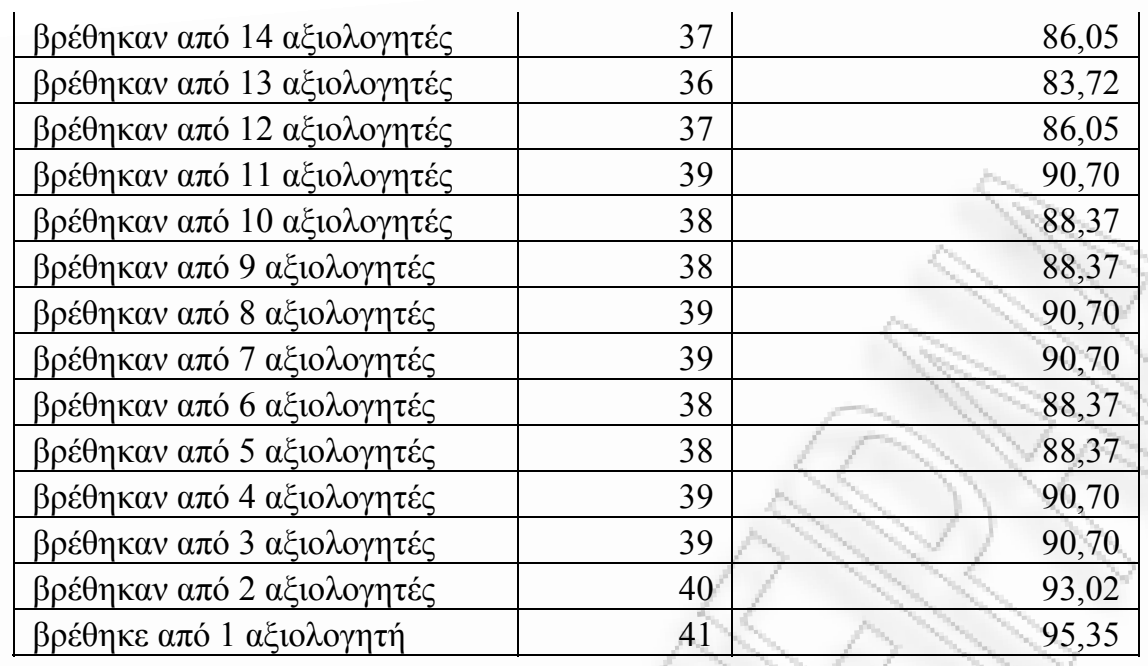

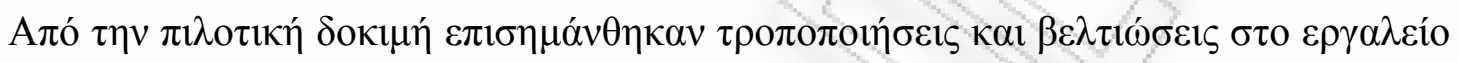

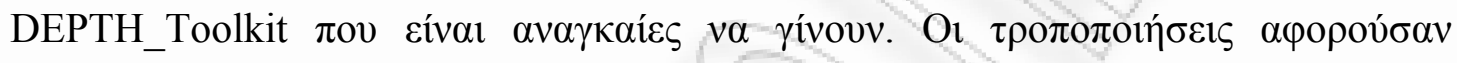

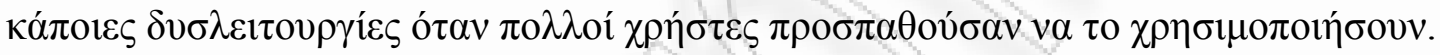




\section{КЕФАМАIO 6

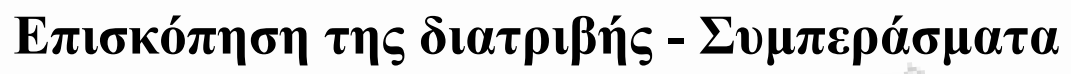

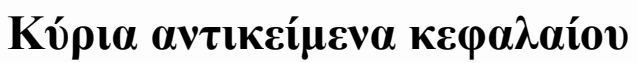

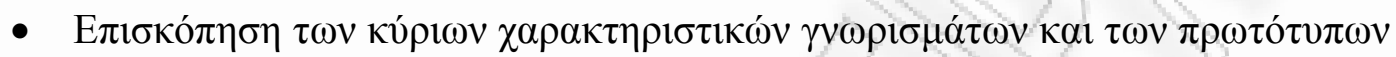

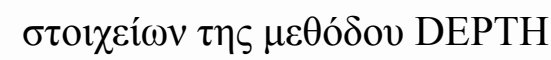

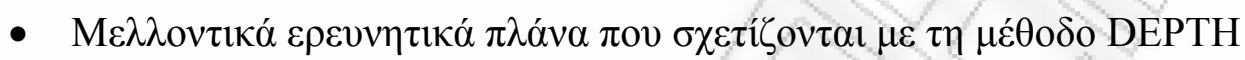

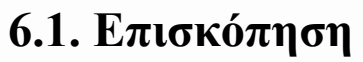

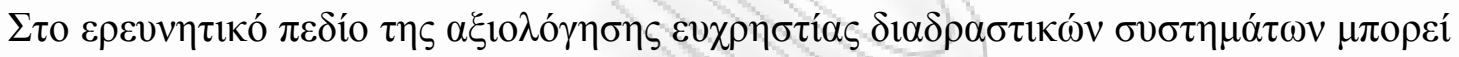

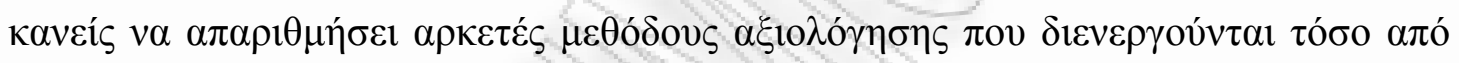

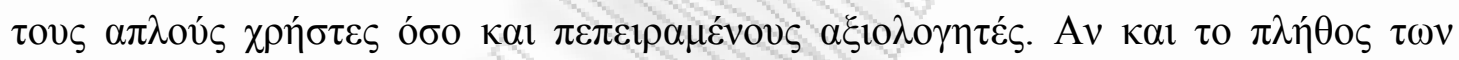

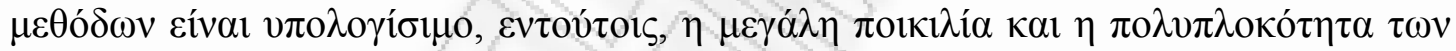

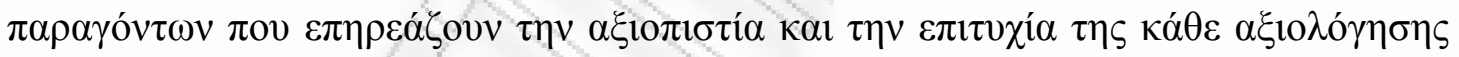

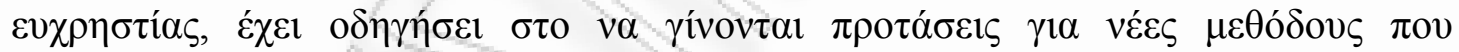

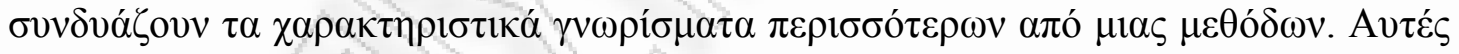

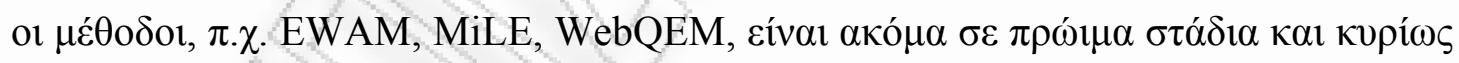

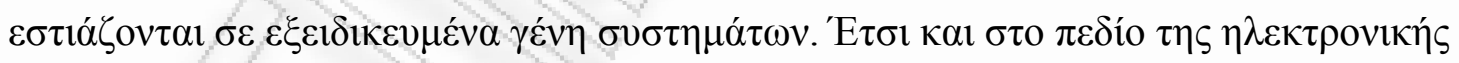

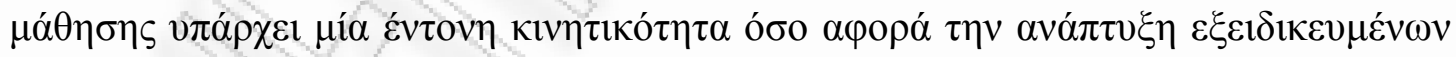

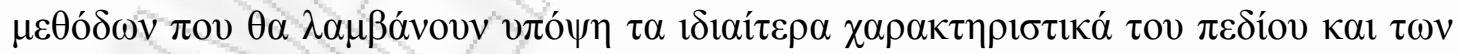
$\varepsilon \mu \pi \lambda \varepsilon \kappa о \mu \varepsilon ́ v \omega \nu$.

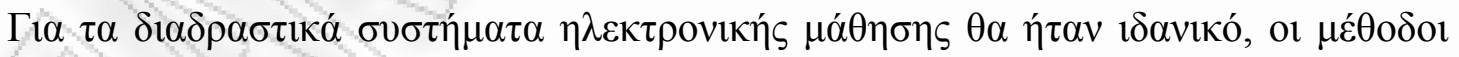

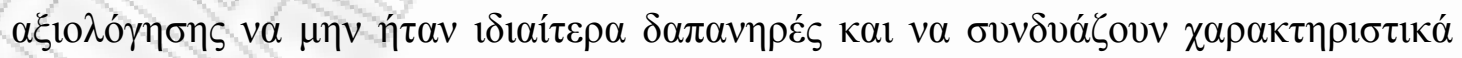

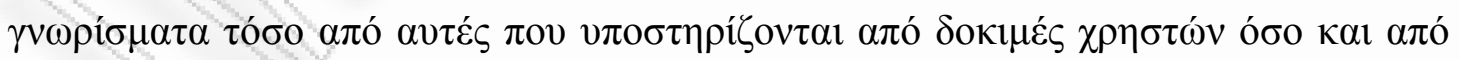

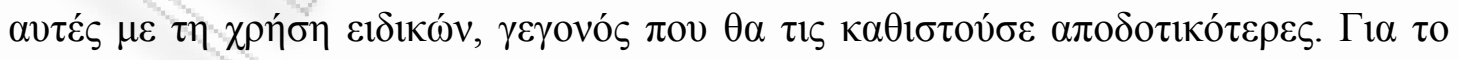
$\lambda$ ó́

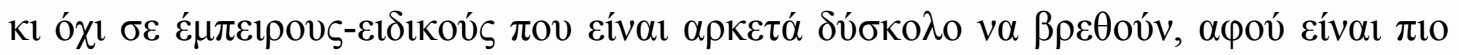

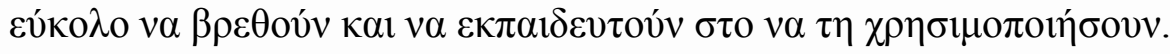




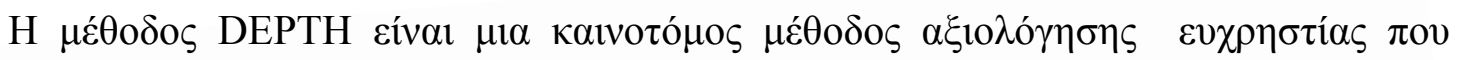

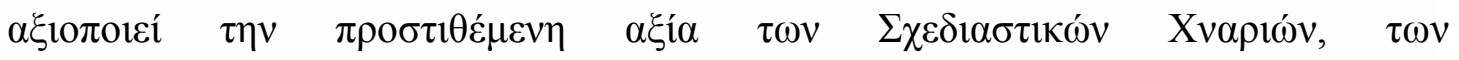

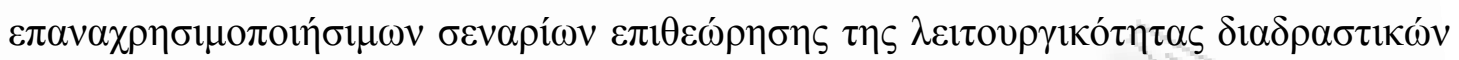

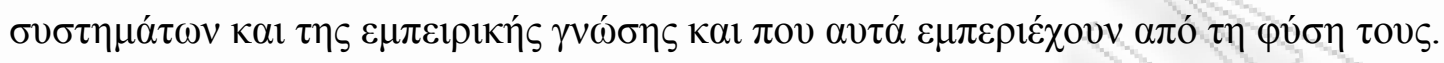

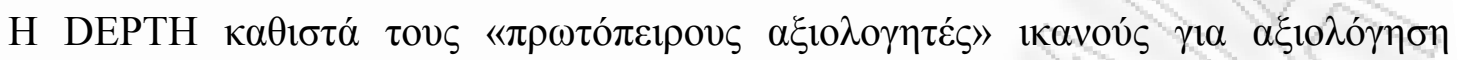

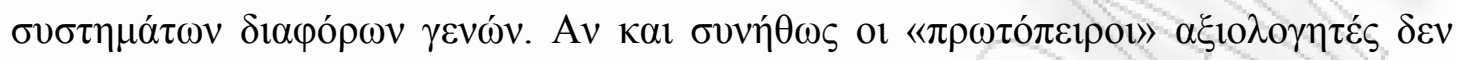

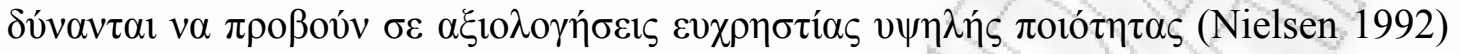

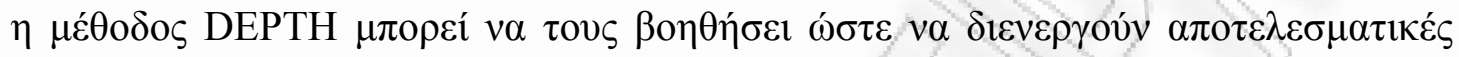
$\mu \varepsilon \lambda \varepsilon ́ \tau \varepsilon \varsigma \alpha \xi 10 \lambda o ́ \gamma \eta \sigma \eta \varsigma$.

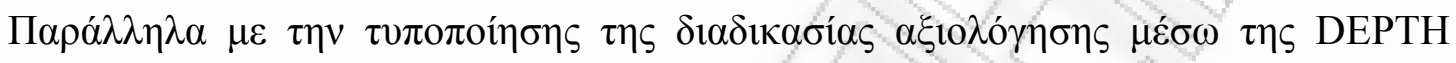

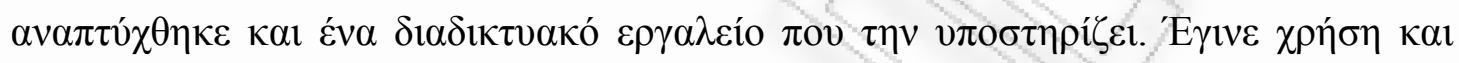

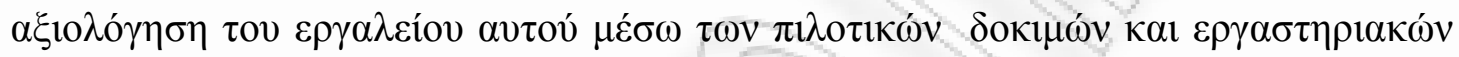

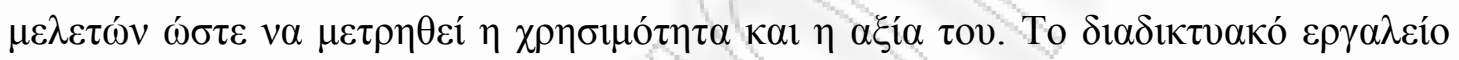

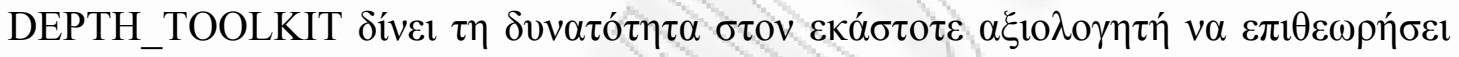

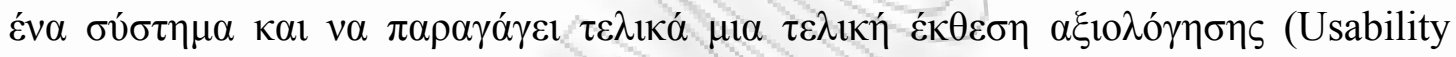

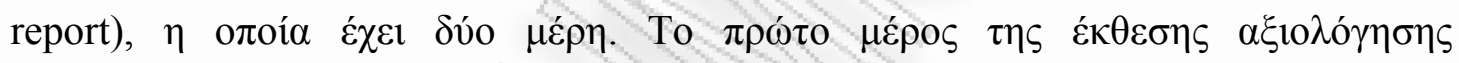

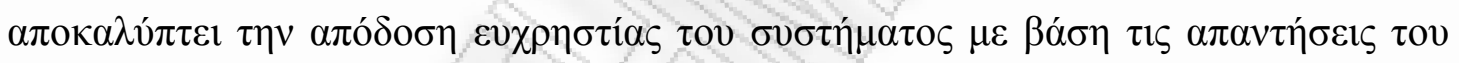

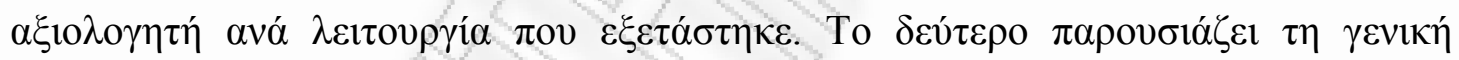

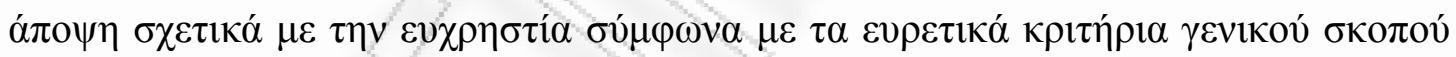
$(\pi \cdot \chi \cdot$ Nielsen$)$.

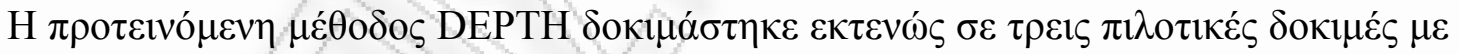

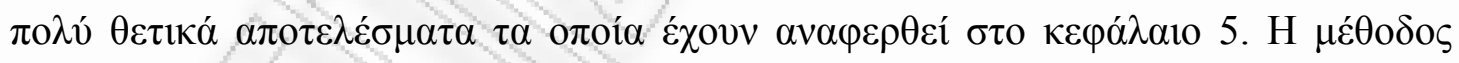

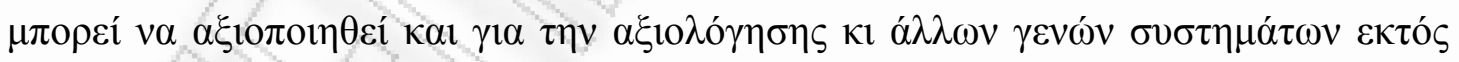

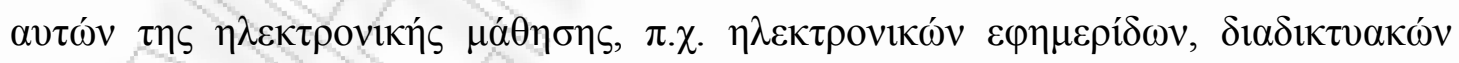

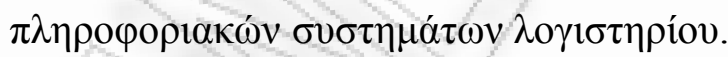

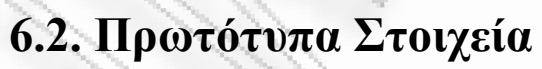

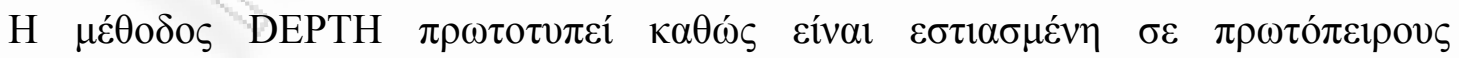

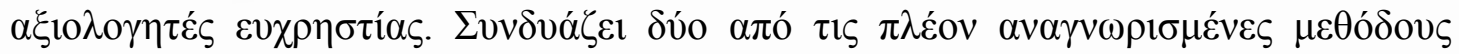

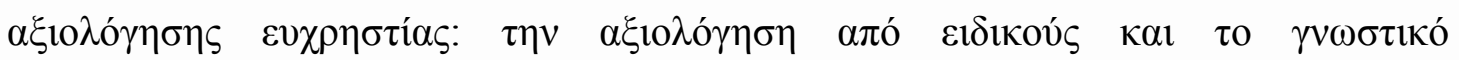

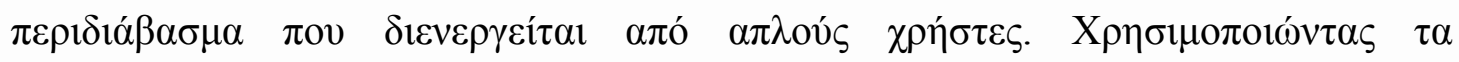




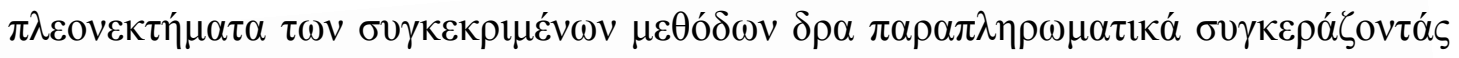

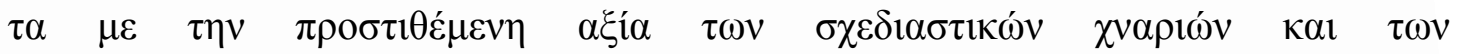

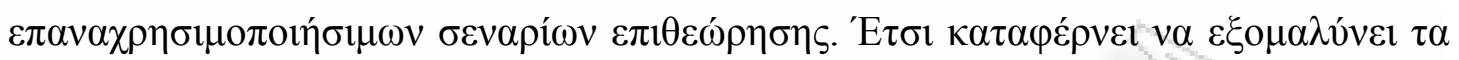

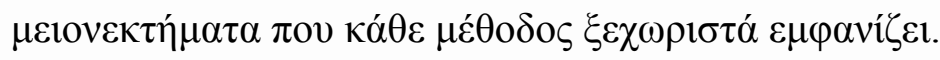

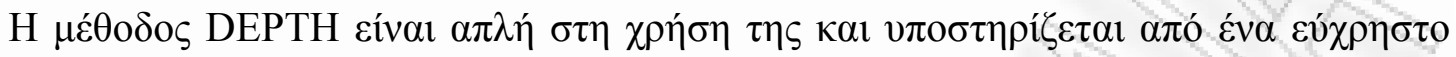

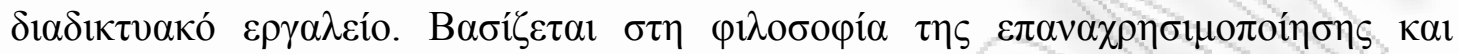

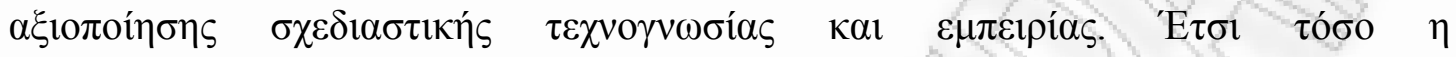

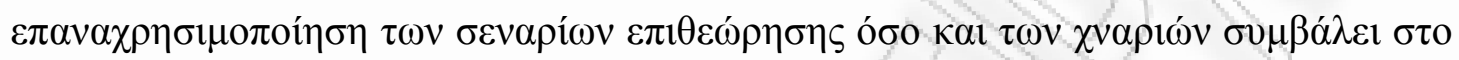

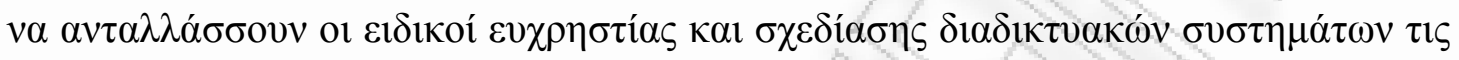

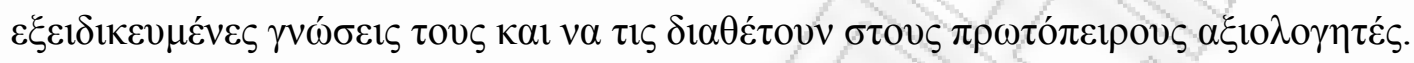

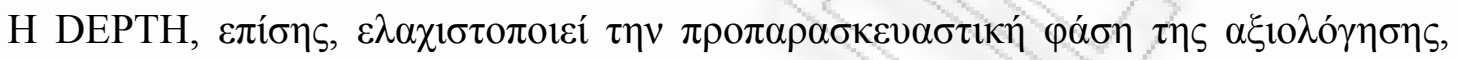

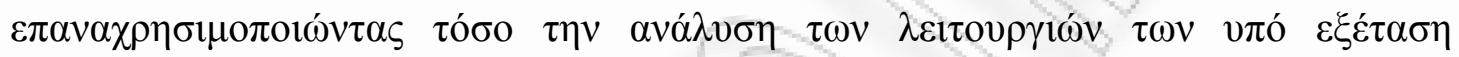

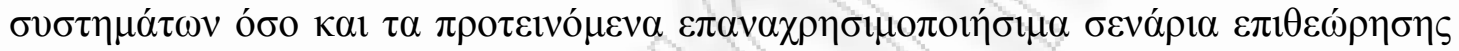

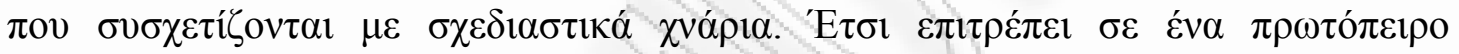

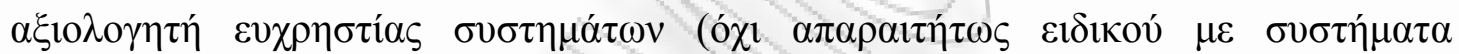

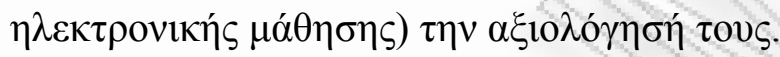

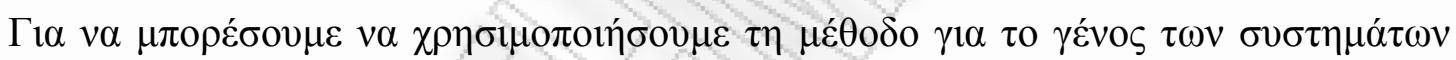

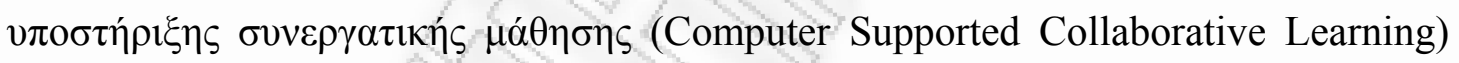

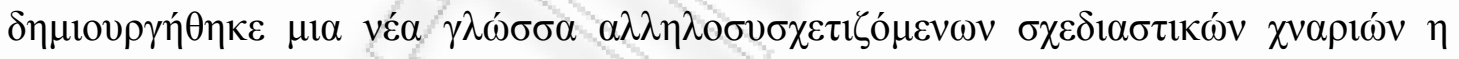

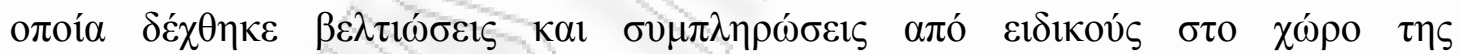

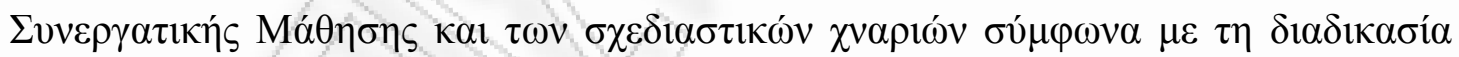

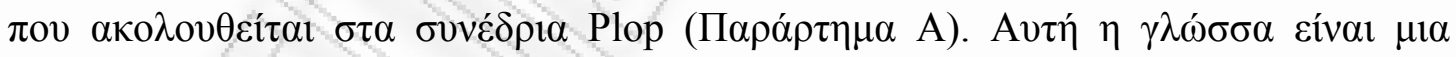

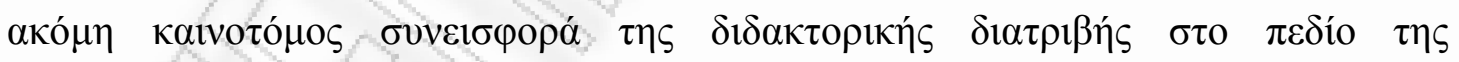

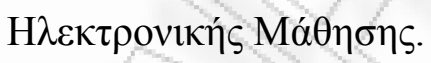

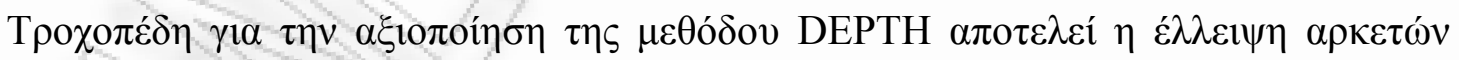

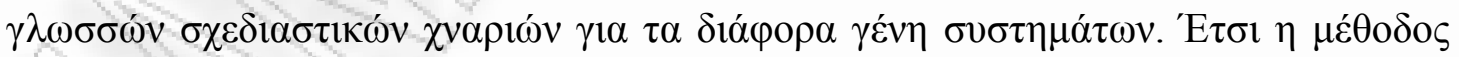

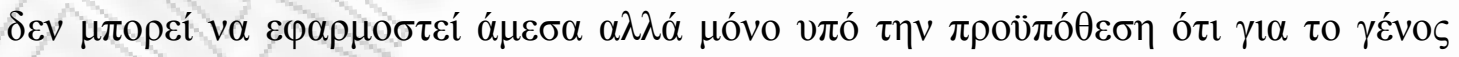

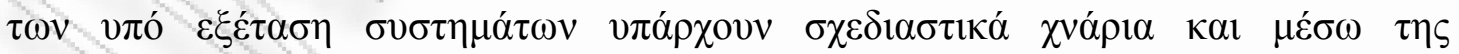

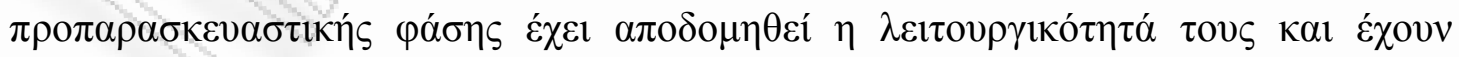

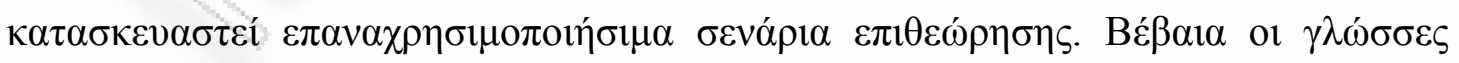

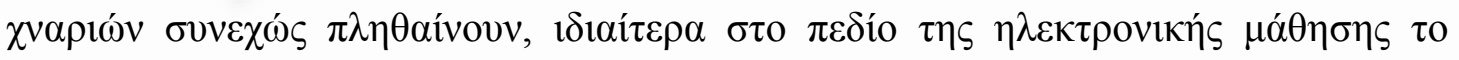

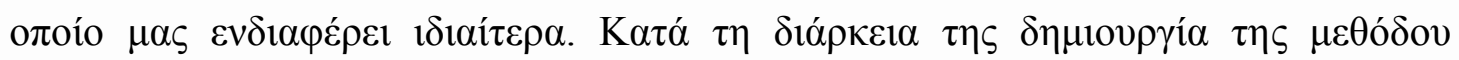

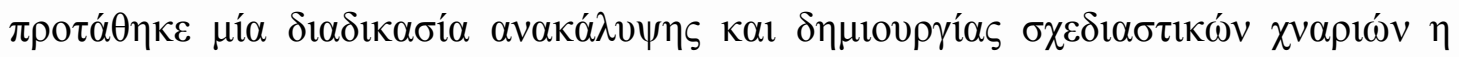

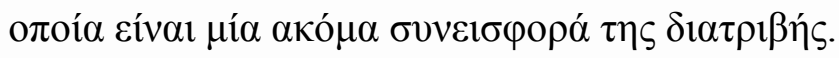




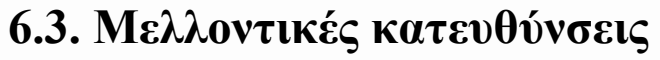

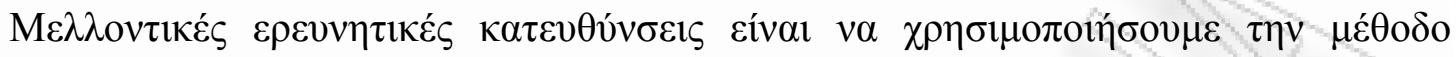

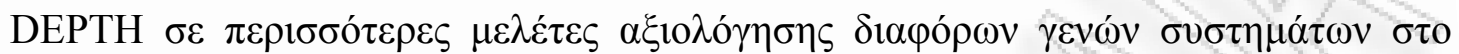

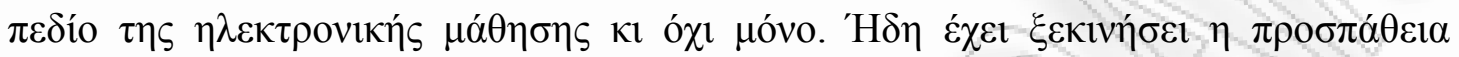

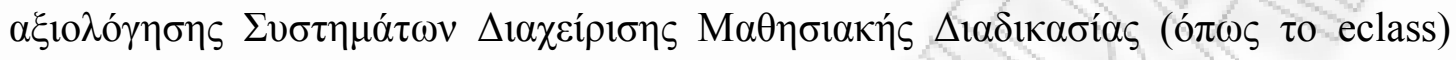

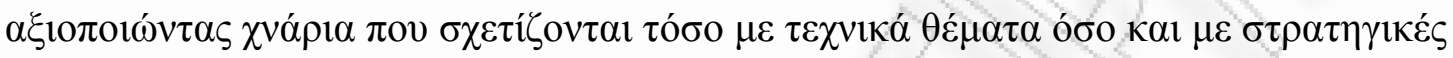

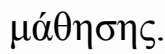

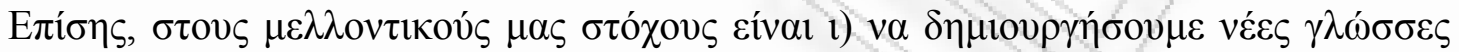

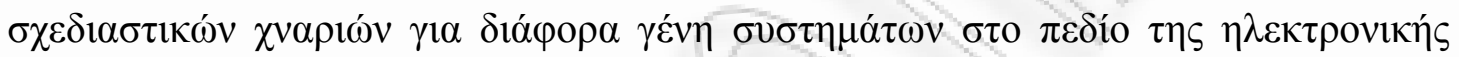

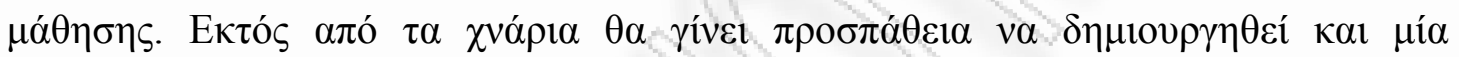

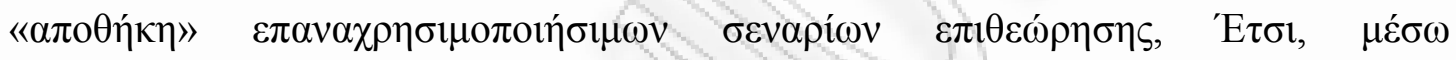

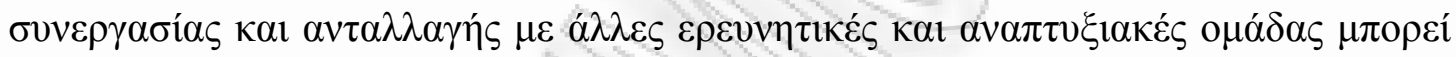

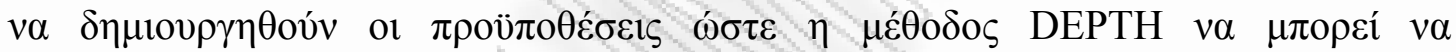

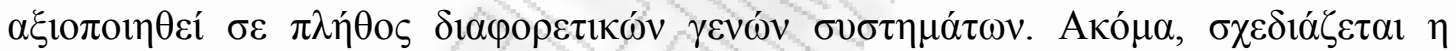

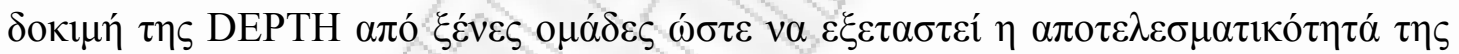
$\sigma \varepsilon \delta 1 \alpha-\pi \circ \lambda \imath \tau \imath \sigma \mu \imath \kappa o ́ ~ \varepsilon \pi i \pi \varepsilon \delta o$.

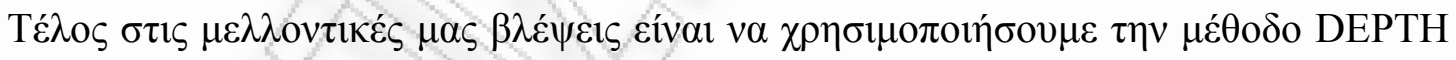

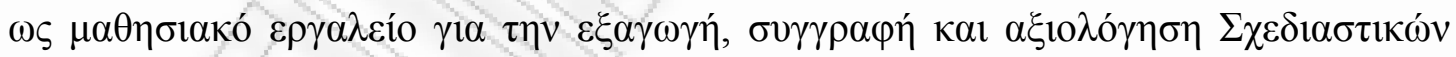

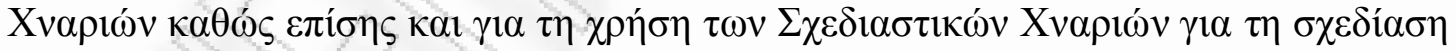

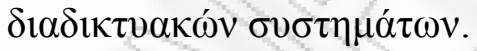




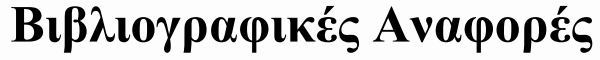

Agarwal R, Venkatesh V, 2002, Assessing a firm's web presence: a heuristic evaluation procedure for the measurement of usability. Information Systems Research, 13, 168-186.

Alexander, C., Ishikawa, S., Silverstein, M., Jacobson, M., Fiksdahl-King, I. \& Angel, S. (1977), “A Pattern Language: Towns, buildings, constructions”, Oxford University Press, New York, USA

Alexander, C. (1979) “The Timeless Way of Building”, Oxford University Press, New York, USA Angeli AD, Matera M, Costabile MF, Garzotto F, Paolini P, 2003, On the advantages of a systematic inspection for evaluating hypermedia usability. International Journal of HumanComputer Interaction, 15, 3, 315-335.

Appleton, B. (2000). Patterns and Software: Essential Concepts and Terminology. http://www.enteract.com/ bradapp/docs/.

Ardito C., Costabile M., De Marsico M., Lanzilotti R., Levialdi S., Roselli T., Rossano V. (2006). An Approach to Usability Evaluation of e-Learning Applications. Universal Access In The Information Society. vol.4(3), pp. 270-283. ISSN: 1615-5289.

Avgeriou, P., Papasalouros, A. and Retalis, S. (2001), Web-based learning Environments: issues, trends, challenges, in Proceedings of the 1st IOSTE symposium in Southern Europe, Science and Technology Education, Paralimni, Cyprus

Avgeriou, P. Papasalouros A., Retalis S. and Skordalakis E. (2003). "Towards a Pattern Language for Learning Management Systems", Educational Technology \& Society, ISSN 1436-4522, Volume 6, Issue 2, pp. 11-24, 2003 http://ifets.ieee.org/periodical/6-2/2.html (ISSN1436-4522).

Avouris N., Tselios N., Tatakis E.C. (2001), Development and evaluation of a computerbased laboratory teaching tool, Journal Computer Applications in Engineering Education, vol. 9 (1), pp. 8-19 
Avouris N., Tselios N., Fidas C., Papachristos E., (2003) Website evaluation: A usabilitybased perspective, in Manolopoulos Y. et al. (ed.) Advances in Informatics, LNCS No 2563, pp. 217-232, Springer Verlag, Berlin.

Baggetun, R., Rusman, E., \& Poggi, C. (2004). Design patterns for collaborative learning: From practice to theory and back. In Cantoni, L. \& McLoughlin, C. (Eds.)

Baker, F., \& Rachlin, H. (2002). Teaching and learning in a probabilistic prisoner's dilemma. Behavioural Processes, 57(2/3), 211.

Bennett, J., 1979. The commercial impact of usability in interactive systems. 1-17. In: Shackel, B., Man/computer Communication: Infotech State of the Art Report, vol. 2. Infotech International, Maidenhead, UK.

Berry B, 2003, Adapting heuristics for notification systems. Proceedings of 41st Annual ACM Southeast Conference, Savannah, GA, 144-149.

Bersin \& Associates (2007), LMS 2008: Facts, Practical Analysis, Trends, and Vendor Profiles, http://store.bersinassociates.com/lms.html

Bevan, Nigel (1995): Usability is Quality of Use. In: Proceedings of the Sixth International Conference on Human-Computer Interaction July 9-14, 1995, Tokyo, Japan. pp. 349-354.

Bevan Nigel, (2006): Practical issues in usability measurement. Interactions, Volume 13, Issue 6 (November + December 2006), SPECIAL ISSUE: Waits \& measures Pages: 42 - 43, ISSN:1072-5520.

Bolchini D., Paolini P., (2002): Capturing Web Application Requirements through GoalOriented Analysis, in Proc. of 5th Workshop on Requirements Engineering WER'02, Valencia, Spain, pp.16-28

Brouns, F., Koper, R., Manderveld, J., Van Bruggen, J., Sloep, P., Van Rosmalen, P., Tattersall, C., \& Vogten, H. (2005). A first exploration of an inductive analysis approach for detecting learning design patterns. Journal of Interactive Media in Education, 2005/03, retrieved October 25, 2005 from http://www-jime.open.ac.uk/2005/03/brouns-2005-03.pdf.

Brusilovsky, P., Karagiannidis, C. and Sampson, D. (2004) 'Layered evaluation of adaptive 
learning systems', Int. J. Cont. Engineering Education and Lifelong Learning, Vol. 14, Nos. 4/5, pp.402-421.

Buschmann F., Meunier R., Rohnert H., Sommerlad P., Stal M. (1996), Pattern-Oriented Software Architecture A System of Patterns, John Wiley and Sons Ltd, Chichester, UK, ISBN 0-471-95869-7

Cagnin, M. I., Braga, R. T. V, Germano, F. S. R., Chan, A., Maldonado, J. (2005). Extending Patterns with Testing Implementation. In: Fifth Latin American Conference on Pattern Languages of Programs, 2005, Campos do Jordão - SP. Proceedings do SugarLoafPLoP 2005, 2005. v. 1

Caroll J. M., (1995 ), "Scenario-based design: envisioning work and technology in system development," Wiley, New York.

Carroll J. M., (2000), Making Use: Scenario-Based Design of Human-Computer Interactions, MIT Press, Cambridge, MA.

Carneiro R., and Nascimbeni F., (2007). Observing the eLearning phenomenon, eLearning Papers ,N 4, March 2007, http://www.elearningpapers.eu/

Chattratichart J., Brodie J., (2002), Extending the heuristic evaluation method through contextualisation", Proceedings of the 46th Annual Meeting of the Human Factors and Ergonomics Society, HFES, September, Baltimore, pp. 641-645.

Cockton G, Woolrych A, 2001, Understanding inspection methods: lessons from an assessment of heuristic evaluation. [in:] Blandford AJ, Vanderdonckt J (eds.) People \& Computers XV, Springer- Verlag, 171-192.

Cockton, G., Woolrych, A., Hall, L. and Hindmarch, M. (2003). Changing analysts' tunes: The surprising impact of a new instrument for usability inspection method assessment. In P. Palanque, P. Johnson, and E. O’Neill (Eds.), People and computers XVII: Designing for society (Proceedings of HCI 2003, pp. 145-162). Berlin, Germany: Springer-Verlag.

Cockton, G. (2006). Designing worth is worth designing. In Proceedings of the 4th Nordic Conference on Human-Computer interaction: Changing Roles (Oslo, Norway, October 14 $18,2006)$. 
Conallen Jim (2002), Building Web Applications with UML Second Edition. Addison Wesley Longman. September 2002.

Cooper J. (2000), Java Design Patterns - A Tutorial, Addison- Wesley, 2000

Coplien, James O., Harrison, Neil B., Organizational Patterns Of Agile Software Development, Prentice Hall PTR, Upper Saddle River, NJ, 2005. Extract available online at http://www.bell-labs.com/cgi-user/OrgPatterns/OrgPatterns?OldOrgPatterns.

Coplien J. O. and Schmidt D. C., (1995), ed. Pattern Languages of Program Design. AddisonWesley, Reading, Mass.

Cunningham W. and Beck K., (1987), Using Pattern Languages for Object-Oriented Programs, Technical Report CR-87-43, Tektronix, Inc., September 17, 1987. Presented at the OOPSLA'87 workshop on Specification and Design for Object-Oriented Programming.

Cunningham, I (1994) The wisdom of strategic learning: the self-managed learning solution Maidenhead, McGraw-Hill

De Angeli A., Matera M., Costabile M., Garzotto F., Paolini P. (2003). On the Advantages of Systematic Inspection for Evaluating Hypermedia Usability. International Journal Of HumanComputer Interaction. vol. 15, No 3, pp. 315-355 ISSN: 1044-7318.

Di Blas N., C. Poggi (2006). "3D for Cultural Heritage and Education: Evaluating the Impact". In D. Bearman \& J. Trant (Eds.) Museums and the Web, Selected Papers from an International Conference. Toronto, Canada: Archives \& Museum Informatics,141-150. http://www.archimuse.com/mw2006/papers/diblas/diblas.html

DiGiano, C., Yarnall, L., Patton, C., Roschelle, J., Tatar, D., and Manley, M., (2002), "Collaboration design patterns: conceptual tools for planning for the wireless classroom," Proceedings of the IEEE International Workshop on Wireless and Mobile Technologies in Education (WMTE'02).

Dimitriadis, Y., Asensio-Pérez, J.I., Hernández-Leo, D., Roschelle, J., Brecht, J., Tatar, D., Chaudhur, S., DiGiano, C., Patton, C.M. (2007), From socially-mediated to technologymediated coordination: A study of design tensions using Group Scribbles Proceedings of the 
Computer Supported Collaborative Learning 2007 Conference, CSCL 2007, New Jersey, USA.

Dix A., Finlay J., Abowd G., Beale R., (2003): Human-Computer Interaction ( ${ }^{\text {rd }}$ Edition). Prentice Hall, ISBN 0-13-046109-1

Donker, A., Markopoulos, P. (2001). Assessing the effectiveness of usability evaluation methods for children. Proc PCHCI 2001, Patras, Greece, pp. 409-410, Typorama publ.

Doubleday A, Ryan M, Springett M, Sutcliffe A (1997) A Comparison of Usability Techniques for Evaluating Design. In: Proceedings of the 1999 Symposium on Designing Interactive Systems: Processes, Practices, Methods and Techniques, Amsterdam, the Netherlands, August, pp 101-110

Dumas, J. S. \& Redish, J. C. (1993). A practical guide to usability testing. Norwood, NJ: Ablex Publishing.

E-LEN (2004). Design patterns and how to produce them, retrieved from http://www2.tisip.no/ELEN/documents/ELEN-Deliverables/booklet-elen_design_experience.pdf.

Erikson, H., Penker, M. (2000): Business Modeling with UML: Business Patterns at Work. OMG Press John Wiley \& Sons

Feldstein S. B., (2002), Fundamental mechanisms of PNA growth and decay. Quart. J. Roy. Meteor. Soc., 128, 775-796.

Filho César Olavo de Moura, Derycke Alain (2006), Pedagogical Patterns and Learning Design: When Two Worlds Cooperate, Proceedings of the UNFOLD/Prolearn joint workshop, Valkenburg, September 22nd-23rd, 2005. Heerlen: Open University of The Netherlands, $\Delta$ la $\theta \dot{\sigma} \sigma \mu \mathrm{o}:$ http://dspace.ou.nl/bitstream/1820/474/9/09_DBU_review.pdf

Fincher, S., \& Utting, I. (2002). Pedagogical Patterns: their Place in the Genre. Paper presented at the ITiCSE 2002, Aarhus, Denmark.

Frizell, S. and Hübscher, R. (2002). Supporting the Application of Design Patterns in WebCourse Design.Proceedings of the World Conference on Educational Multimedia, Hypermedia, and Telecommunications. Denver, CO. 
Frøkjær, E. and Hornbæk, K. (2002). Metaphors of human thinking in HCI: Habit, stream of thought, awareness, utterance, and knowing. In Proceedings of HF2002/OzCHI 2002, Melbourne, Australia, Nov. 25--27.

Gagne, R.M., K.L. Medsker, (1996) The Conditions of Learning Training Conditions, Harcourt Brace College Publishers, TX

Gamma E., Helm R., Johnson R. and Vlissides J. (1995). Design Patterns-Elements of Reusable Object-Oriented Software. Reading, Massachusetts: Addison-Wesley

Garzotto F, Matera M, Paolini P, (1998), Model-based heuristic evaluation of hypermedia usability. Proceedings of the Working Conference on Advanced Visual Interfaces, May 2427, L’Aquila, Italy.

Garzotto, F., Paolini, P., Bolchini, D. \& Valenti, S. (1999). "Modeling-by-patterns" of web applications. Advances in Conceptual Modeling, P. Chen, D. Embley, J. Kouloumdjian \& S. Little (eds.), Lecture Notes in Computer Science 1727, Springer, 293-306.

Garzotto F., Retalis S., Cantoni I., Papasalouros A. (2004), Patterns for designing adaptive/adaptable e-learning experiences. In: Proceedings of the First International Workshop on Authoring of Adaptive and Adaptable Educational Hypermedia at the IASTED Interna-tional Conference on Web-based Education. Innsbruck.

Georgiakakis P., Papasalouros A., Retalis S., Siassiakos K. (2004), "A frame in the evaluation of the usability of Web - based Learning Management Systems",, 4th Hellenic conference information and communication technologies in education, 29th September - 3rd October 2004, Athens, Greece

Georgiakakis, P., \& Retalis, S. (2005). Demystifying the asynchronous network supported collaborative learning systems. International Journal of Computer Applications in Technology, Special Issue on Patterns for Collaborative Systems.

Georgiakakis P., Retalis S. \& Psaromiligkos Y. (2007) "Using design patterns for evaluating computer supported collaborative learning tools", Conference Workshop on CSCL Design Patterns at Computer Supported Collaborative Learning (CSCL) 2007, New Jersey, USA, July $16-21$. 
Goodyear, P. (2002), Psychological Foundations for Networked Learning, In C. Steeples \& C. Jones (Eds), Networked Learning: Perspectives and Issues. London: Springer-Verlag.

Goodyear, P., Banks, S., Hodgson, V., McConnell, D (2004a) Advances in Research on Networked Learning, Dordrecht: Kluwer Academic, Chapter 5, pp 91 - 121.

Goodyear, P., Avgeriou, P., Baggetun, R., Bartoluzzi, S., Retalis, S., Ronteltap, F., \& Rusman, E. (2004b). "Towards a pattern language for networked learning", Proceedings of Networked Learning 2004, pp. 449-455.

Goodyear, P. (2005). Educational design and networked learning: Patterns, pattern languages and design practice. Australasian Journal of Educational Technology, 21(1), 82-101. http://www.ascilite.org.au/ajet/ajet21/goodyear.html

Graham I. (2003), A Pattern Language for Web Usability, Addison-Wesley

Grigoriadou, M., Papanikolaou, K., Kornilakis, H. and Magoulas, G. (2000), In: INSPIRE: an intelligent system for personalized instruction in a remote environment. Proceedings of $3 \mathrm{rd}$ Workshop on Adaptive Hypertext and Hypermedia, pp. 13-24.

Grimaldi, L. 1998. Disminuyen Accidentes por uso de Plaguicidas. Prensa Libre. Guatemala City, 18-19.

Gutwin, C. and Greenberg, S. (2000). The Mechanics of Collaboration: Developing Low Cost Usability Evaluation Methods for Shared Workspaces. IEEE 9th Int'1 Workshop on Enabling Technologies: Infrastructure for Collaborative Enterprises (WET-ICE'00).

Hartson, H. R., Andre, T. S., \& Williges, R. C. (2001). Criteria for evaluating usability evaluation methods. International Journal of Human-Computer Interaction, 13, 4, 373-410. http://research.cs.vt.edu/usability/publications/evalg\%20uems\%20(ijhci).pdf

Hartson H.R., Andre T.S. and Williges R.C., (2003) Criteria for evaluating usability methods, International Journal of Human-Computer Interaction 13 (2003), pp. 373-410.

Hassenzahl, M. (2001). The effect of perceived hedonic quality on product appealingness. International Journal of Human-Computer Interaction, 13, 4, 481-499. 
Hayes, R. (2000). Exploring discount usability methods to assess the suitability of online course delivery products. The Internet and Higher education 2 (2-3), 119-134.

Hernández-Leo, D., Villasclaras-Fernández, E. D., Asensio-Pérez, J. I., Dimitriadis, Y., Jorrín-Abellán, I. M., Ruiz-Requies, I., \& Rubia-Avi, B. (2005). COLLAGE, a Collaborative Learning Design Editor Based on Patterns. Educational Technology \& Society.

Hernández D., E.D. Villasclaras, I.M. Jorrín, J.I. Asensio, Y. Dimitriadis, I. Ruiz, B. Rubia (2006). COLLAGE, a Collaborative Learning Design Editor Based on Patterns, IEEE LTC Educational Technology and Society, 9 (2006)

Hertzum, M. and Jacobsen, N.E., (2001) The Evaluator Effect: A Chilling Fact about Usability Evaluation Methods, Int. Journal of Human-Computer Interaction, vol. 13, no. 4 (2001), pp. 421-443

Holleran, P.A. (1991). A methodological note on pitfalls in usability testing. Behaviour and information technology, 10 (5), 345-357.

Horton W. and Norton K. (2003), E-learning tools and technologies, Wiley Publishing, Inc; Indianapolis USA,

Hudlicka, E. (2003). To feel or not to feel: The role of affect in human-computer interaction. International Journal of Human-Computer Studies, 59: 71-5.

Hutchins EL, Hollan JD, Norman DA (1985), Direct manipulation interfaces. HumanComputer Interaction, 1:311-338

IEEE Learning Technology Standards Committee (LTSC)/IEEE P1484.3 Glossary Working Group. (2001). Draft Standard for Information Technology --- Learning Technology --Glossary. http://ltsc.ieee.org/doc/wg3/Glossary-20010309.doc

IMS LD (2003). IMS Learning Design Best Practice and Implementation Guide. IMS Global Learning Consortium, Inc.

ISO FDIS 9241-11 (1997). Ergonomic Requirements for Office Work with Visual Display Terminals (VDTs), Part 11: Guidance on Usability Specification and Measures. Technical report,. 
ISO/IEC. 13407 (1999), Human-Centred Design Processes for Interactive Systems, ISO/IEC 13407: 1999 (E),.

Jeffries R, Desurvire HW (1992) Usability Testing vs. Heuristic Evaluation: Was There a Context? ACM SIGCHI Bulletin, 24(4):39-41

Jeffries R, Miller J, Wharton C, Uyeda KM, (1991) User Interface Evaluation in the Real Word: A Comparison of Four Techniques. In: Proceedings of the ACM International Conference on Human Factors in Computing Systems, New Orleans, USA, pp 119-124

Jonassen, D. H. (2003). Using cognitive tools to represent problems. Journal of Research on Technology in Education, 35(3), 362-381.

Jones, C. (2004) Theory and the practices of learning technology. In Banks, S., Goodyear, P., Hodgson, V., Jones, C., Lally, V., McConnell, D and Steeples, C. (Eds) Networked Learning 2004: Proceedings of the Fourth International Conference on Networked Learning 2004. Lancaster: Lancaster University and University of Sheffield pp 22 - 27.

Jones, A., Scanlon, E., Tosunoglu, C., Morris, E., Ross, S., Butcher, P. and Greenberg, J., (1999). Contexts for evaluating educational software. Interacting with Computers 11 (3), pp. 499-516.

Jorgensen, A.H. (1990). Thinking-aloud in user interface design: a method promoting cognitive ergonomics. Ergonomics, 33, (4), 501-507.

Karat, C.-M., Campbell, R. L., \& Fiegel, T. (1992). Comparison of empirical testing and walkthrough methods in user interface evaluation. Proceedings of CHI 92, 397-404. New York, NY: ACM.

Kantner L, Rosenbaum S (1997) Usability Studies of WWW Sites: Heuristic Evaluation vs. Laboratory Testing. In: Proceedings of the ACM 1997 International Conference on Computer Documentation, Snowbird, USA, pp 153-160

Kim, K. S. \& Allen, B. (2002). Cognitive and Task Influences on Web Searching Behavior, Journal of the American Society for Information Science and Technology, 53(2), 109-119.

Kirakowski, J. Claridge, N. (1998). Human Centered Measures of Success in Web Site Design. Fifth Human Factors and the Web meeting, June 1998, NY. 
Koper R. (2006), Building Learning Networks for Lifelong Learners: Challenges, Models, Technologies and Standards. ICALT 2006: 1098-1099

Koutsabasis, P. Spyrou, T. and Darzentas, J. (2007) Evaluating usability evaluation methods: criteria, method and a case study, 12th International Conference on Human-Computer Interaction, Beijing, China, 2007, Lecture Notes in Computer Science, Vol. 4550, Springer.

Kreimeier, B. (2003). Game Design Methods: A 2003 Survey, Gamasutra.

Kurosu, M., Matsuura, S., \& Sugizaki, M. (1997). Categorical inspection method: Structured heuristic evaluation (sHEM). Proceedings of 1997 IEEE International Conference on Systems, Man, and Cybernetics, October 12-15, 1997, Orlando, Florida, USA, 2613-2618.

Kurosu, M., Sugizaki, M., \& Matsuura, S. (1998). Structured Heuristic Evaluation (sHEM): A more productive method to find out usability problems. Proceedings of UPA 98, 3-5.

Kurosu, M., Sugizaki, M., \& Matsuura, S. (1999). A comparative study of sHEM (structured heuristic evaluation method). In Bullinger, H.-J,, \& Ziegler, J. (Eds.), Human-computer interaction: Ergonomics and user interfaces, 938-942. Proceedings of HCI International 99, Volume 1. Mahwah, NJ: Lawrence Erbaum Associates.

Lanzilotti R., Costabile M., Ardito C., De Angeli A. (2006). eLSE Methodology: a Systematic Approach to the e-Learning Systems Evaluation. Educational Technology \& Society. Vol. 9(4), pp.42-53. ISSN: 1176-3647.

Laurillard, D., (2000). students and the curriculum. In: Scott, P., Editor, Higher Education Re-formed, Falmer Press, London, pp. 133-153.

Lewis JR (1995) IBM Computer Usability Satisfaction Questionnaires: Psychometric Evaluation and Instruction for Use. Human-Computer Interaction, 7(1):57-78

Lewis, C., Polson, P., Wharton, C., Rieman, J. (1990) Testing a Walkthrough methodology for Theory-Based Design of Walk-Up-and-Use Interfaces. Proc. of ACM CHI1990,Seattle, Washington. pp: 235-242.

Lewis. C., Rieman, J. (1994) Task centered User Interface Design- A practical introduction. $\Delta 1 \alpha \theta \varepsilon ́ \sigma 1 \mu о \sigma \tau$ o http://www.hcibib.org. 
Lilly, S. (1996), Patterns for Pedagogy. Object Magazine, 5(8)

Lohr.L.L. (2000), Designing the instructional interface, Computers in Human Behavior 16 161-182.

Lukosch S., Schümmer T., (2004), Communicating Design Knowledge with Groupware Technology Patterns: The Case of Shared Object Management, CRIWG, 3198: 223-237, 2004.

Magoulas G., Papanikolaou, K., Grigoriadou, M., (2003), Adaptive web-based learning: accommodating individual differences through system's adaptation. British Journal of Educational Technology. v34 i4. 511-527.

Manns, M. L., Sharp H., McLaughlin P., Prieto M., (1998) 'Capturing successful practices in OT education and training', Journal of Object-Oriented Programming, Vol 11, No 1, March/April.

Mariage C, Vanderdonckt J, (2000), A Comparative usability study of electronic newspapers. Proceedings of International Workshop on Tools for Working with Guidelines, October 7-8, Biarritz, Springer-Verlag, London, 325-337.

Matera M, Costabile MF, Garzotto F, Paolini P, (2002), SUE inspection: An effective method for systematic usability evaluation of hypermedia. IEEE Transactions on Systems, Man, and Cybernetics-Part A: Systems and Humans, 32, 1, 93-103.

Mayer, R. E., \& Wittrock, M. C. (1996). Problem-solving transfer. In R. Calfee \& R. Berliner (Eds.), Handbook of educational psychology (pp. 47-62). New York: Macmillan

McGrow K, Brennan AH, Preece J, 2004, Development of a tool for heuristic evaluation of healthcare information systems. Computers, Informatics, Nursing, Journal of Hospice \& Palliative Nursing.

McCormack, C., Jones, J. D., (1997). Building a web-based education system. Wiley Computer.

McGorry, S. Y. (2003). Measuring quality in online programs. The Internet and Higher Education, 6(2), $159-177$. 
Mezaros, Gerard, and James Doble (1998). A Pattern Language for Pattern Writing," in Pattern Languages of Program Design-3, Addison-Wesley, Reading MA, 1998, pages 529574.

Miller M. J. (2005), Usability in E-Learning, URL: http://www.learningcircuits.org/2005/jan2005/miller.htm

Miller, J. (2006), "Usability Testing: A Journey, Not a Destination," IEEE Internet Computing, vol. 10, no. 6, pp. 80-83, Nov/Dec,

Mislevy, R., Hamel, L., Fried, R., G., Gaffney, T., Haertel, G., Hafter, A., Murphy, R., Quellmalz, E., Rosenquist, A., Schank, P., Draney, K., Kennedy, C., Long, K., Wilson, M., Chudowsky, N., Morrison, A., Pena, P., Songer, N., Wenk, A. (2003). Design patterns for assessing science inquiry (PADI Technical Report 1). Menlo Park, CA: SRI International.

Mohan P., Daniel B. K. (2006) Towards Object-Oriented Design Patterns for Reusability of Learning Objects. ICALT 2006: 1025-1027

Molich R, Nielsen J (1990) Improving a Human-Computer Dialogue. Communications of the ACM, 33(3):338-348.

Molich R., Ede M. R., Kaasgaard K., Karyukin B., (2004), Comparative usability evaluation, Behaviour \& Information Technology, v.23 n.1, p.65-74.

Mor Y., Winters N. (2007), Design approaches in technology enhanced learning, Interactive Learning Environments 15 (1) 2007 (2006) 61-75

Mørch A., Morgan K., Bratteteig T., Ghosh G., and Svanaes D., (2006), Eds. NordiCHI '06, vol. 189. ACM, New York, NY, 165-174.

Nielsen J (1992) The Usability Engineering Lifecycle. J IEEE Computer, 25(3):12-22

Nielsen J (1993) Usability Engineering. Academic Press, Cambridge, MA.

Nielsen J (1994a), Special Issue on Usability Laboratories. Behavior and Information Technology, 13(1)

Nielsen J (1994b) Heuristic evaluation. In Nielsen, J., and Mack, R.L. (Eds.), Usability Inspection Methods. John Wiley \& Sons, New York, NY. 
Nielsen, J. (2000). Security \& Human Factors. Jakob Nielsen's Alertbox, November 26, 2000 http://www.useit.com/alertbox/20001126.html

Nielsen J, Landauer TK., (1993) A Mathematical Model of the Finding of Usability Problems. In: Proceedings of the ACM 1993 International Conference on Human Factors in Computing Systems, Amsterdam, Netherlands, April, pp 296-213

Nielsen J, Mack RL., (1994) Usability Inspection Methods. Wiley, New York

Nielsen J, Molich R., (1990) Heuristic Evaluation of User Interfaces. In. Proceedings of the ACM 1990 International Conference on Human Factors in Computing Systems, Seattle, USA, April, pp 249-256

NISE (1997). Doing CL: CL Structures, retrieved October, 2004 from http://www.wcer.wisc.edu/nise/cl1/CL/ doingcl/clstruc.htm",

Norman, D.A., (1988), The Design of Everyday Things. MIT Press

Notess M., (2001), Usability, User Experience, and Learner Experience, Available at http://www.elearnmag.org/

Oorni A (2003) Consumer search in electronic markets: an experimental analysis of travel services. European Journal of Information Systems 12(1), 30-40.

Paolini, P., Garzotto, F., Bolchini, D., Valenti, S., (1999) 'Modelling by Pattern' of Web Applications, in Peter P.S. Chen (Eds). Proc. of Advances in Conceptual Modeling (ER'99) Workshops on Evolution and Change in Data Management, Reverse Engineering in Information Systems, and the World Wide Web and Conceptual Modeling, Paris , France , November 15-18.

Parlangeli O.; Marchigiani E.; Bagnara S, (1999). Multimedia systems in distance education: Effects of usability on learning. Interacting with Computers. Volume 12, Number 1, September 1999, pp. 37-49(13).

Paternò F., Ballardin G., (2000), "RemUSINE: a Bridge between Empirical and Model-based Evaluation when Evaluators and Users are Distant", Interacting with Computers, Vol.13, N.2, pp. 229-251, Elsevier. 
Poggi, C. \& Torrebruno, A. (2007). From the Dead Sea Scrolls to Social Sports, passing through European History: an Effective Pedagogical Format Based on 3D Worlds. In C. Montgomerie \& J. Seale (Eds.), Proceedings of World Conference on Educational Multimedia, Hypermedia and Telecommunications 2007 (pp. 4240-4248).

Polson P., Lewis C., Rieman J., Wharton C., (1992), Cognitive Walkthrough: A Method for Theory-based Evaluation of User Interfaces. Man-Machine Studies, 36:741-773

Pond, W. K. (2002). Twenty-First Century Education and Training: Implications for Quality Assurance. The Internet and Higher Education. 4, 185-192

Preece, J. (1999) Combating Social Exclusion in University Adult Education. Hampshire: Ashgate.

Preece, J., Rogers, Y., Sharp, H., Benyon, D., Holland, S. \& Carey, T. (1994), Humancomputer interaction, Workingham. England: Addison-Wesley.

Quinn, C. N. (1996). Pragmatic Evaluation: Lessons from Usability. 13th Annual Conference of the Australasian Society for Computers in Learning in Tertiary Education, Australasian Society for Computers in Learning in Tertiary Education, Adelaide.

Reeves, T. C., Benson, L., Elliot, D., Grant, M., Holschuh, D., Kim, B., (2002). Usability and Instructional Design Heuristics for E-Learning Evaluation. Paper presented at the Proceedings 14th, Denver, Colorado.

Retalis S., Georgiakakis P., Dimitriadis Y., (2006 ) "Eliciting design patterns for e-learning systems", Computer Science Education, Volume 16, Number 2, pp. 105-118(14), Routledge, part of the Taylor \& Francis Group.

Roschelle, J. Kaput, J., Stroup, W., and Kahn, T. M.(1998). Scalable Integration of Educational Software: Exploring the Promise of Component Architectures. Journal of Interactive Media in Education (6) (Oct, 1998) pp. 1-31

Rossi, G., Schwabe, D. and Garrido, A. (1997) "Design Reuse in Hypermedia Applications".Eighth ACM Conference on Hypertext Technology, Southampton, UK (ACM Press), pp. 57-66 
Rosson, M. B., Carroll, J. M., Cerra, D. D., (2001), “Usability Engineering: Scenario-Based Development of Human Computer Interaction”, Morgan Kaufmann Publishers, 1st edition, October 15, 2001, ISBN: 1558607129.

Rusman, E., Van Bruggen, J., \& Koper, R. (2007). From Pattern To Practice: Evaluation Of A Design Pattern Fostering Trust In Virtual Teams. Paper Presented at the CSCL 2007.

Shackel, B., (1991). Usability-Context, framework, definition, design and evaluation. In: B. Shackel \& S. J. Richardson (Eds.), Human factors for informatics usability, Cambridge: Cambridge University, 21-37

Sears A., (1997), Heuristic walkthroughs: Finding the problems without the noise. International Journal of Human-Computer Interaction, 9, 3, 213-234.

Schuemmer, T., Evolving a Groupware Pattern Language. In ECSCW2003 Workshop "From Good Practices to Patterns", (Helsinki, Finland, 2003).

Schneiderman B., (1992), Designing the user interface: Effective strategies for effective human-computer interaction. 2nd ed., Reading, Mass.: Addison-Wesley.

Shneiderman B., (1998). Designing the User Interface. Reading, MA, Addison Wesley Longman.

Shneiderman B., Plaisant C., (2005). Designing the User Interface: Strategies for Effective Human-Computer Interaction: Fourth Edition, Addison-Wesley Publ. Co., Reading, MA.

SIG -CHI. (2001), Notes from E-learning Special Interest Group (SIG) Discussion at CHI 2001 http://www.elearnmag.org/

Soloway E., (1996), Interactive learning Environments: Where They've Come From \& Where They're Going, In Proceedings of Computer Human Interaction '96, 384-385

Sommerville 1., (2007). Software engineering, 8th edition, Harlow: Addison Wesley. ISBN 978-0-321-31379-9 ISBN 0-321-31379-8

Squires, D., (1999) Usability and Educational Software Design: Special Issue of Interacting with Computers, Interacting with Computers 11 (5) 463-466, 
Squires D., Preece J., (1999), Predicting quality in educational software: Evaluating for learning, usability and the synergy between them, Interacting with Computers 11 (5) 467-483

Sutcliffe A., (2001), Heuristic evaluation of website attractiveness and usability. Proceedings of the $8^{\text {th }}$ International Workshop on Interactive Systems: Design, Specification, and Verification, Springer-Verlag, London, UK, 183-198

TELL Project, (2005). "Introducing a Framework for the Evaluation of Network Supported Collaborative Learning",(1.37 MB .pdf) TELL Project, Deliverable of WorkPackage 1. Retrieved 2005 from TELL project website: http://cosy.ted.unipi.gr/tell/.

TELL Project, (2006), Towards Effective network supported coLLaborative learning activities: Pattern book Output of WP3 TELL project: "Design patterns for teachers and educational (system) Designers" Design Patterns for Network Supported Collaborative Learning (NSCL): p.121-184. Retrieved 2006 from TELL project website: $\underline{\text { http://cosy.ted.unipi.gr/tell/ }}$

Tselios N., Avouris N., Dimitracopoulou A., Daskalaki S. (2001). Evaluation of Distancelearning Environments: Impact of Usability on Student Performance. International Journal of Educational Telecommunications, 7(4), pp. 355-378.

Tselios N., Avouris N., Komis V., (2008), The Effective Combination of Hybrid Usability Methods in Evaluating Educational Applications of ICT: Issues and challenges, Education and Information Technologies Journal, vol. 13 (1), pp. 55-76.

Tselios N., Komninou M., Ayouris N. (2002). Usability of Educational Software: Problems and Proposals, Proc. 3rd Pan-Hellenic Conference on Information and Communication Technologies in Education, Rhodes, Volume I, pp. 775-784.

Tselios N., Komis V., Avouris N. (2004). Heuristic Evaluation of open learning environments by simulated experts, Proceedings 4th ETPE Conf., vol. A., pp. 587-596, Athens.

Van Duyne, D.K., Landay, J.A. and Hong, J.I. (2003): The Design of Sites: Patterns, principles, and processes for crafting a customer-centered web experience. Boston, AddisonWesley, Pearson Education. 
Van Welie, M. and Van der Veer, G. C. (2003). Pattern Languages in Interaction Design: Structure and Organization. Interact 2003.

Van Wilie, M (2005). The News Box. Retrieved 2005 from "The collection of Web Design patterns", http://www.welie.com/patterns

Wong, B., Nguyen, T. T., Chang, E., \& Jayaratna, N. (2003). Usability Metrics for ELearning. Lecture Notes in Computer Science, 2889, 235-252.

World Bank, (2003), Lifelong Learning in the Global Knowledge Economy: Challenges for Developing Countries. A World Bank Report. Washington, DC: World Bank. 140 pp. ISBN $0-8213-5475-2$

Yang, D.F. \& Goodyear, P. (2004). Pattern languages and genres for writing computer science discourse. In R. Atkinson, C. McBeath, D. Jonas-Dwyer \& R. Phillips (Eds), Beyond the comfort zone: Proceedings of the 21st ASCILITE Conference (pp. 961-967). Perth, 5-8 December. http://www.ascilite.org.au/conferences/perth04/procs/yang.html

Zaharias, P. (2004). A Usability Evaluation Method for E-Learning Courses. PhD Thesis. Athens University of Economics and Business.

Zhang, Z., Basili, V., Shneiderman B., (1999) Perspective-based usability inspection: An empirical validation of efficacy. Empirical Software Engineering, 4, 1, 43-69. 


\title{
ПАРАРТНМА А
}

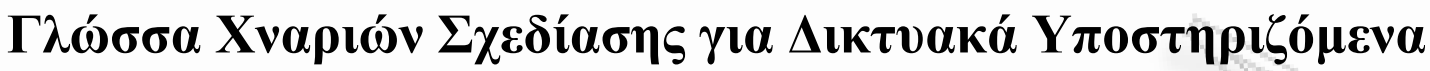

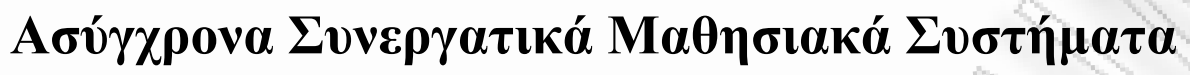

\author{
Problem \\ Asynchronous Network-Supported \\ Collaborative Learning (ANSCL) has \\ emerged as a distinct field of research \\ and practice that emphasises the role \\ of social interactions in the \\ construction of knowledge. ANSCL is \\ more ambitious than traditional \\ approaches to e-learning, and thus \\ more difficult to design, apply and \\ assess. Applying ANSCL designs in \\ authentic situations emerge the need of \\ innovative ANSCL systems that must \\ grand specific requirements. What are \\ the requirements for a system to be \\ considered an ANSCL system, and \\ what are the steps that a \\ development team should follow to \\ build them? \\ Forces ANSCL systems must effectively support teachers and students in \\ designing and performing collaborative learning tasks. These systems \\ will adopt features from more general systems that have been used for \\ asynchronous communication (e.g. e-mail systems, newsgroups, etc.) \\ as well as from others that have been used in the area of computer- \\ supported co-operative work (CSCW) but they should emphasise on \\ the learning aspect of collaboration and co-operation. \\ Thus, ANSCL systems must: Promote learning: the purpose is not just \\ to perform a task - as in CSCW systems - but to promote (intentional \\ and/or incidental) learning during and after the collaborative \\ interaction. Typically, collaborative learning involves two or more \\ peers, with shared learning goals, who interact in a technology- \\ mediated learning environment, exchange resources, co-develop \\ artifacts in order to acquire new capabilities or enhance \\ understanding. \\ ANSCL systems must also enable collaboration: collaboration is \\ distinguished from simple interaction between participants. \\ Collaboration involves more than communication; more than the \\ simple exchange of ideas, information or material. Many distance/e- \\ learning technologies support information exchange but not \\ collaboration. Collaboration implies working together, on the creation \\ and improvement of (cognitive) artifacts (see e.g. Goodyear 2005; \\ Koschmann, 1996).
}


ANSCL systems must promote collaboration: It isn't enough for an ANSCL system to allow collaboration. The design of tasks and technology needs to promote and support collaboration, to raise awareness, to allow mentoring, group formation and assessment of learning progress. This is because (a) commitment to ANSCL arises from a belief in the potential of collaboration to enhance learning; (b) realization of this potential can be impeded by a number of factors, including (c) the fact that individualization and competition are powerful forces at work in the world (TELL 2003).

ANSCL systems must be the technological foundation of a convivial e-learning environment: The construction of convivial e-learning environments is a demanding task involves the design of learning tasks and their sequence, the linkage of tasks with learning resources, the creation of social paces and organizational forms that should be monitored and sustained during the course lifecycle as well as that provide security and protection along with a usable environments for management of tightly and loosely-coupled collaboration (Pinelle et al., 2003).

Solution ANSCL systems should be used to create a new type of convivial learning environment, where tangible objects and people/peers sit alongside virtual computer-based spaces, objects and the outcomes of interactions. ANSCL systems serve as an integrated environment that facilitate the formation of virtual learning communities (either large or small, i.e. groups) (GROUP MANAGEMENT), and support the interaction between users by maintaining common spaces for the posting and organization of messages (DISCUSSION SPACES FOR ANSCL SYSTEMS), as well as for the sharing, re-using and annotating resources (SPACES FOR COLLABORATIVE WORK WITH ARTIFACTS). Functionalities that are important for ANSCL systems are those that facilitate the representation of participants' identities, status, tasks, progress and presence (AWARENESS) along with the examination of the frequency of interactions across the individual learner or among members of groups (EVALUATION) that can reveal many of the behaviours associated with collaborative learning.

Typical user tasks that ANCSL systems supports:

- DISCUSSION SPACES FOR ANSCL SYSTEMS. This functionality is related to the provision of tools for asynchronous communication: Email, threaded discussion fora, etc. Students and instructors can post their messages on particular subjects, establishing a space for discourse. Moreover, electronic documents and files can be attached to specific messages, thus providing an additional space for document sharing. More elaborated forms of discussion supported by certain tools provide frameworks for dialog.

- AWARENESS. An important functionality in ANSCL systems is representation of participants' identities, status, tasks, progress and presence (Erickson 2002). In order to satisfy these needs, the concept of collaboration awareness was introduced, defined as "an understanding of the activities of the others 
which provides context for your own activity" (Dourish \& Belloti 1992).

- Evaluation. Examination of the frequency of interactions across the individual learner or among members of groups can reveal many of the behaviours associated with collaborative learning. Generally, interaction data concern the number of the messages read, the postings to a discussion board, the file uploads, the annotations to the uploaded files, etc. In addition, analysis of participants' postings via content analysis techniques can enlighten the evaluators of learners' behaviour, outcomes and experiences (TELL 2003).

- Group management. Asynchronous CSCL systems often support collaboration between members of teams of students working in a common project. The project artifacts are managed in particular "spaces for collaborative work" and are supported by the functionality mentioned above. Group management functionality has to deal with the successful management of student teams related to collaborative projects.

- SPACES FOR COLLABORATIVE WORK WITH ARTEFACTS. This functionality is related to the creation, management and presentation of work-spaces where students can post documents and files together with annotative comments on these documents. These documents are typically the outcomes of collaborative project assignments. Different versions of documents are supported so that the process of document evolution is properly presented.

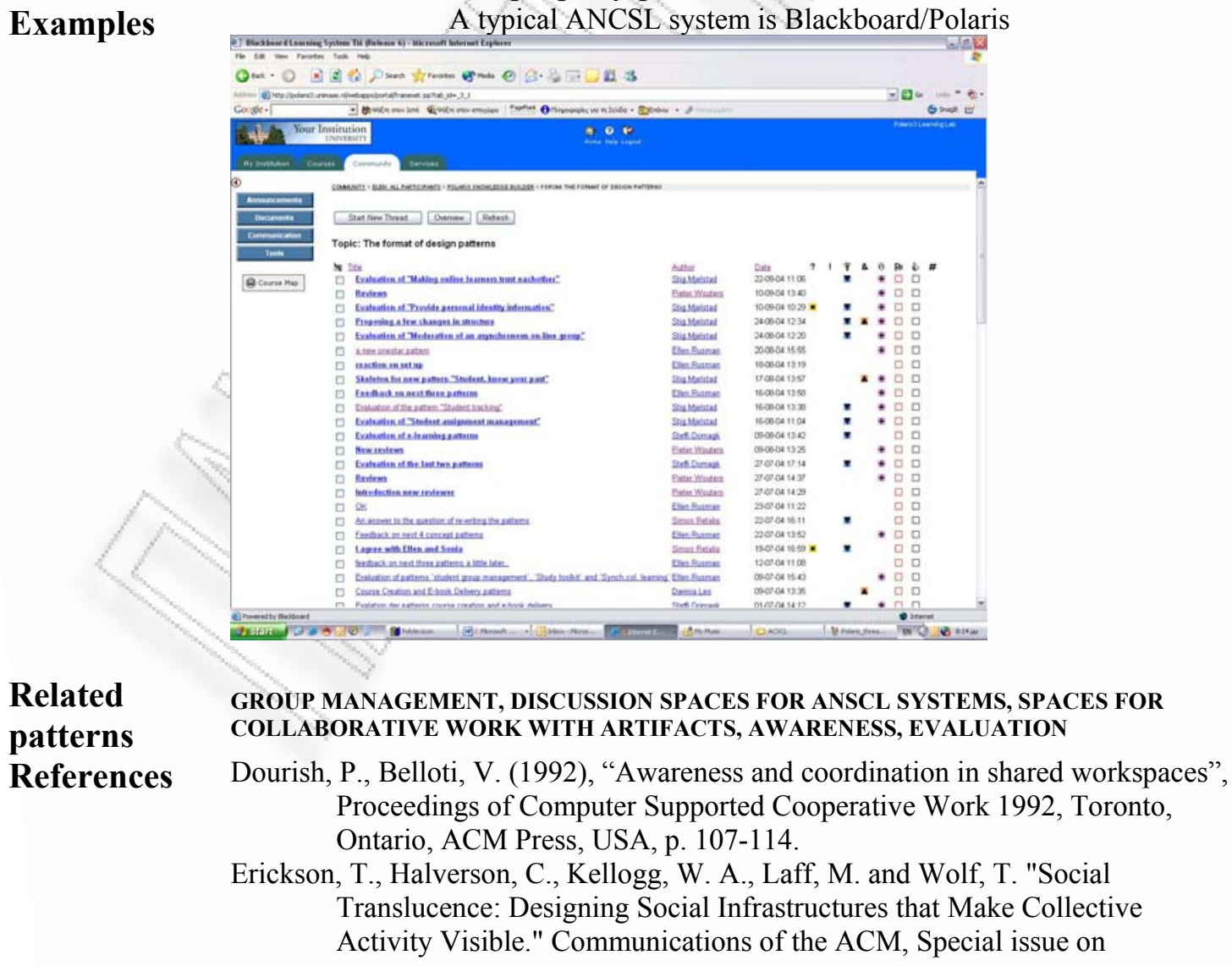


Community, Vol. 45, No. 4, pp. 40-44, 2002

Goodyear, P. (2005). Educational design and networked learning: patterns, pattern languages and design practice. Australian Journal of Educational Technology, 21(1), 82-101.

Koschmann, T. (1996). CSCL: theory and practice of an emerging paradigm. Malwah, NJ, USA: Lawrence Erlbaum

Pinelle, D., Gutwin, C., Greenberg, S. (2003) Task Analysis for Groupware Usability Evaluation: Modeling Shared-Workspace Tasks with the Mechanics of Collaboration. Transactions on Computer-Human Interaction (TOCHI), ACM Press, 10(4), 2003, pp. 281-311. Short summary in interactions, ACM Press, 11(2), 2004, pp. 7-8.

TELL Project. (2003). Introducing a Framework for the Evaluation of Network Supported Collaborative Learning, WP1 Deliverable, Project number: EAC/61/03/GR009 eLearning Initiative, EU: European Commission http://cosy.ted.unipi.gr/tell/media/WP1_deliverable.pdf 


\section{Design patterns for Discussion Spaces}

\begin{tabular}{|c|c|}
\hline $\begin{array}{l}\text { Pattern } \\
\text { Name }\end{array}$ & $\begin{array}{l}\text { Discussion Spaces for ANSCL } \\
\text { systems }\end{array}$ \\
\hline Problem & $\begin{array}{l}\text { Users need a communication along } \\
\text { with a space that will contain tools } \\
\text { for the creation, sharing and } \\
\text { improvement of cognitive artifacts } \\
\text { between the community members. } \\
\text { Students and instructors need to post } \\
\text { their messages on particular subjects, } \\
\text { establishing a space for discourse. } \\
\text { How should the Discussion Spaces } \\
\text { for ANSCL systems be designed? }\end{array}$ \\
\hline Forces & $\begin{array}{l}\text { The need for continuous education and training has brought into } \\
\text { education people with various time and place constraints that are not } \\
\text { easily met by traditional means such as lectures, scheduled } \\
\text { laboratories etc. Discussion spaces should facilitate simple interaction } \\
\text { between participants, i.e. posting and reading e-mails with or without } \\
\text { attachment on discussion topics either triggered by the instructor or by } \\
\text { peers who have queries. Collaboration for learning, however, involves } \\
\text { more than communication or simple exchange of ideas, information or } \\
\text { material. It implies peers working together on the creation and } \\
\text { improvement of (cognitive) artefacts. Thus spaces be organised in } \\
\text { such a way that would become areas for the completion of } \\
\text { collaborative learning tasks/assignments. Furthermore, participants } \\
\text { might also like to structure the discussion in phases thus annotating } \\
\text { messages (motivating question, criticism, feedback comments), as } \\
\text { well as managing the timing of the discussion spaces (i.e. "opening } \\
\text { hours" of various rooms), etc. }\end{array}$ \\
\hline Solution & $\begin{array}{l}\text { Discussion spaces for ANSCL systems should provide tools for } \\
\text { asynchronous communication: Participants should be able to access } \\
\text { and the postings via various means such as threaded discussion fora, } \\
\text { email, etc., (READ MESSAGES). Students and instructors can post their } \\
\text { messages (POST MESSAGES) on particular subjects, establishing a space } \\
\text { for discourse. Moreover, electronic documents and files can be } \\
\text { attached to specific messages (TACK A FILE), thus providing an } \\
\text { additional space for document sharing (SPACES FOR COLLABORATIVE } \\
\text { wORK WITH ARTEFACTS). More elaborated forms of collaborative } \\
\text { discourse supported by certain tools provide frameworks for dialog } \\
\text { (THREADED DISCUSSION MANAGEMENT). Each message posted to a forum } \\
\text { might be accompanied by a specific annotation (ANNOTATION ON POSTED } \\
\text { MESSAGE) depending on the learning phase it belongs to (sharing ideas, } \\
\text { commenting, feedback, etc) or its type (comment, disagreement, etc.). }\end{array}$ \\
\hline $\begin{array}{l}\text { Related } \\
\text { Patterns }\end{array}$ & $\begin{array}{l}\text { READ MESSAGES, POST MESSAGES, TACK A FILE, SPACES FOR COLLABORATIVE } \\
\text { WORK WITH ARTEFACTS, THREADED DISCUSSION MANAGEMENT, ANNOTATION ON } \\
\text { POSTED MESSAGE }\end{array}$ \\
\hline
\end{tabular}




\section{Post Messages}

\begin{tabular}{|c|c|c|}
\hline $\begin{array}{l}\text { Pattern } \\
\text { Name }\end{array}$ & Post messages & \\
\hline Problem & $\begin{array}{l}\text { Users in order to improve their } \\
\text { collaborative tasks need means to } \\
\text { easily communicate asynchronously } \\
\text { and manage the whole process of } \\
\text { messages' exchange. Which } \\
\text { features should an ANSCL system } \\
\text { provide to learners to facilitate the } \\
\text { posting of messages? }\end{array}$ & \\
\hline Forces & \multicolumn{2}{|c|}{$\begin{array}{l}\text { In asynchronous collaborative learning peers belong to the same convivial } \\
\text { learning environment and share learning goals. They should have usable } \\
\text { means for creating, posting and exchanging messages and ideas that are } \\
\text { related to questions, or answers to specific issues or contribution to } \\
\text { ongoing discussions. Learners might also want to start a new discussion } \\
\text { tread (given that they have the appropriate rights). Since discussion is } \\
\text { asynchronous, participants should have some time to preview and modify } \\
\text { a posting before it is posted to a discussion area. They might even } \\
\text { accompany the message with an annotation (e.g. urgent, strong } \\
\text { disagreement) in order to motivate peers to read it asap and to trigger the } \\
\text { interaction on specific issues. In fact, the annotation schema should } \\
\text { contain symbols easily recognised and that won't cause information } \\
\text { overload. }\end{array}$} \\
\hline Solution & \multicolumn{2}{|c|}{$\begin{array}{l}\text { ANSCL systems must allow users post a message to group (POST MESSAGE } \\
\text { TO GROUP) with attachment files (TACK A FILE). System must also allow } \\
\text { authors to preview the message before being posted as well as making } \\
\text { changes just after posting it (CHANGEABLE POSTINGS). Tools could also give } \\
\text { users the option to annotate a message (ANNOTATION ON POSTED MESSAGE) } \\
\text { and help them to organize and study their incoming postings. Users can } \\
\text { personalize their messages by inserting 'emoticons' (I FEEL LIKE). }\end{array}$} \\
\hline $\begin{array}{l}\text { Related } \\
\text { Patterns }\end{array}$ & \multicolumn{2}{|c|}{$\begin{array}{l}\text { POST MESSAGE TO GROUP, TACK A FILE, CHANGEABLE POSTINGS, ANNOTATION ON } \\
\text { POSTED MESSAGE, I FEEL LIKE }\end{array}$} \\
\hline
\end{tabular}




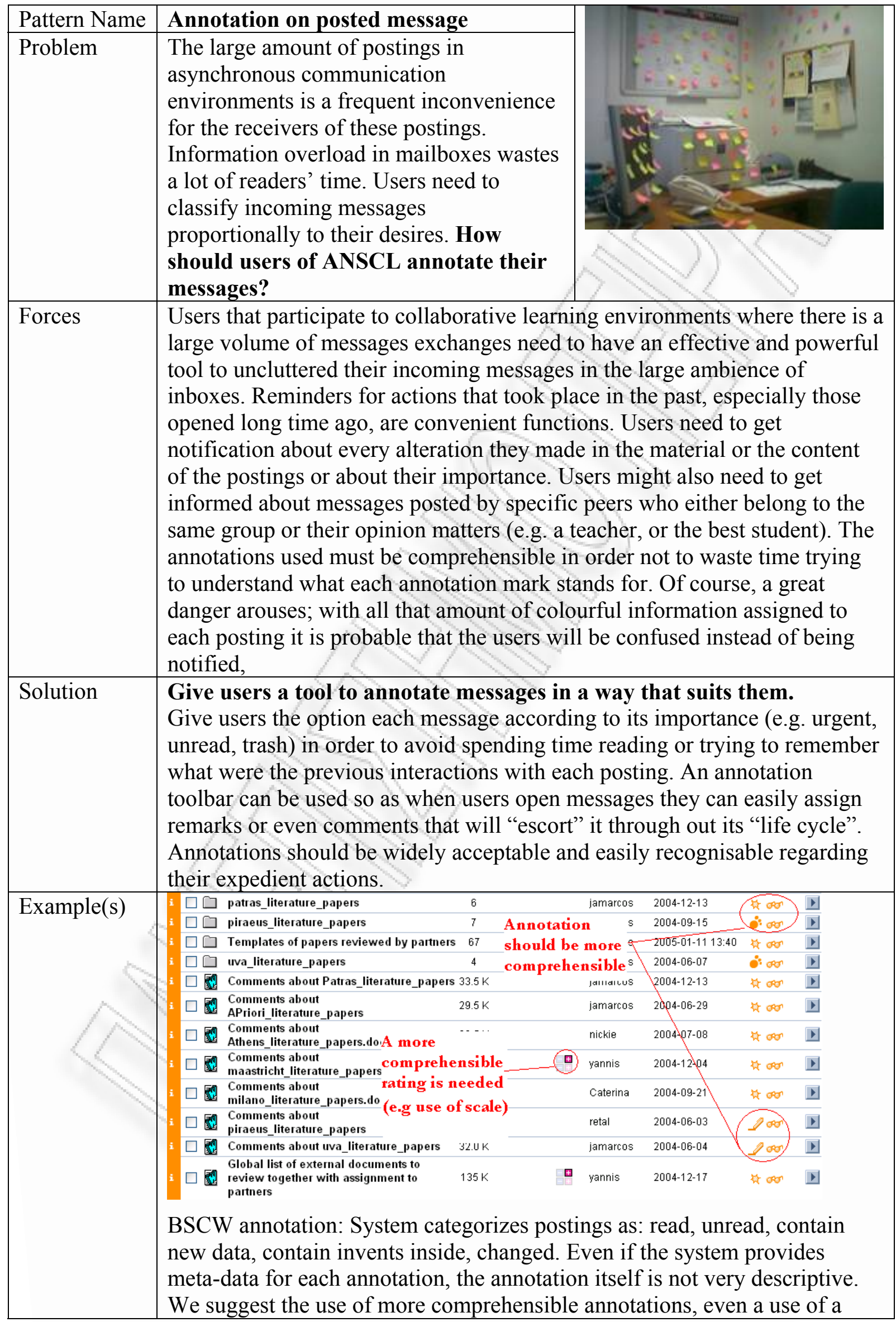




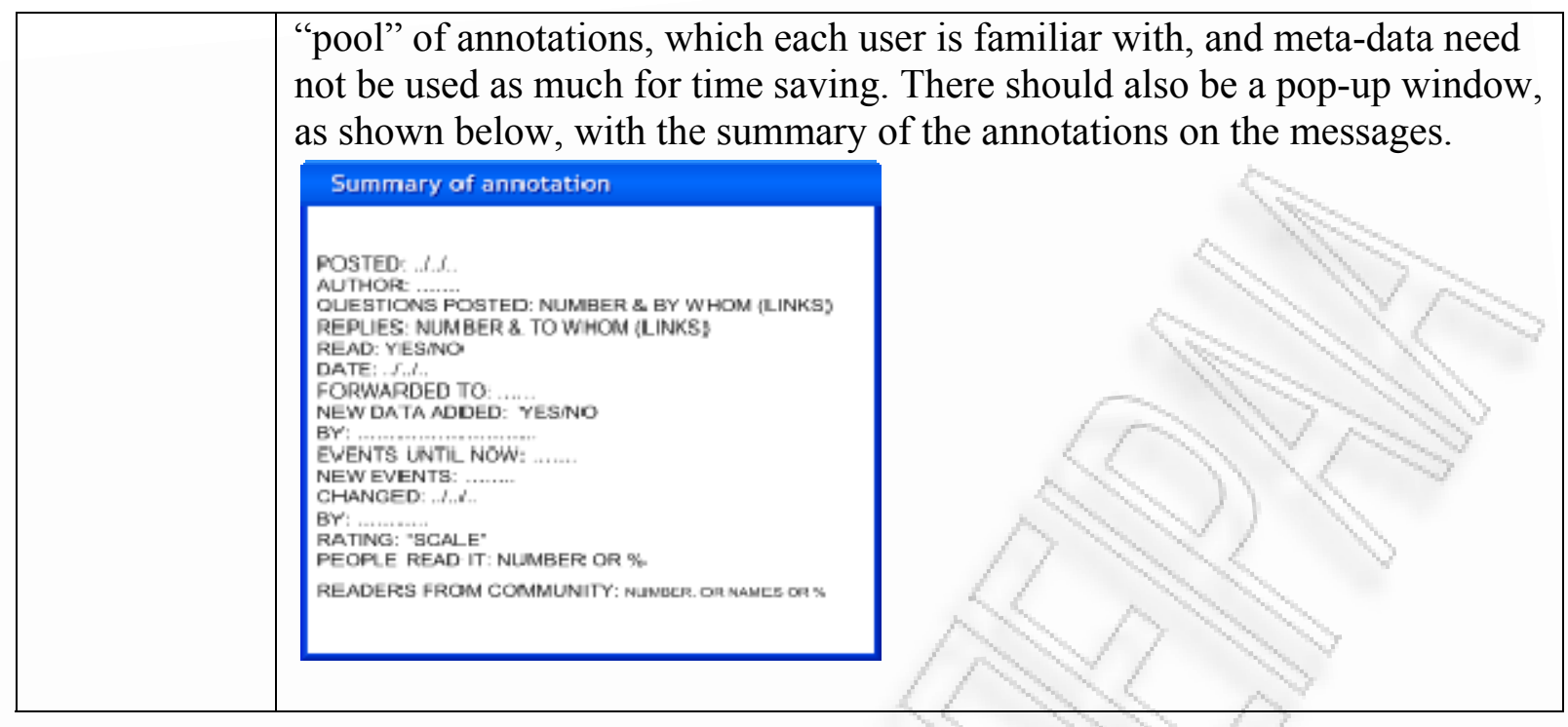




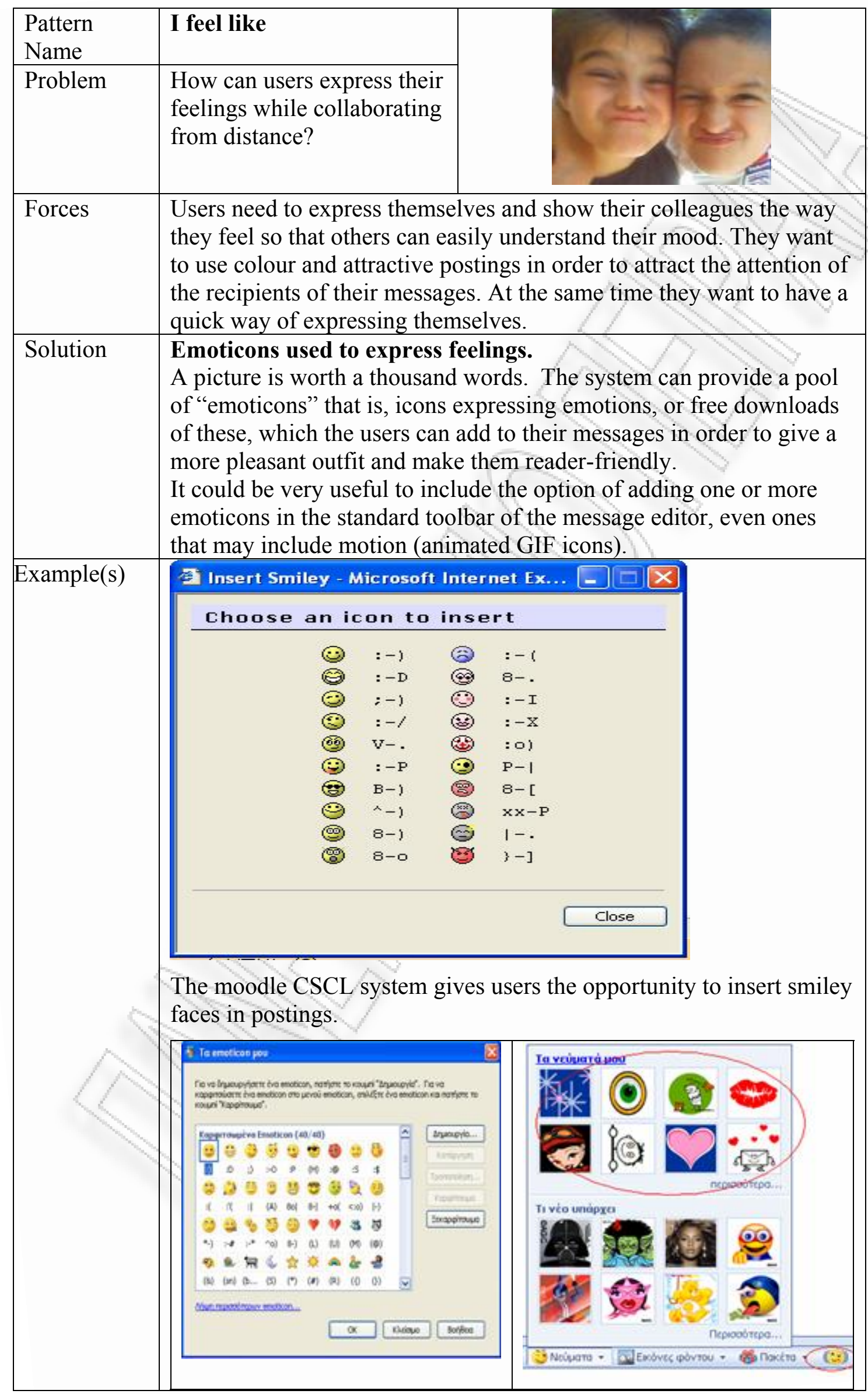


MSN Messenger provides a large amount of emoticons (most of them have animation) which can be used during chat. It also allows users to create and manage customized menus of icons by using downloaded as well as icons provided by the user. MSN Messenger also provides animated emoticons with sound that pop up from messages upon user selection. Furthermore there is an option to "vibrate" online peers, choosing the option to send a tremble notification to them. 


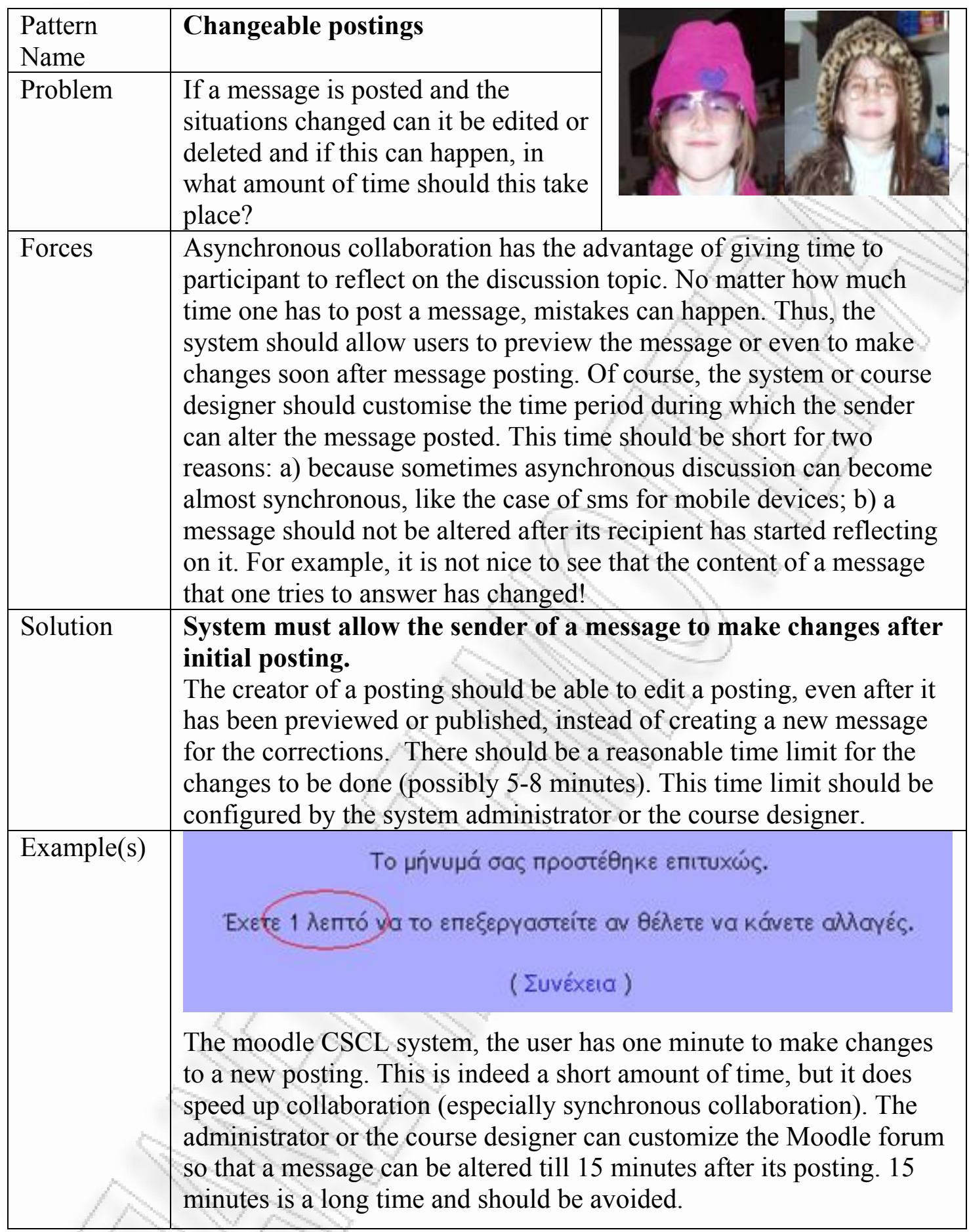




\begin{tabular}{|c|c|c|c|c|c|}
\hline $\begin{array}{l}\text { Pattern } \\
\text { Name }\end{array}$ & \multicolumn{3}{|c|}{ Tack a file } & & \\
\hline Problem & \multicolumn{3}{|c|}{$\begin{array}{l}\text { How can users share their resources with } \\
\text { other colleagues? Can files be attached in } \\
\text { discussion spaces? Is there a way to be } \\
\text { informed about the technical characteristics } \\
\text { of resource files? }\end{array}$} & & \\
\hline Forces & \multicolumn{5}{|c|}{$\begin{array}{l}\text { Bibliography, resources and references, are a very important part of an } \\
\text { educational system, and need to be provided to the students in efficient ways. } \\
\text { Several times, authors want to add a resource to a message which can be } \\
\text { useful to the rest of colleagues in order to facilitate collaboration. If authors } \\
\text { have access to a file that contains the information they want to mention, } \\
\text { reusing of such a file is time saving and the recipient(s) can avoid searching } \\
\text { for the document, especially in cases when unauthorized access is not } \\
\text { possible. Also editors want to provide information in various types of } \\
\text { supported formats and let the recipients easily identify the types of the } \\
\text { attached files. }\end{array}$} \\
\hline Solution & \multicolumn{5}{|c|}{$\begin{array}{l}\text { The system must allow attachments to messages. Provision for multiple } \\
\text { attachments to the same message should be considered. Also the system } \\
\text { should be flexible with regards to the format of the attachments (URLs, } \\
\text { spreadsheets, text, presentations, video, audio, etc.). The location of the } \\
\text { attached files should not be limited to the author's own folders, but also } \\
\text { include any folder where access is permitted. }\end{array}$} \\
\hline \multirow[t]{5}{*}{ Example(s) } & \multirow{2}{*}{\multicolumn{5}{|c|}{$\begin{array}{l}\text { Add } \text { th Item } \\
\text { In Blackboard Polaris the editor can choose what kind of resource to add to } \\
\text { each posting. }\end{array}$}} \\
\hline & & & & & \\
\hline & \multirow{2}{*}{\multicolumn{3}{|c|}{ 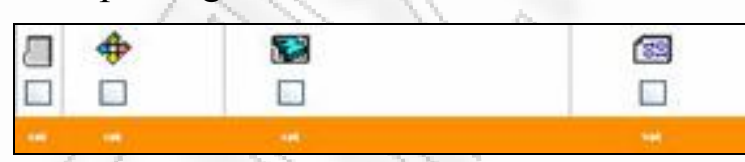 }} & 四 & \\
\hline & & & & & \\
\hline & \multicolumn{5}{|c|}{$\begin{array}{l}\text { In BSCW the user is also informed by the system about the type of available } \\
\text { documents with appropriate annotations. Nevertheless the icons used are not } \\
\text { too comprehensible. }\end{array}$} \\
\hline
\end{tabular}




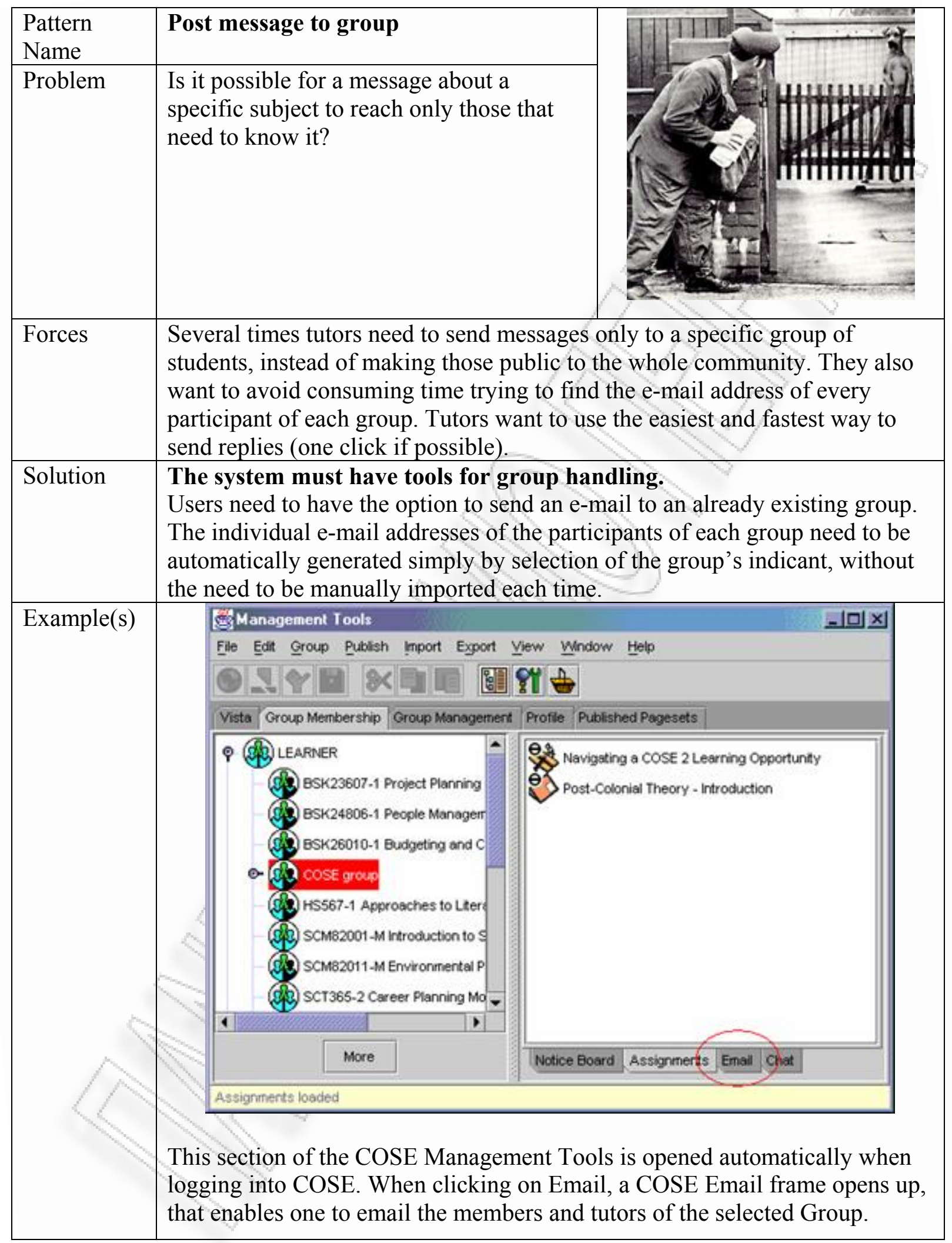




\section{Read messages}

\begin{tabular}{|c|c|}
\hline Pattern Name & Read messages \\
\hline Problem & $\begin{array}{l}\text { When users want to read a } \\
\text { message or a file, can they keep } \\
\text { margin comments or different } \\
\text { kinds of annotations on it? Can } \\
\text { they find what they are looking } \\
\text { for in the vast pool of knowledge } \\
\text { that has been built? }\end{array}$ \\
\hline Forces & $\begin{array}{l}\text { Postings from peers at an e-learning environment could be } \\
\text { valuable learning resources. Learners learn from each other while } \\
\text { discussing a topic. One can also learn from the reply that a } \\
\text { teacher gave to a fellow learner or from the technical solution that } \\
\text { the support team gave to given technical problem. Thus, handling } \\
\text { messages as recourses, learners often need to underline, highlight } \\
\text { or even add comments either the content of messages or to the } \\
\text { specific documents attached to them. Since large volume of } \\
\text { messages can be exchanged, participants to an asynchronous } \\
\text { discussion often need to search for postings related to specific } \\
\text { learning tasks or subjects, of specific type (e.g. replies, questions, } \\
\text { etc.) or from a particular peer (e.g. teacher or group member). } \\
\text { However, sometimes learners or teachers just post messages for } \\
\text { people to read but not necessarily react with another posting. } \\
\text { They should be given the permission to disallow peer to the reply } \\
\text { to some sort of messages (e.g. the case of an announcement). } \\
\text { Most probably users might want to be excluded from the list of } \\
\text { recipients of messages of specific nature (e.g. ongoing discussion } \\
\text { about technical problems, or group discussions, etc.). }\end{array}$ \\
\hline Solution & $\begin{array}{l}\text { The ANSCL system must give tools to users to annotate a } \\
\text { specific message while reading (ANNOTATE MESSAGE ON READING). } \\
\text { Also the system must provide a more personalised search } \\
\text { machine that can be customized to users' needs (MY SEARCH). } \\
\text { Thus the physical/digital resources, technology, tools, artefacts, } \\
\text { readings, etc that been made available to them, which (in } \\
\text { conjunction with things they provide themselves) are used by } \\
\text { them to customise or 'fit out' their individual learnplaces. Finally } \\
\text { when a message is posted, the user should be able to customise } \\
\text { the option of prohibiting replies (DO I WANT A REPLY?). }\end{array}$ \\
\hline Related Patterns & ANNOTATE MESSAGE ON READING, MY SEARCH, DO I WANT A REPLY? \\
\hline
\end{tabular}




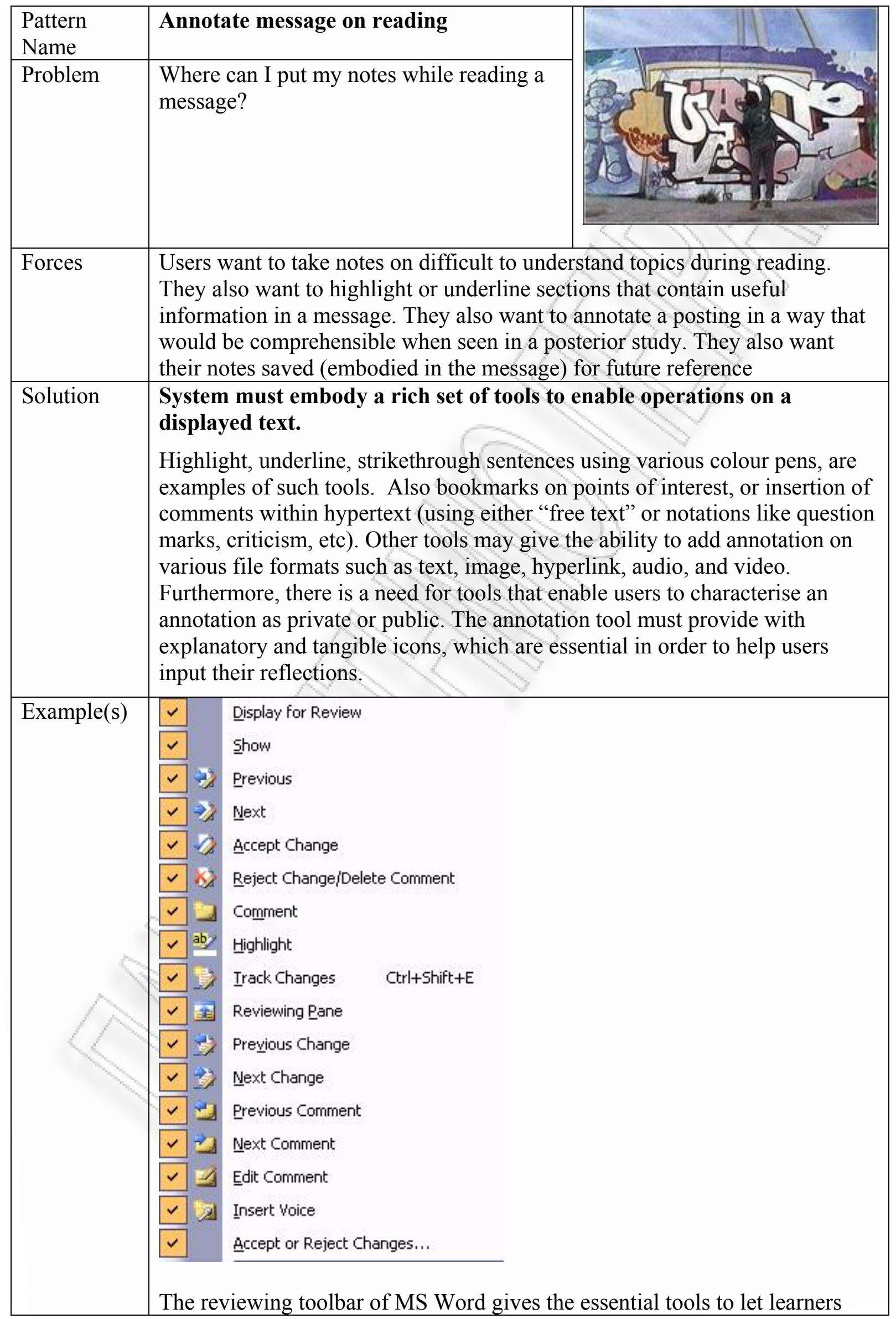


actively engage in written texts and learn from such.

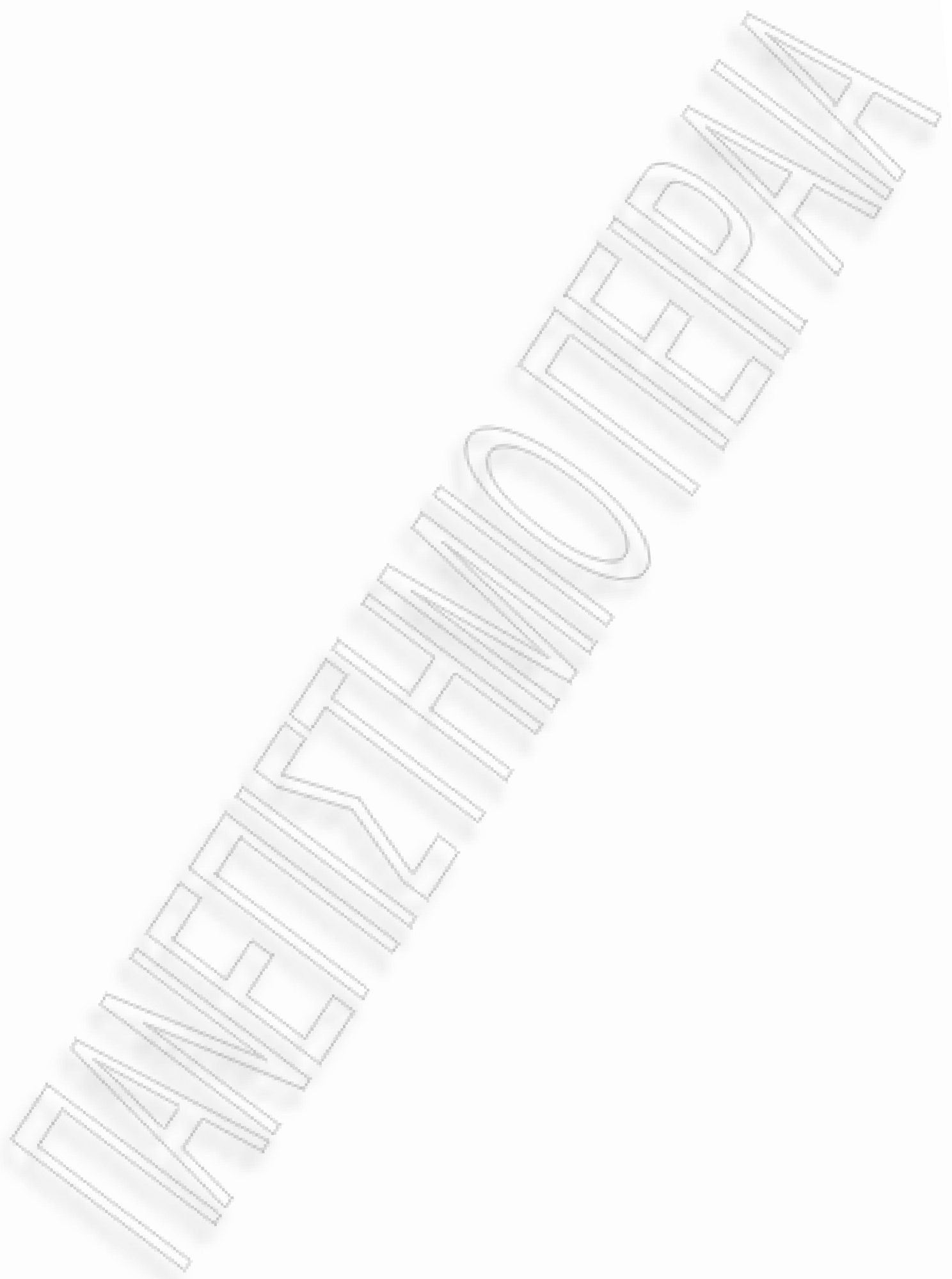




\begin{tabular}{|l|l|}
\hline Pattern & My search \\
\hline Problem & $\begin{array}{l}\text { Are search options for several kinds of } \\
\text { documents available to users? }\end{array}$ \\
\hline Forces & $\begin{array}{l}\text { Users often want to search for resources (documents, video files, pictures } \\
\text { etc.) that are relative to their subjects in repositories that are not easily found } \\
\text { neither by a powerful search engine nor but in specialized system. Many } \\
\text { times their search is much easier when they can include the use of parameters } \\
\text { in their search such as the date range or the item posted or produced, file } \\
\text { format preferred type, language, author etc. They also want to use keywords } \\
\text { that they need to be found in the search results or search for annotations by } \\
\text { making queries with respect to the annotation type. While searching through } \\
\text { the engine, users often need to find similar pages or links from the whole } \\
\text { WWW or from specific URLs. They also want to rate the quality of the } \\
\text { documents they found in order to prompt colleagues to read them or view the } \\
\text { ratings of other members of the online community for specific issues }\end{array}$ \\
\hline Solution & $\begin{array}{l}\text { Let the users search only within the area of their interests. } \\
\text { Users need a more studiously search engine exclusively in system's files and } \\
\text { folders. They don't want to delve into the vast information of all the web } \\
\text { repositories but only in postings or documents that the specific educational } \\
\text { community provides. Search with parameters (e.g. note title, author, } \\
\text { keywords, date of issue, file type, language, specific folder, urgency, even } \\
\text { "unwords" - words that should not be contained in the document) is } \\
\text { necessary, in order to diminish the time spent for the search. Filtering of } \\
\text { unwanted material shall also be supported. A search engine should also } \\
\text { remember previous inquiries of the same user and prompt users to re-visit } \\
\text { these folders, since probably the same topic will be visited many times. }\end{array}$ \\
\hline
\end{tabular}




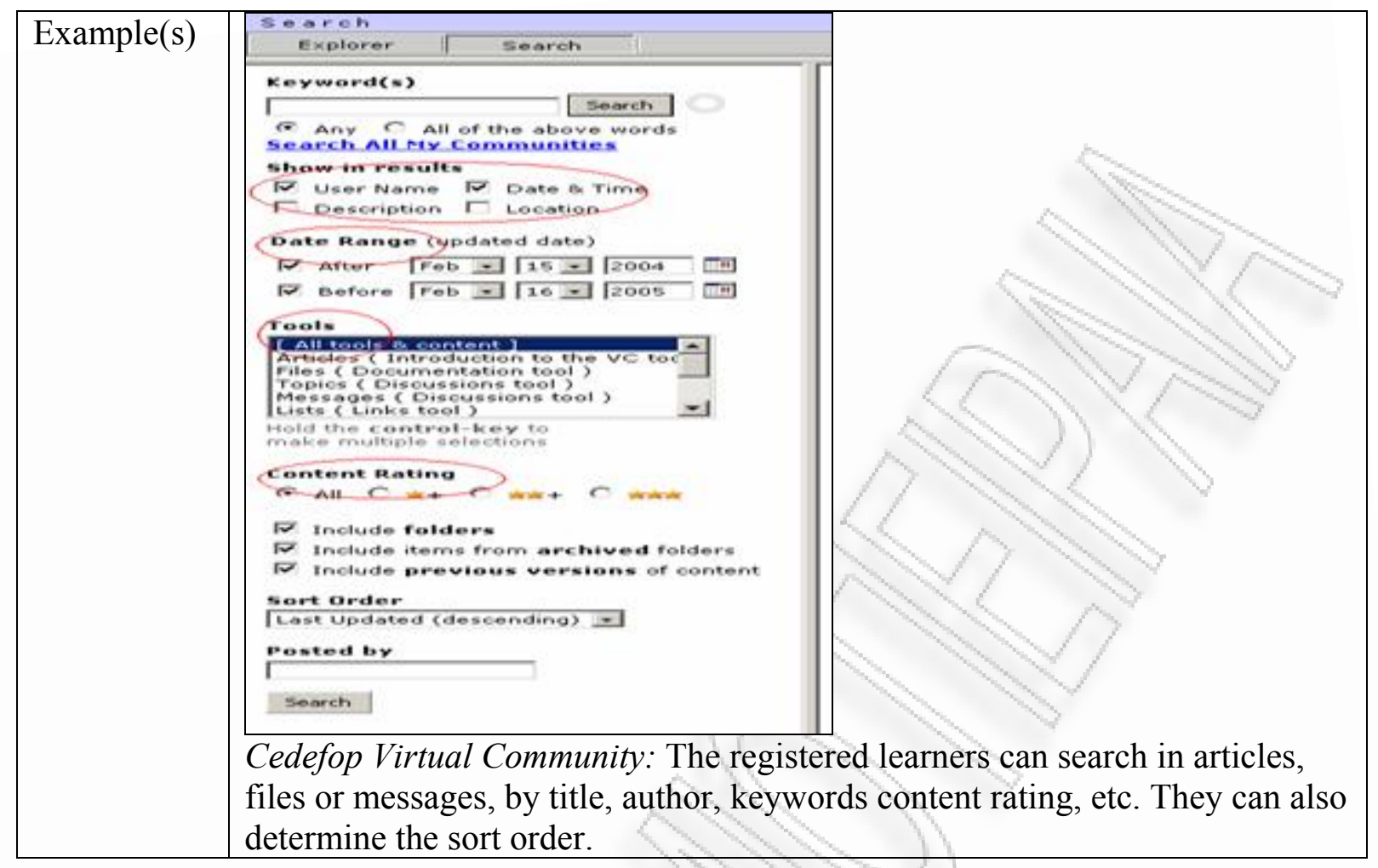




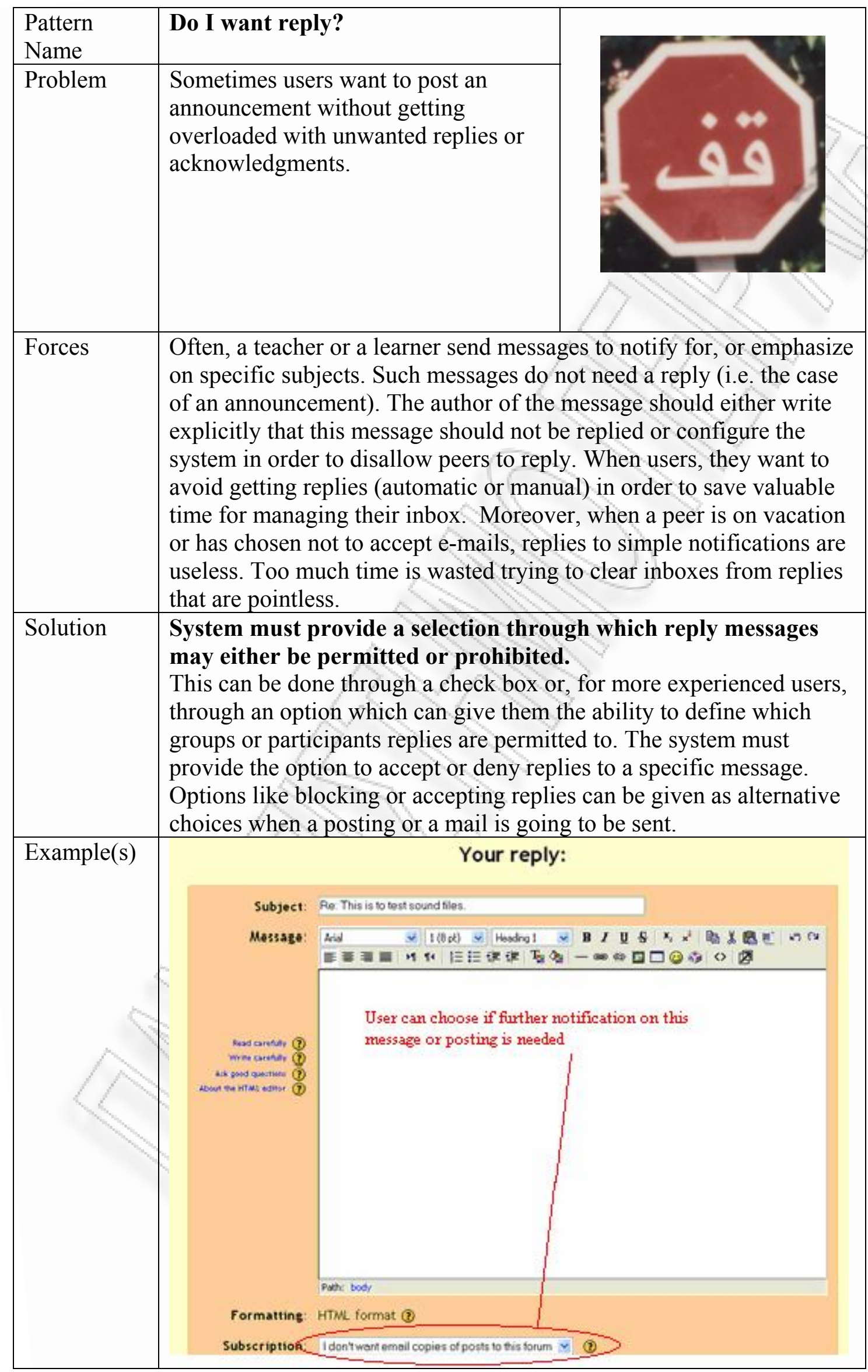




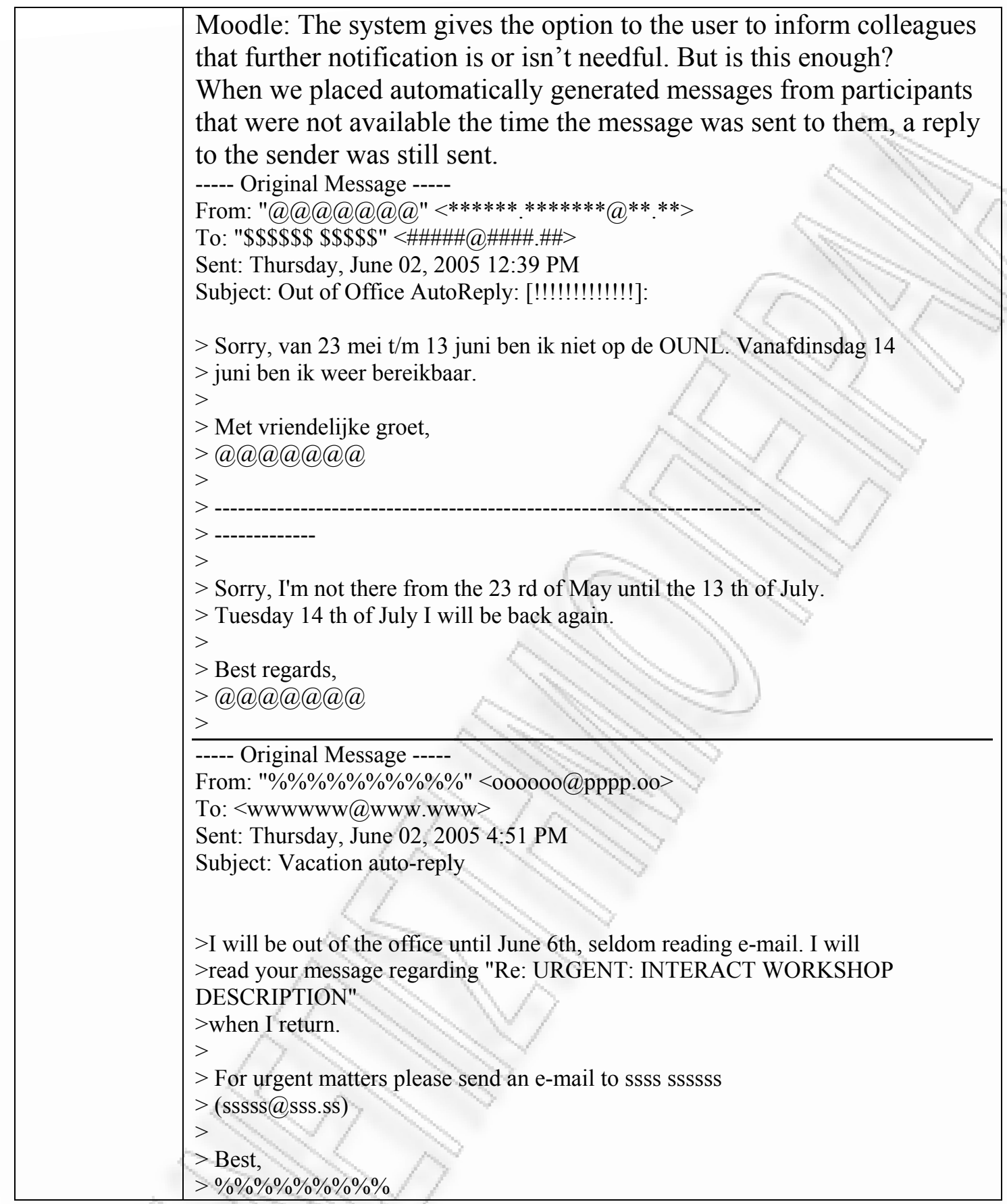




\section{Threaded Discussion Management}

\begin{tabular}{|c|c|}
\hline Pattern Name & $\begin{array}{l}\text { Threaded Discussion } \\
\text { Management }\end{array}$ \\
\hline Problem & $\begin{array}{l}\text { How can tutors manage } \\
\text { discussions? }\end{array}$ \\
\hline Forces & $\begin{array}{l}\text { The learning effectiveness of collaboration depends on the } \\
\text { formation of virtual learning communities (either large or small, } \\
\text { i.e. groups), and the support of the interaction between users by } \\
\text { maintaining common spaces for the posting and organisation of } \\
\text { messages, as well as for the sharing, re-using and annotating } \\
\text { resources. Collaborative learning tasks can be: Setting up } \\
\text { research questions, posting arguments or critical comments on } \\
\text { specific topic, exchanging messages for constructing working } \\
\text { theories or finding solutions to a given problem, etc. Tools that } \\
\text { empower tutors to manage this way of communication between } \\
\text { participants (either in groups or at a plenary session) are crucial } \\
\text { for the effectiveness of collaboration. Handling issues of privacy } \\
\text { of ideas exchanged are of major importance to build trust as well } \\
\text { as avoid unauthorised use of sensitive data. }\end{array}$ \\
\hline Solution & $\begin{array}{l}\text { ANSCL system must give the option to easily create a new } \\
\text { discussion topic (CREATE A NEW DISCUSSION TOPIC) where treaded } \\
\text { discussion can occur. In every threaded discussion user must be } \\
\text { able to sort messages by selected criteria (e.g. date, sender) } \\
\text { (soRTING THREADED DISCUSSION). The system must provide an } \\
\text { option to facilitate the automatic threading of answers to } \\
\text { messages (SHORT MESSAGES BY CATEGORY). Tutors should be able to } \\
\text { associate a discussion forum to a particular group (WHISPER) and } \\
\text { determine when a discussion can be viewed (SET FORUM VISIBILITY) } \\
\text { and by whom (SET FORUM AVAILABILITY). Attachments to a } \\
\text { discussion message must be of limited size (LIMIT ATTACHMENT } \\
\text { MESSAGE SIZE) in order to facilitate downloading of each page. } \\
\text { System must provide space in the message board or in other } \\
\text { specific area, where user can forward important messages for } \\
\text { further analysis (MY MESSAGE PORTFOLIO). Issues of security and } \\
\text { message privacy should be handled. System must enable tutors to } \\
\text { select the users who have access to the discussion spaces as well } \\
\text { as to make an invitation to non registering ones in case needed, } \\
\text { i.e. invitation of an expert to participate to a short term discussion } \\
\text { (INVITE NON REGISTERED uSER). }\end{array}$ \\
\hline Related Patterns & $\begin{array}{l}\text { CREATE A NEW DISCUSSION TOPIC, SORTING THREADED DISCUSSION, SHORT } \\
\text { MESSAGES BY CATEGORY, WHISPER, SET FORUM VISIBILITY, SET FORUM } \\
\text { AVAILABILITY, LIMIT ATTACHMENT MESSAGE SIZE, MY MESSAGE } \\
\text { PORTFOLIO, INVITE NON REGISTERED USER }\end{array}$ \\
\hline
\end{tabular}




\begin{tabular}{|c|c|}
\hline Pattern Name & Sort messages by category \\
\hline Problem & Can I keep my message board organized? \\
\hline Forces & $\begin{array}{l}\text { The plethora of postings that are not connected with each other can lead to } \\
\text { anarchy on the message board. There is a need for filing and sorting them, in } \\
\text { order to avoid cascading through all the messages one by one when doing a } \\
\text { search or opening the inbox. Categorization of postings by topic as a better } \\
\text { study of a specific theme can be efficient, if the main topic and all the } \\
\text { contributions from colleagues fall into the same category. Better } \\
\text { organization, makes the message board more user friendly and manageable. } \\
\text { Of course, the way of defining the categories where messages will be sorted } \\
\text { is not an easy task and appropriate names should be found in order that the } \\
\text { user can easily recognise it and recall the messages that belong to that } \\
\text { category. }\end{array}$ \\
\hline Solution & $\begin{array}{l}\text { The system must provide an option to facilitate automatic threading of } \\
\text { answers to messages. } \\
\text { Each new posting shall be assigned to a new link on the message board. } \\
\text { Users can create their own folders and move, delete or reorganize every } \\
\text { posting in their board. }\end{array}$ \\
\hline Example(s) & 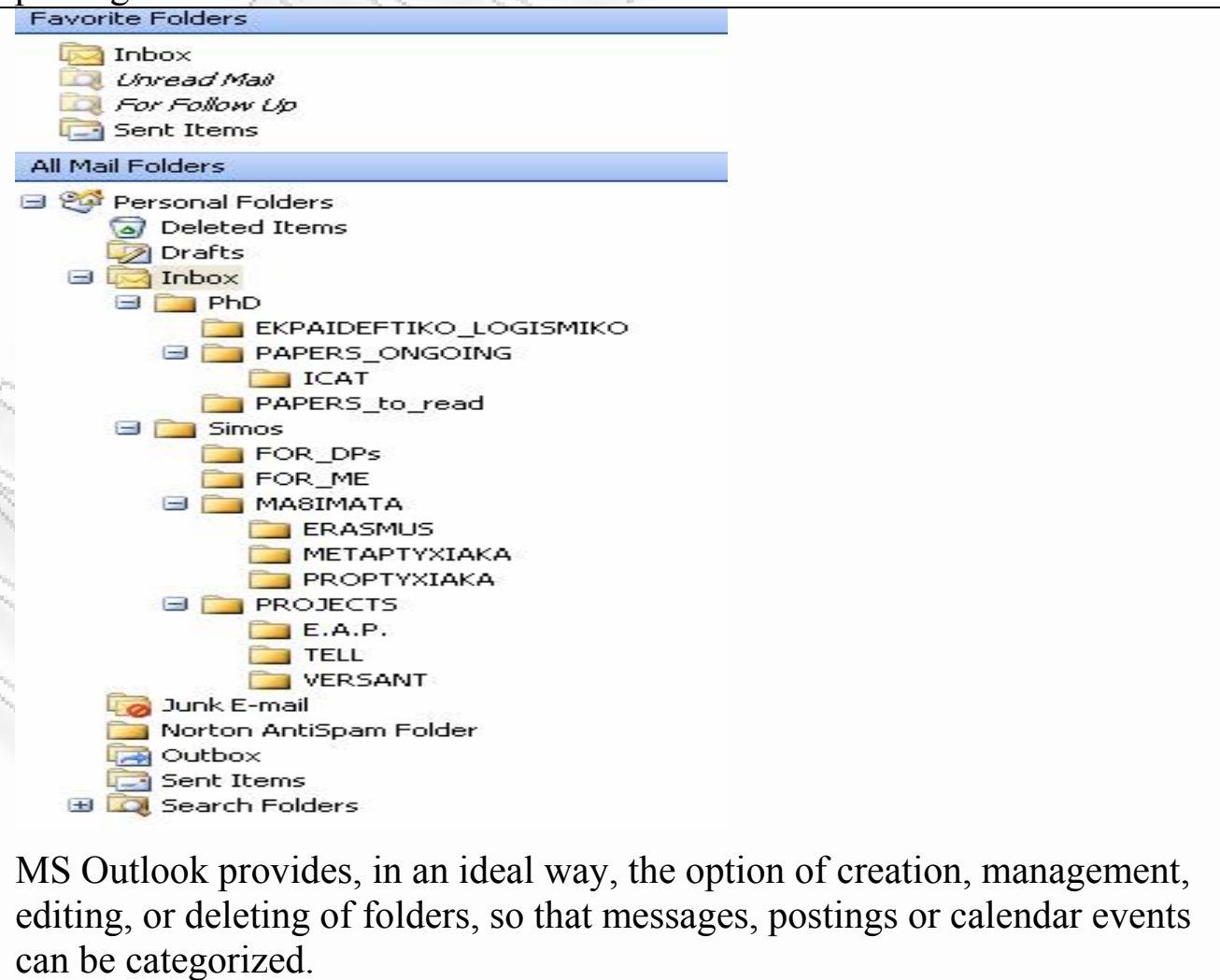 \\
\hline
\end{tabular}




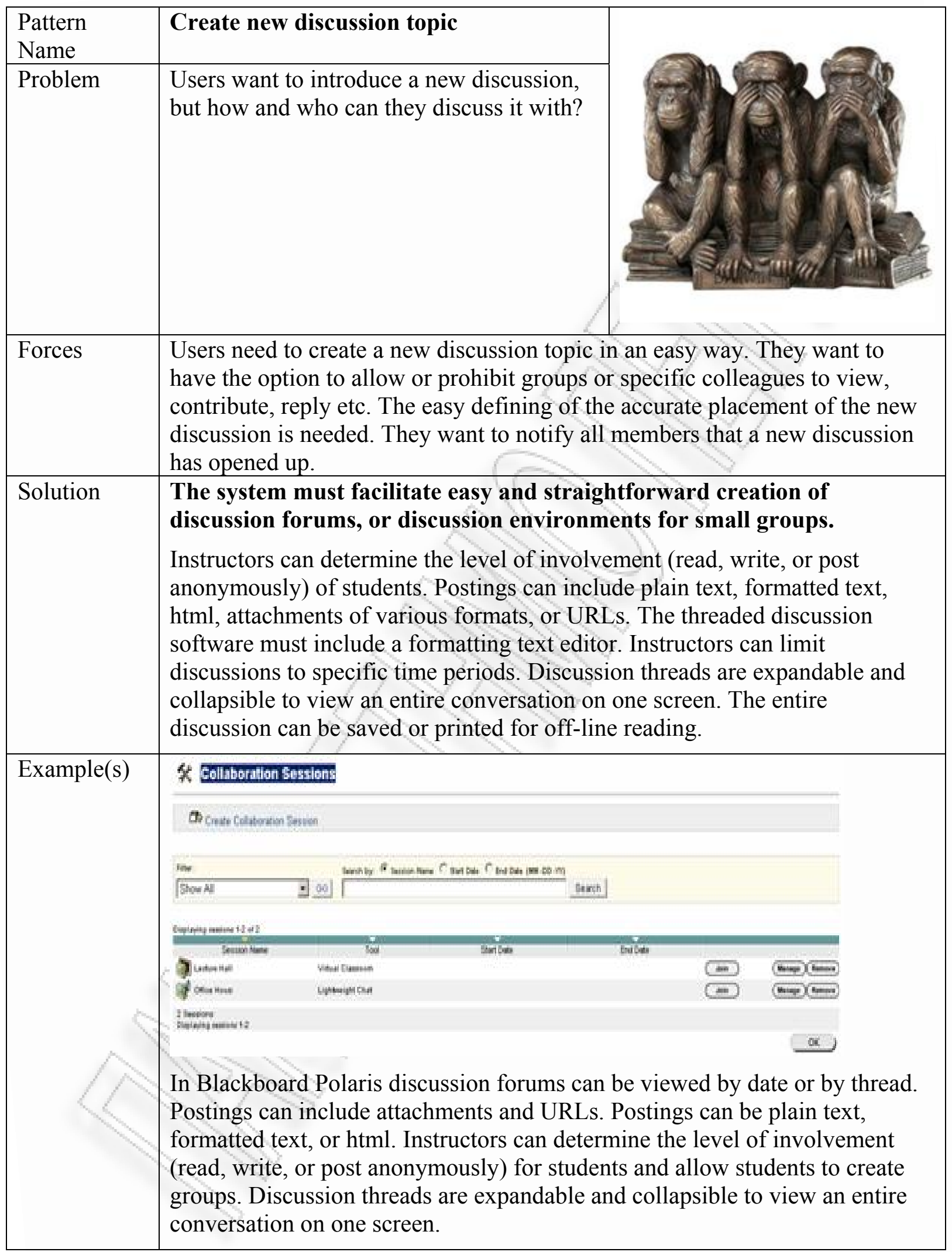




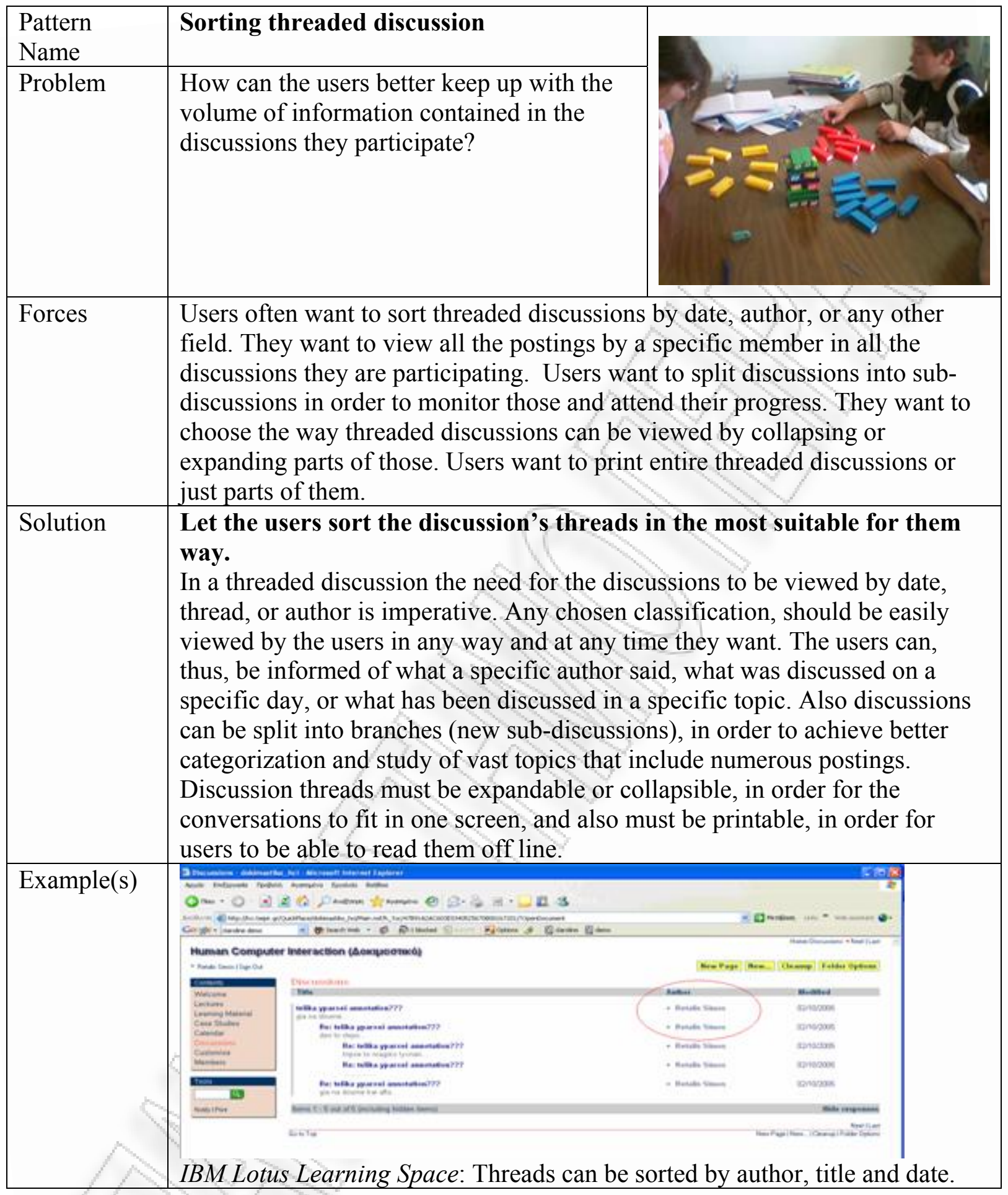




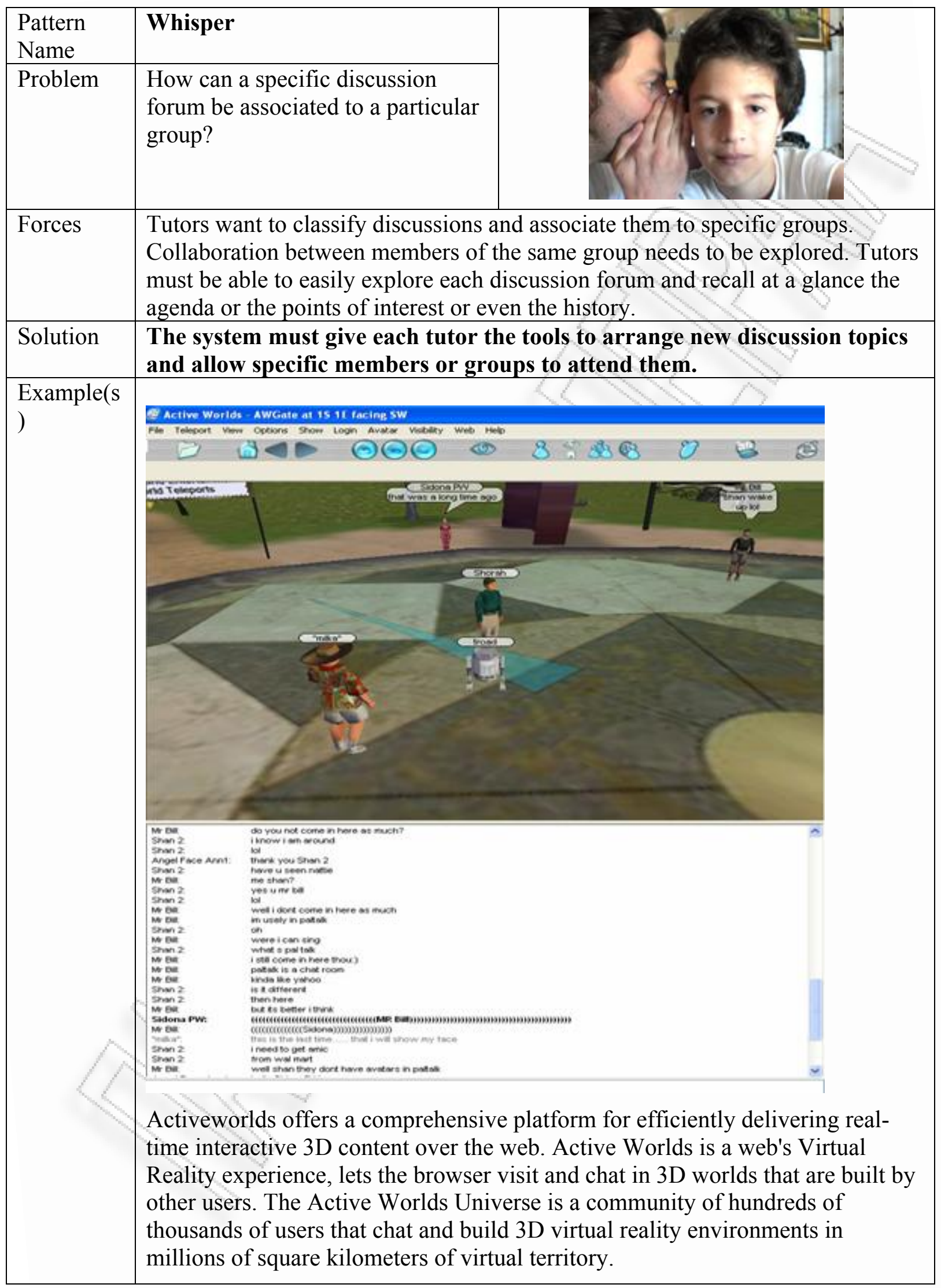




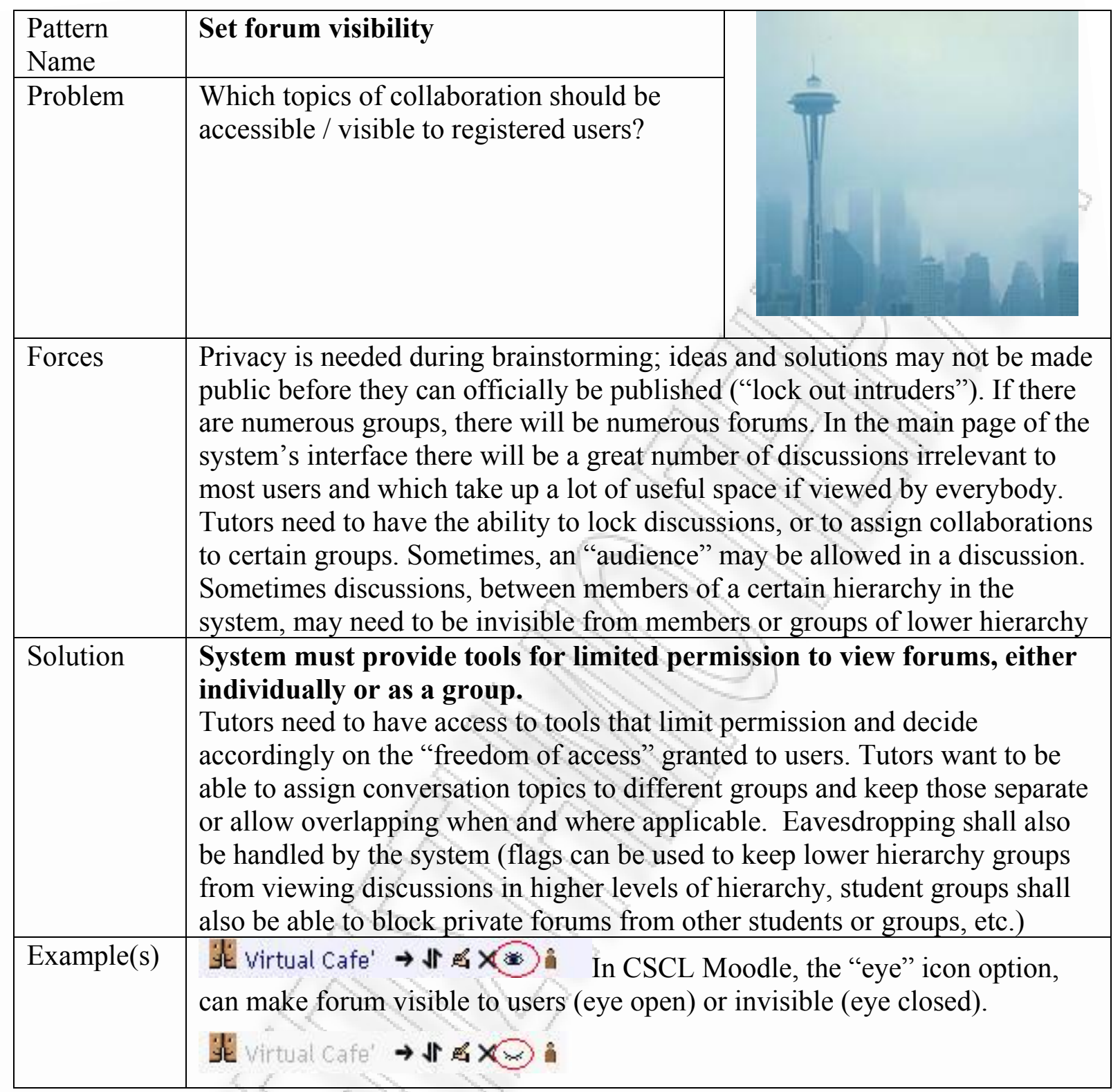




\begin{tabular}{|c|c|c|c|}
\hline $\begin{array}{l}\text { Pattern } \\
\text { Name }\end{array}$ & \multicolumn{2}{|c|}{ Set forum availability } & 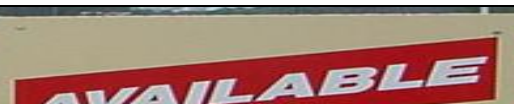 \\
\hline Problem & \multicolumn{2}{|c|}{$\begin{array}{l}\text { Who shall be able to attend, participate and } \\
\text { interact with the others in a forum? }\end{array}$} & \\
\hline Forces & \multicolumn{3}{|c|}{$\begin{array}{l}\text { Tutors may want, in order to promote collaboration and to obtain qualitative } \\
\text { outcomes, to create specific forums for specific users. In these forums } \\
\text { registration will be permitted only if prerequisites are completed successfully. } \\
\text { The control of forum accessibility in order to minimize outside interference is } \\
\text { needed. }\end{array}$} \\
\hline Solution & \multicolumn{3}{|c|}{$\begin{array}{l}\text { The system must provide tools to the tutor/administrator for selecting } \\
\text { users and authorizing accessibility in regards with each forum. } \\
\text { Tutors must have the power to allow or prohibit access to any participant. } \\
\text { Tutors may also be able to associate with a specific forum several kinds of } \\
\text { users and, sometimes, more users than those registered. Interference from } \\
\text { third parties should be easily controlled. }\end{array}$} \\
\hline \multirow[t]{8}{*}{ Example(s) } & \multicolumn{3}{|c|}{ (1) Set Area Properties } \\
\hline & Area Type: & \multicolumn{2}{|l|}{ Announcements } \\
\hline & Area name: & \multicolumn{2}{|c|}{ Announcerenents } \\
\hline & & \multicolumn{2}{|c|}{ 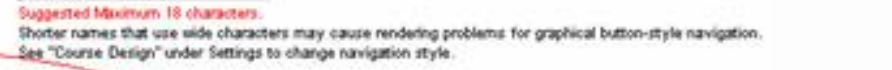 } \\
\hline & Allow guest & \multicolumn{2}{|c|}{$\nabla / \gamma$} \\
\hline & Allow obser & \multicolumn{2}{|l|}{$\nabla$} \\
\hline & $\begin{array}{l}\text { Make avail } \\
\text { Student }\end{array}$ & \\
\hline & \multicolumn{3}{|c|}{$\begin{array}{l}\text { In Blackboard Polaris specific areas can be available and accessible from } \\
\text { different kind of participants if selected. }\end{array}$} \\
\hline
\end{tabular}




\begin{tabular}{|c|c|c|}
\hline $\begin{array}{l}\text { Pattern } \\
\text { Name }\end{array}$ & Limit attached message size & \\
\hline Problem & $\begin{array}{l}\text { How can the size of the attached messages } \\
\text { be controlled in order to save time in } \\
\text { downloading in case user has a low } \\
\text { bandwidth. }\end{array}$ & \\
\hline Forces & \multicolumn{2}{|c|}{$\begin{array}{l}\text { The size of an attachment or file, affects the time it takes for the user to } \\
\text { download it; files should be as small/compact as they can get. Users don't } \\
\text { usually have high speed internet connections, which mean that they don't } \\
\text { have the convenience to download fast. The bigger the size of an attachment } \\
\text { or even an animation or a photograph contained in the web page, the longer it } \\
\text { will take for it to be downloaded, which is a nuisance to the user. On the } \\
\text { contrary sometimes tutors need to upload attachments larger than the } \\
\text { maximum upload size in order to provide more sufficient material. }\end{array}$} \\
\hline Solution & \multicolumn{2}{|c|}{$\begin{array}{l}\text { Tutors must have the option to select a limit in the size of the } \\
\text { attachments that can be uploaded. } \\
\text { Also they must have the option to designate types of users that may be } \\
\text { allowed to upload larger attachments. }\end{array}$} \\
\hline \multirow[t]{5}{*}{ Example(s) } & \multicolumn{2}{|c|}{ 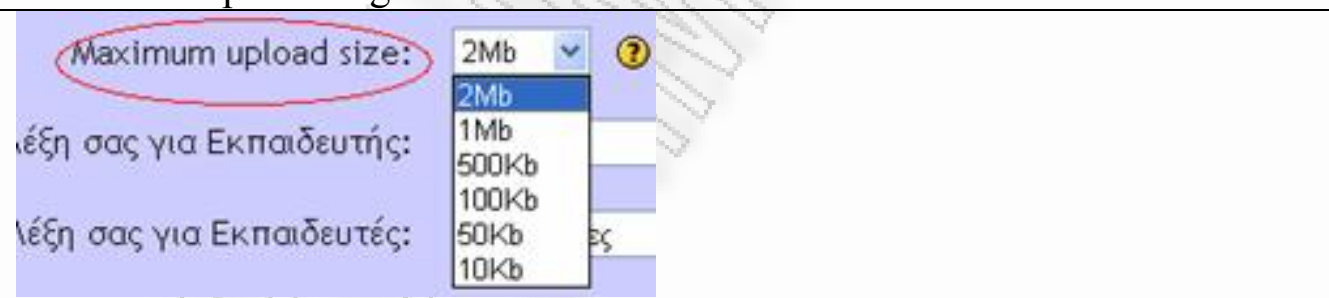 } \\
\hline & \multicolumn{2}{|c|}{$\begin{array}{l}\text { In CSCL Moodle, the administrator or instructor can configure the maximum } \\
\text { size of an uploaded file from a prearranged scale of sizes }\end{array}$} \\
\hline & \multicolumn{2}{|c|}{ 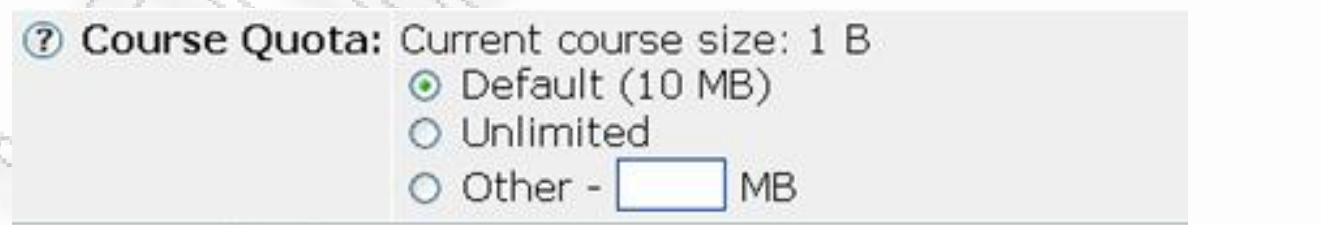 } \\
\hline & \multicolumn{2}{|c|}{$\begin{aligned} \text { Max File Size: } & \odot \text { Default (1 MB) } \\
& \bigcirc \text { Maximum system allows (2 MB) } \\
& \bigcirc \text { Other - } \square \mathrm{MB}\end{aligned}$} \\
\hline & \multicolumn{2}{|c|}{$\begin{array}{l}\text { In CSCL ATutor, the instructor can configure not only the maximum file size } \\
\text { to be uploaded, but also the overall (extended) size of the whole course. }\end{array}$} \\
\hline
\end{tabular}




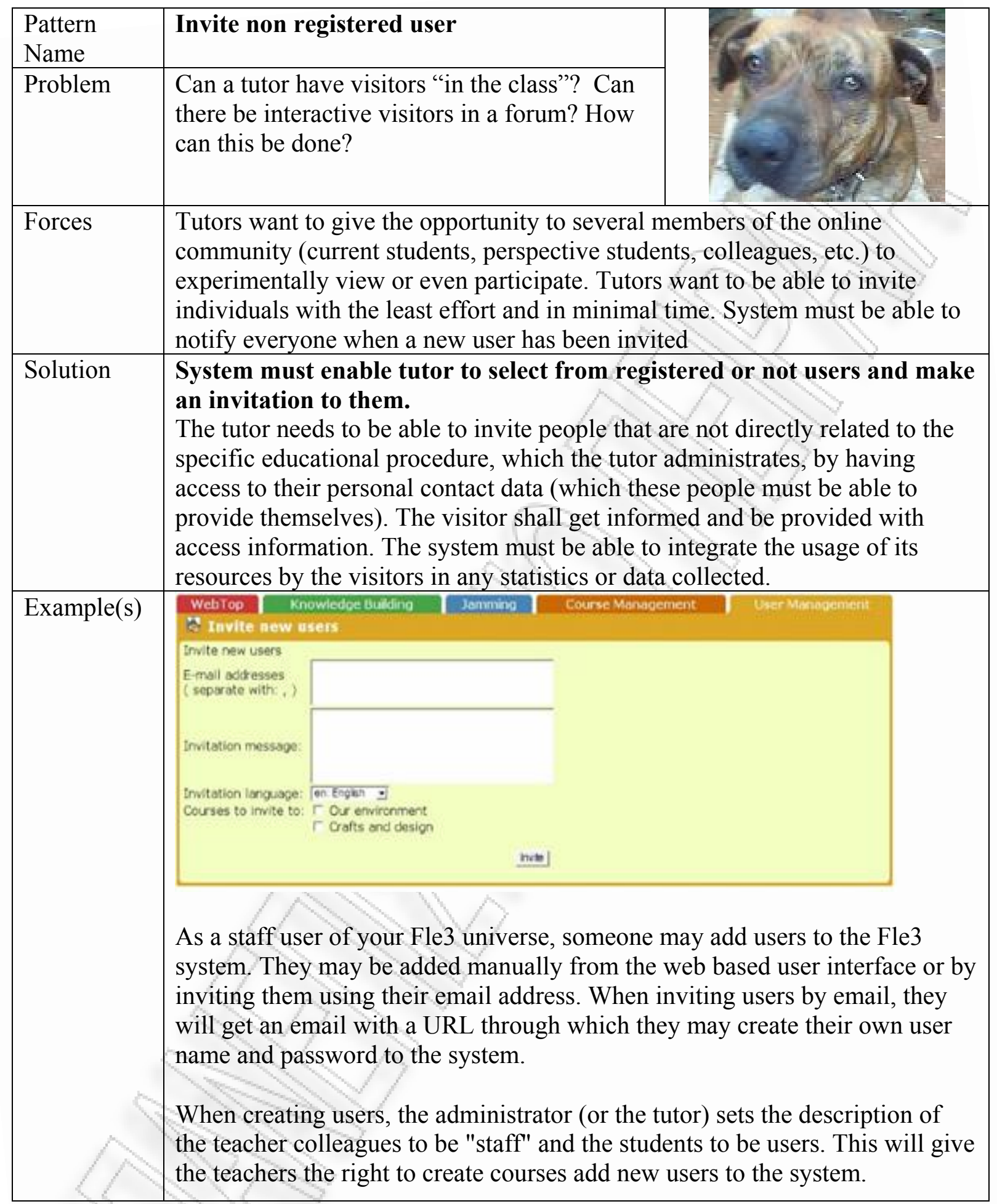




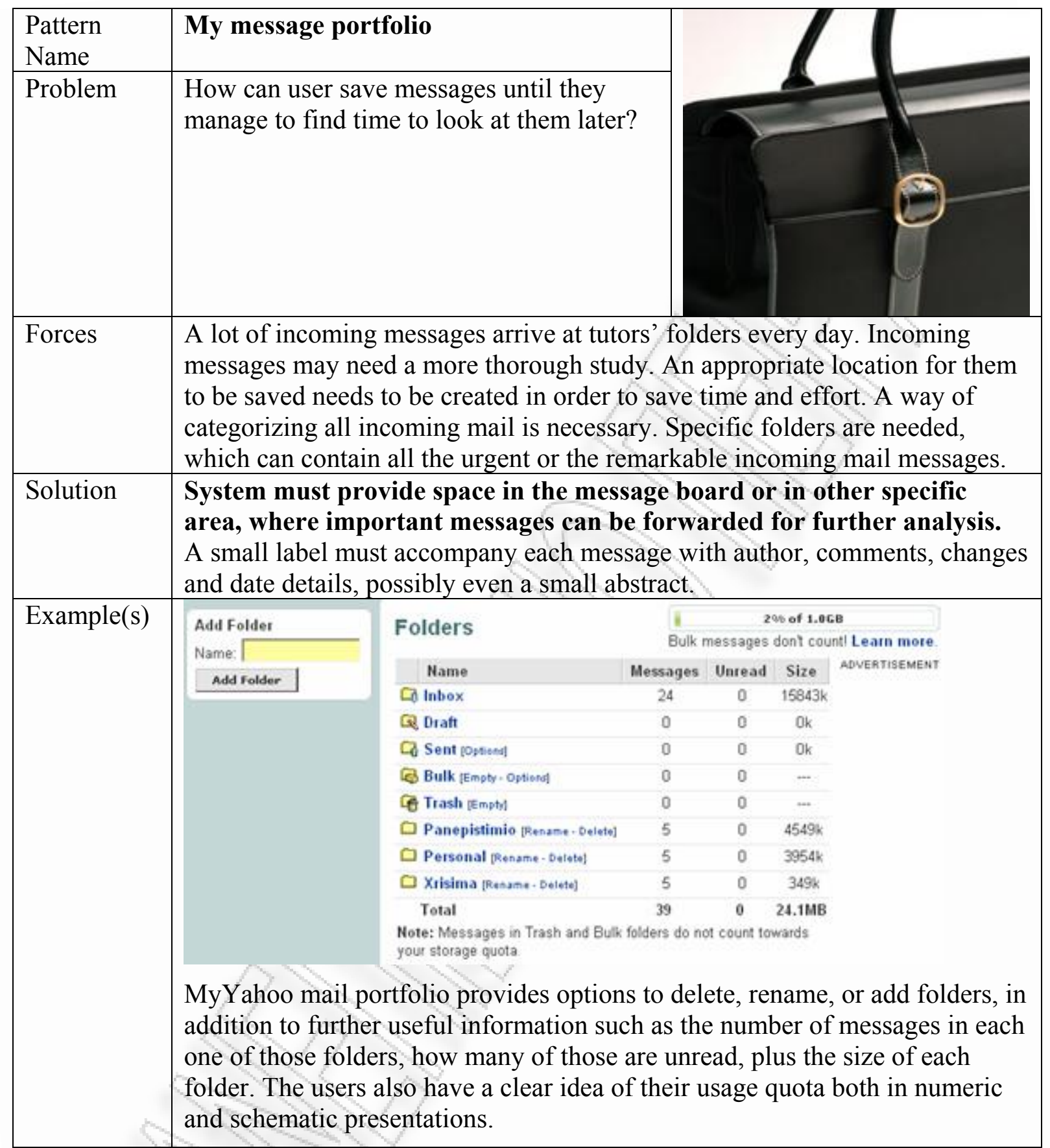


Design Patterns for Spaces for collaborative work with artifacts

\begin{tabular}{|c|c|c|}
\hline Pattern Name & $\begin{array}{l}\text { Spaces for collaborative work } \\
\text { with artifacts }\end{array}$ & \\
\hline Problem & $\begin{array}{l}\text { Where can users create, manage, } \\
\text { and present the outcomes of } \\
\text { collaborative project assignments? }\end{array}$ & \\
\hline Forces & \multicolumn{2}{|c|}{$\begin{array}{l}\text { Collaborative learning tasks often has to do with the joint development of } \\
\text { artifacts or the co-manipulation of shared learning objects. In order users } \\
\text { to produce deliverables or create artefacts during the collaborative } \\
\text { learning process need spaces that support the process of documents } \\
\text { creation, evolution, and presentation. }\end{array}$} \\
\hline Solution & \multicolumn{2}{|c|}{$\begin{array}{l}\text { Creation, management and presentation of work-spaces (ASSIGN } \\
\text { WORKSPACE TO GROUP) where students can post documents and files (FILE } \\
\text { MANAGEMENT) together with annotative comments on these documents } \\
\text { (ANNOTATION ON DOCUMENTS) that are typically the outcomes of } \\
\text { collaborative project assignments is need. Different versions of } \\
\text { documents are supported so that the process of document evolution is } \\
\text { properly presented (VERSION CONTROL OF ARTEFACTS). Management of disc } \\
\text { quota and access rights for folders and documents can be easily modified } \\
\text { by tutor (SET LEARNER / GROUP ACCESS RIGHTS). Document uploading, } \\
\text { support of uploaded document versions and annotations of documents are } \\
\text { functionalities that promote collaboration. System should give the option } \\
\text { to composers of an artefact to permit or block reviewers (PEER REVIEW / } \\
\text { ASSESS OF ARTEFACTS). }\end{array}$} \\
\hline Related Patterns & $\begin{array}{l}\text { ASSIGN WORKSPACE TO GROUP, FILE MAN } \\
\text { VERSION CONTROL OF ARTEFACTS, SET LE }\end{array}$ & $\begin{array}{l}\text { GEMENT, ANNOTATION ON DOCUMENTS, } \\
\text { RNER / GROUP ACCESS RIGHTS }\end{array}$ \\
\hline
\end{tabular}




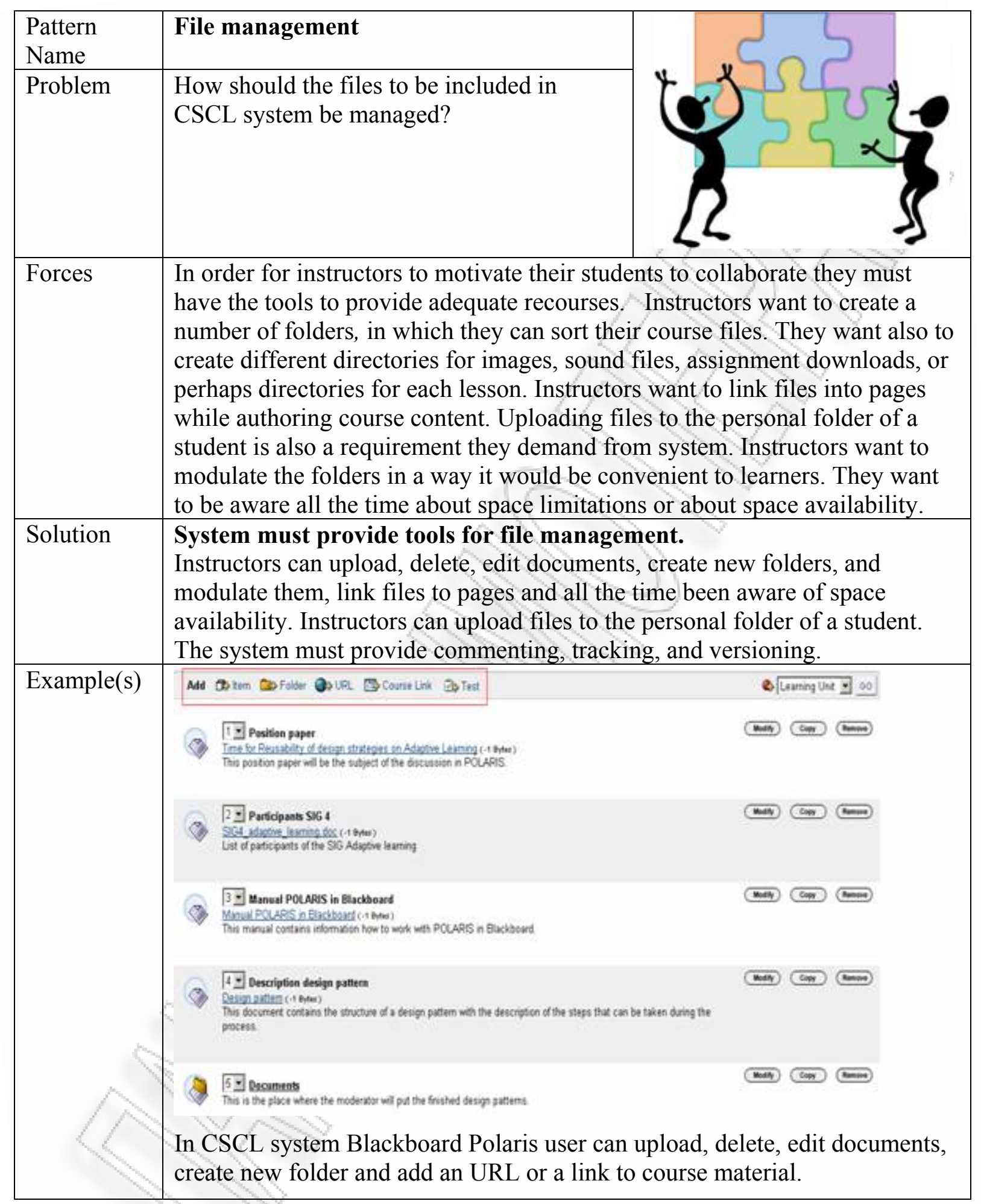




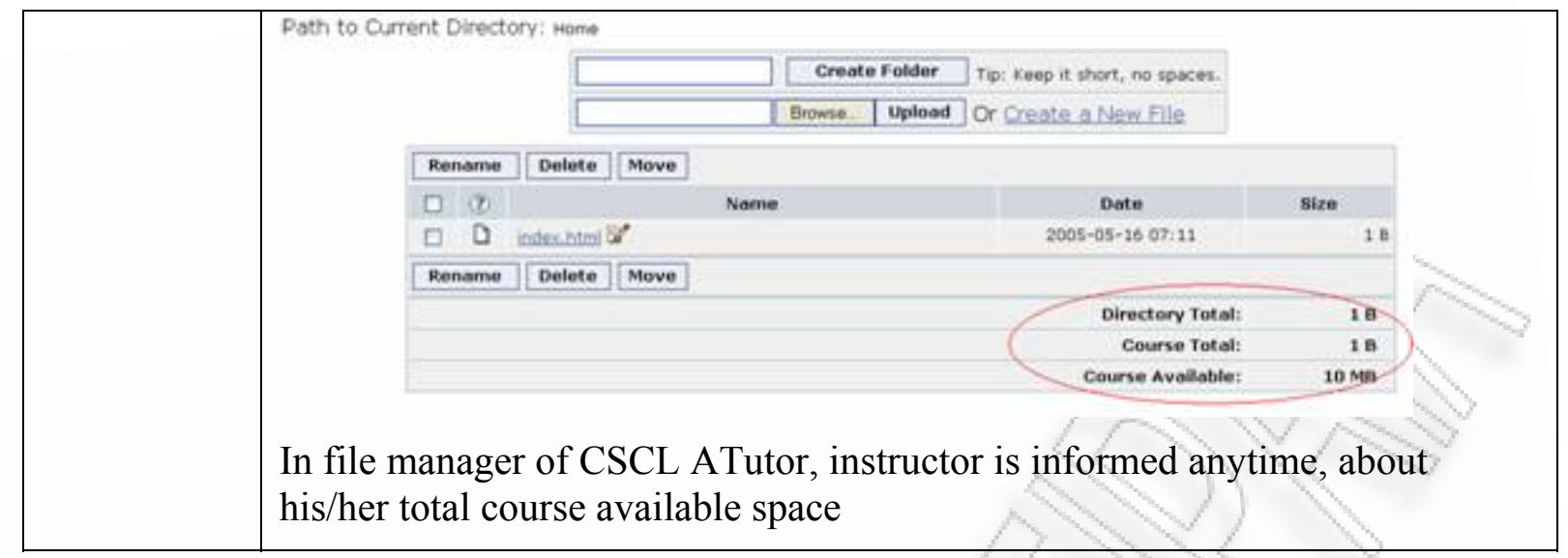




\begin{tabular}{|c|c|c|}
\hline $\begin{array}{l}\text { Pattern } \\
\text { Name }\end{array}$ & Version control of artifacts & \\
\hline Problem & $\begin{array}{l}\text { How can all versions of an artefact be } \\
\text { handled optimally? }\end{array}$ & 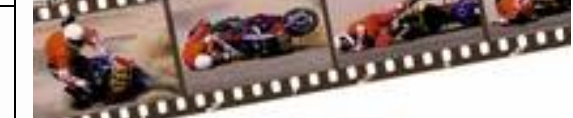 \\
\hline Forces & \multicolumn{2}{|c|}{$\begin{array}{l}\text { Users need to have easy access to the latest version of an artefact. They need } \\
\text { to be able to access all previous versions as well. After an upgrade, in each } \\
\text { artefact, a newer version needs to be declared. After a period of time users } \\
\text { may want to revise their postings, delete them or enhance them. }\end{array}$} \\
\hline Solution & \multicolumn{2}{|c|}{$\begin{array}{l}\text { The system must automatically generate the latest version of the artefact, } \\
\text { after contributors have made corrections to or have edited the current } \\
\text { version. } \\
\text { In the main page of postings the latest version should be kept on the top. } \\
\text { Also some kind of annotation needs to be used to indicate to the other } \\
\text { members of the community that the message has been reissued. The older } \\
\text { version shall be automatically saved and together with all previous versions } \\
\text { can be kept in a history folder. Date of version and modifier-id should be } \\
\text { accompanying each posting. Furthermore, an automatically generated e-mail, } \\
\text { informing all the potential readers (individuals or groups), of the issuance of a } \\
\text { newer version, needs to be sent as part of the administrative procedure. }\end{array}$} \\
\hline Example(s) & \multicolumn{2}{|c|}{ Show History } \\
\hline & \multicolumn{2}{|c|}{ 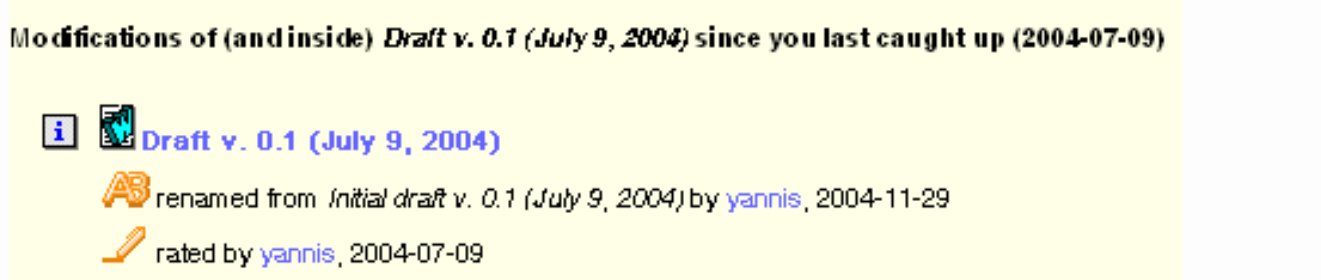 } \\
\hline & \multicolumn{2}{|l|}{ Catch up Cancel } \\
\hline & \multicolumn{2}{|c|}{$\begin{array}{l}\text { In CSCL system BCSW users can collaboratively author a document using a } \\
\text { version control tool. }\end{array}$} \\
\hline
\end{tabular}




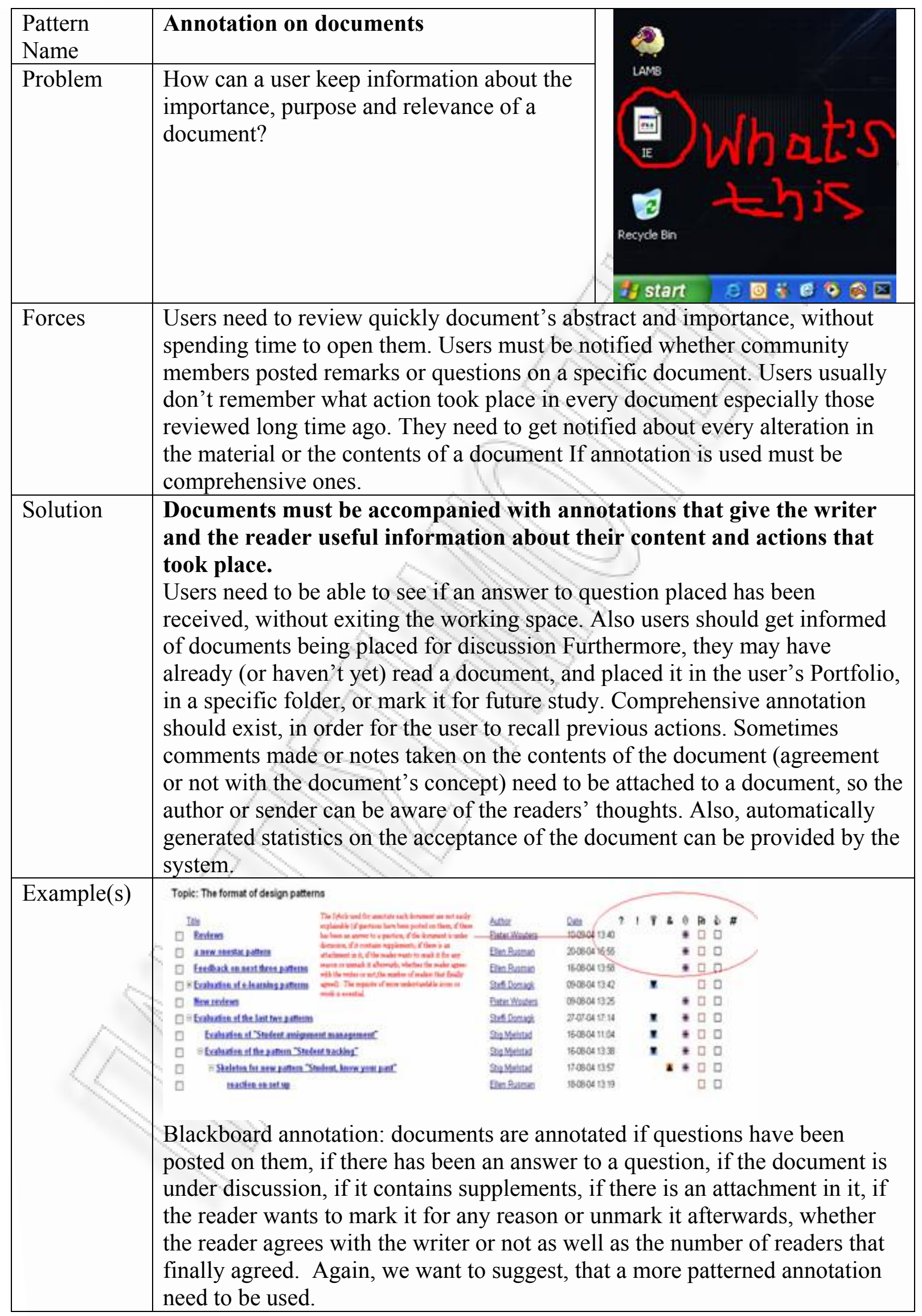




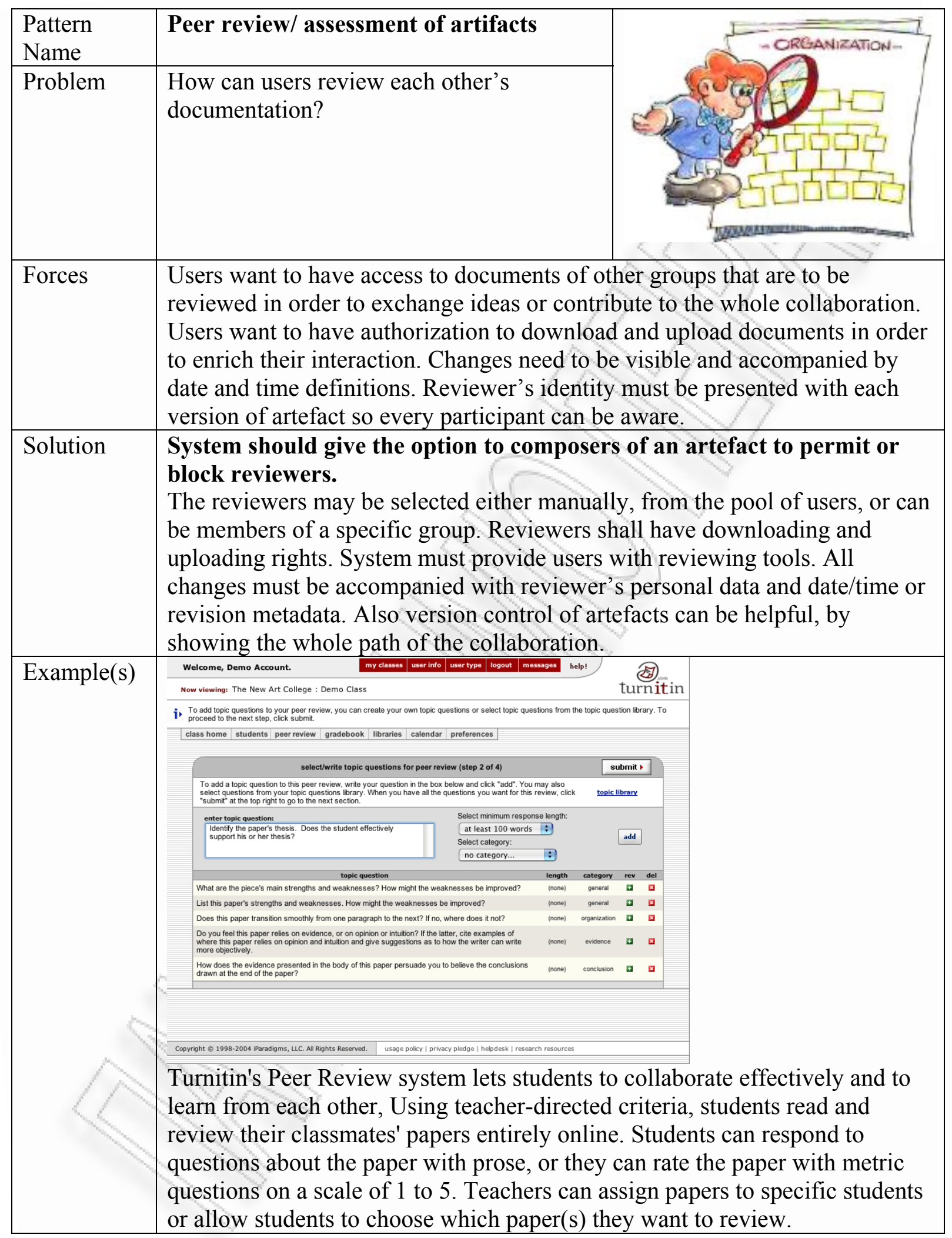




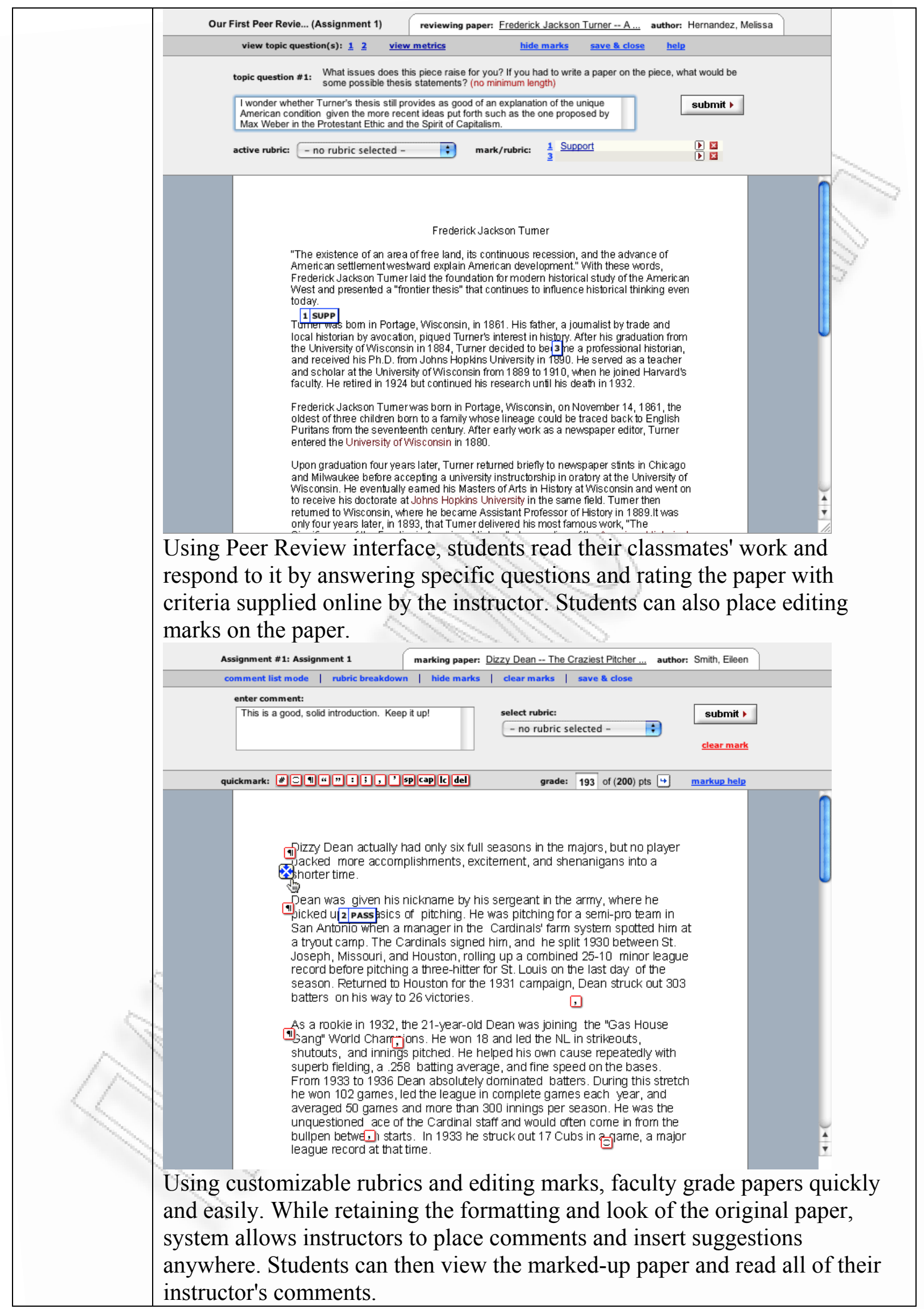




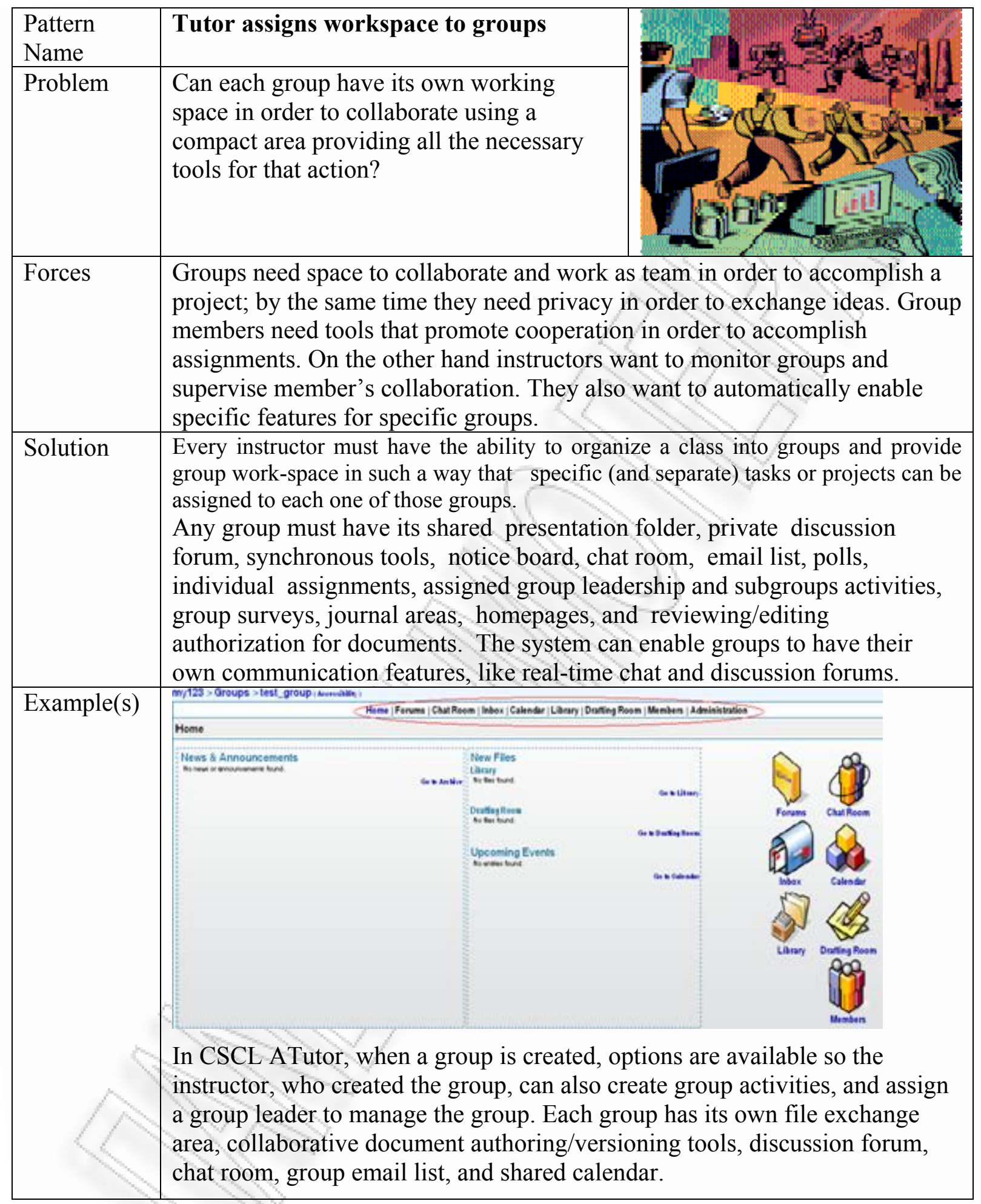




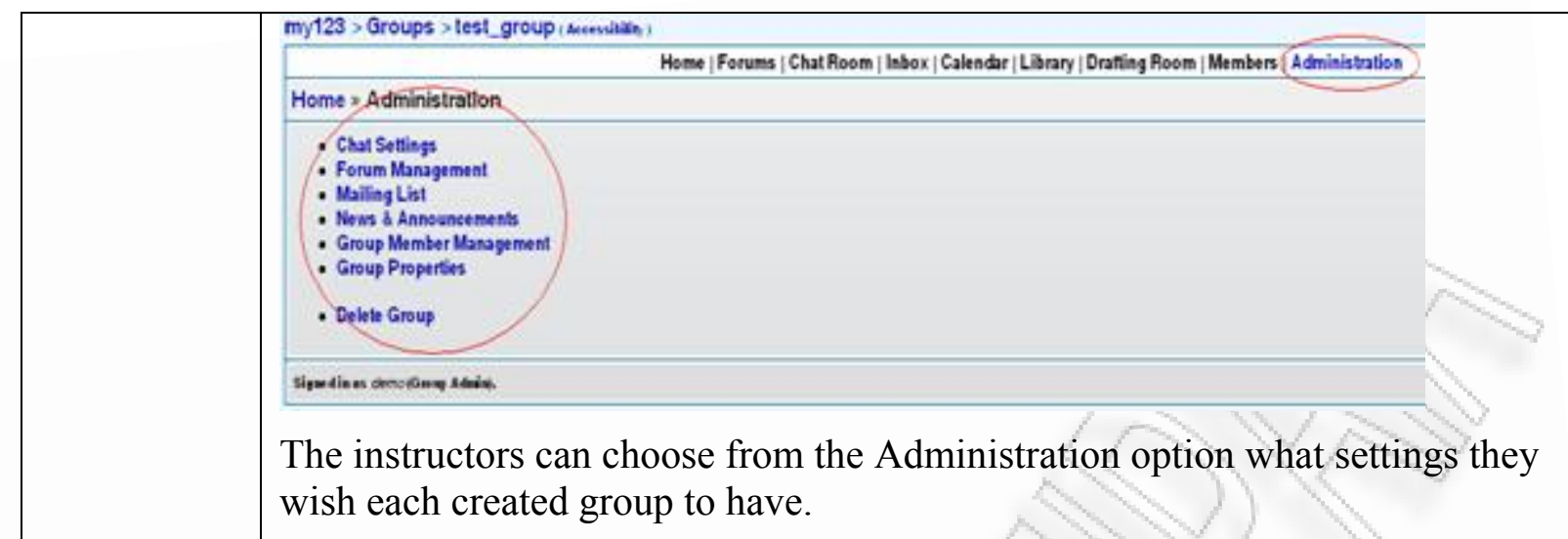
wish each created group to have. 


\begin{tabular}{|c|c|c|}
\hline $\begin{array}{l}\text { Pattern } \\
\text { Name }\end{array}$ & Set learner/group access rights & \\
\hline Problem & $\begin{array}{l}\text { How can tutors predefine a set of access } \\
\text { rights capability to any user group? How } \\
\text { can access violations be treated and } \\
\text { conflicts resolved? }\end{array}$ & $\begin{array}{l}\text { EMERGENCY } \\
\text { PROGRESS }\end{array}$ \\
\hline Forces & \multicolumn{2}{|c|}{$\begin{array}{l}\text { Every individual or group interacting with the system has a specific role, } \\
\text { which needs to be clear and simplified, in order for system management to be } \\
\text { straightforward and the performance of the application optimal. At user level } \\
\text { administrator has to prevent conflicts that are conclusions of access violation. } \\
\text { Every type of participant must be demarcated. Sometimes during the } \\
\text { educational procedure roles can be twofold or can be modified, regarding } \\
\text { tutors educational prospect. }\end{array}$} \\
\hline Solution & \multicolumn{2}{|c|}{$\begin{array}{l}\text { The system administrator or each instructor separately, must be able to } \\
\text { determine the level of interaction between each registered participant } \\
\text { and the system resources. There are different types of users in a } \\
\text { collaborative system, such as tourists, guests, teaching assistants, tutors, } \\
\text { administrators, staff, students, observers, etc. For example the roles of an } \\
\text { instructor and a student, will most probably be different in different courses } \\
\text { and group contexts. Administrators can create an unlimited number of } \\
\text { custom organizational units and roles with specific access privileges to course } \\
\text { content and tools. Administrators and Instructors can assign to students } \\
\text { different levels of access to the system or to specific courses based on pre- } \\
\text { defined roles. They can also delegate administration of the system to different } \\
\text { organizational entities within an institution or consortia. }\end{array}$} \\
\hline \multirow[t]{5}{*}{ Example(s) } & \multicolumn{2}{|c|}{$\begin{array}{l}\text { Unix permissions specify which operations can be performed for any } \\
\text { ownership relation with respect to the file. In Unix to set/modify a file's } \\
\text { permissions you need to use the chmod program. Of course, only the owner } \\
\text { of a file may use chmod to alter a file's permissions. chmod has the following } \\
\text { syntax: chmod [options] mode file(s) or better chmod [-r] permissions } \\
\text { filenames }\end{array}$} \\
\hline & \multicolumn{2}{|c|}{$\begin{array}{l}-r \\
\text { Of the directory that you are currently in. }\end{array}$} \\
\hline & \multicolumn{2}{|c|}{$\begin{array}{ll}\text { permission } & \begin{array}{l}\text { Specifies the rights that are being granted. Below are the } \\
\text { different rights that you can grant in alpha a numeric } \\
\text { format. }\end{array}\end{array}$} \\
\hline & \multicolumn{2}{|c|}{\begin{tabular}{l|l} 
filenames & File or directory that you are associating the rights with.
\end{tabular}} \\
\hline & \multicolumn{2}{|c|}{$\begin{array}{l}\text { Permissions } \\
\mathrm{u} \text { - User who owns the file. } \\
\mathrm{g} \text { - Group that owns the file. } \\
\mathrm{o} \text { - Other. } \\
\mathrm{a} \text { - All. } \\
\mathrm{r} \text { - Read the file. } \\
\mathrm{w} \text { - Write or edit the file. } \\
\text { x - Execute or run the file as a program. } \\
\text { Numeric Permissions: }\end{array}$} \\
\hline
\end{tabular}




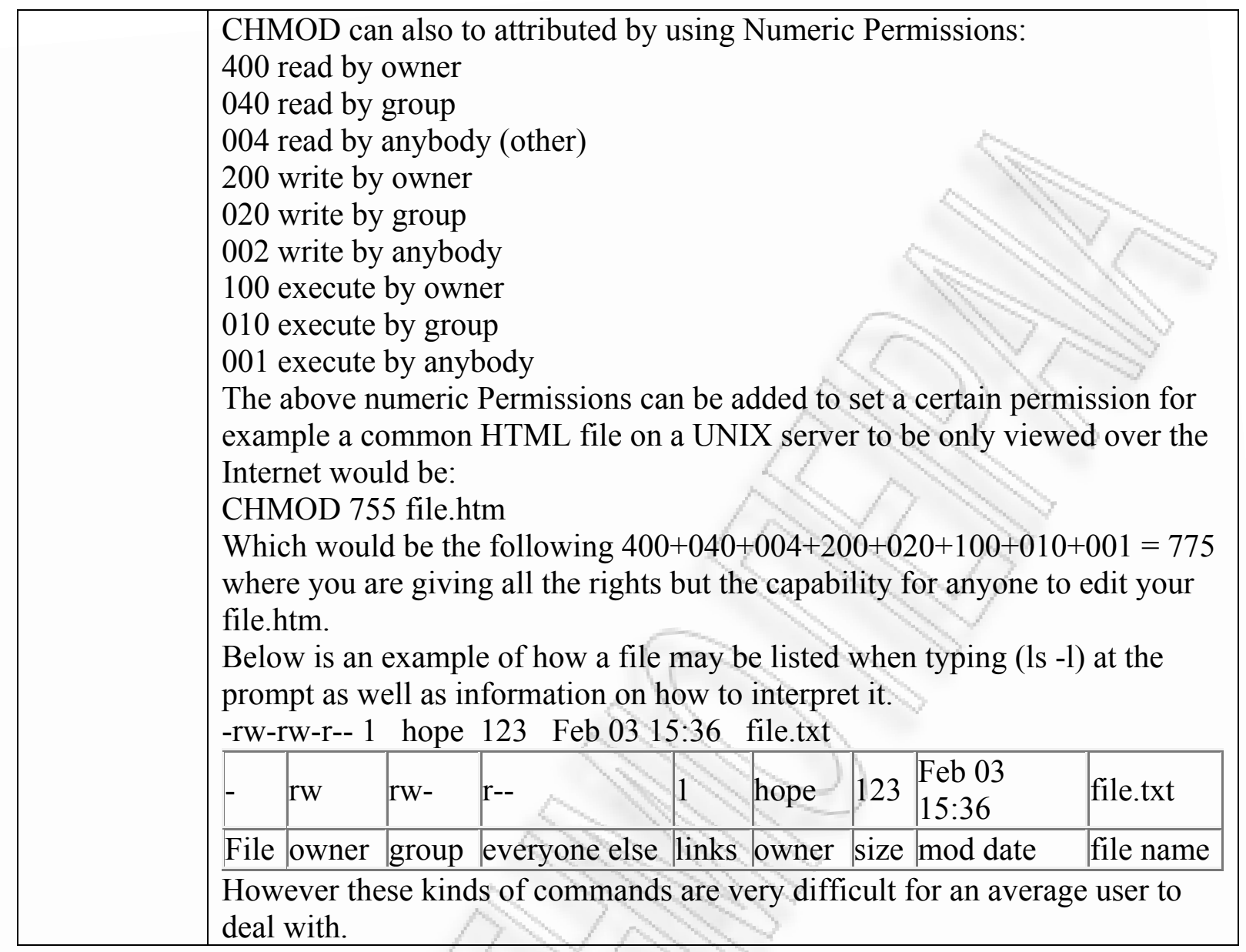


Programma_final_v3 Properties

General Security Custom Summary

무료

programma_final_v3

Type of file:

Microsoft Word Document

Opens with:

VA Microsoft Dffice Word

Change...

Location: C: Documents and Settings'Petros Georgiakakis \M

Size: $169 \mathrm{~KB}[173.056$ bytes]

Size on disk: $172 \mathrm{~KB}[176.128$ bytes]

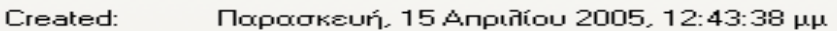

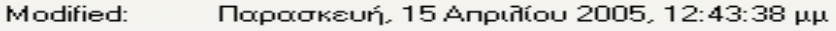

Accessed:__Texóptn, 29 louviou 2005, 1:04:45 $\mu \mu$

Attributes: $\square$ Read-only $\square$ Hidden $\quad$ Advanced...

\section{Advanced Attributes}

EIt Choose the options you want for this file.

Archive and Index attributes

$\checkmark$ File is ready for archiving

For fast searching, allow Indexing Service to index this file

Compress or Encrypt attributes

$\square$ Compress contents to save disk space

$\square$ Encrypt contents to secure data

MS Windows give to authors the option to apply advanced attributes on specific documents 
Design patterns for Awareness

\begin{tabular}{|l|l|l|}
\hline Pattern Name & Awareness \\
\hline Problem & $\begin{array}{l}\text { Can the understanding of the } \\
\text { activities of the other group } \\
\text { participants provide context for } \\
\text { users' own activity? }\end{array}$ \\
\hline Forces & $\begin{array}{l}\text { Colleagues want to be aware of the social connections within the } \\
\text { group or team, to be aware of the other learners' interactions with } \\
\text { the workspace and also to be aware about how to complete the } \\
\text { common task. }\end{array}$ \\
\hline Solution & $\begin{array}{l}\text { The representation of participants' identities (PERSONAL } \\
\text { confIGURATION), status, tasks (POSTING NOTIFICATION), progress and } \\
\text { presence (GETTING FAMILIAR wITH COLLEAGUES) is an important } \\
\text { functionality in ANSCL systems. }\end{array}$ \\
\hline Related Patterns & $\begin{array}{l}\text { PERSONAL CONFIGURATION, POSTING NOTIFICATION, GETTING FAMILIAR } \\
\text { WITH COLLEAGUES }\end{array}$ \\
\hline
\end{tabular}




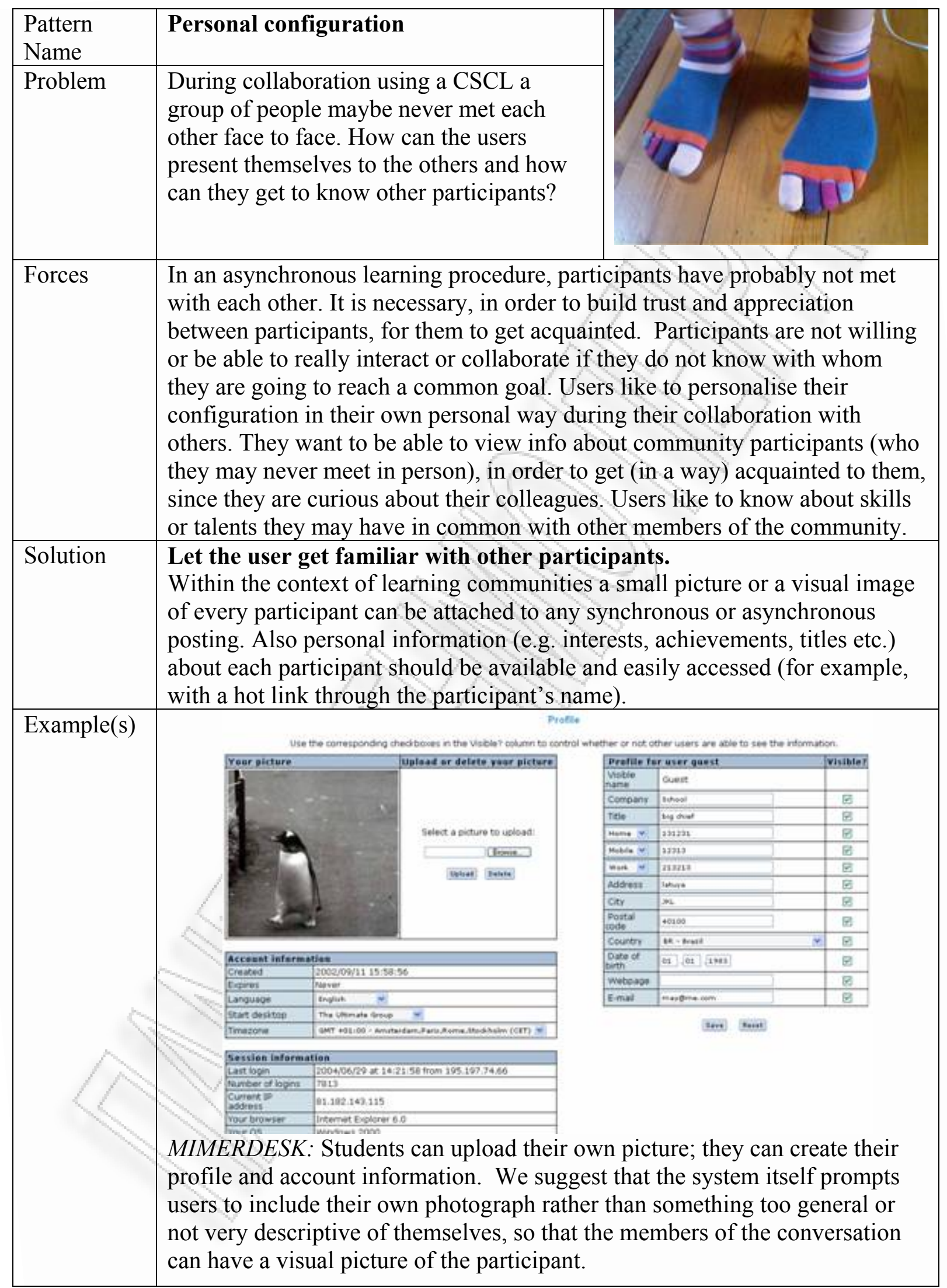




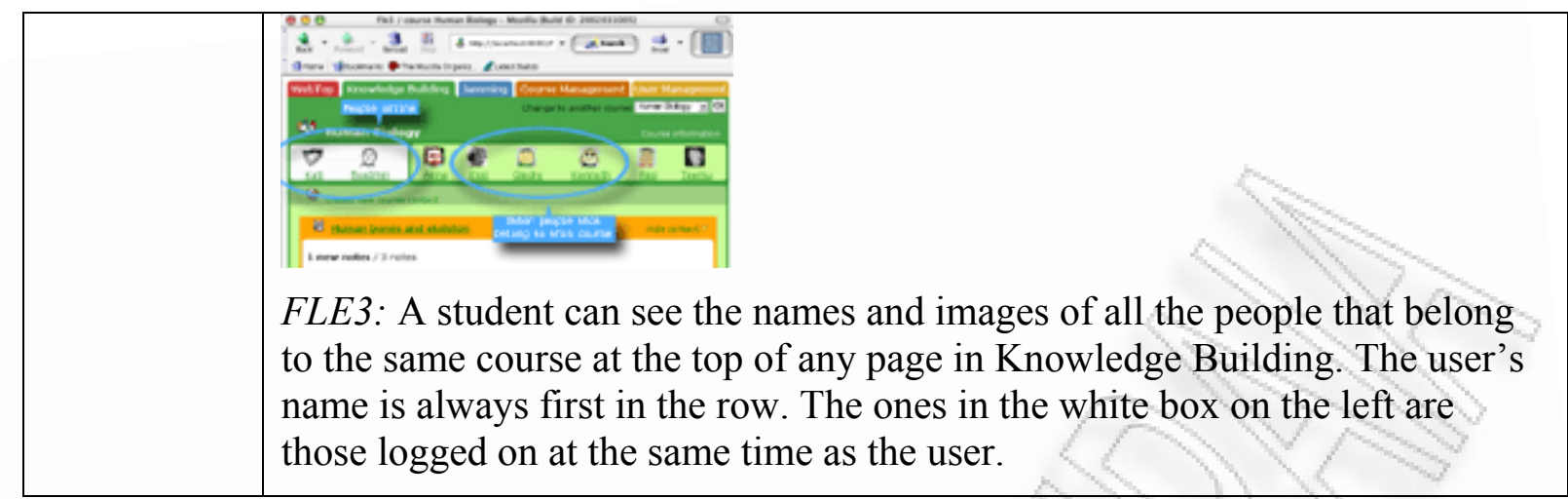




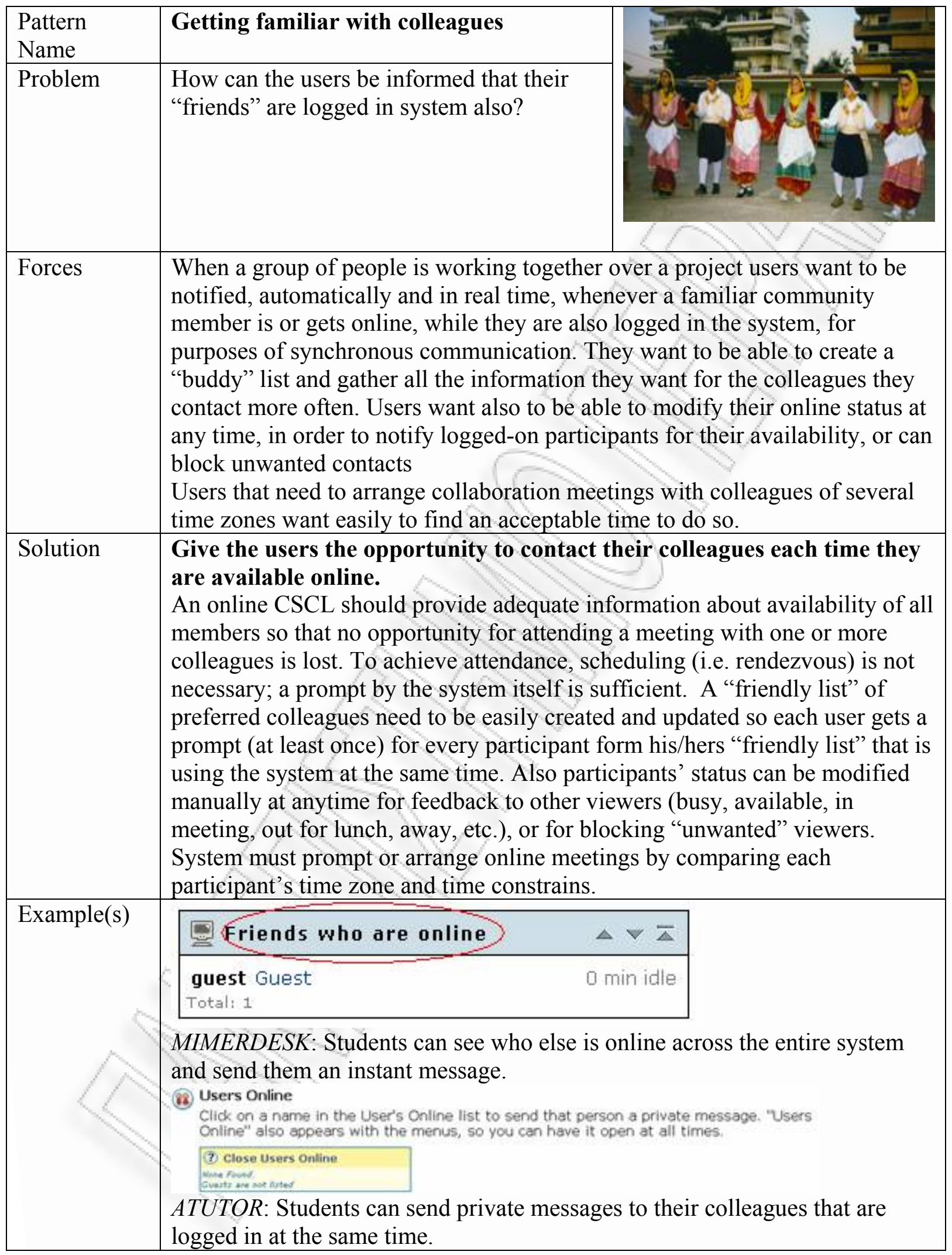




\begin{tabular}{|c|c|}
\hline $\begin{array}{l}\text { Pattern } \\
\text { Name }\end{array}$ & Posting notification \\
\hline Problem & $\begin{array}{l}\text { Instead of the users looking for news, can } \\
\text { the latest news reach those interested? }\end{array}$ \\
\hline Forces & $\begin{array}{l}\text { Keeping the educated aware for what happens in an asynchronous discussion } \\
\text { is very important in order to increase its effectiveness. Tutors and learners } \\
\text { don't want to waste time searching through the CSCL every day for new } \\
\text { postings. If it is possible they want to be notified, every time a new posting is } \\
\text { added (One step mail reading is). Users must be kept aware of proceedings } \\
\text { and outcomes of asynchronous discussions in their area of interest. Users } \\
\text { should be informed when a teacher adds files with important material for the } \\
\text { learners. Users sometimes need to block some community members so they } \\
\text { won't receive specific notification from them. }\end{array}$ \\
\hline Solution & $\begin{array}{l}\text { Awareness of activity increases the quality of asynchronous } \\
\text { collaboration. } \\
\text { Automatic briefing, for all kinds of newly placed statements, via notification } \\
\text { of their choice (email, or SMS), can give the participants the opportunity to } \\
\text { be notified about all the events that took or will take place, without them } \\
\text { having to spend any time visiting the system. Also the choice of briefing or } \\
\text { no briefing, according to subject, sender, or time period, should be an option } \\
\text { (in the case the participant doesn't want to get this kind of briefing). }\end{array}$ \\
\hline Example(s) & 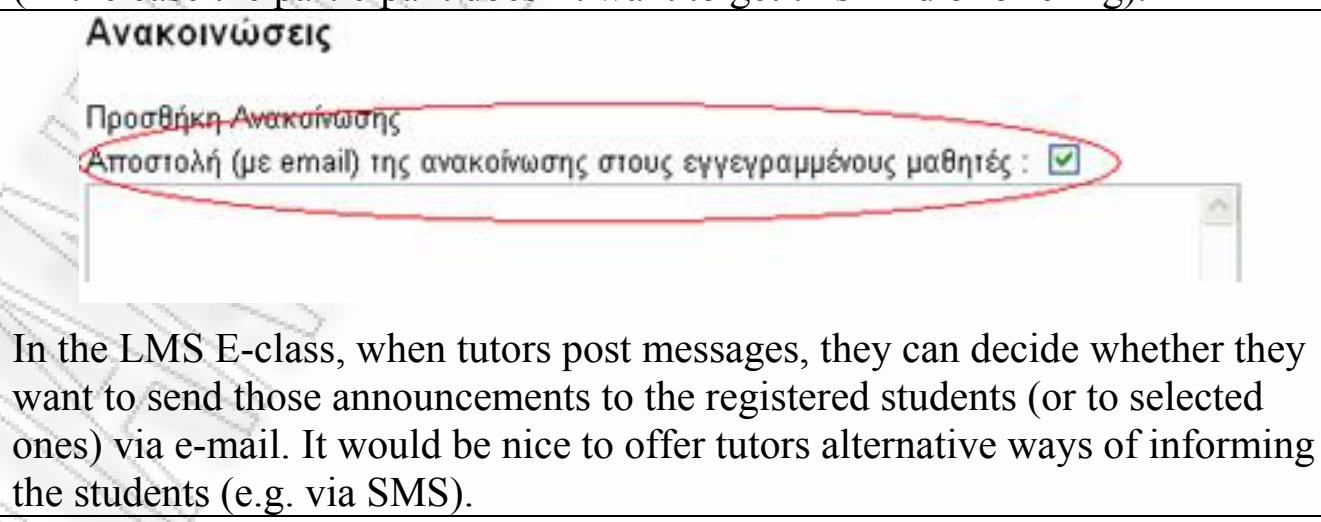 \\
\hline
\end{tabular}




\section{Design patterns for Evaluation}

\begin{tabular}{|c|c|c|}
\hline Pattern Name & Evaluation & \\
\hline Problem & $\begin{array}{l}\text { Monitoring the process of } \\
\text { collaborative learning is an open } \\
\text { and difficult issue. } \\
\text { How can tutors be informed that } \\
\text { learners are working as a team } \\
\text { in the various types of } \\
\text { collaborative tasks (shared } \\
\text { problem solving, problem } \\
\text { discussion, group forming, etc) } \\
\text { or how can they identify lurkers } \\
\text { or learners that dominate the } \\
\text { discussion? }\end{array}$ & cesdesign_sericess services_evaluation.htm! \\
\hline Forces & \multicolumn{2}{|c|}{$\begin{array}{l}\text { The analysis of collaborative activity in shared collaborative } \\
\text { learning spaces is based on the idea of observing and recognizing } \\
\text { user actions and indicating specific patterns of activity and } \\
\text { interaction. The scope of this analysis to feed this information } \\
\text { either to the teacher in order to keep track of the level of } \\
\text { interaction thus helping identifying lurkers, dominant learners, } \\
\text { etc., or back to the learners by making them more ware of the } \\
\text { trends over time. Interaction analysis can also be used for } \\
\text { revealing learners behaviour associated with collaborative learning } \\
\text { tasks thus helping the assessment of the individual participation. } \\
\text { Visualization techniques for highlighting trends over time and } \\
\text { average, showing the frequency of interactions among the learners } \\
\text { or among members of groups can be a valuable tool for the teacher } \\
\text { as well as the course evaluator who will see whether specific } \\
\text { collaborative techniques have been applied by the learners. }\end{array}$} \\
\hline Solution & \multicolumn{2}{|c|}{$\begin{array}{l}\text { In general, interaction data concern the number of the messages } \\
\text { read, the postings to a discussion board, the file up-loads, the } \\
\text { annotations to the uploaded files, etc.(GENERATE STATISTICS). In } \\
\text { addition, analysis of participants' postings via content analysis } \\
\text { techniques can enlighten the evaluators about learners' behaviour, } \\
\text { outcomes and experiences (MAKE QUERIES). Also log files can be } \\
\text { captured, decoded and analyzed for providing statistics of } \\
\text { interaction as well as activity patterns. }\end{array}$} \\
\hline & \multicolumn{2}{|l|}{ GENERATE STATISTICS, MAKE QUERIES } \\
\hline
\end{tabular}




\begin{tabular}{|c|c|c|}
\hline $\begin{array}{l}\text { Pattern } \\
\text { Name }\end{array}$ & Generate course usage statistics & \\
\hline Problem & $\begin{array}{l}\text { How can tutors get feedback from the } \\
\text { system regarding successful or } \\
\text { unsuccessful usage of course materials and } \\
\text { resources? How can they be sure what they } \\
\text { provide sufficient or proper material? }\end{array}$ & \\
\hline Forces & \multicolumn{2}{|c|}{$\begin{array}{l}\text { Instructors offer resources to learners that they consider necessary for the } \\
\text { latter to accomplish the collaborative learning tasks. However, they would } \\
\text { like to monitor whether learner frequently accessed the recourses how much } \\
\text { time they spent on reading them. They need to know if students collaborated } \\
\text { for the joint development of deliverables, and which students had been more } \\
\text { active in modifying the deliverables before their submission. By collecting } \\
\text { statistics and visual data, tutors can check which learners had been more } \\
\text { active, which recourses need to be improved, which collaboration patterns } \\
\text { occurred during the collaborative learning process (either in groups or in } \\
\text { plenary sessions). Of course statistics cannot show the full image of the } \\
\text { interaction. It is highly probably that a learner might create the first draft and } \\
\text { then sit back and watch its evolution by the fellow learners via several rounds } \\
\text { of interaction. In this case, the statistics will show the first learner was less } \\
\text { active than the rest. In fact, creating a good first draft is sometimes very } \\
\text { important. Thus, the teacher/evaluator should not entirely depend on the } \\
\text { statistics or assign weights to the various parameters of the interaction } \\
\text { process. The same can happen when a learner posts few but very accurate and } \\
\text { thorough messages about a discussion topic. Perhaps other learner might be } \\
\text { more active in the discussion space. Their participation, however, might not } \\
\text { trigger further exchange of ideas. Thus, the evaluator should correlate the } \\
\text { findings from statistical analysis of the interaction with content analysis. This } \\
\text { is not always feasible and easy to be made. }\end{array}$} \\
\hline Solution & $\begin{array}{l}\text { The system must have tools for creation o } \\
\text { need to offer descriptive statistics about the } \\
\text { page accesses, time of day, date and frequen } \\
\text { individually or all students together as a gro } \\
\text { course units, discussion forums and assignm } \\
\text { to get reports in tabular or chart formats sho } \\
\text { each student on course content, specific cour } \\
\text { forums. Instructors must be able to set a flag } \\
\text { to track the frequency of student access on th } \\
\text { summarize all discussion postings to date by } \\
\text { can share this kind of tracking information }\end{array}$ & $\begin{array}{l}\text { statistic reports. These reports } \\
\text { nteraction and handle number of } \\
\text { cy of access by each student } \\
\text { ap in relation to a specific course, } \\
\text { ents. Instructors must also be able } \\
\text { ving the amount of time spent by } \\
\text { se units and/or discussion } \\
\text { on individual course components } \\
\text { ose components. Instructors can } \\
\text { group or by student. Instructors } \\
\text { ith students. }\end{array}$ \\
\hline
\end{tabular}




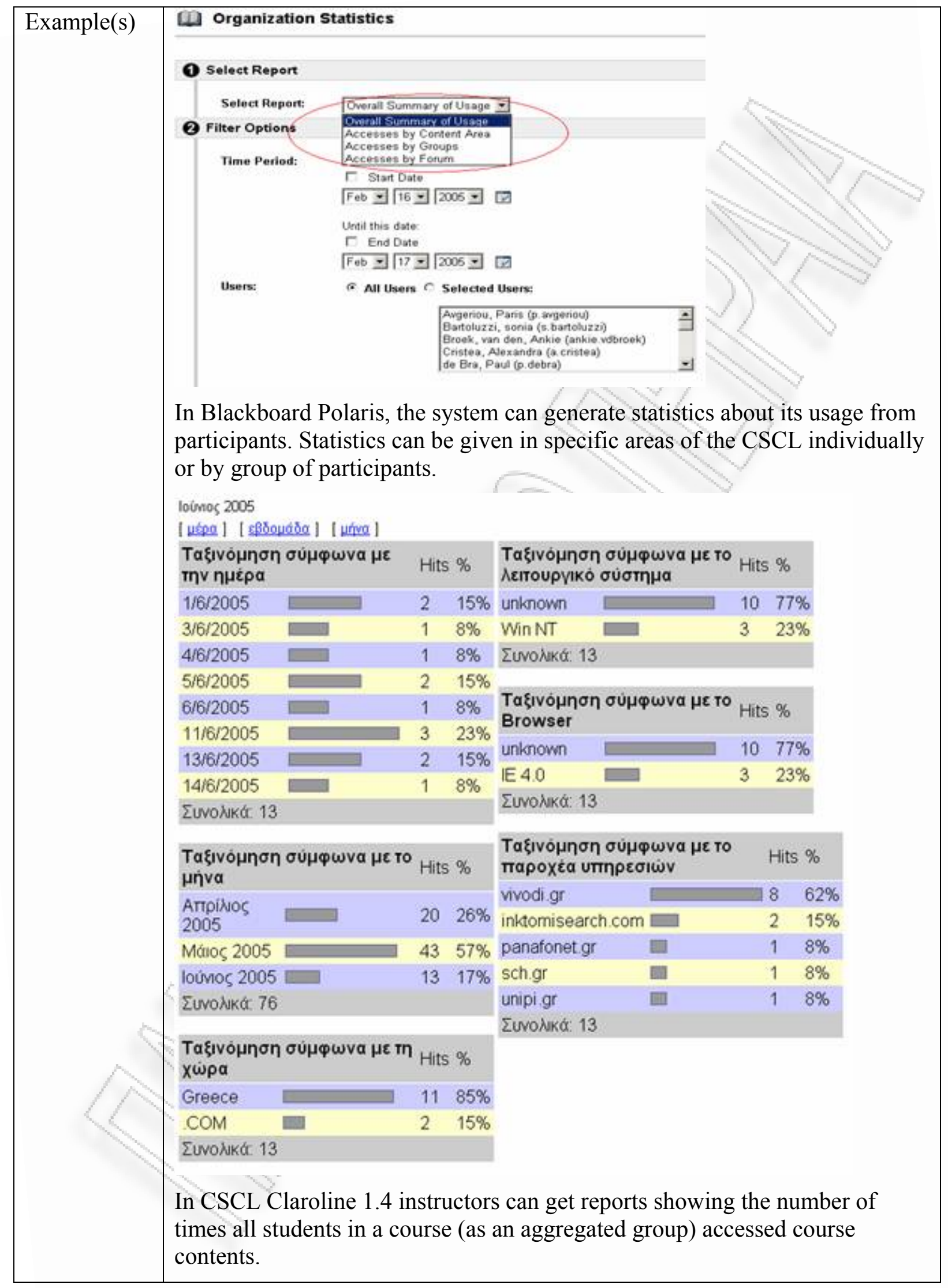




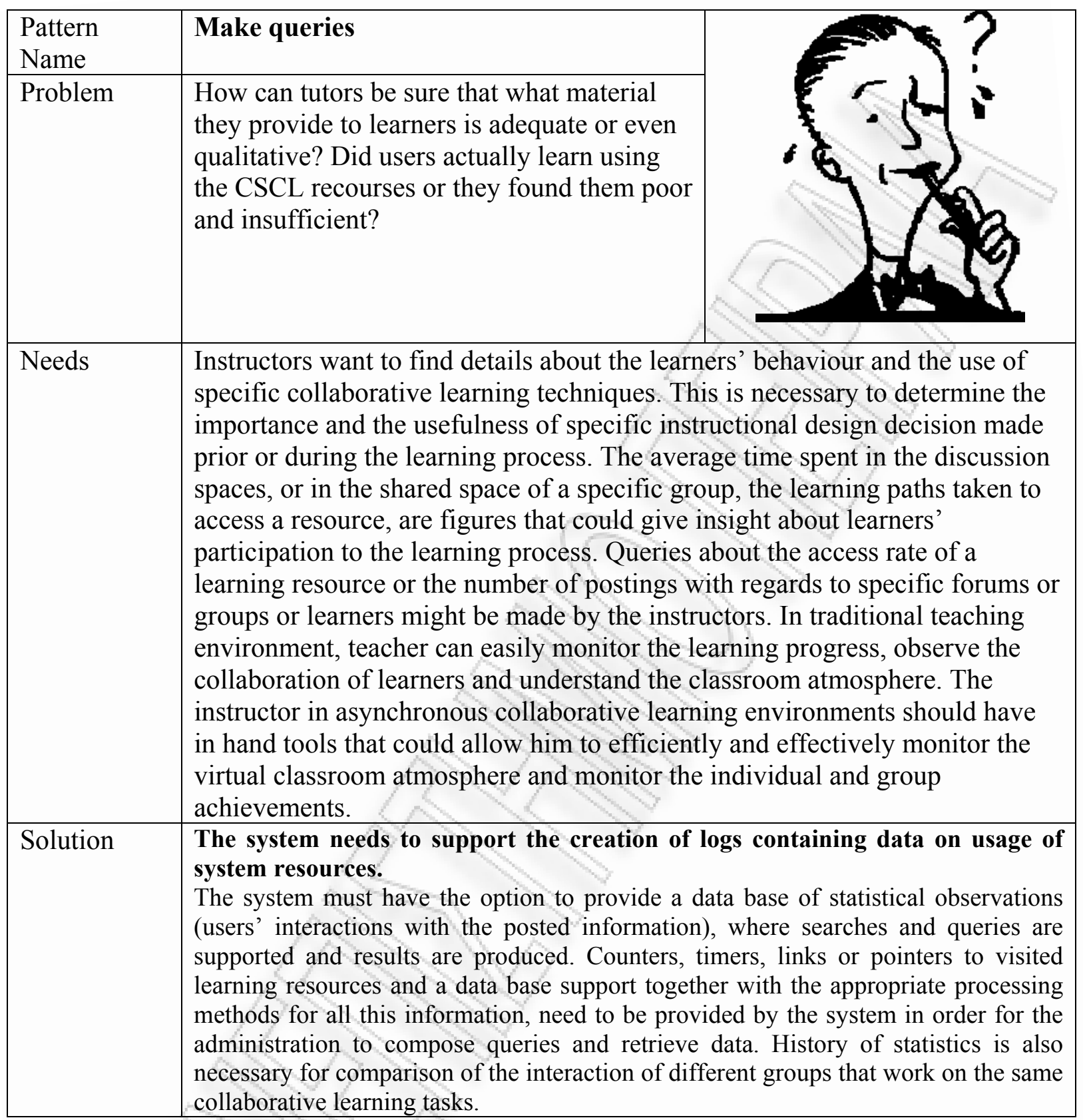




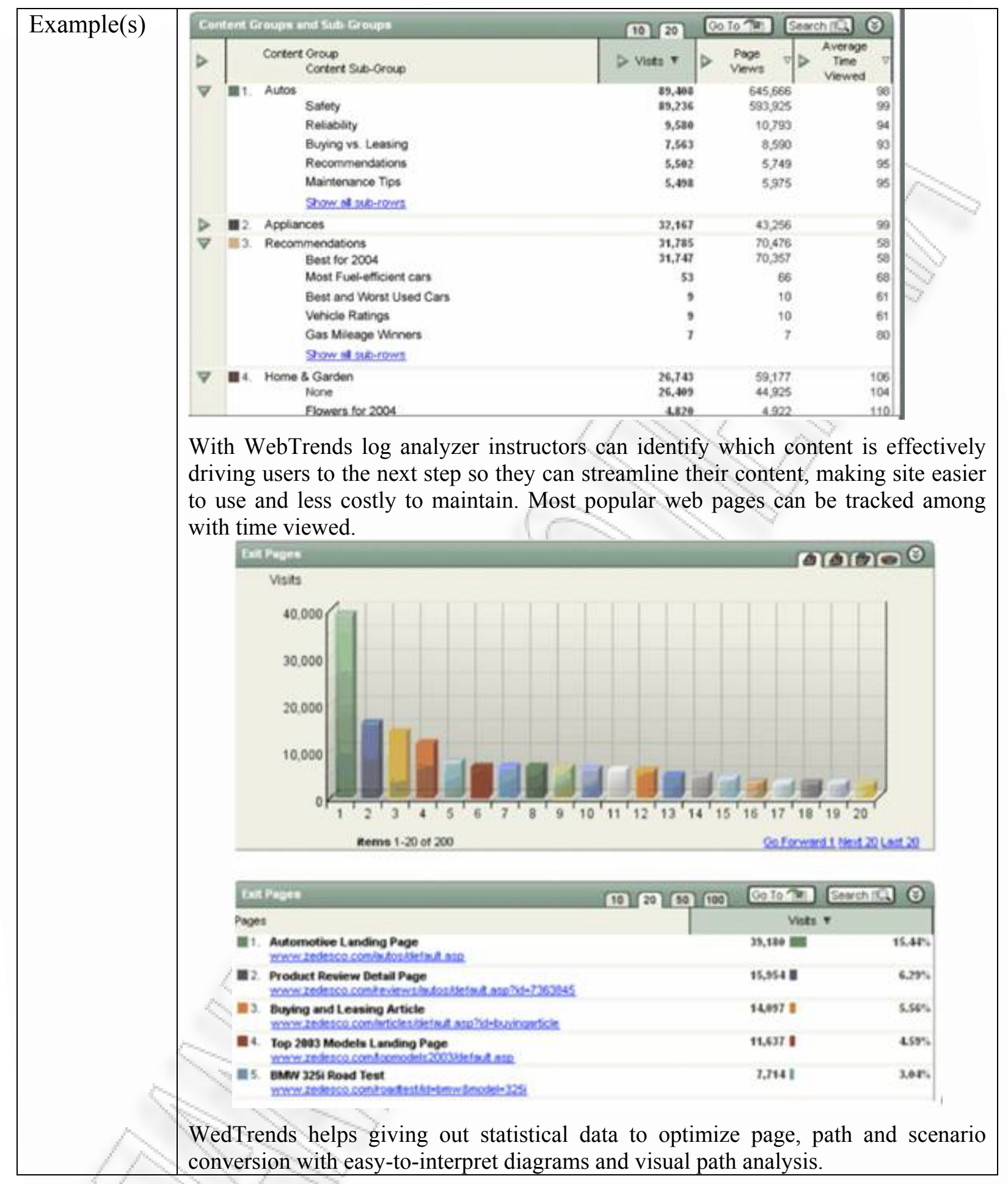




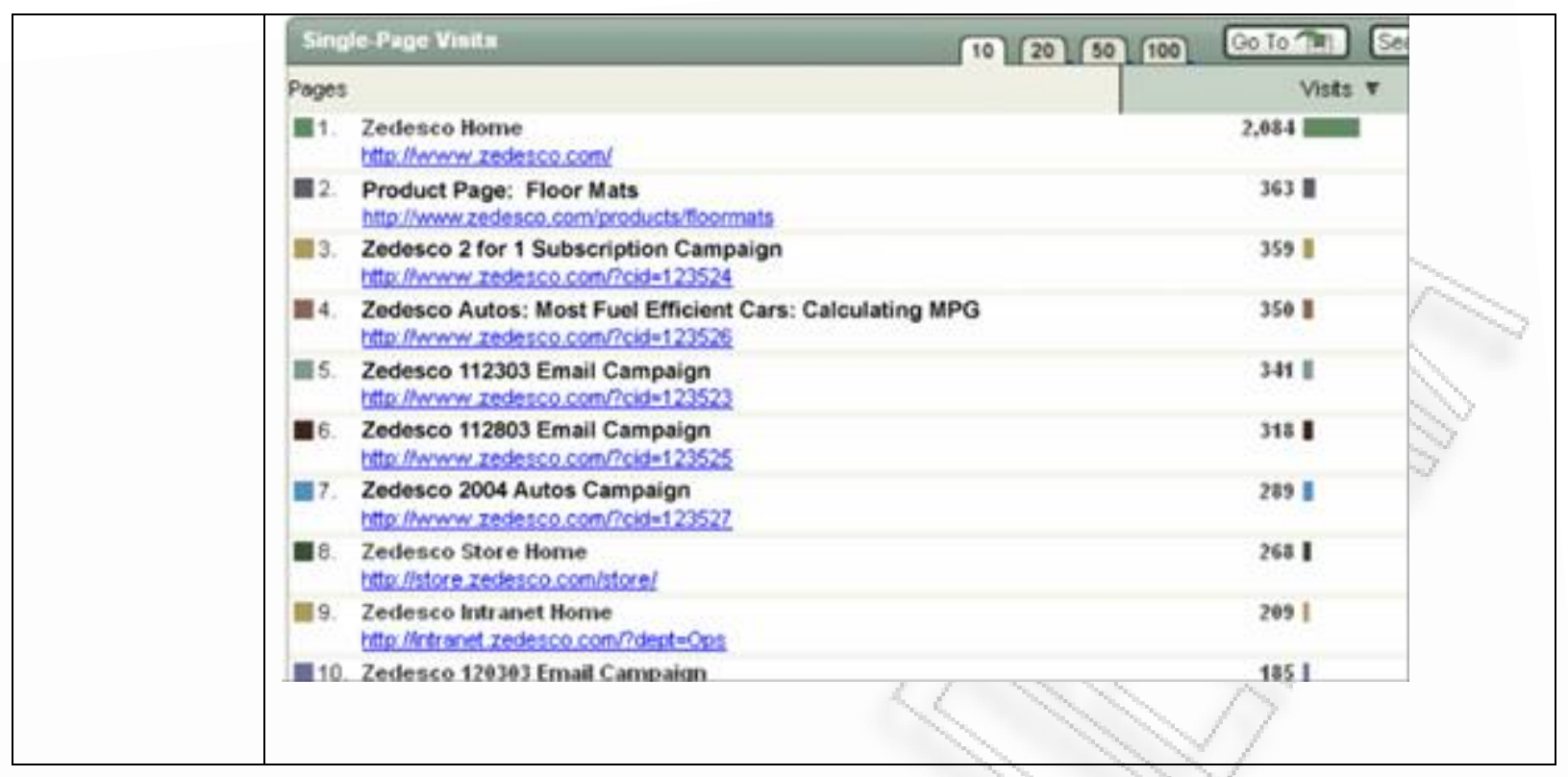




\section{Design Patterns for Group management}

\begin{tabular}{|c|c|c|}
\hline Pattern Name & Group management & \\
\hline Problem & $\begin{array}{l}\text { How can a well functioning group } \\
\text { for collaborative learning in an } \\
\text { educational context be successfully } \\
\text { managed? }\end{array}$ & $8 \times 2-2$ \\
\hline Forces & \multicolumn{2}{|c|}{$\begin{array}{l}\text { In order to make collaborative learning as constructive as possible, } \\
\text { students should work in groups, either formed by them or by a tutor. } \\
\text { Decisions of various short should be made: what sort of group to be form } \\
\text { (informal, formal or base, heterogeneous or homogenous), the group } \\
\text { size, etc. Aspects like these have crucial results on the successfulness of } \\
\text { each group, the building of trust, the mode of interaction, and the } \\
\text { effectiveness of the whole group or project based collaboration process. }\end{array}$} \\
\hline Solution & \multicolumn{2}{|c|}{$\begin{array}{l}\text { Asynchronous CSCL systems often support collaboration in groups } \\
\text { where students work on a common project. The project artefacts are } \\
\text { managed in particular "spaces for collaborative work" (GROUP } \\
\text { MANAGEMENT). Group management has to deal with the successful } \\
\text { management of student teams related to collaborative projects (ASSOCIATE } \\
\text { FORUM TO GROUP). Thus, group management is related to collaborative and } \\
\text { project-based learning. Modifications on group members and roles need } \\
\text { to be done (MODIFY GROUP MEMBERS AND ROLES) in order to emphasise on } \\
\text { collaborative aspects of learning as well as individual ones, an } \\
\text { identification of social interactions as an important element of } \\
\text { knowledge construction, a focus on the learner(s) and their activities. }\end{array}$} \\
\hline Related Patterns & \multicolumn{2}{|c|}{$\begin{array}{l}\text { GROUP MANAGEMENT, ASSOCIATE FORUM TO GROUP, MODIFY GROUP MEMBERS AND } \\
\text { ROLES }\end{array}$} \\
\hline
\end{tabular}




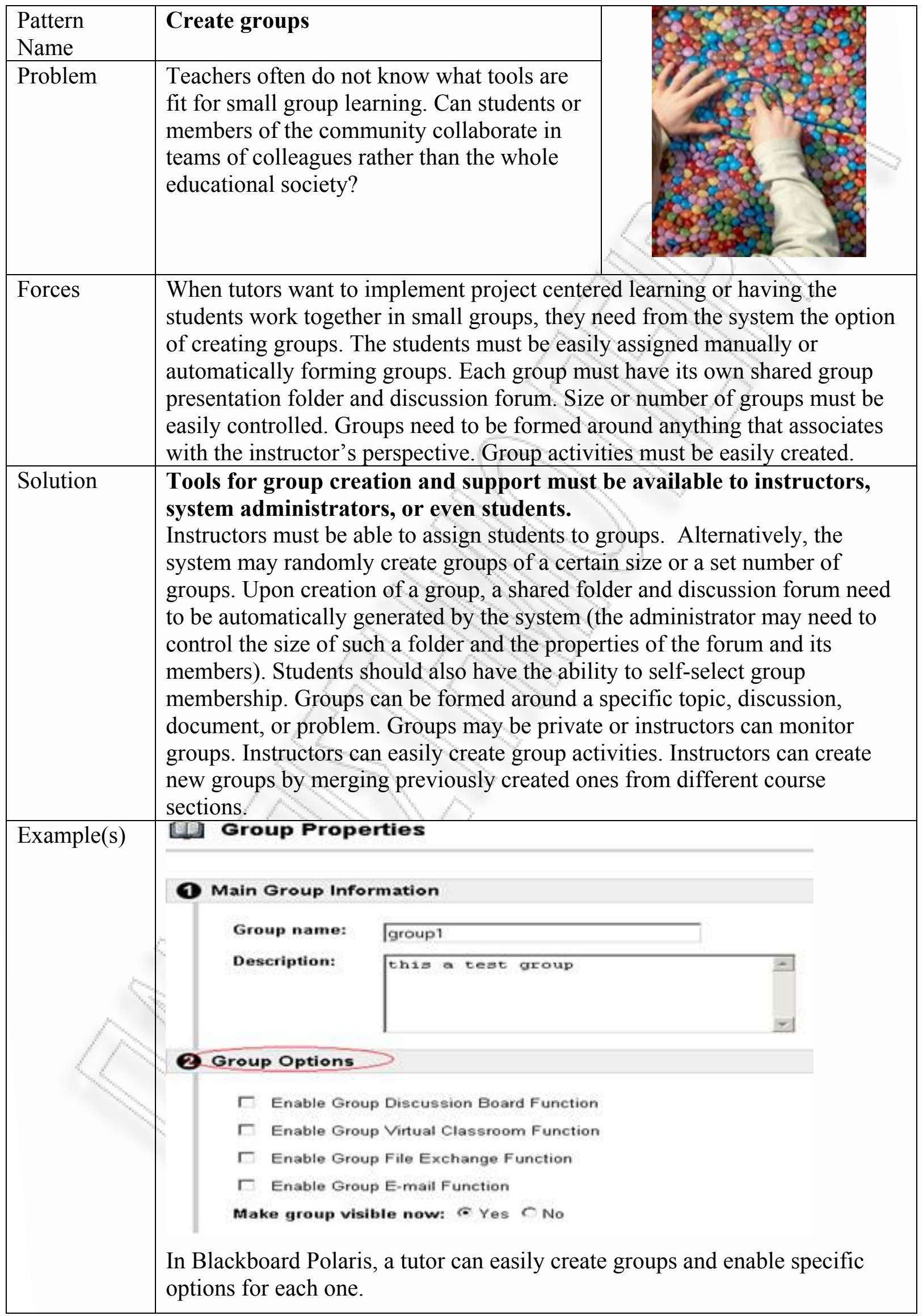




\begin{tabular}{|c|c|c|}
\hline $\begin{array}{l}\text { Pattern } \\
\text { Name }\end{array}$ & Modify group members and roles & \\
\hline Problem & $\begin{array}{l}\text { How can tutors support changes within a } \\
\text { group? Are the groups flexible or new ones } \\
\text { must be created every time a change occurs? } \\
\text { Can tutors modify group member role? }\end{array}$ & ? \\
\hline Forces & \multicolumn{2}{|c|}{$\begin{array}{l}\text { When tutors create several groups they want to assign group leaders in order } \\
\text { to minimise their interference in learners' collaboration. Several times } \\
\text { instructors need to modify roles of group members and giye privileges to or } \\
\text { take from specific users. }\end{array}$} \\
\hline Solution & \multicolumn{2}{|c|}{$\begin{array}{l}\text { Instructors need to be able to supervise membership and activity in } \\
\text { groups. } \\
\text { They must be able to assign students to groups of a certain size, assign group } \\
\text { leaders, create and manage groups. Groups can either be defined at the course } \\
\text { level and apply across all the activities that support those, or at the individual } \\
\text { activity level. }\end{array}$} \\
\hline Example(s) & \multicolumn{2}{|l|}{$\begin{array}{l}\text { Forum User Settings: } \\
\qquad \begin{aligned} 2 & =\text { Forum Administrator privileges } \\
a & =\text { Permanent Forum Administrator } \\
b & =\text { User is blocked from posting }\end{aligned}\end{array}$} \\
\hline & \begin{tabular}{||l||} 
Avgeriou, Paris (p.avgeriou) \\
Bartoluzzi, sonia (s.bartoluzzi) \\
A. Broek, van den, Ankie (ankie.vdbroek) \\
Cristea, Alexandra (a.cristea) \\
de Bra, Paul (p. debra) \\
Domagk, Steffi (s.domagk) \\
Georgiakakis, Petros (geopet) \\
Horgen, Svend Andreas (sa.horgen) \\
Koehorst, Andre (a.koehorst) \\
Lutgens, Gaby (g.lutgens) \\
Niegemann, Helmut (h. niegemann) \\
Papanikolaou, Kyparisia (s.papanikolaou) \\
\end{tabular} & $\begin{array}{l}\text { ADMIN } \\
\text { NORMAL } \\
\text { BLOCK UNBLOCK }\end{array}$ \\
\hline & \multicolumn{2}{|c|}{$\begin{array}{l}\text { In Blackboard Polaris administrator or leader of the course can modify group } \\
\text { members and their roles simply by marking them from all the available users } \\
\text { choosing to give or deprive rights to them by clicking to the corresponding } \\
\text { box. }\end{array}$} \\
\hline
\end{tabular}




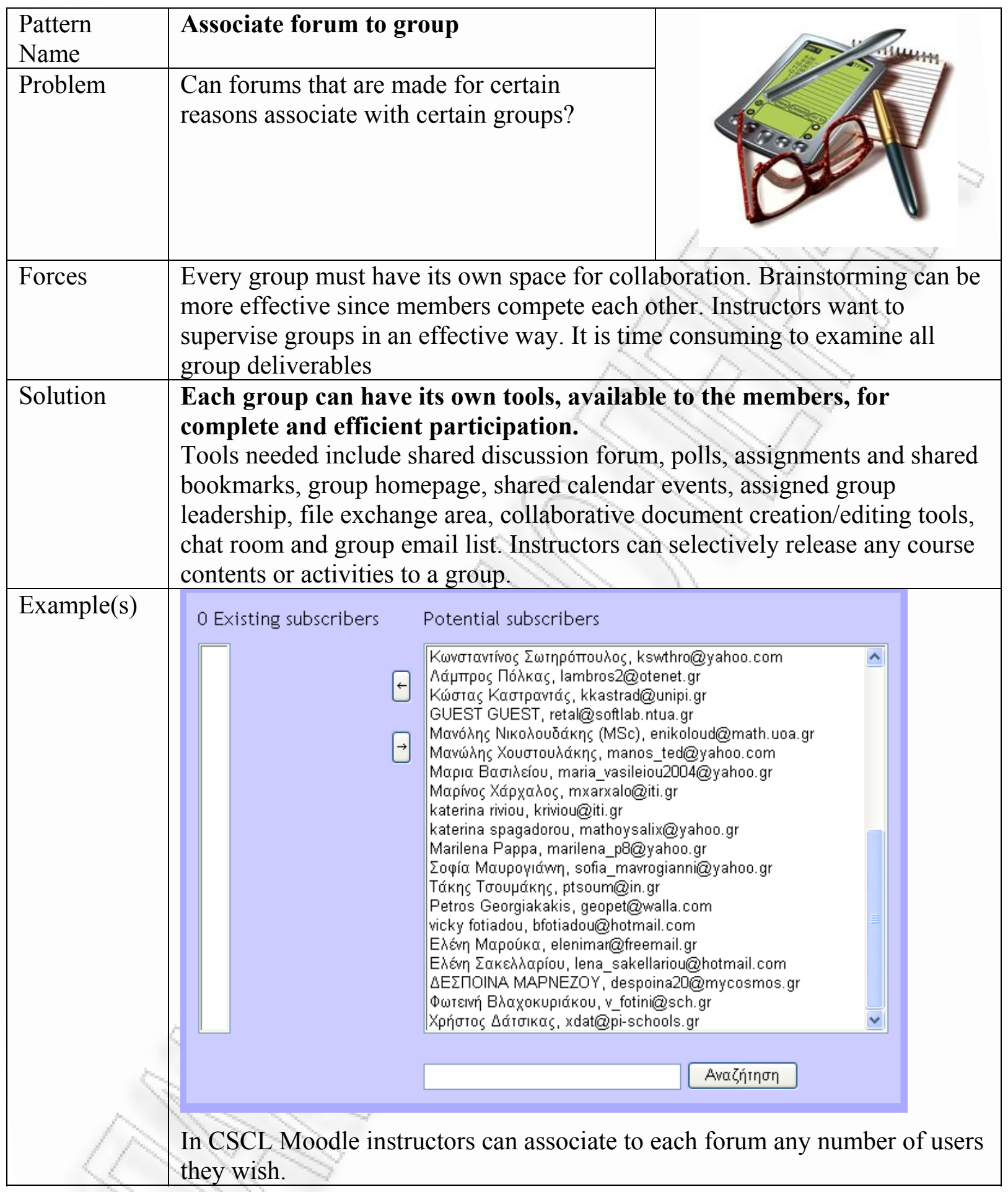




\section{ПАРАРТНМА В}

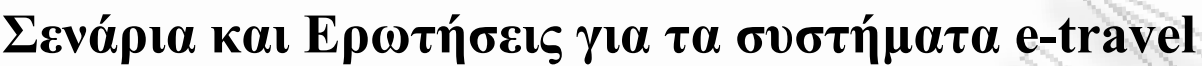

\section{(S1)Description:}

Task:

Questions:

\section{Booking}

You are looking for the price of a ticket in association with date and time factors. Determine the item you wish to book or buy among probably some other options given. Select town or even airport you wish to leave from and going to. If you don't know the names or the abbreviations find out from information given from the site. Choose the departing date, the time, the booking class, and the people traveling with you. Because you are looking for the best available price and you don't mind to be precise to the dates you've given choose these dates to be flexible but near to what you want. Submit tour choices.

- Have you easily found the searching area where you can place your options?

- In the area where you can choose the departing and the arriving airport or town did you had to write down to the form the name or the abbreviation or you could select from a list given?

- If you should write down was there any help to find out the correct abbreviation or spelling?

- Were there any error messages in case something wrong has been typed?

- In the departing and returning date forms was there the help of a calendar or you should type yourself the exact date?

- Could you select flexible dates for example \pm 3 days from what you've chosen?

- When you select the persons traveling was it obvious what they meant with the term Adult or Senior, or Children?

- Could you search for a one way trip if you wised?

- When you submitted your choices and a search in their databases was performed were you informed that some action was taken place?

- Did some results come out?

(S2)Description: Double Tab Navigation

Task:

Travel sites are not only offering tickets but also a large number of conveniences for people traveling. Suppose you are interested for something more than a ticket. Try to find the page that you can rent a car. Try to find a page that you can personalize the site according to your wishes. Try to find hotels in the area near the airport you are landing or near a specific address.

Questions: - Was it obvious that Travel site was offering more than tickets?

- Could you see all these groups of information?

- By selecting one of those the others disappeared? 
- Were the colors used indicate where you are or the use of them was rather than helpful?

- If your choice had second level option could they easily visualized?

- Was it fast to jump to another category if wished?

\section{(S3)Description: Shortcut Box}

Task:

Questions:

\section{(S4)Description: Language Selector}

Task:

Try to change the site language.

Questions:

- Can you find the list of the different languages provided by the site?

- In what way they are presented (by names, by flags)?

\section{(S5)Description: Newsletter}

Task:

Try to register in the Travel Site for receiving a newsletter from it. It the place where you can register you should also have to possibility to unsubscribe. Signing up is done in a 'signup-box' that consists of a field to enter the email address and a button labeled 'signup'. In the same place there should be an indication of how to unsubscribed or manage the subscription (for changing the mail address or delivery options).

Questions:

- Was it easy to find the registration area?

- Was the unsubscribe area obvious?

- Is the user informed of when delivery takes place?

- Was there a clear distinction between the newsletter and the site (if there is one) so that it doesn't look like you get a "site" in the mail?

- Were there options about personalizing the updating content of the newsletter?

- Is the newsletter site why and from whom you received it and make it clear how to unsubscribe from it (optionally how to change settings)?

(S6)Description: Purchase Process

Task:

You have finally decided which ticket suites you and you want to buy it. You are guided to the purchase page where your personal data must be given. If you have already registered then give your user name and password. If not fill in the forms and create an account. Then select the way of purchase and before the last click abort the checkout procedure.

Questions:
- Did the system actually guided you for checking out or did you have to find it yourself?

- Could you abort the procedure at any step?

- When you try to check out again later did you started from the beginning?

- Where the numbers of web pages used a lot or just one or two?

- The page layout was simple or you've been distracted? 
- When you created your account was it simple and in small steps?

- If you were registered and after login did your personal data automatically generated or you had to fill them again in the forms?

- In order to purchase did the system oblige you to make an account in advance?

- Did the system informed you that after purchase a confirmation via email or SMS should be sent?

- Was there all the time a way to let you know where you are in a hierarchical structure?

\section{(S7)Description: Login}

Task:

Questions:

(S8)Description: Search Results

Task:

Questions:

Task:

Questions: button?

\section{(S9)Description: Breadcrumbs}

Don't register yet. Try to find the login screen. Log in using a username and a password you wish.

- Was it easy to find the login page?

- Was there a solid reason that motivated you to login?

- Did it came up during the procedure of booking a ticket or somewhere else that was not necessary?

- Could you use the TAB key to go from the username field to the password field and press ENTER instead of selecting the "Log in"

- Was there a link to help users who have forgotten their password displayed directly under the password field?

- Was there an option whether you are a new or returning customer?

- If you weren't registered was there a link connecting you with the registration page?

- If you weren't registered and you entered a username/password was there an error message?

- Could you also try to start the registration, at least on the error page, using the username/password that was already submitted?

- Did the system assure you that your activities are safe enough?

Go to the Booking page to request a flight, complete all the necessary fields and submit. After the processing of your request view your selection outcome. You are not satisfied and you want to make some additional combinations. Try to change some of your choices.

- Did your results presented with a numbered list, sorted by relevance and starting with the most relevant?

- If the results were too many were there shown in batches?

- If you didn't find what you wanted could you refine your query?

While you are in the ticket booking function each time you submit your preferences you are doing hierarchal actions in order to finally achieve your goal . Try to traverse through this hierarchy. Jump several steps back instead of following the hierarchy. Try to find marks from what location you started an in what you should be arrive.

- Did the site have a main navigation scheme that allows users to traverse the hierarchy? 
- Was there a path showing the location of the current page in the total information structure?

- Was each level of the hierarchy labeled and functioned as a link to that level?

- Did the current page you've been marked in order to give feedback about where you are now?

- Did the path indicated that a top-down path is traversed by using appropriate separators such as $>$ or $\backslash$ that suggest a downward motion?

- If the path became too long to fit in the designated place, did some of the steps replaced by an elipsis e.g. "..."?

- Did the path placed in a separate "bar" that spanned the entire width of the content area?

- Was it placed close to the content area, preferably above the content area but below the page header?

(S10)Description: Stepping

Task:

Questions:
While booking a ticket and you have already chosen the departure and destination cities among with the dates you wish to travel, try to go back or front without using the Explorer's navigation key (back or front).

- Was there any 'next' link that took you to the next item in the sequence while the 'previous' link took you on step back?

- Was these links placed close to the object they belongs to (e.g. above the object)?

- Did you have to risk scrolling to them?

- Was the next/previous links always presented in the same place? While you were clicking through a list did you have to move the mouse-pointer?

- Was there a 'previous' link at the beginning or a 'next' link at the end of the list? Were the labels 'next' and 'previous' extended with further specifications of what there is to find next, e.g. "next image", "next page" etc?

\section{(S11)Description: Processing Page}

Task:

Try to check the flight-availability about a trip you are interested in. Go to the booking page complete all the necessary fields and submit for starting your search.

Questions:

- Did the system give some feedback telling you that your request is being performed and that you'll have to wait a bit?

- Was there an animation or real progress feedback?

\section{(S12)Description: Table Sorter}

Task:

Try to search for a trip that is very common e.g. Athens-New York. We want to see a large number of options and decide among them.

Questions:
- Did the outcome presented in an effective way?

- Were the possible flights sorted in tables?

- Could these tables been sorted by different ways (price, total duration, departure time, arrival time, number of stops etc.)?

- Is it visible which table you can choose? 
(S13)Description: Color Coded Section

Task:

Open the web page of a Travel site and navigate just by pressing the several "sub sites" or "sub categories" that are presented in the Double Tab Navigation. Try also to search for an air ticket by choosing the option to be flexible about the departing and returning dates.

Questions:

- Could you recognize in what "sub site" or "sub category" you are?

- Are the colors used pleasant and related to the topic of the section?

- Are there some colors been used more than one time?

- If you managed to view a color guide about the several fares was it useful?

- Did colors help you to recognize faster and more effective the information you searched for?

(S14)Description: Date Selector

Task:

Try to put a date of birth in a Registration form or try to select the start and the end date while Booking a trip.

Questions:

- Did you have to type the date in the edit box? If yes was there a hint placed next/under the edit box to indicate the expected format?

- Was there a month-based clickable calendar presented?

- Were the days presented in a table with rows of weeks?

- At the top of the table was there a selector of month that allows you to move to the next/previous month as well as to jump to a specific month using a combo-box? When you clicked on one of them that date entered in the form?

- If some days where not available were the 'possible' days click able and visually distinctive?

(S15)Description: Constraint Input

Task:

Try to choose dates and airports for departure and arrival. Try to put invalid data.

Questions:

- Did the fields for each data element of the structure presented?

- Did each field labeled with the name of the data unit?

- Did the syntax been explained with an example or a description of the format?

(S16)Description: Registration

Task:

When the system prompts you register. If it doesn't please find the registration page.

Questions:

- Were you forced to register?

- Did the system prompt you to register when starting or completing the checkout process?

- Did you require much additional effort during the registration procedure?

- Was the amount of data filled in the forms as minimum as it could be?

- Were there provided the benefits of registering and information about sites' privacy policy?

- Were there provided information about how to un-register?

- Was there an email sent with a confirmation-link that activates your account? 
- If not were you taken back to the place where the registration was started?

(S17)Description: Form

Task:

Questions:

\section{(S18)Description: Input Error Message}

Task:

Questions:

Task:

Questions:
Find a form that you should fill. Use the TAB key to move from one field to the other. Use the ENTER key to submit.

- Did you understand what you've been asked?

- Were there any examples for re-enforcing the meaning of the field?

- Were elements placed in a logical order?

- Was there any distinction between mandatory and optional fields?

- Did the layout and the input elements fulfill your needs?

- Did the input elements (radio buttons, list boxes, edit boxes, check boxes etc.) were used in a proper way?

- When you put invalid data was there any response from the system?

- Could you use the TAB key to go from one field to the other and the ENTER key as a shortcut for "confirm", submit, save etc.?

- When the page was loaded the cursor was already in the first field?

When filling a form do some willful mistakes like forgetting the @ in your email, or instead of your name type 1234 or even don't fill in anything.

- Did an error message appear?

- If so was it displayed in a way that was easily recognizable (colored letters, colored box, pop up window)?

- Did the notice point out what the problem was and what the users can do to repair it (e.g. going to the "problem"-field and changing the input)?

- Did the system when you typed an error input prevented somehow?

\section{(S19)Description: Printer-friendly Page}

When searching for a flight or after booking one you want to keep the provided information in a piece of paper. Booking codes or several combinations of prices and trips are often hardly to remember.

- Was there a link, next to the page content, to a printer-friendly version of the page you were viewing?

- Was there a "print" button on that page that actual sends the page to the printer?

- Did the page to be print used a different layout than the other pages on the site? Was there the entire width of the page used so that the page can be scaled appropriately in the printing process? 


\title{
ПАРАРТНМА $\Gamma$
}

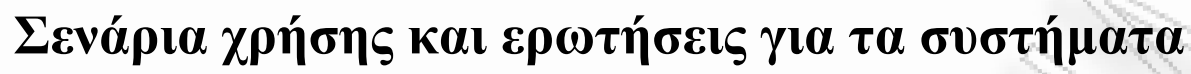 Learning Brockerege Platforms}

\author{
S1)Description: Language Selector \\ Task: $\quad$ Try to change the site language. \\ Questions: $\quad$ - Can you find the list of the different languages provided by the site? \\ - In what way they are presented (by names, by flags)?
}

\section{(S2)Description: Doormat Navigation}

Task:

Understand the main categories and most of the elements of the homepage. Open the desired homepage and spend a moments navigating on it.

Questions: - At first sight were you able in a quick and informative overview to understand the primary choices this site enables you to make?

- Were important aspects hidden?

- Were all options accessible in one click?

(S3)Description: Breadcrumbs

Task:

While you are in the booking or the searching function each time you submit your preferences you are doing hierarchal actions in order to finally achieve your goal. Try to traverse through this hierarchy. Jump several steps back instead of following the hierarchy. Try to find marks from what location you started an in what you should be arrive.

Questions: - Did the site have a main navigation scheme that allows users to traverse the hierarchy?

- Was there a path showing the location of the current page in the total information structure?

- Was each level of the hierarchy labeled and functioned as a link to that level?

- Did the current page you've been marked in order to give feedback about where you are now?

- Did the path indicate that a top-down path is traversed by using appropriate separators such as or $\backslash$ that suggest a downward motion?

- If the path became too long to fit in the designated place, did some of the steps replaced by an elipsis e.g. "..."?

- Did the path placed in a separate "bar" that spanned the entire width of the content area?

- Was it placed close to the content area, preferably above the content area but below the page header?

(S4)Description: Double Tab Navigation

Task:

Learning Brokerage Platforms are not only offering courses but also a large number of conveniences for people willing to learn. Suppose you 
Questions:

are interested for something more than purchasing a simple course. Try to find the page that you can do that. Try to find a page that you can personalize the site according to your wishes.

- Was it obvious that Learning Brokerage Platform site was offering more than tickets?

- Could you see all these groups of information?

- By selecting one of those the others disappeared?

- Were the colors used indicate where you are or the use of them was rather than helpful?

- If your choice had second level option could it be easily visualized?

- Was it fast to jump to another category if wished?

\section{(S5)Description: Main Navigation}

Task:

Questions:
Users need to know where they can find what they are looking for. Try navigating though the system searching for different types of courses provided or specific requirements for each subject of these courses.

- Was the information you were searching for accessible?

- When you were navigating did you have any help that informed you about the structure of the site and telling you where you are and where you can go?

- Where you in a matter of speaking 'educated' about the structure of the site and being helped to be more effective in any of the activities you may wish to perform?

- Was it easy to navigate (go up and down the hierarchy of the site or navigating on the same level)?

\section{(S6)Description: Search area}

Task:

In order to find a specific course or any kind of information don't use the main navigation scheme and try to find a secondary functionality to search for information.

Questions:

- Did you found easily a dedicated area with different kinds of search functionality?

\section{(S7)Description: Search Box}

Task:

Let's try to search for something simple; an item or specific information. Go to the search box and type a word or a sentence for something that is relevant with your interests.

Questions:

- Is the offered search functionality consisting of a search label, a keyword field, a filter if applicable and a "go" button?

- Pressing the return key has the same function as selecting the "go" button?

- Are there provided any Search Tips and examples in a separate page? A link to that page is it placed next to the search functionality?

- Is the edit box for the search term large enough to accommodate 3 typical user queries (typically around 20 characters)?

- Were the search results presented on a new page with clear label? The search function was repeated in the top-part of the page with the entered keywords, so that the user knew what the keywords were?

- Did each search result show a link to the item itself and a snippet of 
text to explain the item? Did the structure of the "result" typically show any or all of the Page Title, the Description, the Categorization, the URL, the Size, the Date created?

\section{(S8)Description: Advanced Search}

Task:

Questions:

(S9)Description: Task:

Questions:
Try to put in your searching something very common so a large collection of items can arouse. Try to find a specific item in this collection by using full control over the search engine so that you can be more successful in achieving your goals. Keep in mind that you have already an additional knowledge of the data that is available and it is hence only for experienced users like you.

- Was there an advanced search button or option that leads you in a new more advanced search tool with more options?

- Was in these options included AND/OR functionality together with exclusion functionality? If so could you use with the same convenience the functionalities "match all terms" and "match any term" instead of AND and OR operators in the text field?

- Could you limit the possible Search Results by defining the conditions that a result must meet (for example, results from a particular subsite, item types (articles, video, audio...), or item properties (title, date, location, size, author...)?

\section{$\underline{\text { Search Results }}$}

Go to the search page to request for a course, complete all the necessary fields and submit. After the processing of your request view your selection outcome. You are not satisfied and you want to make some additional combinations. Try to change some of your choices.

- Did your results presented with a numbered list, sorted by relevance and starting with the most relevant?

- If the results were too many were there shown in batches?

- If you didn't find what you wanted could you refine your query?

\section{(S10)Description: Overview By Detail}

Task:

Try to find a set of courses that are presenting all together. Now you need to inspect objects of this set. Try to show an overview of all objects and display object details of a selected object on a new page.

Questions:

- Were there any image collections browseable?

- Was the overviews contained enough information in order to select objects for a particular purpose?

- Could you pick an item in order to have a detailed view of it? Is this view show the object with all the details that cannot be shown otherwise, because they are not relevant or because it is too much information to show in the available space?

\section{(S11)Description: Shopping Cart}

Task:

You want to collect and purchase several items in one transaction.

Locate the shopping cart/Shopping Basket. Search for a product (either manually or using search mechanism) and if found add it in the basket. Add in the basket a new product that is advertised in the home page. Search for a new product, other than those already included in the 
Questions:

(S12)Description: Task:

Questions: basket. Browse through the store. Delete one item from the shopping cart. Increase the number of items of a product included in the cart from 1 to 2 . Select one of the products that exist in the cart and see its price. While viewing the shopping basket contents, try to locate a link related to shipping and handling costs and their calculations. While viewing the shopping basket contents, try to locate a link related to the return policy

- Is the name of the shopping cart used appropriate?

- Is shopping Basket easily located?

- Are you able to add in the basket a product advertised in the home page? How easy did you find this operation?

- While viewing search results are you able to see the contents of the shopping basket?

- Could the operation of searching for a new product other than those already included in the basket, be executed with zero or one click / move? Are you still able to view the contents of the shopping basket?

- Was it easy to delete items from the shopping basket? Was it easy to modify their quantity? Was the total price automatically recalculated?

- Was it easy to see the price of any product item that included in the cart if you selected it?

- Was it easy to locate the link related to shipping and handling costs and their calculations? Was the information provided satisfactory?

- Was it easy to locate the link related to the return policy? Was the information provided satisfactory?

\section{Purchase Process}

You have finally decided which courses combinations suites you and you want to purchase them. You are guided to the purchase page where your personal data must be given. If you have already registered then give your user name and password. If not fill in the forms and create an account. Then select the way of purchase and before the last click abort the checkout procedure.

- Did the system actually guided you for checking out or did you have to find it yourself?

- Could you abort the procedure at any step?

- When you try to check out again later did you started from the beginning?

- Where the numbers of web pages used a lot or just one or two?

- The page layout was simple or you've been distracted?

- When you created your account was it simple and in small steps?

- If you were registered and after login did your personal data automatically generated or you had to fill them again in the forms?

- In order to purchase did the system oblige you to make an account in advance?

- Did the system informed you that after purchase a confirmation via email or SMS should be sent?

- Was there all the time a way to let you know where you are in a hierarchical structure? 